
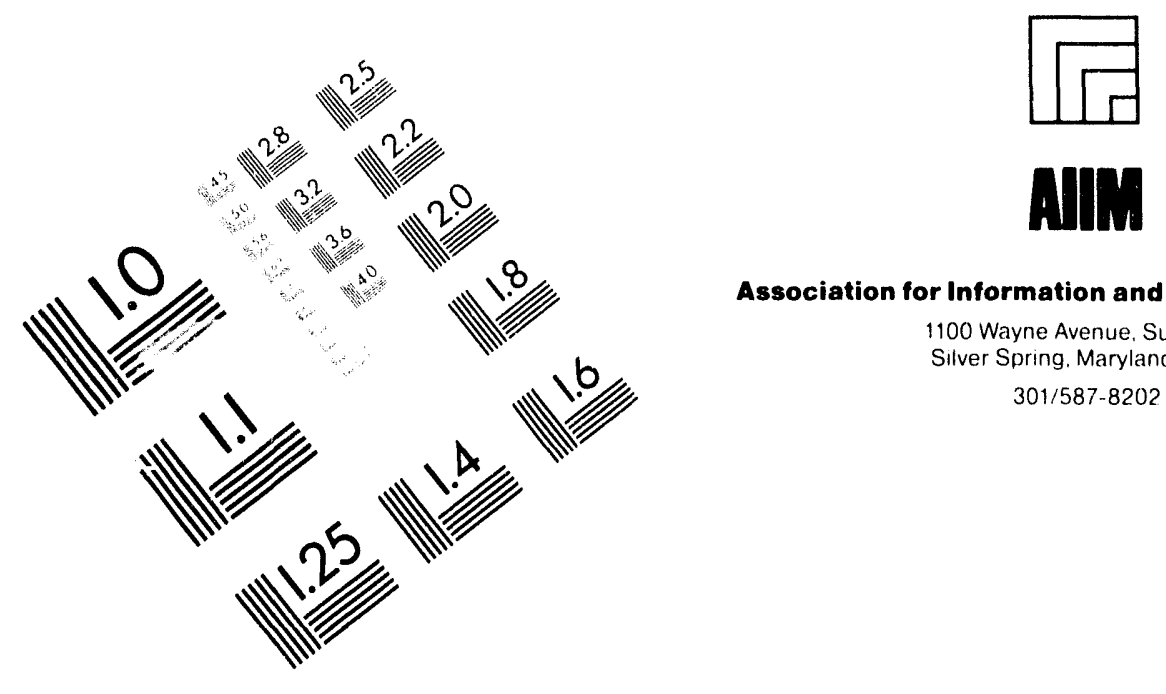

Association for Information and Image Management

1100 Wayne Avenue. Suite 1100

Silver Spring. Maryland 20910

301/587-8202

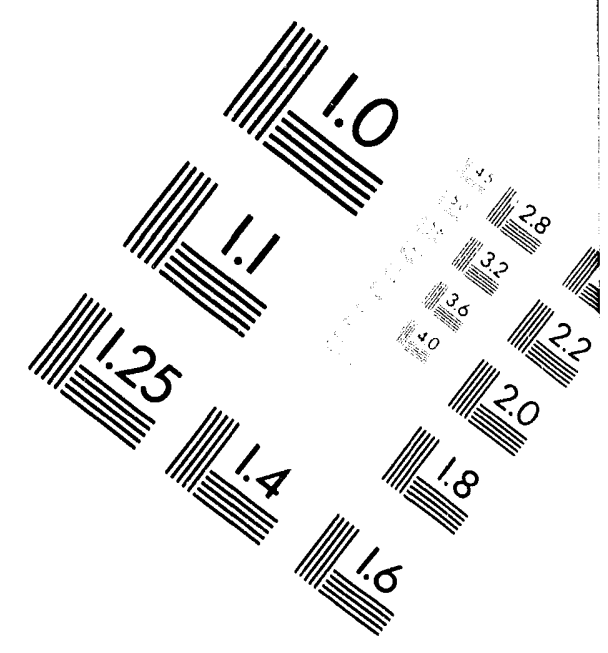

\title{
Centimeter
}

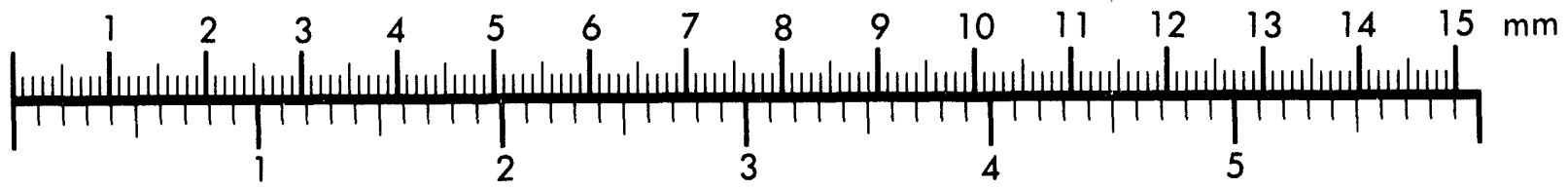
Inches
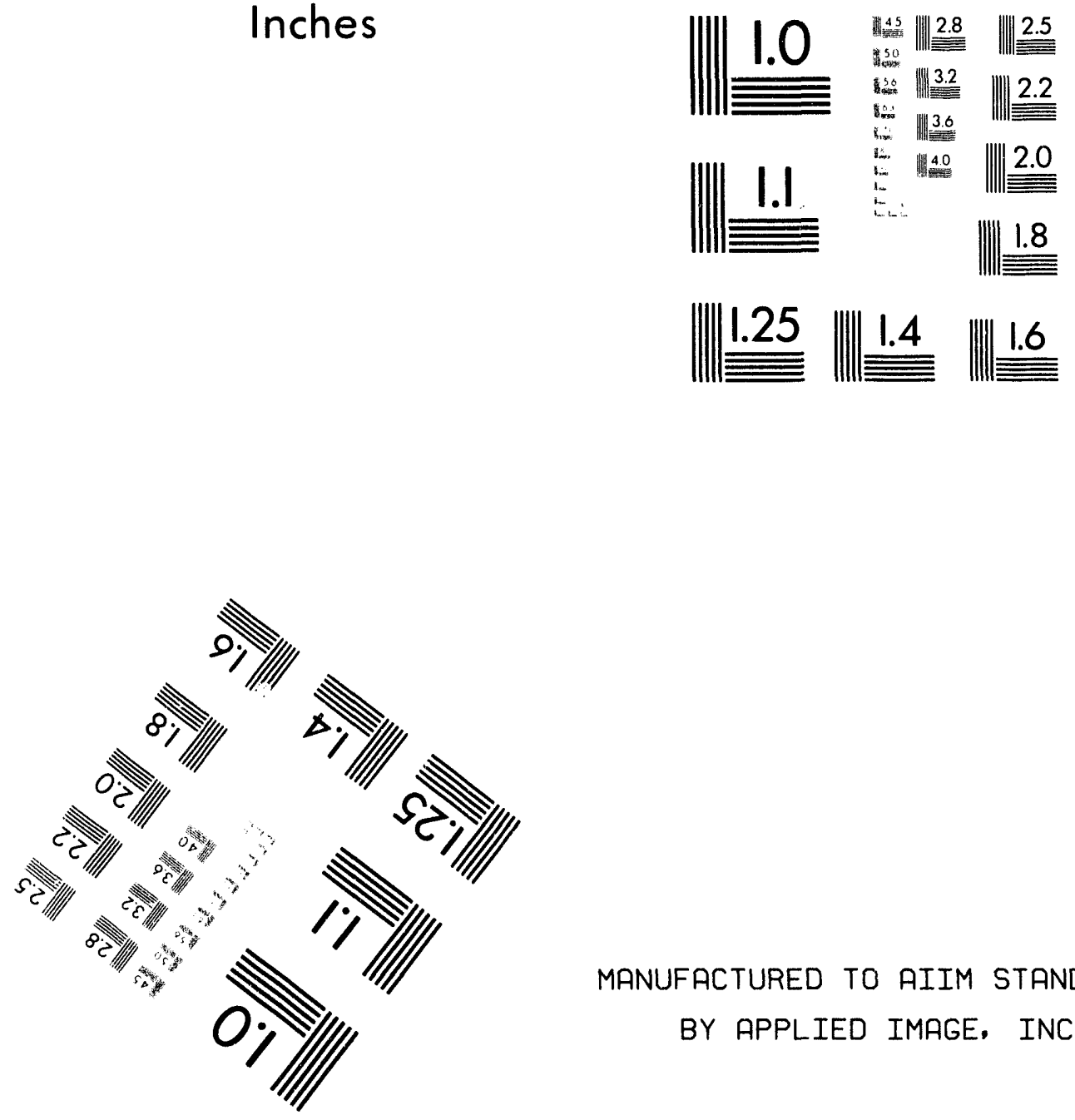

MANUFACTURED TO AIIM STANDARDS BY APPLIED IMAGE. INC.

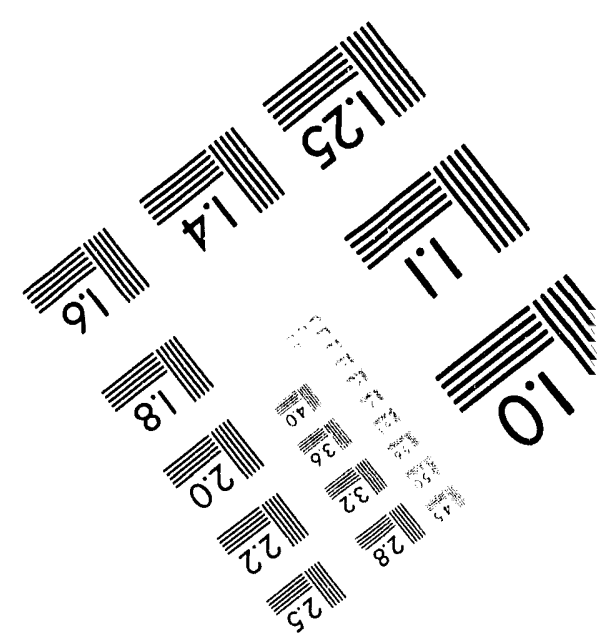



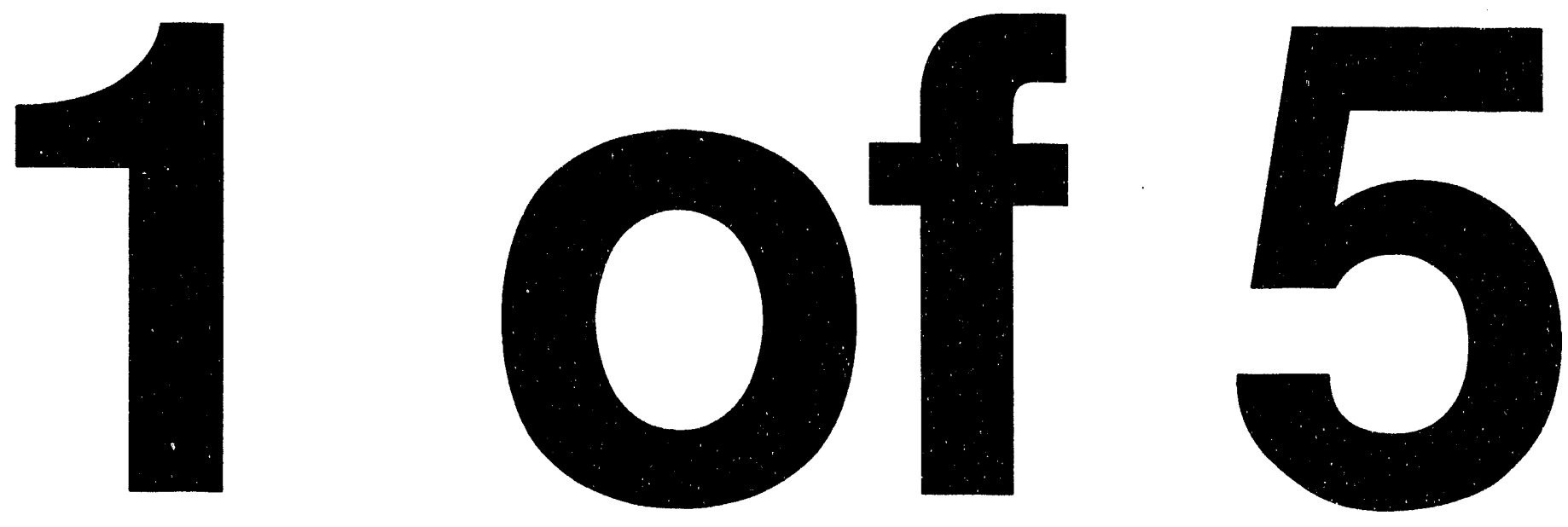

1 1 


\section{Proceedings of the Third Atmospheric Radiation Measurement (ARM) Science Team Meeting}

March 1-4, 1993

Norman, Oklahoma

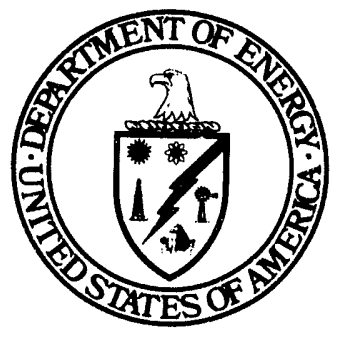

United States Department of Energy Office of Energy Research Office of Health and Environmental Research Environmental Sciences Division Washington, DC 20585

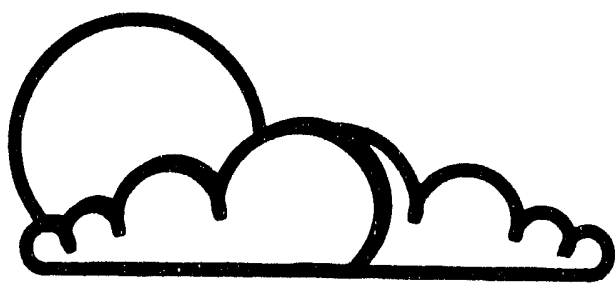




\title{
History and Status of the Atmospheric Radiation Measurement Program - March 1993
}

\author{
P. Lunn U.S. Department of Energy \\ Washington, D.C. \\ T. Cress and G. Stokes \\ Pacific Northwest Laboratory \\ Richland, WA 99352
}

This document contains the summaries of papers presented at the 1993 Atmospheric Radiation Measurement (ARM) Science Team meoting held in Norman, Oklahoma. To put these papers in context, it is useful to consider the history and status of the ARM Program at the time of the meeting.

The history of the project has several themes. First, the Program has from its very beginning attempted to respond to most critical scientific issues facing the United States Global Change Research Program. Second, the Program has been strongly coupled to other agency and international programs. The Program reflects an unprecedented collaboration among the various elemenis of the national research community, including a significant level of cooperation among the U.S. Department of Energy's (DOE) national laboratories. Next, ARM has always attempted to make the most judicious use of its resources by collaborating and leveraging existing assets and has managed to maintain an aggressive schedule despite budgets that have been much smaller than planned. Finally, the Program has attracted some of the very best scientific talent in the climate research community and has, as a result, been very productive scientifically. This introduction covers the first three points - we believe the papers themselves speak to the last point.

\section{Initial Concept}

The initial concept for ARM came out of a series of studies that fell under the auspices of the Intercomparison of Radiation Codes in Climate Models (ICRCCM). ICRCCM pointed to several key issues that are now central to the ARM approach and strategy. First, ICRCCM was based on an assertion that one must understand the quality of the physics inside a climate model if one is to understand the quality of the climate model itself. Next, it showed that it is possible, and in fact necessary, to understand the relatively coarse representations of physics contained in a climate model in terms of a hierarchy of process models. For radiation, this hierarchy ranges from the highly detailed line-by-line codes to the highly parameterized forms of the radiation codes used in climate models. Finally, the hierarchy of models that leads to the parameterizations of processes in climate models must be built on a sound base of experimental verification.

Concurrently with the release of the ICRCCM results, it was becoming clear that the radiative transfer of energy in the atmosphere and the impact of clouds was, and remains, one of the greatest sources of error and uncertainty in the current generation of general circulation models (GCM) used for climate research and prediction. With this as a starting point, DOE proposed a major program targeted at improving the understanding of the role and representation of atmospheric radiative processes and clouds in models of the earth's climate. Initially, the DOE Program focused on the radiative aspects of the climate prcblem. As the scientific issue was studied in more detail, however, it was obvious that a study of radiative processes associated with clouds could not be decoupled from the problem of representing the processes by which clouds form, are maintained, and dissipate in climate models. As a result, the ARM Program was proposed to the then Committee on Earth Sciences of the Federal Coordinating Council on Science Engineering and Technology with two basic objectives:

- to improve the treatment of radiative transfer in climate models under all relevant conditions 
- to improve the treatment of clouds in climate models, including the representation of the cloud life cycle and the prognosis of cloud radiative properties.

\section{The "Approved" Plan}

The ARM Program Plan was subjected to peer review in the fall of 1989. The key element of the proposed ARM effort was to be the Cloud and Radiation Testbed (CART). This user facility was to consist of four to six semipermanent observational facilities desigried to allow detailed investigation of process models used in climate research. These more permanent facilities were to be supplemented with a mobile facility that would allow related measurements to be made at other locations on a campaign-oriented basis. The facility would include a data management and communications system capable of acquiring and qualitycontrolling site data; acquiring data from sources outside the program, particularly satellites; and communicating that data to a Science Team. This Science Team would be selected through a peer review process open to all investigators nationally and internationally.

Based on the peer review, the climate subcommittee of the Committee on Earth Sciences approved the Plan, noting severalkey things about how it should be carried out. First, the scope was broadened beyond radiative transfer to include clouds and cloud processes, a change deemed necessary to support the level of effort proposed by DOE. Next, the Committee recommended that the DOE implementation of this program involve the talents of other federal agencies to the extent possible and that an interagency steering group be formed to assist in that process. Finally, the relevance of ARM to several other climate programs was noted, and DOE agreed to tie its deployment of facilities to the schedules of other national and international programs, most notably the Tropical Ocean Global Atmosphere-Coupled Ocean Atmosphere Response Experiment (TOGA-COARE).

\section{The Early Implementation}

The implementation of ARM began in January 1990, proceeding on two coupled but parallel tracks. First, a multi-laboratory team was formed to plan the detailed implementation of the ARM facilities. The second track involved the formation of the Science Team. However, the science drivers were so important to the design of the facility, concurrent with the initiation of a solicitation process intended to assemble a Science Team, a series of scientific workshops were held in the spring and summer of 1990 to clarify the scientific foundations of the program.

As these two tracks moved forward, several features of the Program emerged, the most significant of which was the pattern of collaboration with other programs. This collaboration was characterized on one hand by a series of collaborations with field campaigns and, on the other, by involvement in program planning. In the field collaborations, ARM attempted to bring a value-added contribution to another agency's or group's planned effort, while at the same time trying to gain operational experience necessary to guide its own field deployment.

This strategy resulted in collaborations with the Federal Aviation Administration's Winter Icing and Storms Program (WISP) and First ISCCP Regional Experiment (FIRE) activities in Coffeyville, Kansas, and the Azores. In Coffeyville, early ARM concepts were tested in the jointly funded NASA-DOE Spectral Radiance Experiment (SPECTRE). It also led to ARM-fostered projects such as the Boardman-ARMRegional Flux Experiment which tested key aspects of surface and surface flux characterization.

From the standpoint of planning, ARM attempted to gain early involvement in the program planning of other programs that would be evolving in parallel with it. Most notable among these planning collaborations was the Global Energy and Water Experiment (GEWEX). One of these joint planning activities culminated in the field deployment of the Pilot Radiation Observation Experiment (PROBE) to Kavieng, Papua New Guinea, as part of TOGA-COARE in the winter of 1992-3.

A key convergence between science and facility planning tracks was the selection of a siting strategy for the ARM facilities. This process resulted in the identification of five areas of the world, locales in which ARM should locate its semi-permanent facilities, and a comparable number of secondary locales in which the program should consider shorter, campaign-like activities. The primary locales in the order of their intended occupation were the Southern Great Plains of the United States, the Tropical Western Pacific, the North Slope of Alaska, marine stratus zones of either ihe Atlantic or Pacific Ocean, and the Gulf Stream. 


\section{Budget Realities}

While ARM was planned as a decade-long program with a cumulative funding level of almost $\$ 500 \mathrm{M}$, it has always been clear that the annual rate of expenditure would not reach projected levels and that the Program's schedule would be drawn out. This reality has been approached in several ways and needs to be understood in terms of several competing concerns: the cost of acquiring equipment, the tradeoff between capital and operating budgets, and the relative costs of design and deployment versus operating costs.

Earty in the Program, capital equipment resources were inadequate to acquire the instrumentation necessary for the first site and the construction of the associated data system. As a result, the deployment to the first site has been heavily phased, supporting one aspect of the program, the radiative transfer segment, over the cloud life cycle segment. Similarly, the Program has sought opportunities to take advantage of existing equipment and data. This approach led in no small way to the decision to deploy the first site in the North Central Oklahoma/South Central Kansas area, to take advantage of the existing National Oceanic and Atmospheric Administration (NOAA) profiler and radar facilities as well as the Oklahoma Mesonet.

The operational budgets also lagged early on, which led to a series of joint development activities. For example, rather than building a new data system for field data acquisition, the Program has instead developed a collaboration with the National Center for Atmospheric Research (NCAR), which has allowed us to build the data system around $Z e b$, their campeign data management system.

Finally, the project has been rescoped annually. This rescoping has resulted in several substantive changes including the cancellation of the mobile facility and sharply diminished support for campaign activities. It has also led to a continuing delay in the implementation of a data archive generally available to the wider scientific community.

Despite the budgetary concerns and what seemed to be an endless environmental assessment process, deployment began near Lamont, Oklahoma, in the Southern Great Plains in May 1992, only one month later than planned. The initial deployment was meager, a single borrowed portable meteorological station. By the fall of 1992 , however, most of the infrastructure for the instrumentation was in place, and the major equipment was beginning to emerge out of the procurement system.

\section{Project Status at the Time of the Norman Meeting}

Despite the delays in the deployment of instrumentation, by the time of the Norman meeting many members of the Science Team had made major progress in addressing key ARM issues. Most of this work has been based on either preliminary modeling investigations or the many pilot field programs and campaigns. A major ARM session was organized at the January 1993 American Meteorological Society Meeting. At the time of the ARM meeting in Norman, there were several collaborations that had not existed before the formation of the ARM Program. Most notably, the degree to which DOE staff were iniegrated with university and other investigators was higher than in any other DOE atmospheric science program. The PROBE experiment as part of TOGA-COARE was probably the most striking of these collaborations and offered a vision of what we expected to develop over the next several years of the program.

These collaborations and, in fact, much of the collective work of the ARM Science Team had developed around a series of working groups that had been formed around the concept of a general measurement strategy. These strategies include nearly all of the modeling problems addressed by ARM, and they have proven very useful as a starting point in specifying the sets of observations needed to meet the requirements of Science Team experiments. These General Measurement Strategies are

- single column model - Many of the key process models and parameterizations that compose GCM-based climate models appear to be testable by extracting a single vertical array of cells from the model and operating the array in what is referred to as a single-column model. This single-column core retains the subgrid scale physics that must be represented in GCMs, and it offers a promising approach to testing the parameterization of this physics. The CART facility around Lamont, Oklahoma, was designed to support this strategy. 
- hierarchical diagnosis - GCMs may be viewed as part of a hierarchy of models and modeling systems whose representation of atmospheric physics ranges from the highly aggregated to the highly specific. GCMs and single-column models are necessarily characterized by highly aggregated physics and by coarse spatial and temporal resolution. To understand the limitations imposed by such aggregation and, ultimately, to improve the models, observations must be analyzed and interpreted using higher resolution models. A critical part of the ARM implementation is deciding how to select and obtain the observations on a finer space and time scale. Equally important is deciding which variables not normally carried in a GCM must be observed in order to credibly construct a sound parameterization of a meteorological process in a GCM.

- data assimilation - Meteorological observations are, by necessity, made at discrete locations and times, and they sample only limited portions of the atmosphere. However, the data needed to test models on a variety of scales and to run a single-column model must be continuous in space and time. Consequently, much of the data supplied to ARM experimenters will be in the form of objectively analyzed fields produced by standard four-dimensional data assimilation techniques.
- instantaneous radiative flux - Accurate treatment of radiation is essential in climate models, and testing of radiation transfer models is central to the objectives of ARM. In principle, testing of radiation transfer models calls for complete specification of the state of the atmosphere and the surface. The ICRCCM (Ellingson and Fouquart 1991) has emphasized that the state-ofthe-art in radiative modeling cannot be advanced by further model intercomparisons. Progress can be achieved only by supplementing intercomparisons with field observations. CART provides a facility for such observations.

These strategies are also the basis of the organization of much of the report. The remainder of the papers cover the development of the CART facilities and the ARM Instrument Development Program (IDP). The first products of the IDP program were just going to the field at the time of the meeting and some early results were covered in the papers presented - including data that were just a fow days old.

\section{Reference}

Ellingson, R., and Y. Fouquart. 1991. The intercomparison of radiation codes in climate models: An ovenviow. $J$. Geophys. Res. 96:8925-8927. 


\section{Acronyms and Abbreviations}

$\begin{array}{ll}\text { 1-D RCM } & \text { one-dimensional radiative-convective model } \\ \text { 2-D RCAM } & \text { two-dimensional radiative-advective-convective equilibrium model } \\ \text { 4DDA } & \text { four-dimensional data assimilation } \\ \text { ACARS } & \text { Aircraft Communication and Recording System } \\ \text { AGCM } & \text { atmosphere general circulation model } \\ \text { AER } & \text { Atmospheric and Environmental Research, Inc. } \\ \text { AERI } & \text { Atmospheric Emitted Radiance Interferometer } \\ \text { AERI-FEP } & \text { AERI Front End Processor } \\ \text { AFGL } & \text { Air Force Geophysics Laboratory } \\ \text { AGL } & \text { above ground level } \\ \text { ALFA } & \text { AER Local Forecast and Assimilation (model) } \\ \text { AMIP } & \text { Atmospheric Model Intercomparison Project } \\ \text { ARC } & \text { Ames Research Center } \\ \text { ARCS } & \text { Atmospheric Radiation and Cloud Stations } \\ \text { ARM } & \text { Atmospheric Radiation Measurement } \\ \text { A-S } & \text { Arakawa-Schubert } \\ \text { ASCOT } & \text { Atmospheric Studies in Complex Terrain Program } \\ \text { ASTER } & \text { Atmosphere-Surface Turbulent Exchange Research } \\ \text { ASTEX } & \text { Atlantic Stratocumulus Transition Experiment } \\ \text { ATDD } & \text { Atmospheric Turbulence and Diffusion Division } \\ \text { AVHRR } & \text { Advanced Very High Resolution Radiometer } \\ & \\ \text { BAO } & \text { Boulder Atmospheric Observatory } \\ \text { BBHIR } & \text { broadband hemispherical IR flux radiometer } \\ \text { BBHSR } & \text { broadband hemispherical solar radiometer } \\ \text { BBSS } & \text { balloon-bome sounding system } \\ \text { BMRC } & \text { Bureau of Meteorological Research Center (Australia) } \\ \text { BW } & \text { bulk water } \\ & \\ \text { CAO } & \text { cold air outbreak } \\ \text { CART } & \text { Cloud and Radiation Testbed } \\ \text { CCM0 } & \text { Community Climate Model at the National Center for Atmospheric Research } \\ \text { CCM2 } & \text { version } 2 \text { of the NCAR Community Climate Model } \\ \text { CCN } & \text { cloud condensation nucleus } \\ \text { CEM } & \text { cumulus ensemble model } \\ \text { CEPEX } & \text { Central Equatorial Pacific Experiment } \\ \text { CGCM } & \text { coupled atmosphere/ocean general circulation model } \\ \text { CKE } & \text { cumulus kinetic energy } \\ \text { CLARET } & \text { Cloud Lidar and Radar Exploratory Test } \\ \text { CLASS } & \text { Cross-chain Loran Atmospheric Sounding System } \\ & \end{array}$




\begin{tabular}{|c|c|}
\hline \multicolumn{2}{|c|}{ ARM Science Team Moeting } \\
\hline $\begin{array}{l}\text { COARE } \\
\text { CPRS } \\
\text { CRS } \\
\text { CSU } \\
\text { CW }\end{array}$ & $\begin{array}{l}\text { Coupled Ocean Atmosphere Response Experiment } \\
\text { Cloud Profiling Radar System } \\
\text { cavity-ringdown spectrometer } \\
\text { Colorado State University } \\
\text { continuous wave }\end{array}$ \\
\hline $\begin{array}{l}\text { DAR } \\
\text { DBS } \\
\text { DISORT } \\
\text { DMSP } \\
\text { DSP } \\
\text { DUNDEE }\end{array}$ & $\begin{array}{l}\text { Division of Atmospheric Research (Australia) } \\
\text { Doppler beam swinging } \\
\text { Discrete Ordinate (model) } \\
\text { Defense Meteorological Support Program } \\
\text { digital signal processing } \\
\text { Down Under Doppler and Electricity Experiment }\end{array}$ \\
\hline $\begin{array}{l}\text { EBBR } \\
\text { EC } \\
\text { ECLIPS } \\
\text { ECMWF } \\
\text { EMEX } \\
\text { EML } \\
\text { ENSO } \\
\text { EOF } \\
\text { EOS } \\
\text { ERBE } \\
\text { ET }\end{array}$ & $\begin{array}{l}\text { energy balance Bowen ratio } \\
\text { eddy correlation } \\
\text { Experimental Cloud Lidar Pilor Study } \\
\text { European Centre for Medium-Range Weather Forecasts } \\
\text { Equatorial Mesoscale Experiment } \\
\text { Environmental Measurements Laboratory } \\
\text { El Nifo-Southern Oscillation } \\
\text { empirical orthogonal function } \\
\text { Earth Observing System } \\
\text { Earth Radiation Budget Experiment } \\
\text { evapotranspiration }\end{array}$ \\
\hline $\begin{array}{l}\text { FARS } \\
\text { FDI } \\
\text { FFT } \\
\text { FGGE } \\
\text { FIRE } \\
\text { FOV } \\
\text { FTIR }\end{array}$ & $\begin{array}{l}\text { Facility for Atmospheric Remove Sensing } \\
\text { field data investor } \\
\text { Fast Fourier Transform } \\
\text { First GARP Global Experiment } \\
\text { First ISCCP Regional Experiment } \\
\text { field of view } \\
\text { Fourier transform infrared radiometer }\end{array}$ \\
\hline $\begin{array}{l}\text { GALE } \\
\text { GARP } \\
\text { GATE } \\
\text { GCM } \\
\text { GENESIS } \\
\text { GEWEX } \\
\text { GISS } \\
\text { GMT } \\
\text { GOALS } \\
\text { GOES } \\
\text { GSL }\end{array}$ & $\begin{array}{l}\text { Genesis of Atlantic Lows Experiment } \\
\text { Global Atmospheric Research Program } \\
\text { GARP Atlantic Tropical Experiment } \\
\text { general circulation model } \\
\text { Global ENvironmental and Ecological Simulation of Interactive Systems } \\
\text { Global Energy and Water Experiment } \\
\text { Goddard Institute for Space Studies } \\
\text { Greenwich Mean Time } \\
\text { Global Ocean Atmosphere Land System } \\
\text { Geostationary Operational Environmental Satellite } \\
\text { Gulf Stream Locale }\end{array}$ \\
\hline
\end{tabular}




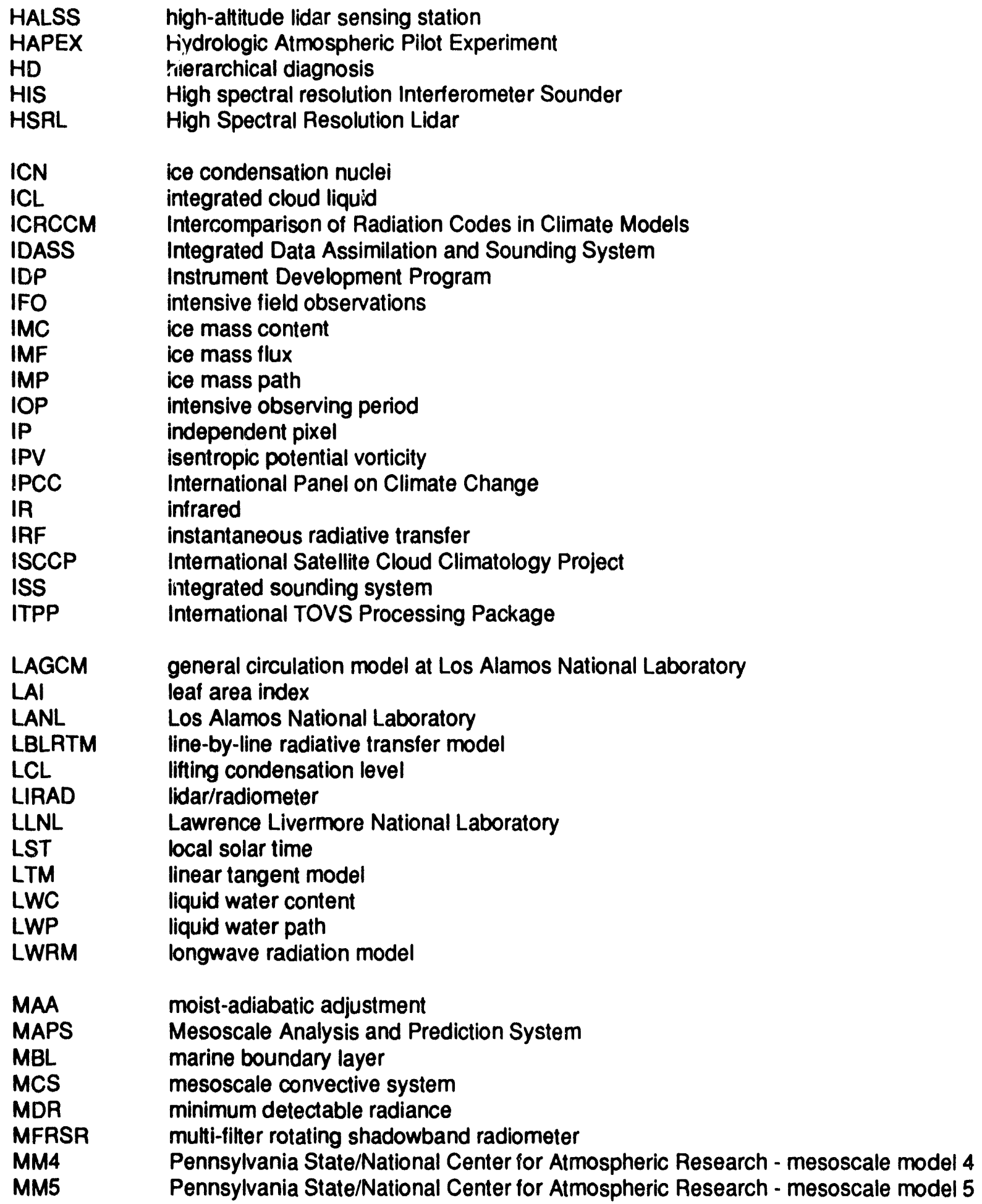




\begin{tabular}{|c|c|}
\hline $\begin{array}{l}\text { MIRSL } \\
\text { MPL }\end{array}$ & $\begin{array}{l}\text { Microwave Remote Sensing Laboratory } \\
\text { Marine Physical Laboratory } \\
\text { Micro-Pulse Lidar }\end{array}$ \\
\hline & $\begin{array}{l}\text { Motion-Sensing Platiorm } \\
\text { microwave radiometer }\end{array}$ \\
\hline MWSR & microwave water substance ra \\
\hline
\end{tabular}

NASA National Aeronautics and Space Administration

NCAR National Center for Atmospheric Research

NEPA National Environmental Policy Act

NGM Nested Grid Model

$\mathrm{NH}$

NMC

NOAA

NWP

northern hemisphere

NWS

National Meteorological Center

National Oceanic and Atmospheric Adminstration

numerical weather prediction

National Weather Service

OGCM

ocean general circulation model

OMWOG

Ocean-based Measurements Working Group

ORNL

Oak Ridge National Laboratory

OSSE

observation system simulation experiment

$\begin{array}{ll}\text { PAM } & \text { Portable Automated Mesonet } \\ \text { PBL } & \text { planetary boundary layer } \\ \text { Pdf } & \text { probability density function } \\ \text { PDL } & \text { polarization diversity lidar } \\ \text { PRF } & \text { pulse repetition frequency } \\ \text { PRT } & \text { precision radiation thermometer } \\ \text { PROBE } & \text { Pilot Radiation Observation Experiment } \\ \text { PSU } & \text { Pennsylvania State University } \\ \text { PV } & \text { potential vorticity } \\ \text { PW } & \text { precipitable water } \\ \text { PWV } & \text { precipitable water vapor } \\ & \\ \text { RASS } & \text { radio-acoustic sounding system } \\ \text { RAMS } & \text { Regional Atmcspheric Modeling System } \\ \text { RH } & \text { relative humidity } \\ \text { RI } & \text { radar interferometry } \\ \text { r.m.S. } & \text { root mean square } \\ \text { RRTM } & \text { rapid radiative transfer model } \\ \text { RSR } & \text { rotating shadowband radiometer } \\ \text { RSS } & \text { rotating shadowband spectroradiometer } \\ \text { RWVL } & \text { Raman Water Vapor Lidar }\end{array}$




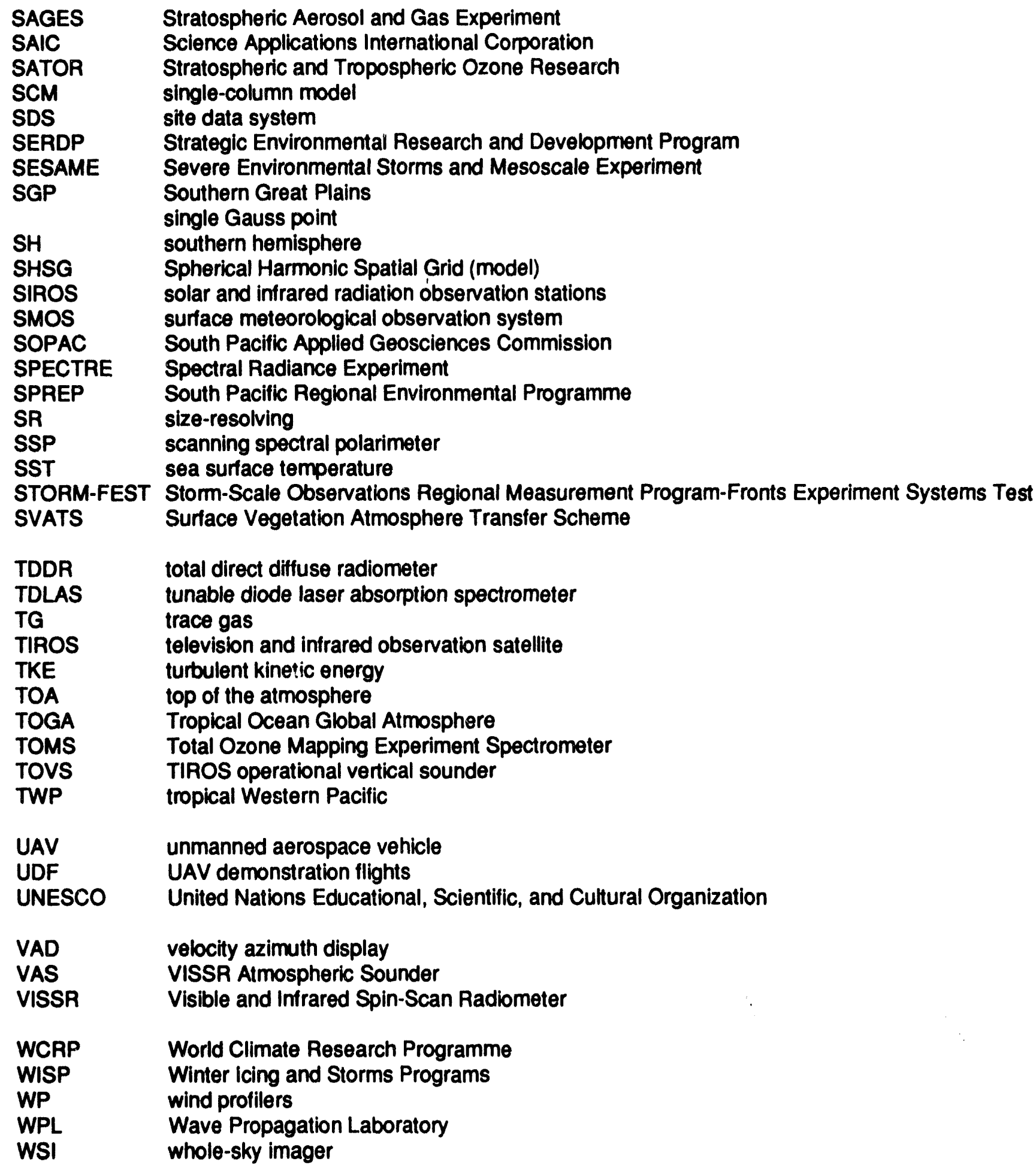




\section{Contents}

History and Status of the ARM Program - March 1993

Acronyms and Abbroviations

\section{Clear Skles}

A Study of Longwave Radiation Codes for Climate Studies: Validation with

Observations and Tests in General Circulation Models-an Update

R. G. Ellingson, F. Baer

Radiative Transfer Model Development in Support of the Atmospheric Radiation

Measurement Program

S. A. Clough

General Circulation Model Feedback Sensitivity Assessment

A. A. Lacis

Long-Pathlength Infrared Absorption Measurements of Line and Continuum Features

in the 8-to 14- $\mu \mathrm{m}$ Atmospheric Window

T. J. Kulp, J. Shinn

\section{Cloudy Skies}

Radiative Properties of Nonuniform Clouds

P. H. Daum, L. I. Kleinman, G. L. Stephens

A Stochastic Formulation of Radiative Transfer in Clouds

G. L. Stephens, P. M. Gabriel, K. F. Evans, D. Duda.

Two Complementary Multifractal Analysis Techniques for Non-Stationary Atmospheric Processes

with an Application to Cloud Liquid Water Content

W. Wiscombe, A. Davis, A. Marshak.

Treatment of Cloud Radiative Effects in General Circulation Models

W.-C. Wang, X.-Z. Liang, M. D. Dudek, M. Ding, L. Zhu, J. Everette, S. Cox

Cloud Effects on Radiation at the Top of the Atmosphere and at the

Surface: Observations and Modeling Studies

C. Gautier, D. Lavallée, W. O'Hirok, T. Figel, M. Landfield, P. Ricchiazzi,

S. Yang, D. Lubin, S. Lovejoy, C. Lamder, G. Lewis, S. Pecknold,

P. Silas, D. Schertzer, R. Borde, Y. Tessier, F. Schmitt.

Analysis of Cloud Radiative Forcing and Feodback in a Climate General Circulation Model

A. A. Lacis 
Stochastic Radiation Transport for Climate Models

R. N. Byme, D. N. Arion, F. Malvagi, Y. Serra,

R.C.J. Somerville, G. C. Pomraning, BU Su

A Model of the Microphysical Evolution of a Cloud

J. Zinn

Data Assimilation

The Sensitivity of Flux Parameterizations to Surface Characteristics

J. C. Doran

Areally Averaged Estimates of Surface Heat Flux from Field Studies

for the Atmospheric Radiation Measurement Program

R. L. Coulter, T. J. Martin, D. R. Cook

Surface Fluxes Important to Cloud Development

F. J. Barnes, W. Porch, K. E. Kunkel

An Ultra-High Frequency Boundary Layer Doppler/Interferometric Profiler

J. S. Van Baelon

System Design and Implementation of the Integrated Sounding System

C. L. Martin, A.-L. Barrett

Single-Column Modeling

Single-Column Model and Cumulus Ensemble Model Simulations of

GARP Atlantic Tropical Experiment Data

D. A. Randall, K. $-M . X u$

Diagnostic Modeling of the Experimental Site of the Atmospheric

Radiation Measurement Program

R.C.J. Somerville, S. F. lacobellis

A Strategy for Testing the Impact of Clouds on the Shortwave Radiation

Budget of General Circulation Models: A Prototype for the Atmospheric

Radiation Measurement Program

R. D. Cess

A Stratiform Cloud Parameterization for General Circulation Models

S. J. Ghan, L. R. Leung, C. C. Chuang, J. E. Penner, J. McCaa

Factors Influencing Regional-Scale Cloud Cover: Investigations Using

Satellite-Derived Cloud Cover and Standard Meteorological Observations

C. J. Walcek

Single-Column Sub-Grid Cumulus Model for the Atmospheric

Radiation Measurement Program

R. B. Stull 


\section{Four-Dimensional Data Assimilation}

Single-Column Data Assimilation for the

Atmospheric Radiation Measurement (ARM) Program

Interactions Between Aerosols and Clouds

O. B. Toon, A. Ackerman, E. Jensen

Are Ground-Based Measurements Sufficient to Quantify Atmospheric Radiative Properties?

S. Kinne, R. Bergstrom, T. Ackerman

Advances in the Development of an Integrated Data Assimilation and Sounding System

W. F. Dabbendt, D. Parsons, Y.-H. Kuo, J. Dudhia, Y.-R. Guo, J. Van Baelen, C. Martin, S. Oncley

Modeling of Clouds and Radiation for Development of Parameterizations

for General Circulation Models

D. Westphal, B. Toon, E. Jensen, S. Kinne, A. Ackerman, R. Bergstrom, A. Walker

The Role of Atmospheric Radiation in the Generation and Maintenance of

Circulations of Different Scales

M. J. Leach, S. Raman

Vegetation Forcing and Thermally Induced Generation of Mesoscale Circulation

X. Hong, M. J. Leach, S. Raman

Boundary-Layer Observations Over the Southern Great Plains Cloud and Radiation

Testbed Site During the November Flux-Profiler Test

S. P. Oncley, J. Van Baelen

Comparison of Mesoscale Model and Tower Measu:ements of

Surface Fluxes During Winter Icing and Storms Program/Atmospheric

Radiation Measurement 91

S. P. Oncley, J. Dudhia

\section{Hierarchical Diagnosis}

A Hierarchical Approach to Improved Cloud Radiation Parameterization for

Climate Models Through the Atmospheric Radiation Measurement Program

J. T. Kiehl, M. W. Moncrieff, J. J. Hack, W. Grabowski, V. Ramaswamy

Development of a Parameterization Scheme of Mesoscale Convective Systems

W. R. Cotton

Development of a Radiative Cloud Parameterization Scheme of Stratocumulus and Stratus Clouds Which Includes the Impact of Cloud Condensation Nucleus on Cloud Albedo

W. R. Cotton, G. L. Sievens, D. Duda, B. Stevens, R. L. Walko, G. Feingold

Regional Climatology Sensitivity Studies

J. E. Bossert, C.-Y.J. Kao, J. L. Winterkamp, J. O. Rhoads, S.-C. Chen 
ARM Science Team Meoting

Simulation of Cloud/Radiation Interaction Using a Second-Order

Turbulence Radiative-Convective Model

C.-Y.J. Kao, W. S. Smith

Prognostic Cloud Water in the Los Alamos General Circulation Model

J. E. Kristjánsson, C.-Y.J. Kao

Factors Influencing the Parameterization of Anvil Clouds Within General Circulation Models

J. M. Leone, Jr., and H.-N. (Steve) Chin

Modeling Studies of the Indo-Pacific Warm Pool

T. P. Barnett, N. Schneider, M. Tyree, J. Ritchie, V. Ramanathan,

S. Shenwood, G. Zhang, M. Flatau.

Cloud Studies in Climate Research Programs with 8-Millimeter Wavelength Doppler Radar

R. A. Kropfli, S. Y. Matrosov, T. Uttal, B. W. Orr .

Parameterization of Cirrus Microphysical and Radiative Properties in Larger-Scale Models

A. J. Heymsfield, J. L. Coen .

Thre日-Dimensional Cloud Characterization from Paired Whole-Sky Imaging Cameras

M. Allmen, W. P. Kegelmeyer, Jr.

Complex Researches of Tropospheric and Stratospheric Ozone Performed

at the Institute of Atmospheric Optics as a Basis of a Complete Radiation Experiment

V. V. Zuev

Theoretical Studies of Radiative Properties of Broken Clouds

G. A. Titov

Radiation Experiment in Support of the Atmospheric Radiation Measurement Program

V. E. Zuev, V. V. Zuev, G. A. Titov.

\section{Radiometry}

Technical Progress in the Atmospheric Radiation Measurement

Instrument Development Program

J.W. Griffin

High Spectral Resolution Fourier Transform Infrared Instruments

for the Atmospheric Radiation Measurement Frogram

H. E. Revercomb, W. L. Smith, R. O. Knuteson, F. A. Best,

R. G. Dedecker, T. P. Dirkx, R. A. Herbsleb, J. F. Short,

H. B. Howell, D. Murcray, F. Murcray.

Development of Rotating Shadowband Spectral Radiometers and General Circulation Model Radiation Code Test Data Sets in Support of the Atmospheric Radiation Measurement Program J. Michalsky, L. Harrison, M. Beik, W. Berkheiser III, J. Schlemmer 
Development of a Balloon-Borne Stabilized Platform for Measuring Radiative

Flux Profiles in the Atmospheric Boundary Layer

C. D. Whiteman, J. M. Alzheimer, G. A. Anderson, W. J. Shaw.

Boundary Layer Heat Budgets from Wind Profiler/Radio Acoustic

Sounding Systems Data: A Feasibility Study

M. Furger, C. D. Whiteman

Accuracy of Net Radiation Profiles from a Self-Leveling Balloon-Borne Platform

W. J. Shaw, C. D. Whiteman

Narrow-Beam Fast Filter Radiometry and the Use of the Lidar/Radiometer

Method in the Atmospheric Radiation Measurement Program

C.M.R. Platt, J. W. Bennett, S. A. Young, M. D. Fenwick,

P. J. Manson, G. R. Patterson, B. Petraitis

Aerosol Characterization Using Stellar Imagery

P. G. Weber

\section{Remote Sensing}

Laser Remote Sensing of Water Vapor: Raman Lidar Development

J.E.M. Goldsmith, M. Lapp, S. E. Bisson, S. H. Melfi,

D. N. Whiteman, R. A. Ferrare, K. D. Evans

Cloud and Aerosol Characterization for the Atmospheric Radiation

Measurement Central Facility: Multiple Remote Sensor Techniques Development

K. Sassen

Development of a Dual-Frequency, Millimeter-Wave Cloud Profiling Radar System

R. E. Mclntosh.

\section{Instrumentation for Southern Great Plains}

Implementation of Instruments and Facilities at the Southern Great Plains

Cloud and Radiation Testbed Site

D. L. Sisterson, M. L. Wesely

\section{Tropical Western Pacific}

The Tropical Western Pacific: Status Report

T. Ackerman, F. J. Barnes, W. Clements, D. Renné,

E. R. Westwater, T. Barnett

\section{Gulf Stream Locale}

VAS-Derived Cloud Observations over the Gulf Stream

Locale During the Winter Months

R. J. Alliss, S. Raman

Scientific Issues in the Gulf Stream Locale

P. Michael, M. L. Daum, S. Raman, M. Leach 339 


\section{Summary of Breakout Sessions}

Summary of Combined Hierarchical Diagnosis and Single-Column Model Breakout Session

D. A. Randall, J. T. Kiahl, M. Bradlay

\section{ARM/Unmanned Alr Vehicle/Satellites}

Unmanned Aerospace Vehicle System Concept

W. R. Bolton

The Atmospheric Radiation Measurement Unmanned Aerospace Vehicle Program: An Overview

P. A. Crowley, J. Vitko, Ji.

Strawman Payload for the Unmanned Aerospace Vehicle Demonstration Flights

J. Vitko, Jr.

\section{Poster Sessions}

Surface Aerosol Measurements at Lamont, Oklahoma

R. Leifer, R. Knuth, H.-N. Le日

Retrieval of Three-Dimensional Cloud Structure for Estimating Cloud-Radiative Forcing

L. Shi, J. del Corral, V. Ramanathan, E. Boer .

Backward Air Mass Trajectory Analysis for the First Cloud and Radiation Testbed Site at Lamont, Oklahoma

H.-N. Lee, R. Leifer

The Effect of Network Resolution on Data Assimilation in a Mesoscale Model

J. Dudhia 359

Radiation Studies with a High-Resolution Mesoscale Model

J. Dudhia

Meso-Beta-Scale Data Assimilation of the Winter Icing and Storms

Program/Atmospheric Radiation Measurement Program 91 Intensive

Observing Period Case on 6 March 1991

Y.-H. Kuo, Y.-R. Guo.

Combined Ground- and Satellite-Based Profiling of Temperature and Water Vapor

B. B. Stankov, E. R. Westwater, J. B. Snider, and J. H. Churnside

Microwave and Infrared Radiometric Observations of Water Vapor and Clouds

During the Pilot Observation Experiment

E. R. Westwater, J. H. Churnside, J. B. Snider 
Clear Skies 


\title{
A Study of Longwave Radiation Codes for Climate Studies: Validation with Observations and Tests in General Circulation Models-an Update
}

\author{
R. G. Ellingson and F. Baer \\ Department of Meteorology \\ University of Maryland \\ College Park, MD 20742
}

\section{Introduction}

Research by the U.S. Department of Energy (DOE) has shown that cloud radiative feedback is the single most important effect determining the magnitude of possible climatic responses to human activity. However, these effects are still not known at the levels needed for climate prediction. Consequently, DOE has launched a major initiative-the Atmospheric Radiation Measurement (ARM) Program-directed at improving the parameterization of the physics governing cloud and radiative processes in general circulation models (GCMs).

One specific goal of ARM is to improve the treatment of radiative transfer in GCMs under clear-sky, general overcast, and broken cloud conditions. In 1990, we proposed to contribute to this goal by attacking major problems connected with one of the dominant radiation components of the problem-longwave radiation. In particular, our long-term research goals are to

- develop for use in GCMs an optimum longwave radiation model that has been calibrated with state-of-the-art observations

- assess the impact of the longwave radiative forcing in a GCM

- determine the sensitivity of a GCM to the radiative model used in it

- determine how the longwave radiative forcing contributes relatively when compared with shortwave radiative forcing, sensible heating, thermal advection and expansion.

Our approach to developing the radiation model is to test existing models in an iterative, predictive fashion. We plan to compare calculations from a set of models with operationally observed data. The differences we find will lead to the development of new models to be tested with new data. Similarly, our GCM studies will use existing GCMs to study the radiation sensitivity problem. We anticipate that the outcome of this approach will provide both a better longwave radiative forcing algorithm and a better understanding of how longwave radiative forcing influences the equilibrium climate of the atmosphere.

This report summarizes the research results obtained since the last ARM Science Team meeting under the categories of Radiation Model Testing Activities and General Circulation Model Testing Activities. Additional details may be obtained from the authors.

\section{Radiation Model}

\section{Testing Activitiés}

Longwave radiation quantities-radiances, fluxes and heating rates-are usually calculated in GCMs as the cloud amount weighted average of the values for clear and homogeneous cloud conditions. For example, the downward flux at the surface, $F \downarrow$, may be written as

$$
F \downarrow=\left(1-N^{*}\right) F_{0} \downarrow+N^{*} F_{c} \downarrow
$$

where $F_{0} \downarrow$ is the flux that would occur if the sky were clear with the observed, non-cloud radiative properties, $F_{c} \downarrow$ is the flux that would occur if the sky were completely covered by a single plane-parallel cloud layer of uniform 
optical properties, and $N^{*}$ is the "effective" fraction of the sky covered by plane-parallel clouds. The equation is deceptively simple, but there are significant problems associated with the calculation of $F_{0} \downarrow, F_{c} \downarrow$, and $N^{*}$.

Our research program is directed at problems associated with each of the three terms. We have made significant progress in determining the

- ability of line-by-line radiation models to calculate the $F \downarrow$ at the surface

- uncertainties in calculating the downwelling radiance and flux at the surface associated with the use of different profiling techniques

- variability among calculations from radiation codes from different climate models and their differences relative to clear-sky radiance and flux observations

- uncertainties associated with estimating $\mathrm{N}^{*}$ from surface longwave flux observations

- sensitivity of calculations to different formulations of finite-sized clouds.

These topics are discussed below.

\section{Clear-Sky Studies}

We have begun to compare clear-sky spectra of the downwelling radiance at the surface observed during SPECTRE (Spectral Radiance Experiment) with FASCOD3P and with narrow- and broad-band radiation models. Examples of such spectra are shown in Figure 1 , and the mean difference between the observed and FASCOD3P calculated radiance for 26 different spectra is shown in Figure 2a. The FASCOD3P line-by-line model calculations were performed using near simultaneous radiosonde temperature and Raman water vapor profiles with surface-based trace gas data and the 1992 line compilation as input.

In general, the line-by-line model captures most of the features in the observed clear-sky spectra. The model tends to underestimate the observed radiance in the window region between 800 to $1000 \mathrm{~cm}^{-1}$ and to overestimate it in portions of the 1200 to $1400 \mathrm{~cm}^{-1}$ region. However, we are not yet in the position to make firm conclusions concerning the spectral and absolute character of the differences because the final instrument calibration is not yet complete. Nevertheless, the differences in many locations is of the order or smaller than the estimated absolute accuracy of the observations ( $1 \%$ full scale of Figure 1).

Our sensitivity analysis of the radiation calculations shows that with the expected $5 \%$ humidity and $0.5 \mathrm{~K}$ temperature accuracies, the uncertainties in the calculations due to errors in the meteorological data should be kept below about $8 \%$ in the regions of relatively strong lines and below about $5 \%$ in weakly absorbing regions. In the transparent region of the spectrum from 800 to $1000 \mathrm{~cm}^{-1}$, narrowband model radiances calculated using the Roberts et al. (1976) continuum and those using the more recent continuum of Clough et al. (1992) used in FASCOD differ by about $30 \%$, and they show a particular spectral signal.

The differences between the observed and calculated spectra in the 800 to $1000 \mathrm{~cm}^{-1}$ region for the highest water vapor amounts seen during SPECTRE ( $2 \mathrm{~cm}$ precipitable $\mathrm{H}_{2} \mathrm{O}$ ) show that the FASCOD continuum yields better results than Roberts et al. The data from the drier cases cannot be used to support this conclusion at this time because the absolute error of the observations is larger than the radiance in the more transparent regions of the 800 to $1000 \mathrm{~cm}^{-1}$ region. However, as the instrument calibrations are completed, it will be possible to use the high relative accuracy to check the spectral signatures of the differences and to put error bars on them.

We recently completed 26 sets of comparisons of Atmospheric Emitted Radiance Interferometer (AERI) observations with FASCOD calculations using near simultaneous water vapor profiles from radiosondes, Westwater's microwave radiometer, and Melfi's Raman lidar. The spectral distribution of the RMS differences (Figure 2b) shows that the Raman data yield a more consistent spectral pattern of differences between observations and calculations than occurs when the radiosonde or microwave data are used in the calculations. As the calibration of the AERI is finalized, it will be possible to use the high relative accuracy of the observations to check the spectral signatures of the differences between observations and calculations and to put better error bars on the absolute differences. The improved calibration data and Cloud and Radiation Testbed (CART) site observations over larger water vapor amounts should allow more stringent tests of the continuum formulations than possible heretofore, particularly if a Raman lidar is used for profiling water vapor. 


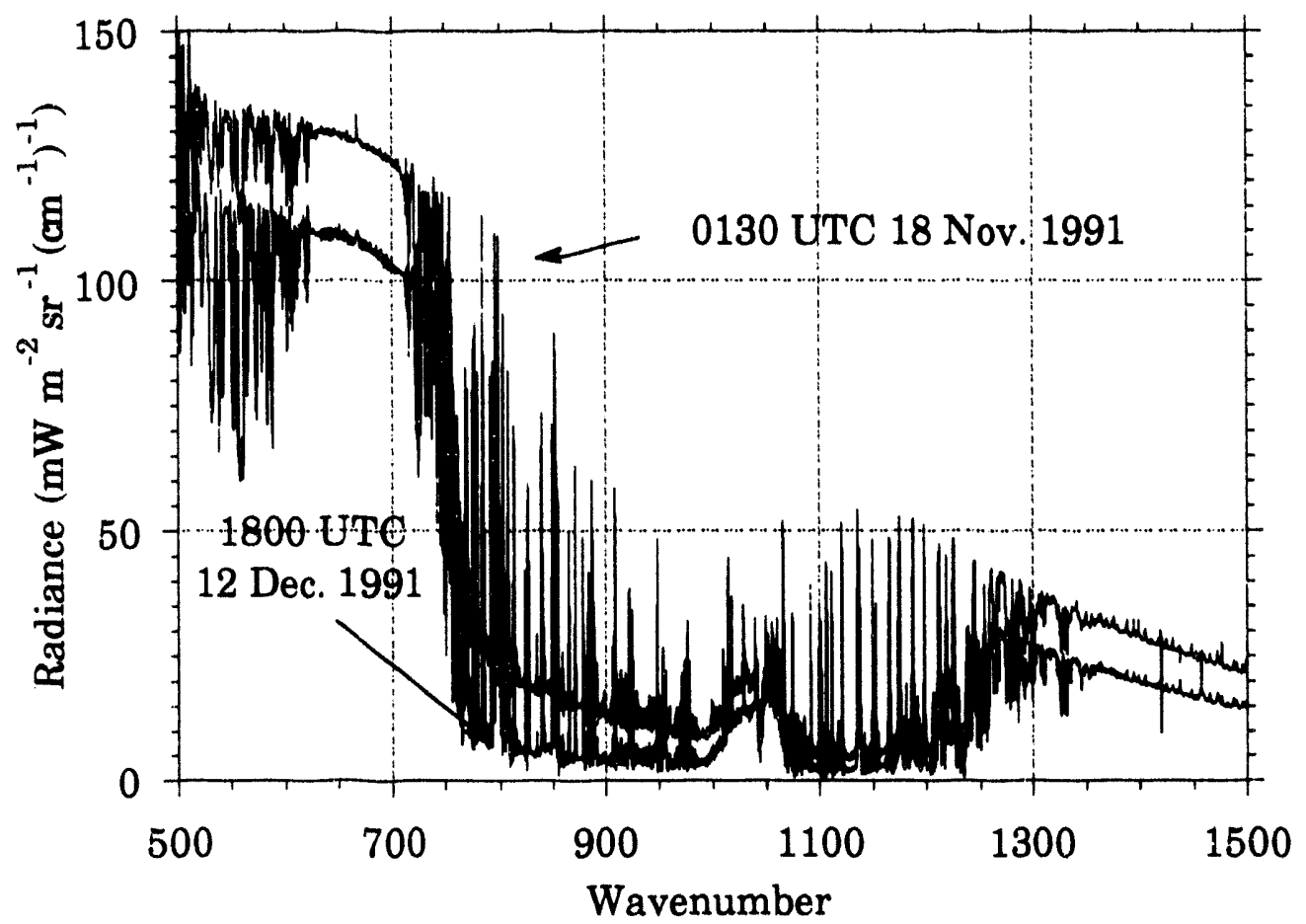

Figure 1. Examples of AERI spectra from SPECTRE.

Climate modelers are also anxious to test the ability of their radiation codes to calculate the total downward flux. The radiance observations can be integrated over the observed portion of the spectrum to yield estimates of the flux uncertainty if one assumes the angular variation is known. Note that for a given plane parallel, horizontally homogeneous atmosphere, the downward flux may be related to the vertically downward radiance $I(0)$ as $F \downarrow=\pi \mid(0) L$ where

$L=\int_{0}^{1} \frac{((\mu)}{1(0)} \mu d \mu$

$\mu=\cos \theta$, and $\theta$ is the local zenith angle.

To first order, we assume $L$ to be a property of the temperature and water vapor distributions and not of the ability to calculate $I$. Thus, the soundings may be used with FASCOD to calculate $L$, and the uncertainty in $F \downarrow$ for the observed spectral interval is given approximately as $\delta F \downarrow=\pi L\left(I_{\text {obs }}(0)-I_{c a l}(0)\right)$

We are in the process of calculating $L$ from (2) using FASCOD3P for the SPECTRE soundings; we will also do this for CART data as they become available. In the interim, we have estimated $\delta F$ using the diffusivity approximation as calculated by MODTRAN. When integrated over the 550 to $1500 \mathrm{~cm}^{-1}$ interval, the comparisons indicate the mean (observed - calculated) flux uncertainty of the FASCOD calculations to be $0.4 \pm 0.2$. $0.5 \pm 0.5$, and $1.2 \pm 0.3 \mathrm{~W} \cdot \mathrm{m}^{-2}$, depending upon the use of Raman, radiosonde or microwave water vapor profiles, respectively. For individual soundings, the results show that the downward flux for this interval may be calculated to within about $\pm 2 W_{0 m}-2$ for any of the sounding types.

The data do not allow us to estimate the uncertainties in the 0 to $550 \mathrm{~cm}^{-1}$ region. However, since this portion of the spectrum is nearly opaque for the conditions we observed during SPECTRE, the uncertainties in calculating the 


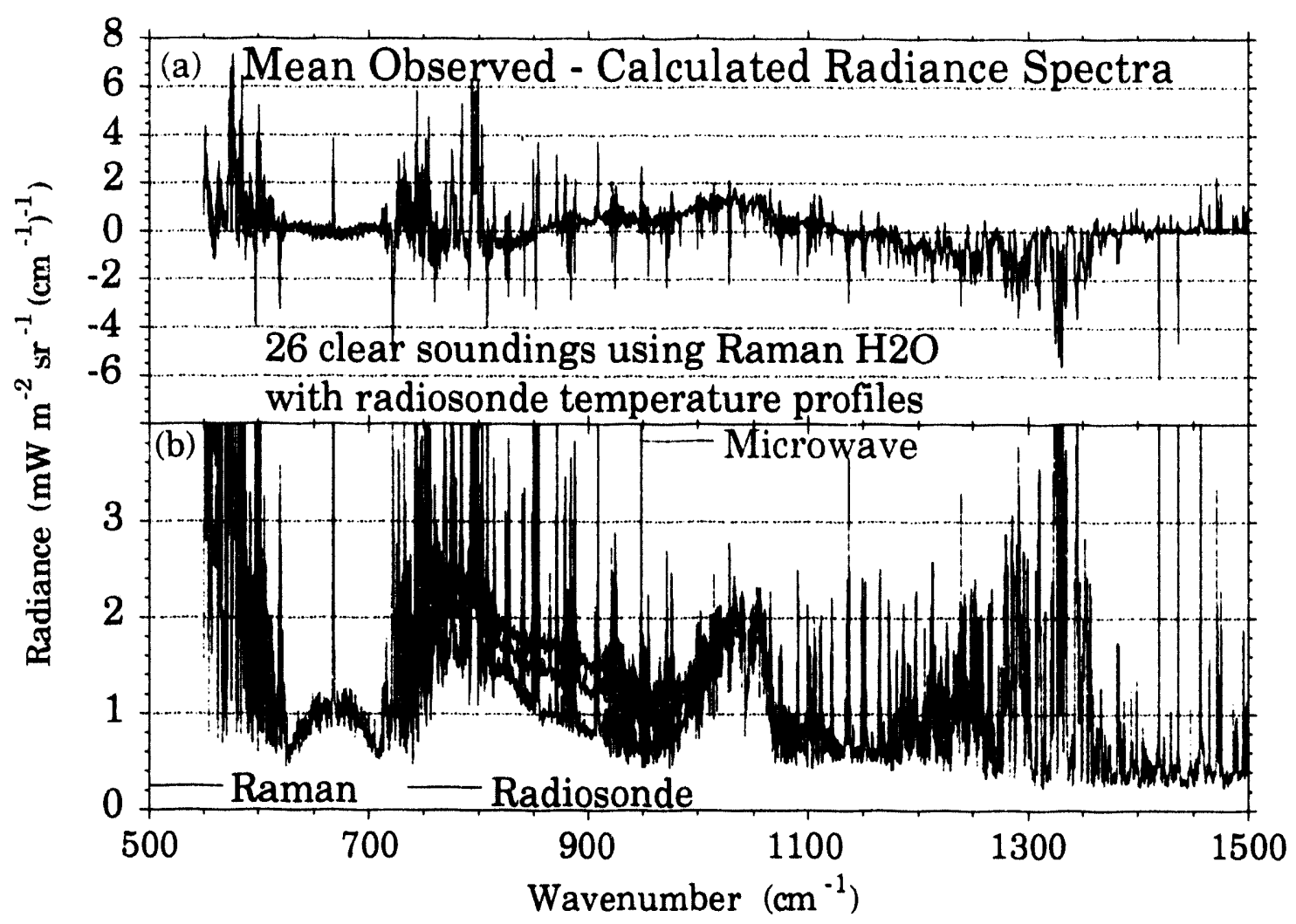

Figure 2. Mean (a) and RMS (b) AERI observed minus FASCOD3P calculated radiance spectra from SPECTRE.

downward flux at the surface from this portion of the spectrum are relatively small. Thus, we plan to estimate the flux for homogeneous clear and cloudy conditions with model calculations for the 0 to $550 \mathrm{~cm}^{-1}$ region and with (1) for the 550 to $3000 \mathrm{~cm}^{-1}$ region and compare the results with observations from the pyrgeometers located at the CART and SPECTRE sites. Since the uncertainties in the interferometer data are smaller than those associated with the pyrgeometers, we believe that the interferometerbased flux data will serve as a baseline calibration of the pyrgeometers for homogeneous clear or cloudy conditions.

A disturbing trend of the differences between the AERI "observed" and calculated fluxes is that differences become more negative as the total preoipitable water (PW) increases, independent of the source of water vapor data. The calculated flux (integrated radiance for the 550 to 1500 $\mathrm{cm}^{-1}$ region) is greater than that observed for six of the nine cases with $\mathrm{PW}>1.4 \mathrm{~cm}$. These differences appear to be correlated primarily with $\mathrm{PW}$ in the $1100.1200 \mathrm{~cm}^{-1}$ region and with surface temperature in the $725-850 \mathrm{~cm}^{-1}$ region. These differences hint at potential problems in the continuum formulation-temperature dependence in the self-broadened term and the magnitude of the continuum coefficients in the foreign broadened regions. Clough et al. (1992) show results consistent with ours in the higher wavenumber regions, although the amount of water in the atmosphere during those observations was considerably less than the amount we found during SPECTRE. We will have to closely examine CART observations at the Southem Great Plains (SGP) and tropical Western Pacific (TWP) sites at higher water vapor amounts and temperatures to see if these differences continue.

During the past 12 months we began preparing for operational testing of a variety of narrow- and broad-band models with CART observations by intercomparing calculations with spectrally integrated AERI observations 
from SPECTRE. The suite of models includes several used in GCMs (including GLA, CCM1, CCC, NMC, RPN and ECMWF) and several detailed models (e.g., the Air Force Geophysics Laboratory's MODTRAN and LOWTRAN7, and Ellingson's narrow-band model). We modified several of the GCM-type models to calculate radiance, rather than flux. Furthermore, we grouped output into common spectral intervals for comparison purposes, although this cannot be done for all models because not all calculate in the same spectral interval and not all can be modified to give results in those spectral intervals sensed by the AERI.

We started our study by examining the more transparent $800-1200 \mathrm{~cm}^{-1}$ region since the Intercomparison of Radiative Codes in Climate Models (ICRCCM) Program indicated that uncertainties associated with the water vapor continuum make this the most suspect portion of the spectrum. Figure 3 shows an intercomparison of some of the statistical properties of the various model calculations and AERI observations in the $800-1200 \mathrm{~cm}^{-1}$ region using the 26 clear-sky radiosonde temperature and water vapor profiles noted above as input.

Some large differences occur in the medians, but these appear to be largely systematic for all models, as the fractional variance of the AERI observations explained by the different models is about the same. The latter result may largely be the result of the small range of $P W$ sensed during SPECTRE. Clearly, some of the models have deficient parameterizations of the water vapor continuum, and these will have to be changed in order for the models to yield correct radiances and fluxes over the full range of atmospheric conditions. Note also that the correlations decreased for each of the models when the 9.6- $\mu \mathrm{m}$ ozone band was included in the comparisons (Figure 3b). We believe this to be a result of a few poor ozonesonde profiles in the sample, but this will have to be studied in greater detail.

Dr. John DeLuisi of the National Oceanic and Atmospheric Administration (NOAA) carried out a pyrgeometer intercomparison during SPECTRE. The data from that study allow the possibility of intercomparing model flux calculations with observations; they also allow the possibility of calibrating the pyrgeometers with the interferumeter. Shown in Figure 4 is an intercomparison of fluxes calculated by the NMC radiation model and those observed by a pyrgeometer at the launch time of the 26 soundings discussed above. No attempt was made to ensure that the sky was completely clearduring these soundings, although clear sky conditions at the zenith were observed.
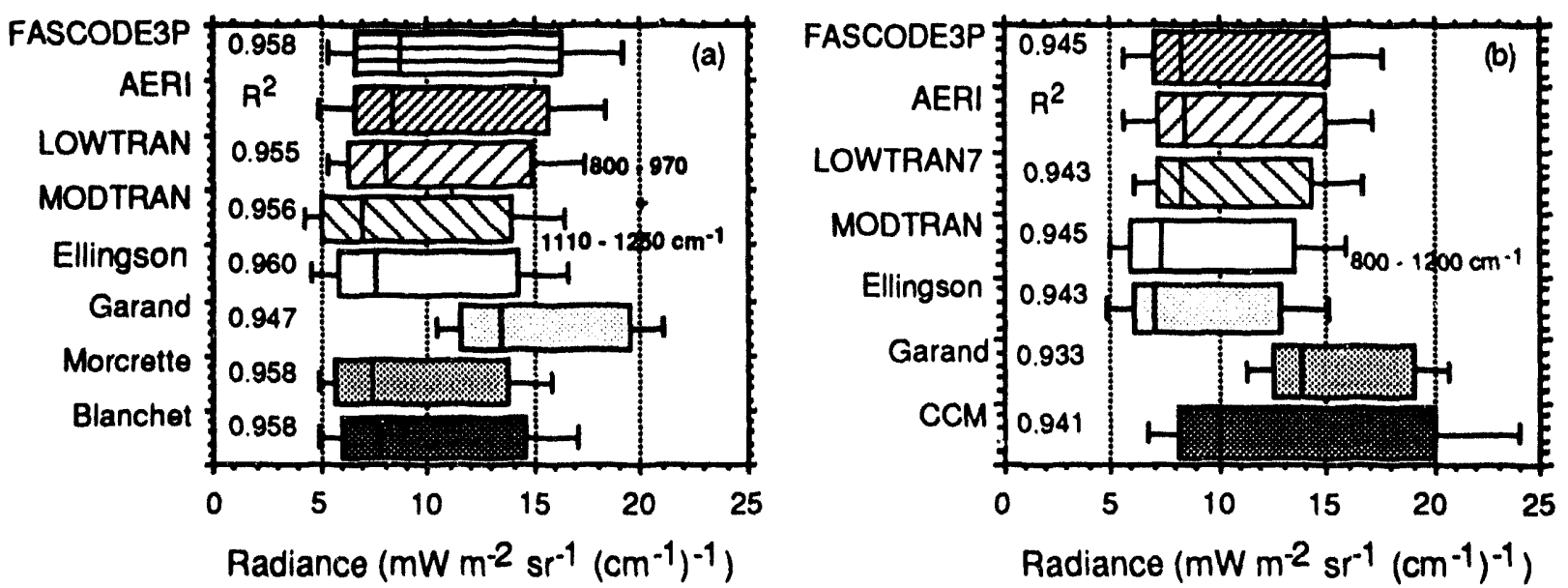

Figure 3. Distributions of vertically downwelling radiance at the surface for 26 clear-sky cases as calculated by several different models and observed by the AERI during SPECTRE. The vertical lines in the boxes show the median values. The edges of the boxes mark the limits of $\pm 25 \%$ of the population, and the lines extending from the boxes mark the minimum and maximum values. The fraction of the AERI variance explained by each model is shown in the column marked $\mathrm{A}^{2}$. 

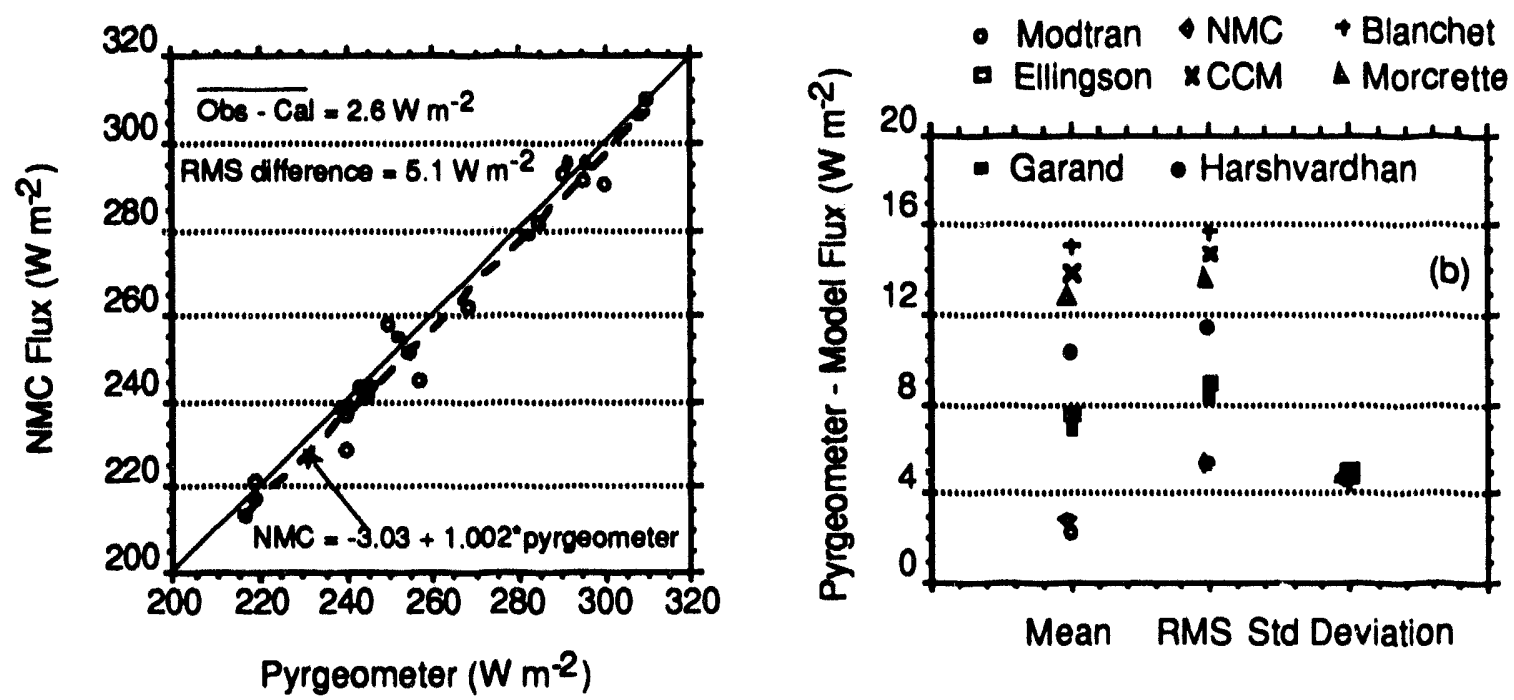

Figure 4. Comparisons of model calculated with pyrgeometer observed clear-sky downward fluxes at the surface during SPECTRE. (a) NMC model; (b) Statistics from other models.

Overall, the results are quite impressive. For this set, the NMC model underestimates the observed fluxes by about $3 \mathrm{~W} \cdot \mathrm{m}^{-2}$ in the mean, and the RMS difference is about $6 \mathrm{~W} \cdot \mathrm{m}^{-2}$. The other models have somewhat greater biases, but all models have about the same RMS error when the biases are removed-about $6 \mathrm{~W} \cdot \mathrm{m}^{-2}$. These comparisons are very consistent with the model-interferometer comparisons. Since the nominal accuracy usually ascribed to pyrgeometer data is about $\pm 5 \%$, the comparisons hint that the uncertainties in the pyrgeometer data may not be as large as thought when great care is exercised in the observations.

\section{Broken Cloud Cover Studies}

Since liquid-water clouds are often nearly black in the thermal infrared, cloud geometry dominates the longwave broken cloud problem. Our research in this area is directed at testing the accuracy of parameterizations of $\mathrm{N}^{*}$ in terms of bulk geometric factors such as the absolute cloud amount, aspect ratio, thickness, spacing, and the distribution of clouds on the horizontal plane. However, a number of difficulties are associated with research on this problem, including
- Finite-size cloud effects on $F_{0} \downarrow$ at the surface are generally within the $5 \%$ accuracy of pyrgeometer observations.

- There are no standard methods for estimating the required cloud properties.

If clouds were black and randomly distributed, the quantities necessary to perform the radiation calculations are the probability of a clear line of sight through the atmosphere at all angles and the probability of seeing a cloud between given altitude regions at all angles. The major difficulty is determining the probability functions.

Although some work has been done on the effects of cloud geometry in the thermal infrared (e.g., Harshvardhan and Weinman 1982; Ellingson 1982), the work is not extensive. We recently completed a comprehensive study of the effect of shape and spatial distribution of cumulus clouds on longwave flux (Killen and Ellingson 1993) in which we were able to derive equations for $\mathrm{N}^{*}$ in terms of geometric cover for several models of clouds with different spatial and size distributions, different aspect ratios, and different shapes. Overall, the study has found that $\mathrm{N}^{*}$ is sensitive to cloud shape and aspect ratio but is insensitive to the cloud spatial distribution or distribution of cloud area. This is 
because the cloud distribution must be normalized to geometric cover, and this is the dominant factor. $\mathrm{N}^{*}$ is also very sensitive to the thermal gradient between the cloud top and base.

Our research on using observations for testing models of $\mathbf{N}^{*}$ is following two different approaches. One approach will determine $\mathrm{N}^{*}$ from a combination of flux and radiance observations and/or calculations using a variation of the spatial correlation technique used for determining cloud amount from satellite data. We will then compare the estimated $N^{*}$ with those calculated by the theoretical models using data from the three-dimensional mapping network.

The uncertainty in estimating $N^{*}$ depends primarily on the uncertainty of the measured flux components [i.e., $F, F_{0}$ and $F_{c}$ in (1)]. Assuming the error of the flux components to be random with equal standard deviation $\sigma_{f}$, the standard deviation of the uncertainty of $N^{*}, \sigma_{8 N^{*}}$, for one cloud layer may be written as

$\sigma_{8 N^{*}}=\frac{\sigma_{f} \sqrt{2}}{F_{0}-F_{c}} \sqrt{\left[1+N^{*}\left(N^{*} \cdot 1\right)\right]}$

Note that $F_{0}$ depends upon the temperature and water vapor distributions, and $F_{c}$ depends additionally on the altitude of the cloud base. We have calculated $\sigma_{8 N}$. for the five McClatchey (1971) soundings and cloud base altitudes ranging from 0.5 to $3 \mathrm{~km}$. The results for $\sigma_{1}=5 \mathrm{~W} \cdot \mathrm{m}^{-2}$ are shown in Figure 5a. The variation of $N^{*}$ with $N$ for different cloud shapes is shown in Figure $5 b$ for comparative purposes.

In general, the results show that the uncertainty of individual estimates of $\mathrm{N}^{*}$ is of the order of 0.1 when the flux observations have uncertainties of the order of $5 \mathrm{~W} \cdot \mathrm{m}^{-2}$. This magnitude of uncertainty will not allow us to distinguish between the formulations for random distributions of cubes or cylinders at $N=0.4$, for example. However, it will allow us to validate the general shape of the form of the variation of $N^{*}$ with $\mathbf{N}$ for different size clouds.

Our second approach to estimating $N^{*}$ from observations is to use scanning lidars, cloud radars, and cloud imagery to develop empirical probability statistics. This is similar to performing Monte Carlo simulations on a computer, but here the atmospheric physics change the cloud parameters and a lidar tracks the photons. The observed probability statistics will be compared with those calculated from simple geometrical considerations.

Dr. Ezra Takara, an expert in Monte Carlo radiation calculations for engineering applications, has recently joined our group and is working with us to develop our ability to interpret the anticipated cloud observations. In particular, Dr. Takara will be generating clear and cloudy line-of-sight probability statistics for clouds of mixed
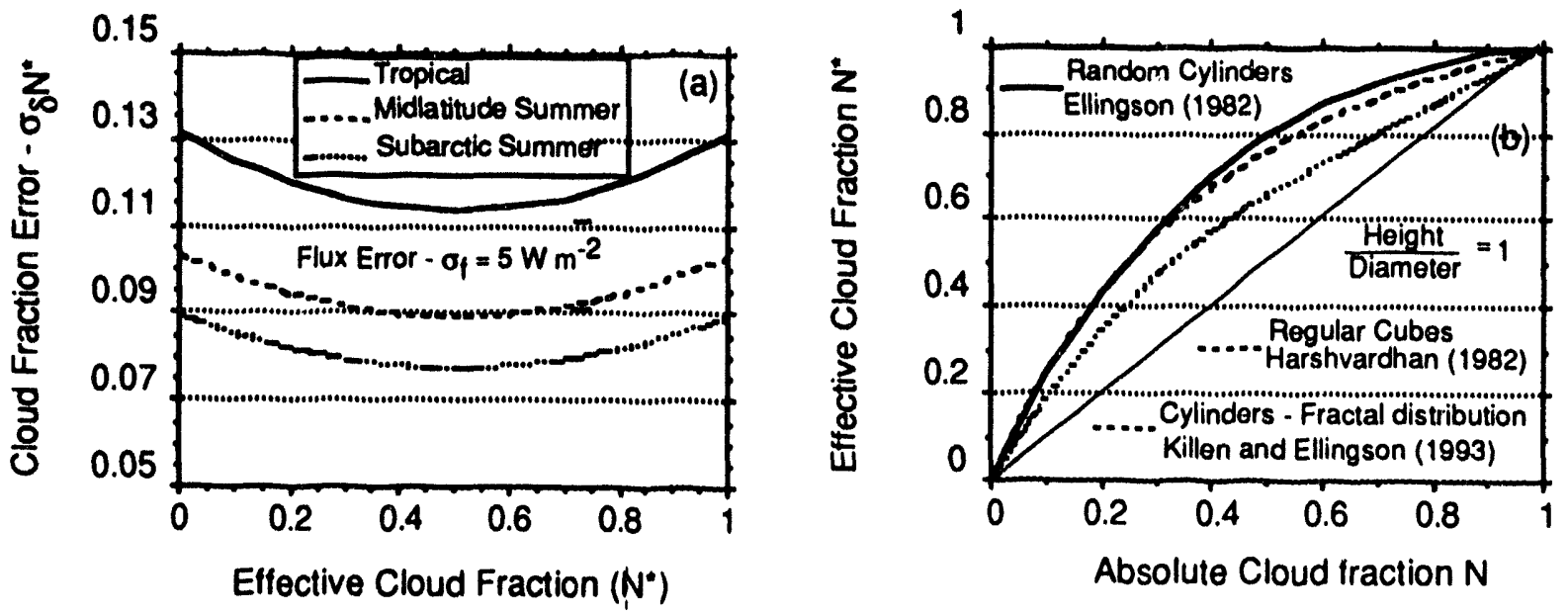

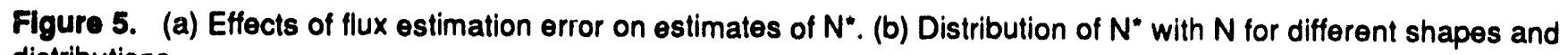
distributions. 
geometry and will be calculating fluxes which include the effects of multiple scattering. These calculations are necessary because clouds are not completely opaque in the IR, and because our analytic emission studies give results for average type of effects. The Monte Carlo simulations should allow us to determine the manner of interpreting the cloud imagery, radar and lidar observations when they become available. Such data when combined with the approach using the flux data should allow us to more precisely determine the manner by which the effective cloud fraction varies with cloud parameters.

As information concerning the cloud and aerosol properties becomes more readily predictable in GCMs, it will be necessary for the longwave radiation models to include the effects of multiple scattering as well as thermal emission. Including multiple scattering effects is relatively straightfonward if one uses a k-distribution technique. However, there are potential drawbacks to this technique as concerns the number of k's and the temperature and pressure scaling. An alternative approach is to use the efiective beam-length as done in mariy heat transfer studies (e.g., Yuen 1990; Yuen and Takara 1990). This type of approach was discussed by Taylor and McCleese (1976) with application to some remote sensing problems, but its application to climate problems has not been extensive. The advantage of the approach is that current techniques for calculating longwave transfer may be used by modifying the absorber amount and/or effective pressure. Ms. Anne Costolanski, a Ph.D. student in our group, is studying the feasibility of such techniques as part of her dissertation research, and we plan to continue this during the next fow years.

\section{General Circulation Model Testing Activities}

Since longwave radiation plays a crucial role in the evolution of climate, its introduction into climate models must be as accurate as possible. We have studied the sensitivity of models which calculate longwave radiative heating to variability of input conditions and to the structure of the model algorithms. In addition, we have begun to assess how GCMs respond to the variability in heating rates provided by the longwave radiation algorithms.

To understand the sensitivity of heating rates to longwave radiation model algorithms (LWRMs), we have intercompared seven such models-most of which are currently used in a unique GCM. The institutions involved are CCC (Canada), ECMWF (United Kingdom), National Center for Atmospheric Research (NCAR) (United States), Colorado State University (CSU) (United States), UMCP (United States), RPN (Canada), and NMC (United States). Input data to each of these models include vertical soundings of temperature, water vapor mixing ratio, ozone, and clouds when applicable (as of this date we have tested the models primarily under clear sky conditions). To establish systematic tests of the selected algorithms, we have prepared several data sets. For reference to previous studies, we have used the standard McClatchey (1971) soundings. For more careful analyses, we have complled 100 soundings for each of four regional/seasonal domains based on a large data set presented by Phillips etal. (1988). These soundings allow us to look at mean profiles as well as standard deviations from those means. The four Phillips data sets show significant variability in the temperature and moisture profiles, both among themselves in the mean and in the standard deviations within each set.

The heating rates produced by the algorithms for the available data sets show significant variability, frequently varying in excess of $0.5 \mathrm{~K} /$ day over a large range in the vertical. If one uses more levels in the vertical, the profiles are smoother, but the general shape is reasonably preserved when a 30 -level calculation is compared with an 18-level one.

We discuss results with 18 levels because it is in the range of current models, in particular, the NMC model which produces weather forecasts. The horizontal profiles show considerable differences between the McClatchey soundings and the means of the Phillips soundings when comparing over seasons and regions. Analysis of the heating rates and their standard deviations produced by the Phillips soundings shows pronounced variability within each of the four data sets. Finally, if one compares the heating rates produced by the different algorithms using correlation coefficients, large differences appear. One can thus conclude that substantially different heating rates for the same sounding data are made available to a GCM, depending only on which algorithm the GCM applies. Since this conclusion is based on clear sky analysis, one can speculate that cloudy sky calculations would exacerbate this situation.

Does this sensitivity play a role when the chosen LWRM is introduced into a GCM? A systematic test to identify such 
sensitivity would require the introduction of various LWRMs into the same GCM and integrations to be performed for a variety of climate scenarios. We plan an experiment of this type but as a preliminary study, we used archives of climate runs and compared the data derived therefrom to give some indication of sensitivity. Currently available to us are extended climate runs with the NCAR CCM1 and CCM2. The archives include profiles of output of the LWRM heating rates in the model over the entire globe and at frequent intervals during the integration. We have analyzed heating rates for those models for a JanuaryFebruary period taken from the archives for the CCM1 (R15), CCM1 (T42), and CCM2 (T42). For these three models, the data are taken for the same time period. We have analyzed the data over the two months and calculated standard deviations from the means. The data are presented on each model level and in terms of the expansion coefficients of the spectral components, effectively yielding the amplitudes of the heating rates in terms of spatial scales. To compare the vertical resolution, vertical means were also calculated (external mode), and deviations from those means were evaluated (internal mode).

The results of these calculations, although striking, were not unexpected. Most pronounced was the difference in the horizontal and vertical distribution of heating rates produced by the R15 and T42 models of the CCM1. We can fairly report that these heating rates were completely different, although the total amplitude was in the same range. This was true for all spatial scales except the overall global value with height and the vertical distribution in the mean zonal field. Moreover, the amplitude as a function of meridional scale did not decay with decreasing scale in the R15 model, subjecting that model to potentially serious nonlinear computational errors. The heating rates generated by the T42 model did decay with decreasing scale.

To a limited extent, we were able to test the interannual variations relative to the intraannual variations by analyzing a second annual season (a different January-Fobruary archive) for the CCM1. If we define the difference in the winter mean of the two annual seasons as the inierannual variation and compare it with the standard deviation of heating rates for one of the winter seasons, we find that the intraannual variation (standard deviation) is considerably larger than the interannual variation. We can thus feel more comfortable in an analysis of one of the annual winter pariods.
Analysis of the January-February period taken from the CCM2 (T42) shows distributions which diffor to some extent from the CCM1 (T42) but have more similarities than the comparison of the CCM1 runs based on truncation. Nevertheless, changes in the CCM2 result in heating rates which decrease more rapidly with decreasing scale when compared with the CCM1.

We currently are setting up a plan to test various LWRMs in a specific GCM, hopefully, one available at Lawrence Livermore National Laboratory. Concurrently, we are collecting heating rate data generated from several models which have produced data for the Atmospheric Model Intercomparison Project (AMIP) and plan to intercompare those data to assess model sensitivity.

\section{References}

Clough, S. A., M. J. lacono, and J-L. Moncet. 1992. Lineby-line calculations of atmospheric fluxes and cooling rates: Application to water vapor. J. Geophys. Res. 97:15761-15785.

Ellingson, R. G. 1982. On the effects of cumulus dimensions on longwave irradiance and heating rates. J. Atmos. Sci. 39:886-896.

Harshvardhan, and J. A. Woinman. 1982. Infrared radiativo transfer through a regular array of cuboidal clouds. J. Atmos. Sci. 39:431-439.

Killen, 7. M., and R. G. Ellingson. 1993. The effect of shape and spatial distribution of cumulus clouds on longwave irradiance. Submitted to J. Atmos. Sci. April 1993.

Lacis, A. A., and V. Oinas. 1991. A description of the correlated $k$-distribution method for determining nongray gaseous absorption, thermal emission, and multiple scattering in vertically inhomojeneous atmospheres. J. Geophys. Res. 96:9027-9074.

McClatchey, R. A., R. W. Fenn, J.E.A. Solby, F. E. Volz, and J. S. Garing. 1971. Optical Properties of the Atmosphere. Rep. AFCL-71-0279, Air Force Cambridge Res. Lab., Bedford, Massachusetts.

Phillips, N., J. Susskind, and L. McMillin. 1988. Results of a joint NOAANASA sounder simulation study. J. Atmos. Ocean Tech. 5:44-56. 
Roberts, R. E., J.E.A. Selby, and L. M. Biberman. 1976. Infrared continuum absorption by water vapor in the 8-12 $\mu \mathrm{m}$ window. Appl. Opt. 15:2085-2090.

Taylor, F.W., and D. J. McClesse. 1976. Multiple scattering in broad spectral bands. Radiation in the Atmosphere, H.-J. Bolle, ed., p. 271-273. Science Press, Princeton, Now Jersey.

Yuen, W.W. 1990. Development of a network analogy and evaluation of mean beam lengths for multi-dimensional absorbing/isotropically-scattering media. AMSE J. Heat Transfer 112:408-414.
Yuen, W. W., and E. Takara. 1990. Development of a generalized zonal method for the analysis of radiative heat transfer in absorbing and anisotropically scattering media. Numerical Heat Transfer, eds. K. Vafai and J.L.S. Chen, ASME HTD 130:123-132. 


\title{
Radiative Transfer Model Development in Support of the Atmospheric Radiation Measurement Program
}

\author{
S. A. Clough \\ Atmospheric and Environmental Research, Inc. \\ Cambridge, MA 02139
}

The objective of this research effort is to develop radiative transfer models that are consistent with Atmospheric Radiation Measurement (ARM) Program spectral radiance measurements for clear and cloudy atmospheres. Our approach is to develop the model physics and related databases with a line-by-line model in the context of available spectral radiance measurements. The line-byline model then functions as an intermediate standard to both develop and validate rapid radiative transfer models appropriate to GCM applications.

\section{Line-by-Line Multiple Scattering Code: CHARTS}

CHARTS (Code for High-Resolution Atmospheric Radiative Transfer with Scattering), J.-L. Moncet lead investigator, is now fully functional for applications in the thermal infrared. The code is optimized to calculate the spectral radiance and spectral transmittance for a scattering atmosphere with the viewing angle and position specified. These are the anticipated conditions under which the code will be applied to ARM spectral observations: ground, aircraft or satellite-based spectral radiance and transmittance measurements. CHARTS restricts the multiple scattering calculation to the atmospheric regime in which the scatterer is included. A line-by-line radiative transfer model, in this case LBLRTM, is used for the non-scattering atmosphere in the altitude regime above the clouds, providing the spectral dependent boundary condition for the CHARTS calculation.

Figure 1 demonstrates the effects on the downwelling spectral radiances due to multiple scattering from an optically, thin cirrus cloud in the altitude regime from 10 $12 \mathrm{~km}$ for a range of cloud water paths. This case ${ }^{(\mathrm{a})}$ is

(a) S. T. Ackerman, private communication, 1993 consistent with downlooking data taken with the high spectral resolution interferometer sounder (HIS) (Smith et al. 1983) from $20 \mathrm{~km}$ and with simultaneous data taken with the atmospheric emitted radiance interferometer (AERI) on the ground as part of the Spectral Radiance Experiment (SPECTRE). Ackerman has calculated the spectral scattering parameters for ice spheres with a $15-\mu$ mode radius. The cloud, covering the altitude regime from $10-12 \mathrm{~km}$, has a mass path $5 \mathrm{~g} / \mathrm{m}^{2}$. Validations are being performed using these data sets and comparing the results of our model with results from more rapid but less rigorous treatments of the multiple scattering. Extension of the model to solar spectral transmittance measurements is being initiated.

\section{Radiation Models: LBLRTM and RRTM}

Extensive effort has gone into two aspects of the Line-byLine Radiative Transfer Model (LBLRTM): 1) applying the model to real-time spectral validations for ARM measurements, including the implementation of an $\mathrm{ftt}$ package to model spectral instrument functions and 2) adapting the model to provide the necessary interface with CHARTS. LBLRTM is a vectorizable line-by-line model based on the FASCODE algorithms and includes the capability to calculate atmospheric fluxes and heating rates. The model has also been adapted to function as the forward model in a retrieval algorithm to retrieve atmospheric profiles of temperature and trace gas profiles from highresolution spectral radiance data. LBLRTM has been extensively validated with HIS measurements, e.g., Clough ot al. (1992).

In the course of developing a rapid radiative transfer model (RRTM), we have performed spectral band comparisons of cooling/heating rate results from the NASA-AMES/ Penn State model (Toon et al. 1989), with line-by-line 


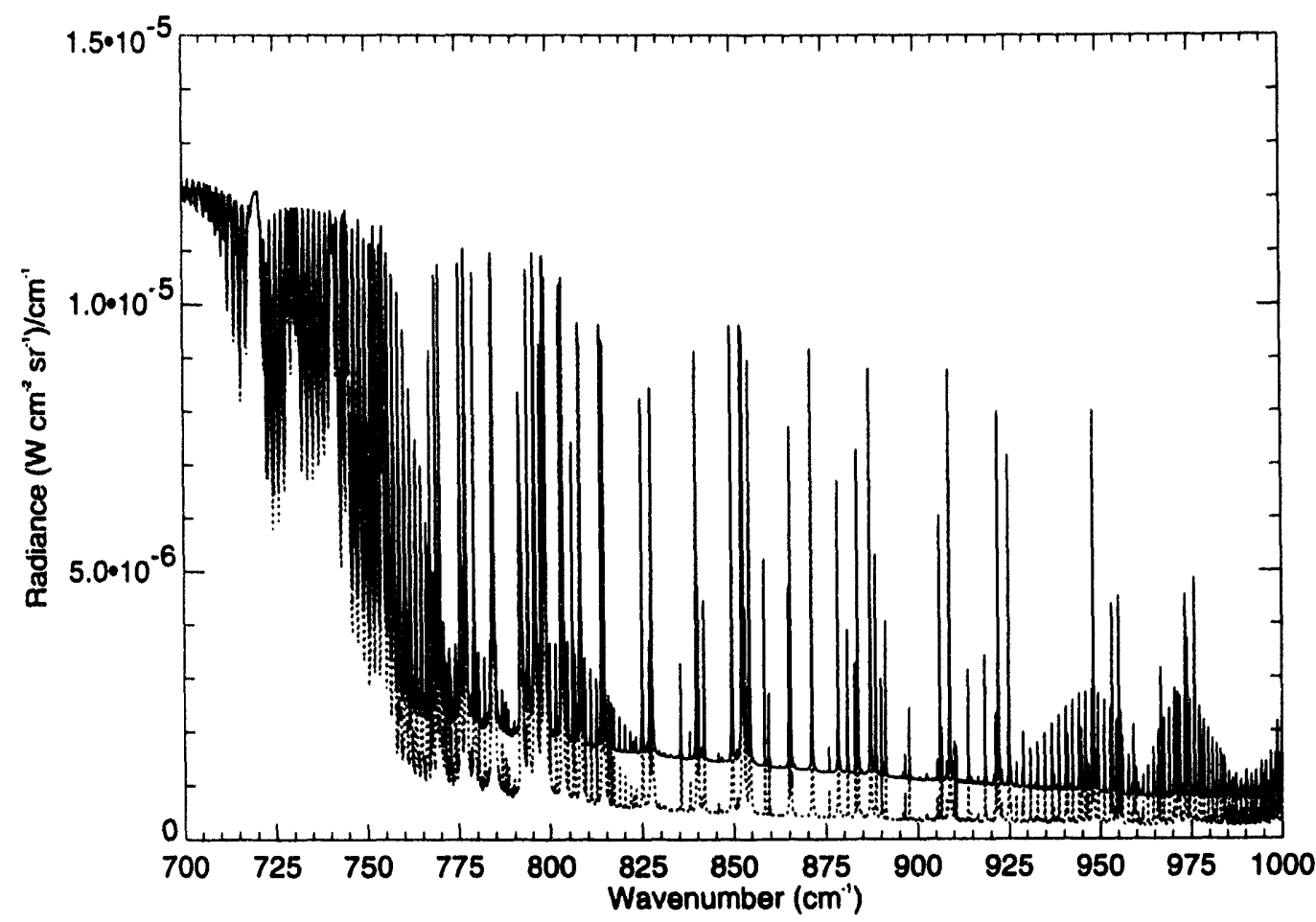

Figure 1a. The downwelling spectral radiance at the surface for an atmosphere with a 10-12 $\mathrm{km}$ optically thin cirrus cloud with $15 \mu$ mode radius and mass path of $5 \mathrm{~g} / \mathrm{m}^{2}$ (solid curve) and for a clear atmosphere (dotted curve).

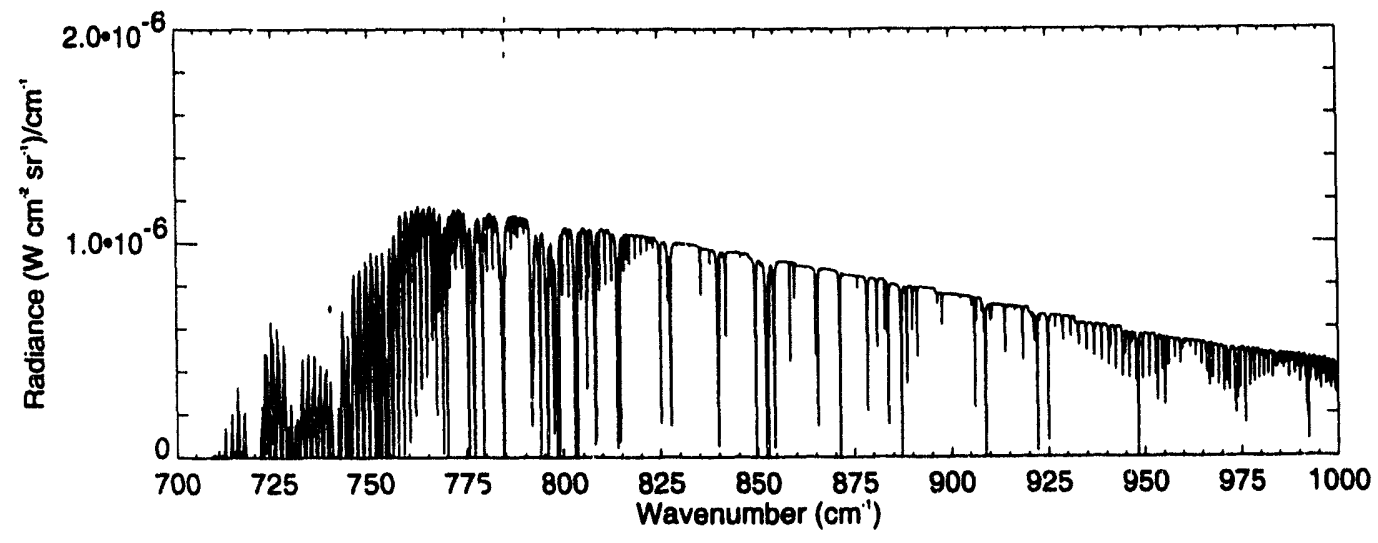

Figure 1b. Difference in spectral radiances of Figure 1a, cloud - clear. 
results obtained from LBLRTM. The results at this stage of the effort indicate that the treatment of the continuum and the k-distributions for the line contribution in this rapid model are not consistent with LBLRTM. At the surface, there is a difference of $10 \mathrm{w} / \mathrm{m}^{2}$ in the net flux for the midlatitude summer atmosphere. Since the goal of the current research effort is to attain accuracy performance from the rapid model that is consistent with LBLRTM and measurements to be taken at the ARM sites, an initiative is under way to develop anew RRTM that uses a continuum formulation and k-distributions that provide results as consistent as possible with the line-by-line calculations.

\section{Revised Continuum Model}

The water vapor continuum plays an important role in atmospheric radiative transfer, providing increased opacity between spectral lines over the full spectral region from the microwave to the visible. The continuum has a significant influence on atmospheric fluxes and cooling rates. Additionally, the continuum is important to the, physical solution of the inverse problem, the remote sensing of atmospheric state to retrieve temperature, water vapor, surface properties and other state parameters.

The continuum has two components: the self-broadened continuum, dependent on the square of the partial pressure of water vapor, and the foreign-broadened continuum, dependent on the product of the water vapor partial pressure and the $\mathrm{dry}$ air pressure. As a consequence, the selfbroadened continuum tends to be more important in the lower atmosphere, while the foreign-broadened continuum tends to be more important in the middle to upper troposphere.

A comprehensive continuum model based on a single line shape for all transitions from the microwave to the visible has provided generally acceptable results (Clough et al. 1989 and Clough et al. 1980; hereafter CKD). The nadir and zenith spectral radiometric measurements with the University of Wisconsin HIS instrument have been particularly useful in establishing the general level of accuracy for the continuum. For most spectral regions, the apparent error in the continuum is of the order of $10 \%$ or less. The term "apparent" is used because of the difficulty of unambiguously characterizing the atmosphere, particularly with respect to aerosol loading and to subvisual cloud effects. However, for special conditions of atmospheric state, measurements indicate that for specific and very limited spectral regions, errors in the CKD continuum may be significant.

To address this situation and to improve overall accuracy of atmospheric radiance calculations, we have developed an improved but preliminary water vapor continuum model, designated CKD-1. The approach has been to more carefully model the laboratory measurements of Burch and to validate the refined model with up- and downlooking spectral radiance data. The full data set of Burch and coworkers $(1985,1984,1981)$ has been reanalyzed using a self-consistent approach for the contribution of local lines. In contrast to the earlier model, this reanalysis provides full consistency with the line shape decor, position used for the LBLRTM model.

Our intention had been to develop a new water vapor line shape which, when applied to all water vapor lines, would provide agreement with the laboratory and atmospheric measurements. Eviderice is mounting that, for the accuracy required for ARM radiation measurements and for climate change considerations, a continuum model based either on semi-empirical analytic line shape functions (CKD) or those obtained from collisional theory is not likely to prove adequate. Using the quasistatic approximation, $\mathrm{Ma}$ and Tipping (1992) have developed a line shape which they have used to obtain a self and foreign water vapor continuum. (a) In our judgment, their results are not sufficiently accurate to be used directly to describe the continuum; a calculation using the molecular potential of the water molecule would not be expected to be valid to the required level of accuracy.

The continuum absorption coefficient for a homogeneous path, $k_{c}$, may be expressed in terms of the self and foreign continuum coefficients, $C_{s}$ and $C_{p}$, as

$$
k_{c}=v \tanh \left(h c v / k T\left[\left(\rho_{h_{2}}\right) \bar{C}_{s}(T)+\left(\rho_{\text {air }} / \rho_{0}\right) \bar{C}_{1}(T)\right]\right.
$$

where $v$ is the photon wavenumber value, $T$ is the temperature, $\rho_{h_{2}}$ is the density of water vapor, $\rho_{\text {alr }}$ is the density of dry air and $\rho_{0}$ is the reference density at one atmosphere.

Figures $2 a$ and $2 b$ provide the self and foreign continuum coefficients, respectively, for the longwave region. The symbols represent Burch's measurements with the local

(a) Private communication. 


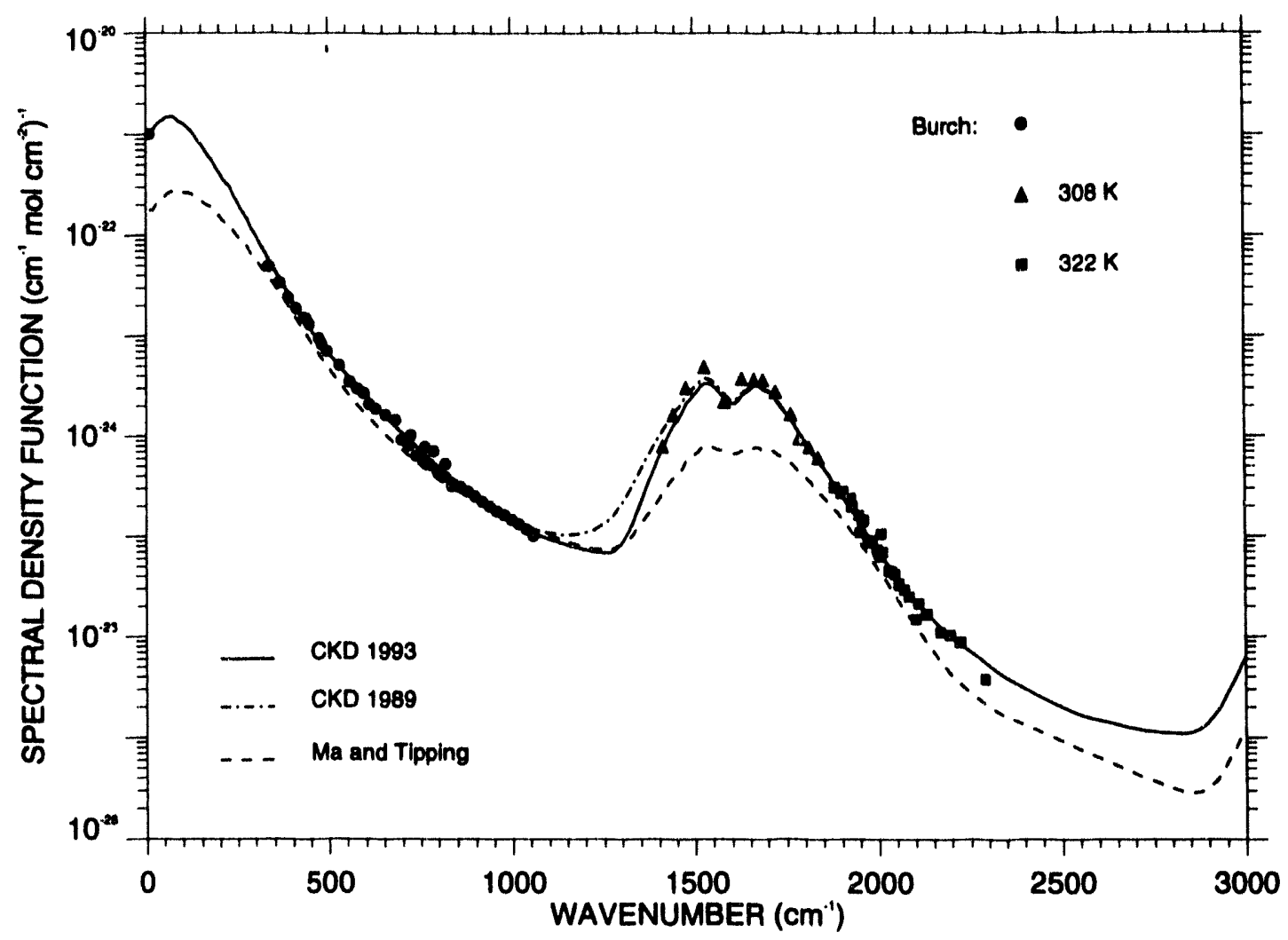

Figure 2a. The water vapor continuum coefficients at $296 \mathrm{~K}$ for the self-broadening case.

line contribution removed using the LBLRTM local line definition at the indicated temperature. In both the self and foreign cases the CKD continuum does not model the data well at the low wavenumber side of the $v_{2}$ band in the $1300 \mathrm{~cm}^{-1}$ region. The CKD-1 continuum has been analytically adjusted to improve agreement. A validation of this modification is provided in Figure 3.

Figure $3 a$ provides the upwelling spectral radiances measured at $20 \mathrm{~km}$ with the HIS instrument, Figure $3 \mathrm{~b}$ provides the spectral residuals with the CKD continuum, and Figure $3 \mathrm{c}$ provides the spectral residuals with the revised CKD-1 continuum. Significant improvements in the spectral residuals are apparent. The remaining residuals in the $1300 \mathrm{~cm}^{-1}$ region are not attributable to the continuum since removal of the continuum does not reduce these residuals (which are most likely attributable to an incorrect methane profile). The upwelling radiance provides an important validation of the treatment of the temperature dependence of the continuum since the radiation is emanating from altitude regimes associated with colder temperatures. The temperature and water vapor profiles have been obtained from radiosonde measurements; the profiles for the uniformly mixed species have been obtained from climatology; and the line-by-line calculations are from LBLRTM. Similar conclusions may be drawn from the downwelling radiances from HIS ground-based observations. Preliminary results from laboratory measurements by Kulp(a) indicate essential confirmation of the CKD-1 continuum from $700-1300 \mathrm{~cm}^{-1}$ at $296 \mathrm{~K}$.

The CKD water vapor continuum model provides calculated spectra in significantly better agreement with observed

(a) Private communication, 1993. 


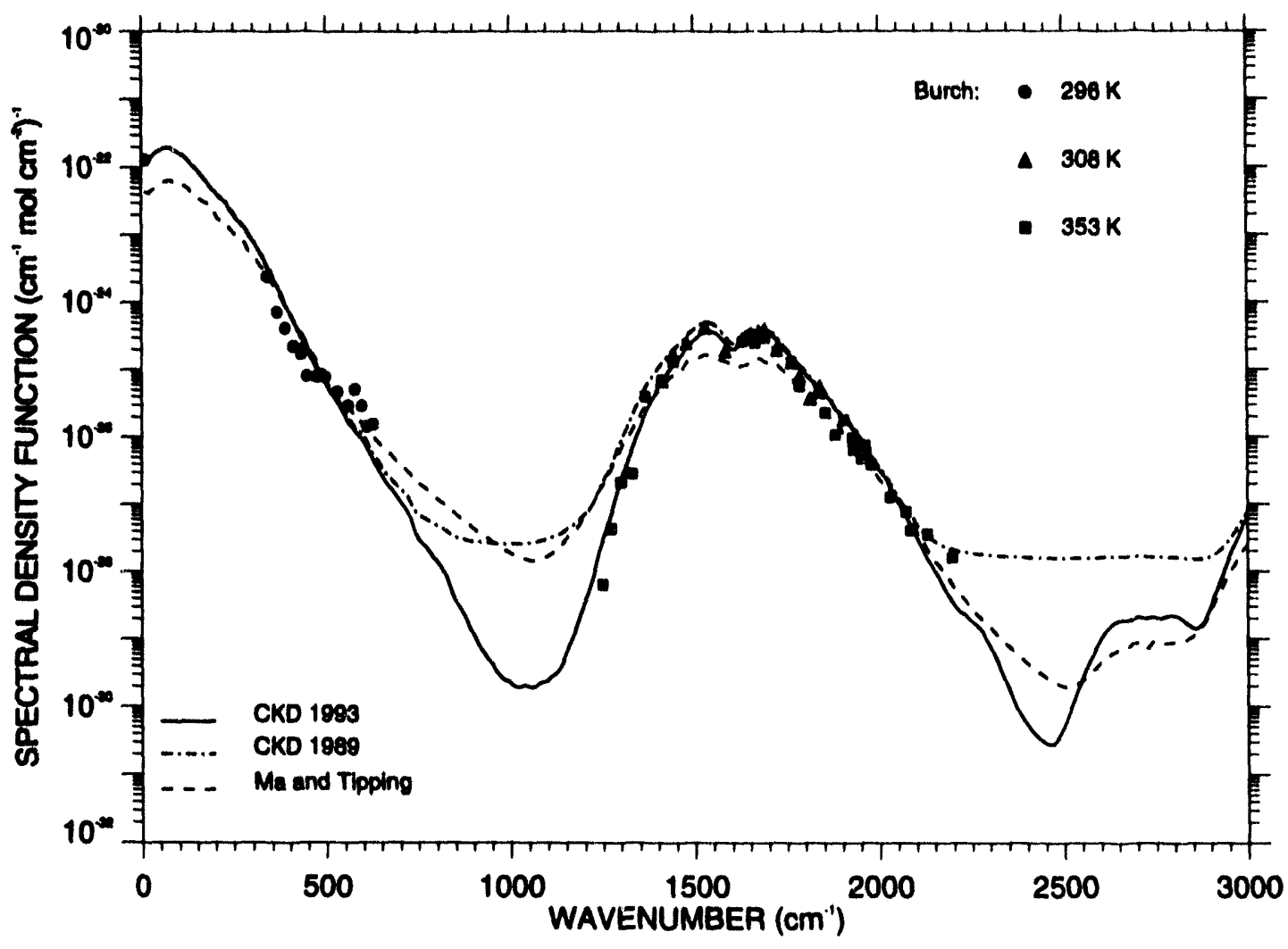

Figure 2b. The water vapor continuum coefficients at 296K for the foreign-broadening case.

spectral radiance data than the Roberts et al. model (1976), which is used in many radiative models for studies related to climate change. Because of its analytical form the Roberts of al. model has a cortain appeal related to simplicity of application; nevertheless, the model reflects incorrect physics, does not properly treat the foreign continuum, and is not sufficiently accurate to meet present requirements.

The CKD-1 continuum is available to the general scientific community in a stand-alone program via anonymous ftp at AER. The numerical nature of the CKD model makes it readily tractable for application to correlated $k$-distribution radiative transfer models. The model will continue to undergo validation and refinement. We anticipate that the spectral radiance data to be obtained at the Western Pacific ARM site will be especially valuable in assessing continuum model performance for tropical atmospheres, a matter of particular importance for climate considerations.

\section{Acknowledgments}

We would like to acknowledge the important contributions of several AER scientists including Jean-Luc Moncet, Bob Worsham and Pat Brown. We are also grateful for the many interactions wo have had with ARM scientists, especially the U. of Wisconsin group under Bill Smith, who has been most generous in providing data that have enabled us to improve modeling performance. 


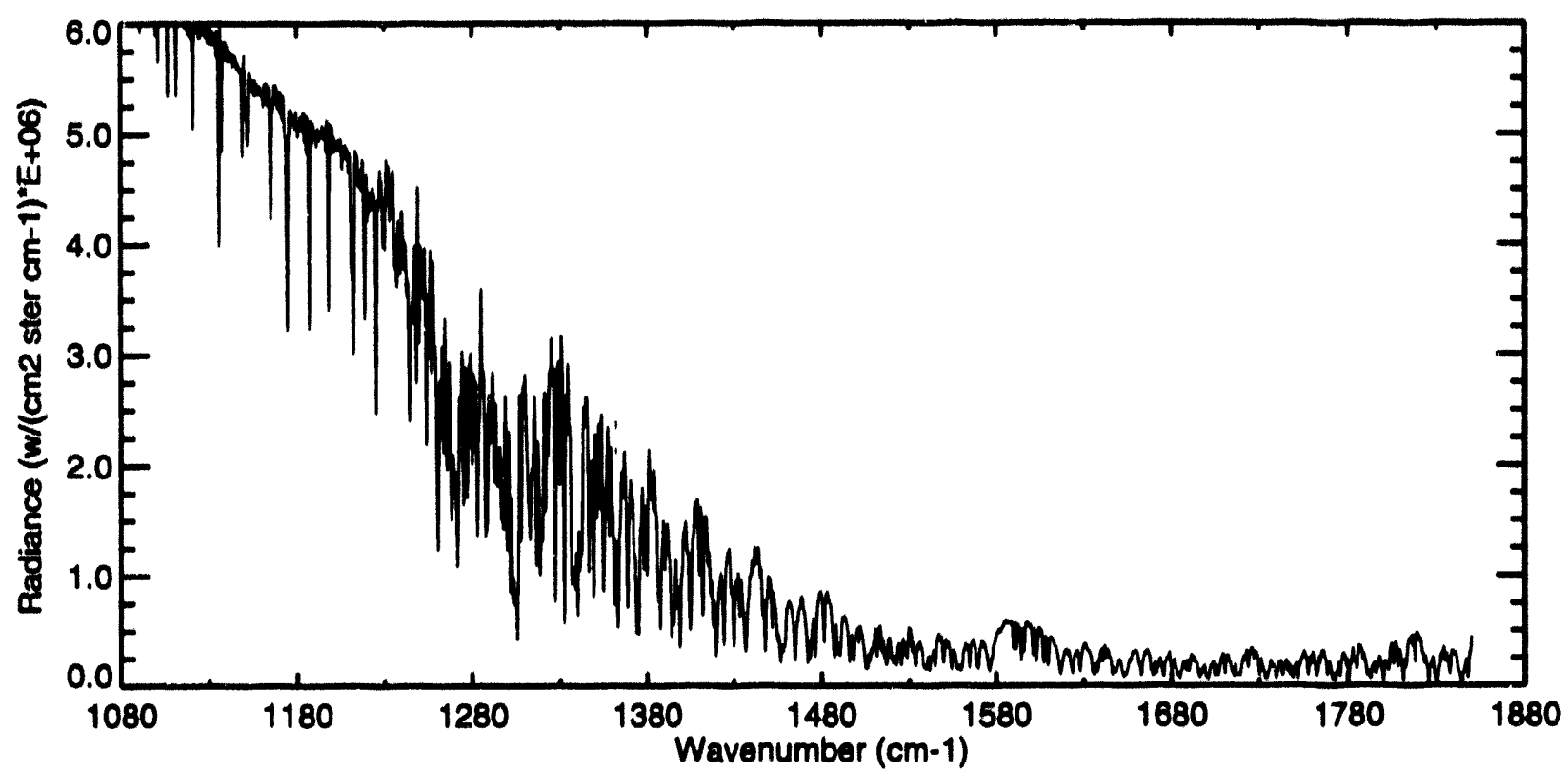

Flgure 3a. Spectral radiances from the 14 April $1986 \mathrm{HIS}$ measurement from $19.6 \mathrm{~km}$.

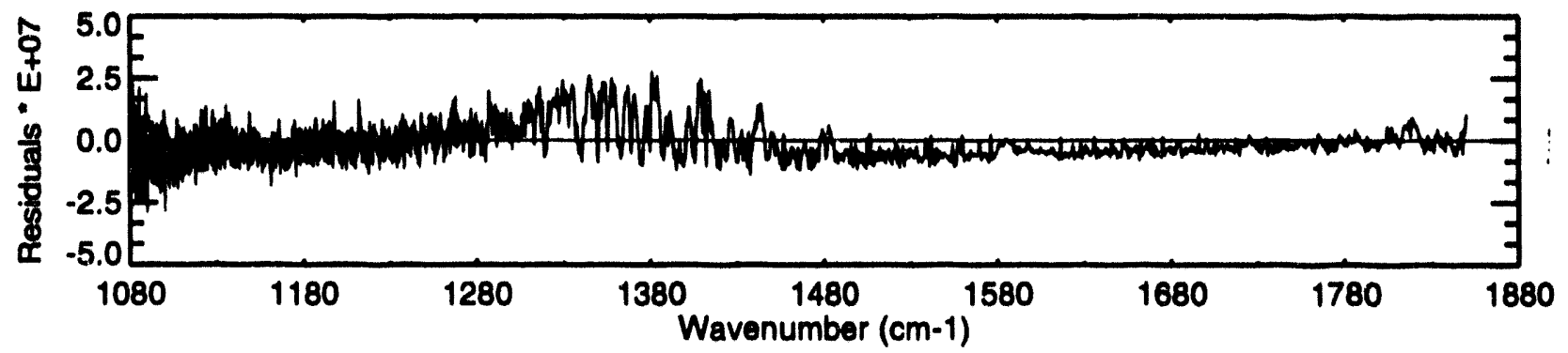

Figure 3b. Spectral residuals from the difference of the observation of Figure $3 a$ and an LBLRTM calculation using the CKD continuum.

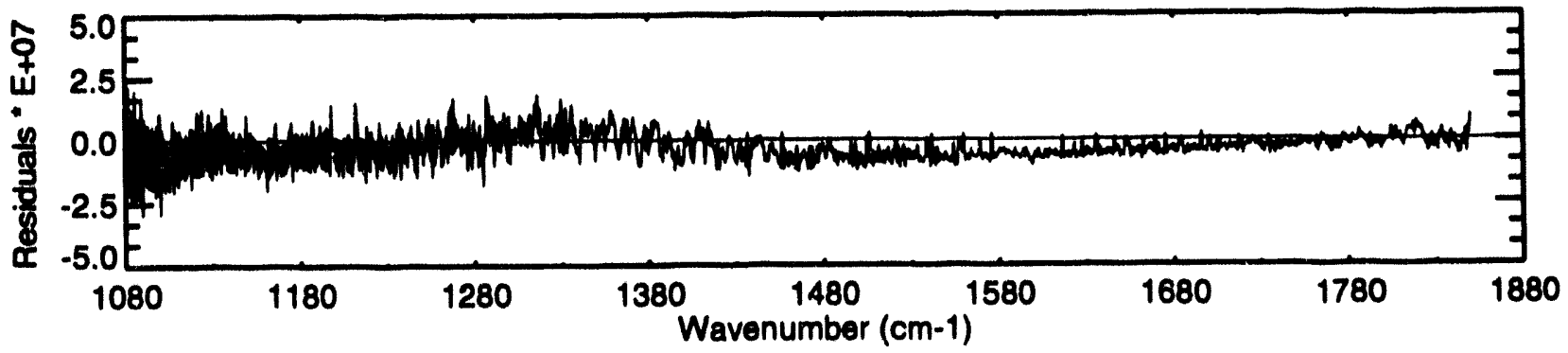

Figure 3c. Spectral residuals from the difference of the observation of Figure $3 a$ and an LBLRTM calculation using the CKD.1 continuum. 


\section{References}

Burch, D. E. 1981. Continuum absorption by $\mathrm{H}_{2} \mathrm{O}$. Rep. AFGL-TR-81-0300, 32 pp. Air Force Geophys. Lab., Hanscom Air Force Base, Massachesetts.

Burch, D. E., and R. L. Alt. 1984. Continuum abeprption in the $700.1200 \mathrm{~cm}^{-1}$ and $2400-2800 \mathrm{~cm}^{-1}$ windowe. Rep. AFGL-TR-84-0128, 46 pp. Air Force Geophys. Lab., Hanscom Air Force Base, Massachesette.

Burch, D. E. 1985. Absorption by $\mathrm{H}_{2} \mathrm{O}$ in Narrow Band windows between $3000 \mathrm{~cm}^{-1}$ and $4200 \mathrm{~cm}^{-1}$. Rep. AFGLTR-85.0036, 38 pp. Air Force Geophys. Lab., Hanscom Air Forco Base, Massachesetts.

Clough, S. A., F. X. Knelzys, R. W. Davies, R. Gamache, and R. H. Tipping. 1980. Theoretical line shape for $\mathrm{H}_{2} \mathrm{O}$ vapor: Application to the continuum. Atmospheric Water Vapor, ods. A. Deepak, T. D. Wilkerson, and L. H. Rhunke, pp. 25-46. Ace tomic Press, London.

Clough, S. A., F. X. Knelzys, and R. W. Davies. 1289. Line shape and the water vapor continuum. Atmos. Res. 23:229. 241.

Clough, S. A., R. D. Worsham, W. L. Smith, H. E. Revercomb, R. O. Knuteson, G. P. Anderson, M. L. Hoke, and F. X. Kneizys. 1989. Validation of FASCODE
Calculations with HIS Spectral Radianco Measuremonts. IRS '88: Current Problems in Atmospheric Radiation, uds. J. Lenoble and J. F. Geleyn, Pp. 376-379. A. Deepak Publishing, Hampton, Virginia.

Clough, S. A., M. J. lacono, and J.-L. Moncot. 1982. Line. by-Line Calculations of Atmospheric Fluxes and Cooling Rates: Application to Water Vapor. J. Geophys. Res. 97:15761-15785.

Ma, Q., and R. H. Tipping. 1892. A far wing line shape theory and its application to the water continuum absorption in the infrared region (II). J. Chem. Phys. 96:8655-8663.

Roberts, R. E., J.E.A. Solby, and L. M. Biberman. 1978. Infrared continuum absorption by atmospheric water vapor In the 8.12 mm window. Appl. Opt. 15:2085-2090.

Smith, W. L., H. E. Revercomb, H. B. Howell, and H. M. Woolf. 1983. HIS - A satellite instrument to observe temperature and humidity profiles with vertical resolution. Proc. Fith Conterence on Atmospheric Radiation, American Moteorological Society, Boston, Massachesetts.

Toon, O. B., C. P. Mckay, T. P Ackerman, and K. Santhanam. 1989. Rapid Calculation of Radiative Heating and Photodissociation Rates in Inhomogeneous Multiple Scattering Atmospheres. J. Geophys. Res. 94:16,287-16,301. 


\title{
General Circulation Model Feedback Sensitivity Assessment
}

\author{
A. A. Lacis \\ NASA Goddard Space Flight Center \\ Institute for Space Studies \\ Now York, NY 10025
}

\section{Feedback Effects in Climate GCMs}

Estimating the magnitude of the climate system response to a radiative forcing perturbation is made difficult by feedback contributions that can magnity or diminish the initial effect of the forcing. A comparative study of radiative forcing parameterizations in 12 different general circulation models (GCMs) by Coss of al. (1993) showed that GCM forcing for doubled $\mathrm{CO}_{2}$ ranged from 3.38 to $4.74 \mathrm{Wm}^{-2}$, but that the spread in the global mean surface temperature response to the rediative forcing ranged from $1.7^{\circ}$ to $5.3^{\circ} \mathrm{C}$. This larger spread in the GCMs' responses is a clear indication of their greater diversity in toedback sensitivity than in their radiative forcing. As climate changes in response to the initial forcing, foedback contributions arise from the additional radiative effects that are generated by

1. changes in water vapor amount and distribution

2. changes in cloud cover, height and optical depth

3. changes in snow/ice cover, l.e., surface albedo

4. changes in advected energy transports.

Since the feedbacks that contribute to the total change in global temperature are produced by complex interactions between different physical processes, it is very difficult to isolate and evaluate the strength of individual foedbacks directly from the GCM output, even in carefully constrained climate experiments.

\section{Radlative-Convective- Advective Equilibrium}

Whenever the local atmospheric temperature gradients become unstable because of solar heating of the ground, "convective adjustment" takes place. Energy is transported upward within the troposphere and, in the process, the "critlcal lapse rate" of tropospheric temperature is established. Similarly, in response to the seasonally driven change in solar forcing, there is a latitudinal redistribution ofenergy by dynamical transports resulting in the meridional advection of energy. In view of the continuous change in diurnal and seasonal radiative forcing, the atmosphere is never actually allowed to reach equilibrium. However, on an annually averaged basis, there is reason to expect that the net Top-of-the-Atmosphere solar and thermal fluxes and the meridionally advected sensible, latent, and geopotential energy musi be in balance at each latitude when the global climate is in an equilibrium state. On this basis, it is appropriate to consider a detalled 1-Dimensional Radiative-Convective Model (1.D RCM) type energy balance analysis for each individual latitude zone.

\section{Feedback Analysis}

The relative strengths of the different climate feedbacks can be determined by computing the magnitude of the change in equilibrium surface temperature attributable to specific changes in atmospheric structure and/or to changes in the concentration and distribution of the radiatively active constituents. This information can be obtained by using a 2-Dimensional Radiative-Convective-Advective equilibrium model (2-D RCAM) to analyze the changes in the latitudinal energy balance that take place between the GCM experiment and the GCM control run. The input information required for this analysis consists of the zonally averaged annual average temperature and water vapor profiles, advected energy transports, cloud cover, and surface albedo from the GCM experiment and control runs. 
As the first step, we need to exprese the radiative forcing perturbation in terms of the equivalent equilibrium tomperature change, $\Delta T_{0}$, that would restore the radiative energy balance in the abeence of feedback contributions. The lattudinal dependence of this radiative forcing term, $\Delta T_{0}$, is shown in the upper panels of Figure 1 by the heavy red lines for the $2 \%$ solar constant and doubled $\mathrm{CO}_{2}$ experiments, respectively. The net feedback factor, f, can then be identified as the multiplication factor that relates the radiative forcing term to the total change in equilibrium tomperature. Thus,

$\Delta T_{1}=\mid \Delta T_{0}$

where $\Delta T_{1}$ is the total change in equilibrium surface temperature with all foedback contributions included, and $\Delta T_{0}$ is the no-feedbecks forcing. Since $\Delta T_{1}$ consists of both forcing and feedback components, we can write

$\Delta T_{1}=\Delta T_{0}+\Delta T_{\text {rencounde }}$

If we assume that the foedback contributions can be separated into portions that are identifiable with specific foedback procosses, then

$$
\Delta T_{\text {fencoude }}=\Delta T_{w}+\Delta T_{c}+\Delta T_{q}+\Delta T_{a}+\ldots
$$

where the subscripts $w, c, s, a$ designate the broad categorles of water vapor, cloud, snow/ice, and advective feedbacks, respectively. (The water vapor feedback, for example, may be subdivided further into specific $\Delta T$ components associated with changes in column amount, changes in vertical distribution, and lapse rate changes in the temperature profile.) By implication, the climate foedbacks are assumed ultimately to be temperature driven, and their response is assumed to be the total change in temperature that is encountered. Hence the relative strength of the feedback contribution of the th feedback process is given by the ratio

$g_{1}=\Delta T_{1} / \Delta T_{1}$

where $\Delta T_{1}$ denotes the equillbrium temperature change contribution (as obtained with the 2-D RCAM) for the specified change in the amount and/or distribution of the radiative constituent associated with the th feedback process. If follows, then, that the individual feedback strengths, $g_{1}$, are additive quantities, but feedback factors combine non-linearly as

$$
1 \cdot\left(1 \cdot \Sigma g_{1}\right)^{-1}
$$

Thus, with the help of a 2-D RCAM, this approach yields the latitudinal dependence of the radiative forcing and of the climate foedback sensitivity shown in Figure 1. Hansen of al. (1984) carried out a similar analysis using globally averaged annual average GCM output and running the GCM radiation code as a 1-D RCM to determine the global average feedback sensitivity of the GISS GCM for the $2 \%$ solar constant and the doubled $\mathrm{CO}_{2}$ experiments. While this approach yields only information on the GCM global foedback sensitivity, the analysis is greatly simplified in that globally averaged, the advected energy fluxes are zero, and knowledge of the annually averaged latifudinal solar zenith angle Jependence is not required.

\section{Model Results}

The upper panels in Figure 1 show the latitudinal dependence of radiative forcing, $\Delta T_{0}$, and of the principal foedback components for the $2 \%$ solar constant and doubled $\mathrm{CO}_{2}$ experiments, respectively. Even though the radiative forcing (heavy red lines) is concentrated in the tropics for the solar constant change and is latitudinally uniform for $\mathrm{CO}_{2}$ forcing, the latitudinal temperature response of the GCM (heavy black lines) and the overall foedback contributions (multi-colored lines) of water vapor, snow/ ice, cloud, and advective feedbacks are remarkably similar for the two forcings. The green and yellow lines demonstrate the general ability of the 2-D RCAM to reproduce the actual GCM temperature changes in terms of annually averaged GCM climatological information. The green line represents the sum of individual $\Delta T_{1}$ responses computed separately, and the yellow line is the combined response, $\Delta T_{1}$ to all constituent changes computed together in the 2-D RCAM; the relatively small differences indicate that non-linearities in feedback interactions are comfortably small.

The bottom panels in Figure 1 show the magnitude and latiludinal variability of the individual components of the advective and water vapor feedbacks. These can be 

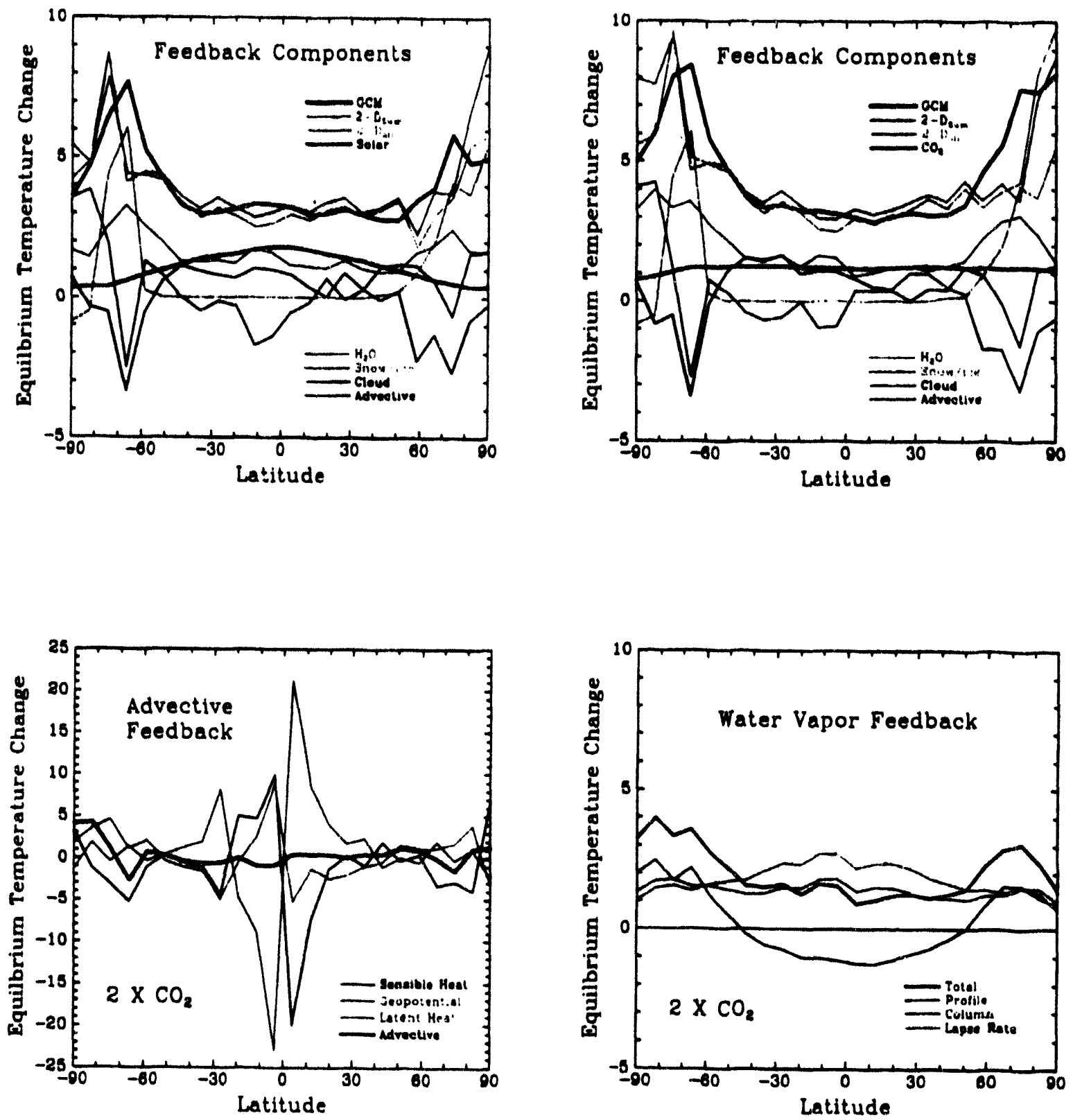

Flgure 1. The upper panels show GCM surface temperature response (heavy black) and radiative forcing (heavy red), for $2 \%$ solar constant and doubled $\mathrm{CO}_{2}$ experiments, respectively. The blue, grey, purple, and magenta lines represent feedback contributions to the equilibrium temperature change from water vapor, snow/ice, cloud, and advective feedbacks, respectively. The green line is the sum of individual $\Delta T$, responses computed separately, while the yellow line is the combined response, $\Delta T_{p}$ to all constituent changes taken together. The bottom panels show the individual components of the advective and water vapor feedbacks. 
separately analyzed and used as a diagnostic tool to investigate changes in dynamical energy transport and in the hydrological cycle in response to climate forcing perturbations. As expected, there is large-scale cancellation between the advective transports of geopotential onergy and of sensible and latent heat. Within the water vapor teedback components, feedback enhancoment is increased by changes in the vertical distribution of water vapor, which, in low to mid-latitudes, is largely canceled by the negative lapee rate foedback due to moist convection. However, at polar latitudes the change in lapse rate provides positive foedback.

\section{Conclusions}

By using the zonally averaged, annual average climatologies of the GCM experiments as input data to the 2-D RCAM (which uses the same radiation code as the GCM), we can closely reproduce the latitudinal dependence of the annual average atmospheric thermal structure and surface temperature changes that were obtained in GCM climate change simulations. Since the radiative input parameters of the 2-D RCAM can be changed at will, the equilibrium surface temperature change can be evaluated separately for each specified change in amount or vertical distribution of individual radiative constituents, thus defining the magnitude of Individual relative feedback strengths.

Water vapor feedback is strongly positive and roughly uniform with latitude. Positive feedback at low to middle latitudes is enhanced by the vertical shift in water vapor, which is largely canceled by the negative lapse rate feedback due to moist convection. Snow/ice albedo feedback is associated primarily with changes in sea ice and is confined to polar latitudes. Cloud feedback is strongly positive at low to middle latitudes, due primarily to adecrease in low clouds and an increase in cirrus. Negative cloud feedback occurs in the polar regions. Sensible, latent, and geopotential energy feedbacks are individually large, but tend to cancel each other. The net advected energy feedback is highly variable with latilude and is strongly anti-correlated with cloud foedback.

As demonstrated, a quantitative measure of GCMresponse to radiative forcing and the latitudinal dependence of foedback sensitivity can be obtained from 2-D RCAM analysis of annually averaged GCM climatologies from climate change experiments. This type of analysis can provide important diagnostic information regarding GCM performance and would be sultable for conducting intercomparisons of GCM feedback sensitivity. Such intercomparisons would help to clarity the reasons for the $1.7^{\circ}$ to $5.3^{\circ} \mathrm{C}$ range of $\mathrm{GCM}$ response to doubled $\mathrm{CO}_{2}$.

\section{References}

Cess, R. D., M.-H. Zhang, G. L. Potter, H. Barker, R. A. Colman, D. A. Dazlich, A. D. Del Genio, M. Esch, J. R. Fraser, V. Galin, W. L. Gates, J. J. Hack, J. T. Kiehl, A. A. Lacis, X.-Z. Liang, J.-F. Mahfouf, B. J. McAvanoy, V. P. Meleshko, J.-J. Morcrette, D. A. Randall, E. Roeckner, D. A. Sheinin, A. P. Sokolov, K. E. Taylor, W.-C. Wang, and R. T. Wetherald. 1993. Intercomparison of $\mathrm{CO}_{2}$ radiative forcing in general circulation models. Nature (submitted).

Hansen, J. E., A. A. Lacis, D. Rind, G. Russell, P. Stone, I. Fung, R. Ruedy and J. Lerner. 1984. Climate sensitivity: Analysis of feodback mechanisms. Climate Processes and Climate Sensitivity, eds. J. Hansen and T. Takahashi, Geophysical Monograph, 29, AGU, 130-163. 


\title{
Long-Pathlength Infrared Absorption Measurements of Line and Continuum Features in the 8- to 14- $\mu \mathrm{m}$ Atmospheric Window
}

\author{
T. J. Kulp and J. Shinn \\ Geophysics and Environmental Research Program \\ Lawrence Livermore National Laboratory \\ Livermore, CA 94550
}

\section{Introduction}

The accurate characterization of the latent infrared (IR) absorption in the atmospheric window regions continues to be an area of research interest for the global climate modeling community. In the window between 8 and $14 \mu \mathrm{m}$, this absorption can be attributed primarily to water vapor. It consists of 1) weak lines originating from the edge of the water vapor pure rotational band (at low wavenumbers) and the trailing P-branch of the $v_{2}$ rovibrational band (at the high-wavenumber boundary of the window) and 2) the water vapor continuum absorption.

The goal of our project is to improve our quantitative and physical understanding of both of these absorption processes. Specifically, our immediate aims are to fill in gaps in the experimental radiative transfer databases pertaining to the line parameters (i.e., line intensities and broadening coefficients) and to the self- and foreignbroadened water vapor continuum. There are many lines that have not been measured in the laboratory. Similarly, there are many conditions of atmospheric importance under which the continuum absorption has not been determined.

To accomplish our goals, we are making long-pathlength absorption measurements using a Fourier transform infrared spectrometer (FTIR) (for the continuum and line measurements, at low resolution) and a tunable diode laser absorption spectrometer (TDLAS) (for the line measurements, at high resolution). These measurements are being made on gas samples contained in a 400-m maximum pathlength Horn Pimentel multipass cell that was designed and constructed for this project. Accomplishments of our project in the past year are summarized in the following sections.

\section{Completion of the Experimental Apparatus}

The experimental apparatus used to make our measurements consists of the multipass cell and its chamber, the FTIR spectrometer, the TDLAS system, and the necessary data collection apparatus. This equipment was assembled and the chamber was constructed during the first year of our project. The primary thrust of that effort was to design and fabricate the cell and chamber. Special attention was paid to ensuring the mechanical stability of the optics and the ability of the cell to contain stable water vapor samples.

To meet these goals, the chamber was constructed from polished stainless steel and mounted in a way to mechanically isolate it from the cell optics. For a summary of this design, the reader is referred to the last Science Team proceedings (Kulp and Shinn 1992). At the time of that meeting, the chamber construction had not yet been completed. Since then, we accepted delivery of the chamber and installed it in our lab.

Following this, our efforts were directed toward characterizating the stability of the charnber and of the BOMEM MB100 FTIR spectrometer and our TDLAS system. Attention was also pald to developing our sample handling methods, including heating the cell optical surfaces and filling and evacuating the chamber. These efforts extended through March 1992, whereupon our water vapor absorption experiments began. 


\section{Water Vapor Absorption Measurements}

During the past year, IR absorption measurements were made using both FTIR spectroscopy and tunable diode laser absorption spectroscopy (TDLAS). The FTIR measurements are targeted toward the measurement of both the lines and the continuum, while the TDLAS measurements are made with the goal of determining line parameters only. Although the FTIR measurements provide line intensity information, they are not made at a sufficient resolution to resolve the lines. Thus, the TDLAS data give information about lineshapes and widths. In the following two sections, progress in both these measurements will be discussed.

\section{FTIR Measurements of Lines and Continuum in the 8- to 14- $\mu \mathrm{m}$ Window}

The FTIR measurements made during the past year began with the collection of room temperature spectra of pure water vapor absorption. They have provided information about the window region line intensities (albeit at the $1 \mathrm{~cm}^{-1}$ resolution of the MB100) and the self-broadened continuum at room temperature. Measurements were made by coupling the modulated beam from sideport of the MB100 into the chamber and directing the exit radiation from the cell through the MB100 sample area and onto its detector using a set of plane mirrors. Data were collected by first recording a reference spectrum with the chamber evacuated and then filling the chamber with the sample and recording a sample transmission spectrum. These spectra were rationed to determine the water absorption spectrum.

At the present time, data have been collected using water vapor samples at several pressures. Figure 1 contains a representative spectrum of the entire window region, obtained at a pressure of 8.1 torr, a temperature of $297 \mathrm{~K}$ and a pathlength of $252 \mathrm{~m}$. It represents an average of four runs (i.e., four different fills of the chamber), each being an average of 150 scans. The spectral resolution is $1 \mathrm{~cm}^{-1}$, apodized, using the BOMEM cosine apodization function. Note the line spectra and the underlying continuum. The scan near zero absorbance is a spectrum in which pure nitrogen was introduced at a pressure of 8.1 torr, rather than water vapor. The purpose was to measure the system (cell + spectrometer) drift during the time of a measurement.
Figures 2a-c contain enlargements of three regions of the spectrum in Figure 1, magnified to better show the line spectra. Figures 3a-c show these regions further magnified to feature the continuum. The nitrogen spectrum is also shown in Figures 2 and 3. The nitrogen spectra in Figures 3a-c shows the baseline drift of the system to be less than $10^{-3}$ absorbance units.

Our data are being compared with calculations generated by the Line-by-Line Radiative Transfer Model (LBLRTM) developed by Atmospheric and Environmental Research (AER, Cambridge, Massachusetts). These comparisons are being done in collaboration with Tony Clough of AER. Examples of these calculations are also plotted in Figures $2 a-c$ and $3 a-c$. The spectrum was calculated by $A E R$ assuming a homogeneous horizontal path containing only water vapor under the conditions of our measurement. The line-by-line calculations were convolved with an instrumental response function (assuming the BOMEM cosine apodization) to generate the experimental spectrum.

Using LBLRTM and our data, we were able to generate an empirical self-broadened continuum. This was accomplished by using LBLRTM to calculate the window region lines-only contribution (assuming line wings out to $20 \mathrm{~cm}^{-1}$ from line center) and subtracting this from our measured window region spectrum. This is plotted in Figure 4. Also plotted there is the LBLRTM continuum and an empirical self-broadened continuum calculated using the self-broadening coefficients measured by Burch ot al. (1981a,b) and Burch and Alt (1984). The Burch values represent an empirical fit to ten data points within the range covered by that curve.

In general, the comparison of calculations and measurements indicate that errors remain in the HITRAN 92 line parameter database We have also compared AERI - LBLRTM simulation residuals (obtained from Tony Clough) with our residual from LBLRTM and were able to correlate line errors in both sets of measurements. This further substantiates the need to correct these lines. The fit to the continuum is fairly good and tends to support the Burch measurements (over other past measurements that differed from his). It should be noted, however, that our data should be considered preliminary at this time and that we are in the process of testing its accuracy. That accomplished, we will move on to the measurement of the foreign-broadened continuum and to measurements at lower temperatures. 


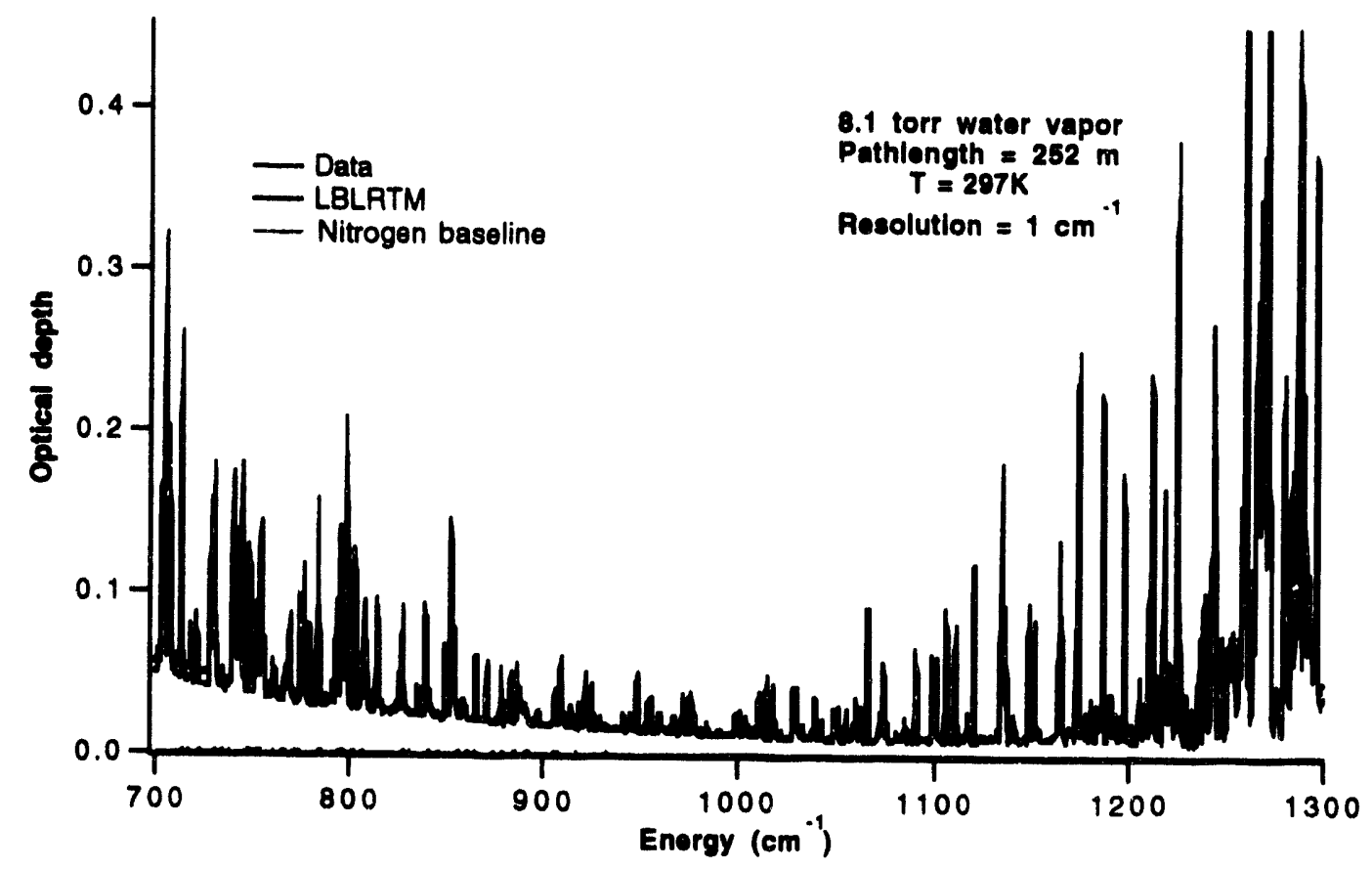

Figure 1. Water vapor spectrum of the window region obtained under conditions indicated, showing entire region between 700 and $1300 \mathrm{~cm}^{-1}$ at lowest magnification.

\section{Water Vapor Line Absorption Measurements}

TDLAS absorption measurements of a number of weak lines were carried out between March and August of 1992. Table 1 lists the lines of which spectra have been taken and the conditions under which the measurements were made. Spectra of each line were made under a number of nitrogen pressures to determine their nitrogen-broadening coefficients. As with the self-broadened continuum data, we are now preparing a publication on the line data acquired to date. TDLAS line measurements will continue for the remainder of this fiscal year. In the proposed continuation of our work, we hope to accelerate our line measurements using a high resolution FTIR (BOMEM DAB) that will be made available to us and used in concert with the TDLAS system.

\section{Future Work}

In our future work, we will continue our line and continuum measurements to expand our understanding of the window region absorption to other atmospheric conditions. In particular, we will begin the foreign-broadened continuum measurements and will also begin lower temperature measurements. Initially, we will use our existing FTIR and TDLAS instruments.

We are also, however, proposing to improve ourcapabilities in two ways: 1) using a high resolution FTIR (BOMEM DAB) that will be made available to accelerate our line measurements and 2) incorporating a cavity-ringdown spectrometer (CRS) into our apparatus. CRS is a highly sensitive laser-based method (O'Keefe and Deacon 1988; Ramponi et al. 1988) that can measure absorption in relatively small cells with an effective total pathlength of up to $6 \mathrm{~km}$. We propose to use CRS to improve the sensitivity and precision of our continuum measurements, particularly at low temperatures.

Both new approaches will be implemented in our existing chamber in such a way that they can operate simultaneously with the existing approaches. This will allow validation of the new methods against the current ones. 


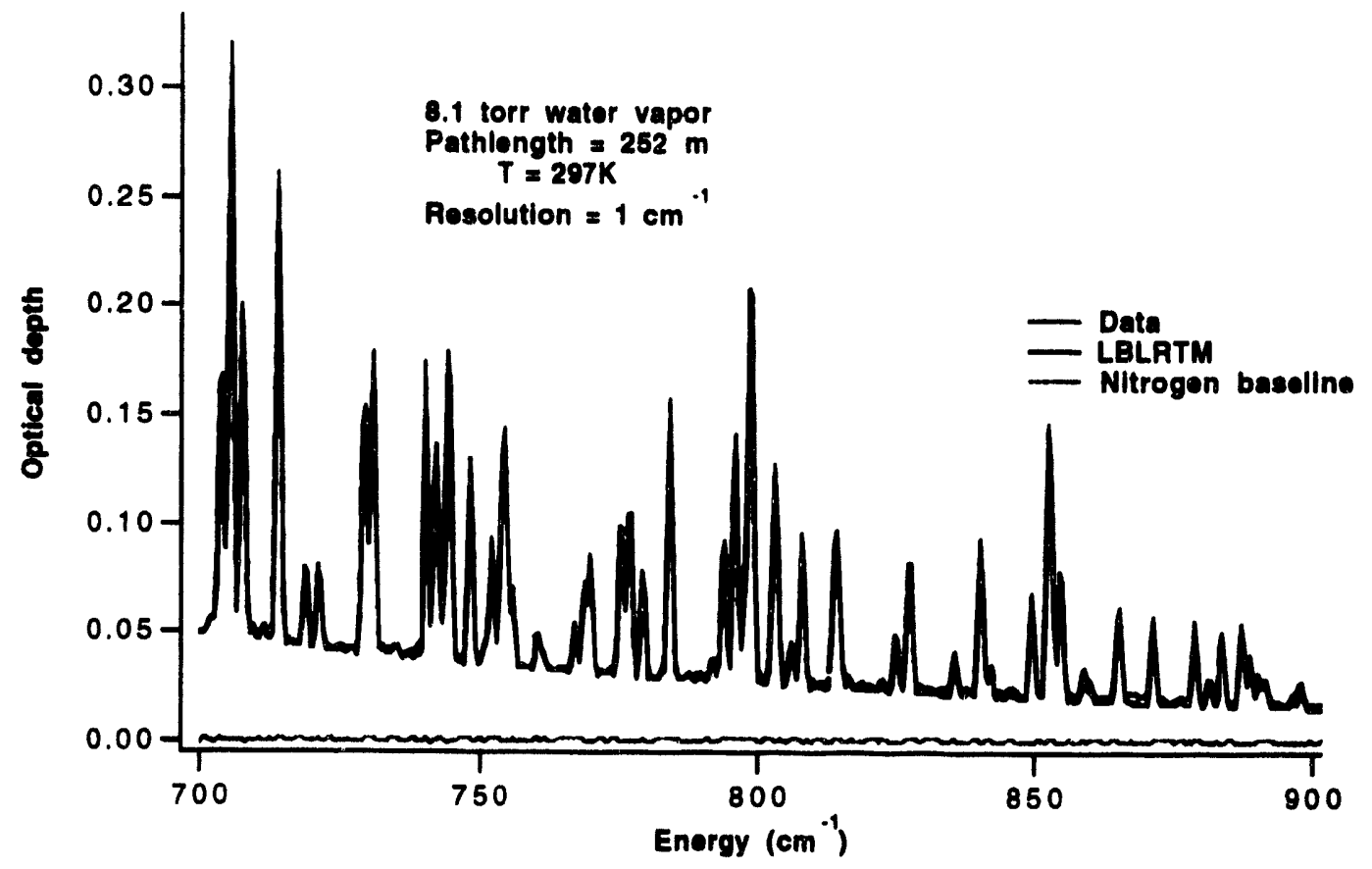

Flgure 2a. Portion of the spectrum between 700 and $900 \mathrm{~cm}^{-1}$.

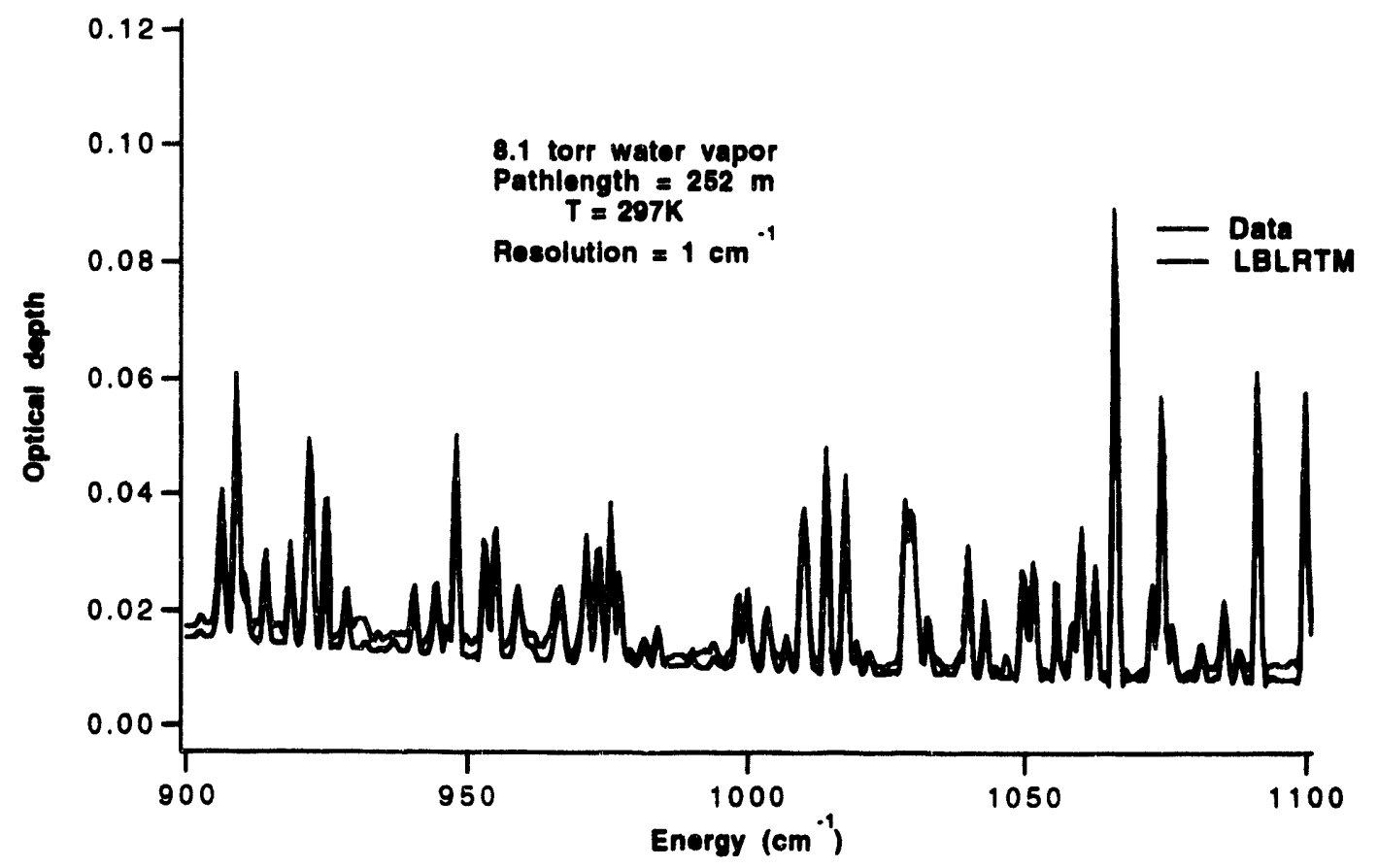

Figure 2b. Portion of the spectrum between 900 and $1100 \mathrm{~cm}^{-1}$. 


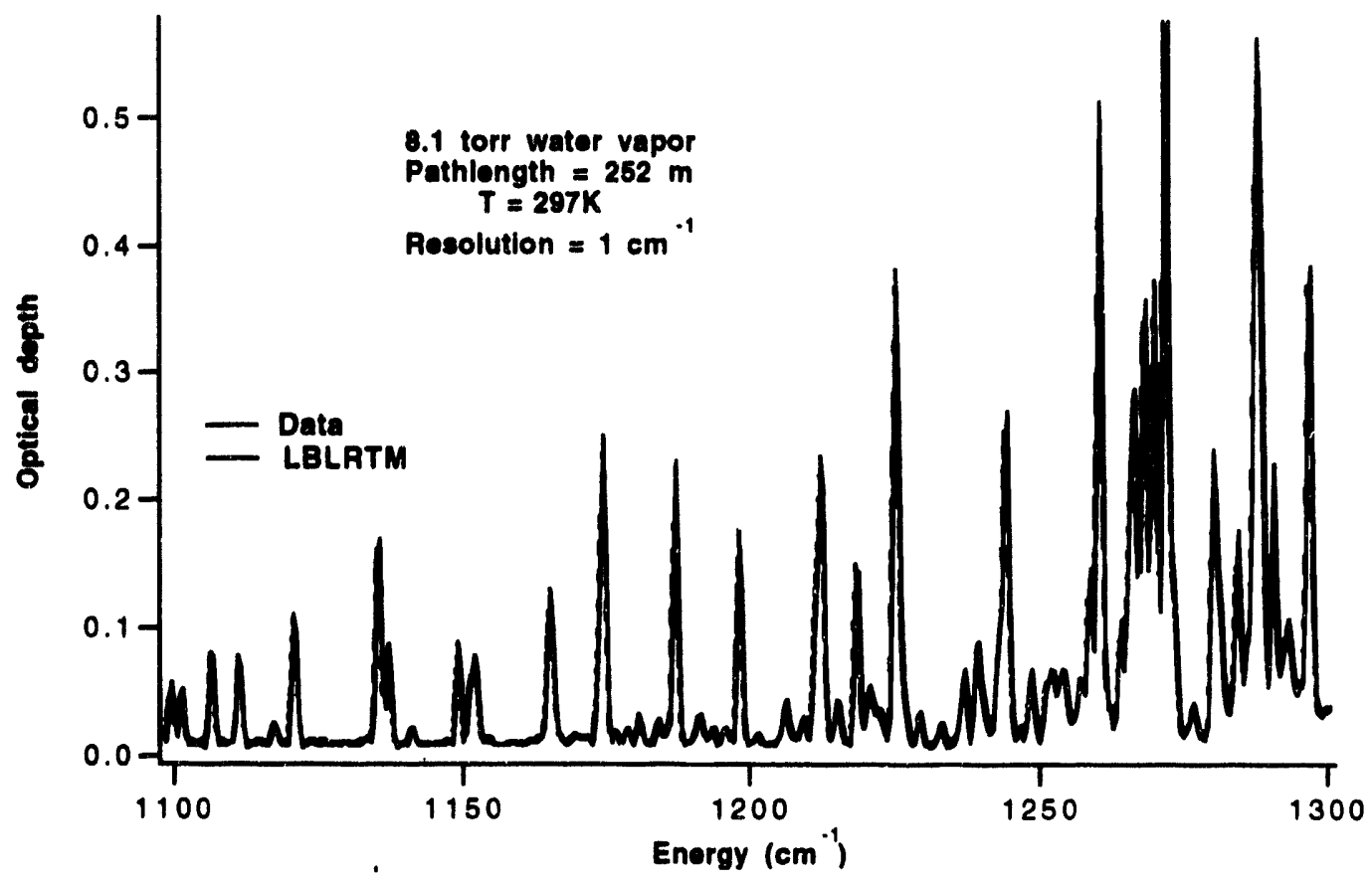

Figure 2c. Portion of the spectrum between 1100 and $1300 \mathrm{~cm}^{-1}$.

Figure 2. Water vapor spectrum of Figure 1 magnified to show lines. The spectrum is overlaid with the LBLRTM simulation run for the experimental conditions.

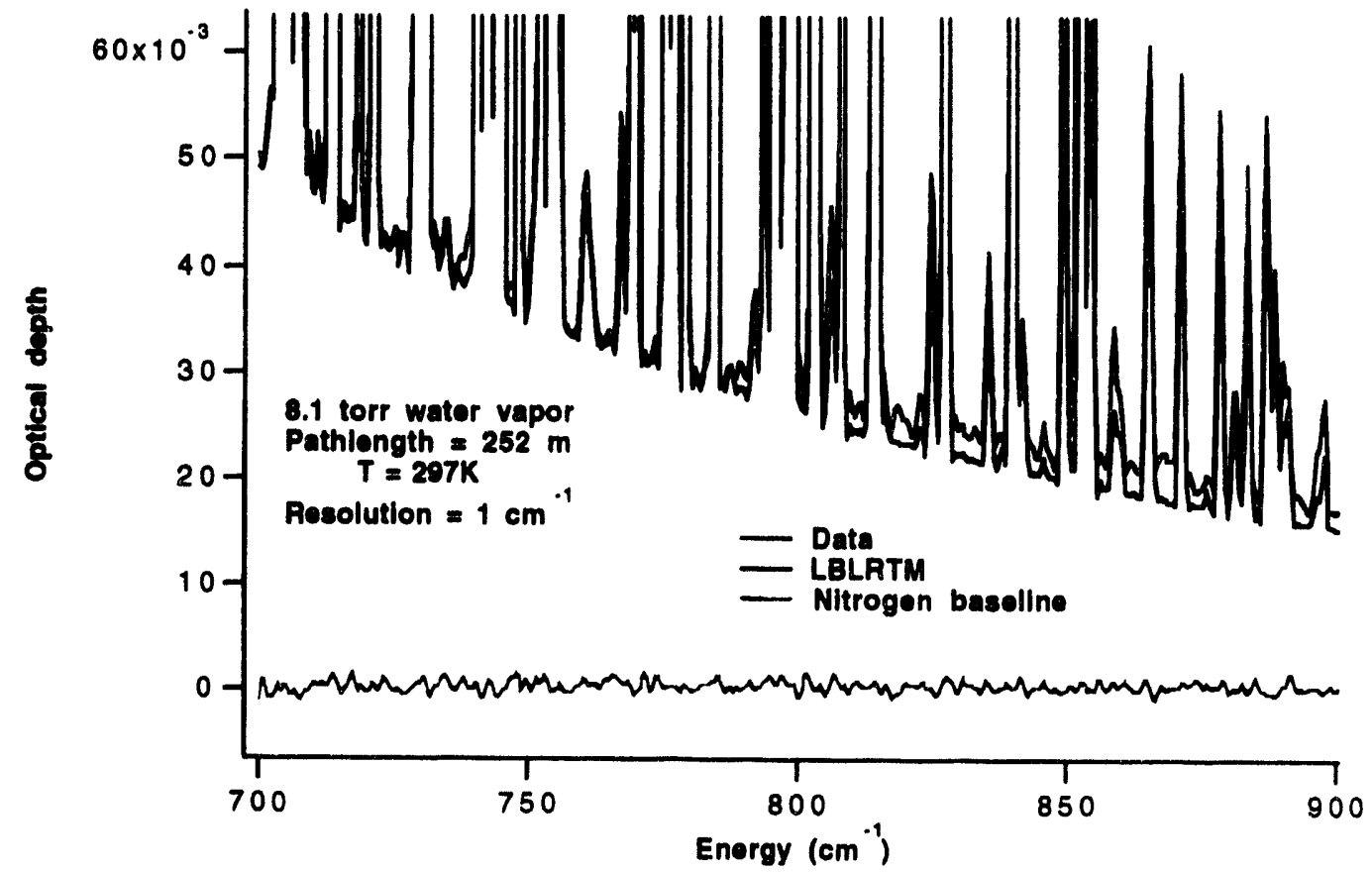

Figure 3a. Portion of the spectrum between 700 and $900 \mathrm{~cm}^{-1}$. 


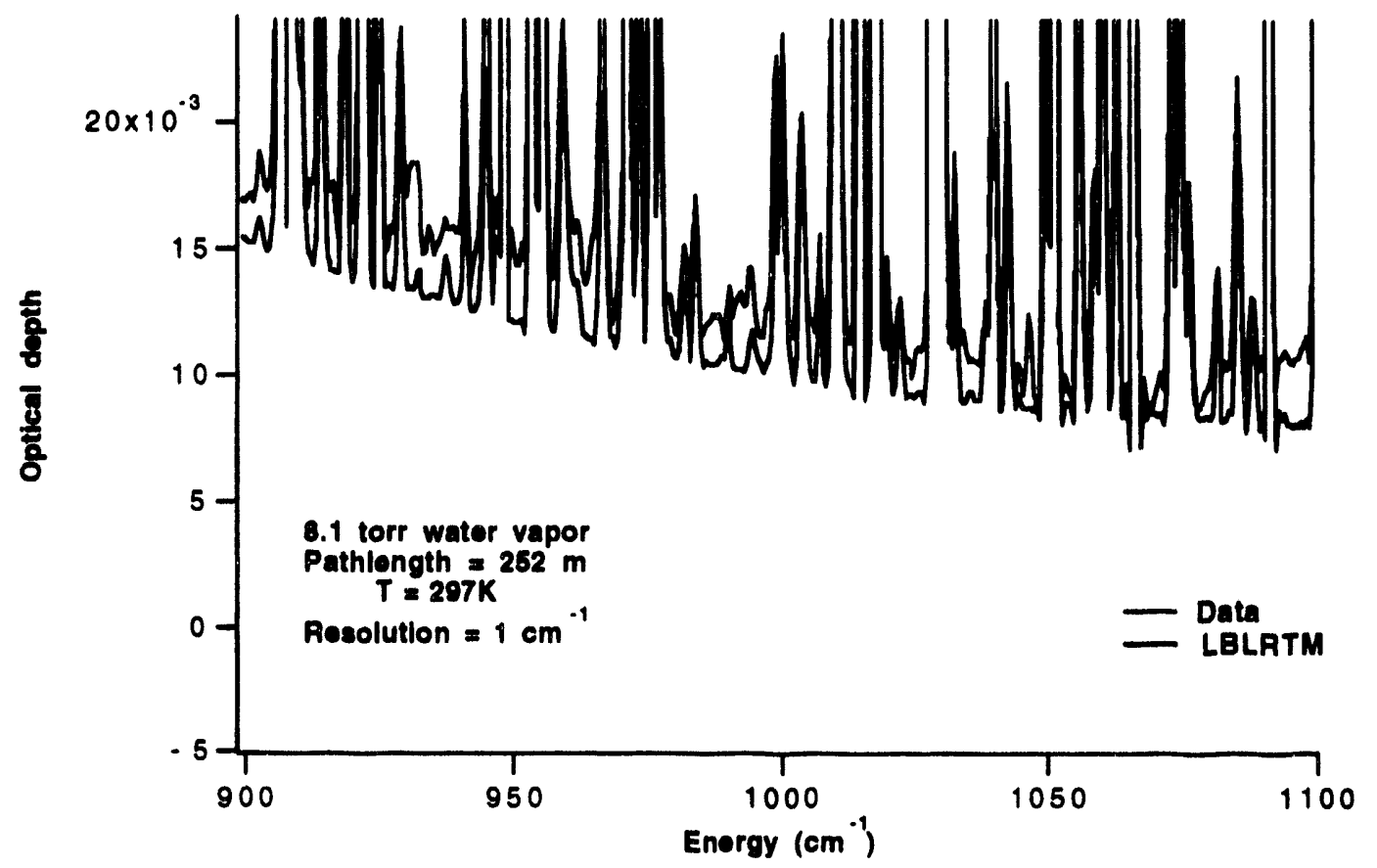

Figure 3b. Portion of the spectrum between 900 and $1100 \mathrm{~cm}^{-1}$.

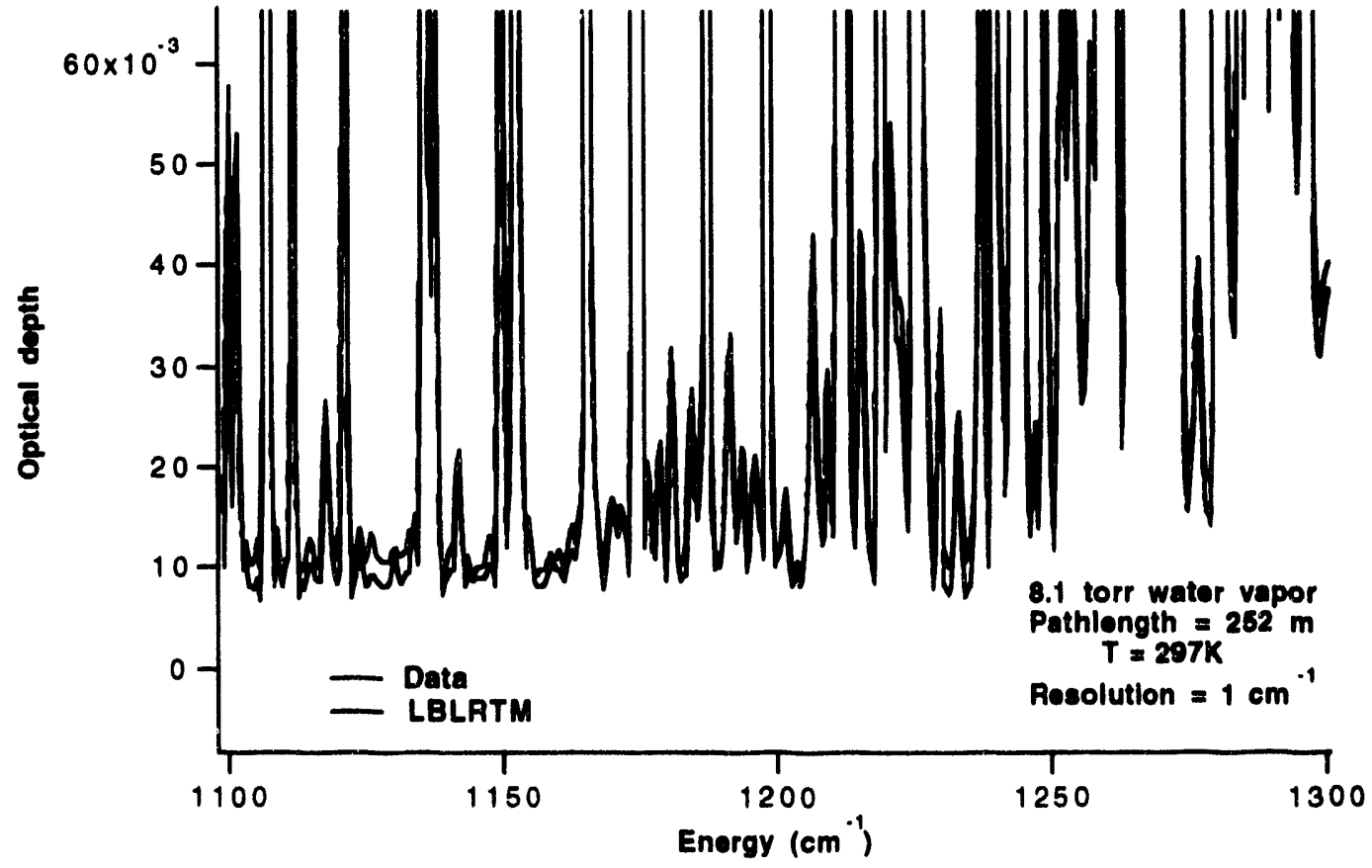

Figure 3c. Portion of the spectrum between 1100 and $1300 \mathrm{~cm}^{-1}$.

Flgure 3. Water vapor spectrum of Figure 1 at highest magnification to show continuum. The spectrum is overlaid with the LBLRTM simulation run for the experimental conditions. 


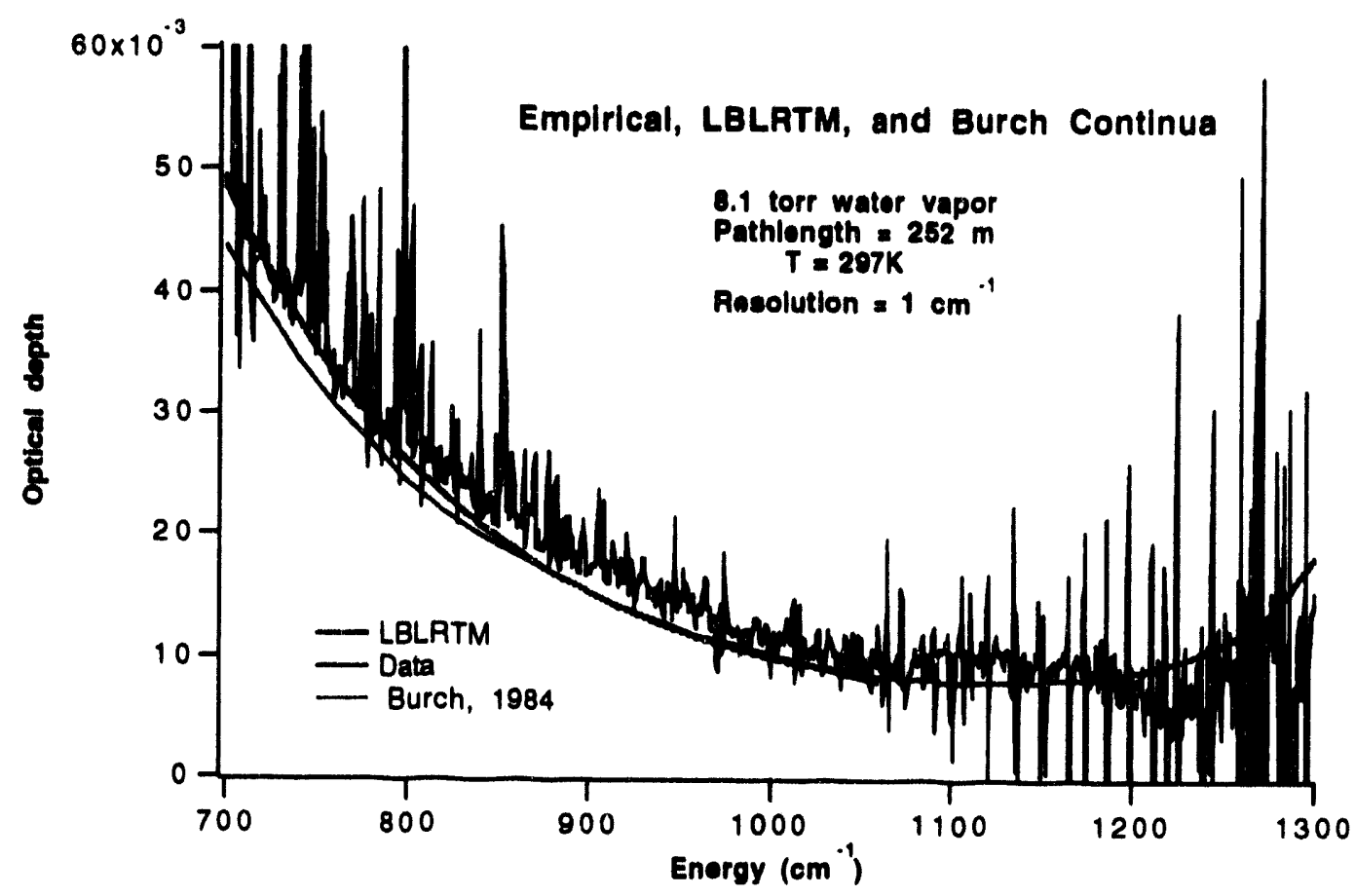

Figure 4. Plot of the empirical continuum derived from the data in Figure 1. Also overlaid on this is the LBLRTMcontinuum and the continuum derived for these conditions from the data of Burch and Alt (1984).

Table 1. Listing of lines measured during the existing ARM project and the conditions of the measurements.

\begin{tabular}{rr} 
Energy $\left(\mathrm{cm}^{-1}\right)$ & $L(\mathrm{~m})$ \\
\cline { 3 - 3 } 922.142 & 180 \\
948.260 & 108 \\
1000.2910 & 252 \\
1003.5673 & 252 \\
1003.6887 & 252 \\
1007.2390 & 252 \\
1010.8132 & 252 \\
1011.6211 & 252 \\
1011.6418 & 252 \\
1014.4751 & 252 \\
1019.6611 & 252 \\
1028.2706 & 180 \\
1028.3125 & 180 \\
1028.6842 & 180 \\
1029.4977 & 180 \\
1029.6974 & 180 \\
1032.6844 & 180
\end{tabular}

\begin{tabular}{r}
$P\left(N_{2}\right.$, tor $)$ \\
\hline $0-300$ \\
$0-293$ \\
$0-40$ \\
$0-80$ \\
$0-90$ \\
$0-70$ \\
$0-400$ \\
$0-60$ \\
$0-60$ \\
$0-400$ \\
$0-70$ \\
0 \\
0 \\
$0-200$ \\
$0-200$ \\
$0-200$ \\
$0-80$
\end{tabular}

\begin{tabular}{r}
$P\left(H_{2} \mathrm{O}\right.$, lort $)$ \\
\hline $1.20-6.00$ \\
$2.17-11.07$ \\
$1.49-6.96$ \\
$3.10-7.22$ \\
$3.10-7.22$ \\
$2.04-8.01$ \\
$2.95-6.96$ \\
$1.95-6.11$ \\
$1.95-6.11$ \\
$0.93-4.95$ \\
$1.56-6.99$ \\
$0.91-7.56$ \\
$0.91-7.56$ \\
$2.97-9.04$ \\
$1.45-5.17$ \\
$1.45-7.44$ \\
$1.39-9.00$
\end{tabular}

\begin{tabular}{r}
$N$ \\
\hline 8 \\
9 \\
8 \\
9 \\
9 \\
11 \\
11 \\
6 \\
6 \\
9 \\
11 \\
5 \\
5 \\
8 \\
12 \\
14 \\
12
\end{tabular}

$L=$ pathlength; $p=$ pressure; $N=$ number of measurements 


\section{References}

Burch, D. E. et al. 1981a. Continuum Absorption by Atriospheric $\mathrm{H}_{2} \mathrm{O}$. Proc. Soc. Photo-Instrum. Eng. 277:2839.

B.rch, D. E. of al. 1981b. Continuum Absorption by Atmospheric $\mathrm{H}_{2} \mathrm{O}$. Repont AFGL-TR-81-0300 by Ford Aeronutronic to Air Force Geophysics Laboratory, Hanscom Air Force Base, Massachusetts.

Burch, D. E., and R. L. Alt. 1984. Continuum Absorption by $\mathrm{H}_{2} \mathrm{O}$ in the $700-1200 \mathrm{~cm}^{-1}$ and $2400-2800 \mathrm{~cm}^{-1}$ Windows. Report AFGL-TR-84-0128 to the Air Force Geophysics Laboratory, Hanscom Air Force Base, Massachusetts.

Kulp, T. J., and J. Shinn. 1992. Spectroscopic Study of Water Vapor Absorption in the 8- to $14-\mu \mathrm{m}$ Atmospheric
Window: Measurement of Now Line and Continuum Parameters and Investigation of Far-Wing Phenomena. Proceedings of the Second Atmospheric Radiation Measurement (ARM) Science Team Meoting. CONF9110336, United States Department of Energy, Office of Health and Environmental Research, Washington D.C.

O'Keofe, A., and D.A.G. Deacon. 1988. Cavity Ring-Down Spectrometer for Absorption Measurements using Pulsed Laser Sources. Rev. Scl. Instrumen. 59:2544.

Ramponi, A. J., F. P. Milanovich, T. Kan, and D. Deacon. 1988. High Sensitivity Atmospheric Transmission Measurements using a Cavity Ringdown Technique. Appl. Opt. 27:4606. 
Cloudy Skies 


\title{
Radiative Properties of Nonunlform Clouds
}

\author{
P. H. Daum and L. I. Kloinman \\ Environmental Chemiatry Division \\ Brookhaven National Laboratory \\ Upton, NY 11973 \\ G. L. Stephens \\ Colorado Stato Univereity \\ Department of Atmospheric Scionces \\ Fort Collins, CO 80523
}

We report here preliminary results from our first two experiments for the Almospheric Radiation Monitoring (ARM) Program. The first experiment is mechanistic in character and focuses on testing general circulation models (GCM) parameterizations of cloud radiative properties. In this experiment, Cloud and Radiation Testbed (CART) measurernents of solar flux and integrated liquld water content are used to obtain an empirical relationehipbetween the diffuse transmission of stratus clouds and the liquid water path (LWP). Dittuse cloud transmission is defined as

$$
T_{c}=F_{c t} / F_{c t}
$$

where $F_{\text {cb }}$ is the downwelling shortwave flux at cloud base, and $F_{f f}$ is the downwelling shortwave flux at cloud top. A plot of the experimentally derived cloud transmittance vs liquid water path is shown in Figure 1 for several days of data trom the Southern Great Plains CART site in Oklahoma. Each point on this plot consists of a 5-minute average of the relevant data. The solid line is a plot of the empirically derived relationship between LWP and cloud transmittance from Derr et al. (1990).

The shortwave fluxes necessary to compute the cloud transmission in Figure 1 were estimated in the following way. Because no measurements of $F_{c t}$ were made, $F_{c t}$ was estimated from measurements of the downwelling shortwave flux at the surface under clear sky conditions. Clear sky conditions chosen for these estimates were restricted to days adjacent to those when the cloudy sky measurements were made. Since $F_{\mathrm{ct}}$ varies with time of day, measurements of $F_{c t}$ were time-matched with radiation measurements mede during cloudy periods. $F_{c t}$ was assumed to equal the measured shortwave flux at the surfaco. Estimates of $F_{c t}$ and $F_{c b}$ made in this way introduce some orror; the extent of this error la a subject for future calculations. An alternate procedure would be to calculate $F_{e t}$ and $F_{c b}$ using avallable fiold data and a radiative transter model.

It is apparent from Figure 1 that the data follow the general functional form of the empirical parameterization developed by Dorr of al. (1990). However, there is significant scatter about the line that best describes the Derr relationship between LWP and cloud transmittanco, and there appears

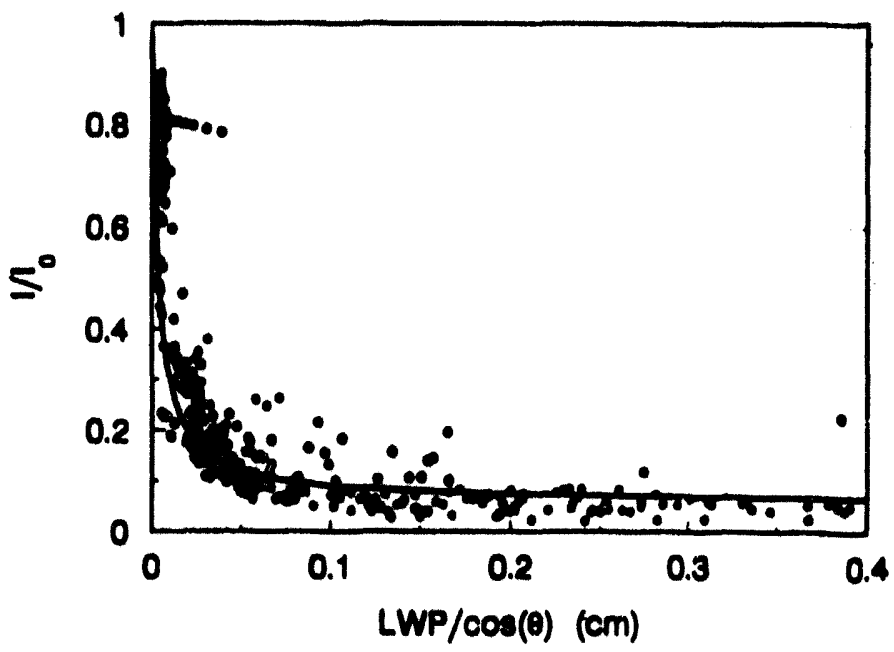

Figure 1. The diffuse solar tranemittance as a function of liquid water path derived from CART data. The solld line is the empirical relationship of Dorr et al. (1990). 
to be some bles in the data. While if is premature to draw conclusions on the basis of such a limited data set, eeveral comments regarding this variablity and blas aro in order. For a fixed llquild wator path, variations in the equivalent redlus of the cloud droplote can influenca the tranemittance. Larger numbers of emallor drope for the same liquid wator path load to decreased cloud tranemittance. Thus, knowledge of the droplet size spectrum may be neceseary to reduce the scatter and blas in the plot. Small scab variabilly in the liquid wator path may also contributo to the scattor. We also note that measurements of LWP from the microwave radiomoter currently used at the CART site is only marginally useful for exploring the LWP.T. parametorization at low LWPs where $T_{c}$ is changing mosi rapidly. Subsequentially, we plan to compare the experimentally derived cloud transmittance to the tranemittances calculated using now paramotorization scheme developed in this project and to extend this type of analysis to relationshipe such as between cloud emiselvity and LWP, albodo and LWP.

Our second experiment is aprototype of asot of experiments in which we seok to explore broad climatological relations between radiative properties of the atmosphere and other key atmospheric parameters, both as a general test of parameterizations and as a way of introducing now diagnostic analysis procedures which can be used to test global climate models. In our first effort, we are evaluating a simple graybody relationship (Stephens and Greenwald 1991) between the downwelling longwave flux at the surface, $F_{g}$ the outgoing longwave flux at the top of the atmosphere, $F_{\infty}$; and the column integrated water vapor content, $\omega, v i z$.,

$F_{0}=F_{\infty}(c+d w)$

Stephens et al. (1993) used an analysis system developed by Slingo and Wobb (1992), (Simulation and Analysis of Measurements from Satellites using Operational a NalysesSAMSON) to establish the constants of the relationship. This model incorporates data from the European Centre for Medium-Range Weather Forecasts (ECMWF) operational analyses into a radiative transfer model to simulate the radiation fields. The results of this simulation gave values for the constants $c$ and $d$ and suggested that simultaneous satellite observations of $\omega$ and clear sky $F_{\text {. }}$ may provide a way of predicting downwelling longwave flux af the eurface with an eccuracy comparable to the best current moasurements of these fluxes $\left(15 \mathrm{~W} / \mathrm{m}^{2}\right)$.

Independent evaluation of the flux ratio relationship is difficult eince thore are fow if any concurrent mosesuremonts of $F_{Q} F_{w}$ and $w$ over the ccean where satellite retrlevals of wuaing SSMImoseurements are poseible (e.9., Greenwald of al. 1993). Our offorts so far uee data collocted during coveral oceanographic cruless, the RN Alliance cruises in the Moditorranean in the summor of 1890 and the fall of 1991, and the USCGC cruise in the polar sea in the summer of 1992. The relevant data from these crulses consiat of moasurements of the downwelling longwave radiation at the surface, and concurrent soundings of water vapor and tomperature. Unfortunately, no satelitio measurements of $F_{\infty}$ are avallable so, for purposes of this analysis, $F_{\infty}$ ls calculated uaing a radiative transfor model.

Our evaluation consists of three steps:

1. The $F$ calculated from the radiative transfer model using the measured water vapor and tomperature profiles is compared with the observed $F_{Q}$.

2. The flux ratio $F_{g} / F_{\text {. calculated using the radiative }}$ transfor model and the experimental soundings is compared with the relationship derived by Stephens ot al.

3. The relationship $F_{\theta} / F_{\infty}$ determined from the measured values of $F_{p}$ and $\omega$, and $F_{\infty}$ calculated from the radiative transfer model is compared with the Stephens of al. relationship.

The first two steps in the evaluation are tests of the radiative transfer model's ability to calculate the fluxes correctly. The third step in the evaluation is a direct test of the relationship derived by Stephens of al., albeit with calculated values of $F_{\infty e}$. Figures $2 a$ and $2 b$ show the results of this ovaluation.

In Figure 2a, we see that the observed and calculated values of $F$ agree quite well, with an average deviation for all of the data on the order of $10 \mathrm{w} / \mathrm{m}^{2}$. This result gives us confidence that the radiative transfer model is correctly calculating the fluxes. In Figure 2b, we show the results of 


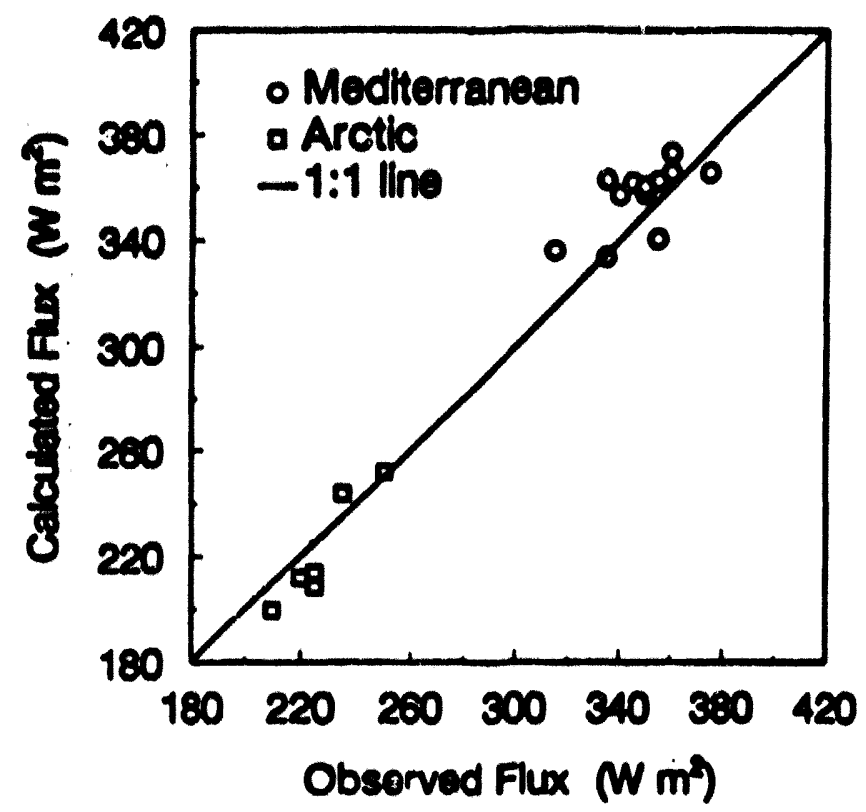

Figure 2a. Comparison of calculated and observed downwelling longwave fluxes.

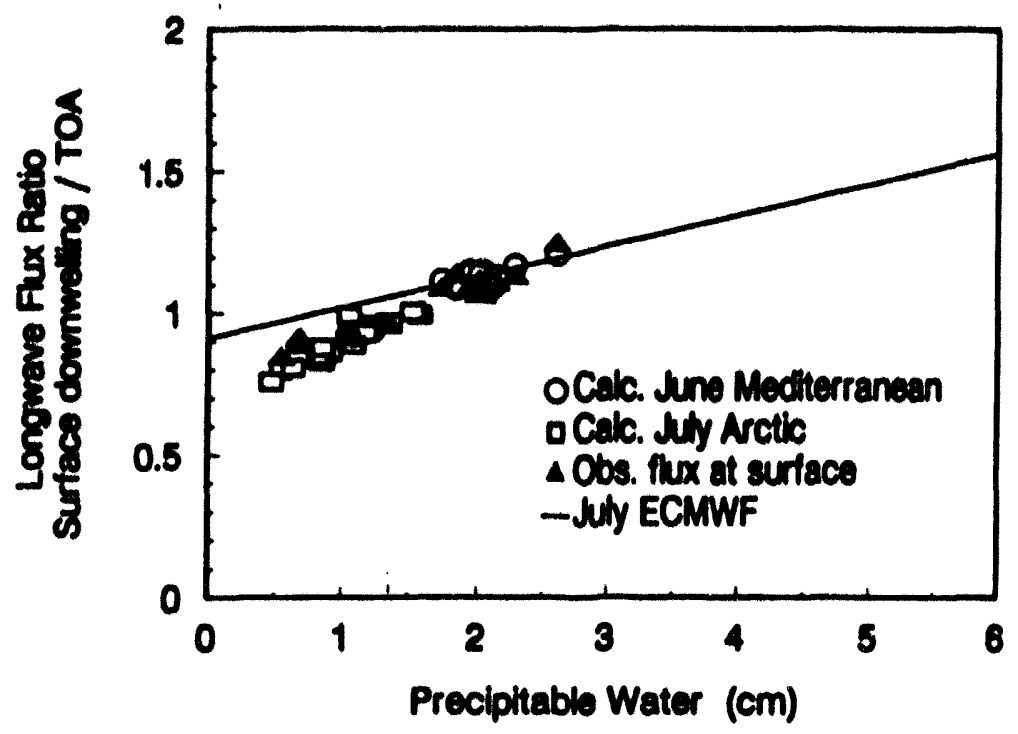

Flgure 2b. $F_{d} / F_{\infty}$ calculated using the radiative transfer model and the experimental soundings and using the measured values of values of $F_{0}$ and $\omega$ and $F_{\infty}$ compared to the Stephens et al. relationship. 
the calculations described in steps 2 and 3 above superimposed on a plot of the relationship computed from the ECMWF data. Again, we obeerve generally good agreement including the ratio's curve of growth at smaller values of $\omega$. The cause of this curvature is the subject of current investigation. These prellminary results are very oncouraging, and we plan to continue this analysis using CART data. Of particular interest are data from the Tropical Western Pacific locale because of the high values of $\omega$ at that location.

\section{References}

Derr, V. E., R. S. Stone, L. S. Fedor, and H. P. Hanson. 1990. A parameterization for the shortwave transmiseivity of stratiform water clouds based on empirical data and radiative transfer theory. J. Atmos. Scl. 47:2774-2783.
Greenwald, T. J., G. L. Stephens, T. H. Vonder Haar, and D. L. Jackson. 1993. A physical retrieval mothod of liquid water over the global oceans using SSMI observations. J. Geophys. Res. (to be published).

Slingo, A., and M. J. Wobb. Simulation of clear-sky outgoing longwave radiation over the oceans using operational analyses. Quart. J. Roy. Mot. Soc. (10 bo publishod).

Stephens, G. L., A. Slingo, and M. Wobb. 1983. On measuring the greenhouse effect of the earth. High Spectral Resolution Infrared Remote Sensing for Earth's Weather and Climate Studios, eds., A. Chedin and M. T. Cahine. NATO ASI Sories. 


\title{
A Stochastic Formulation of Radiative Transfer in Clouds
}

\author{
G. L. Stephens, P. M. Gabriel, K. F. Evans, and D. Duda \\ Colorado State University \\ Department of Atmospheric Science \\ Ft. Collins, CO 80523
}

\section{Introduction}

The research conducted as part of this project breaks down into three broad areas:

- deterministic radiative transfer

- remote sensing

- stochastic radiative transfer.

The approach pursued in this research employs different forms of radiative transfer models in one, two, and three dimensions in an attempt to understand radiative transfer in clouds with realistic spatial structure and to determine the key geometrical parameters that influence this transfer. A key focus is understanding the relative importance of these geometrical effects in contrast to the microphysical effects of clouds. The main conclusion is that geometry has a profound influence on all aspects of radiative transfer and the interpretation of this transfer.

\section{Deterministic Radiative Transfer}

Our research on radiative transfer has produced three different formulations and solutions of the radiative transfer problem. The first is an efficient Monte Carlo model (O'Brien 1992) that has been used as a benchmark for testing the other two numerical models. The two different numerical solutions are in excellent agreement with Monte Carlo results. These models are discussed in detail by Gabriel of al. (1993) for the Fourier-Riccatl method and Evans (1993) for the Spherical Harmonic Spatial Grid method.

The Fourier-Riccati model was used to study the effects of geometry on solar heating rate. The essential conclusions of this work are given in Figure 1, showing the horizontal disposition of the heating at different depths in the cloud compared with the plane parallel heating. The heating is highly distributed and significantly exceeds the plane parallel values in the densest regions of the cloud. The two panels differ in the microphysics assumed in the simulation. Clearly, both microphysics and geometry affect the heating in important ways.

\section{Remote Sensing}

We also examine the effects of geometry on spectral reflectance and, using procedures published in the literature, interpret these effects in terms of retrieved optical properties of clouds. An example of this work is presented in Figure 2, which shows the bispectral plot of radiances derived from a simulation of radiative transfer through a 2D variable cloud. The particle size distribution was fixed in this cloud, and only number concentration was varied (thus, the effective radius was also fixed at all levels).

The scatter of points of Figure 2 is a direct result of the geometrical influences on spectral reflectances. This scatter is erroneously interpreted as changes in both optical depth and effective radius, the former varying by as much as $20 \%$ and the latter by as much as $30 \%$ to $50 \%$.

\section{Stochastic Radlative Transfer}

These and many other studies clearly indicate that geometrical factors profoundly influence the transfer of radiation through clouds. It is unlikely that all of the relevant scales of spatial variability will be resolved in climate models, or even cloud models. Furthermore, it is the mean radiative quantities and not the details of the radiation field that are important for the climate system. 

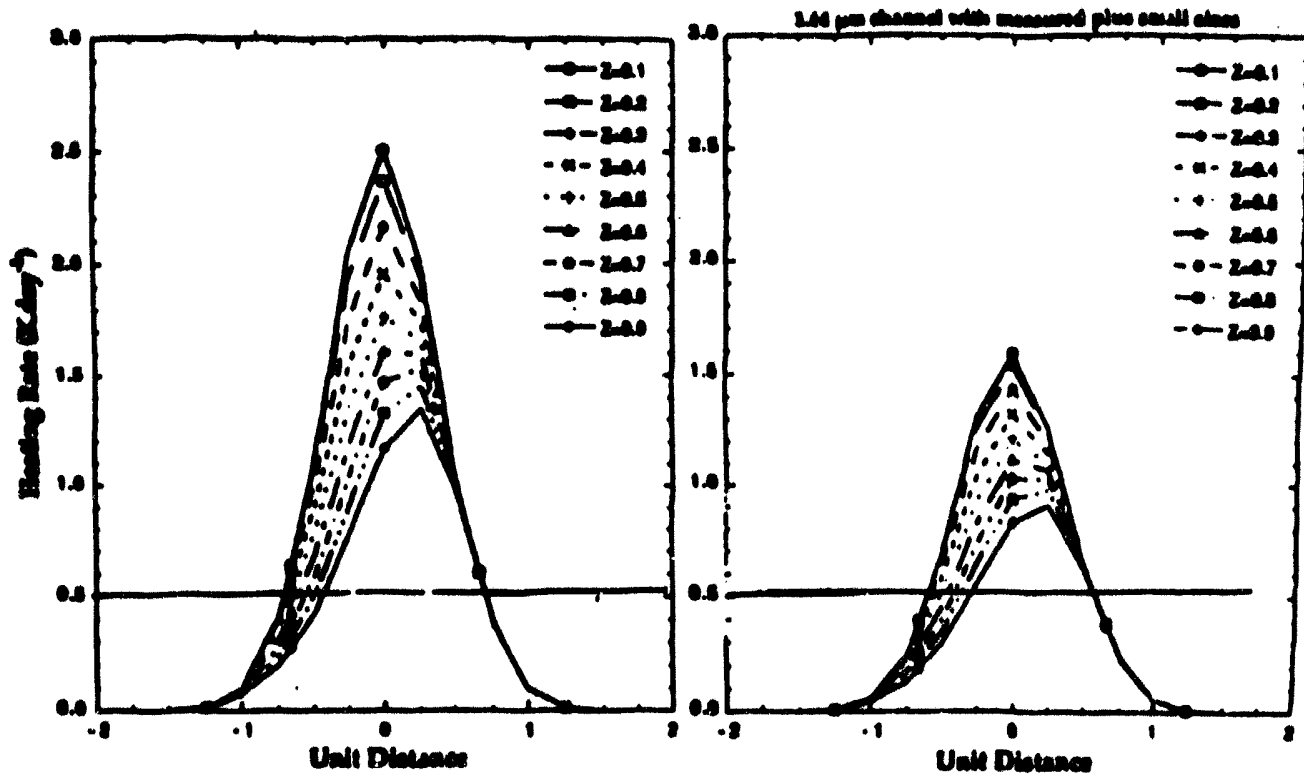

Figure 1. Two-dimensional heating rates calculated by Fourier-Raccati method. Cloud has a Gaussian extinction function and is illuminated by 1.64 micrometer radiation at a solar zenith angle of 30 degrees. Solid horizontal ine is the heating rate as calculated by a plane-parallel model using a domain averaged optical depth of 1.0. Differences in figures are due to differences in the microphysics. Microphysics of the cloud in the left figure is based on measurements where the smallest resolvable particle is approximately 17 micrometers. The cloud on the right includes small particles. The particle size is distributed according to a gamma distribution.

The question remains as to what is the relevant structure information and how this information should be incorporated into radiative transfer. Stochastic transfer methods attempt to address these questions by investigating the radiative effects of ensembles of cloud structures described by probability distributions. The previous work on stochastic radiative transfer has been limited in its applicability to the atmosphere by assumptions such as small amounts of variability, no internal structure in clouds, or inappropriate spatial distribution functions.

We have developed a new method of stochastic radiative transfer that applies to any sort of spatial variability in clouds (Evans 1993). The new method is based on the backward Monte Carlo solution of radiative transfer, using O'Brien's (1992) method. The radiance or flux exiting a surface is expressed as an order of scattering series. Each scattering is associated with an integral over transmission along a path and an angular integral over direction to the next path.
The fundamental idea behind this new method of stochastic radiative transfer modeling is to do backward Monte Carlo integration of the order of scattering integral with additional integrations over probability distributions of path lengths. The spatial variability information is expressed in path probability density functions (pdf). With the assumption of homogeneous and isotropic statistics, a single path pdf has the form $f(s \mid T)$, which is the conditional density of distance(s) given transmission (T). Since successive paths are not independent, higher order information such as the

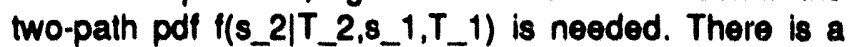
hierarchy of approximations based on the order and form of the path pof's.

The accuracy of these approximations was tested by computing deterministic Monte Carlo radiative transfer in many realizations of $3 D$ isotropic lognormal-multifractal extinction fields. In simulations of thermal radiative transfer in cirrus clouds and solar radiative transfer in boundary 


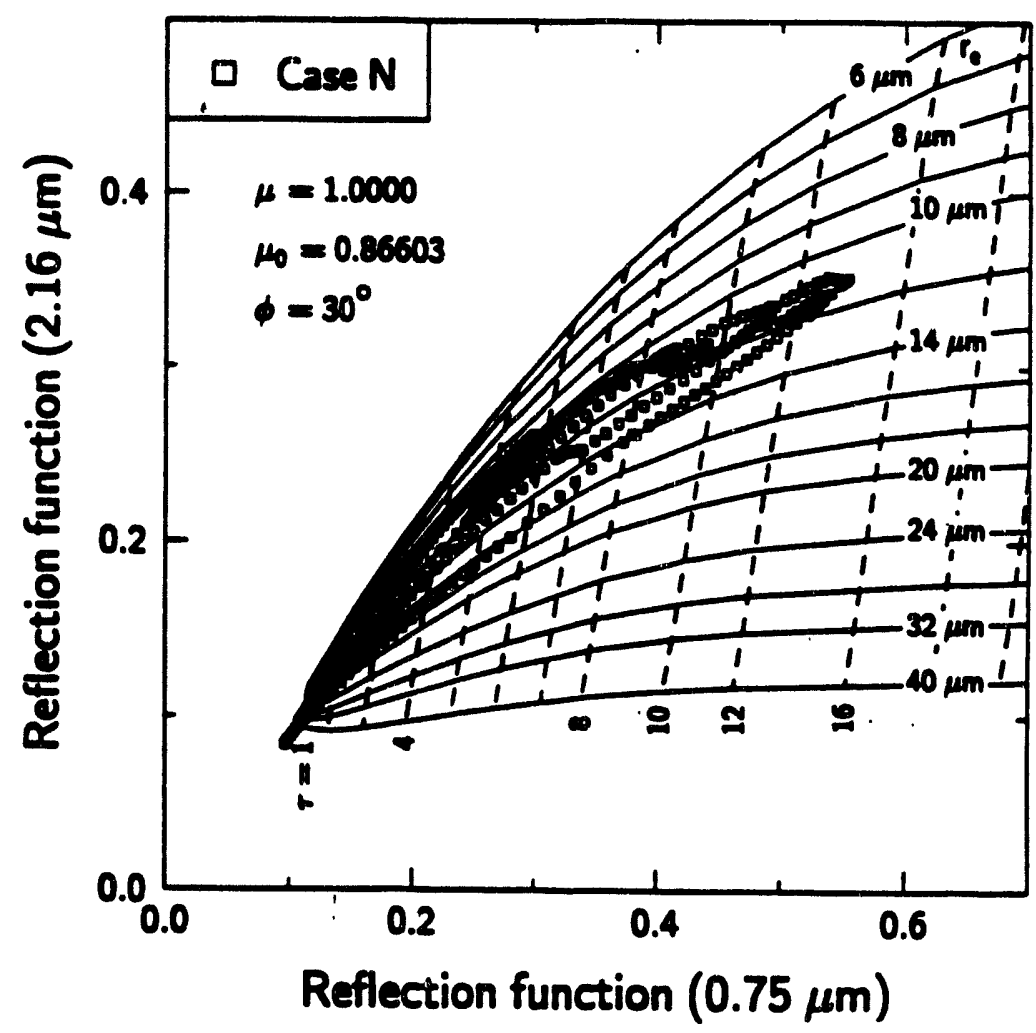

Figure 2. A bispectral scatter plot of simulated reflectance on a plane-parallel $\tau_{e} \tau$ grid. The scatter arises solely from geometrical effects.

layer clouds, each with a range of variability, the stochastic method with two-path pdf's gave accurate mean flux results, while use of the single-path poffs usually did not. Besides providing a practical and general method of stochastic radiative transfer, this work also indicates what cloud structure information is important for radiative transfer. Path pdfs can be obtained from in situ measurements using aircraft, as well as from cloud radars and lidars. Such observations combined with further modeling should lead to radiation parameterizations for climate models that incorporate the effect of heterogeneities in clouds.

\section{Publications}

Duda, D., G. L. Stephens, K. F. Evans, and W. R. Cotton. 1993. The effect of microphysics on the albedo and reflectivity of two dimensional cloud fields. In preparation for J. Atmos. Sci.
Duda, D., and G. L. Stephens. 1993. Radiative heating in two dimensional cloud fields. In preparation for J. Atmos. Sci.

Evans, K. F. 1993. Two-dimensional Radiative Transfer in Cloudy Atmospheres: The Spherical Harmonic Spatial Grid Method. In press, J. Atmos. Sci.

Evans, K. F. 1993. A General Solution to the Stochastic Radiative Transfer Problem. In preparation.

Evans, K. F., and G. L. Stephens. 1993. A Theoretical Foundation for Microwave Remote Sensing of Cirrus Clouds from Space. In preparation for J. Atmos. Sci.

Gabriel, P., S-C Tsay, and G. L. Stephens. 1993. A Fourier Riccati Approach to Radiative Transfer, Part I: Foundations. In press J. Atmos. Sci. 
O'Brien, D. M. 1992. Accolorated quasi-Monte Carto integration of the radiative transfer equation. J. Quant. Spoctrosc. Radiat. Transfer. 48:41-59.

Stephens, G. L. 1993. The remote sensing of the lower atmosphere: An Introduction. Oxford University Press. To appear Summer 1993.
Tsay, S-C., P. Gabriel, and G. L. Stephens. 1993. A Fourier Riccati Approach to Radiative Transfer, Part 2: Applications. In preparation for J. Atmos. Sci. 


\title{
Two Complementary Multifractal Analysis Techniques for Non-Stationary Atmospheric Processes with an Application to Cloud Liquid Water Content
}

\author{
W. Wiscombe and A. Davis \\ NASA Goddard Space Flight Center \\ Climate \& Radiation Branch \\ Greenbelt, MD 20771 \\ A. Marshak \\ SSAI \\ Lanham, MD 20706
}

Standard Gaussian-type statistics are not really adequate to describe atmospheric variability; this traditional approach implicitly dismisses fluctuations as "noise" of use only in computing a standard deviation which, together with the mean, presumably varies 80 little in space and time that one can meaningfully speak of "climatological" values. In geophysics, unfortunately, this approach often fails. Moans and standard deviations not only exhibit no "climatology," but depend on the scale of epacentime sampling.

We adopt the opposite viewpoint: variability is in fact the signal, not the noise. This variability teaches us about the fundamental physics at work. Even simple laboratory or computational systems (and a fortiori geophysical systems) are typically attracted into dynamical equilibria characterized by a large range of longth- and/or timescales, power-law energy spectra, and fractal geometrical properties. For such systems, the concepts of scaleinvariance and/or multifractality provide the most productive framowork for data analysis and simulation.

Typical atmospheric signals exhibit non-stationary behavior. Our first task is therefore to define the most interesting stationary aspects of such non-stationary datasets. One method for doing this, called "Singular Measures," focuses on the absolute values of the gradient field, which is more likely to be stationary; as we progressively degrade the resolution rof this field, taking powers qand averaging, the results appear as power-laws in terms of the resolution $r$ where the exponent is a function of q. Another method, called "Structure Functions," focuses on the absolute values of the differences that occur in the data over arbitrarily large or small scales. For detailed descriptions of both methods, we refer to Davis et al. (1993a) for a graphically based tutorial and to Davis ot al. (1993b and refers therein) for a wavelet-based approach.

Singular Measures are currently attracting more attention than the once more popular Structure Functions. This is largely due to their close connection with multiplicative cascade models, first invented to describe turbulentenergy cascades. While there is no completely general connection between the two methods, for a specific physical process or stochastic model, a relation may exist. However, in a typical data analysis situation, we have no inkling whether such a relation exists; in fact, one of our goals is to find a statistical connection between the fluctuations of the field and the fluctuations of its gradients. (Wo procesed by analogy with classical theory, where a complete description of the system calls for both energy [field] and flux [gradient] terms.) We therefore advocate the use of both methods of analysis, either independently (Davis ot al. 1993a) or jointly (Davis of al. 1993c). Indeed the two techniques reveal quite different and, in many respects, complementary aspects of the data and, hence, of the underlying physical processes.

Structure Functions characterize the non-differentiability or "roughness" of the data, as well as its degree of stationarity. As an example, Figure 1a shows a time-series of cloud liquid water content (LWC) measured during the Atlantic Stratocumulus Transition Experiment (ASTEX) 

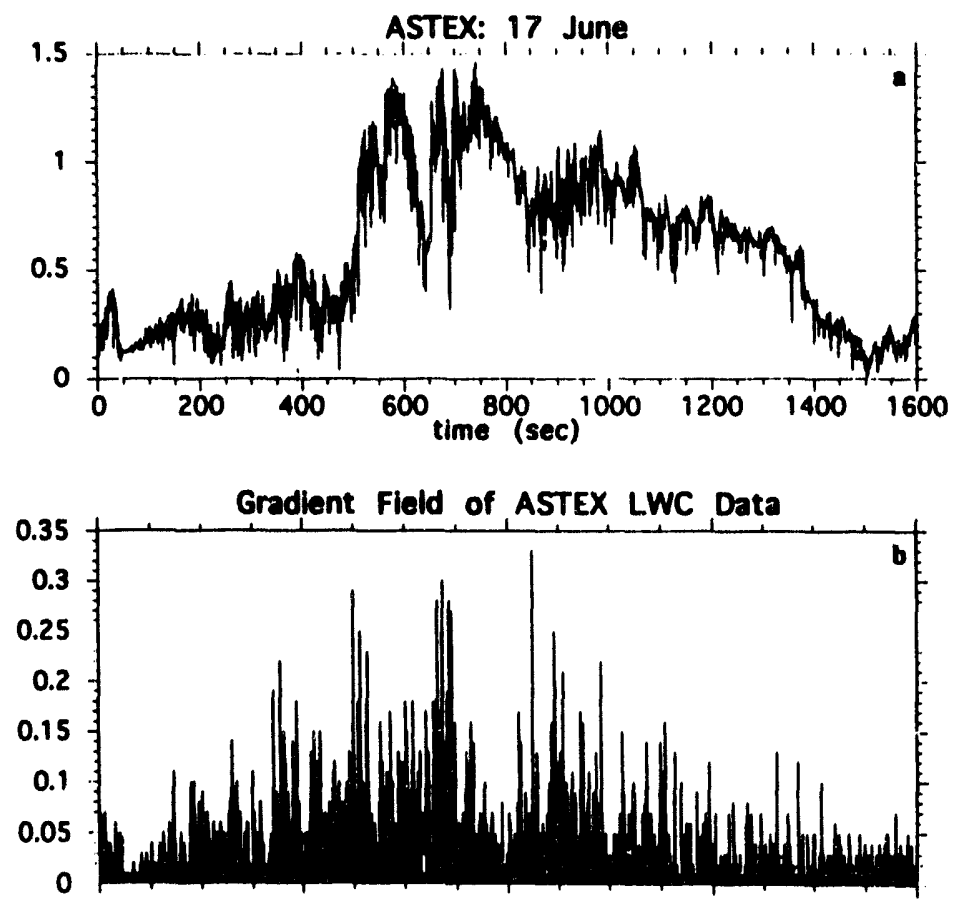

Figure 1. $10 \mathrm{~Hz}$ LWC data collected with the Gerber PVM-100 during an ASTEX research flight (Gerber 1992). (a) a segment of LWC data from 17 June 1992. (b) Absolute small scale gradient field of the dataset in (a).

field program in June 1992. The most straightforward measure of the roughness of this graph is its fractal dimension $D_{\rho}$ (Mandelbrot 1977): if $D_{\mathrm{g}}=1$, then the graph is smooth (differentiable), while, at the other extreme, if $D_{\text {, }}$ $=2$, the graph is so discontinuous that it fills a whole region of two-dimensional space. An alternative quantification of the "smoothness" of the data is $H_{1}=2-D_{0}$, which ranges from 0 to 1 ; at $H_{1}=1 / 2$, one finds, for example, the case of a one-dimensional random walk. Another, a priori independent, measure of non-differentiability, possibly discontinuity, is the power spectrum exponent (e.g., $-5 / 3$ for Figure 1a). One can define an infinite number of other exponents, all of which are organized in a "multifractal" hierarchy (Parisi and Frisch 1985).

The Singular Measure method highlights the "intermittency" present in the typically very spiky absolute gradient dataset; see Figure 16 for that associated with the LWC data in
Figure 1a. The spike. ("singularities") tend to cluster and to concentrate on sparse subsets of space ("fractals"). Usually many different degrees of singularity are observed in the gradient data, indicating the presence of a multifractal field, so, here too, an infinite number of exponents are needed to describe the data statistically. The simplest of these exponents, the "information" dimension $D_{1}$ (e.g., Hentschel and Procaccia 1983), measures the size of the set occupied by those singularities that contribute most significantly to the mean (of the absolute gradients). If $D_{1}$ $=1$, this set has the same dimension as the full time interval and there is no intermittency; if $D_{1}=0$, this set consists of only a finite number of points (including the case where the gradient field is reduced to a Dirac $\delta$-function). Following Schertzer and Lovejoy (1987), it is convenient to use $C_{1}=$ $1-D_{1}$, which ranges from 0 to 1 as the intermittency of the gradient field increases from norie to the $\delta$-function case. 
In Figure 2, we summarize our ideas using regions in a plane. The axes correspond to our two fundamental exponents: horizontally the smoothness parameter $H_{1}$, and vertically the intermittency parameter $C_{4}$. We call this the " $q=1$ multifractal plane" since for starters we are dealing only with first-order statistical moments. We have indicated the loci of various models found in the literature and of various LWC datasets that we have analyzed. Along the vertical axis, we find multiplicative cascade models which are discontinuous and stationary, hence $H_{1}=0$. Along the horizontal axis, we find stationary Gaussian noises (at $H_{1}=0$ ), Mandelbrot's (1977) additive models known as "fractional" Brownian motions (at $\left.0<H_{1}<1\right)$ that generalize standard Brownian motion found at $H_{1}=1 / 2$, and, finally, differentiable functions typical of dynamical models (at $H_{1}=1$ ). All of these have non-intermittent Gaussian or even bounded gradient fields, hence $C_{1}=0$. Four different LWC datasets are represented: the ASTEX one in Figure 1a and three from the 1987 First ISCCP(a) Regional Experiment (FIRE) marine stratocumulus experiment.

These LWC datasets typically have small but non-trivial $C$,'s and $H_{1} \approx 1 / 3$ (see Marshak et al. [1993b] for further details). This suggests modeling the LWC data using a class of "hybrid" stochastic models having both multiplicative and additive ingredients in order to give non-vanishing projections on both axes in Figure 2.(b) Schertzer and Lovejoy (1987) invented one such model involving a lowpass power-law filtering (also called a "fractional integration") of a singular multiplicative cascade model. Cahalan et al. (1989) invented another such model, involving smoothing a multiplicative cascade by making the dispersion of the weights decrease with scale; this results in a bounded cascade process. All the models indicated on the figure have power spectrum exponent $-5 / 3$, as do the empirical data (Cahalan and Snider 1989). Bounded cascade models have very small, essentially residual $C_{1}$-values (Marshak et al. 1993a), as does one of the datasels. (By "residual" we mean that, because of the finite range of scales involved, we cannot distinguish them statistically from purely additive models having $C_{1}=0$, according to a simple criterion developed by Davis et al. [1993d].)

(a) International Satellite Cloud Climatology Project.

(b) In the physics and turbulence communities, such models are known as "multi-affine" (Viscek and Barabasi 1991).
In conclusion, we aim to characterize the 3D structure of clouds (as sampled by 1D transects of LWC) using extensions of simple, robust, and turbulence-proven multifractal statistical methods and models. We are aiming for the simplest possible stochastic models with the minimum number of free parameters, yet one capable of capturing variability on the widest possible range of scales. This is obviously an important prelude to the theoretical and empirical study of cloud radiation problems. Beyond that, we believe there will be fruitful applications of these general-purpose scale-invariant data analysis methods for many other Atmospheric Radiation Measurement (ARM) Program purposes: $\theta .9 .$, comparisons of model output with ARM observations at a much deeper level than traditional mean-variance; and interpolations and extrapolations in space and time scale, to mention just a few possibilities.

\section{References}

Cahalan, R.F., and J.B. Snider. 1989. Marine stratocumulus structure. Remote Sens. Environ. 28:95-107.

Cahalan, R.F., M. Nestler, W. Ridgeway, W. J. Wiscombe, and T. Bell. 1989. Marine stratocumulus spatial structúre. Proc. 4th Int. Moeting on Statistical Climatology, pp. 19-25.

Davis, A., A. Marshak, W. Wiscombe, and R. Cahalan. 1993a. Multifractal Characterizations of Non-stationarity and Intermittency in Geophysical Fields, Observed, Retrieved or Simulated. J. Geophys. Res. (submitted).

Davis, A., A. Marshak, and W. Wiscombe. 1993b. Waveletbased multifractal analysis of non-stationary and/or intermittent geophysical signals-To appear in Applications of Wavelet Transforms in Geophysics, Foufoula. Georgion and P. Kumar. Academic Press.

Davis, A., A. Marshak, and W. Wiscombe. 1993c. Joint Bimultifractal analysis and multi-affine modeling of nonstationary geophysical processes, application to turbulence and clouds. Fractals (accepted probability distributions for the gradients and the increments of non-stationary processes (In preparation).

Davis, A., A. Marshak, W. Wiscombe, and R. Cahalan. 1993d. A simple test for the hypothesis of multiplicity in scaling of statistical properties in geophysical signals. (In preparation). 


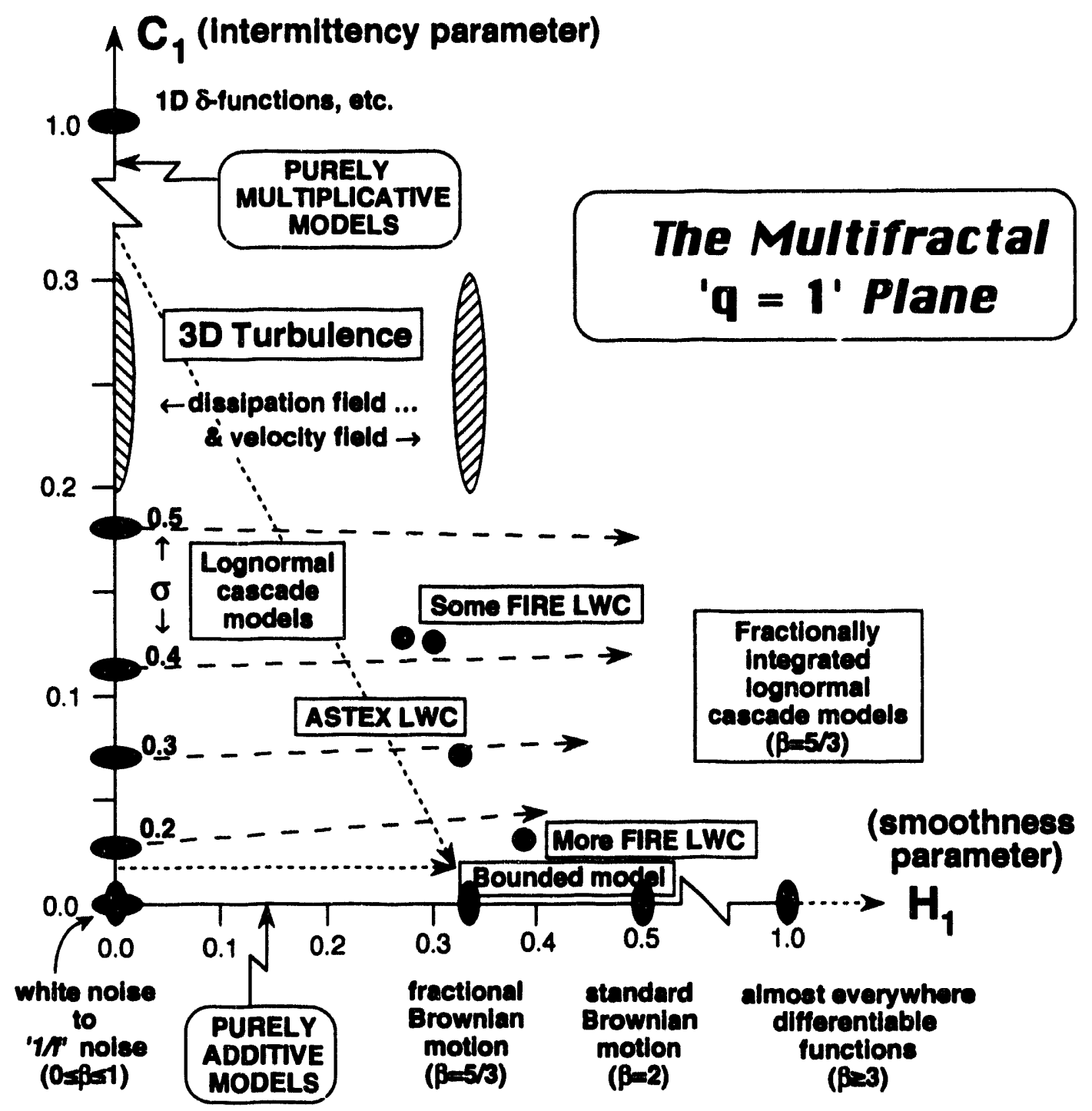

Figure 2. Multiplicative and additive models populate the axes; whereas, "hybrid" or "multi-affine" models populate the plane itself. This new class of models contains, as a simple example, randomly positioned Heaviside steps; they are almost everywhere differentiable $C_{1}=3 D 1$, but their gradients are $\delta$-functions $\left(C_{1}=3 D 1\right)$. The arrows indicate the effect of "turning on" the smoothing parameter in two other hybrid models discussed in the text. Note that turbulent velocity or passive scalar transects typically have about the same $H_{1}$ but a higher $C_{1}$ than we find for our LWC data. 
Gerber, H. 1992. New microphysics sensor for aircraft use. 1992. Preprint Volume, Proc. 11th. Intem. Conf. on Clouds and Precipltation, Montreal, Canada, Aug. 17-21, 1992, pp. 942-944.

Hentschel, H.G.E., and I. Procaccia. 1983. The infinite number of generalized dimensions of fractals and strange attractors. Physica D. 8:435-444.

Mandelbrot, B. B. 1977. Fractals: form, chance, and dimension. W. H. Freeman and Company, San Francisco.

Marshak, A., A. Davis, R. Cahalan, and W. Wiscombe. 1993a. Bounded cascades as non-stationary multifractals. Physical Roviow E (in press).

Marshak, A., A. Davis, W. Wiscombe, and R. Cahalan. 1993b. The scale-invariant structure of marine stratocumulus deduced from observed liquid water distributions; Part 1: Spectral properties and stationarity issues; Part 2: Multifractal properties and model validation. J. Atmos. Scl., submitted.

Parisi, G., and U. Frisch. 1985. A multifractal model of intermittency. Turbulence and predictability in geophysical fluid dynamics and climate dynamics, eds. M. Ghil, R. Benzi, and G. Parisi, pp. 84-88. North-Holland, Amsterdam.

Schertzer, D., and S. Lovejoy. 1987. Physical modeling and analysis of rain clouds by anisotropic scaling multiplicative processes. J. Geophys. Res. 02, (D8):9693-9714.

Viscek, T., and A.-L. Barabasi. 1991. Multi-affine model for the velocity distribution in fully turbulent flows. J. Phys. A: Math. Gen. 24:L845-L851. 


\title{
Treatment of Cloud Radiative Effects In General Circulation Models
}

\author{
W.-C. Wang, X.-Z. Llang, M. P. Dudek, M. Ding, \\ L. Zhu, J. Everette, and S. Cox \\ Atmospheric Sciences Research Center \\ State University of Now York \\ Albany, NY 12205
}

We participate in the Atmospheric Radiation Measurement (ARM) Program with two objectives: 1) to improve the general circulation model (GCM) cloud/radiation treatment with a focus on cloud overlapping and tis cloud optical properties and 2) to study the effects of clouoiradiationclimate interaction on climate simulations. This paper summarizes the project progress since the second ARM Science Team meeting October 26-30, 1991, in Denver, Colorado. Four graduate students participated in the research.

\section{GCM Radiation Model Development}

During this period, we completed the radiation modelmeasurement comparison using the Albany site data. The measurement data ir slude

- the total direct and diffuse solar radiation reaching the surface (15-minute intervals) for the periods 10/86, (4, $5,7,10) 10 / 87$, and 10/91 through 10/92

- the longwave radiation reaching the surface for the periods 10/91-6/92 (15-minute intervals)

- the meteorological data at the surface (hourly data) and in the upper air ( 6 am and $6 \mathrm{pm}$ ) by the National Weather Service. The meteorological data consist of temperature, humidity, wind, and cloud cover and ceiling height for the same pericds as the solar radiation.

Using these data as inputs, we evaluated the longwave and solar radiative codes by comparing the calculated radiative fluxes reaching the surface with measurements for both clear and cloudy sky conditions. These codes were used in the general circulation models CCM1 (Wang ot al. 1991 and 1992) and the Global ENvironmental and Ecological Simulation of Interactive Systems (GENESIS) for climate simulations. Both GCMs were developed at the National Center for Atmospheric Research. The GENESIS includes the diurnal cycle and much improved physics (clouds, moisture transport, convection, etc.) and surface models for soil and snow/ice.

Figure 1 shows the comparison of longwave and solar radiation for clear and cloudy sky. Note that the cases for longwave radiation are restricted to the time of the day when radiosonde data are available. Note also that the cases of cloudy sky are limited to one layer of cloud either at low level $(<3 \mathrm{~km}$ ) or at the middle level (between 3 and $6 \mathrm{~km}$ ), which is determined through examining the humidity profile. For clear sky, the model calculations are in good agreement with measurements for longwave radiation, and the contribution of the observed trace gases of $\mathrm{CH}_{4}$, $\mathrm{N}_{2} \mathrm{O}, \mathrm{CFC}-11$, and CFC-12 is 2 to $3 \mathrm{Wm}^{-2}$.

The GCM radiation parameterization calculates systematically higher values for solar radiation. Our sensitivity calculations indicate that the effects of column ozone amount and surface albedo are on the order of 10 to $20 \mathrm{Wm}^{-2}$. The likely cause for the larger values is related to the omission of aerosols in the flux calculations, as well as the measurement uncertainty.

Large differences are found in the comparison for the cases of cloudy sky, and the differences are particularly large for solar radiation. These differences can be attributed to the radiation model's internally assigned cloud albedo and emissivity and, thus, a lack of interactive cloud optical propertles. For the latter, work is already under way to use observations such as the First ISCCP Reglonal Experiment 
Surface Incident Radiation Flux $\left(\mathrm{Wm}^{-2}\right)$ Albany, NY
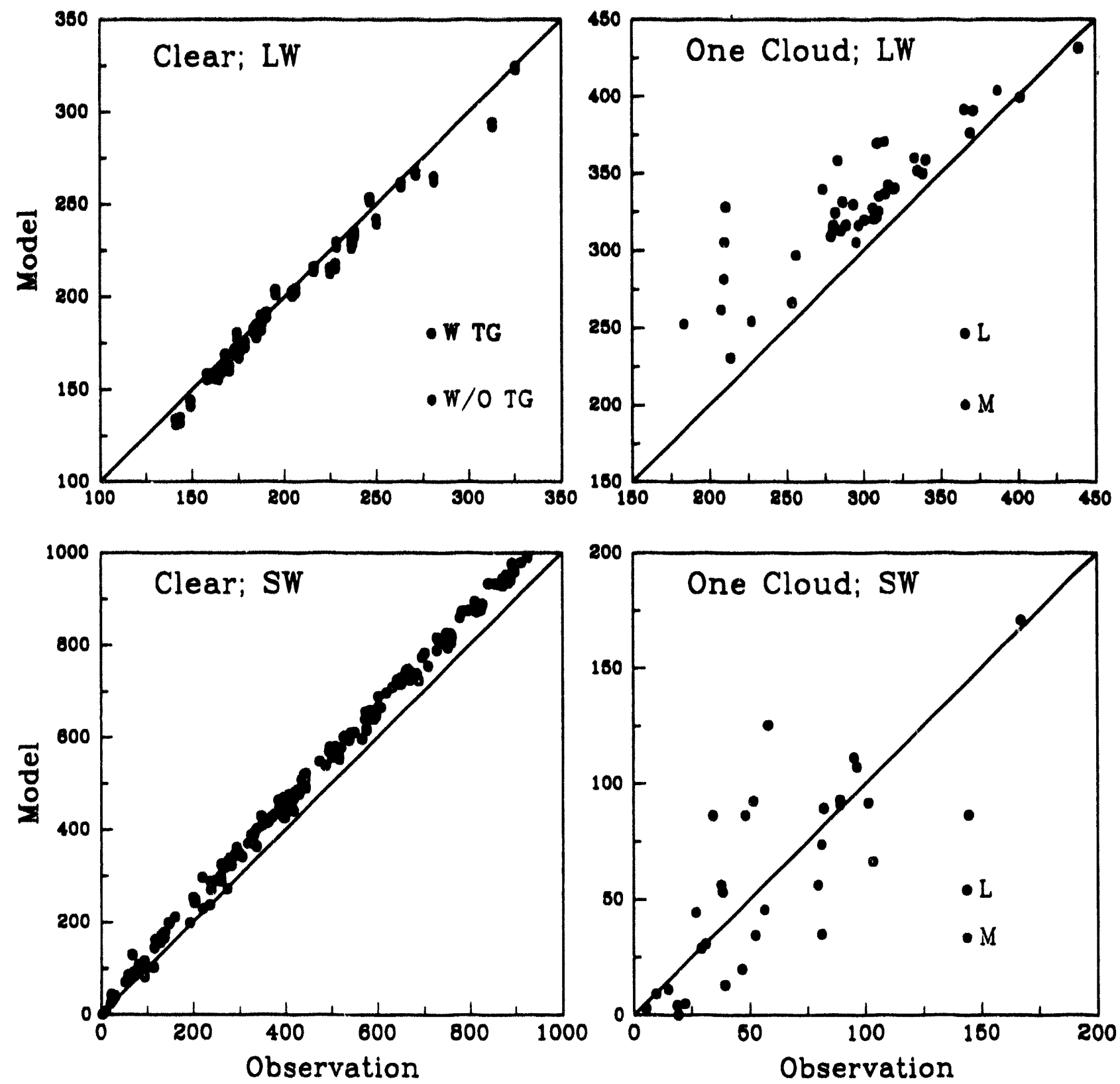

Figure 1. GCM radiation model-observation comparison of the downward radiation fluxes reaching the surface at Albany for cloar (with and without trace gases, TG) and cloudy (Low or Middle lovel cloud) sky conditions. Note that cloudy sky calculations include the trace gases. 
(FIRE) and Internationa/ Batellib Cloud Climatology Project (I8CCP) data to dovalop rediative paramotorizations for cirrus and stratiform clouds with interactive microphysice.

We plan to continue the research uaing the data from the Southern Great Plains CART alto, which are now becoming avallable.

\section{Resolution Dependence of GCM Cloud-Radiation Parameterization}

A semi-prognostic climate model has been developed to study the resolution dependence of physical paramotorizations ueed in GCMs. The climatic model, consisting of the basic physical paramoterizations from GENESIS, reads in the three-dimensional atmospheric stato for the specified reglon and computes a consistent eot of diegnostic quantities (such as cloudinese, precipitation or surface radiation quantitios) and instantaneous local tims bendencies (such as radiation or condensation heating ratos within the atmosphere).

We have tested the seml-prognostic model using the standard radiosonde network around the Southern Great Plains CART aite. The model was run for grid size of $60 \mathrm{~km}$, $120 \mathrm{~km}, 180 \mathrm{~km}, 240 \mathrm{~km}, 360 \mathrm{~km}$, and $720 \mathrm{~km}$. For each grid size, the horizontally averaged basic state quantities are firet calculated and used as inputs to the model to calculate the diagnostic quantities and local time tendencies. Comparison of the averages obtained using different horizontal resolutions will allow an assossment of the dependence of the results as the horizontal resolution changes. If the model physics are unchanged across a range of resolutions, the only source of differences in the final averages for the region will be the horizontal resolution of the calculations. In particular, since the semi-prognostic approach allows no dynamical interactions within the model, the effect of changes in horizontal resolution on the physical parameterizations can be isolated froin changes in the dynamics of the model.

Figure 2 shows the total cloudiness and net upward longwave radiation evaluated from the semi-prognostic model. For the 60-km grid resolution (upper panels), the horizontal distribution of cloud cover (upper left panel) varies from $100 \%$ to 0 , with corresponding net longwave radiation (upper right pand) from 0 to $110 \mathrm{Wm}^{-2}$ over the region. Most of the change in surface radiation budget corresponds to cloudy versus clear areas. Figure 2 also shows the resolution dependence of the frectional cloud cover (lower bett panel) and the longwave radiation (lower right panel). The fractional cloudiness varies from 0.3 on the $60-\mathrm{km}$ grid to no clouds for the $720-\mathrm{km}$ grid. This result implies that for a very large-scale model, the cloud parameterization would diagnose no clouds over the region. As horizontal resolution increases, the diagnosed fractional cloud cover also increases, Indicating the cloud parameterization contains a strong dependence on horizontal resolution. The resulting longwave radiation dependence on the resolution follows the similar change in cloudiness and varies from 72 to $92 \mathrm{Wm}^{-2}$ as the horizontal resolution changes from 60 to $720 \mathrm{~km}$.

We plan to conduct more detalled study using Southern Great Plains CART site data and calibrate the radiation calculations with measurements.

\section{GCM Simulations}

\section{Atmospheric Ozone}

The radiation model-measurement comparisons reported in the first section of this paper indicato that the atmospheric ozone can substantially afiect the solar radiation reaching the surface, with subsequent effect on climate simulations. Currently, the ozone climatology used in GENESIS is the data corresponding to the 1970 s and, more importantly, does not account for the longitudinal variations. We have used a combination of the satellite data from Total Ozone Mapping Spectrometer (TOMS) and Stratospheric Aerosol and Gas Experiment (SAGES) and the ozonesonde station data to update the GENESIS model ozone climatology.

Comparison of the ozone column between the two climatologies indicates that large differences exist over the middle-and high-latitude continental regions during winter. To make a preliminary evaluation of the effect of the updated ozone climatology on the climate simulations, we run perpetual January and July simulations.

The differences in the latitudinal-longitude surface temperature between the old and the updated ozone climatologies are shown in Figure 3. The difference can be as large as $6^{\circ}$ to $8^{\circ} \mathrm{C}$ over North America and Central 

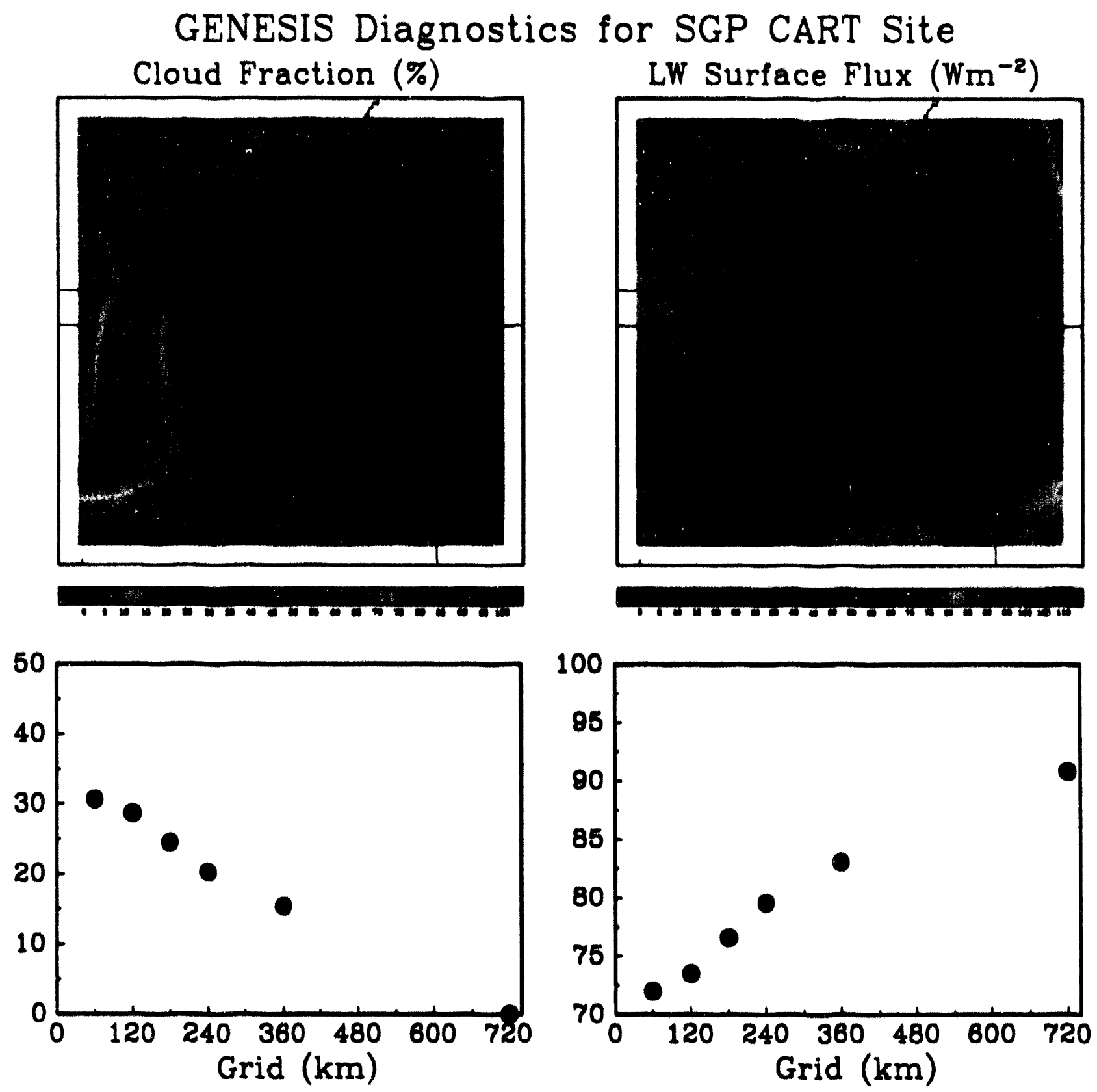

Figure 2. The total fractional cloudiness (lop left panel) and the net upward longwave radiation flux at surface (top right panel) diagnosed at the Southern Great Plains CART site for 12 GMT 12 June 1885 on a 60-km grid. The diagnosis is conducted using the GENESIS which includes the Slingo and Slingo (1991) cloud parameterization. Most of the cloudiness within the region is middle clouds, with some vertical overlapping with low clouds in the southeastern corner of the domain. The two lower panels are the domain-averaged cloudiness and net longwave radiation fluxes as a function of horizontal resolution of the computation grid. 

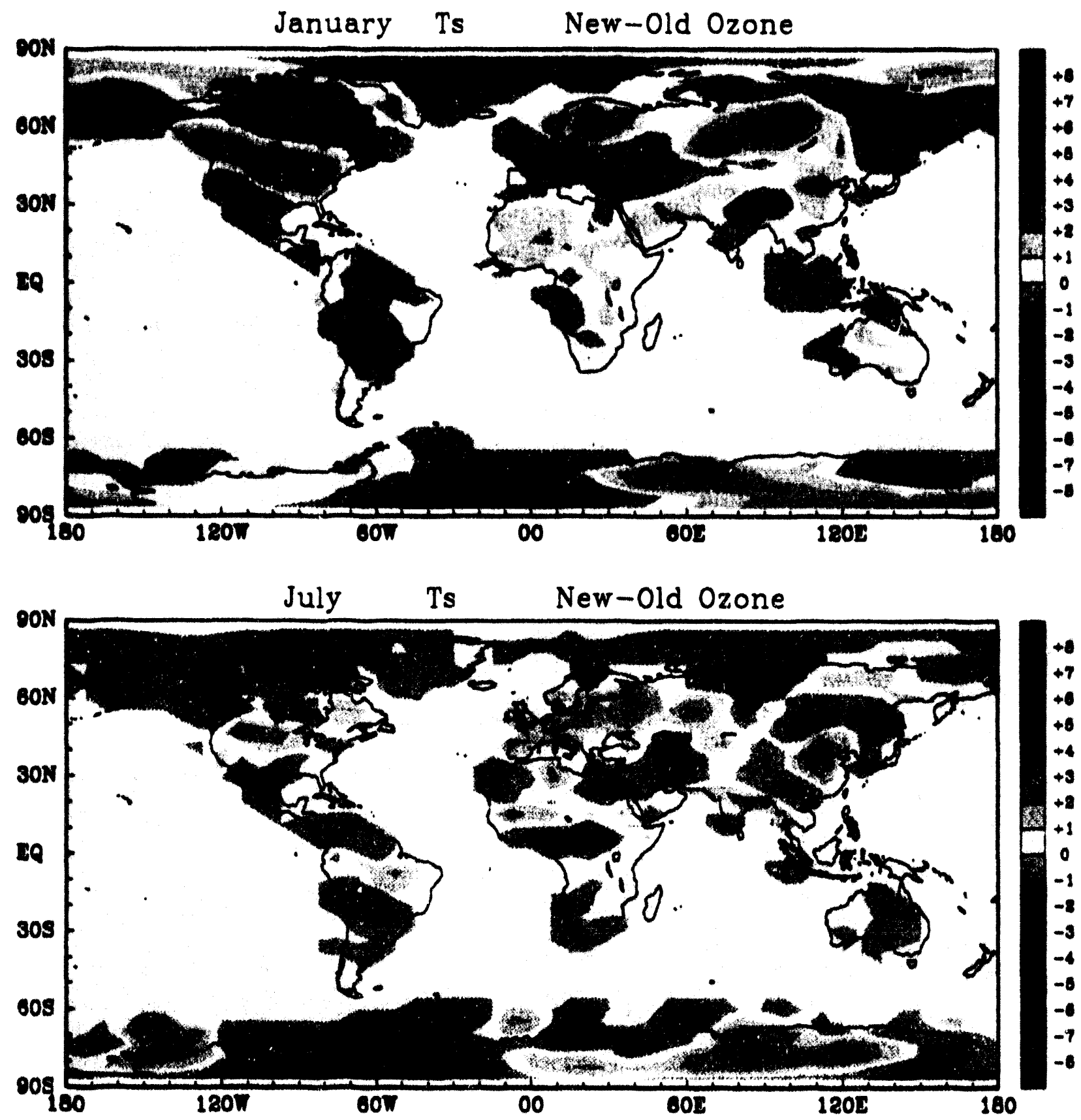

Flgure 3. Effect of atmospheric ozone on the January and July surface air temperature as simulated from GENESIS. The new ozone climatology is based on the TOMS, SAGES, and 0zonesonde station data, while the old climatology is zonal mean and, thus, does not account for the longitudinal variation. Perpotual January and July experiments were conducted here. 
Eurada in January. GCM simulations with full seasonal cycles are currently boing conducted.

\section{Sulfate Aorosols}

As mentioned earlier, the aerosols can also have a significant offect on the solar radiation with subeequent climaticeffects. The posable cooling effect of anthropogenic sulfate sorosols has been a toplc of considerable interest recontly.

According to recont estimates by Charison of al. (1992) and Wigloy and Raper (1892), the radiative forcing due to arosol is substantial but opposite in sign to the radiative forcing dus to the enhanced greenhouse gas concentrations todats. Langner and Rodhe (1991) have calculated monthly average anthropogenic sulfate aerosol burdens, which oxhiblt strong geographical and seasonal variations.

We have incorporated these burdens into CCM1. Preliminary results suggest that the sulfate forcing induces regional climate responses markedly different from those caused by the greenhouse gases. We will continue the study by carrying out longer simulations.

\section{References}

Charteon, R. J., S. E. Schwartz, J. M. Halos, R. D Coss, J. A. Coakley, J. E. Hansen, and D. J. Hotmann. 1992. Climate forcing by anthropogenic aerosols. Science 255:423-430.
Langner, J., and H. Rodhe. 1991. A global three. dimensional model of the tropospheric sulfur cycle. J. Atmos. Chem. 13:225-263.

Slingo, A., and J. M. Slingo. 1991. Response of the NCAR CCM to improvements in the representation of clouds J. Geophys. Res. 96:15341-15357.

Wang, W.-C., M. P. Dudek, X.-Z. Llang, and J. T. Kiohl. 1991. Inadequacy of effective $\mathrm{CO}_{2}$ as a proxy in simulating the greenhouse offect of other radiatively active gases. Nature 350:573.577.

Wang, W.-C., M.P. Dudek, and X.-Z. Liang. Inadequacy of effective $\mathrm{CO}_{2}$ as a proxy to assess the greenhouse effect of other radiatively active gases. Geophys. Res. Lett. 19:1375-1378.

Wigley, T.M.L., and S.C.B. Raper. 1992. Implications for climate and sea level of revised IPCC emissions scenarios. Nature 357:293-300. 


\title{
Cloud Effects on Radiation at the Top of the Atmosphere and at the Surface: Observations and Modeling Studies
}

\author{
C. Gautier, D. Lavalibe, W. O'Hirok, T. Figel, \\ M. Landfield, P. Ricchiazzi, and S. Yang \\ Univereity of California, Santa Barbara \\ Santa Barbara, CA 93111 \\ D. Lubin \\ University of California, San Diego \\ La Jolla, CA 92093-0221 \\ S. Lovejoy, C. Larnder, G. Lowis, S. Pecknold, and P. Silas \\ McGill University \\ Montreal, Quebec, Canada \\ D. Schertzer, R. Borde, Y. Tessier, and F. Schmitt \\ Laboratoire de Mótéorologle Dynamique \\ Université Pierre of Marie Curie \\ Paris, France
}

\section{Introduction}

Our project's main goal is to study the effects of clouds, particularly those with inhomogeneous spatial properties, on radiation at the top of the atmosphere and at the surface. To accomplish this goal, we use a combination of observations from space and at the surface and models to diagnose procesess and predict relationships botween cloud properties and radiation fields. In addition, we develop theoretical foundations for our main analysis procedure, which is based on multifractal theory and analysis tochniques.

Our project is thus multi-faceted as is reflected in this abstract and our presentation. Preliminary analysis of data from the Atmospheric Radiation Measurement (ARM) Program involves computing the surface shortwave irradiance data from the Visible and Infrared Spin-Scan Radiometer (VISSR) on the Geostationary Operational Environmental Satellite (GOES), as well as estimating the multifractal properties of the cloud radiance field observed by the satellite. The analysis of non-ARM data includes satellite and in-situ observations over the Antarctic, as well as multispectral (visible, IR and microwave) satellite observations over the globe. The modeling activities encompass diagnostic studies with a number of radlative transfer codes, particularly the Discrete Ordinate model of Stamnes ot al. (1988) (DISORT), and the development of a highly flexible Monte Carlo model to compute the threedimensional (3-D) radiation field for any cloud (liquid water) distribution. In addition, a number of multifractal analyses have been performed with satellite data, and new radiative transfer simulations have been achioved.

\section{Role of Clouds on Surface Solar Irradiance over ARM Central Site}

We have developed an operational procedure to compute the downwelling and net shortwave irradiance at the surface from hourly GOES VISSR visible data over the contral ARM Site at $1-\mathrm{km}$ resolution. These computations are performed with a version of the simple radiative transfer model of Gautier et al. (1980) and require surface and cloud albedo. Hourly surface albedos are computed from 
clear brightness values obtained by applying a minimum brightness technique on a sequence of brightness values over a several day period for each pixel. Such fields will be used to investigate the effects of clouds on the spatial variability of the surface shortwave irradiance.

The spatial variability of the first mean field, computed for sixteen (16) daily values obtained in January and February 1993 is rather small with values ranging from about $100 \mathrm{Wm}^{-2}$ to $140 \mathrm{Wm}^{-2}$. This result can be explained by the fact that the effects of the cloud field are rather random, when averaged over a 16-day time period. The largest values of the field are found in the northwest corner of the analyzed area and correspond to high surface albedo regions. This results from the multiple cloud-base-tosurface reflections which enhance the downwelling surface shortwave irradiance.

\section{Multifractal Data Analysis}

Atmospheric observations are characterized by extreme variability over a wide range of scale. The scaling symmetry observed in geophysical fields can be characterized with the help of multifractal dimensions. These dimensions give a complete statistical description of the geophysical fields that is scale independent. Multifractals are also characterized by their scaling exponents, and when generated by canonical cascade processes, they generally belong to specific universal classes. In this case, the scaling exponents are specified by two parameters, the Lévy index $\alpha$ and the codimension of the mean singularity $c_{1}$.

Modeling and statistical analysis of clouds and their associated radiance fields require knowledge of the basic length scales involved. The standard model of atmospheric dynamics involves isotropic 3-D turbulent regimes at small scales, separated by an isotropic two dimensional (2-D) turbulent regime at large scales. It is supposed that these regimes have qualitatively different types of scaling in the mesoscale that define a basic length scale separating small and large scale motions and structures. However, new ideas about generalized scale invariance lead to the simpler assumption of a single anisotropic scaling regime spanning the entire range of meteorologically significant motions; this is the "unified scaling model."
Clouds and their radiance fields provide sensitive tests of these ideas. Using Meteorological Satellite (METEOSAT), LANDSAT and Advanced Very High Resolution Radiometer (AVHRR) data at visible, IR, and near IR wavelengths, we presented the first systematic analysis of satellite cloud radiances over the ocean. By calculating energy spectra, we were able to show that over the range of $=160 \mathrm{~m}$ to $-4000 \mathrm{~km}$, as predicted by the unified scaling model, these radiances display a very nearly power law form. Since wide scaling ranges will lead to multifractal statistics, this study (which must be followed up by studies of the smaller and larger scales) provides an essential justification for multifractal modeling of clouds and the associated radiative transport phenomena. In further support of the unified scaling model, we also reported on a series of recent multifractal analyses indicating that the radic.nce fields are special types of ("universal") multifractals also predicted by the theory.

\section{GOES IR Radiance Field over the Central Site}

Using the "Double Trace Moment" that allows direct estimate of the $\alpha$ and $C_{1}$ parameters, the scaling behavior of GOES IR data over the ARM site has been analyzed.

The results confirmed that the scale invariance is a fundamental feature of the atmosphere that is independent of the instrument.

With a value of $\alpha:=1.36$, IR GOES data have the same degree of multifractality as the IR satellite data $\alpha=1.35$ obtained from other satellite observations (e.g., AYHRR). This value is larger for IR satellite data than for visible data $(\alpha: \approx 1.1)$, but smaller than for microwave satellite data ( $\alpha$ : - 1.64).

The fact that $\alpha$ varies with wavelength suggests that the radiances probability distributions are not the same. This finding is not so surprising, since quantities associated with each sensor may be governed by different dynamical processes. This result is important since the data analyzed are for land surface conditions, and it suggests that the analysis is not overly sensitive to the background (ocean vs. land). 
Simulated and Observed AVHRR Images Over Antarctica
Using Plane Parallel Clouds

We have performed a radiative transfer modeling and observational study over Antarctica with the goal of better understanding the interaction between radiation and clouds over highly reflecting surfaces. In-situ observations have been provided by ground-based spectral (corresponding to channel 1 and 2 of the AVHRR instrument) and broadband radiometers. Satellite radiances have been obtained from the AVHRR instrument on NOAA 10 and 11. To interpret the satellite observations in terms of cloud properties, we have estimated the surface albedo using the AVHRR data in clear conditions and computed the top of the atmosphere radiance that AVHRR would see under the assumption of plane parallel clouds for a number of cloud optical thicknesses. The modeling was performed with the DISORT model (Stamnes et al. 1988). One of the reasons for performing these comparisons is to test the adequacy of DISORT's plane parallel approximation over the maritime Antarctic, a region where stratiform clouds are expected to be typical.

Comparisons between observed AVHRR (Ch. 1) radiance and simulated AVHRR radiances for different cloud optical thicknesses under plane-parallel assumption were presented for two days: days 281 and 285. On Day 281, the observed satellite image displays a low radiance contrast and the appearance of full cloud coverage over most of the image. On the contrary, on Day 285, the observed satellite image displays more variability in the cloud field. The simulated data show that a very large optical thickness (far larger than that suggested by the surface instruments) is needed to reproduce the observed low contrast. An analysis of the cross section for that day further suggests that the observed radiance gradient is opposite from that simulated.

Some processes, not included in the plane parallel simulations, act to decrease the cloud radiance over snow and ice. We believe these processes are related to the clouds' 3-D geometry and expect to resolve this issue with the Monte-Carlo radiative transfer model described in the next section.

\section{Top of the Atmosphere Simulated Radiance Field for Fractal Clouds}

A Monte Carlo model has been developed. It is based on a structure of unlimited spatially variable cells capable of modeling the interactions of photons with the major radiative constituents of the atmosphere. By varying the size of individual cells, we can enhance the resolution for areas being investigated. For more homogeneous areas, a reduction in resolution lessens processing time and provides a buffer effect on the boundaries of the model structure.

Each cell can be individually addressed and assigned differing amounts and mixtures of atmospheric gases, aerosols and cloud properties. Cells in the lower boundary of the model can be assigned a multitude of complex surface features including semitransparent cells capable of simulating vegetation canopies.

Input to the model is achieved through one-, two- and three-dimensional overlays allowing the direct entry of satellite images, spatial classifications and theoretically derived cloud parameters. Dividing the Monte Carlo calculations among many processors allows numerous complex simulations to be run in relatively short time. Individual locations and trajectory vectors of photon absorption, scattering and exiting from the model's structure are all stored to provide maximum flexibility in post-run analysis.

\section{Antarctic Cloud Properties Derived From Surface FTIR Measurements}

Spectral resolved radiometric measurements of middle infrared atmospheric emission can be used in conjunction with detailed radiative transfer calculations to retrieve cloud emissivity and to estimate cloud liquid water path (LWP), optical depth, and equivalent radius of the droplet size distribution. Using a discrete-ordinates radiative transfer formulation, we have developed an algorithm to retrieve these properties from Fourier transform infrared 
(FTIR) data. The algorithm has been successfully applied to a 4-month Antarctic data set provided by the CalSpace FTIR Spectroradiometer.

Radiative transfer calculations sulficient to bracket values expected in the field were performed to estimate spectral cloud emissivity for a range of cloud optical depth, liquid water content, and equivalent radius.

These calculations made use of bi-modal droplet size distributions actually observed in Antarctic clouds. A leastsquares algorithm is used to choose a theoretical cloud emission spectrum that best reproduces a given measured brightness temperature spectrum. The results show marked differences in cloud emissivity between high and low overcast layers and between clouds with and without precipitation. The results also suggest that the emissivity of a maritime Antarctic cloud deck should be smaller for a given LWP than the parameterization frequently used in general circulation models.

\section{Radiative Transfer Modeling}

The simplest nontrivial scaling models of cloud heterogeneity are the monofractal models which assume the optical density is constant on a fractal sel, zero elsewhere. This already leads to bulk (averaged) albedo and transmission properties which differ from plane parallel theory by factors which become arbitrarily large as the clouds get thicker. To study the transfer in the more realistic multifractal models, we developed a series of approaches: numerical simulations, orders of scattering, and radiative diffusion.

\section{Numerical Simulations}

To understand the relations between the cloud and radiation fields, we sought to statistically relate the multifractal singularities of the various fields. From a numerical point of view, this is very demanding: the existence of rare but extremely optically dense regions can lead to spurious negative intensities if the radiative transfer equations are not integrated with great care. Monte Carlo techniques avoid this problem, but require enormous numbers of simulated photons in order to yield good estimates of the internal cloud fields.
We therefore developed a robust semi-implicit scheme. This scheme was used to numerically test theoretical predictions that vertical fluctuations in an appropriately defined relative intensity can be approximately related to the horizontally averaged mean optical density. If this relationship can be substantiated by further numerical and theoretical work, it will be possible to predict the overall multifractal statistical properties of the radiation field from those of the cloud field.

\section{Orders of Scattering Approach}

The relation between cloud and radiation fields is relatively simple in two limiting situations: the optically thin (linear) regime and the optically thick regime. The latter can be tackled analytically by deriving asymptotic expansions for the scattering of various orders in the (large) extinction coefficient. In the case of log-normal multifractal clouds, we presented specific results for photon path statistics, and we indicated how to extend them using functional integration.

\section{Radiative Diffusion}

In optically thick clouds, the complex radiative transfer equation is often replaced by the much simpler diffusion equation. While there now exists a considerable body of literature concerning diffusion in monofractal systems, diffusion on multifractals is a completely new paradigm for diffusion.

Using Monte Carlo simulations, we presented the very first results. These indicate that, in spite of the existence of multifractal radiation fields, the transport is apparently dominated by a single fractal component. In addition, the resulting diffusion is systematically slower than normal diffusion ("subdiffusion"), indicating that photons tend to get "rrapped" in low optical density regions surrounded by denser regions. 


\title{
Analysis of Cloud Radiative Forcing and Feedback in a Climate General Circulation Model
}

\author{
A. A. Lacis \\ NASA Goddard Space Flight Conter \\ Institute for Space Studies \\ Now York, NY 10225
}

The principal objecilves of the Atmospheric Radiation Measurement (ARM) Program research at the Goddard Institute for Space Studies (GISS) are 1) to improve and validate the radiation parameterizations in the GISS general circulation model (GCM) through model intercomparisons with line-by-line calculations and through comparisons with ARM observations, 2) to improve the GCM diagnostic output to enable more effective comparisons to global cloud/radiation data sets, and 3) to use ARM Cloud and Radiation Testbed (CART) data to develop improved parameterization of clouds in the GCM and to study the interaction of dynamics and radiation.

\section{Improvement of GCM Radiation}

We have tested and evaluated several different approaches for modeling particle-size-dependent multiple scattering in the GISS GCM shortwave algorithm. These involve modifications to the current Single Gauss Point (SGP) doubling-adding algorithm that is used to calculate cloud and aerosol radiative properties. We have explored the use of two- and three-dimensional interpolation schemes in the radiative input parameters to the SGP doubling algorithm. These schemes have been least-squares fitted to reproduce accurately the reflected and absorbed radiation for small and large optical thicknesses and for particle sizes characteristic of clouds and aerosols. In addition, they have been fitted to reproduce the albedo dependence on solar zenith angle. We have also explored using a fourdimensional interpolation scheme of cloud albedos as a function of solar zenith angle, optical depth, cloud particle size, and single scattering albedo.
Each approach has different advantages and limitations in precision, speed, or storage requirements that need to be weighed and optimized. Upgrading the current GCM treatment of multiple scattering is needed to fully implement the interactive cloud prediction scheme that we have been developing for the GISS GCM. More flexibility is also required to enable handling of particle-size-dependent scattering effects to accommodate the different types of aerosols being included in the GCM simulations.

For the longwave calculations, cloud particle size dependence has been included by mapping the spectral dependence of the cloud absorption cross-sections (obtained from Mie scattering calculations) into the 25 spectrally non-contiguous k-distribution intervals used for gaseous absorbers. The resulting cloud absorption coefficients are then tabulated and interpolated as functions of particle size. A corresponding table of emissivity correction factors is also generated as part of the longwave algorithm to correct the outgoing radiation at cloud-top level for multiple scattering effects that impart a finite reflectivity to clouds at thermal wavelengths. A similar set of absorption cross-section tables is also being generated to model the radiative effects of aerosols at thermal wavelengths.

The theoretical formulation for the continuum absorption by water vapor is one of the major accomplishments of our ARM Program participation. Based on the quasistatic approximation for far-wing absorption, and the binary collision approximation of one absorber molecule and one "bath" molecule, the continuum absorption was numerically calculated using an interaction potential consisting of an isotropic Lennard-Jones part and anisotropic dipole-dipole part, together with the measured line strengths and positions 
of allowed transitions. The basic results obtained to date were summarized in the ARM Program Poster Session at the American Meteorological Society meeting in Anaheim, California (Ma and Tipping 1993).

The theoretical formalism is applicable over the full range of frequencies and temperatures encountered in the atmosphere and has been compared and validated against available observational data. The continuum results are important not only for providing a theoretical model for the water vapor continuum absorption as a function of wavelength and temperature, but also for improving our basic understanding of the physical mechanism of continuum absorption and of spectral line-shape and farwing absorption.

We have incorporated the $\mathrm{Ma}$ and Tipping formulation of continuum absorption into our multiple scattering line-byline model and have used this model to compare synthetic spectra against the Nimbus-4 IRIS measurements. We have been testing the performance of our line-by-line model against this high spectral resolution Fourier transform infrared (FTIR) satellite data in preparation for the ARM Cloud and Radiation Testbed (CART) Atmospheric Emitted Radiance Interferometer (AERI) and AERI-X measurements.

We have demonstrated the feasibility of using this line-byline model to retrieve cloud information such as optical depth, effective particle size, and cloud-top temperature from IR spectra. The absence of significant line absorption in the thermal window region above typical cirrus altitudes permits accurate cloud property retrievals with minimum complications from line absorption. The ultimate goal is to validate the performance of the GCM radiation code through intercomparisons with line-by-line calculations. Thus, the line-by-line model serves to bridge the gap between the GCM radiation model and observational data.

\section{Improvement of GCM Diagnostics}

We have rewritten parts of the GCM diagnostics package to facilitate GCM intercomparisons. This includes extracting from the GCM output cloud radiative forcing camponents in Method II and Method III formats to enable more accurate intercomparisons with other GCM results and with observational data from the International Satellite
Cloud Climatology Project (ISCCP) and the Earth Radiation Budget Experiment (ERBE). We have also developed a two-dimensional radiative-convective-advective equilibrium model (2-D RCAM) to analyze the latitudinal dependence of GCM foodbacks (Lacis and Sato 1993).

In the 2-D RCAM analysis, zonally averaged annual mean GCM climatological information for $1.02 \mathrm{~S}_{0}$ and doubled $\mathrm{CO}_{2}$ experiments were used to determine the latitudinal dependence of feedback sensitivity. Although the applied radiative forcing was very different in the two experiments, the latitudinal dependence of the GCMsurface temperature response and of the feedback sensitivity was remarkably similar. In both cases, atmospheric water vapor was the principal positive feedback, and snowv/ice albedo was a strong positive feedback at high latitudes. Clouds were a positive feedback at low to middle latitudes, but produced negative feedback in the polar regions. Advected energy foedbacks showed significant latitudinal changes with substantial cancellation of cloud feedback contributions.

We also made intercomparisons of several satellite data sots (ISCCP, Nimbus-7, ERBE) for data diagnostic purposes and for comparison to GCM results. An empirical orthogonal function (EOF) analysis of high clouds in the ISCCP and Nimbus-7 data sets showed similar variability in magnitude, phase, and global distribution between the two data sets (Cairns 1993). Thus, both data sets provide effective high cloud validations and were particularly useful in diagnosing the EI Nino-Southern Oscillation (ENSO) high-cloud signature in GCM simulations. EOF analysis was also instrumental in detecting calibration and data artifacts that can impact the variability of cloud diurnal and seasonal cycles (Carlson and Wolf 1993). It was very clear from this analysis that clouds undergo complex diurnal and seasonal amplitude and phase changes and that these variations provide powerful diagnostic tests of both the radiative and cloud prediction parameterizations that are being developed to upgrade the GCM performance.

\section{Improvement of GCM Cloud Treatment}

We have test-run an improved cumulus and stratiform cloud parameterization in the GISS GCM (Del Genio et al. 1993). The new cloud parameterization includes a mass flux computation designed to produce a quasi-equilibrium 
between convective-scale and large-scale motions; it provides for simultaneous deep and shallow convection, transport by cumulus-scale downdrafts, as well as environmental subsidence. Stratiform clouds in the new parameterization are based on a cloud liquid/ice water budget, including a representation of mesoscale cumulus anvils, different microphysical properties for liquid and ice, collection of cloud water by precipitation, diffusional growth of ice, cloud-topentrainment instablity, and variable optical thickness.

The results show enhanced upward moisture transport by the general circulation and increased injection of water vapor and ice at the cumulus cloud top level, producing a strong positive feedback due to water vapor. Also, the new cloud scheme was able to reproduce the El Nino highcloud signature in an Atmospheric Model Intercomparison Project (AMIP) simulation, while the old scheme could not.

\section{Acknowledgments}

The research referenced below (and summarized above) was supported at GISS by the U.S. Department of Energy through an Interagency Agreement under the Atmospheric Radiation Measurement Program.

\section{References}

Cairns, B. 1993. Inter- and intra-annual cloud variations from satellite based climatologies. Fourth Symposium on Global Change Studies, pp. 245-248, January 17-22, 1993. Anaheim, California. American Meteorological Society, Boston, Massachusetts.
Carlson, B. E., and A. B. Wolf. 1993. Spatial and temporal characterization of diurnal cloud variability. Fourth Symposium on Global Chango Studies, pp. 98-103, January 17-22, 1993, Anahoim, California. American Meteorological Society, Boston, Massachusetts.

Del Genio, A. D, M.-S. Yao, and C. E. Wendell. 1993. GCM foedback sensitivity to interactive cloud water budget parameterization. Fourth Symposium on Global Change Studies, pp. 176-181, January 17-22, 1993, Anahoim, California. American Meteorological Society, Boston, Massachusetts.

Lacis, A. A, and M. Sato. 1993. GCM feedback assessment with a 2-D Radiative-Convective-Dynamic equilibrium model. Fourth Symposium on Global Change Studies, pp. 198-202, January 17-22, 1993, Anaheim, California. American Meteorological Society, Boston, Massachusetts.

Ma, Q., and R. H. Tipping. 1993. Theory and results for water vapor continuum absorption. Fourth Symposium on Global Change Studies, pp. 214-219, January 17-22, 1993, Anaheim, California. American Metenrological Society, Boston, Massachusetts. 


\title{
Stochastic Radiation Transp.ort for Climate Models
}

\author{
R. N. Byrne, D. N. Arion, and F. Malvagi \\ Science Applications International Corporation \\ San Diego, CA 92121-1578 \\ Y. Serra and R.C.J. Somerville \\ Scripps Institution of Oceanography \\ University of Callfornia, San Dlego \\ La Jolla, CA 92093-0224 \\ G. C. Pomraning and BJ SU \\ School of Engineering and Applied Science, UCLA \\ University of California, Los Angeles \\ Los Angeles, CA
}

\section{Thesis}

Computation can and will, in the near future, predict cloud morphology based upon knowledge of the local intrinsic variables such as heating rate and humidity profiles. Among the desired variables are the size distribution, in space and optical depth, of the clouds.

The radiative effects depend upon both the amount and the morphology of cloud. Data from the Atmospheric Radiation Measurement (ARM) Program will allow us to parameterize morphology in short run and will lead to adequate predictive theory and models in longer run.

Our approach, therefore, is to use ARM data to determine the statistics of observed cloud fields and to calculate the resulting radiation transport in a statistical mannier; that is, we hope to predict the average energy balance from a knowledge of the average statistics. Because radiant energy transfer is a nonlinear function of the medium traversed, this is a nontrivial problem.

\section{Areas of Research}

The four general areas of research are described below:

1. Functional Cloud Cover. This is our first attempt at a simple correction to common general circulation model (GCM) radiative treatments.
2. Theory of Stochastlc Transfor. We have shown that the Titov and Pomraning-Levermore solutions to a certain problem in stochastic transfer, though apparently very different, can be reconciled.

3. Effect on Model Calculations. We have used the Scripps Single Column Model as a stand-in for a GCM in an investigation of the effect of our first functional correction tables on climate prediction.

4. Tests of stochastle Theory. We have performed some numerical tests of stochastic transfer theory, and haveconstructed a radiation scene generator that should allow better tests to be performed. This prototype machine does not produce data, but it does demonstrate that a useful experiment could be designed and performed in a similar apparatus.

\section{Functional Cloud Cover}

coal

Our goal is to derive a method of handling the cloudradiation problem that will improve the accuracy of GCM codes and lead to increased accuracy in the prediction of global climate change. We want a method that is

- simply implemented in existing codes, to increase the chance that it will be actually used, and 
- very fast to run, because of the large number of scenes that must be considered in every time step of a GCM.

\section{Technique}

Our technique is to apply some new results of stochastic radiation transfer theory to the problem of radiation transport through clouds, for both the scattering-dominated shortwave solar and the absorption-dominated long-wave terrestrial reradiation. We are using the ARM observations for two purposes: 1) to choose a statistical description of the clouds over the AFM site, and 2) to test the results of our model calculations against observations in situ. The results will be presented in tabular form, allowing a GCM to replace its calculated cloud cover with one which is functionally equivalent to the real cloud cover for the purposes of radiative energy balance, given the radiative approximations of the GCM.

\section{Stochastlc Radiative Transfer}

The general equation in this one-dimensional geometry for the average radiation intensity at direction $\operatorname{cosin} \theta \mu$ is

$$
\begin{aligned}
& \mu \frac{\partial}{\partial z}\left(p_{i} l_{l}\right)+\sigma_{i} p_{i} l_{l}= \\
& \int_{-1}^{1} d \mu^{\prime} \sigma_{i}^{s}\left(\mu, \mu^{\prime}\right) p_{i} l_{i}\left(\mu^{\prime}\right)+p_{i} s_{i}+\frac{p_{j} \bar{l}_{l}}{\lambda_{j}} \cdot \frac{p_{i} \bar{l}_{l}}{\lambda_{i}}
\end{aligned}
$$

where we distinguish two media, clpud and clealr sky, and label them by $i$ andj. $\sigma$ stands for the attenuation coefficient, $\sigma$ for its scattering part, and $\lambda$ is the scale length for transition from sky to cloud or vice versa. The barred l's stand for the conditional probabilities, when making the transition from one $r$. idium to the other. This equation is exact as it stands, within the stated approximations, but is incomplete since we do not know how to relate the barred I to the unadorned one.

If we have a Markov distribution of cloud and no scattering, then we can use the simple closure, in which case the problem reduces to a simple set of two differential equations in two unknowns and can be solved readily. This is such a strong condition that the answer is of little interestscattering is always present to some extent, the cloud size distribution is not Markovian, and a simple binary distinction of cloud/clear is unsupportable.
Pomraning has suggested a simple modification of this closure that seems to give much more accurate solutions, in aset of numerical test problems. Supplement Equation (1) by the closure:

$\mu \frac{\partial}{\partial z}\left(p_{1} I_{1}\right)+\sigma_{1} p_{1} I_{1}=$

$\int_{-1}^{0} \sigma_{i}\left(\mu, \mu^{\prime}\right) p_{1} I_{1}\left(\mu^{\prime}\right) d \mu^{\prime}+\int_{0}^{1} \sigma i\left(\mu \mu^{\prime}\right) p_{1} I_{1}\left(\mu^{\prime}\right) d \mu^{\prime}+p_{1} s_{1}+\frac{p_{1} I_{1}}{\lambda_{1}}-\frac{p_{1} I_{1}}{\lambda_{1}}$

for $\mu$ along the direction of $I$, and with the integral limits reversed othenwise. These two equations give an accurate, but still approximate, average solution to the set of problems, not the solution of the average problem.

\section{Results}

Our results, so far, include a computation of sample correction functions based, in the absence of ARM data, on a simple model of cloud morphology that has little to recommend it beyond simplicity. They have been implemented in the SIO Single Column Model and the effect of these changes has been determined and will be presented. below. We do not claim accuracy at this stage, but we have demonstrated feasibility of the functional technique. The geometry of the stochastic calculation we use as a first case is shown in Figure 1.

We have taken a semiinfinite layer of thickness $L$ and assumed a uniform randomly distributed population of elliptical clouds with horizontal extent $D$ and vertical height $H$. Their volume fraction is p. Scatterers (shortwave mockup) and absorbers (longwave) are both modeled. Both diffuse and narrow beam (at solar angles of $0^{\circ}, 30^{\circ}$, and $60^{\circ}$ ) have been studied.

Figure 2 shows the transmission expected of a particular set of parameters as calculated using the simple butwidely used fractional cloud cover model and the more accurate model given above.

\section{Conclusion}

We have found so far that this formulation of the stochastic transfer problem is tractable. We have been able to construct tables using this method, as described below. Our investigation as to number and accuracy of tables required is proceeding. 


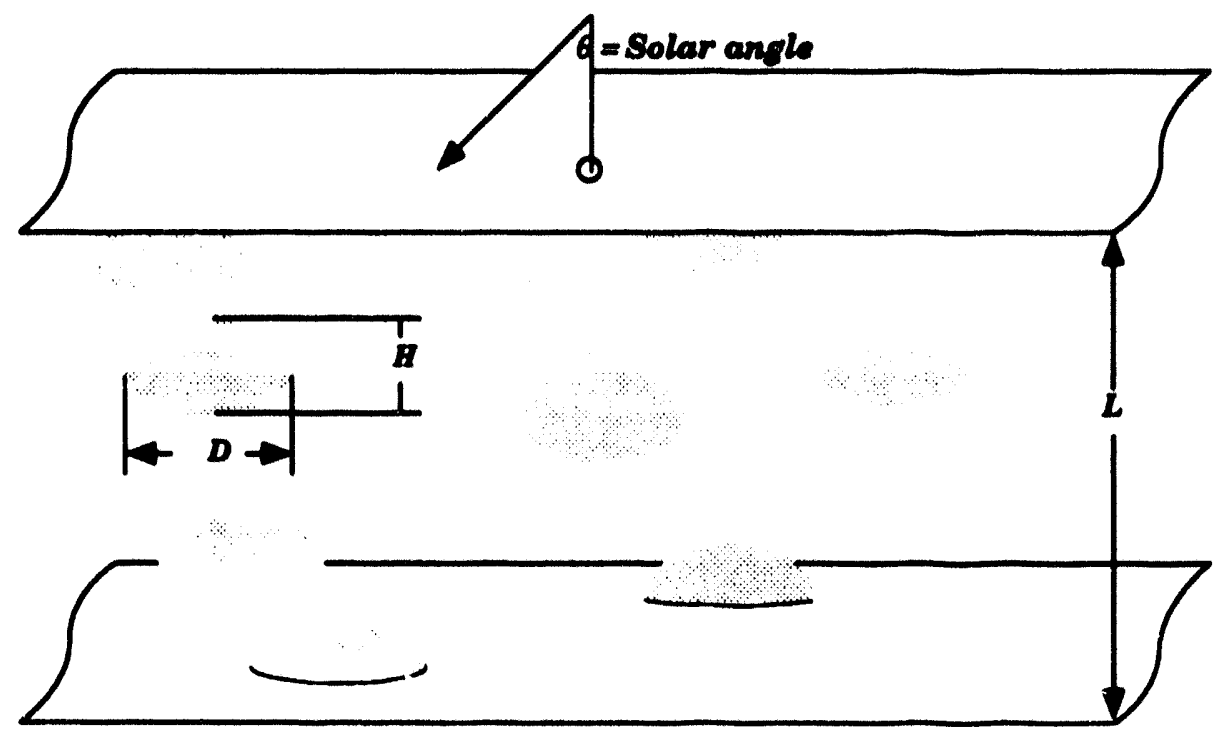

Figure 1. Genmetry of model system.

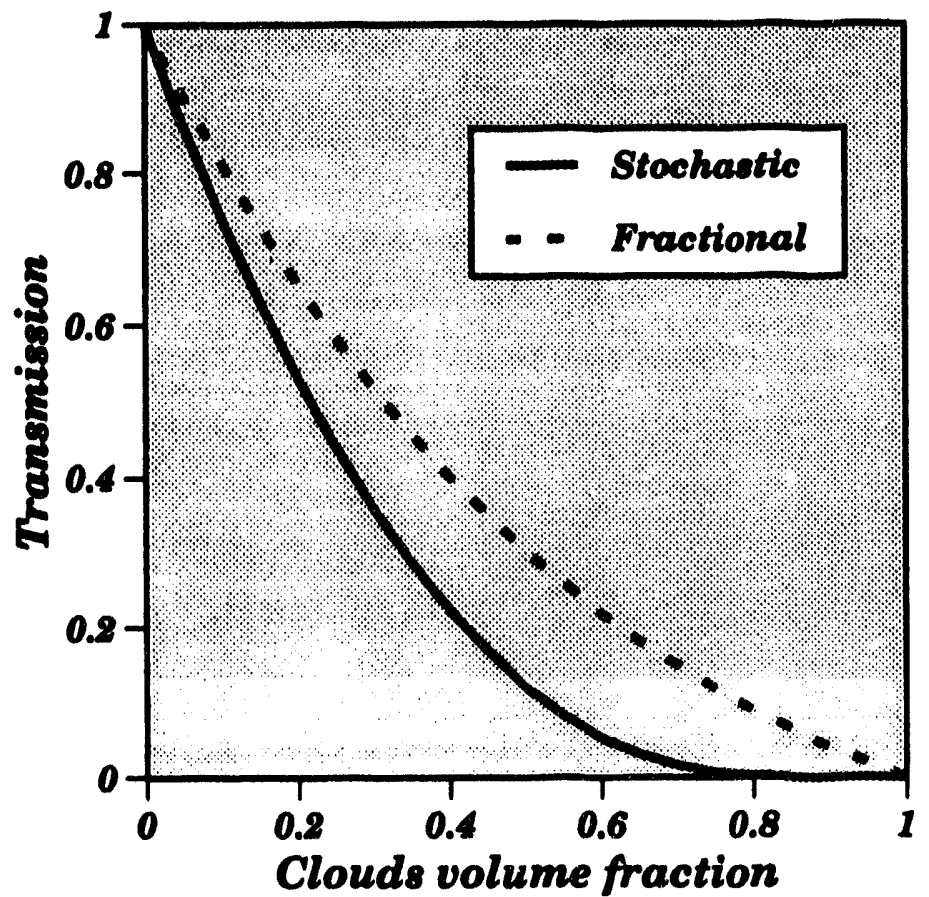

Figure 2. Transmission through absorbing cloud cover. 


\section{Stochastic Transfer Theory}

\section{Reconclilation: THov-Pomraning}

Titov has solved the problem of a Markovian distribution of clouds in a binary mixture of clear sky and cloud, derlving aset of integral equations. The above formulation, in torms of differential equations, comes from a very different set of considerations, yot both approaches should bo correct for this problem, sinss each is an exact solution for this geomotry, assuming that the clouds are pure absorbere and that the clear sky ls perfectly tranemiselve. Wo have been able to show that, as hoped, the two solutions agree in this case. The differential method we espouse as more generality, however.

\section{Effect on Model Calculations \\ Sonstivitiy of Singlo Column Model (SCM)-Ocean}

We wish to estimate the effect of this new stochastic treatment on GCMs. As a simple, relatively controllable stand-in for a GCM we use the Scripps Single Column Model, which has the general physics packages of the standard GCMs in it, but gains simplicity through replacement of the three dimensional fluid dynamice calculation by a one-dimensional column in which horizontal divergences are specifled and only the vertical calculations are performed, making it a goometric mean of a radiativeconvective model and a full three-dimensional GCM. In the absence of ARM data, we assumed a general form for a functional correction table and made it depend on a single parameter. As a function of this parameter, the functional correction table (there was only one, assumed to apply both to longwave and shortwave) is shown in Figure 3.

Some results of this calculation, for the Indian Ocean at the start of the monsoon season, are shown in Figure 4.

\section{Tests of Stochastic Theory Numerical}

We have tested the new closure prescription by running a large number of computer calculations. For a given set of statistical parameters, we generate many problems with input chosen at random from the desired distribution, then

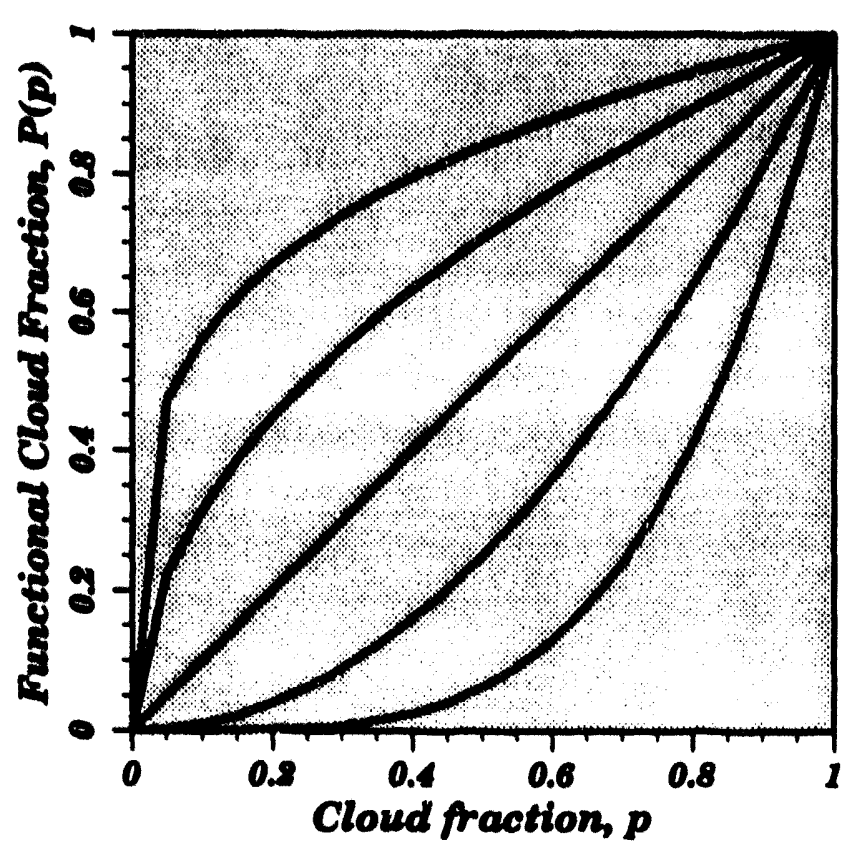

Figure 3. Functional forms used in the study.

solve them numerically. With this technique wo can include both scattering and absorbing modia, as well as arbitrary mixtures. Computer time constraints limit us to onedimensional geometries, th.sugh. We have solved both rod and slab systems. The resultis generally indicate increased accuracy for the new closure as far as global quantities such as transmission and absorption are concerned, but the spatial distribution of absorption is not completely understood.

\section{Exporimental}

In part because the large number of problems we must run on a computer to get a meaningful average of the solution (which effectively restricts us to one-dimensional systems) and in part because of the desire to proceed with the research in the period before actual ARM data is avallable, we investigated the possibility of bullding a laboratoryscale machine that could be used to test stochastic transport theories. For a parts cost of under $\$ 200$ we put together the apparatus shown in Figure 5. 


\section{HEAT BUDGETS - Ocean Data}
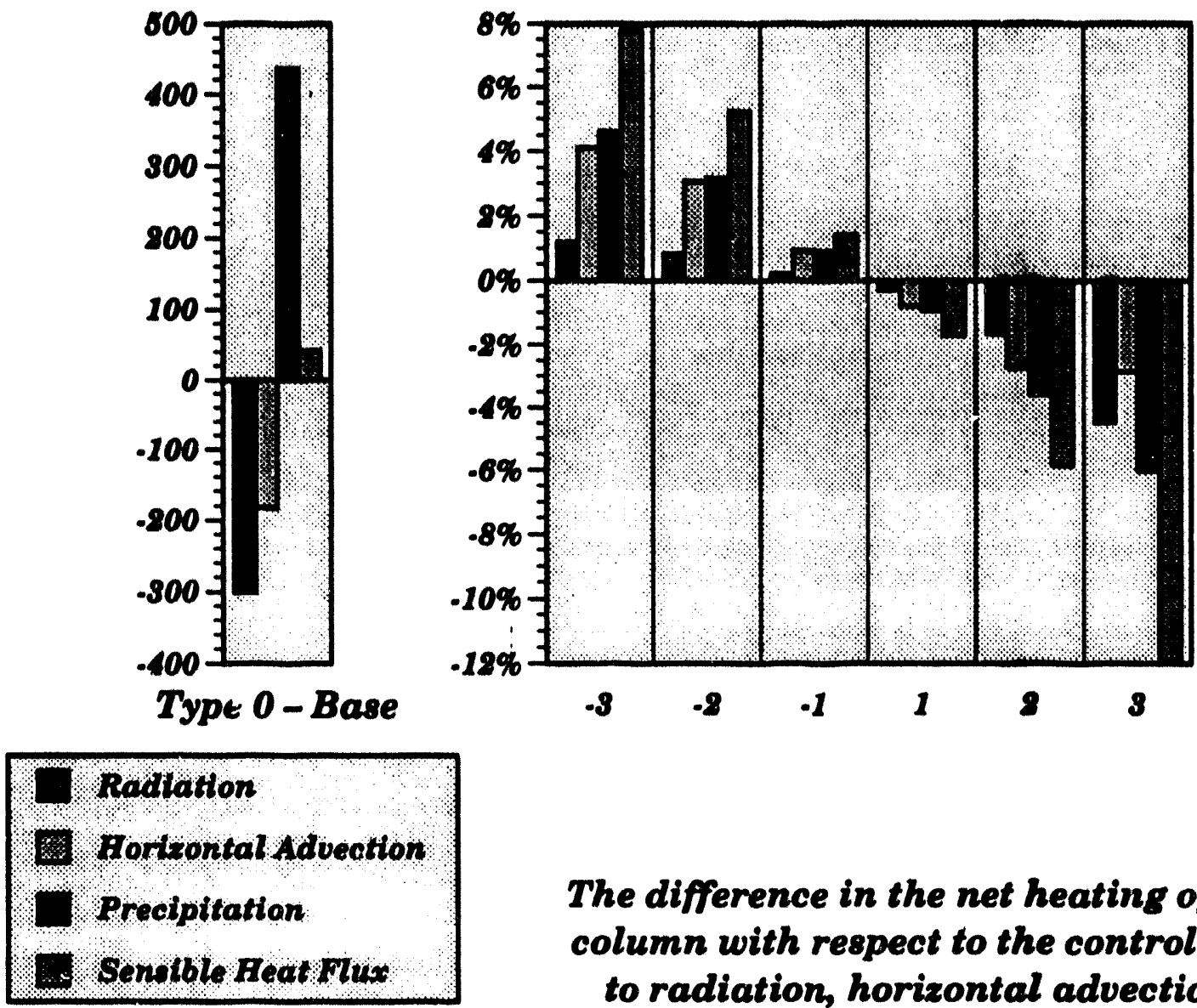

\section{The difference in the net heating of the column with respect to the control due to radiation, horizontal advection, precipitation, and sensible heat fluxes.}

Figure 4. Results: heat budgets over ocean.

This machine is a cylinder lined with shiny foil, containing a controlled number of styrofoam beads. These are very white and very light. They are agitated by a stream of air introduced from below, and are contained by a set of screens at the top and bottom of the experimental volume. A small halogen bulb is at the focus of a (borrowed) telescope mirror, which produces a parallel beam of test radiation. Photodiodes are located at the top, midplane, and bottom. The air flow is regulated by varying the fan speed, and the resulting agitation of the spheres ranges from a slow heave to a completely volume-filling random motion.

The resulits, shown in Figure 6, indicate that the output of the photodiodes is stable over a period long enough with respect to that required for the radiation transfer problem to be completely redefined. Actually, we observe no significant drift in the optical properties of this simple system for periods exceeding half an hour. 


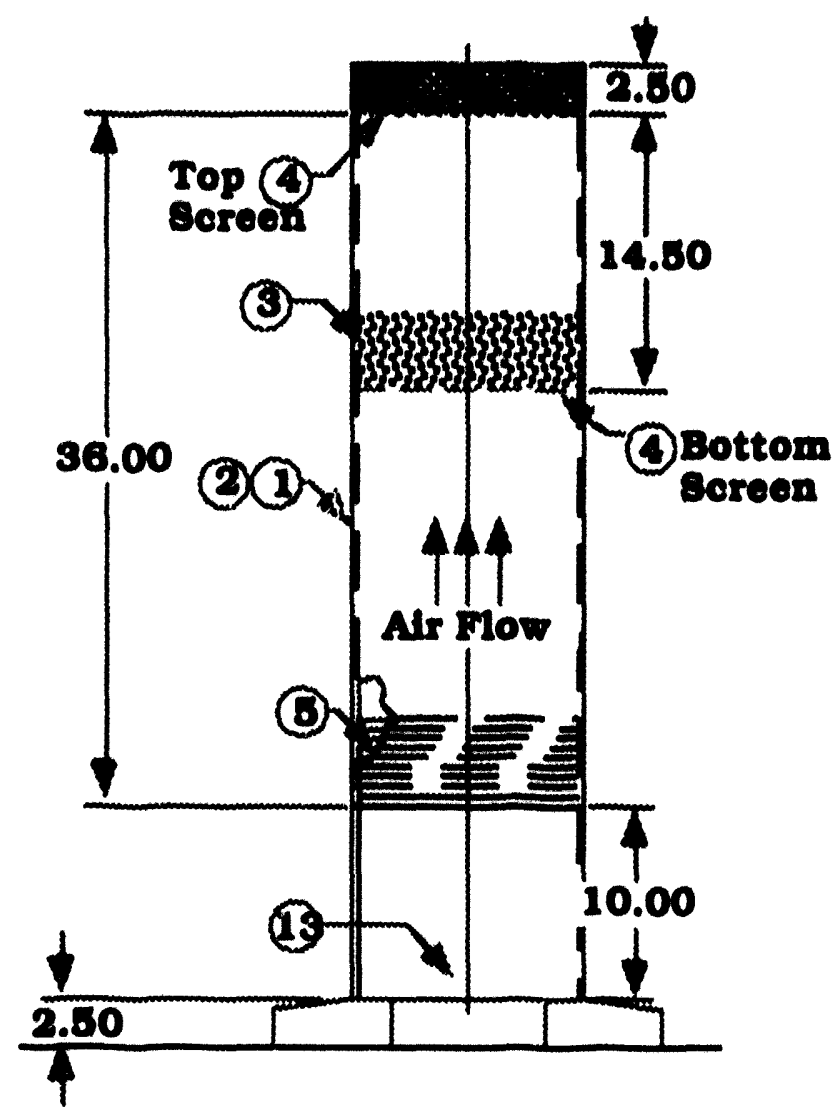

Flgure 5. MicroARM machine.
We conclude that this concept is capable of development into a dovice that could perform useful service in experimental stochastic radiation transfor. The diagnostice on the prototype are inadequate for anything other than demonstrating that the machine works and would have to be supplemented with a bottor set bofore useful data could be obtained, but wo have shown that the experiment is posalble, that the output le ateady over many decorrelation times, that the measuremente are preclse and reproduclble, and that the input (air flow, density of spheres) affects output; although this is not documented here, it has been obeerved in the lab.

Although the styrofoam spheres are not the water droplets found in clouds, the radiation problem they present is related, and this is a more controlled experiment than is easily reproduced elsewhere.

\section{Conclusion}

Wo have made progress in the area of functional cloud cover (correction tables for GCMs), in theoretical stochastic transier (especially the reconcillation of the Titov and Pomraning approaches to the Markov problem), in the effect of improved cloud-radiation interaction on GCMs through the application of the Scripps code, and to numerical and experimental tests of stochastic theory. 


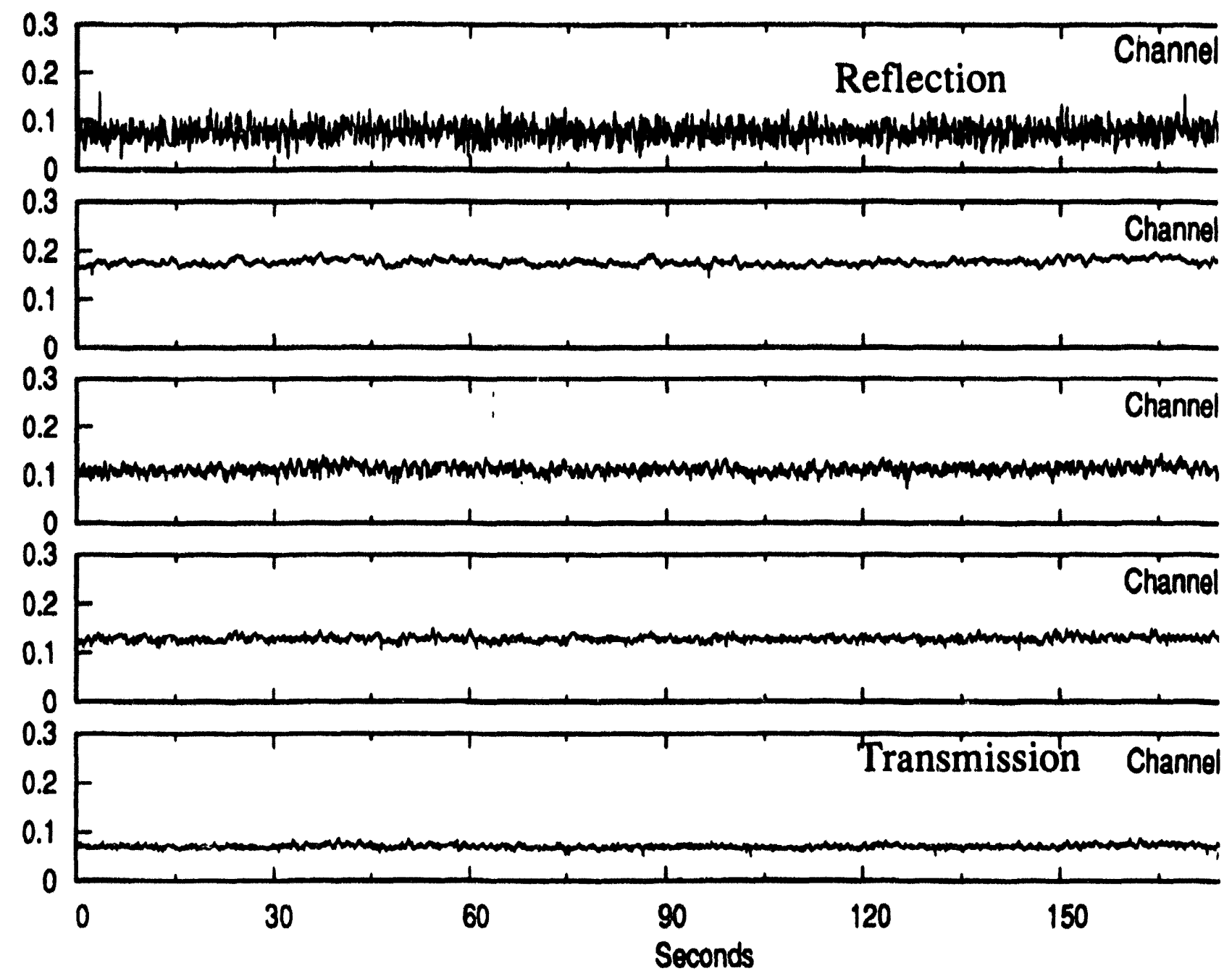

Figure 6. Sample results of radiation experiment. 


\title{
A Model of the Microphysical Evolution of a Cloud
}

\author{
J. Zinn \\ Space Science and Technology Division \\ Los Alamos National Laboratory \\ Los Alamos, NM 87545
}

The earth's weather and climate are influenced strongly by phenomena associated with clouds. Therefore, a general circulation model (GCM) that models the evolution of weather and climate must include an accurate physical model of the clouds. This paper describes our efforts to develop a suitable cloud model. It concentrates on the microphysical processes that determine the evolution of droplet and ice crystal size distributions, precipitation rates, total and condensed water content, and radiative extinction coefficients.

We assume a fixed temperature, a cloud vertical thickness, and concentrations and size distributions of cloud condensation nuclei (CCN) and ice condensation nuclei (ICN). The computation starts at time $\mathrm{t}=0$ with a given concentration of precipitable water entirely in the form of vapor. As time advances, we compute the evolution of the number and size distributions of the liquid droplets and ice crystals along with their evaporation/condensation rates, collision rates, vertical falling rates, and rates of loss from outfluxing. We then compute the resulting evolution of the cloud water vapor, liquid and ice concentrations. As a byproduct, the precipitation rate and the cloud optical extinction coefficient are computed.

The evaporation/condensation rate expression for liquid drops is based on the well-known Kohler equations, which include the effects of vap.. pressure reduction due to dissolved CCN material (Raoult's law) and enhancement due to droplet surface tension (Gibbs-Thomson effect). An inforence from these equations is that for small positive values of the vapor phase supersaturation, there is a limited range of CCN masses for which equilibrium between the liquid droplets and the vapor phase can exist. For larger CCN mass above a certain value, $m_{\text {CNerit, and }}$ corresponding droplet radil above the value $r_{\text {crit }}$, no equilibria are possible, and the droplets tend to grow without limituntil the vapor phase supersaturation is reduced.
Even in the absence of ICN orice crystals, these equations describe a surprisingly complex range of phenomena. For a given initlal value of the vapor-phase supersaturation 8 greater than zero (relative to liquid water), the smallest droplets tend to grow faster than the larger ones, leading to a droplet size distribution function that ovolves rapidly toward a delta function-1.e., a single droplet size, until continued condensation onto the droplets causes the supersaturation to drop to a point where $r_{\text {crit }}$, which varies as $1 / 8$, increases to a value larger than the prevaling droplet radius. At that point, some of the droplets begin to reevaporato- the smallest first, causing the small dropsize (haze) part of the distribution to be repopulated. (See Figure 1.) At the same time, the largest droplets are falling more rapidly than the smaller ones, resulting in collisions and coalescence which accelerate the growth of the large drops at the expense of the smaller ones.

Figure 1 shows the computed evolution of the droplet size distribution function $d N_{\text {cropo }}$ /dogr vs $r$ for a sequence of times for a system with only liquid water and vapor (with $T$ $=270 \mathrm{~K}$ and an initial saturation ratio of 1.255). Condensation onto the CCN is essentially complete within about 5 seconds. The subsequent evolution is dominated by droplet coalescence and reevaporation.

Aquantity of particular interest is the computed precipitation rate and the associated rate of water loss from the cloud. The computed rate of development of precipitation in clouds that include only liquid water with no ice phase is quite slow-somewhat slower than the observed rate of ovolution of clouds over land. For a more realistic representation of terrestrial cloud systems, the ice phase must be included.

With the inclusion of ice, at temperatures below about $266 \mathrm{~K}$, we obtain a more rapid development of precipitation (Bergeron-Findeisen effect). Because the ice crystals have 


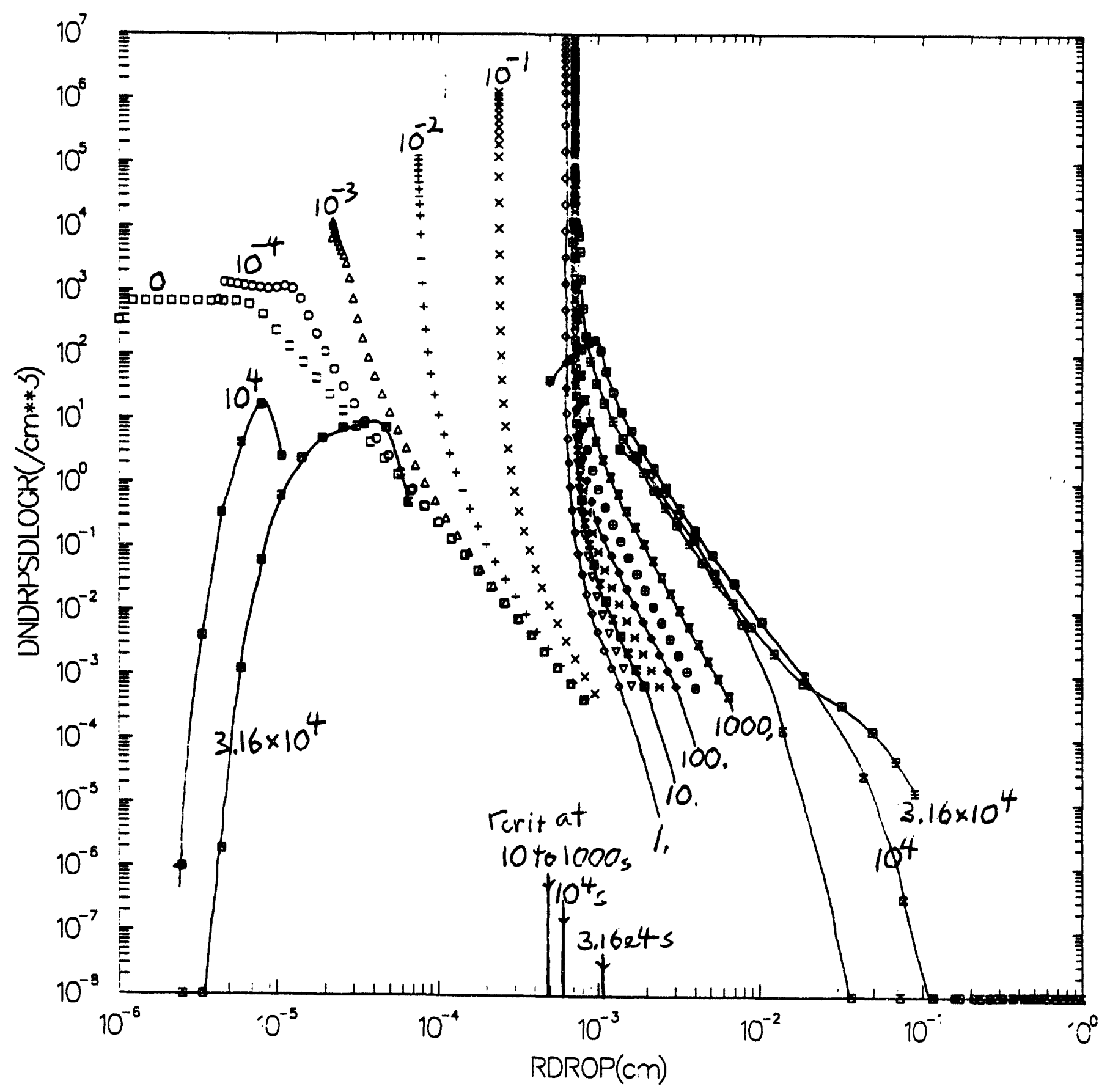

Figure 1. Liquid drop number density vs log-radius distribution function $d N / d \log r\left(\mathrm{~cm}^{3}\right)$ vs $\log r$ for a cloud at $270 \mathrm{~K}$ with no ice condensation nuclei-for a sequence of times from zero to $3.162 \times 10^{4} \mathrm{~s}$. The times are indicated on the curves. Also shown along the $r$ axis are the Kohler critical droplet radii at selected times. The initial water vapor concentration in this case was 1.255 times the concentration of saturated vapor. 
lower equilibrium vapor pressures than the liquid water drops, water tends to evaporate off the liquid drops and condense on the ice crystals, causing the latter to grow rapidly. As the ice crystals grow, they fall with increasing speed, coalescing with liquid drops in an accelerating rate. In this model, coalescence between an ice crystal and a liquid drop is assumed to result in a larger ice crystal, with the mass of the two particles combined.

Figures $2 a$ and $2 b$ show the computed evolution of the droplet size distribution function and the ice crystal size distribution function when both liquid and ice are included and the initial supersaturation is quite large $(T=264 \mathrm{~K}$, initial saturation ratio 1.915 ). Here again, condensation onto the liquid droplets is essentially complete after 5 seconds. For the next half hour, the dominant process is growth of the ice crystals by condensation and coalescence with liquid drops at the expense of the liquid drops. During the succeeding half hour, there is very rapid growth of the ice crystals and strong precipitation, resulting in substantial drying out of the cloud. For the next 8 hours, the cloud continues to evolve, with continuing precipitation and with the total number of droplets decreasing continually because of coalescence.

During most of the cloud's life, from 10 to $10^{4} \mathrm{~s}$ the predominant liquid drop radius is $10 \mu \mathrm{m}$, as is typical in real clouds. The ice crystals grow steadily for the first half hour, reaching a radius of $1.4 \mathrm{~mm}$, at which point they are precipitating rapidly.

Over the 8-hour period of the computation, the droplet number density drops from an initial value of about 600 per $\mathrm{cm}^{3}$ to a final value of 3 per $\mathrm{cm}^{3}$. The ice crystal density is about 1.3 per $\mathrm{cm}$. The optical extinction coefficient drops gradually over a 9 -hour period to $1.9 \times 10^{-5} \mathrm{~cm}^{-1}$.

In a real cloud, of course, we never observe a state consisting of dry CCN and highly supersaturated water vapor. In the model, this hypothetical state is simply an artificial reference point from which we start the computation. The computations show that by condensation of liquid water onto the CCN, the system evolves into a fairly realistic state within 3 to 10 seconds. After that, the initial liquid-vapor equilibration is essentially complete, and the supersaturation is reduced to between $0.01 \%$ to $0.02 \%$. For comparisons with real clouds the initial 10 seconds of the computation should be ignored.
These computed results depend, of course, on the assumed concentration and size distribution of $C C N$ and ICN. The distributions that we use in this model are analytic fits to data quoted by Pruppacher and Klett (1978). The effective ICN concentration is a very strong function of the degree of supercooling. We have performed parameter studies in which we vary the $\mathrm{CCN}$ or ICN concentrations by a factor of 10 above or below the assumed standard concentrations, or alternatively, where we set the ICN concentration to zero. The model also assumes an idealized uniform cloud of fixed vertical extent. We have also done some parameter studies with varying the vertical extent.

These computations were intended to provide a basis for developing parameterized descriptions of clouds and precipitation for use in a GCM. We have not yet finished this task. Even within the context of this idealized model, the variable parameters include temperature (T), CCN concentration, ICN concentration, cloud vertical thickness, initial saturation ratio $\left(S^{0}\right)$, and time $(t)$.

So far, only a few useful generalizations are evident. There are three distinct classes of systems to be considered:

- Type 1-unsaturated air masses $\left(S^{0}<1\right)$

- Type lla-supersaturated air masses $\left(S^{0} \geq 1\right)$ with $T>$ $273.1 \mathrm{~K}$

- Type llb-supersaturated air masses with $T<273.1 \mathrm{~K}$.

Unsaturated systems are relatively simple, and give rise to haze, with no cloud droplets. Wo will concentrate on Type II (supersaturated) systems and only on processes that occur within 8 hours.

In Type II systems, by the end of 10 seconds the initial phase of condensation of water vapor onto the CCN is essentially complete; the supersaturation is of order $10^{-4}$; the water vapor concentration $n_{\text {vap }}$ is very close to the saturation concentration, $n_{s a r}$ and the liquid concentration $n_{1 q}\left(\mathrm{molec} / \mathrm{cm}^{3}\right)$ is very close to $n_{\text {sat }}\left(S^{0}-1\right)$.

The processes of droplet coalescence, gravitational settling, and formation of ice crystals can be considered to begin from this point. The computations show that gravitational settling fluxes of liquid droplets are insignificant (less than about $3 \times 10^{-7} \mathrm{~g} / \mathrm{cm}^{2} . \mathrm{s}$ ) when the liquid water density is less than $2 \times 10^{15} \mathrm{~cm}^{-3}$, and the fluxes are roughly proportional to the 1.5-power of $n_{1 q}$ for larger values of $n_{1 q}$. Thus the 


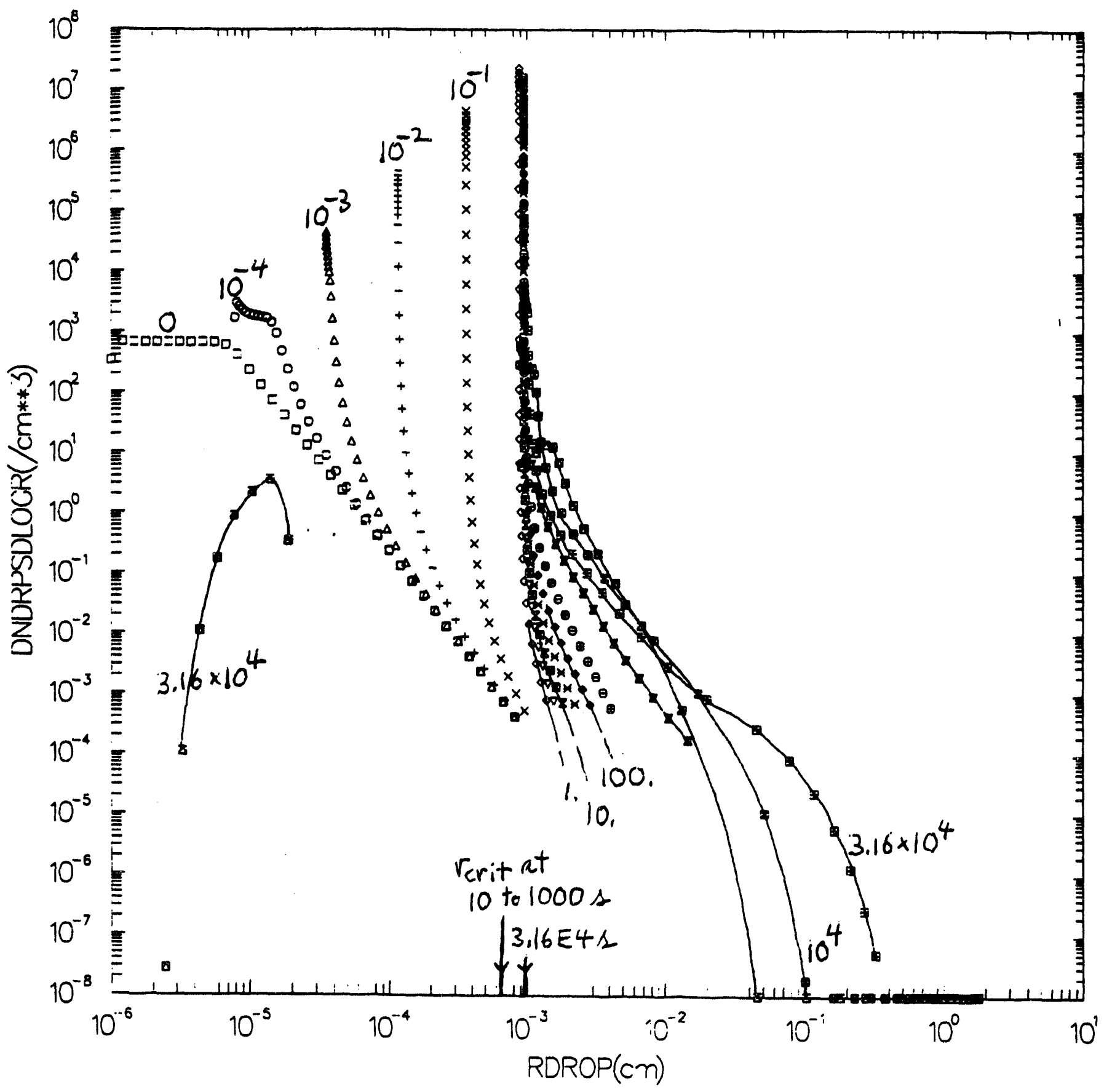

Figure 2a. Liquid drop number density vs log-radius distribution function $d N / d \log r\left(\mathrm{~cm}^{3}\right)$ vs logrfor a cloud at $264 \mathrm{~K}$ with the "standard" number density distribution of ice condensation nuclei-for a sequence of times from zero to $3.162 \times 10^{4} \mathrm{~s}$. The times are indicated on the curves. Also shown along the $r$ axis are the Kohler critical droplet radil at selected times. The initial water vapor concentration in this case was 1.915 times the concentration of saturated vapor. 


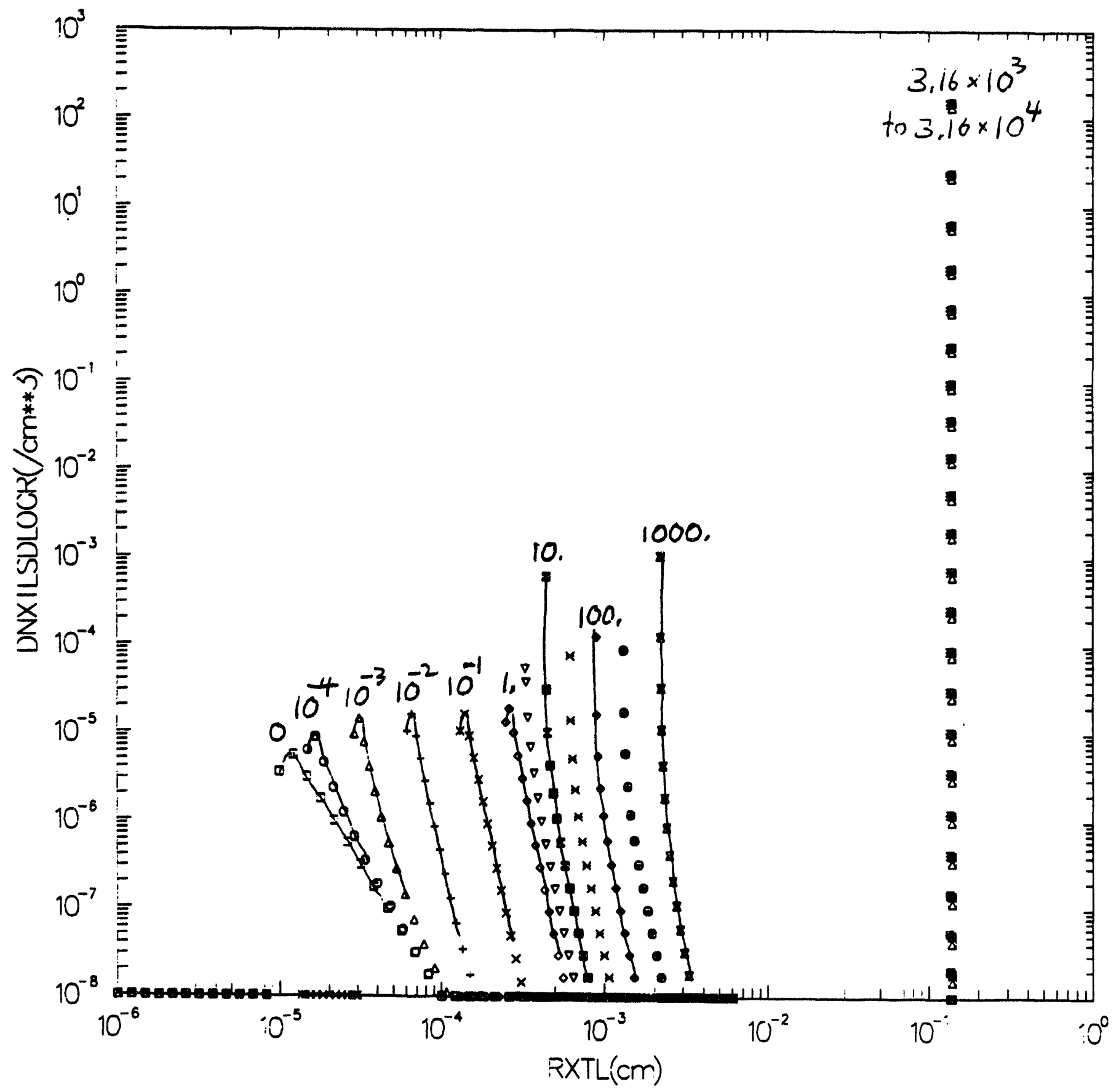

Figure 2b. Ice crystal number density vs log-radius distribution function $d N / d \log r\left(\mathrm{~cm}^{3}\right)$ vs logr for a cloud at $264 \mathrm{~K}$ with the "standard" number density distribution of ice condensation nuclei-for a sequence of times from zero to $3.162 \times 108$. The times are indicated on the curves. The initial water vapor concentration in this case was 1.915 times the concentration of saturated vapor. 
water a cloud loses through falling droplets is insignificant if $n_{\text {eat }}\left(5^{0}-1\right)<2 \times 10^{15}$. $n_{\text {eat }}$ can be computed from the Clausius-Clapeyron equation, so this sets a practical lower limit to the range of $S^{0}$ for clouds that can precipitate or change shape because of gravitational settling of droplets.

lce crystals begin to have a significant effect on precipitation rates and/or total settling fluxes only at temperatures below about $266 \mathrm{~K}$. For temperatures above $262 \mathrm{~K}$ the ice concentration $n_{i c o}$ (molec/ $/ \mathrm{cm}^{3}$ ) (for times less than 8 hours) is smaller than the $n_{\text {rep }}$ and the evolution of the ice distribution does not materially affect $n_{1 q}$ or the average droplet radius or the liquid drop settling flux. Nevertheless, the ice crystals continue to grow, both by condensation from the vapor and by collisions with liquid droplets, leading to ice crystal precipitation and settling rates $\left(\mathrm{g} / \mathrm{cm}^{2} . \mathrm{s}\right)$ that exceed those of the liquid water.

The results of computations that start from large values of $S^{0}$ exceeding about 1.2 show strong precipitation, which tends to dry out the cloud. In 8 hours such a cloud seems to evolve toward a fairly "typical" state in which the liquid water concentration, average drop size, relative humidity, and optical extinction coefficient are all fairly constant, depending only weakly on the temperature, the precise value of $S^{0}$, the ICN concentration, or the cloud vertical thickness. Typical 8-hour values of the liquid concentration, average drop radius, supersaturation, and extinction coefficient at temperature $273 \mathrm{~K}$ are $1.2 \times 10^{15} \mathrm{molec} / \mathrm{cm}^{3}$, $12 \mu \mathrm{m}, 7 \times 10^{-5}$, and $2 \times 10^{-5}$, respectively.

Although the cloud vertical thickness has relatively little effect on the final concentrations of liquid water and vapor, it can have a pronounced effect on the total precipitation rate.

\section{Reference}

Pruppacher, H. R., and J. D. Klett. 1978. Microphysics of Clouds and Precipitation. D. Reidell Publishing Co., Dordrecht, Holland. 
Data Assimilation 


\title{
The Sensitivity of Flux Parameterizations to Surface Characteristics
}

\author{
J. C. Doran \\ Pacific Northwest Laboratory \\ Richland, Washington 99352
}

The problem of measuring or calculating appropriate surface heat and moisture fluxes for use in general circulation models (GCMs) and single-column models (SCMs) is an important one. This is particularly true at Cloud and Radiation Testbed (CART) sites, where an accurate treatment of the surface boundary conditions is essential if SCMs are to be used to study cloud and radiation processes in detail. For this discussion, three issues will be considered:

1. From measurements of surface fluxes at a finite (and relatively small) number of points, such as will be available at the CART site, how can one interpolate/ extrapolate to get the average flux over an area covered by an SCM or a GCM grid cell?

2. How are fluxes parameterized in models? Are these parameterizations consistent with what is known about the behavior of the planetary boundary layer (PBL) over inhomogeneous terrain? Can one deduce the correct average surface fluxes from knowledge of averaged boundary layer quantities such as mean winds or temperatures at a model level?

3. How much does it matter if fluxes are not correctly parameterized? What might some of the consequences be if the flux values used in models are incorrect?

To address these questions, some results from both observations and model simulations will be described. These results show differences in boundary layer properties over adjacent areas with differing surface characteristics. Such differences can have important consequences for the determination of area-averaged flux values from point measurements.

Several laboratories carried out measurement programs for the Atmospheric Radiation Measurement (ARM) Program in the springs of 1991 and 1992 in northeastern Oregon near the town of Boardman. The region is characterized by two distinct surface types - dry sagebrush and grassland steppe and heavily irrigated agricultural areas.

The winds during the 1992 experiment had three principal patterns. In the first pattern, the winds were moderately strong (up to $10 \mathrm{~m} / \mathrm{s}$ near the surface) from the westsouthwest and persisted through most or all of the day. In the second pattern, winds would start out from the westsouthwest in the morning at speeds of $4.7 \mathrm{~m} / \mathrm{s}$, decrease, and around 1100 PST would shift and blow out of the north or even northeast. In the third pattern (which happened once) the winds were west-southwest for almost the whole day but the speeds were only $3-7 \mathrm{~m} / \mathrm{s}$. Winds from the west or southwest blew first over a dry fetch of $\sim 18 \mathrm{~km}$ before blowing over the instrumented farm site.

Despite the relatively small scales of the adjacent disparate surface types, it was possible to detect differences in the boundary layer characteristics over the wet and dry areas for all three wind patterns. For strong wind cases, increased evaporation produced cooler temperatures over the irrigated area than over the dry area in the lowest 50 . $100 \mathrm{~m}$. For lighter winds, the temperature contrasts near the surface were smaller but temperature differences could still be seen over depths of several hundred meters. The winds over the dry area were stronger, primarily due to the lower roughness length in that region. However, the reduced wind speeds over the farm were also consistent with the development of a weak secondary circulation arising from the thermal contrasts between the two surface types. On the day with the third wind pattern described above, additional suggestions of the development of a "farm breeze" were seen in the wind speed and direction profiles obtained from three sodars operated during the experiment.

From the results of the Boardman experiment, it is apparent that in calculating fluxes over different areas or in 
interpolating fluxes from point values to area averages, knowledge of the soll properties (vegetation, soil moisture, soil type, etc.) is a necessary but not sufficient condition. One may also have to take into account the variations in the wind, temperature, and cloud fields that help determine the surface fluxes. Even over a scale as small as that for Boardman, with an experimental domain less than $30-\mathrm{km}$ long, such factors were found to vary substantially and had significant influences on the local flux values. Over a CART site, the scales will be larger and the effects may be greater.

Possible effects of heterogeneity in subgrid-scale surface properties can also be simply illustrated by considering results from some two-dimensional simulations carried out with the Colorado State University Regional Atmospheric Modeling System (RAMS) nonhydrostatic mesocale model. The issue add ressed in these simulations is the consistency of the parameterizations used in GCMs or SCMs when the model resolution is insufficient to explicitly account for subgrid-scale variations in surface fluxes. In the simulations, the average soil moisture over the modeling domain $(200 \mathrm{~km}$ in extent) was held fixed. A central $100-\mathrm{km}$ region was then modified so that half of the region was wetter than the average and half was drier by the same amount. The dry and wet halves were broken up into patches ranging from 12 to $50 \mathrm{~km}$ in size, and the order in which the ambient wind blew over the dry and wet patches was varied. In one case, a 50-km dry patch was encountered first and, in the other case, a 50-km wet patch was first. Contour plots of the resulting distributions of horizontal wind speeds and of mixing ratios are shown in Figures 1 and 2, respectively.

The figures clearly show that the responses of the boundary layer in the two cases differ. Because the differences in sensible heat fluxes over the wet and dry areas generate secondary circulations, the spatial variations of the wind and moisture fields change substantially as the orientation of the different surface types relative to the mean wind is changed. Averages of wind speeds or mixing ratios over the $100-\mathrm{km}$ central region differ as well. Temperature fields are similarly affected.

Two conclusions may be drawn from this example. First, properties of the boundary layer derived from averages of the surface conditions will not, in general, be the same as the average of the conditions derived over two different surfaces. Second, the order in which the individual patches are encountered may also be important. These findings are consistent with results obtained from the Boardman experiments. The implication for SCMs is important: to handle surface inhomogeneities correctly, one must know not only the percentages of each land surface type, but the characteristic sizes of the patches and their orientation relative to the prevailing winds.

How much does it really matter if the flux distributions are not treated correctly in the models? There are at least three reasons why a knowledge of the details of the boundary layer structure may be important for issues of concern to the ARM Program. First, the surface energy budgets are obviously dependent on the surface characteristics and the local meteorological conditions, which include the properties of the boundary layer. The surface energy budget, in turn, determines the surface temperature and the longwave radiation from the surface. Second, the height of the boundary layer and the distribution of aerosols and moisture in the boundary layer can clearly play an important role in the radiation budget; these distributions are determined largely by the turbulent mixing of heat, momentum, and moisture in the boundary layer, which, in turn, depend on the surface fluxes driving them. Finally, local "hot spots" may tend to favor the formation of cumulus clouds preferentially in some areas of the CART domain and not in others. All of these boundary layer features must be represented properly in SCMs and GCMs if their treatments of clouds and radiation are to be tested and improved.

\section{Acknowledgment}

This research was supported by the U.S. Department of Energy under Contract DE-AC06-76RLO 1830 at Pacific Northwest Laboratory. Pacific Northwest Laboratory is operated for the U.S. Department of Energy by Battelle Memorial Institute. 


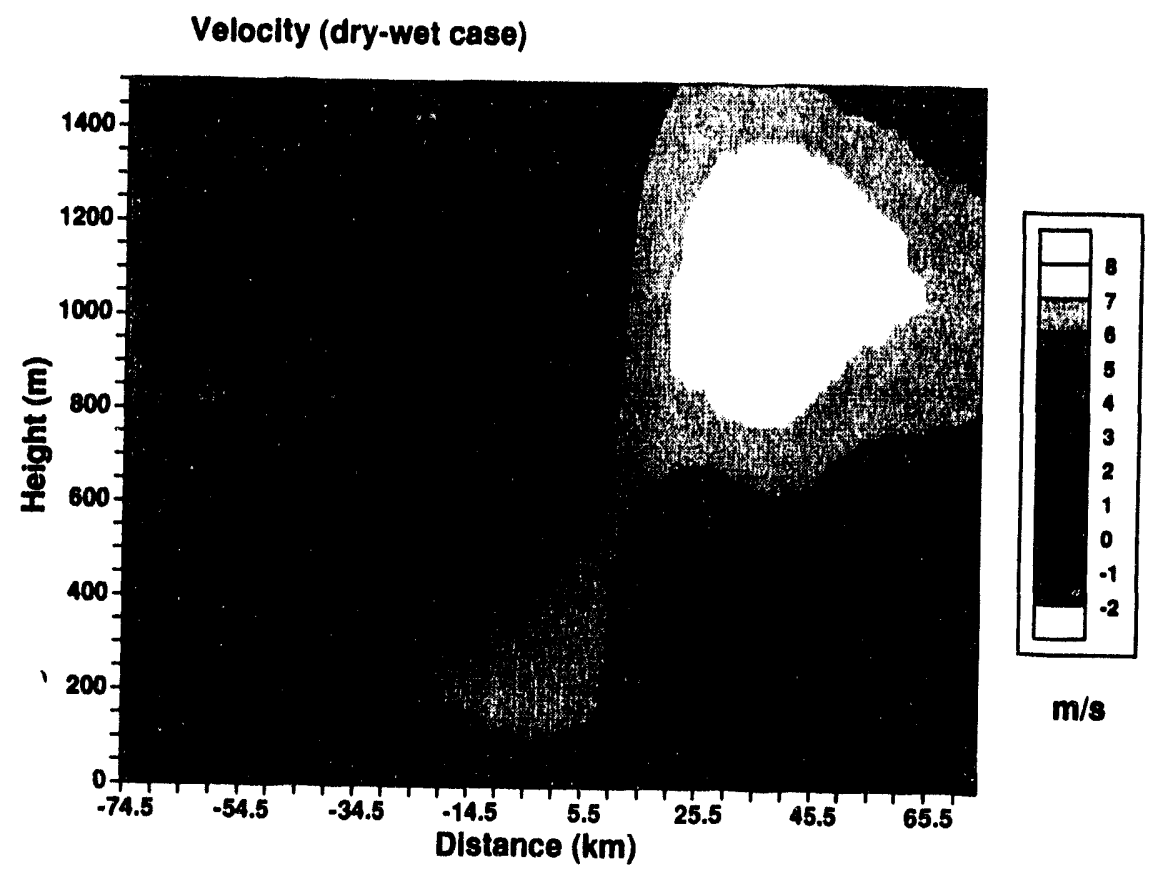

Velocity (wet-dry case)

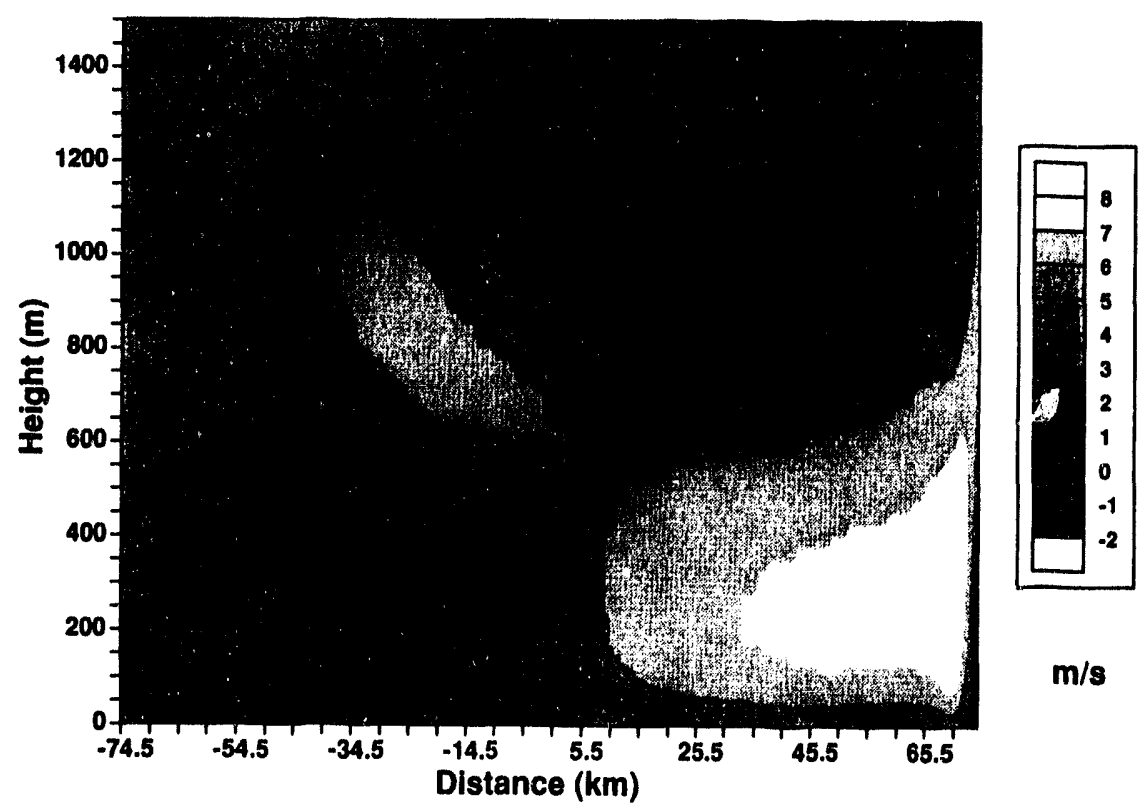

Figure 1. Contour plots of simulated variations in the u-component of velocity over dry-wet and wet-dry surfaces. 
Mixing Ratlo (dry-wot case)

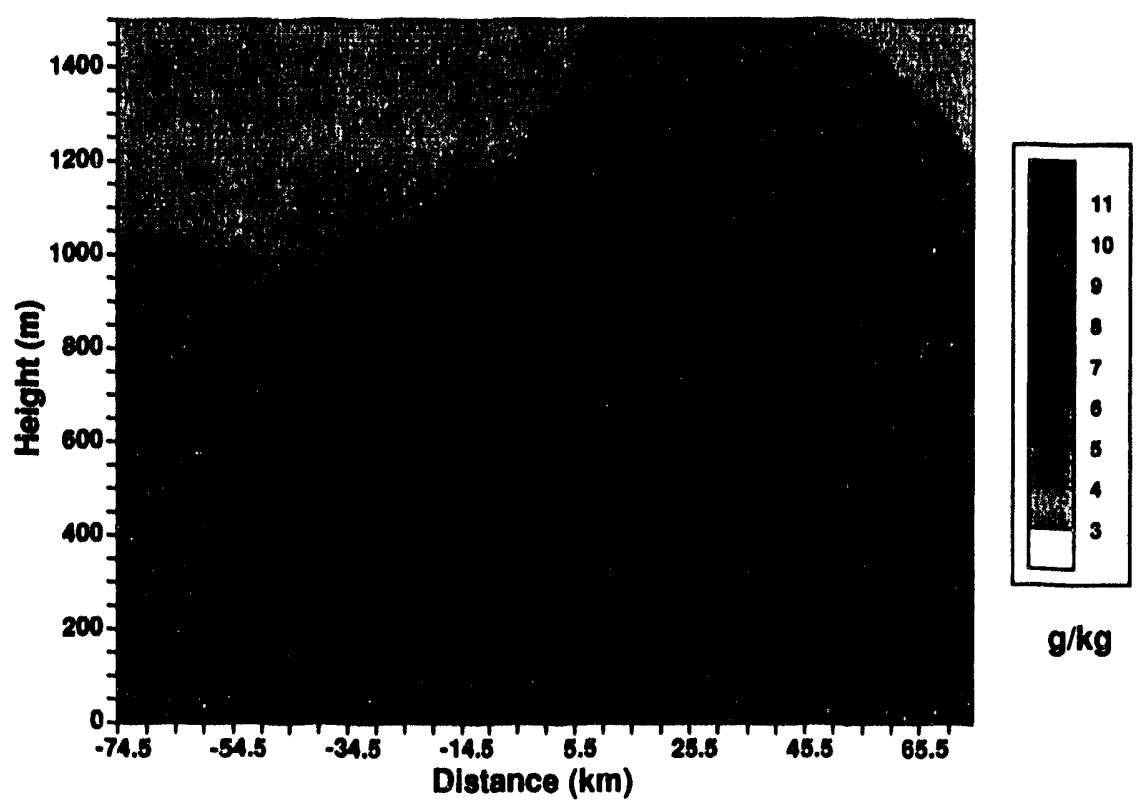

Mixing Ratlo (wot-dry case)

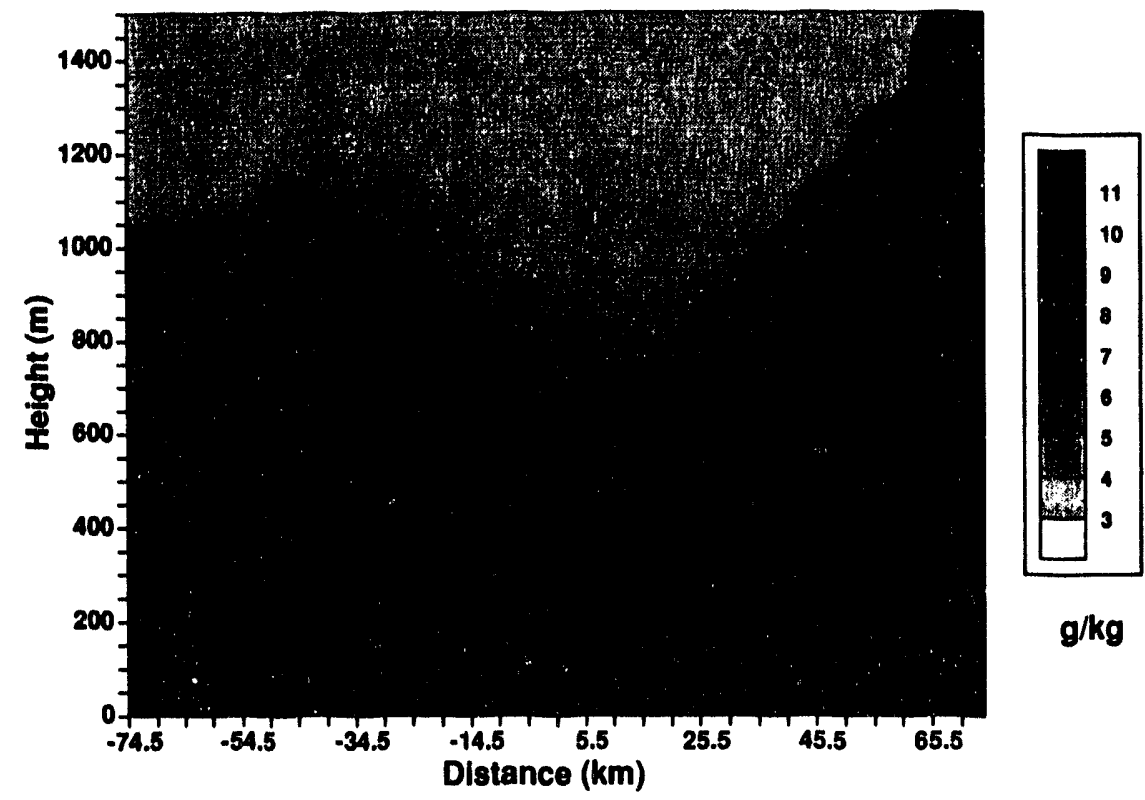

Figure 2. Contour plots of simulated variations in mixing ratios for flow over dry-wet and wet-dry surfaces. 


\title{
Areally Averaged Estimates of Surface Heat Flux from Field Studies for the Atmospheric Radiation Measurement Program
}

\author{
R. L. Coulter, T. J. Martin, and D. R. Cook \\ Argonne National Laboratory \\ Argonne, IL 60439
}

\section{Introduction}

The determination of areally averaged surface fluxes is a problem of fundamental interest to the Atmospheric Radiation Measurement (ARM) Program. The Cloud and Radiation Testbed (CART) sites central to the ARM Program will provide high-quality data for input to and verification of general circulation models (GCMs). The extension of several point measurements of surface fluxes within the heterogeneous CART sites to an accurate representation of the areally averaged surface fluxes is not straightfonward. As the CART sites become fully operational, several different types of measurements, including vertical profiles of meteorological variables, can potentially be used synergistically with point measurements to provide rellable estimates of surface fluxes averaged over large areas.
To investigate these problems, ARM science team members conducted two fleld studies near Boardman, Oregon, during June of 1991 and 1992. The site was chosen to provide strong contrasts in surface moisture while minimizing the differences in topography. The region, described in detall by Doran et al. (1992), consists of a substantial dry steppe (desert) upwind of an extensive area of heavily irrigated farm land, $15 \mathrm{~km}$ in width and divided into 800-m-diameter circular fields in a close packed array, in which wheat, alfalfa, corn, or potatoes were grown (Figure 1). A full rotation of the irrigation arm was completed approximately once every 28 or $35 \mathrm{~h}$ during the growing season. This region provides marked contrasts, not only on the scale of farm-desent $(10-20 \mathrm{~km})$ but also within the farm $(0.1 .1 \mathrm{~km})$ because different crops transpire at different rates, and the pivoting irrigation arms

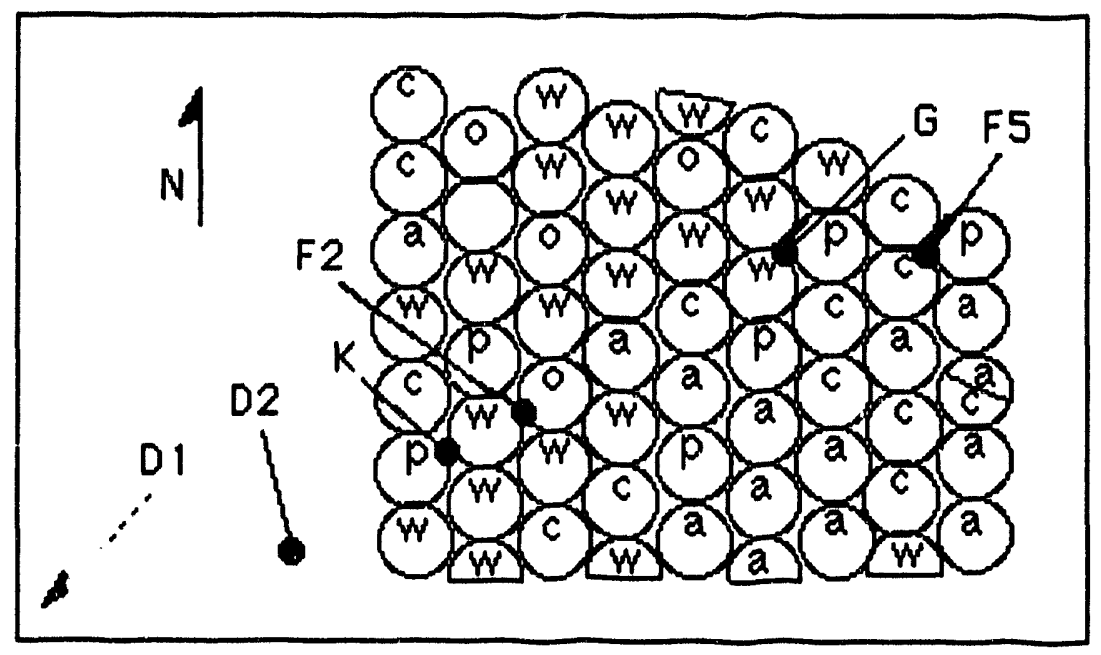

Figure 1. Farm crop locations and instrument locations (solid dots) in 1992. Field types are $w$ (wheat), a (alfalfa), $c$ (corn), or $p$ (potatoes). With prevailing wind from the southwest, the crop sampled is to the southwest of instrument site. The D1 location, not to scale, is $8 \mathrm{~km}$ west of the farm. Site $G$ is the location of the permanent minisodar. Capital $D$ and $F$ stand for desert and farm, respectively. 
provide an ever-changing pattern of heavy surface moisture throughout the farm area. This paper primarily discusses results from the 1992 field study.

\section{Thermal Plumes}

Convection is the principal mechanism by which the atmosphere transports energy from the surface layer throughout the mixed layer. Thermal plumes, large regions of buoyant, rising air whose maximum height is limited by the top of the mixed layer, dominate the convective boundary layer. They scale with the height of the mixed layer $\left(z_{1}\right)$, are the large-scale source of energy input to the turbulence spectrum (Stull 1989), and provide the mechanism for transferring energy from the surface through the mixed layer and into the capping inversion. When conditions are appropriate, fair-weather cumulus clouds develop at the top of the thermal plumes. In this sense, small cumulus clouds provide a visual description of the size, location, and lifetime of mature, active thermal plumes in a deep mixed layer. Traveling with the mean wind, thermal plumes act as a conduit for heat and moisture between the surface and lower troposphere.

We can define thermal plumes either as organized regions of rising air or more generally as organized regions of enhanced "thermal turbulence" that can be depicted by large values of the temperature structure parameter $C_{T}{ }^{2}$, which can be evaluated with sodar techniques (Coulter and Wesely 1980). This more general description includes some regions of entrainment into the plume and is more descriptive of the organization of large scale turbulent transfer of heat and moisture.

The convective velocity scale $\mathrm{w}$. is defined as

$W^{*}=\left[\frac{g z_{i}}{\theta_{v}} \overline{w^{\prime} \theta_{v}}\right]^{1 / 3}$

where $\mathrm{g}$ is acceleration of gravity, $\theta_{v}$ is virtual potential temperature, $w$ is vertical velocity, and primed quantities are deviations from the mean. The vertical velocity fluctuations within thermal plumes can be treated as a measure of $w^{*}$, which ties the surface heat flux and the mixed layer together precisely as does a thermal plume.
Measurements have shown a positive difference in both temperature and moisture between plume and amblent air, as this definition of $w^{*}$ implies.

The potential usefulness of thermal plumes in quantifying areal averages of surface heat flux resulis from the relatlvely long lifetime of a coherent structure that, in traveling with the mean wind, is representative of the integrated surface heat flux input from all the surfaces over which it has passed. A plume lifetime of 30 min with a mean wind speed of $5 \mathrm{~m} / \mathrm{s}$ results in a $9 . \mathrm{km}$ path. One can also surmise that the relationship of the profile of either $C_{T}{ }^{2}$ or $w$ to surface forcing is height dependent; that is, the near-surface portion of the profile is closely identified with "nearby" surfaces, while the upper portion is an integrated sample of more distant surfaces.

Two minisodars were used in 1992 to sample relative heat flux contributions within the study area. One minisodar, permanently stationed upwind (most of the time) of an actively growing wheat field (site G) measured three components of the wind and $C_{T}{ }^{2}$. The second, a portable, single-axis system, measured only vertical velocity and $\mathrm{C}_{T}{ }^{2}$ and was placed at various locations (solid dots in Figure 1) for periods ranging from several hours to a day or two. Thus, a benchmark was maintained at site $G$ to facilitate intercomparisons among different fields. Averaging times varied from as long as $30 \mathrm{~s}$ (about 24 samples) to $0 \mathrm{~s}$ (every pulse recorded). The data reported here were not averaged prior to this analysis, permitting the maximum detail in thermal plume description.

\section{Farm Versus Desert}

Profiles of both signal intensity and $w$ (Figure 2) on 4 June show much larger values over the desert than over wheat, as expected. The near-surface difference in $C_{T}{ }^{2}$ between the sites corresponds to a factor of nine in heat flux. Note that at elevations above 80 to $100 \mathrm{~m}$ the intensity and vertical velocity profiles become more similar, perhaps reflecting the importance of input from larger scales.

For 5 June, the results are much different. The intensity profile over wheat is much larger than that over desent, and the vertical velocities are similar below $40 \mathrm{~m}$. This result is due to a northwest wind direction at site $G$ that appare itly allows the desert-like boundary region between fields to dominate at very low levels. The steep fall-off of the 

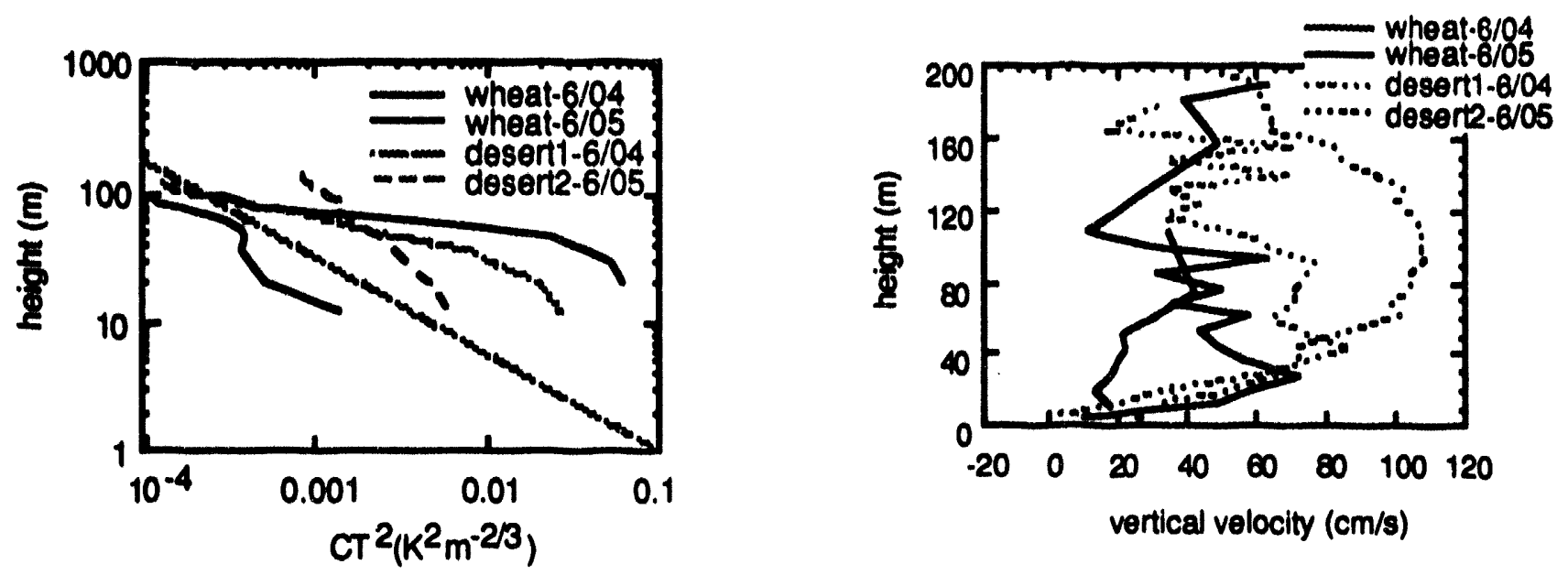

Figure 2. Signal intensity and vertical velocity proflles within plumes above wheat and two desert sites. The desert2 site was located about $0.5 \mathrm{~km}$ west of the farm. The dashed straight line is a reference line with slope proportional to $z^{-A / 3}$.

intensity and velocities above 40 m corresponds to sampling of the wheat field to the northwest of site G. In addition, this desert site, unlike that on 4 June, was located very near the eastern edge of the desert and was under the influence of the easterly "farm breeze" during this averaging time. Hence, the low-level intensity profile is depressed; however, the upper lovels reflect a steep increase in values, probably coinciding with wind direction reversal to southwesterly above the farm breeze.

\section{Farm Versus Farm}

Comparisons of wheat (at G), potatoes and corn are shown in Figure 3. Near-surface values of intensity and $w$ over wheat and potatoes are similar; however, even though the vertical velocities become very similar at larger altitudes, the intensities at larger altitudes above potatoes rapidly become very small. This is surprising, because the desert is upwind of the potato field.
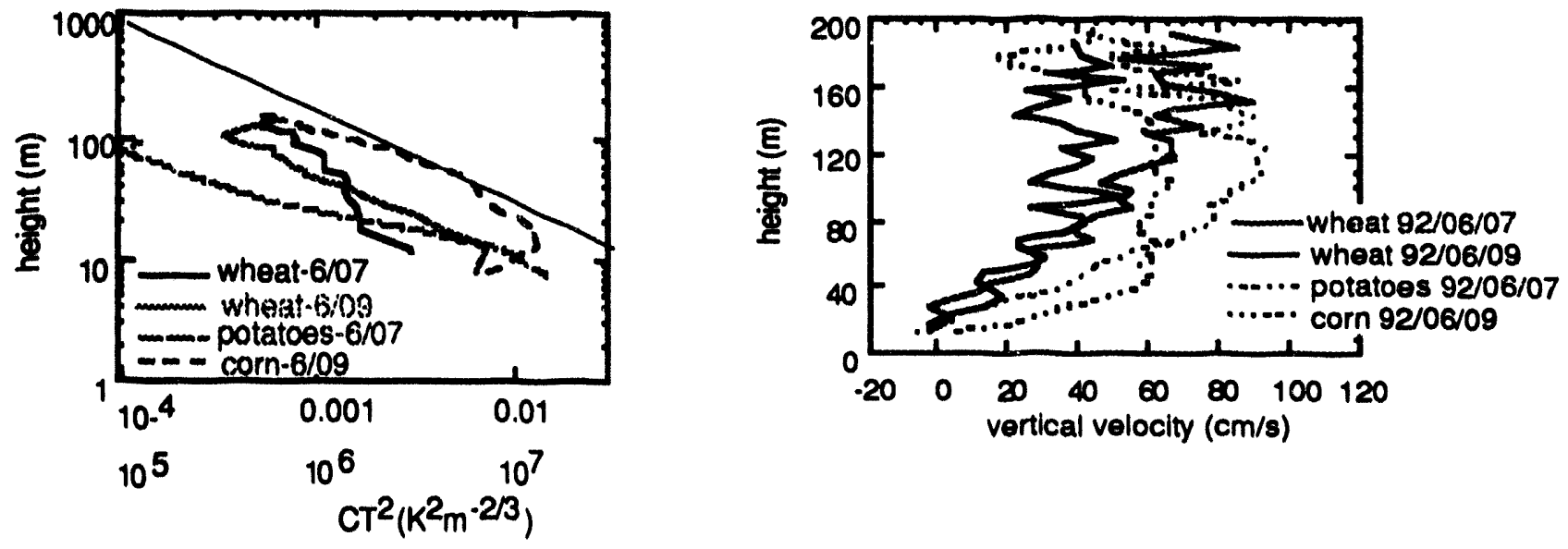

Figure 3. Signal intensity and vertical velocities within plumes above wheat, potatoes, and corn. 
Well-behaved profiles abovs both wheat and corn translate to about $70 \%$ more heat flux above corn than wheat. The wheat crop was much denser than the corn; preliminary eddy correlation values of heat flux above the wheat field were quite small, usually much less than $50 \mathrm{~W} / \mathrm{m}^{2}$. Profiles well above both crops tend to increase in intensity (relative to the $z^{-4 / 3}$ slope). Vertical velocities gradually merge to similar values at larger heights.

Comparison between two wheat fields at different locations within the farm (Figure 1) shows profiles of both intensity and vertical velosity (not plotted) that are almost identical; slightly smaller near-surface values and similar velocities and slightly larger intensities above $100 \mathrm{~m}$ appear at site $G$, but these differences may not be significant.

Estimates of the ratio of $w^{*}$ values at the control site to values at the other locations are given in Table 1. The values of $w^{*}$ were determined by taking the maximum values within the profiles.

These values probably underestimate the maximum values within the thermal plumes because the limits of the plumes were determined by large values of intensity rather than by large $w$. Actual values of $w^{*}$ will be larger than those calculated from these data.

Conditional sampling of minisodar data was used to produce vertical velocity profiles within thermals that were extrapolated to the middle of the mixed layer in order to produce estimates of the mean vertical velocity in the thermal plume. These estimates were then used as measures of $w^{*}$ along with estimates of the capping inversion height determined from airsonde releases to produce estimates of $\mathrm{H}$ from (1). We note (Figure 4) that

Table 1. Estimated ratios of convective velocity scale and heat flux at selected sites to those at the permanent wheat site G. 1991 estimates are from eddy correlation values.

\begin{tabular}{|c|c|c|c|c|}
\hline & Desert1 & Desert2 & Potatoes & Corn \\
\hline$w^{*} /(w .)_{w}$ & 1.67 & 1.83 & 1.36 & 1.44 \\
\hline$H / H_{w}$ & 4.66 & 6.13 & 2.52 & 3.01 \\
\hline$H / H_{w} 1991$ & 4.5 & - & $1.5-2$ & $1.8-2$ \\
\hline
\end{tabular}

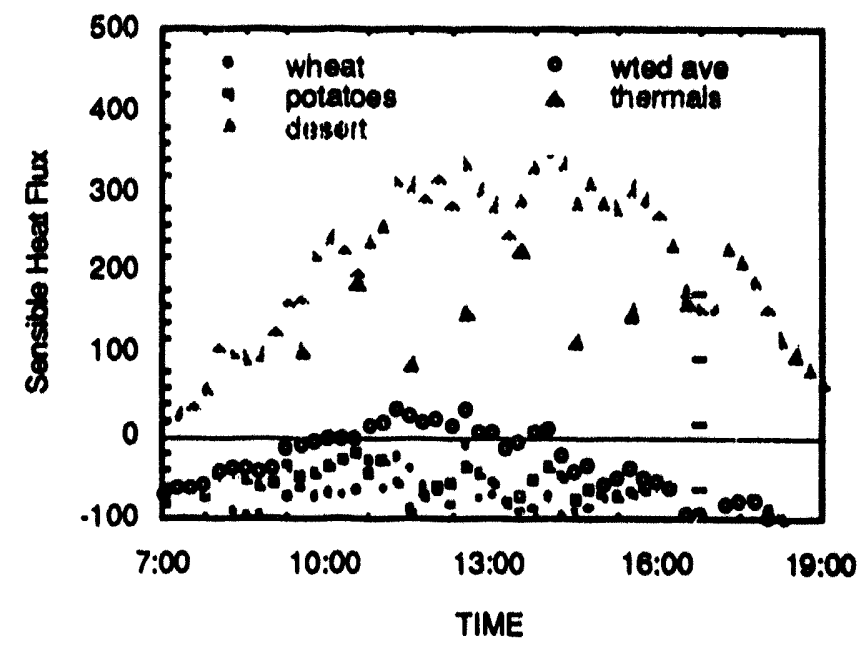

Figure 4. A comparison of heat fluxes via direct, local measurements $4 \mathrm{~m}$ above wheat, potatoes and desert on 7 June 1992, with an areally weighted average from the farm and values derived from thermal plumes representative of the mixed layer located approximately $1 \mathrm{~km}$ from the farm-desert interface.

the coherent structure values are again representative of an average of the underlying surfaces. The values from coherent structures are very appealing as a measurement tool because they are derived from structures that have a definite lifetime, during which they travel with the mean wind over a significant distance. The conditional sampling procedure concentrates the analysis within those portions of the atmosphere most responsible for vertical transport and mixing.

\section{Transfer Functions}

If one assumes, for the farm-steppe system, an exponential form for the relative downward influence of horizontally separated surface inputs and assumes that the horizontal sampling of a measurement at height $z$ is limited to a distance 10z, a relationship for the surface heat flux "seen" at height $z$ is

$H(z)=\frac{1}{\int_{0}^{t} e^{-\theta x / 2}}\left(H_{1} \int_{0}^{d} e^{-\alpha x / z}+H_{0} \int_{0}^{L} \theta^{-\theta x / z}\right)$. 

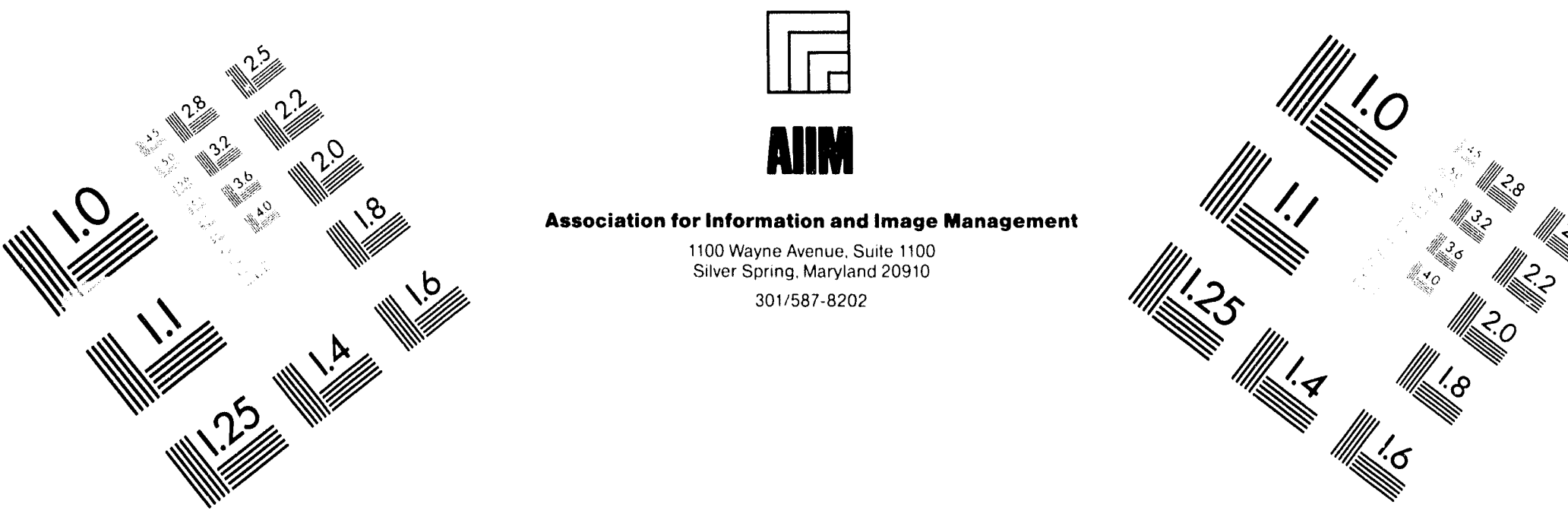

Centimeter

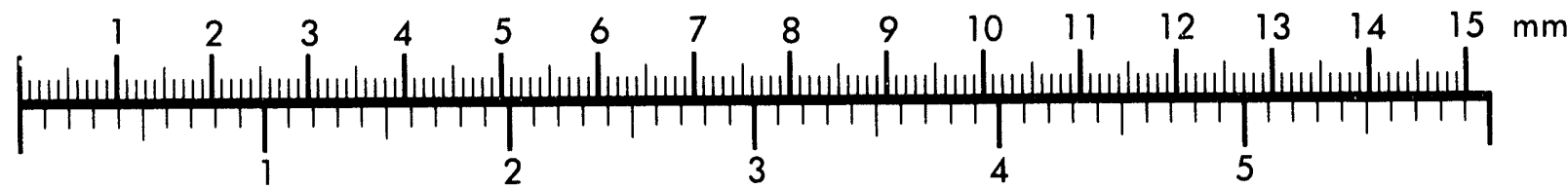

Inches
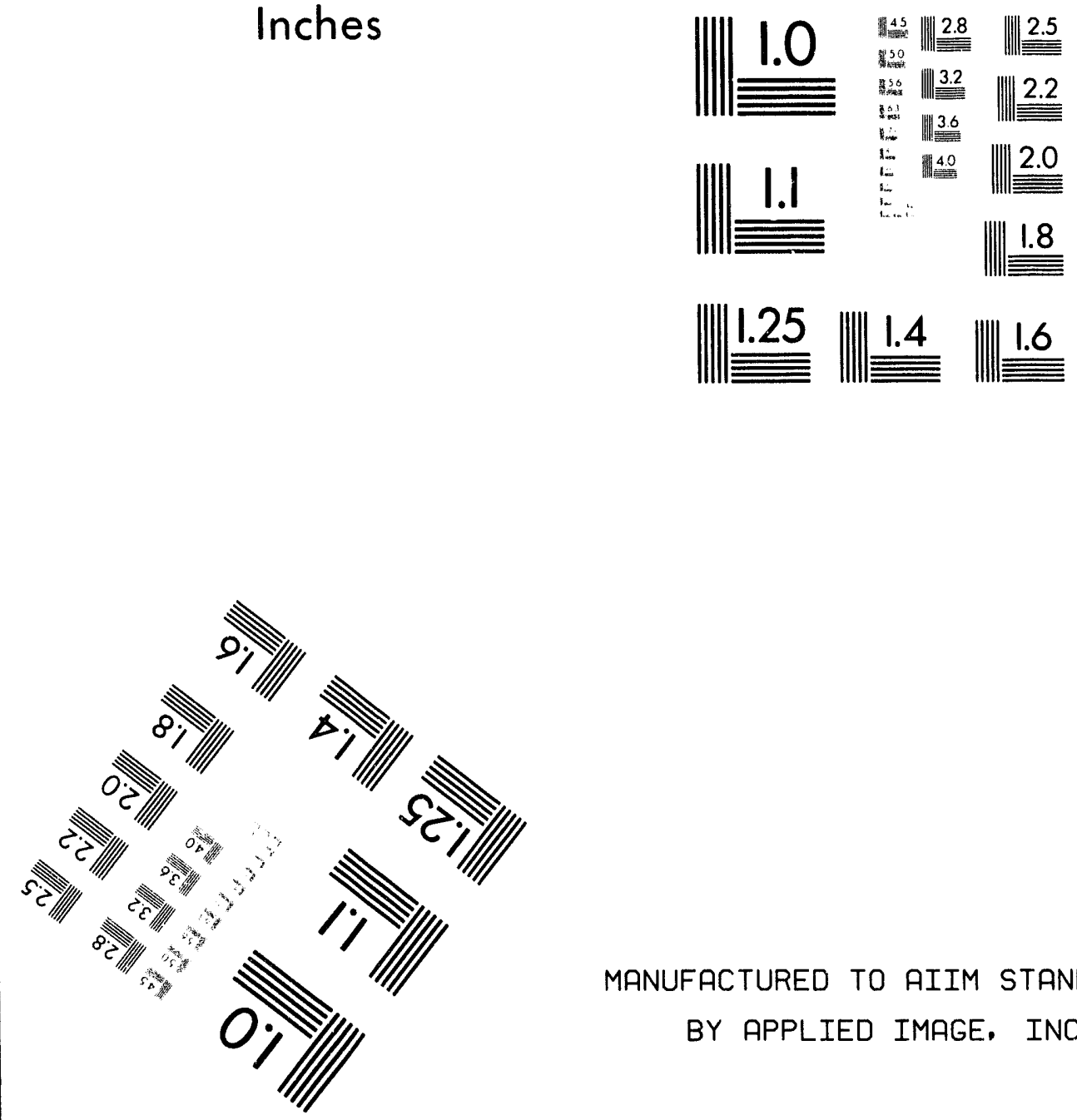

MANUFACTURED TO AIIM STANDARDS

BY APPLIED IMAGE. INC.

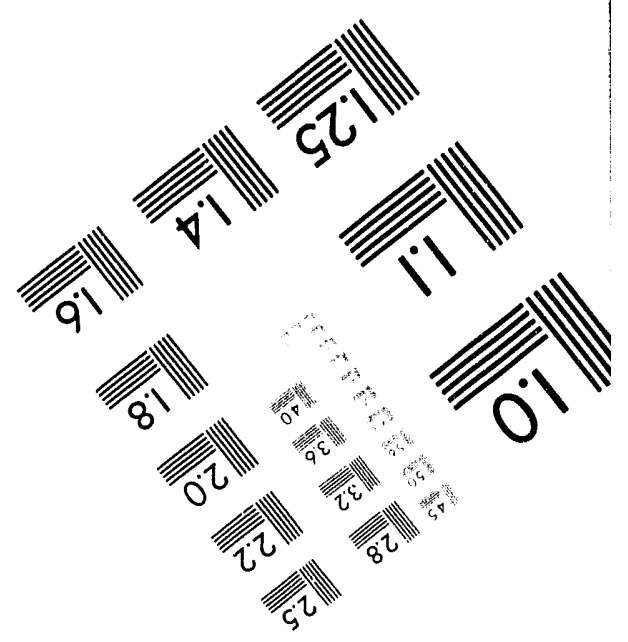



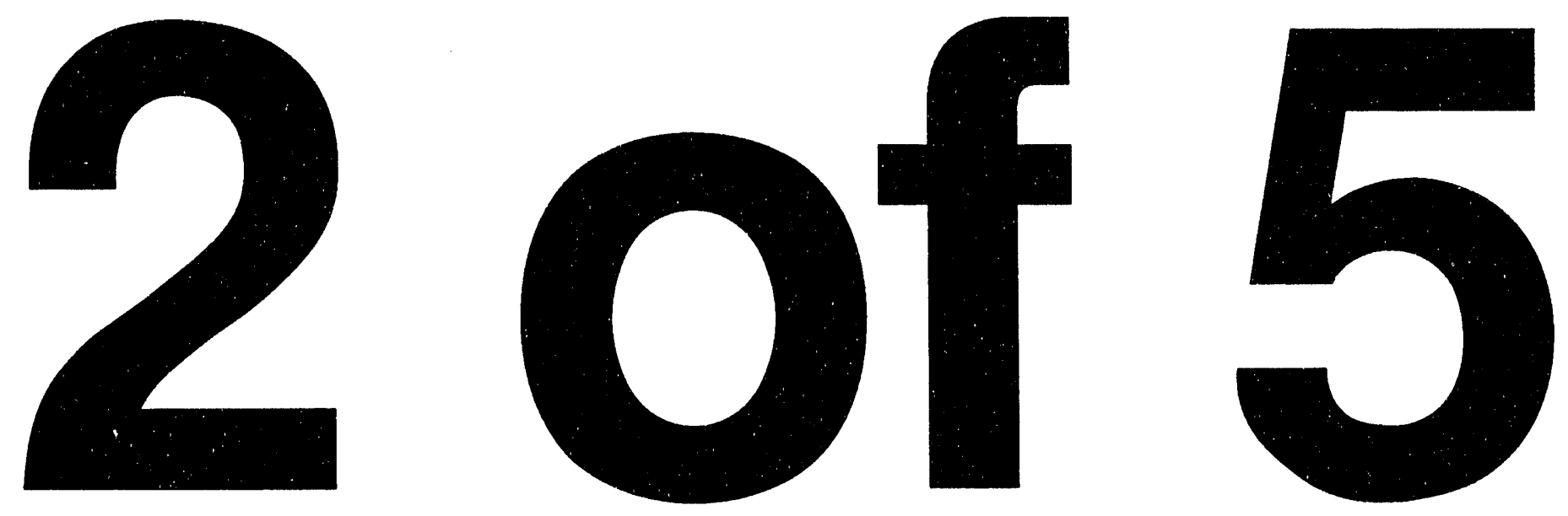
which becomes

$H(z)=\frac{H_{f}\left(1-e^{-\frac{g d}{2}}\right)+H_{d}\left(e^{-\frac{g d}{2}}-\theta^{-g m}\right)}{1-\theta^{-g m}}$.

where $H_{f}$ and $H_{d}$ are the average values across the farm and desert, respectively; $g$ is a distance constant determining the relative horizontal importance with height; and $L=m z$ is the horizontal integration distance. Indeed, $H_{f}$ could be calculated by using a similar expression with more terms to represent each irrigation plot.

Figure 5 illustrates how this type of transfer function would appear over the farm portion of the study region in the case

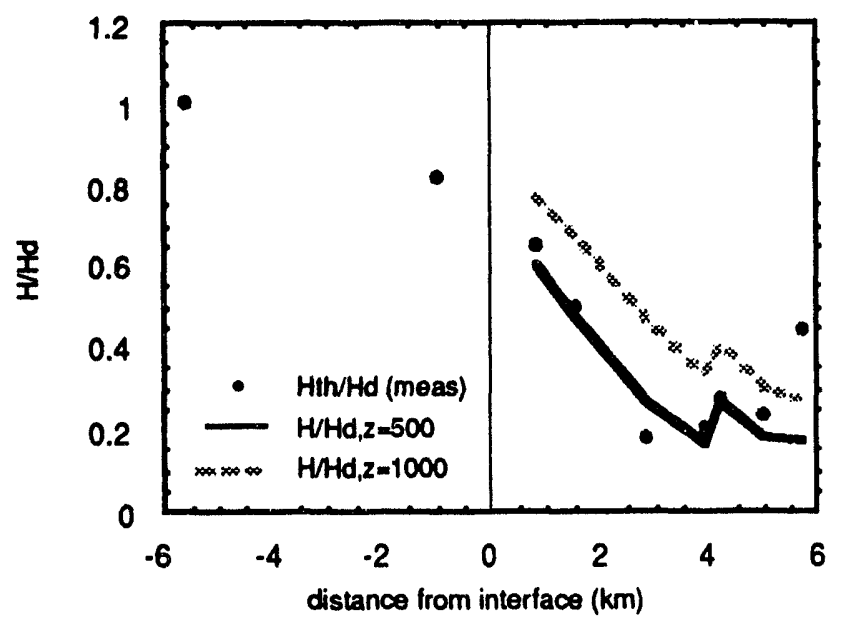

Figure 5. Variation of $\mathrm{H}$ measured from thermal plumes (Hth) normalized by measured heat flux over the desert and $\mathrm{H} / \mathrm{Hd}$ calculated from Equation (3) with different assumptions of effective measurement height. The values of $\mathrm{Hf} / \mathrm{Hd}$ used in Equation (3) are those determined from measured values over desert and farm. where the farm is downwind of the desert. The plotted points are "average" values determined from coherent structures over farm locations at different distances from the farm-desert interface (not on the same day). The farm has an apparent effect on desert values just upwind of the interface. This may be due to effects of the local farm breeze circulation prevalent on the day of these measurements. This effect would not be accounted for with this transfer function, because it assumes the farm is downwind of the desert.

This work was supported by the U.S. Department of Energy Research, Office of Health and Environmental Research, under contract W-31-109-Eng-38.

\section{References}

Coulter, R. L., and M. L. Wesely. 1980. Estimates of Surface Heat Flux from Sodar and Laser Scintillation Measurements in the Unstable Boundary Layer. J. Appl. Meteorol. 19(10):1209-1222.

Doran, J. C., F. J. Barnes, R. L. Coulier, T. L. Crawford, D. D. Baldocchi, L. Balick, D. R. Cook, D. Cooper, R. J. Dobsy, W. A. Dugas, L. Fritschen, R. L. Hart, L. Hipps, J. M. Hubbe, W. Gao, R. Hicks, R. R. Kirkham, K. E. Kunkel, T. J. Martin, T.P. Meyers, W. Porch, J. D. Shannon, W. J. Shaw, E. Swiatek, and C. D. Whiteman. 1992. The Boardman Regional Flux Experiment. Bull. Amer. Met. Soc. 73:1785-1795.

Stull, R. B. 1989. An Introduction to Boundary Layer Meteorology. Kluwer Academic Publishers, Boston, Massachusetts. 


\title{
Surface Fluxes Important to Cloud Development
}

\author{
F. J. Barnes and W. Porch \\ Los Alamos National Laboratory \\ Los Alamos, NM 87545 \\ K. E. Kunkel \\ Illinois State Water Survey \\ Champaign, IL 61820
}

\section{Introduction}

To address some of the issues in scaling and averaging of measurements, U.S. Department of Energy laboratories funded under the Atmospheric Radiation Measurement (ARM) Program conducted collaborative field campaigns in June 1991 and 1992. We selected a site in Boardman, Oregon, with two distinct regions where the sensible and latent heat fluxes would differ sharply and where each region was sufficiently extensive enough to allow full development of boundary layers and use of aircraftmounted instrument systems (Barnes et al. 1992, Doran et al. 1992). Measurements were clustered along a 16-km transect across adjoining irrigated farmland and semi-arid rangeland that allowed the collaborating teams to conduct a variety of studies relating to overall goals. The Los Alamos team focused on assessing the effects of different surface characteristics on fluxes of heat and water vapor.

Progress from the Boardman experiments and preliminary work at the Southern Great Plains (SGP) Oloud and Radiation Testbed (CART) site (see p. 3) has contributed to the ability to model and measure fluxes over a wide range of scales. Extrapolating surface flux measurements and exploring aggregation of measurements of fluxes and surface characteristics at different scales will be important at CART sites in order to model the influence of surface variability on cloud formation and radiative transfer. Our approach is unique in that it integrates modeling and diverse field methodologies as well as expertise in ecological and atmospheric sciences. This is important not only for the surface characterization and Surface Vegetation Atmosphere Transfer Scheme (SVATS) modeling that will be required for the CART sites, but also in the use of remote sensing tools to test the aggregation of tower flux measurements.

\section{Fine Scale Variability in Fluxes Over Range and Farm}

The Los Alamos team members focused on the following measurements over the course of the 3-week campaigns:

1. Intercomparison of micrometeorological instrument performance.

2. Determination of fine-scale variability of surface fluxes over dry grassland (1991) and farmland (1992) and its relationship to variability in surface soil moisture, crop type and leaf area index or biomass.

3. Comparison of spatially averaged optical measurements of heat fluxes and convergence with micrometeorological measurements, high-frequency Doppler acoustic measurements, and multi-spectral cloud images and cloud height measurements.

4. Determination of evapotranspiration (ET) from water balance estimates of 4 crops in the agricultural area and comparison to micrometeorological measurements of ET.

\section{Results}

A survey of the rangeland site (Barnes et al. 1992) showed that one grass and two shrub associations were most important in spatial coverage of the transect area in the northern portion of the rangeland. The grassland areas were dominated by needle-and-thread, a perennial bunchgrass. Aboveground green vegetative cover on the grassland sites ranged from $20 \%$ to $40 \%$. Biomass estimates for three sites in this community ranged from 13 to $55 \mathrm{~g} / \mathrm{m}^{2}$. The two shrub communities were dominated 
by either rabbitbrish or bitterbrush, with understories of needle-and-thread and/or cheatgrass. Overstory canopy cover on the shrub sites was about $14 \%$. At the time of the study, the perennial grasses had largely completed vegetative growth and were starting to set seed, while the annual grasses were senescent with no green follage evident. The shrubs were still in an active vegetative growth phase. Conditions across the rangeland were extremely dry. During the study period, gravimetric soil moisture determined on samples from 0 to $15-\mathrm{cm}$ depth ranged from $1.1 \%$ to $4.1 \%$ volume, and from $2.5 \%$ to $6.4 \%$ volume in the $15-$ to $30-\mathrm{cm}$ depth samples.

Mean latent and sensible heat fluxes were calculated for the shrub (4 sites) and grass (5 sites) communities. Mean maximum daily sensible heat fluxes for both communities ranged from 270 to $450 \mathrm{~W} \mathrm{~m}^{-2}$, and about 45 to $80 \mathrm{~W} \mathrm{~m}^{-2}$ for latent heat fluxes. There was no discernible trend in the effect of vegetative cover type on the mean fluxes. Inspection of the daily flux rates for each site showed that the sensible heat flux rates were remarkably uniform among sites. However, the latent heat flux from the bitterbrush site was significantly $(P<0.01)$ higher than over the other shrubsites. This trend cannot be explained by the effect of surface soil moisture, which tended to be lower at this site. Although the overall vegetative cover on this site was similar to the other shrub sites $(14 \%)$, the dominant shrub species (bitterbrush) has a larger growth form and higher green biomass per shrub than the rabbitbrush growing on the other shrub sites. The potentially higher green biomass per unit ground area and deeper rooting (likely with a larger-sized shrub and resulting in the vegetation accessing soil moisture deep in the profile) could account for the higher ET from the site.

\section{Results}

Results (Barnes et al. 1993) showed that eddy correlation measurements of sensible and latent heat fluxes over the farm were very sensitive to wind speed. ET determined from Bowen Ratio energy balance data was not as sensitive to wind speed. Preliminary water balance calculations suggest that differences in ET can be linked primarily to crop type. This may be a result of both leaf area index (LAI) and stomatal conductance differences between the crops. However, it is also possible that the assumptions in the water balance calculations heavily biased the results since micrometeorological methods of determining ET did not show clearcut differences between crops. Effects of crop type, LAl, and synoptic weather patterns on energy balance components will be further explored using the full micrometeorologica! data set on 15 fields. Hydrologic modeling of the agricultural area is underway and results will be compared with data obtained from aircraft-mounted flux instruments (Doran et al. 1992).

We have also applied techniques to provide spatiallyaveraged flux and wind convergence measurements and relate these to cloud variability. Results are summarized in Porch et al. (1992, 1993) and Porch and Shaw (1993). These measurements covered scales of hundred of meters over a dry grassland with high heat flux. The 1991 experiment focused on comparisons of wind convergence measured by optical cross-wind sensors in a 200-m triangle and vertical velocities measured with a high frequency Doppler acoustic sour.der (Coulter et al. 1992). These measurements showed that vertical velocities were highly correlated with convergence. Also, when wind blew from the predominant wind direction (southwest to west), net divergence and downward vertical velocities were observed. Since net vertical velocities (either up or down) imply a net advective flux, it is important to determine how convergence changes under the wider variety of wind conditions observed in 1991.

In 1992, a second triangle with 400-m legs was added using optical furbulence saturation resistant systems, and we determined the short-term effect of passing clouds on surface heat flux. Results showed first that path-averaged optical heat ilux determination allowed much faster determination of heat fluxes than can be obtained from a tower. Passing cloud shadow effects could be defined with high temporal resolution using optical turbulence-based heat flux and turbulent energy dissipation over paths of 100 to $150 \mathrm{~m}$. Second, convergence of the wind into two triangles defined by optical cross-wind sensors is relatively independent of scale between 200 and $400 \mathrm{~m}$. Though we were unable to determine a land-use-induced daytime convergence in the dry grassland, we were able to detect smaller scale topographic effects that may at times stimulate or inhibit cloud formation. We also showed that net divergence and downward velocities observed the previous year under relatively high wind conditions were not observed under light wind conditions in 1992. 


\section{Modeling of Surface Fluxes Over SGP CART}

In 1992, we hosted Mr. Dean Jordan, a DOE Global Climate Fellow from the $U$. of Wyoming, for a 3-month practicum. His presence at Los Alamos gave us the opportunity to address the issue of modeling surface fluxes (using RAMS, the Regional Atmospheric Modeling System) at the SGP CART site ahead of schedule. Our objectives were to 1) use data bases currently available, characterize the soils and vegetation with maximum detail, and enter the data into the ARC/INFO Geographic Information System (GIS) software (Environmental Systems Research Institute, Redlands, California); 2) aggregate the surface features at different scales; and 3) compare results from modeling experiments with the surface features aggregated at different scales, using a control simulation with uniform vegetation and soils as the baseline. We made progress toward completing the first two objectives, as well as performing initial runs comparing the results from a uniform surface parameterization to a scenario with soils and vegetation aggregated into a few classes at $16-\mathrm{km}$ resolution (Jordan et al. 1993).

Soil texture classes, obtained from Soil Conservation Service maps for Kansas and Oklahoma, were aggregated to eight soil texture classes. Careful checking across state lines, and with county maps was required to obtain a satisfactory map that could be digitized and imported in the GIS.Dr.R. McMillan (Atmospheric Turbulence and Diffusion Division, National Oceanic and Atmospheric Administration) provided us with an Advanced Very High Resolution Radiometer (AVHRR) image processed to show vegetation classification. I nis classification was aggregated into just three vegetation types, crop/mixed farming, tall grass/ pasture, and mixed woodland, plus open water. Both soil and vegetation layers were aggregated to various scales using the ARC/INFO resident algorithm (Figure 1). The control model run was parameterized with uniform crop/ mixed farming and sandy loam soil.

Initial conditions for RAMS were established with dry soils (20\% field capacity) on the summer solstice. Initial atmospheric conditions were horizontally uniform with a vertical temperature and moisture profile typical of summertime conditions. The model was run through $24-h$ diurnal cycles, with energy and moisture fluxes, air and soil temperatures, and winds being generated by local conditions.
Results showed that spatial variability in surface characteristics, particularly vegetation type, had a strong influence on atmospheric boundary layer processes. Maps of surface temperature showed a strong correspondence to vegetation type, with localized cool areas over woodland/ open water, in marked contrast to the uniform temperature fields in the control run. Analysis of the results of the model runs is still under way.

From exploring aggregation at difference scales, we found that features can be eliminated, depending on the scheme and scale used (Figure 1). These differences in surface characteristics are often the very ones that induce localized sharp gradients in surface heating. Thus, the method of aggregating surface features for studying the effects of surface variability on mesoscale circulation needs to be carefully assessed.

\section{References}

Barnes, F. J., W. Porch, S. Tarbox, K. E. Kunkel, R. Scott, W. A. Dugas, R. Hicks, P. Larson, and C. Riemann. 1993. Energy balance components of irrigated crops in NorthCentral Oregon, Fourth Symposium on Global Change Studies, American Meteorological Society Annual Meeting, January 17-22, 1993, Anaheim, California. American Meteorological Society, Boston, Massachusetts.

Barnes, F. J., K. E. Kunkel, W. Porch, D. Cooper, L. Hipps, and E. Swiatek. 1992. Variability of surface fluxes over a heterogeneous semi-arid grassland. American Meteorological Society Annual Meeting, Third Symposium on Global Change Studies, pp. 63-67, January 5-10, 1992, Atlanta, Georgia. American Meteorological Society, Boston, Massachusetts.

Coulter, R. L., W. Gao, P. J. Martin, J. D. Shannon, J. C. Doran, J. M. Hube, W. M. Shaw. 1992. Evolution of the lower planetary boundary layer over strongly contrasting surfaces. 3rd Symposium on Global Climate Change Studies, American Meteorological Society Annual Meeting, Atlanta, Georgia, January 5-10, 1992. American Meteorological Society, Boston, Massachusetts.

Doran, J. C., F. J. Barnes, R. L. Coulter, T. L. Crawford, D. D. Baldocchi, D. R. Cook, D. Cooper, R. J. Dobosy, W. A. Dugas, L. Fritschen, R. L. Hart, R. Hicks. L. Hipps, J. M. Hubbe, W. Gao, R. R. Kirkham, K. E. Kunkel, T. J. Martin, T. J. Meyers, W. Porch, J. D. Shannon, W. J. Shaw, 
E. Swiatek, and C. D. Whiteman. 1992. The Boardman Regional Flux Experiment. Bull. Am. Met. Soc. 73(11):178596.

Jordan, D. N., F. J. Barnes, and J. E. Bossert. Influence of Surface Spatial Scale on Regional Atmospheric Fluxes. Abstract, Eighth Annual U.S. Landscape Ecology Symposium, April 1993. Oak Ridge National Laboratory, Oak Ridge, Tennessee.

Porch, W., F. Barnes, M. Buchwald, W. Clements, D. Cooper, C. Doran, J. Hubbe, W. Shaw, R. Coulter, T. Martin, and K. Kunkel. 1992. Spatially averaged heat flux convergence measurements at the ARM Regional Flux Experiment. Third Symposium on Global Changes Studies, pp. 125-131. American Meteorological Society Annual Meeting, January 5-10, 1992, Atlanta, Georgia. American Meteological Society, Boston, Massachusetts.
Porch, W., F. Barnes, M. Buchwald, J. Stephens, and J. Archuleta. 1993. Application of spatially averaging optical techniques to the study of short-term flux changes associated with clouds. Fourth Symposium on Global Change Studies, American Meteorological Society Annual Meeting, January 17-22, 1993, Anaheim, California. American Meteorological Society, Boston, Massachusetts.

Porch, W., and W. Shaw. 1993. Application of optical remote sensing to the study of surface fluxes related to cloud formation. Optical Remote Sensing of the Atmosphere, Sixth Topical meeting, Optical Society of America, Washington, D.C., March 8-12, 1993, Salt Lake City, Utah. 


\section{S GP / CART Veget a t i o n}

Crop/Mixed Farming Inland Water
T a 1 l G I a s s
a Mi x e d Wo o d l a n d

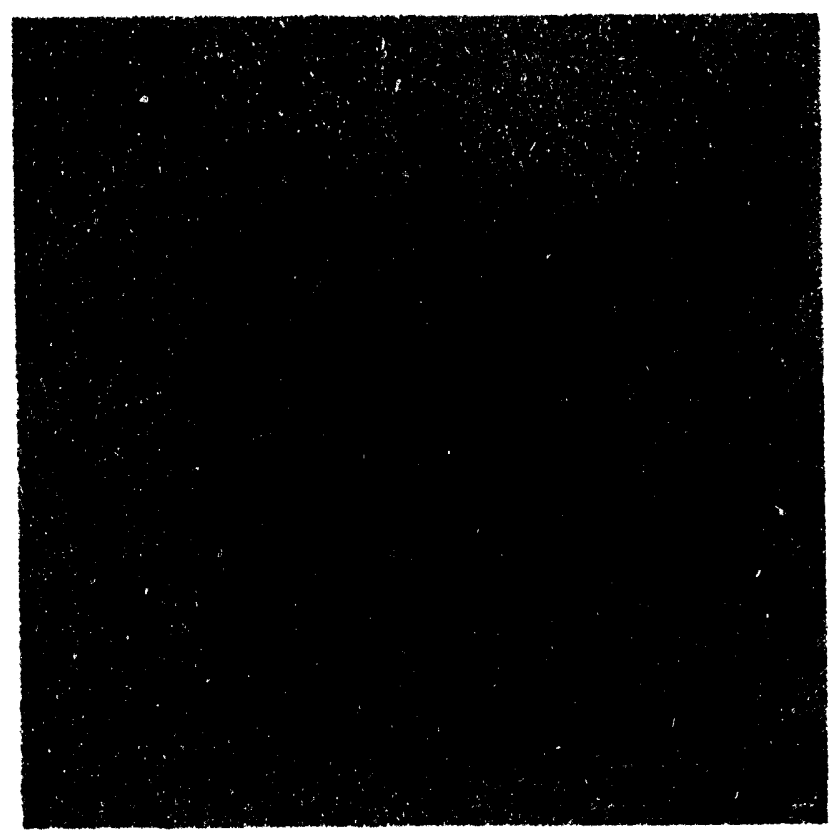

1 km Res ol ut i o n

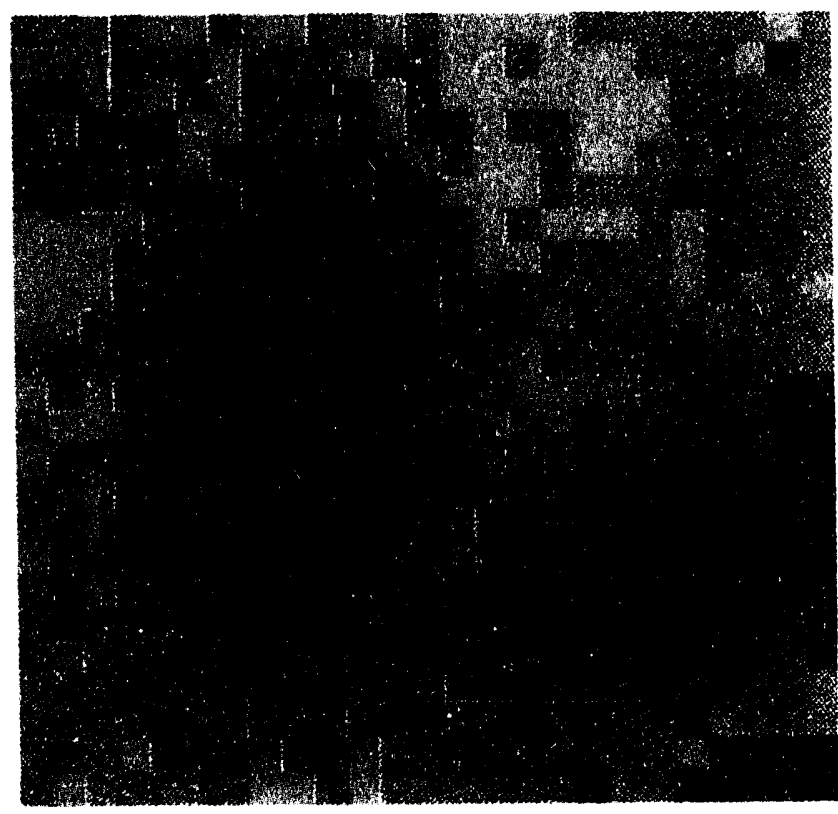

16 km Res olut i o n

Figure 1. Vegetation classification at the SGP CART site, aggregated into three land use types, at 1-km and 16-km resolutions. Note differerices in distribution and extent of inland water and woodland classes between the two scales of resolution. 


\title{
An Ultra-High Frequency Boundary Layer Doppler/Interferometric Profiler
}

\author{
J. S. Van Baelen (a) \\ National Center for Atmospheric Research(b) \\ Boulder, CO 80307-3000
}

\section{Introduction}

The planetary boundary layer (PBL) is that portion of the earth's atmosphere that is directly influenced by the earth's surface. The forcings include heat transfers, frictional drag, pollutant emissions, and moisture transfers through evaporation and transpiration. The PBL can be vigorously turbulent and quite variable in depth ranging from heights of only a few hundred meters to a few kilometers. Also, solar energy which ultimately drives atmospheric circulations is primarily absorbed at the earth's surface and transmitted to the free atmosphere through boundarylayer processes. Water vapor follows a similar path from its source at the earth's surface to the free atmosphere, while in contrast the PBL is a sink of atmospheric kinetic energy with approximately half of the kinetic energy dissipated within the PBL.

An accurate portrayal of these transfers within the PBL is crucial to understand and predict many atmospheric processes from pollutant dispersion to numerical weather prediction and numerical simulations of climatic change. Despite the importance of these transfers within numerical models, confirmation of these turbulent parameterizations is difficult since there are relatively few simultaneous observations available over the depth of the PBL, particularly for the highly disturbed and often spatially varying conditions under which these parameterizations are applied.

The primary product of wind profiling radars is obviously the 3-D wind vector profiles, but recent development of the radio acoustic sounding system (RASS) technology also predicts that those systems will be able to provide reliable virtual temperature profiles. Finally, the possible capability

(a) New affiliation: Centre National de la Recherche Scientifique, Centre National de la Recherche Météorologiques, 31057 Talouse, France.

(b) NCAR is sponsored by the National Science Foundation. of wind profilers to provide accurate estimates of the momentum and heat fluxes might be their most important contribution yet to the field of atmospheric dynamic studies, especially when those measurements can be ingested into circulation models. In particular, flux measurements in the planetary boundary layer can provide critically needed information on the PBL turbulent structures and their effect on the large and mesoscale systems of the atmosphere. However, as the technology moves toward operational wind, temperature, and eventually flux profiling, it is crucial to address the accuracy of the techniques to be used, their reliability for consistently providing valid measurements, and to keep developing and evaluating complementary or alternate profiling techniques.

In this paper we will describe and discuss wind profiling techniques and RASS applications and review past efforts to measure fluxes within the PBL. Then, we will outline a new radar system for accurately measuring both mean and flux quantities, as well as wind field divergence and acoustic wave propagation.

\section{Measurement Methods}

\section{Wind Profiling Techniques}

Generally one can divide wind profilers into two groups: Doppler radars and multiple receiver systems. Both techniques derive their iniormation from radar echoes off irregularities in the refractive index of the atmosphere (Briggs 1980; Röttger 1980). These irregularities arise from fluctuations in temperature and humidity in the troposphere-stratosphere region (Gage and Balsley 1980). In both regions, echoing mechanisms can involve turbulent scattering, as well as reflection and scatter from stratified structures (Röttger 1989). 
The Doppler technique uses narrow beams pointed in different directions. The Doppler shift measured in each beam direction provides the corresponding radial velocity, a projection of the background wind vector along the beam line of sight (Balsley 1981). It is customary to use a vertical beam and two or more non-collinear oblique beam directions to resolve the three-dimensional wind vector.

The limitations of the Doppler technique reside in the fact that different atmospheric volumes are sequentially sampled in the different beam directions. In the presence of nonhomogeneous wind fields, the retrieved wind velocities cannot be trusted, as the wind estimate is contaminated by contributions from divergence terms (Clark et al. 1986). It is therefore crucial to use more than three beams (one vertical and two off-zenith in orthogonal directions) to have a diagnostic capability which identifies such breakdowns.

Other complications arise if the wind field is evolving during the time necessary for sampling in these various directions. Also, if the echo power distribution is not centered on zenith in the vertically directed beam (i.e., when there is anisotropy in the aspect sensitivity), the associated vertical velocity derived by the Doppler technique will be tainted by a projection of the horizontal wind field into the measured radial velocity (Röttger and lerkic 1985; Larsen and Röttger 1991; Van Baelen and Richmond 1991). The associated error can be very significant, as the mean horizontal wind amplitude is typically an order of magnitude larger than the mean vertical wind component (Palmer et al. 1991; Van Baelen et al. 1991).

Finally, if specularity in the backscattering medium is present, a stronger echo power closer to zenith will result in an effective zenith angle for the tilted beams which is less than the physical zenith angle (Tsuda et al. 1986). The result of this reduced zenith angle is an underestimation of the actual wind amplitude. Similarly, the presence of thin enhanced scattering layers can also create what will be perceived as a false wind shear (Fukao et al. 1988a).

To the contrary, multiple receiver techniques use a single transmitted beam but multiple (minimum of 3 ) non-collinear receiving antennas to derive atmospheric motions. Different analyses can be performed in the time domain (spaced antenna full correlation analysis) or in the frequency domain (radar interferometry) but have been shown to provide equivalent information (Briggs and Vincent, in press). In the following paragraphs, we will outline one of the frequency domain methods.
Customarily, the backscattering medium is represented as a collection of point scatterers distributed within the atmospheric volume probed by the radar and advected by the background mean wind. Each scatterer produces a phase difference between two separate receivers (which corresponds to the angle of arrival of its back-scattered signal) and a Doppler frequency (proportional to its radial velocity). In the frequency domain, the phase of the complex cross-spectrum between those two receivers represents the average phase difference between their received signals integrated over the entire atmospheric volume illuminated, with respect to the measured Doppler frequency. The amplitude of the complex cross-spectrum is a measure of the coherency of the returned signals, i.e., it represents whether the contributing scatterers are well localized.

A linear relationship which expresses the variation of the cross-spectrum phase as a function of the measured radial velocity can be defined. The slope of that linear relationship is inversely proportional to the wind amplitude and proportional to the direction of the wind relative to the receiver pair baseline. The intersect of that line with the zero phase axis provides the vertical velocity component plus a phase term related to the anisotropy of the echo power distribution.

Multiple receivers and, thus, multiple baseline directions provide a set of equations which can be solved to derive the 3-D wind vector profile from a single transmitted beam. The detailed theoretical development of this interferometric technique is given in Van Baelen (1990) and Van Baelen and Richmond (1991).

As in the Doppler technique, a homogeneous wind field is assumed but this assumption is much less constraining, as it only extends over a single beam. Turbulence in the wind field and specularity effects can cause overestimation of the background mean horizontal wind. Indeed, turbulence can be seen as adding a random phase component whose statistical mean has zero phase, thus reducing the slope of the calculated linear relationship between phase difference and measured radial velocity.

An important aspect of the interferometric approach is its ability to correctly derive the vertical wind component even when the echo power distribution is not symmetrical with respect to zenith (Palmer et al. 1991; Van Baelen et al. 1991). This ability is due to the fact that the slope and intersect of the linear relationship are not dependent on the echo power distribution within the beam. 
Another potential advantage of the interferometric technique is that it may enable more direct sampling, in that the 3-D wind vector is derived from a single beam; whereas, Doppler requires samples from multiple beams to provide similar information.

\section{Virtual Temperature Profiling}

Because the speed of sound is related to the virtual temperature to a good approximation by the following relationship

$T_{v}=(C a / 20.047)^{2}$

one can derive the virtual temperature profile by tracking acoustic wave fronts by radar and determining their propagation speed (Matsuura et al. 1986; Currier et al. 1988; May et al. 1988). That is done when the Bragg condition is met, i.e., when the acoustic wavelength corresponds to half the radar wavelength.

If a continuous wave (CW) acoustic source is used, the backscattered echo-power will be enhanced at that frequency, regardless of the Bragg frequency, not allowing the derivation of temperature. On the contrary, when short acoustic pulses are used, the backscattered $\theta$ cho will be enhanced at the Bragg frequency, allowing the derivation of the virtual temperature. However, the echo-power strength will be significant only if the acoustic frequency is reasonably close to the Bragg frequency. Thus, in order to cover a wide range of altitudes (i.e., a range of temperatures), multiple acoustic pulses at different frequencies must be used.

The latter process can be done by sending a train of pulses slightly offset in frequency and covering the expected Bragg range for the temperatures considered, or by sweeping the frequency of a continuous acoustic source over the same Bragg domain. More details on the radio acoustic sounding systems (RASS) are given in the tutorial paper by May et al. (1990).

The derivation of the virtual temperature by RASS should also account for the background mean wind (vertical and horizontal) and wind shear effects on the propagating acoustic shells, as evidenced by Peters et al. (1983) and Strauch et al. (1991). Therefore it is beneficial to simultaneously derive both the virtual temperature profile and the three-dimensional wind profile.

\section{Previous Flux Measurement Strategies}

In the boundary layer, estimations of the momentum flux have been performed using the data of velocity azimuth display (VAD) scans from S-band (and also C- and Kband) meteorological Doppler radars. For examples and analysis details, seo Wilson (1970), Kropfli (1986), Frisch et al. (1989), $\mathrm{Xu}$ and Gal-Chen (in press). Although the technique offers great potential, especially when combining conical VAD scans at different elevation angles, its major limitation is the fact that it uses a very expensive and hardly portable instrument, i.e., a meteorological centimeterwavelength Doppler radar, and requires significant signal processing. A similar comment can be made regarding the recent use of Doppler lidar techniques to derive flux quantities (Gal-Chen et al. in press).

Besides the VAD approach, there have been only tower, tethered balloon, and aircraft measurements of momentum fluxes in the PBL (Lenschow 1986). But tower measurements are too limited in height for probing the entire boundary layer and aircraft measurements do hot provide simultaneous vertical coverage. Also aircraft measurements require long flight legs to obtain meaningful statistics, which severely tests the assumption of a nonevolving flow. In view of the limitations of these techniques, researchers have recently turned to ground-based wind profiling radars for boundary layer siudies.

In the troposphere and lower stratosphere, there have also been several attempts at deriving the momentum fluxes with wind profilers (Fukao ot al. 1988b; Fritts et al. 1990; Nastrom and VanZandt, 1991; Yoo et al. 1991). Following the work of Vincent and Reid (1983), pairs of opposite beams (i.e., coplanar beam directions which are pointed in opposite directions at the same zenith angles) are used to estimate the momentum flux components. Fukao et al. (1988b) have shown the advantages of the so-called fourbeam method (the Vincent and Reid method) over the three-beam method with Doppler wind profilers, further advocating the use of more than three beams for Doppler wind profilers.

However, the above technique (as does the VAD scan to some extent) relies on the assumption of a statistically uniform atmosphere over the different volumes probed in the different beam directions. To relax that assumption, Gavrilov et al. (1991) have used wind data derived with a spaced antenna application in which a single volume is 
sampled, illuminated by a vertically transmitted beam. Comparisons (theoretical or experimental) between the two approaches (l.e., four-beam Doppler or single volume spaced receivers) have yet to be performed.

In addition, with the advent of virtual temperature profiling with those radars comes the possibility to infer sensible heat flux measurements when simultaneous measurements of the wind vector are available, as recently investigated by Angevine et al. (1991). Indeed, the only heat flux profiling results so far were obtained by indirect methods based on the similarity theory. Coulter and Wesely (1980) used a power calibrated Sodar to convert echo power profiles into mixed layer heat fluxes, and Weill et al. (1980) inferred mixed layer heat fluxes from profiles of the vertical wind component standard deviation.

The first method is thus restricted to acoustic sounding, but the latter could be applied to Doppler radars. However, both methods are restricted to free convection conditions and are erroneous when mechanical production of turbulence is significant. Finally, these techniques provide only one flux parameter. The profile of flux divergence, which is required to solve the equation of heat conservation necessary for the characterization of the boundary layer fluctuations, cannot be derived that way but only by eddy correlation measurements or related methods.

\section{UHF Doppler/Interferometric Boundary Layer Radar}

To tackle the many questions presented above and, ultimately, to provide accurate means of continuous profiling of the 3-D wind, the virtual temperature, and the fluxes of momentum and sensible heat in the PBL, the National Center for Atmospheric Research is developing, in collaboration with Radian Corporation, a new UHF wind profiling radar. The desired features include capability to simultaneously operate the radar in the Doppler and the interferometric modes, to perform RASS measurements with both modes, and to be part of an integrated sounding system (ISS, i.e., an unattended suite of meteorological instrumentation housed in a portable sea-tainer [Dabberdt et al. 1991]). The radar system being developed is based on Radian's LAP-3000 lower atmosphere profiler, a 915-MHz Doppler wind profiler originally developed by the
National Oceanic and Atmospheric Administration (NOAA) (Eklund ot al. 1988; 1990).

In order to sult ISS integration criteria, a phased array antenna was required for beam steerability. The radar antenna being used consists of four $91 . \mathrm{cm}$ by $91 . \mathrm{cm}$ microstrip panels and can be electronically pointed in five preset directions (one vertical and four orthogonal at $21^{\circ}$ off-zenith).

In the original Doppler wind profiling mode, the different panels were connected in parallel for both transmit and receive. However, to implement radar interferometry, the four panels are connected to separate receiving channels. Thus, the entire antenna array is used to transmit a single narrow beam (half-power beam width of $9^{\prime \prime}$ ) while the retumed signals are sampled in parallel by the individual antenna quadrants and fed into their corresponding receivers.

The digitalized signals can then be used to calculate the cross-spectra for the different pairs of receivers and, thus, to implement the interferometric method. Similarly, those signals can be added up for Doppler analysis with what is then equivalent to using the entire array as a single receiving antenna. Ey doing so in the five beam directions, it is possible to derive the 3-D wind profile using the Doppler method but also the 3-D wind vector in each beam direction through interferometry.

One concern arises from the phase consistency between the different receivers. The different receiving channels are expected to be very stable with regard to phase but they cannot be expected to be of identical path length. Therefore, we are considering calibration techniques with online software correction of the received signal phase. To experiment with the different technique parameters in order to find a compatible setup for efficient (time resolution versus accuracy and reliability of the results) simultaneous measurements in both modes of operation, the interferometric analysis is done in post-processing as coherently integrated data are recorded, while on-line Doppler analysis is performed.

\section{Projected Research}

At UHF there is little specularity, if any, while turbulent scattering dominates the echoing mechanism (Röttger 
1989). Therefore, the Doppler technique should not experience the effective zenith angle problem. Its only limitation is the possible breakdown of the homogeneous wind field assumption (a fact that by itself warrants further investigation into alternate wind profiling methods). The interferometry technique might be adversely affected by the increased randomness of the returned signals and produce over-estimates of the wind amplitude (Van Baelen and Richmond 1991). Thus, radar interferometry (RI) at UHF offers a compromise: the ability to measure simultaneously the three components of the wind vector in a common volume, at the expense of a less well-understood technique which might provide over-estimates of the wind amplitude. If the RI technique is proven reliable, its greatest contribution would certainly be in the area of flux and field divergence estimation rather than wind profiling as outlined in the following paragraphs.

In any case, our first concern will be to compare the wind profiles obtained with the Doppler and the RI methods. In particular, we will want to assess the accuracy of the RI technique and investigate if it represents a reliable alternative to the traditional Doppler technique when the latter is adversely affected by nonhomogeneity or evolution in the wind field. A possible outcome could be that only a combined system can provide continuously accurate measurements of the wind.

Using the RI capability to derive the 3-D wind vector from each beam direction, we will then study the wind field variability between those five locations and possibly study the local divergence and vorticity. Preliminary calculations indicate that an accuracy of $0.5 \mathrm{~m} \mathrm{~s}^{-1}$ in the derivation of the wind amplitude should permit a meaningful estimation of divergence and vorticity on the mesoscale, assuming an homogeneous wind flow.

We will also compare the momentum flux estimates obtained with the Doppler and the RI methods. Although both are implementations of the eddy correlation technique, the $\mathrm{Rl}$ approach uses wind components derived from a single volume. We will also investigate whether the RI approach can directly provide the divergence of the momentum flux, a very important parameter for circulation models.

This new system will also be equipped with a RASS such that it will be possible to measure virtual temperature profiles, as well as heat fluxes, through the different approaches. Interferometry should prove equally valuable to study the acoustic propagation supportive of the RASS echoes.

Lately, Peters(a) has proposed to use the RASS echoes instead of the clear air echoes in order to derive the vertical flux of horizontal momentum. His work is motivated by the fact that the RASS-mode echoes are not perturbed by ground clutter at low altitudes and can achieve a more complete coverage of the PBL. Recent results by Peters and Kirtzel (1991) using that approach show good promise and call for further investigations. Our radar-RASS system appears very well suited to do so.

\section{Conclusions}

We expect that the addition of the interferometric capability to our Doppler boundary layer radar will enable us to better estimate the many dynamical parameters of the PBL.

\section{References}

Angevine, W. M., D. A. Carter, W. L. Ecklund, and K. S. Gage. 1991. Temperature profiling using a $915 \mathrm{MHz}$ wind profiler with RASS. Lower tropospheric profiling: Needs and technologies, pp. 43-44. Boulder, Colorado, Sept 1013. American Meteorological Society, Boston, Massachusetts.

Balsley, B. B. 1981. The MST technique-a brief review. J. Atmos. Terr. Phys. 43:495-509.

Briggs, B. H. 1980 . Radar observations of atmospheric winds and turbulence: $A$ comparison of techniques. J. Atmos. Terr. Phys. 42:823.

Briggs, B. H., and R. A. Vincent. 1992. Spaced-antenna analysis in the frequency domain. Radio Sci. 27:117-129.

Clark, W. L., J. L. Green, and J. M. Warnock. 1986. Determination of $u, v$, and $w$ from single station Doppler radar radial velocities. MAP Handbook, 20:385-392.

(a) Personal communication, 1990. 
Coulter, R. L., and M. L. Wesely. 1980. Estimates of surface heat flux from sodar and laser scintillation measurements in the unstable boundary layer. J. Appl. Meleorol. 19:199-205.

Currier, P. E., W. L. Ecklund, J. M. Warnock, and B. B. Balsley. 1988. Temperature profiling using a UHF wind profiler and an acoustic source. Lower tropospheric profiling: Neods and tochnologies, pp. 121-122. Boulder, Colorado, May 31-June 3. American Meteorological Society, Boston, Massachusetts.

Dabberdt, W. F., C. Martin, H. L. Cole, J. Dudhia, T. Horst, Y. H. Kuo, S. Oncley, J. Van Baelen, K. S. Cage, W. Eoklund, R. Strauch, E. R. Westwater, H. Revercomb, and W. L. Smith. 1991. An integrated data assimilation and hybrid sounding system. Proc. of the International Conference on Mesoscale Meteorology and TAMEX, Dec. 3-6, 1991, Taipei, Taiwan. American Meteorological Society, Boston, Massachusetts.

Ecklund, W. L., D. A. Carter, and B. B. Balsley. 1988. A UHF wind profiler for the boundary layer: Brief description and initial results. J. Atmos. Ocean. Tech. 5:432-441.

Ecklund, W. L., D. A. Carter, B. B. Balsley, P. E. Currier, J. L. Green, B. L. Weber, and K. S. Cage. 1990. Field tests of a lower tropospheric wind profiler. Radio Sci. 25:899906.

Frisch, A. S., B. E. Manner, and J. S. Gibson. 1989. Measurement of the vertical flux of turbulent kinetic energy with a single Doppler radar. Bound.-Layer Meteorol. 49: 331-337.

Fritts, D. C., T. Tsuda, T.E. VanZandt, S. A. Smith, T. Sato, S. Fukao, and S. Kato. 1990. Studies of velocity fluctuations in the lower atmosphere using the MU radar. Part II: Momentum fluxes and energy densities. J. Atmos. Sci. 47:1-16.

Fukao, S., M. Inaba, I. Kimura, P. T. May, T. Sato, T. Tsuda, and S. Kato. 1988a. A systematic error in MST/ST radar wind measurement induced by a finite range volume effect, 1, Observational results. Radio Sci. 23:59.

Fukao, S., T. Sato, T. Tsuda, S. Kato, M. Inaba, and I. Vimura. 1988b. VHF Doppler radar determination of the momentum flux in the upper troposphere and lower stratosphere: Comparison between the three- and fourbeam methods. J. Atmos. Ocean. Tech. 5:57.
Gage, K. S., and B. B. Balsley. 1980. On the scattering and reflection mechanisms contributing to clear-air radar $\theta$ choes from the troposphere, stratosphere, and mesosphere. Radio Sci. 15:293.

Gal-Chen, T., M. Xu, and W. Eberhard. Estimations of ABL fluxes and other turbulence parameters from Doppler lidar data. J. Geophys. Res. 97(D17):18,409-18, 423.

Gavrilov, N. M., A. D. Richmond, J. S. Van Baelen, T. Tsuda, S. Kato, S. Fukao, and M. Yamamoto. 1991. Investigation of internal gravity wave motions in the troposphere and stratosphere with the MU radar. Preprint volume of the Eight Conference on Atmospheric and Oceanic Waves and Stability, pp. 308-311. Oct 14-18, 1991, Denver, Colorado. American Meteorological Society, Boston, Massachusetts.

Kropfli, R. A. 1986. Single Doppler radar measurements of turbulence profiles in the convective boundary layer. J. Atmos. Ocean. Tech. 3:305-313.

Larsen, M. F., and J. Röttger. 1991. VHF radar measurements of refractivity layer tilt angles and associated vertical-bean radial velocity corrections. J. Atmos. Ocean. Tech. 8:477-490.

Lenschow, D. H. 1986. Probing the atmospheric boundary layer. American Meteorological Society, Boston, Massachusetts.

Matsuura, N., Y. Masuda, H. Inuki, S. Kato, S. Fukao, T. Sato, and T. Tsuda. 1986. Racio acoustic measurement of temperature profile in the troposphere and stratosphere. Nature 323:426-428.

May, P. T., K. P. Moran, and R. C. Strauch. 1988. The altitude coverage of temperature measurements using RASS with wind profiler radars. Geophys. Res. Lett. 15:1381-1384.

May, P. T., R. G. Strauch, K. P. Moran, and W. L. Ecklund. 1990. Temperature sounding by RASS with wind profiler radars: A preliminary study. IEEE Rans. Geosci. Remote Sens. 28:19-28.

Nastrom, C. D., and T.E. VanZandt. 1991. Measurements of vertical momentum fluxes in the troposphere by the Flatland VHF radar. Summaries of the 5th Workshop on technical and scientific aspects of MST radars, pp. 84-85. University College of Wales, Aberystwyth, United Kingdom. 
Palmer, R. D., M. F. Larsen, R. F. Woodman, S. Fukao, M. Yamamoto, T. Tsuda, and S. Kato. 1991. VHF radar interferometry measurements of vertical velocity and the effect of tilted refractivity surfaces on standard Doppler measurements. Radio Sci. 26:417.

Peters, G., and H. J. Kirtzel. 1991. Measurements of the flux and diffusion coefficient of the momentum in the lower atmosphere by RASS. Lower tropospheric profiling: Needs and technologies, pp. 97-98. Boulder, Colorado, September 10-13. American Meteorological Society, Boston, Massachusetts.

Peters, G., H. Timmermann, and H. Hinzpeter. 1983. Temperature sounding in the planetary boundary layer by RASS: System analysis and results: Int. J. Remote Sens. 4:49.

Röttger, J. 1980. Reflection and scattering of VHF radar signals from atmospheric refractivity structures. Radio Sci. 15:259.

Rötiger, J. 1989. The interpretation of MST radar echoes: The present knowledge of the scattering/reflection and the irregularity generation mechanisms. MAPHandbook28:6882.

Röttger, J., and H. M. lerkic. 1985. Post beam sieering and interferometer applications of VHF radars to study winds, waves, and turbulence in the lower and middle atmosphere. Radio Sci. 20:1461.

Strauch, R. C., K. P. Moran, and P. T. May. 1991. RASS temperature errors caused by winds. Preprint volume of the Seventh Symposium on Meteorological Observations and Instrumentation, pp. 51-54. Jan. 14-18, 1991, New Orleans, Louisiana. American Meteorological Society, Boston, Massachusetts.

Tsuda, T., T. Sato, K. Hirose, S. Fukao, and S. Kato. 1986. MU radar observations of the aspect sensitivity of backscattered VHF echo power in the troposphere and lower stratosphere. Radio Sci. 21:971.
Van Baelen, J.S. 1990. Comparison of clear air atmospheric radar techniques for the study of atmospheric dynamics in the troposphere and the stratosphere, Ph.D. cooperative thesis 128, $191 \mathrm{pp}$. University of Colorado and National Center for Atmospheric Research, Boulder, Colorado.

Van Baelen, J. S., and A. D. Richmond. 1991. Radar interferometry technique: 3-D wind measurement theory. Radio Sci. 26:1209-1218.

Van Baelen, J. S., A. D. Richmond, T. Tsuda, S. K. Avery, S. Kato, S. Fukao, and M. Yamamoto. 1991. Radar interferometry technique and anisotropy of the echo-power distribution: First results. Radio Sci. 26:1315-1326.

Vincent, R. A., and I. M. Reid. 1983. HF Doppler measurements of mesospheric gravity wave momentum fluxes. J. Atmos. Sci. 40:1321.

Weill, A., C. Klapitz, B. Strauss, F. Baudin, C. Faupart 1980. Measuring heat flux and structure functions of temperature fluctuations with an acoustic Doppler sodar. J. Appl. Meteorol. 19:1209-1222.

Wilson, D. A. 1970. Doppler radar studies of boundary layer wind profiles and turbulence in snow conditions. Proc. 14th Conf. Radar Meteorol., pp. 191-196. Tucson, Arizona. American Meteorological Society. Boston, Massachusetts.

$X u, M$. and T. Gal-Chen. Study of the convective boundary layerdynamics using single Doppler radar measurements. J. Atmos. Sci., in press.

Yoe,J.G., R. Rüster, and G. Schmidt. 1991. Measurements of momentum fluxes in the troposphere and stratosphere during jet stream passages using the SOUSY VHF radar. Summaries of the 5 th Workshop on technical and scientific aspects of MST radars, p. 83. University College of Wales, Aberystwyth, United Kingdom. 


\title{
System Design and Implementation of the Integrated Sounding System
}

\author{
C. L. Martin and A.-L. Barrett \\ National Center for Atmospheric Research \\ Boulder, CO 80307-3000
}

The integrated sounding system (ISS) combines a suite of in situ and remote sensing systems with local computing capabilities. The resulting integrated instrument provides detailed real-time vertical profiles of the basic atmospheric parameters at a variety of time and space resolutions. One hallmark of the system is its configurability, which allows individual sensing systems to be added or removed as needed. A flexible data system copes easily with evolving and changing observation formats and requirements. $A$ typical ISS configuration might include a wind profiler, a radio acoustic sounding system, a navaid based radiosonde, and a surface meteorological station.

Individual observing platforms in an ISS operate in a standalone mode and communicate via a common network to deliver high-level data products to the central workstation (Figure 1). The observing platforms often support archival of low-level data. Allowing the subsystems to operate independently prevents a fault in one system from affecting the others, and the low-level archives provide data redundancy.

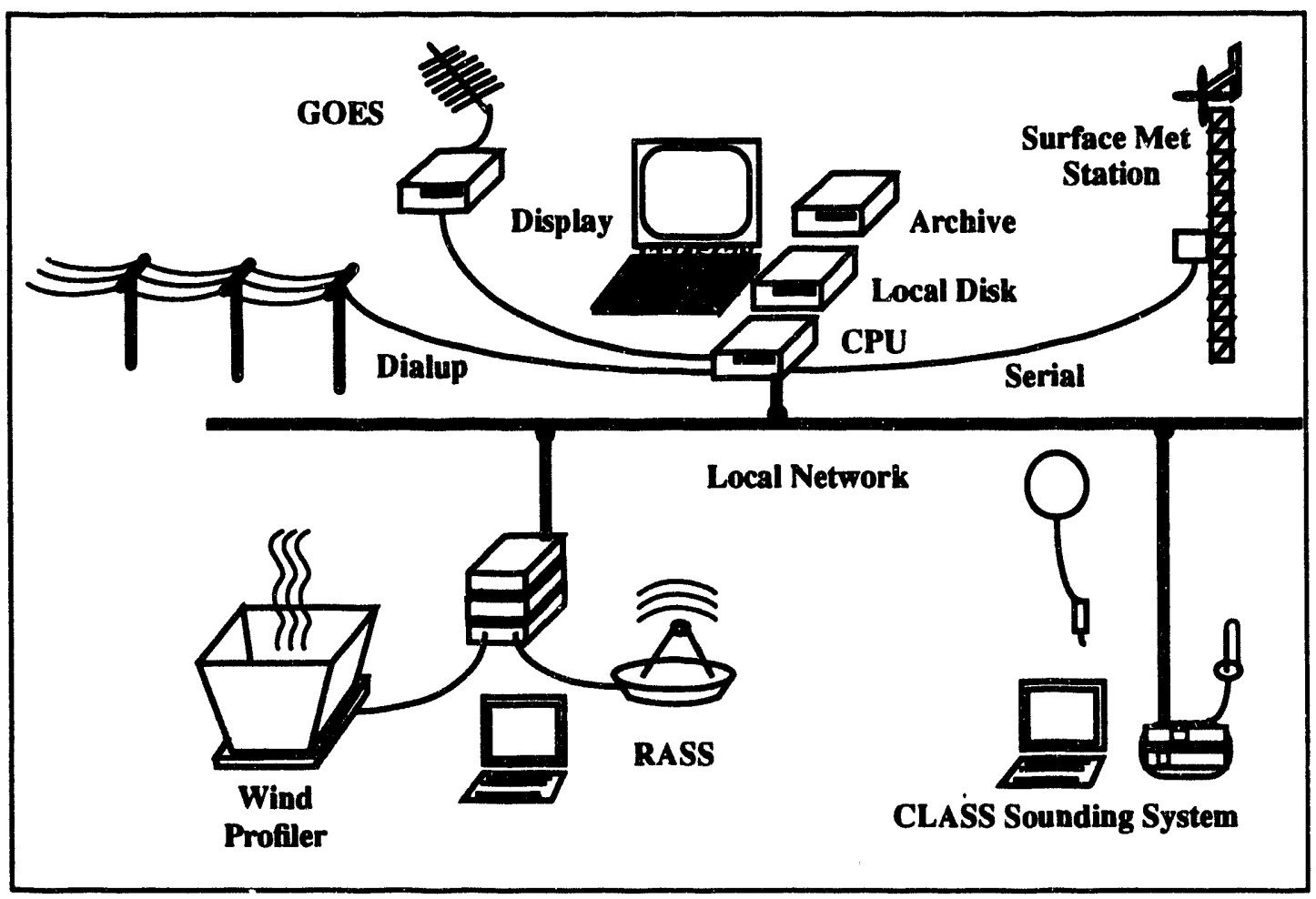

Figure 1. Typical ISS configuration. 
The ISS workstation provides centralized resources to support a wide suite of activities, including system monitoring, data quality control, data archive, graphical display, analysis, and data product communication. Most procedures can operate with either the real-time data or the archived products. On-line storage of many months of observations is feasible in a typical ISS configuration.

The data system is implemented as a collection of cooperating processes. The NCAR Zeb data integration package provides the basic infrastructure supporting these activities. Basic facilities (both low and high level) include the following:

- Interprocess Communication-Message passing provides a generic facility for sharing structured information between processes. Capabilities are provided to multiplex message handling, other $\mathrm{i} / \mathrm{o}$, and compute activities. The message system is used to notify real-time processes when new data are available, coordinate between graphics activities, and synchronize processes within a subsystem such as within the data communications package.

- Data Store-A centralized real-time database defines "platform" objects which represent structured formats appropriate to different data types. The formats support a wide variety of data representations, including multidimensional grids, time series, and imagery. Multiple processes can concurrently attach to the data store and request data in real-time or for specified time periods. Real-time access enables notifications to be sent to processes when new data are placed in the data store. A process requests data notification on a perplatform basis, and data from multiple platforms can be received (or requested) by a single process, providing a mechanism that allows disparate data sets to be merged and analyzed.

- Graphics Processes-Interactive graphics processes display data in a wide variety of representations. Graphics processes operate in both real-time and posttime modes. An unrestricted number of platforms, i.e., data from differing observing systems, can be overlaid on a given plot. Configurable user interfaces allow interactive manipulation of plot features such as scale ranges, time spans, and the dynamic addition and removal of data fields. Sophisticated representations such as contours, vector time-series, and profile timeseries are provided. Multiple graphics processes can simultaneously display in multiple windows, and graphics configurations can be opened and closed, so that a researcher can switch quickly between any number of configurations designed for that particular ISS deployment.

- Ingest Scheduler and Data Ingestors-A configurable process scheduler is provided for controlling data ingest activities and for scheduling other analysis and administrative tasks. Typically, the observing systems will place a data file containing an observation in a networked file directory, where it is noticed by the scheduler. The scheduler then will dispatch a data ingestor to format and transfer the observation to the data store. This scheme allows observing systems to be added to the ISS simply by constructing an appropriate ingestor (or using an existing one) and configuring the ingest scheduler to activate it. The ingest scheduler can also run processes on a periodic or continuous (i.e., "run once") basis. Ingestors are not limited to transferring observations from data files into the data store. Ingestors can access other data inputs; for instance, the surface met station produces a serial data stream which is read by an ingest process and placed directly into the data store.

- Communications-A data communications subsystem provides capabilities for transmitting data products to remote locations. It consists of a communications scheduler and a collection of protocol handlers. Data products for transmission are created by analysis processes and stored in designated data store platforms. The communications scheduler is configured through a table that specifies which platforms and data times are to be transmitted by a given protocol. Protocol handlers are implemented as separate processes. When a data notification is received from the data store for a given platform, the communications scheduler notifies the appropriate protocol handler. The handler then fetches the data product at the appropriate time and performs the transmission. Currently, a protocol handler to use the Geostationary Operational Environmental Satellite (GOES) link has been implemented. Other protocol handlers can be easily added as neөded.

Figure 2 depicts the ISS data flows. The general scheme is that an observing system provides a data file (or serial data stream) which a specific ingestor will transfer to the datastore. This ingestor is activated by the ingest scheduler. 


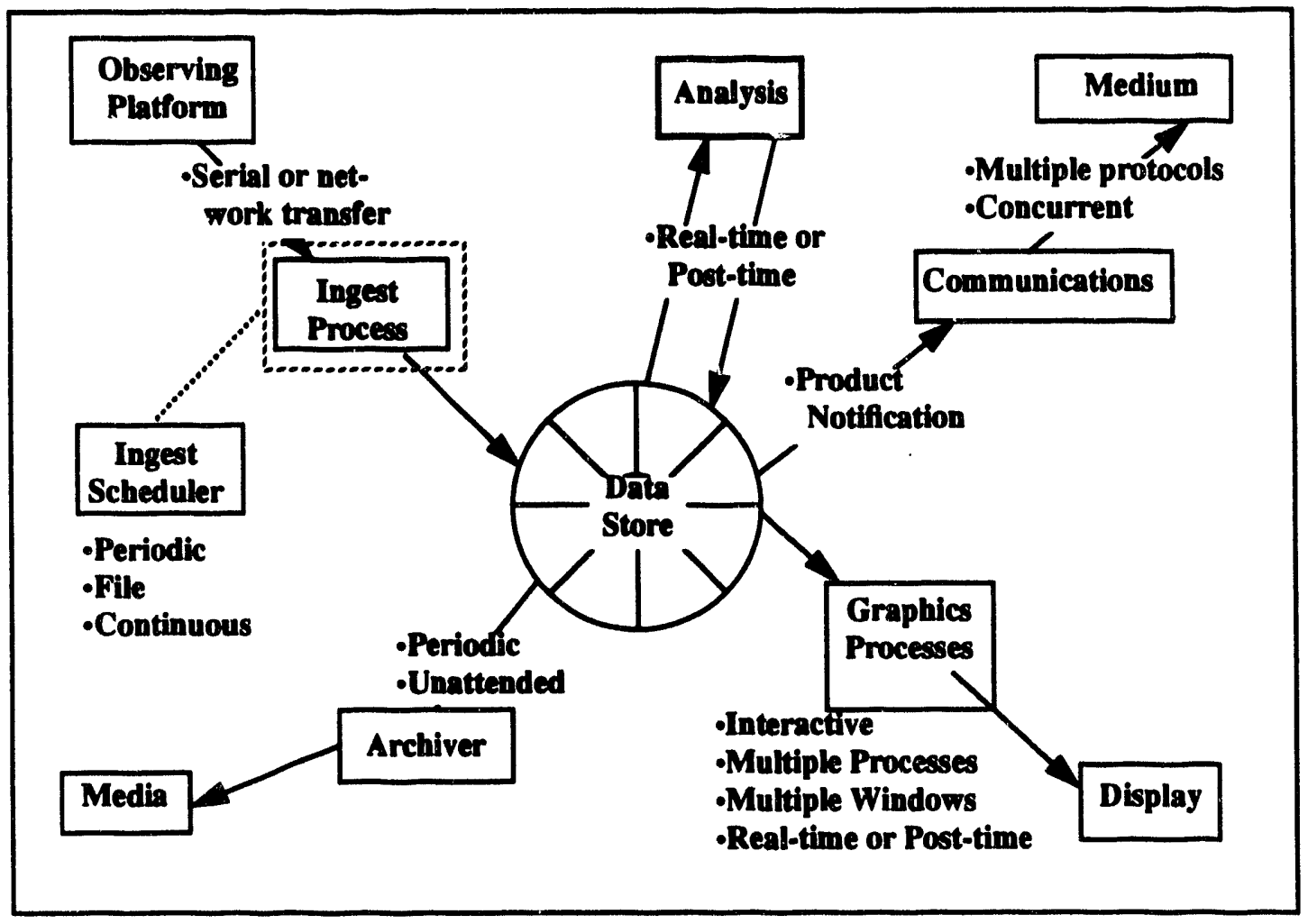

Figure 2. System data flows.

Real-time graphics processes receive notifications and then display the new data. Real-time analyses, e.g., a mesoscale model performing data assimilation, will also receive notification and will automatically obtain data as it becomes available. These models can generate products that are placed back in the data store and can be accessed by the graphics processes just like any other data. The data archiver is activated periodically to save the data store to permanent media. Analysis tasks are also activated to transform new data into products for transmission, and the communications scheduler notifies the protocol handler in order to initiate transfer of the product via the proper medium.

Table 1 outlines the current hardware and software foundation for the ISS computing system. This configuration allows for all source code and development tools to remain available on the system during an ISS deployment. The optical disk is used both for the permanent data archive and the system software distribution.

An ISS base station has been established at NCAR in Boulder to process data products transmitted from a network of ISS sites. Apart from an additional software subsystem which was implemented to reconstruct data products sent via the ISS GOES communications protocol, the base station uses the same software system that is employed in the field ISS. It concurrently processes data sets from the multiple ISS installations and provides for centralized real-time data collection, display and analysis of the observations coming from a multi-site deployment of the ISS. The sharing of software between the Boulder base station and the field systems has greatly reduced the software development and maintenance efforts required to implement and operate the central base station. 
Table 1. The ISS computing system configuration.

Workstation

Archive

Languages

Operating System

Windowing System

Infrastructure

Data File Formats
Sun Microsystems SPARCstation IPX, 24MB Color Graphics

$850 \mathrm{MB}$ disk storage

Erasable, removable optical disk $650 \mathrm{MB}$ capacity per disk

C

C Shell

Fortran (1\%)

SunOS UNIX

MIT X11 Release 5

NCAR RDP Zeb Data Display and Integration Package

Network Common Data Format (NetCDF)

Zeb Native Format (ZNF)

ASCII 


\section{Single-Column Modeling}




\title{
Single-Column Model and Cumulus Ensemble Model Simulations of GARP(a) Atlantic Tropical Experiment Data
}

\author{
D. A. Randall and K.-M. Xu \\ Colorado State University \\ Department of Atmospheric Science \\ Fort Collins, CO 80523
}

\section{Introduction}

OurARM project consists of developing and demonstrating improved cloud formation parameterizations by using both a single-column model (SCM) and a cumulus ensemble model (CEM), together with ARM data. These two models can be driven with "large-scale forcing" (e.g., vertical motion) as observed in ARM; each model produces a field of clouds and the associated radiation and precipitation fields. The SCM does so through its physical parameterizations, while the CEM does so by "directly simulating" convective cloud circulations. The improved parameterizations tested in this way will be further tested and applied in the Colorado State University (CSU) general circulation model (GCM). Figure 1 summarizes the approach.

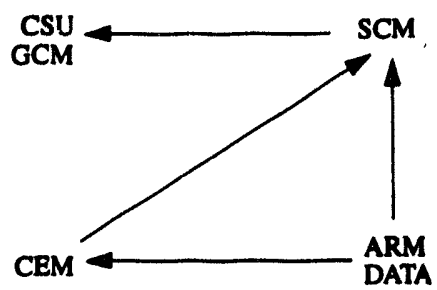

Figure 1. Research strategy followed in this project. ARM data are used to drive the single-column model and the cumulus ensemble model. Parameterizations developed for and tested in the single-column model can then be used in Colorado State University's GCM.

To carry out this research plan, we need to attend to several tasks:

- Develop improved cloudiness parameterizations.

(a) Global Atmospheric Research Program.
- Develop improvements to the CEM, with emphasis on cloud-radiation interactions.

- Drive both models with the ARM data.

- Transfer the improved parameterizations developed in this way to the CSU GCM.

In this brief report, we mention our efforts and progress in each of these areas.

\section{Cloudiness}

\section{Parameterization Development}

\section{Cumulus Convection}

Significant progress has been made in developing improved parameterizations of cumulus convection (Randall and Pan 1992), based on generalization of the parameterization of Arakawa and Schubert (1974). The key idea is to introduce the cumulus kinetic energy as a prognostic variable. This approach allows the strict quasi-equilibrium closure to be relaxed, resulting in drastic simplification and computational economy. The temporal and spatial distributions of cumulus precipitation are smoother with the new parameterization than with the standard Arakawa. Schubert implementation. We are currently working to further generalize the parameterization by allowing cloud bases at multiple levels, simultaneously.

\section{Cloud Microphysics}

We have developed a new prognostic cloud water parameterization that includes not only cloud water but also cloud ice, rain, and snow, all as prognostic variables (Smith and Randall 1992). Cumulus detrainment acts as a 
very important source of cloud ice and cloud water. The microphysics parameterization is based on the work of Rutledge and Hobbs (1983).

\section{Cloud Amount}

We have begun an attack on the difficult problem of physically determining cloud amount, by extending the work of Xu and Krueger (1991). They used the CEM to simulate cloud distributions in response to prescribed large-scale processes. They estimated the cloud amount for low, medium, and high clouds separately, and obtained semi-empirical relations for stratiform cloud amount as a function of the relative humidity and for convective cloud amount as a function of the cumulus mass flux.

When a prognostic cloud water variable is available, it is natural to include it as a key predictor in such a semiempirical scheme. Some preliminary work along these lines has already been carried out, using the CEM. An example is shown in Figure 2. Here the cloud amount is plotted against the average liquid water path, with an averaging distance of $128 \mathrm{~km}$, at an altitude of $1.9 \mathrm{~km}$ above the sea surface. Moderate scatter is apparent. We

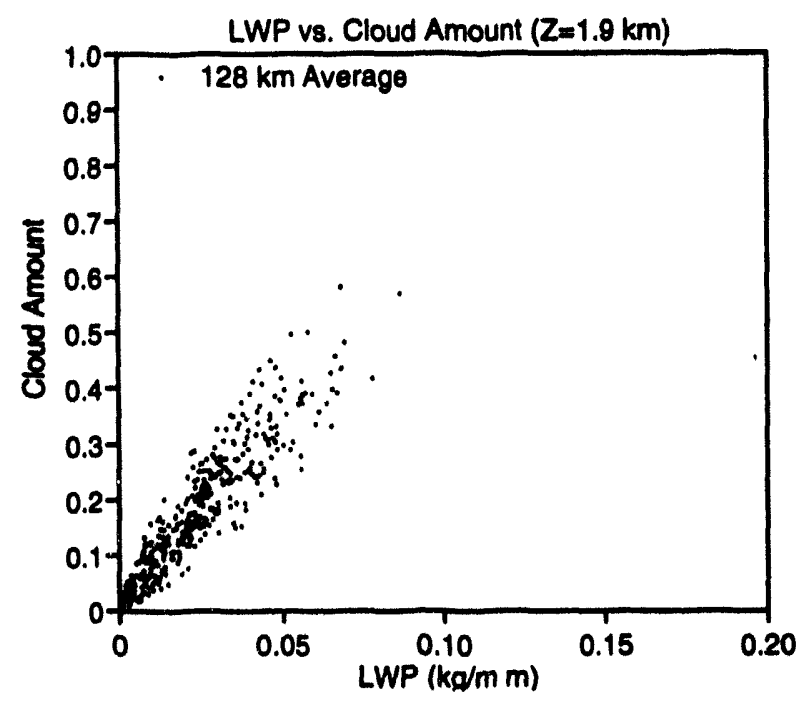

Figure 2. A scatter plot of cloud amount versus liquid water path (LWP), based on high-resolution simulations with the CEM. have found that most of the remaining variance can be accounted for by adding the area-averaged relative humidity as a second predictor. We are currently pursuing this approach to parameterize cloud amount in the GCM. Further discussion is given by XU and Randall (1992).

\section{CEM Development}

We have implemented the radiation parameterization of Harshvardhan et al. (1987) inside the CEM. The cloud optical properties are parameterized following the methods of Stephens (1978). We have performed some sensitivity tests to see the effects of the interactive radiation on the results. These will be discussed below.

In addition, we have modified the CEM to use a more realistic turbulence length scale formulation.

\section{Driving the SCM and the CEM with Data}

Until very recently, we did not have ARM data suitable for driving either model. We have, therefore, been "practicing" with the GARP(a) Atlantic Tropical Experiment (GATE) data, using both the SCM and the GCM. An example is shown in Figure 3. This shows the observed and SCMsimulated precipitation rates for GATE Phase III. The SCM is runhere in afully prognostic modo-nota semi-prognostic test. The agreement between the observations and the simulation is quite encouraging.

Figure 4 shows a similar test performed with the CEM. Again, the results are quite encouraging. Further results were shown at the Science Team meeting.

\section{Tests of the New Parameterizations in the CSU GCM}

We have already successfully tested the microphysics and prognostic cumulus kinetic energy (CKE) parameterizations

(a) Global Atmospheric Research Program. 


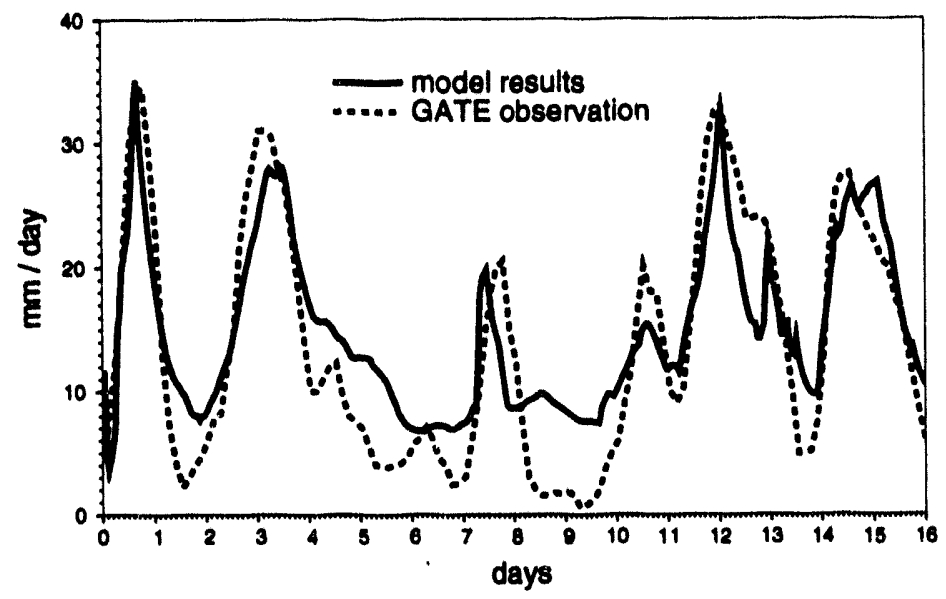

Figure 3. SCM-simulated (solid line) and observed (dashed line) precipitation rate for GATE Phase III.

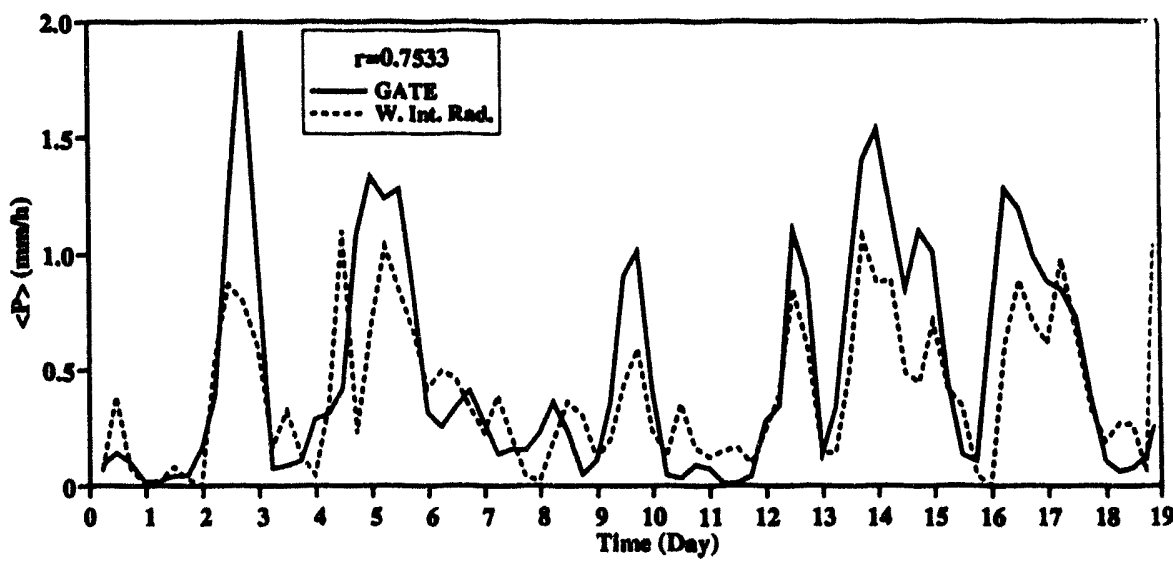

Figure 4. CEM-simulated (dashed line) and observed precipitation rate for GATE Phase III.

in the three-dimensional GCM. At present, we are working on coupling the microphysics parameterization to the radiation parameterization.

For the microphysics, the effects of advection appear to be modest for the resolution currently used ( $4 \times 5$ degrees). The distribution of precipitation at the ground is very similar to that obtained with the conventional "large-scale saturation" parameterization that the microphysics parameterization replaced. Some results are shown in Figure 5. Further results are given by Fowler and Randall (1993).

\section{Conclusions and Plans}

We have developed new parameterizations of convection, cloud microphysics, and cloud amount. The CEM has been endowed with a radiation parameterization. Both the CEM and the SCM have been driven with GATE data and have produced realistic results. We have also tested our new parameterizations of convection and cloud microphysics in the three-dimensional CSU GCM. 

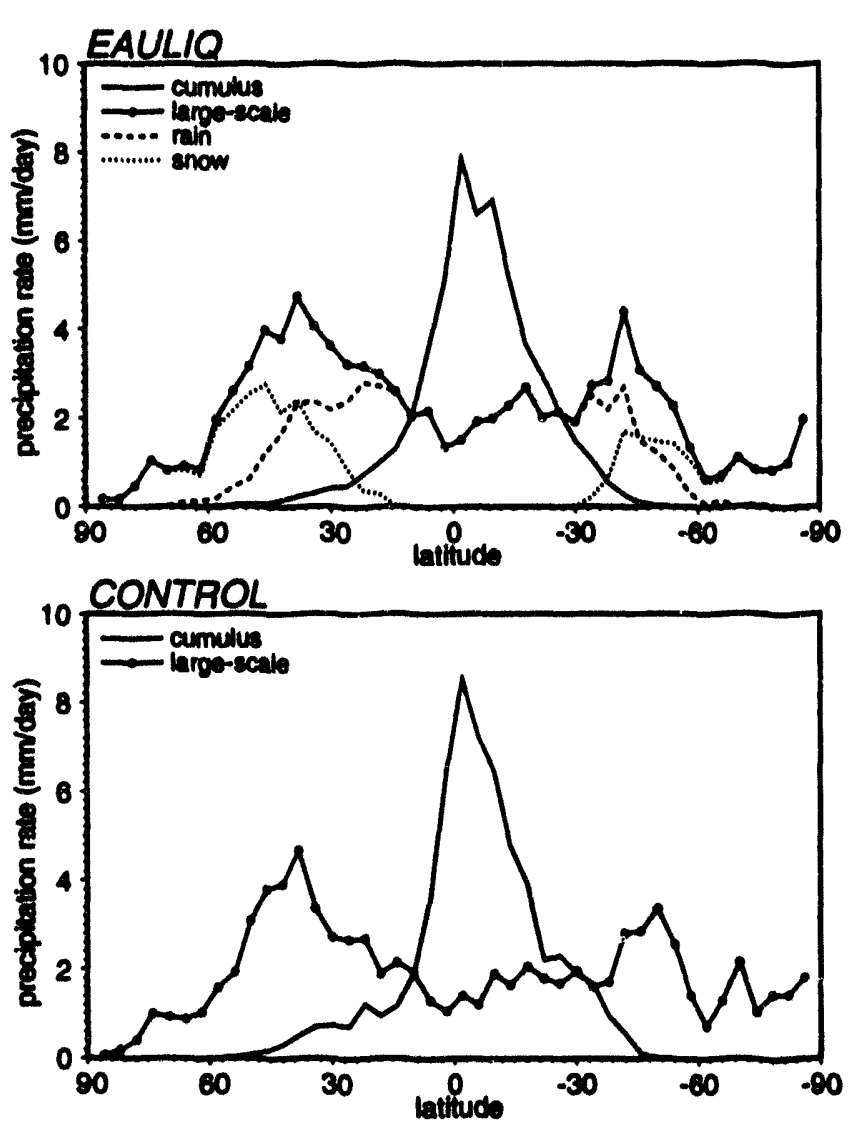

Figure5. Simulated zonally averaged January precipitation rates obtained with the CSU GCM. In the upper panel, a microphysics parameterization has been used. In the lower panel, the conventional "large-scale saturation" parameterization has been used. Snow and rain are shown separately in the upper panel.

Our highest priority for the coming year is to exercise both the SCM and the CEM extensively using real ARM data. In addition, we plan to couple the microphysics parameterization in the SCM and the CSU GCM with the radiation parameterization. Finally, we hope to test our cloud amount parameterization.

\section{References}

Arakawa, A., and W. H. Schubert. 1974. The interaction of a cumulus cloud ensemble with the large-scale environment, Part I. J. Atmos. Sci. 31:674-701.

Fowler, L. D., and D. A. Randall. 1993. Impact of cloud microphysics on the CSU GCM atmospheric moisture budget. Paper presented at the Fourth Symposium Global Change Studles of the American Moteorological Soclety, Anahoim, California, 17-22 January, 1993. American Meteorological Society, Boston, Massachusetts.

Harshvardhan, R. Davies, D. A. Randall, and T. G. Corsetti. 1987. A fast radiation parameterization for general circulation models. J. Goophys. Res. 92:1009-1016.

Randall, D.A., and D.-M. Pan. 1992. Implementation of the Arakawa-Schubert cumulus parameterization with a prognostic closure. To be published in Cumulus Parameterization, ods., K. Emanuel and D. Raymond, a Moteorological Monograph published by the American Moteorological Soclety, Boston Massachusetts.

Rutledge, S. A., and P. V. Hobbs, 1983. The mesoscale and microscale structure and organization of clouds and precipitation in midlatitude cyclones. VIII: A model for the "seeder-feeder" process in warm frontal rainbands. J. Atmos. Sci. 40:1185-1206.

Smith, L. D., and D. A. Randall. 1992. Parameterization of Cloud Microphysical Processes in the CSU General Circulation Model. Atmospheric Science Paper No. 491. Colorado State University, Ft. Collins, Colorado.

Stephens, G. L. 1978. Radiation profiles in extended water clouds. II: Parameterization schemes. J. Atmos. Scl. 35:2123-2132.

Xu,K.-M., and S.K. Kruger. 1991. Evaluation of cloudiness parameterizations using a cumulus ensemble model. Mon. Wea. Rov. 119:342-367.

XU, L.-M., and D. A. Randall. 1992. The Seml-Empirical Basis of a Prognostic Cloud Parameterization for Use in Climate Models. Paper presented at the 11th Intermational Conference on Clouds and Precipitation, Montreal, Canada. American Moteorological Society, Boston, Massachusetts. 


\title{
Diagnostic Modeling of the Experimental Site of the Atmospheric Radiation Measurement Program
}

\author{
R.C.J. Somerville and S. F. lacobellis \\ Climate Research Division \\ Scripps Institution of Oceanography \\ University of California, San Diego \\ La Jolla, CA 92093.0224
}

Our project is centered around a computationally efficient and economical one-dimensional (vertical) model, resembling a single column of a general circulation model (GCM) grid, applied to the experimental site of the Atmospheric Radiation Measurement (ARM) Program. The model contains a full set of modern GCM parameterizations of subgrid physical processes. To force the model, the advective terms in the budget equations are specified observationally from operational numerical weather prediction analyses. These analyses, based on four-dimensional data assimilation techniques, provide dynamically consistent wind fields and horizontal gradients of temperature and moisture.

\section{Model Description}

Our model is diagnostic rather than prognostic. Its input is an initial state, plus the time-dependent advection terms in the conservation equations, provided at all model layers. Its output is a complete heat and water budget for the ARM experimental configuration, including temperature and moisture profiles, clouds and their radiative properties, diabatic heating terms, surface energy balance components, and hydrologic cycle elements, all specified as functions of time.

These model products can then be validated against ARM observations. This validation provides a test of the realism of the model's physical parameterizations and a means of evaluating proposed improvements. In addition, it allows an assessment of the sensitivity of the results to individual elements of the parameterizations. The model thus is a computational testbed which allows progress to be made on the problem of modeling cloud formation as well as cloud characterization. Our research effort is a coordinated program of model development, diagnostic modeling, and linkages with GCM experiments and with observations.

Our model has evolved from the one described by lacobellis and Somerville $(1991 a, b)$. These papers describe the theory and conceptual basis of single-column modeling. Virtually all the parameterizations in our model, however, have been replaced or substantially modified since these papers were written.

\section{Code Development}

Until very recently, ARM observational data were not available. While awaiting the data, we have developed and extensively revised the current version of the model to incorporate state-of-the-art physical parameterizations. At the same time, we have devoted effort to recoding the model for generality, efficiency, and modularity. As an example of generalizing the code, we want to be able to alter the vertical resolution by simply setting one parameter.

We have succeeded in implementing the model on a workstation in interactive form to facilitate testing and funing of alternative parameterizations. We have also made substantial progress in developing plug-compatible code for competing algorithms so as to be able to quickly switch between them. Additionally, we have begun to develop and implement effective graphical displays for the model results. Now that the first ARM site has become operational, we have begun receiving ARM data.

We have spent a significant amount of time improving the model code to facilitate future intercomparisons of the physical parameterizations. For example, the code has been modified to allow the time step to be changed by adjusting a single parameter. This is not a trivial change, 
given the model's dependence on time-dependent observational data. Earlier versions of the model assumed that the observational data would have the same temporal resolution as the model time step. The model can now incorporate observational data with any temporal resolution. Additionally, the different observed variables can have different sampling frequencies, as will be the case in ARM.

The model code and associated software (graphics, data storage, etc.) have been transferred to a high-speed UNIX workstation. The graphics software has been updated to take advantage of the animation and color capabilities of the workstation. Data management procedures are currently being revised to cope with the heterogeneous nature of ARM data. We are also using a data set obtained from the First GARP(a) Global Experiment (FGGE) archives as a surrogate to develop and test the effectiveness of these procedures. At the Science Team meeting, we will show sample results based on these data, applied to an area in Oklahoma near the ARM site.

\section{Physical Parameterizations}

Our research during the past year has concentrated on improving and supplementing the parameterizations and code in the model and, also, on developing procedures and software to facilitate the use of ARM observational data. We have continued to update and improve the model parameterizations.

During the past year we have incorporated and tested the cumulus convection scheme developed by Emanuel (1991). The current model version now includes a choice of three modern and widely used cumulus convection schemes, those of Arakawa and Schubert, Emanuel, and Kuo. This type of software development is essential to ouriobjective of being able to validate and intercompare competing parameterizations using ARM observations.

Our terrestrial radiation parameterization is based on the one developed by Morcrette (1990) for the European Centre for Medium-Range Weather Forecasts (ECMWF) model. We have initiated work to include Morcrette's solar radiation parameterization into our model. A similar version of this shortwave radiation parameterization is currently being used in the ECMWF model. Earlier versions of our

(a) Global Atmospheric Research Program. model incorporated the routine of Lacis and Hansen (1974), which at one time was the standard GCM solar radiation algorithm. Currently, we are using the shortwave parameterization of Fouquart and Bonnel (1980).

The parameterization of clouds and cloud radiative properties is the central focus of our research. We have incorporated a treatment of cloud optical properties adopted by the second-generation GCM of the Canadian Climate Centre (McFarlane et al. 1992), in which optical properties are based on cloud liquid water contents, which in turn are parameterized on temperature and pressure following Betts and Harshvardhan (1987), Platt and Harshvardhan (1988), and Somerville and Remer (1984). Our cloud prediction algorithm at present follows Slingo (1987), but we are examining alternatives.

Our intention is not to advocate any particular physical parameterization a priori. Instead, we intend to create a model in which alternative parameterizations can be selected simply by setting a switch. Then, we will use the ARM data to test the different parameterizations within the model so as to determine those respects in which any given parameterization does or does not conform to reality.

We have been collaborating with other groups in an effort to expand and improve the model. Along with a group from Los Alamos National Laboratory (LANL), we are seeking to develop an improved version of the liquid water prognostication treatment of Sundqvist(1978). The current model does not carry liquid water as a prognostic variable, although recent research has demonstrated the value of doing this so as to be able to predict cloud radiative properties. Thus, the inclusion of the Sundquist-type scheme will give the model added versatility in studying cloud-radiation interactions.

Collaboration has also been ongoing with a group from Science Applications International Corporation (SAIC) headed by N. Byrne. The objective is to implement a statistical treatment of cloud radiative properties within the model (Malvagi ot al. 1993). This collaboration includes a graduate student at Scripps Institution of Oceanography, Ms. Yolande Serra, whose Ph.D. dissertation will be based on our ARM research.

The current version of our model incorporates a land surface parameterization based on that of Deardorff(1978). Ourboundary layer parameterization follows the approach of Benoit (1976). 


\section{Recent Publications}

Three papers describing recent progress on this project and a closely related ARM project (N. Byrne, principal investigator) appeared in the preprint volume of the American Meteorological Society 73rd Annual Meeting and Fourth Symposium on Global Change Studies, Anaheim, California, January 17 - 22, 1993.

F. Malvagi, N. Byrne, G. Pomraning,'and R.C.J. Somerville. Stochastic radiative transfer predictions of functional cloud cover, pp. 149-151.

Y. Serra, N. Byrne, S. F. lacobellis, and R.C.J. Somerville. Effect of varying functional cloud cover on cloud feedback temperature stabilization, pp. 225-227.

R.C.J. Somerville, and S. F. lacobellis. Single-column diagnostic climate modeling in ARM, pp. 82-85.

Additionally, a paper has been published in the referenced literature:

F. Malvagi, R. N. Byrne, G. C. Pomraning, and R.C.J. Somerville. 1993. Stochastic radiative transfer in a partially cloudy atmosphere. J. Atmos. Sci. 50:2146-2158.

\section{References}

Benoit, R. 1976. A comprehensive parameterization of the atmospheric boundary layer for general circulation models. McGill University, Montreal, Quebec. Dissertation Abstracts International 37/11-8:5708.

Betts, A. K., and Harshvardan. 1987. Thermodynamic Constraint on the Cloud Liquid Water Feedback in Climate Models. J. Geophys. Res. 92(7):8483-8485.

Deardorf, J. W. 1978. Efficient Prediction of Ground Surface Temperature and Moisture, With Inclusion of a Layer of Vegetation. J. Geophys. Res. 83(C4):1889-1903.

Emanuel, K.A. 1991. A Scheme for Representing Cumulus Convection in Large-Scale Models. J. Atmos. Sci. 48:23132335.
Fouquart, Y., and B. Bonnel. 1980. Computations of solar heating of the Earth's atmosphere: A new parameterization. Eeitr. Phys. Atmos. 53:35-62.

lacobellis, S.F., and R.C.J. Somerville. 1991a. Diagnostic Modeling of the Indian Monsoon Onset. Part I: Model Description and Validation. J. Atmos. Scl. 48(17):19481959.

lacobellis, S. F., and R.C.J. Somerville. 1991b. Diagnostic Modeling of the Indian Monsoon Onset. Part II: Budget and Sensitivity Studies. J. Atmos. Sci. 48(17):1960-1971.

Lacis, A. A., and J. E. Hansen. 1974. A Parameterization for the Absorption of Solar Radiation in the Earth's Atmosphere. J. Atmos. Sci. 31:118-133.

MicFarlane, N. A., G. J. Boer, J.P. Blanchet, and M. Lazare. 1992. The Canadian Climate Centre SecondGeneration General Circulation Model and Its Equilibrium Climate. J. Clim. 5:1013-1044.

Malvagi, F., R. N. Byrne, G. C. Pomraning, and R.C.J. Somerville. 1993. Stochastic Radiative Transfer in a Partially Cloudy Atmosphere. J. Atmos. Sci. 50:21462158.

Morcrette, J.-J. 1990. Impact of Changes to the Radiation Transfer Parameterizations Plus Cloud Optical Properties in the ECMWF Model. Mon. Wea. Rev. 118:847-873.

Platt, C.M.R., and Harshvardhan. 1988. Temperature Dependence of Cirrus Extinction: Implications for Climate Feodback. J. Geophys. Res. 93(D9):11,051-11,058.

Slingo, J. M. 1987. The development and verification of a cloud prediction scheme for the ECMWF model. Quart. J. Roy. Met. Soc. 113:899-927.

Somerville, R.C.J., and L. A. Remer. 1984. Cloud Optical Thickness Feedbacks in the $\mathrm{CO}_{2}$ Climate Problem. J. Geophys. Res. 89(D6):9668-9672.

Sundqvist, H. 1978. A parameterization scheme for nonconvective condensation including prediction of cloud water content. Quart. J. Roy. Met. Soc. 104:677-690. 


\title{
A Strategy for Testing the Impact of Clouds on the Shortwave Radiation Budget of General Circulation Models: A Prototype for the Atmospheric Radiation Measurement Program
}

\author{
R. D. Cess \\ Marine Sciences Research Center \\ State University of New York \\ Stony Brook, NY 11794-5000
}

Cloud-climate interactions are one of the greatest uncertainties in contemporary general circulation models (GCMs) (Cess et al. 1989, 1990), and the present study has focused on one aspect of this. Specifically, combined satellite and near-surface shortwave (SW) flux measurements have been used to test the impact of clouds on the SW radiation budget of two GCMs. Concentration is initially on SW rather than longwave (LW) radiation because, as will be discussed shortly, in one of the GCMs used in this study an SW radiation inconsistency causes, at least in part, a LW inconsistency. Thus, there'is no logic in testing the LW cloud interactions until the SW problem has been rectified.

The surface data consist of near-surface insolation measured by the upward facing pyranometer at the Boulder Atmospheric Observatory (BAO) tower located approximately $25 \mathrm{~km}$ north of Denver. The tower is surrounded by dry-plains agricultural land typical of the adjoining several hundred square kilometers ranging to the east (Cess et al. 1991). These insolation measurements are provided as hourly means. The satellite data consist of top of the atmosphere (TOA) albedo data, collocated with the tower location, as determined from the Geostationary Operational Environmental Satellite (GOES) SW spinscan radiometer. Although this is an uncalibrated and filtered (i.e., narrow band) instrument, simultaneous calibration and unfiltering were achieved through collocation of GOES and Earth Radiation Budget Experiment (ERBE) pixels. The advantage of using GOES measurements is its high sampling rate. Measurements, collocated with the tower, are made every half hour, with hourly means taken by averaging successive measurements. The combined data are for a 21-day period encompassing 28 June through 18 July 1987 and consist of 202 combined albedo/ insolation measurements.

For current purposes, the tower insolation has been normalized by the cosine of the solar zenith, $\mu$, so as to minimize the dependence on solar zenith angle. Although this dependance remains as asecondary effect, the primary variability is caused by cloudiness variability; low albedo and high insolation/ $\mu$ correspond to clear days, with the reverse coinciding with heavily overcast conditions.

Two GCMs have initially been adopted for comparison with the GOES/tower data; Version 2 of the NCAR Community Climate Model (CCM2) and Cycle 33 of the European Centre for Medium-Range Weather Forecasts (ECMWF). The CCM2, results are for a spectral truncation of T42 and for a perpatual July, and T106 and a seasonal July for ECMWF. For both GCMs, output was selected for a single grid point coinciding with the location of the BAO tower.

In Figure 1, GCM-produced monthly-mean albedos and insolation/ $\mu$ are compared with the 21-day means from GOES and the BAO tower. That CCM2 underestimates the albedo is consistent with its overestimate of insolation/ $\mu$; the model either underestimates cloud cover or cloud brightness. The situation is reversed for ECMWF, although the differences are farmore modest. If we were to stopright here, it would be concluded that ECMWF is the better model. But the data provide far more information.

The albedo histograms in Figure 1 amply demonstrate this. Progressing from left to right corresponds to increasing 

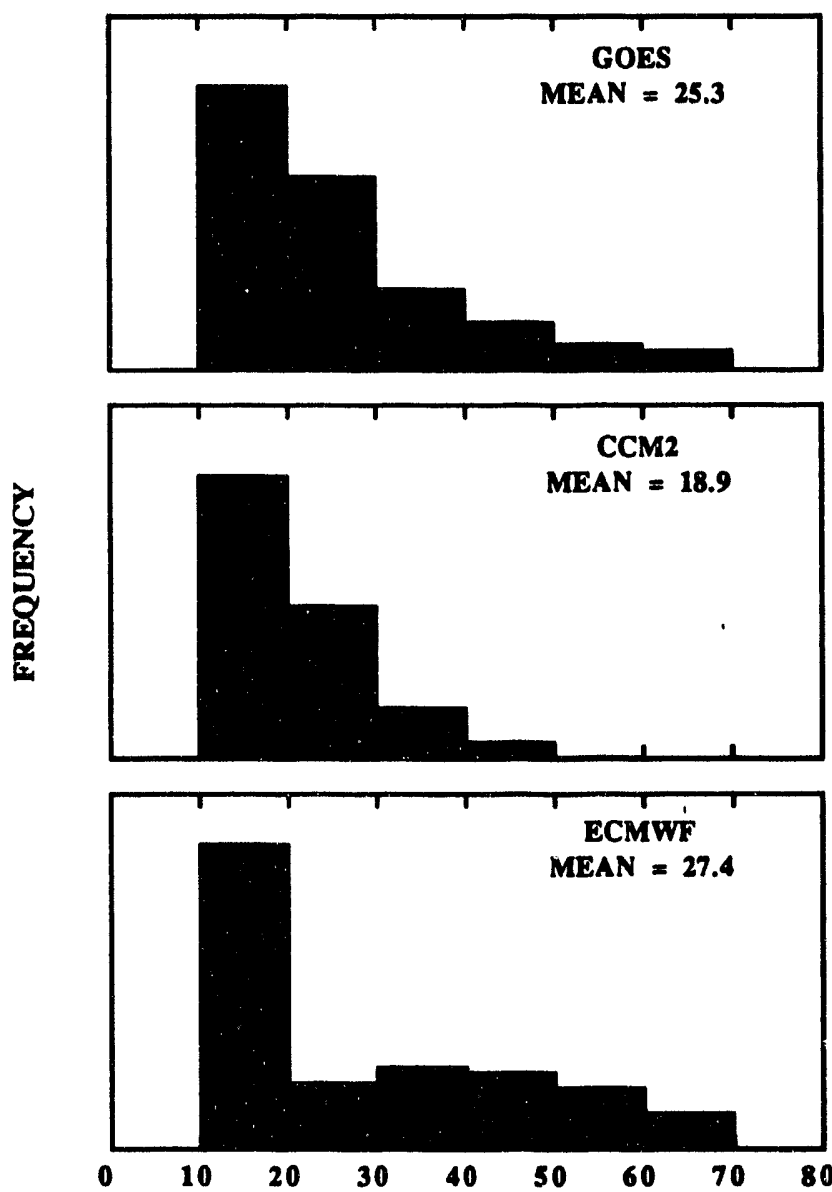

\section{ALBEDO (\%)}

Figure 1. Top of the atmosphere albedos as measured by GOES and from CCM2 and the ECMWF GCM.

cloudiness. The problem with CCM2 is not that it underpredicts cloud cover (its mean cloud-cover fraction at this location is 0.60 ), but rather that it underpredicts a small population of optically thick clouds in the $50 \%$ to $70 \%$ albedo range. The large GOES population in the $20 \%$ to $30 \%$ albedo range indicates a substantial amount of optically thin clouds, and CCM2 is quite consistent with this. Its mean cloud-cover fraction for this range of albedos is 0.77 , so this model has a large amount of optically thin clouds, consistent with the GOES data, while it is underpredicting
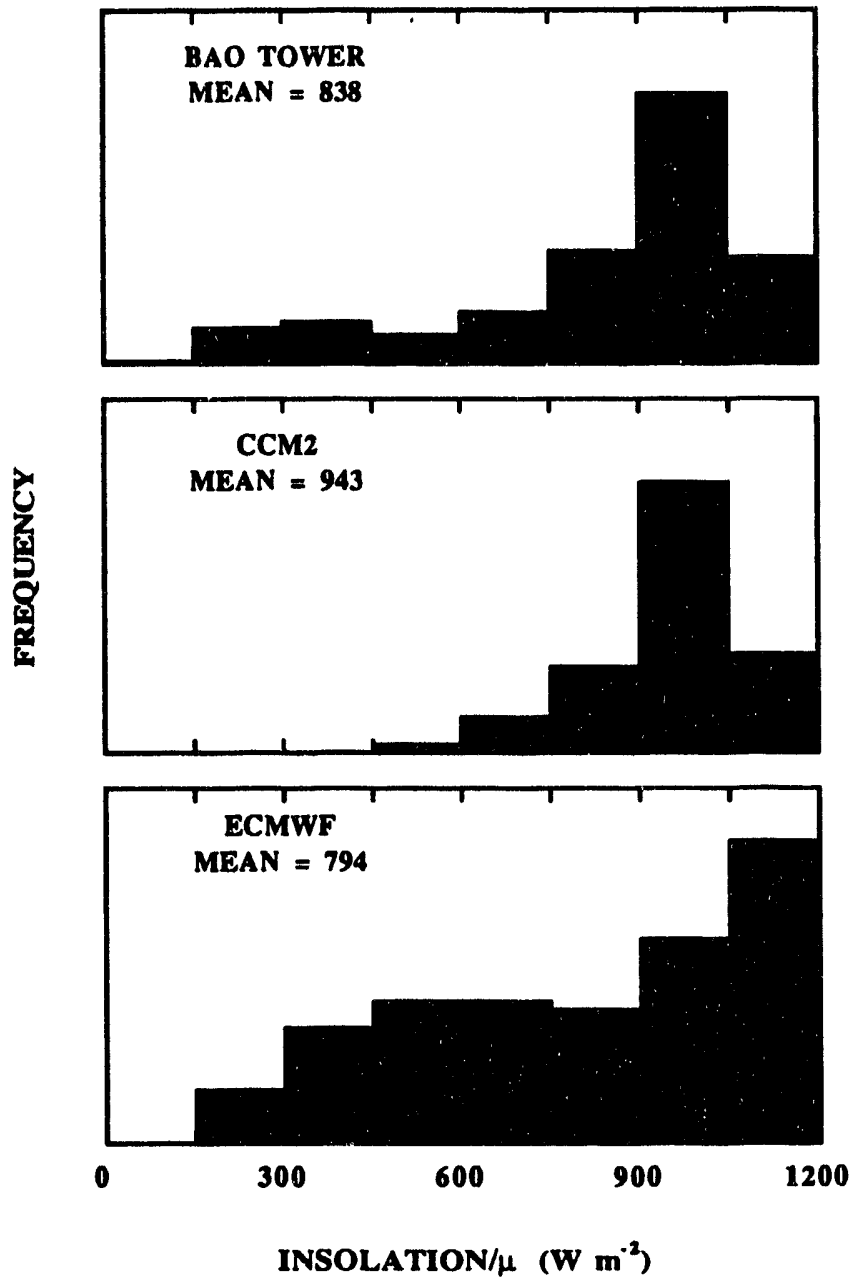

Figure 2. Summary of insolation/ $\mu$ as measured at the $B A O$ tower and from CCM2 and the ECMWF GCM.

a small amount of optically thick clouds. For ECMWF, on the other hand, the situation is reversed; this model is overpredicting optically thick clouds while underpredicting optically thin clouds. These overpredictions and underpredictions, however, tend to be compensatory, so that the model produces a reasonable mean albedo.

Histograms of insolation/ $\mu$ (Figure 2) provide similar information. Here increased cloudiness reduces insolation $\mu$ and so corresponds to a progression from right to left. 
The large 900-1050 $\mathrm{Wm}^{-2}$ insolation/ $\mu$ population for both the tower data and CCM2 is again indicative of large amounts of optically thin clouds, while the minimal populations for $\mathrm{CCM} 2$ below $600 \mathrm{Wm}^{-2}$ again demonstrate an underpopulation of optically thick clouds. And again, the ECMWF GCM is clearly underpredicting optically thin clouds and overpredicting optically thick clouds.

While the two datasets individually provide useful information, collectively they add a further dimension to SW cloud interactions in GCMs. To demonstrate this, scatter plots of albedo versus insolation/ $\mu$ are shown in Figure 3. Points clustering to the right denote clear-sky conditions while increased cloudiness corresponds to a leftward progression.

Although CCM2 and the ECMWF GCM appear to be quite different models with respect to Figures 1 and 2 , they produce virtually identical slopes (Figure 3 ) which differ from that produced by the GOES/tower data. To better understand this difference, the albedo slopes may, to an excellent approximation, be expressed as

$$
\text { Slope }=\frac{\text { Std. Dev. of Albedo }}{\text { Std. Dev. of Insolation } / \mu}
$$

The above standard deviations, normalized to those evaluated from the GOES and tower measurements, are shown in Figure 4. Perfect agreement with the measurements would consist of a normalized standard deviation of unity.

The results in Figure 4 add a further perspective: both models underestimate the increase in SW absorption by the atmospheric column associated with an increase in cloudiness because this increased absorption affects the albedo and insolation/ standard deviations in opposite ways. An increase in cloud SW absorption would reduce cloud albedo and, in turn, the albedo standard deviation. But, simultaneously, this increased absorption by the atmospheric column would reduce surface insolation and thus increase the insolation/ standard deviation. This combination would thus lead to a slope reduction and, as far as this specific aspect is concerned, would provide better agreement of the GCMs with the GOES/tower data (Figure 3). There are several possibilities for implementing such an improvement. But the important issue here is that

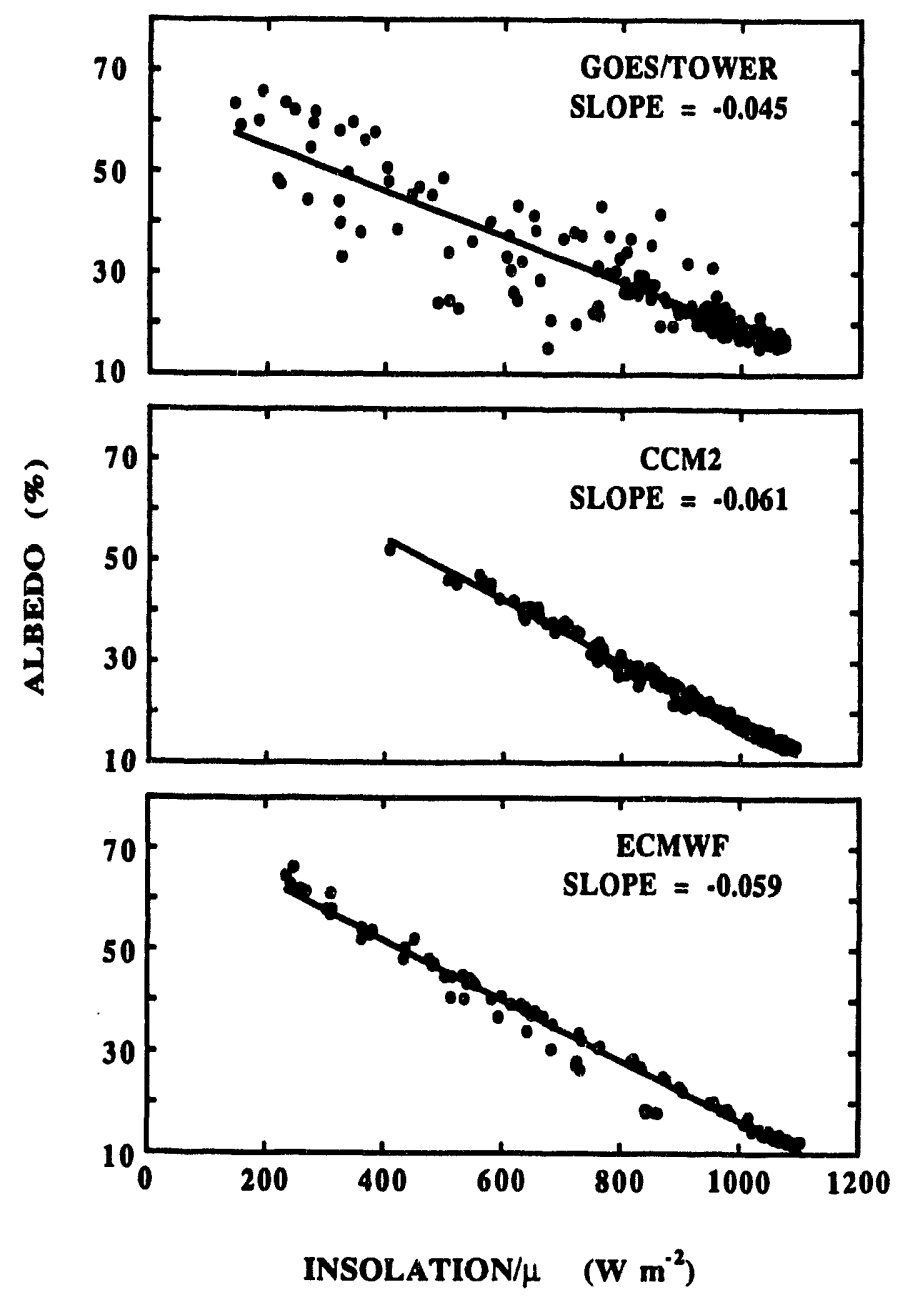

Figure 3. Top of the atmosphere albedo as a function of surface insolation divided by $\mu$. These refer to the combined GOES/tower data, CCM2 and the ECMWF GCM.

the GOES/surface data, both individually and collectively, serve as an extremely useful vehicle for testing cloud $\mathrm{SW}$ radiative interactions in GCMs.

The collocated GOES and BAO tower data serve as a useful prototype for demonstrating what can be done, and in an extended fashion, at ARM sites. The recent addition of Dr. Patrick Minnis to the ARM Program means that GOES measurements will be collocated with ARM sites. 


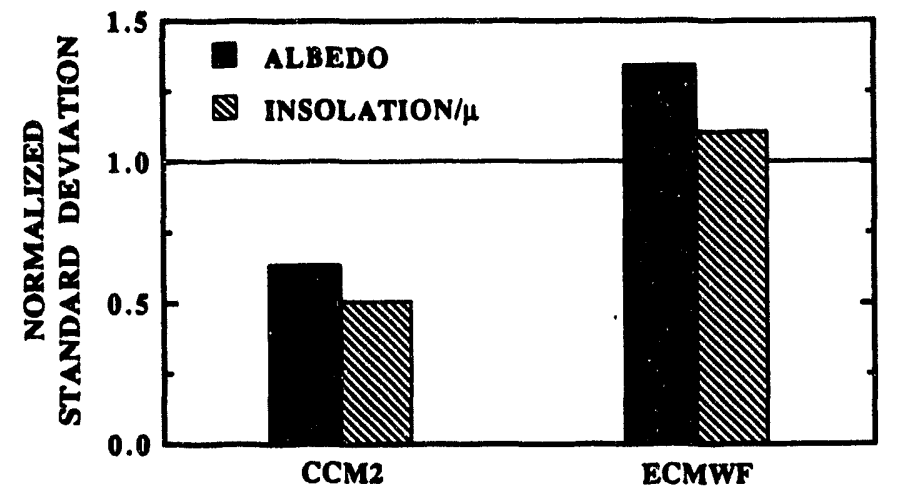

Figure 4. Standard deviation of albedo and insolation $/ \mu$ for CCM2 and the ECMWF GCM. These have been normalized by standard deviations from the GOES and tower measurements.

Because the ERBE scanners are no longer operative, their measurements currently cannot be used to calibrate/ unfilter the GOES measurements. Other options, however, are currently being investigated.
It is thus proposed to pursue the same research at ARM sites as they become operative. What will be advantageous here is that other data, for example cloud optical depth, will be available at these sites. These data will allow a much more comprehensive means of testing and interpreting GCMs than the current research has provided.

\section{References}

Cess, R. D. ot al. 1989. Interpretation of cloud-climate feedback as produced by 14 general circulation models. Science 245:513-516.

Cess, R.D., et al. 1990. Intercomparison and interpretation of climate feedback processes in 19 atmospheric general circulation models. J. Geophys. Res. 95:18,687-18,703.

Cess, R. D., E. G. Dutton, J. J. DeLuisi and F. Jeng. 1991. Determining surface solar absorption from broadband satellite measurements for clear skies: Comparison with surface measurements. J. Clim. 4:236-247. 


\title{
A Stratiform Cloud Parameterization for General Circulation Models
}

\author{
S. J. Ghan and L. R. Loung \\ Pacific Northwest Laboratory \\ Richland, WA 99352 \\ C. C. Chuang and J. E. Penner \\ Lawrence Livermore National Laboratory \\ Livermore, CA 94550 \\ J. McCaa \\ University of Washington \\ Seattle, Washington
}

The crude treatment of clouds in general circulation models (GCMs) is widely recognized as a major limitation in applying these models to predictions of global climate change. The purpose of this project is to develop in GCMs a stratiform cloud parameterization that expresses clouds in terms of bulk microphysical properties and their subgrid variability.

Figure 1 summarizes the various cloud variables and their interactions. Precipltating cloud species are distinguished from non-precipitating species, and the liquid phase is distinguished from the ice phase. The size of the nonprecipitating cloud particles (which influences both the cloud radiative properties and the conversion of nonprecipitating cloud species to precipitating species) is determined by predicting both the mass and number concentrations of each species.

\section{Cloud Microphysics}

The cumulus cloud modeling community has developed several bulk cloud microphysics parameterizations that could, in principle, be applied to stratiform clouds in GCMs. However, because the time step required by such parameterizations is typically 10 seconds, direct application of current cloud microphysics parameterizations to GCMs is computationally impractical.

We have introduced two approximations (Ghan and Easter 1992) that together permit a tenfold increase in the permissible time step of a bulk cloud microphysics parameterization originally developed for mesoscalecloud models (Tripoli and Cotton 1980, Cotton et al. 1982 and 1986, Meyers et al. 1992). These approximations are

- assume precipitating particles fall so fast that the tendency term can be neglected and the concentration diagnosed from the balance between the source/sink terms and the divergence of the fallout

- assume snow melts instantaneously after falling below the freezing level.

The errors resulting from the approximations and the increase in time step are typically $15 \%$ for column cloud water and cloud ice. The resulting increase in efficiency permits application of the bulk parameterization to stratiform clouds in a GCM without greatly increasing the computational demands of the model. For example, when applied to the Pacific Northwest Laboratory's (PNL) version of the National Center for Atmospheric Research (NCAR) Community Climate Model (CCM), the bulk cloud microphysics parameterization increases the computational time by about a factor of two.

The Colorado State University (CSU) bulk cloud microphysics parameterization offers most of the features of the desired treatment of cloud microphysics, but not all. In particular, droplet number concentration is prescribed rather than predicted in the CSU parameterization. To predict droplet numberconcentration, we are in the process of introducing the droplet number as a prognostic variable. 


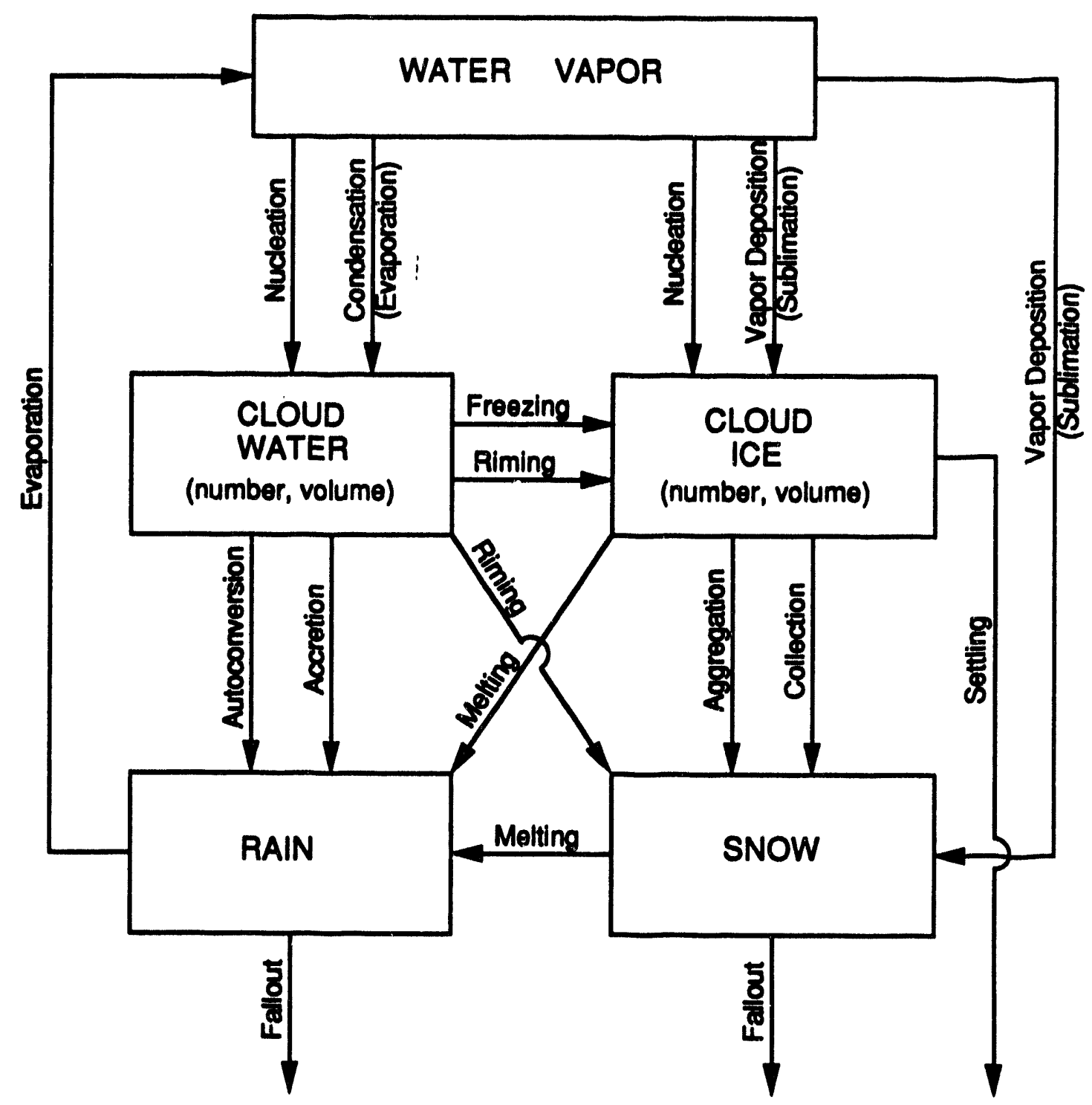

Figure 1. Cloud variables and microphysical processes represented in the stratiform cloud parameterization.

The sink terms in the droplet number balance follow from the sink terms in the cloud water mass concentration, assuming the sink processes affect the cloud water mass and number concentration, but not the average droplet mass. The droplet source reflects the nucleation of cloud droplets near the cloud boundaries and must be parameterized.
To parameterize the droplet nucleation process, we relate the number concentration of droplets nucleated, $N_{n}$, to the vertical velocity $w$ and the aerosol number concentration, $\mathrm{N}_{\mathrm{a}}$, according to the simple expression

$N_{n}=w N_{a} /\left(w+c N_{a}\right)$ 
where $c$ is a coefficient that depends on the temperature, preseure, aerosol composition, and the mode radlus and standard deviation of the aerosol size distribution (Ghan ot al., in press). The relationship shown in Equation (1) can be derlved analytically from a number of approximations, Including

- The aorosol size distribution is log-normal.

- Particle growth is due entirely to diffusion of water vapor.

- The droplet radius at maximum supersaturation can be approximated by the radius at the maximum of the Kohler curve for the aerosol.

The number nucleated according to Equation (1) has been compared with that simulated by a detalled size-resolving nucleation model (Edwards and Penner 1988) (se日 Figure 2). The number nucleated agrees to within $50 \%$ for vertical velocitles ranging from 1 to $500 \mathrm{~cm} / \mathrm{s}$ and aerosol number concentrations ranging from $501050 \mathrm{NO} / \mathrm{cm}^{3}$.

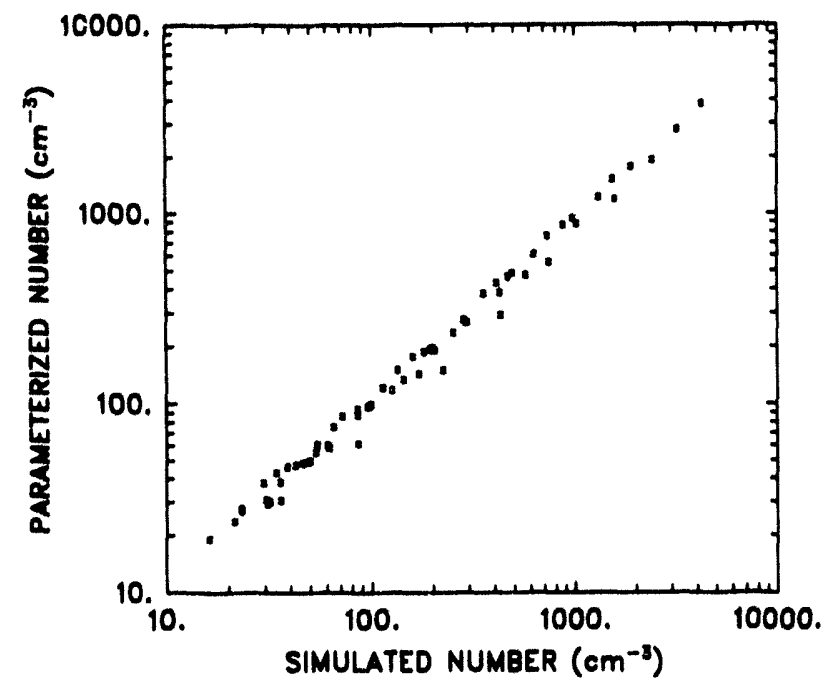

Figure2. Droplet number concentration as parameterized versus simulated by a detalled size-resolving droplet nucleation model, for vertical velocities ranging from 1 to $500 \mathrm{~cm} / 8$ and aerosol number concentrations ranging from 50 to $5000 / \mathrm{cm}^{3}$.

\section{Sub-Grid Cloud Parameterization}

Sub-grid scale variations in cloud microphysical processes must be accounted for in GCMs because cloud procesees are highly nonlinear and are poorly resolved by the coarse grid size of GCMs. We have initiated the development of a statistical formalism that expresses sub-grid scale variatlons in cloud microphysical properties in terms of idealized probability distributlons. Joint probability distrlbutions have beenintroduced to treat the dependence of many cloud microphysical processes on combinations of cloud variables. The Mathematica software is being used to analytically relate the parameters of joint probability distributions to the moments of the cloud variables.

\section{Column Cloud Model}

An essential task in developing cloud parameterization is its verification. Although some aspects of verification will be achieved using climatological simulations with a GCM, more control is possible by applying the cloud parameterization to forecast experiments in the fleld. This project will use the Atmospheric Radiation Measurement (ARM) Cloud and Radiation Testbed (CART) facilities to provide boundary conditions to drive a one-dimensional column cloud model and to provide cloud and radiation observations for model verification. The column model has been constructed and was used to develop the cloud microphysics parameterization. The same parameterizations of radiative transfer and cumulus convection used in the PNL version of the NCAR Community Climate Niodel (CCM1) have also been applied to the column model.

\section{Application to a GCM}

To apply the bulk cloud microphysics parameterization to a GCM, we have replaced the usual prognostic variables temperature $T$ and water vapor mixing ratio $r_{v}$ with the condensation-conserved varlables

$T_{\text {cld }}=T \cdot U c_{p} r_{c}$

and 
$r_{w}=P_{v}+r_{c}$

where $L$ is the latent heat of condensation.

Temperature, water vapor, and the cloud water mixing ratio $r_{c}$ can be dlagnosed from $T_{d c}$ and $r_{w}$ by assuming condensation instantaneously eliminates supersaturations with respect to liquid water. Advection of cloud water is implicitly treated in the advection of $T_{\text {ded }}$ and $r_{w}$, and therefore need not be treated explkitly, thus eliminating probleme associated with advecting a fleld with frequent zeroes. This treatment of cloud water and cloud microphysics has been applied to the PNL version of the NCAR CCM1. A semi-Lagranglan scheme is used to advect total water, cloud ice. loe number, and cloud droplet number.

We have performed two short simulations with the GCM, one with and the other without the prognostic droplet number. Figure 3 shows a scatterplot of instantaneous cloud water concentration versus temperature for the simulation with a fixed droplet number $\left(100 / \mathrm{cm}^{3}\right)$. At

FIXED DROPLET NUMBER

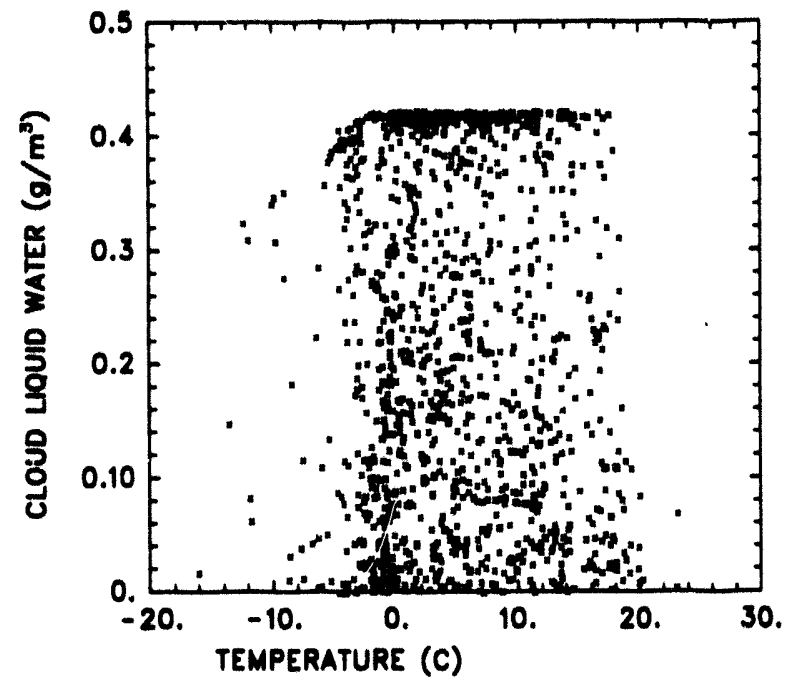

Flgure 3. Instantaneous cloud water concentration versus temperature simulated by the PNL version of the NCAR CCM1 with bulk cloud microphysics and droplet number fixed at $100 / \mathrm{cm}^{3}$. temperafures above freezing, a distinct threshold is evident, which reflects the autoconversion of cloud water to rain when the cloud droplet radius exceeds 10 microns. Some supercooled cloud droplets are present at temperatures as cold $a s \cdot 16^{\circ} \mathrm{C}$.

Figure 4 shows the same scatterplot as Figure 3, but for a simulation with predicted droplet number. The number nucleated at cloud base is prescribed at $100 / \mathrm{cm}^{3}$ rather than parameterized according to Equation (1) because sub-grid scale variations in vertical velocity have not yot been parameterized. The simulated droplet number concentration ranges from $100 / \mathrm{cm}^{3}$ for a new cloud to much smaller concentrations for old cloud layers. Consequently, the threshold radius for autoconversion does not translate to a single threshold liquid water concentration. Cloud water concentrations are generally lower than for the fixed droplet number because the simulated droplet number concentrations are generally lower than the fixed number concentration.

\section{PREDICTED DROPLET NUMBER}

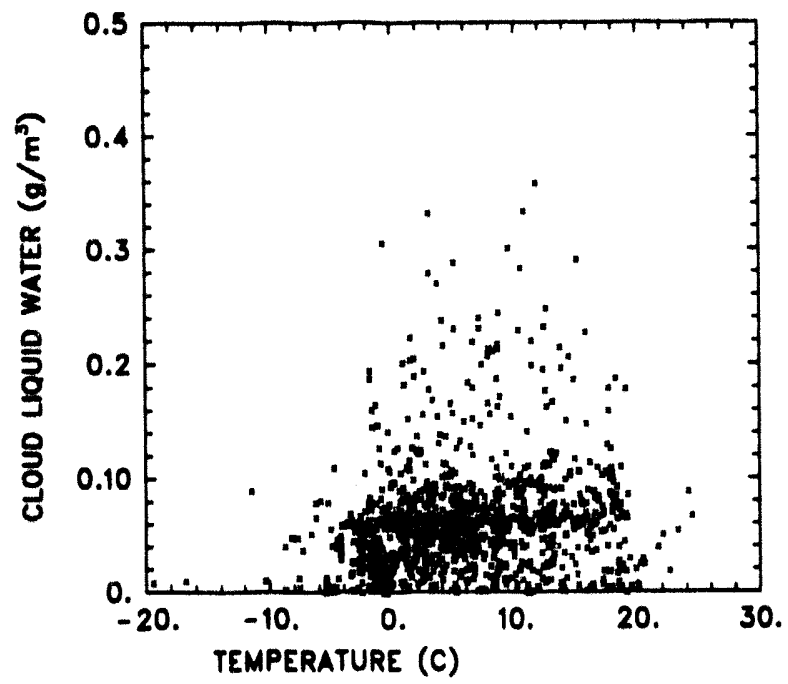

Figure 4. As in Figure 3, but for a simulation with predicted droplet number. 


\section{References}

Cotton, W. R., M. A. Stephens, T. Nehrkorn, and G. J. Tripoll. 1882. The Colorado State University ThreeDimensional Cloud/Mososcalo Model. Part ll: An lce Phase Parameterization. J. Rech. Atmos. 16:295-320.

Cotton, W. R., G. J. Tripoll, R. M. Raubor, and E. A. Mulvihill. 1986. Numerical Simulation of the Effects of Varying lce Crystal Nucleation Rates and Aggregation Proceseses on Orographic Snowtall. J. Clim. Appl. Moteorol. 25:1658-1680.

Edwards, L., and J. E. Penner. 1988. Potential Nucleation Scavenging of Smoke Particles Over Large Fires: A Parametric Study. Aorosols and Climate, ods.P. V. Hobbs and M.P. McCormick, pp. 423-434. A. Deepak Publishing, Hampton, Virginia.
Ghan, S. J., and R. C. Easter, 1992. Computationally Efficient Approximations to Stratiform Cloud Parameterization. Mon Wea Rev 120:1572-1582.

Ghan, S. J., C. C. Chuang, and J. E. Penner. In press. A Parameterization of Cloud Droplet Nucleation, Part I: Single Aerosol Type. Atmos. Res.

Moyers, M.P., P.J. DoMott, and W. R. Cotton. 1892. Now Primary los Nucleation Parameterizations in an Explicit Cloud Model. J. Appl. Moteorol. 31:708-721.

Tripoli, G. J., and W. R. Cotton. 1980. A Numerical Investigation of Several Factors Contributing to the Observed Variable Intensity of Deep Convection over South Florida. J. Appl. Moteorol. 19:1037-1063. 


\title{
Factors Influencing Reglonal-Scale Cloud Cover: Investigations Using Satellite-Derived Cloud Cover and Standard Meteorological Observations
}

\author{
C. J. Walcok \\ Almospheric Sclences Research Center \\ State University of New York \\ Albany, Now York
}

\section{Introduction}

Large-scale numerical models of the atmosphere approximate the heterogeneous or subgrid-scale nature of cloudiness by assuming that a fraction of each grid area is occupled by clouds. This cloud cover fraction is used to apportion cloud effects into a "grid-averaged" forcing within areas that contain a mixture of clear and cloudy regions. Most models of tropospheric dynamics assume that the fractional cloud coverage is determined by the gridaveraged relative humidity, stability, or resolvable-scalo vertical motions (e.g., Slingo 1980).

In this study, we investigate the relationship between cloud cover and other related meteorological factors by comparing observations of clouds and relative humidity, temperature lapse rates, wind shear, and large-scale vertical velocity within various tropospheric lovels.

\section{Cloud Cover Observations}

Cloud observations are derived from the U.S. Air Force operational real-time three-dimensional analysis of cloud cover (3DNEPH). The 3DNEPH is a global analysis of cloud cover that uses surface-based and aircraft reports, logether with visual and infrared satellite imagery, to produce 3-D cloud cover information every 3 hours at 15 vertical layers between the surface and $\sim 16 \mathrm{~km}$ above the surface. Horizontally, the grid size varies from $-25 \mathrm{~km}$ near the equator to $\sim 60 \mathrm{~km}$ at the poles.

In this study, we use five noon-time spring periods (20 to 24 April 1981) analyzed over the northeast United States by the 3DNEPH. During this 5-day period, a relatively intense midlatitude cyclone developed and traversed this domain, allowing one to investigate cloud cover under a wide variety of meteorological environments.

\section{Related Meteorology}

Temperature and molsture observations used in this analysis are taken from the National Meteorological Center global analysis and spatially and temporally interpolated onto an $(80 \mathrm{~km})^{2}$ grid using a hydrostatic mesoscale meteorology model (MM4). During model execution, observations are incorporated into the model calculations in regions near the observation locations. Differences between observed and calculated temperatures, humidities and wind speeds are continuously minimized by "nudging" the calculations towards the observations. In this manner, model calculations agree closely with observations when and where observations are available, and when no observations are available, the meteorological data are dynamically consistent.

\section{Comparisons}

Figure 1 shows contours of the average 3DNEPH cloud cover at 15 tropospheric levels and at various relative humidities. Typically, 1000 to $5000(320 \mathrm{~km})^{2}$ observation pairs were available at each layer, representing cloud cover under cold and warm maritime and continental air masses during this period. The cloud observations are averaged with in $5 \%$ relative humidity increments at each lovel. 


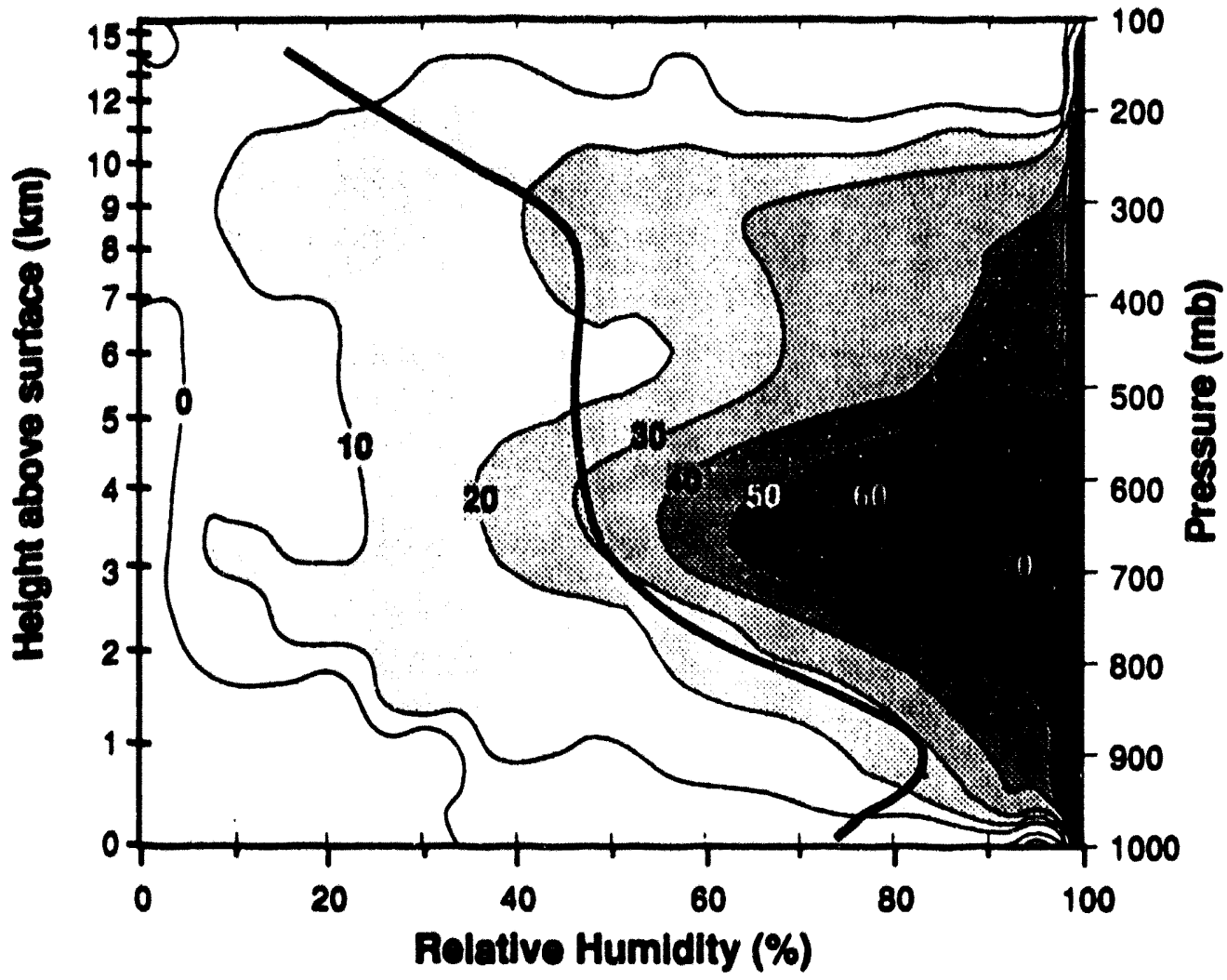

Figure 1. Fractlonal cloud coverage versus relatlve humidity and pressure over the northeast United States during 20 to 24 April 1981.

As expected, cloud amount increases as humidity increases. At a particular relative humidity, cloud amounts are greatest in the $800.10600 \cdot \mathrm{mb}$ layer, a trend that is consistent with earlier approximations (Buriez et al. 1988). The highest cloud amounts occur under high humidities at 800 to $800 \mathrm{mb}$. This foure shows, however, that $10 \%$ to $20 \%$ cloud coverage occurs at humiditios as low as $15 \%$, in contrast to many formulations, which all set zero cloud cover at humidities bolow $50 \% 1080 \%$.

These results suggest that fractional area of cloud coverage decreases exponentially as relative humidity falls below $100 \%$ and that there is no clear "critical relative humidity" where cloud coverage is always zero. We suggest the following approximation for cloud amount $f$ as a function of relative humidity $R h(R h<1)$ and vertical volocity:

$1=\exp \left\{\frac{R h \cdot 1}{\alpha+0.10^{2} w}\right\}$ where $\alpha$ is a function of height in the troposphere and represents the relative humidity depression from $100 \%$ at which cloud amount falls off to $37 \%\left(e^{-1}\right)$ :

$\alpha=\left\{\begin{array}{l}0.2+\sigma / 3 \sigma<0.75 \\ 1.8(1-\sigma) \sigma \geq 0.75\end{array}\right.$

In Equations (1) and (2), $\sigma$ is the pressure relative to surface pressure. Using Equations (1) and (2) to calculate cloud cover from $R h$ and $w$ (averaged over $[320 \mathrm{~km}]^{2}$ areas) produces cloud cover estimates that differ by 10 to 30 percentage points from the 3DNEPH observations.

Based on climate model sensitivity studies, Slingo (1990) estimates that a $15 \%$ change in low cloud cover could potentially counter a double $-\mathrm{CO}_{2}$ warming. If cloud cover changes in response to a change in relative humidity, then there is an important foedback between changing relative humidity and changing cloud cover. 
The dark curve on Figure 1 shows the relative humidity averaged during the observation period considered in this study. The vertical distribution of relative humidity agrees closely with the global mean relative humidity according to various analyees and the NCAR climate model. The averaged cloud cover at any vertical layer can be very accurately calculated from the average relative humidity of the layer simply by choosing the cloud cover corresponding to the mean relative humidity shown in Figure 1. If relative humidity changes ellghtly in a future climate, then the cloud cover change associated with that changing humidity can be approximated by looking at the sensitivity of cloud cover to relative humidity in the present climate.

Figure 2 shows the change in relative humidity that is correlated with a $15 \%$ change in the mean cloud cover. This figure shows that near the middle and top of the planetary boundary layer $(950-900 \mathrm{mb})$, changes in the relative humidity of less than $2 \%$ are correlated with $15 \%$ changes in cloud cover.

\section{Conclusions}

We find that an increase in relative humidity of $\sim 2 \%$ at 950 to $900 \mathrm{mb}$ could lead to a $15 \%$ increase in cloud cover at these layers. Thus, small changes in relative humidity could counter a $\mathrm{CO}_{2}$-induced global warming. These conclusions are based on our comparisons of satellite observations of fractional cloud coverage with collocated related meteorological parameters over the northeast United States.

We find significant correlations between cloud cover and relative humidity and vertical velocity. These comparisons suggest that cloud coverage decreases exponentially as humidity falls below $100 \%$. Relative to other layers in the troposphere, the middle troposphere contains higher cloud amounts at lower humidities. Most parameterizations of cloud coverage calculate smaller cloud amounts than reported by the 3DNEPH observations, especially in the middle troposphere. Furthermore, all cloud cover

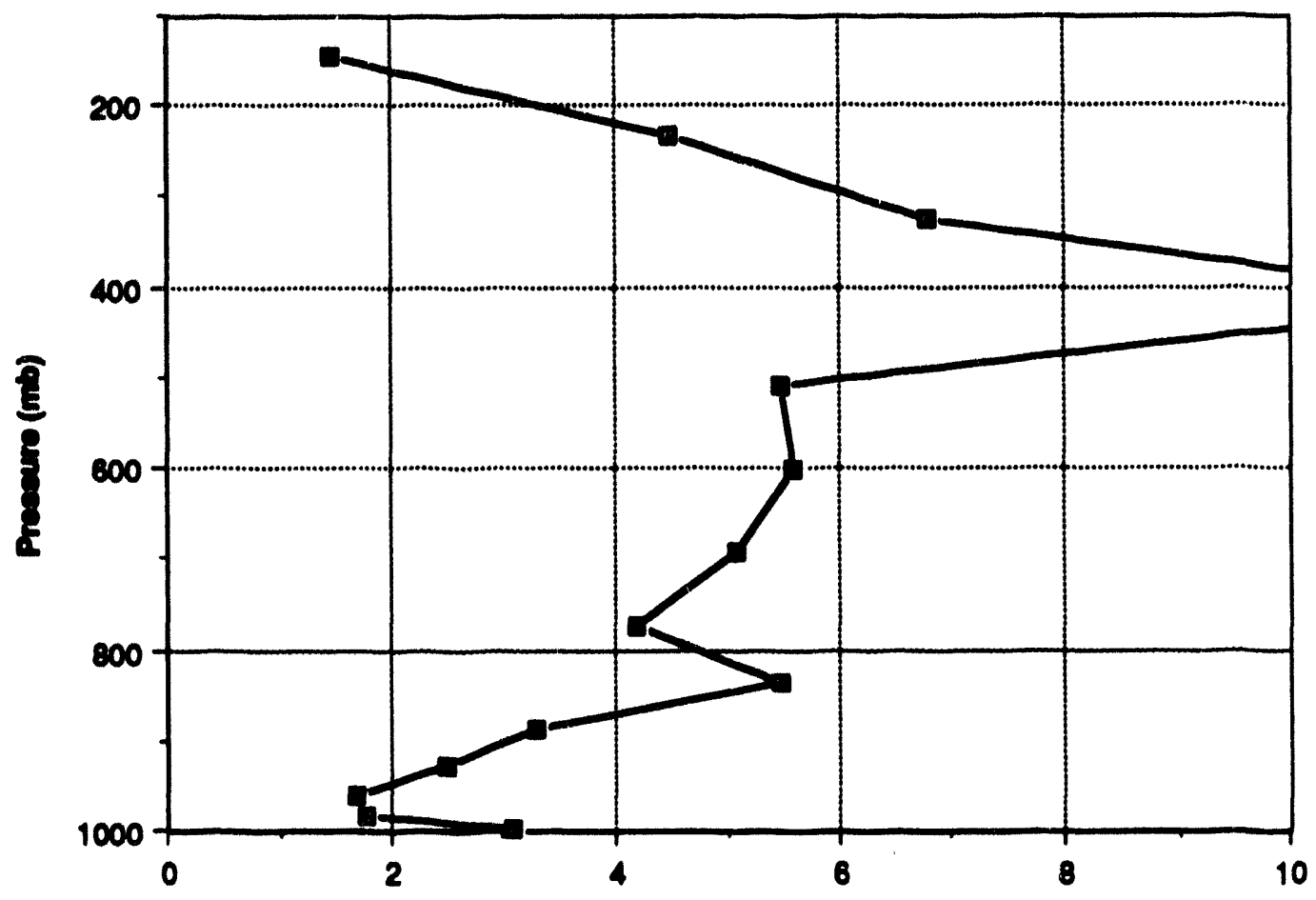

Abeoluto change in relative humldity to change eloud cover by $15 \%$

Flgure 2. Sensitivity of cloud cover to changes in the relative humidity according to the trends reported in Figure 1. This figure shows the change in relative humidity that correlales with a $15 \%$ change in cloud cover. 
parameterizations assume that cloud amount is always zero bolow a "critical" relative humidity, an assumption that is not discernible from this analysis.

These results suggest that current methods of calculating cloud cover within large-scale climate simulations or atmospheric chemical modeling studies are significantly underestimating the effects of clouds. More importantly, current climate models probably cannot adequately estimato the potentially significant changes in cloud cover that can result from small changes in relative humidity under dry conditions.

\section{Acknowledgments}

The author is grateful to the U.S. Department of Energy for supporting the research presented here.

\section{References}

Buriez, J.-C., B. Bonnel, Y. Fouquart, J.-F. Geleyn, and J.-J. Morcrette. 1988. Comparison model-generated and satellite-derived cloud cover and radiation budget. J. Geophys. Res. 93:3705-3719.

Slingo, J. M. 1980. A cloud parameterization scheme derived from GATE data for use with a numerical model. Quart. J. Roy. Mot. Soc. 106:747-770.

Slingo, A. 1990. Sensitivity of the earth's radiation budget to changes in low cloud amount. Nature 343:49-51. 


\title{
Single-Column Sub-Grid Cumulus Model for the Atmospheric Radiation Measurement Program
}

\author{
R. B. Stull \\ University of Wisconsin \\ Madison, WI 53706
}

\section{Heterogeneous Surface Forcings}

Variations in surface albedo, moisture, soll type, vegetation coverage, and other factors cause surface-layer air (air within $50 \mathrm{~m}$ of surface) to be horizontally heterogeneous over land surfaces. This heterogeneity causes patchiness in boundary layer cloud coverage because clouds are formed in thermals of rising surface-layer air.

For climate models, grid cell dimensions are so large that many of these variations are subgrid scale. However, the effect of these variations can be captured via their statistical distributions within each grid cell, and parameters describing these distributions can be used to forecast subgrid scale boundary-layer clouds.

Two phases make up this research: calibration and modeling. First, we use high-resolution turbulence data measured by aircraft over heterogeneous regions to determine the nature of the actual distributions as a function of surface characteristics and solar and atmospheric forcings. Second, the resulting distributions are parameterized using similarity theory and applied in simplified form to forecast cloud coverage and cloud characteristics over other surfaces around the world where no aircraft measurements are feasible.

Prior to the Atmospheric Radiation MeasuremenvCloud and Radiation Testbed (ARM/CART) sites coming on line, we have been using existing data from the Hydrologic Atmospheric Pilot Experiment (HAPEX) fleld experiment in southwest France to develop techniques to determine the statistical distributions. These methods are now refined enough to apply to the preliminary ARMUCART data, such as the data gathered by Steve Oncley from the National Center for Atmospheric Research (NCAR) during his calibration flights in the fall of 1992. We understand that
Oncley's data will become available during March 1993. Data from other research flights over the ARMVCART sites will be used as they become avallable.

\section{Calibration of Subgrid Statistics}

During the HAPEX experiment, one frequently flown flight track was an S-shaped pattern, shown in Figure 1. The track passed over 12 different regions of land use, which can be grouped into five broad classes. From 8 May through 24 July 1986, the NCAR King Air aircraft flow the

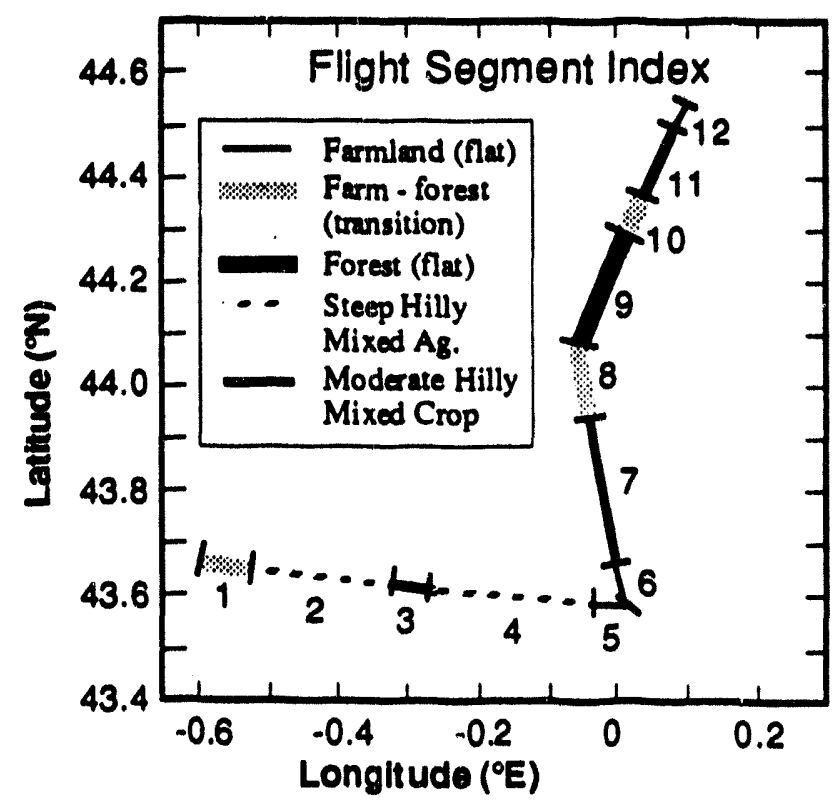

Figure 1. Land usage under HAPEX flight track. 
S-track on 21 days, three times a day. Most of these S-tracks were flown at an altitude of 50 to $100 \mathrm{~m}$ above ground level.

While the scientists' logs indicated approximate starting and ending times for each flight leg, the aircraft was usually not fully stabilized during portions of the beginning and end of each log after or prior to turns or changes in altitude. Detailed post-flight data on aircraft pitch, yaw, roll, altitude, heading, and speed have been analyzed to determine flight stability. The erratic portions of the beginning and ends of flight legs were discarded where necessary.

Although the flight legs were flown over fixed visual landmarks, the recorded data contained navigation errors associated with drift in the aircraft inertial-navigation system. Such navigation errors did not accumulate uniformly during any one flight; hence, end-of-flight measurements of location error were inadequate to make corrections to the mid-flight data. Instead, offseterrors in latitude and longitude were estimated by eye, separately for each full S-track.

The navigationally corrected portions of stabilized flight observations were then split into segments corresponding to the 12 land-use regions. For each segment, the $20-\mathrm{Hz}$ temperature, pressure, and humidity data were analyzed to compute $20 \cdot \mathrm{Hz}$ values of virtual potential temperature, convective available potential energy, and lifting condensation level (LCL). Each data point thus represents an average over roughly $5 \mathrm{~m}$ of air.

Joint frequency distributions of virtual potential temperature versus LCL were computed separately for each segment. As an example, consider the data from segment 6 of the first S-track of Flight 1, flown on 9 May 1986, which is plotted in Figure 2 as a joint relative-frequency distribution (i.e., sum over all bins equals one).

The distribution for any one segment can be modeled with a joint distribution function. At present, we are testing truncated Gaussian distributions, such as the one plotted in Figure 3. Major and minor axes for these distributions are based on the theoretical variation of surface layer air over surfaces with varied Bowen ratio and insolation.

The location and distribution parameters of these distributions are analyzed using maximum likelihood methods. Location parameters (mean LCL and mean potential temperature) are then corrected for nonstationarity, because boundary level temperature and humidity evolve with time during the duration of any one flight track.

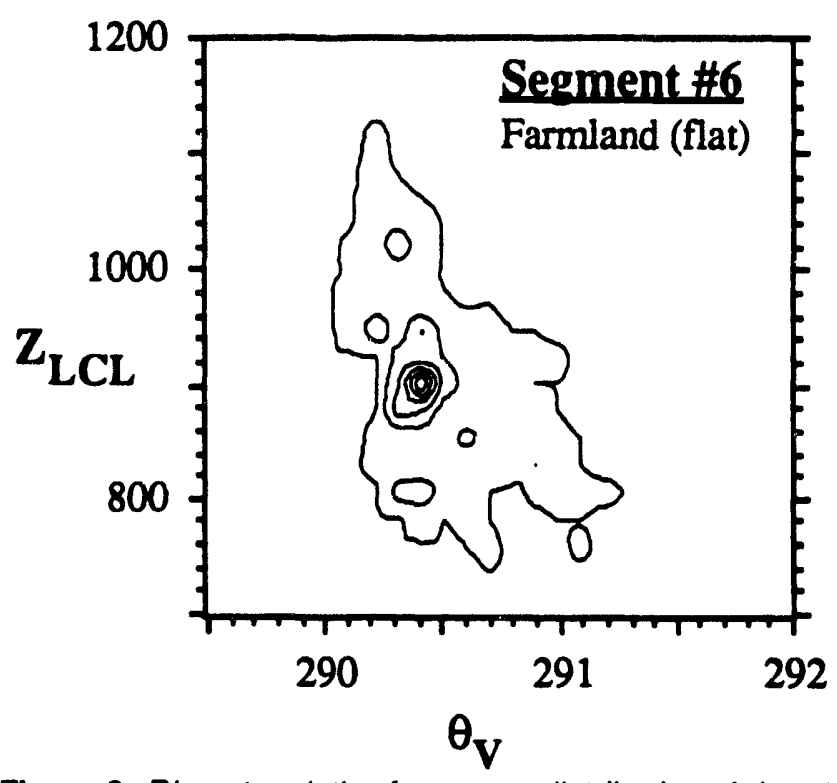

Figure 2. Discrete relative frequency distribution of virtual temperature, $\theta_{y}$, and height of the lifting condensation level, $\mathbf{Z}_{L C L}$, as observed in surface-layer air during segment 6, track 1 , flight 1 of HAPEX. Tic marks indicate the sorting bin size. Contours drawn every 0.02 , except first contour at 0.001 ; $\max$. relative freq. $=0.12$.

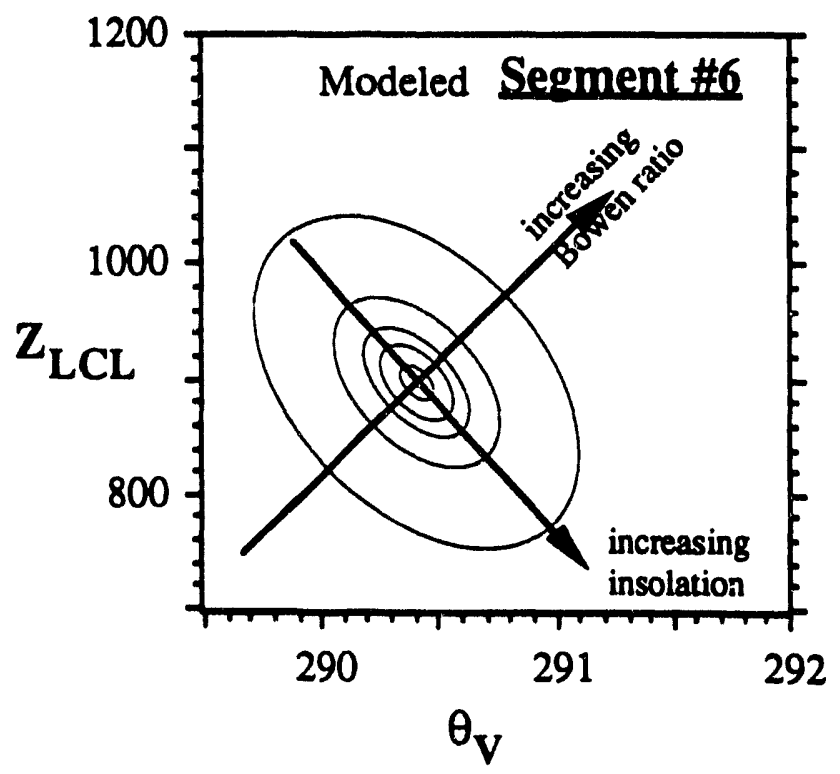

Figure 3. Truncated Gaussian distribution that models the joint distribution data from Figure 2. 
The parameters yielding the best-fit distributions are expected to differ from segment to segment. We will normalize these parameters with respect to boundary layer scaling variables to give dimensionless groups. These dimensionless groups will then be organized by broad land-use class. Within any one land-use class, we expect the dimensionless parameters to change gradually during the modeled months as crops mature and are harvested and expect shorter-term variations associated with rain events. If we can observe these changes, we will include them in the parameterization of the distribution functions.

Any single column of a climate model can contain a wide range of land-use regions. A land-use inventory will allow us to construct a modeled joint frequency distribution that is a composite built from weighted sums of the many individual (single-segment) distributions. This is illustrated in Figure 4, where only a few individual distributions are combined to yield a synthetic grid-average distribution.

\section{Phenomenological Processes}

Only the portion of surface-layer air that is positively buoyant will rise as convective thermals. Most of this air remains undiluted during its rise to the top of the mixed layer. Such a thermal is a physical phenomenon with known characteristics that can bo employed in a cloud model.

Each thermal has a buoyancy based on its surface-layer virtual potential temperature, and thus will rise to a final equilibrium height that depends on the environmental sounding. Also, each thermal will have a computable lifting condensationlevel, based on its surface-layer temperature and humidity.

If the height of a thermal is greater than its lifting condensation level, then it will form a cloud. The fraction of area covered by thermals that reach their LCL equals the fractional cloud coverage.

This process can be graphically represented by plotting the joint frequency distribution of surface-layer virtual potential temperature versus $z_{\mathrm{LCL}}$ (see above), and superimposing on it a graph of environmental sounding virtual potential temperature versus z. For example, a point within the frequency distribution that is to the right of

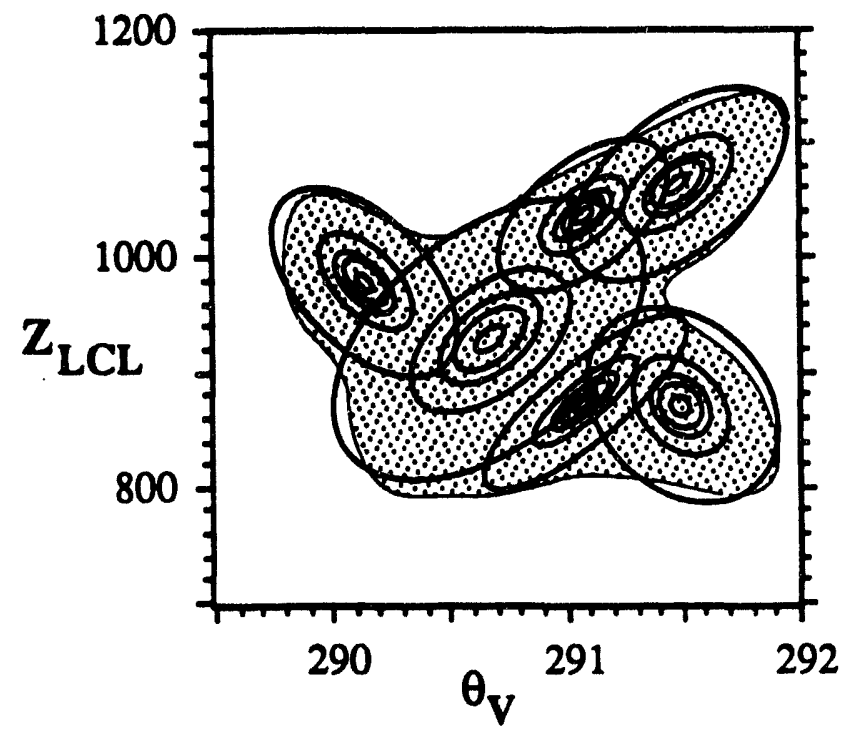

Figure 4. Illustration of a synthetic single-column joint frequency distribution (shaded) of $\theta_{v}$ and $z_{L C L}$, formed as a composite of many simpler (Figure 3 type) distributions.

the sounding represents those air parcels with $\mathrm{z}_{\mathrm{LCL}}$ lower than the top of the thermal, assuming that an air parcel will conserve its virtual potential temperature as it rises until it hits the sounding. This is demonstrated in Figure 5.

In otherwords, the integral over that portion of the frequency distribution that is to the right of the sounding gives the fraction of sky covered by clouds, assuming that the distribution was previously normalized such that an integral over the whole function equals one.

\section{Forecast Model}

To implement this scheme in a single column of a climate model, one must forecast the evolution of both the environmental sounding of Figure 5 and the location of the frequency distribution. The former is easy using a simple slab model of the mixed layer. Such a model forecasts the vertically unresolved mixed-layer top, $z_{1}$, and average mixed-layer temperature $\theta_{M L}$ and humidity $q_{M L}$. The latter is easily forecast by using bulk-transfer relationships to estimate the amount of excess temperature and humidity in the surface layer compared with the mixed layer. 


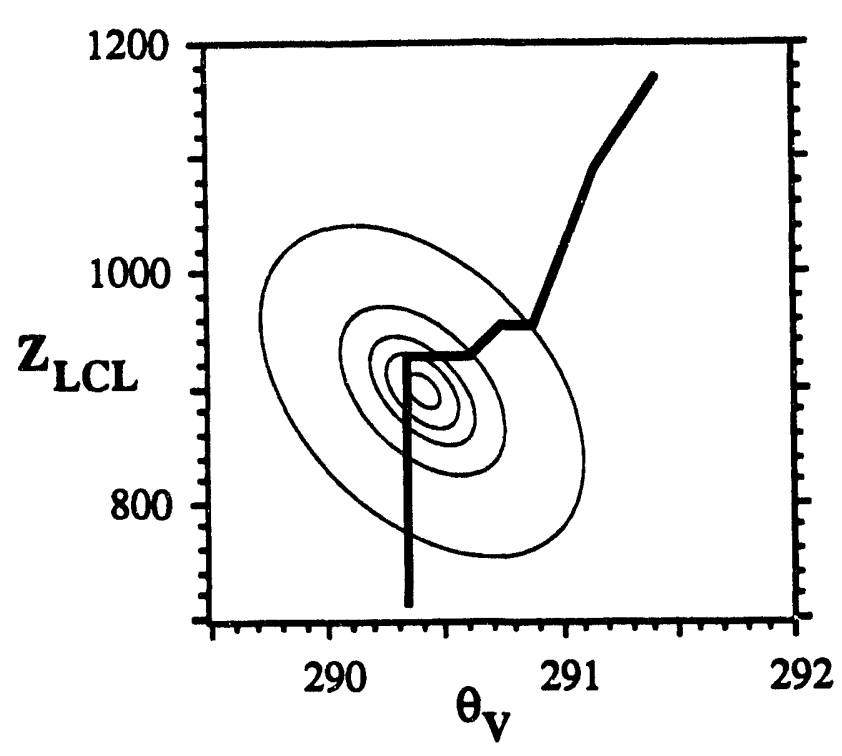

Figure5. Contours represent values of constant frequency distribution of virtual potential temperature $\theta_{v}$ versus $z_{L C L}$ for surface-layer air. Superimposed is a plot of the environmental sounding of $\theta_{v}$ versus height $z$.

Temperature and humidity, $\theta(z)$ and $q(z)$, are known (resolved) in a single-column model in the early morning. This provides data for the initial sounding. Imposed on the boundary layer are surface values of kinematic heat $H$ and moisture Efluxes. Let the mean surface layer (SL) conditions give the location for the frequency distribution. The forecast equations are

$$
\begin{aligned}
& w_{\theta}=0.2 \cdot H\left[\theta\left(z_{1}\right)-\theta_{M L}\right] \\
& d z_{i} / d t=w_{0} \\
& d \theta_{M L} / d t=1.2 \cdot H / z_{i} \\
& d q_{M L} / d t=\left\{E-w_{\theta} \cdot\left[q\left(z_{i}\right) \cdot q_{M L}\right]\right\} / z_{i}
\end{aligned}
$$

$\omega^{*}=\left\{\left(g / \theta_{M L}\right) \cdot z_{i} \cdot\left[H \cdot\left(1+0.61 q_{M L}\right)+0.61 \cdot \theta_{M L} \cdot E\right]\right\}^{1 / 3}$

$\theta_{S L}=\theta_{M L}+H /\left(0.0063 \cdot w^{*}\right)$

$q_{S L}+q_{M L}+E /\left(0.0063 \cdot w^{*}\right)$

As illustration, consider an initial state similar to Figure 5, except that the whole frequency distribution is completely above the sounding. Namely, the surface layer is sufficiently $d r y$ that the lifting condensation levels for all surface-layer air parcels are well above the top of the early morning mixed layer. Thus, there would be no clouds. Later in the day as the mixed layer grows and the LCL distribution moves, a portion of the distribution might be below the sounding, giving a nonzero cloud-cover forecast.

This procedure, except using the composite distribution as in Figure 4, will be used in the single-column model to make the cloud cover forecasts for forced clouds. Separate equations would be added to model the transformation of forced clouds into active and passive ones. The total boundary-layer cloud cover is the sum of these dynamic cloud types.

\section{Summary}

The approach reported here uses subgrid statistics together with phenomenological characteristics of thermals to produce a diagnosis of cloud cover and other characteristics. Hence the name "stochastic-phenomenological" parameterization. When this parameterization is combined with a forecast model of the boundary layer, the result is a computationally efficient yet physically realistic parameterization for subgrid boundary-layer clouds within any single column of a climate-model. 


\section{Four-Dimensional Data Assimilation}




\title{
Single-Column Data Assimilation for the Atmospheric Radiation Measurement (ARM) Program
}

\author{
J.-F. Louis \\ Almospheric and Environmental Research, Inc. \\ Cambridge, MA 02139
}

\section{Why assimilate ARM data?}

The main purpose of the ARM program is to provide the necessary data to develop. test and validate the parameterization of clouds and of their interactions with the radiation field, and the computation of radiative transfer in climate models. For the most part, however, the ARM observations will be imperfect, incomplete, redundant, indirect, and unrepresentative. This is unavoidable, despite the best efforts at equipping the Cloud and Radiation Testbed (CART) site with the best instruments. To understand these limitations, we must consider the structure of a climate model and the observation constraints.

The basic prognostic variables of any climate model are atmospheric temperature, horizontal wind components and humidity, and some surface variables: surface pressure, snow amount, soil temperature, etc. These variables are defined at a set of grid points (or, equivalently, as a set of spectral coefficients) at a small number of vertical levels. The grid boxes generally are a few hundred kilometers on the side, and hundreds to thousands of meters thick. The radiation scheme and cloud parameterization therefore use as input quantities averaged over the model grid box, and produce average fluxes.

By contrast, most of the ARM observations at individual sites will be obtained in a relatively small area compared to the scale of one grid box of a climate model. This is certainly true of radiosonde data, which sample extremely small volumes, both in time and space, but it also applies to most other observation techniques. ARM observations will thus be unrepresentative, meaning that they will be affected by scales of motion that do not exist in climate models. This question of scales and representativity of the data may be particularly serious for the radiative processes, which are highly nonlinear.
Besides fairly infrequent radiosondes, in situ observations of the atmosphere over the ARM sites will not normally be available because of cost considerations. Instead, the ARM plan calls mainly for remote sensing instrumentation. This means that we are not measuring temperature or moisture directly, but indirectly through their effect on electromagnetic radiation.

Although different instrurnents are available and will be used to measure the basic variables of climate modelstemperature, humidity and winds-these measurements are not sufficient to completely validate cloud parameterizations or radiation schemes. Many quantities that would be needed for this purpose will be unavallable, except possibly during campaigns or special observing periods. Vertical profiles of radiative fluxes, cloud droplet distribution, aerosol distribution, turbulent fluxes within clouds, or optical properties of ice crystals are but a fow examples. In many respects, the data will thus be incomplete, but, on the other hand, some quantities will be measured or inferred by several different instruments or methods, with different error characteristics.

We need a way to reconcile conflicting observations. This problem can be illustrated by comparing temperature observations made with two different radiosondes (Figure 1). One is the ARM data on 29 October and the other one the Oklahoma City (OKC) sounding of the National Weather Service (NWS) 4 hours later. One obvious difference is the resolution. In the NWS sounding we only have the data at the mandatory levels. The questions are "Which profile is more appropriate for a climate model?" and "Can the two sets of data be reconciled?"

Finally, measurements are obviously not perfect. Instrument errors can be minimized but not entirely avoided. In addition, the effects (on the measurements) of the scales that are 


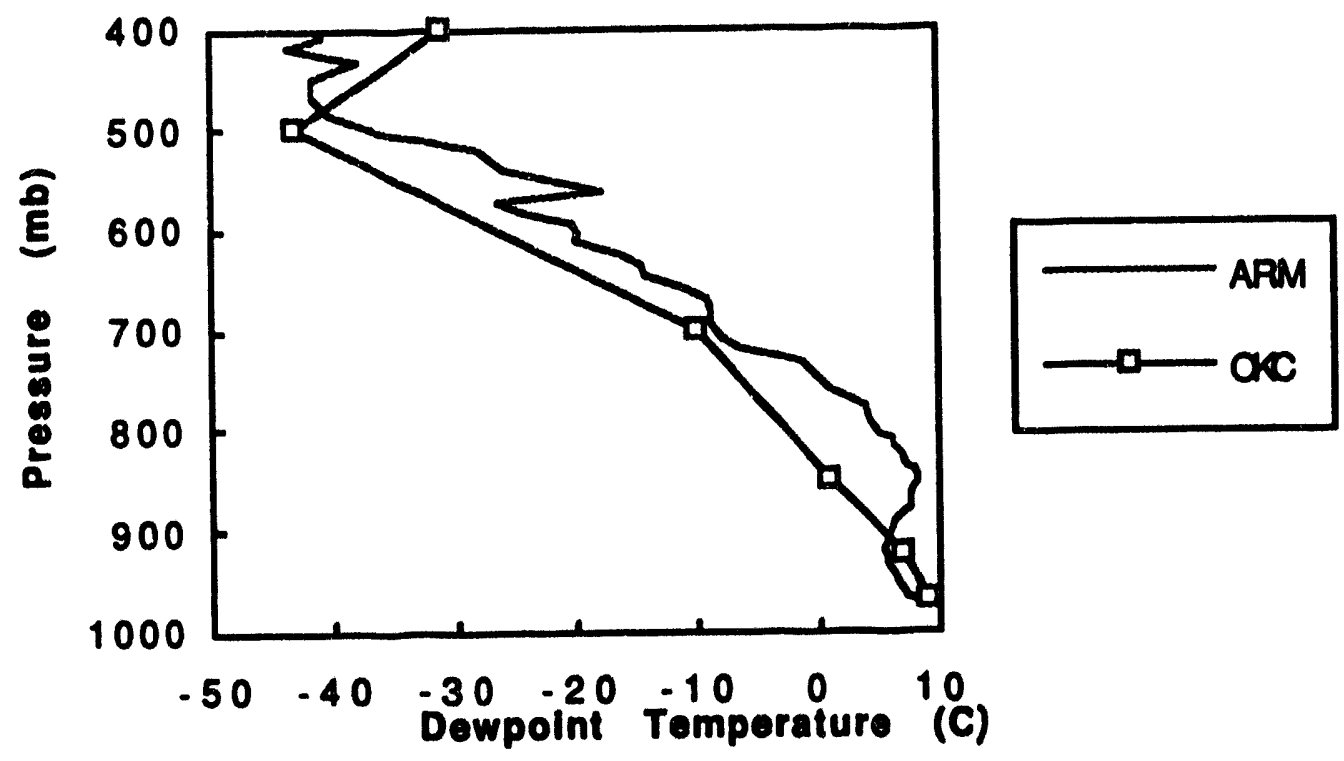

Flgure 1. Dewpoint temperature profile at Oklahoma City (OKC) and at the ARM site, 4 hours apart.

not represented by the climate models cannot be distinguished easily from actual observation errors, except that they may be spatially correlated. Furthermore, when remote sensing is used, the retrieval methods introduce errors which cannotbe avoided. In addition, remole sensing measurements may have exotic error characteristics.

Inaccuracy, incompleteness, redundancy, unrepresentativeness, and indirect measurements are all problems with which the weather forecasting community has been struggling foryears. Various techniques of data assimilation have been developed to deal with them. Common to all the modern techniques of data assimilation is the idea that all the information available about the atmosphere should be used, and that includes not only the observations, but also our knowledge of the physical processes, which can be expressed in a model. The model is used to ensure time continuity and to constrain the analysis to be consistent with the physics of the atmosphere and representative of the desired scales. Estimates of the expected errors of the various data sources can also be used to combine the observations and the model estimate in an optimal way. Finally, the model can provide estimates of quantities that are not observed. While these estimates are not necessarily "the truth," they are compatible both with the other observations and with the model constraints.

The most advanced methods of data assimilation make use of the variational principle, which consists of adjusting some parameters of the model to minimize the difference between the observations and the model simulation. One of the major advantages of this method is that it is quite easy to include indirect measurements, as long as the quantity measured can be simulated by the model. For example, it is not necessary to use a retrieval method to turn the radiances of remotely sensed observations into temperature profiles. The radiances can be used directly because the model can estimate the radiances corresponding to its own temperalure profile. Surface fluxes, precipitation, or cloudiness observations can also be used.

The variational method can be expensive because it requires an iterative minimum search, which involves running the model many times. In the ARM context, though, we are interested in data assimilation at a single 
site. Therefore, we can use a single-column model, which makes the variational method quite feasible, oven with modest computer resources.

Our purpose is twofold: to explore the variational data assimilation tochnique, which has not yet been used in an operational context, and to provide to the ARM community a tool to turn the observations into the measurements that are needed to develop and validate climate model components.

\section{Varlational Data Assimilation}

I introduced the concept of variational data assimilation and adjoint method at the 1992 Science Team meeting. I summarize it here briefly.

A model is used to simulate the evolution of the atmosphere during an assimilation period. The assimilation period should be long enough to include enough observations to constrain the model, but short enough that the evolution of a small perturbation can be described by the linear tangent model (LTM) ${ }^{(a)}$. A period of 12 to 24 hours seems to be reasonable.

As the model is run, we compute a cost function, which is essentially a measure of simulation errors. Typically, it is the weighted sum of the squared differences between observations and model output, the weights being the inverse of the expected errors. Other constraints can be introduced in the cost function as will be seen later.

The method then consists in adjusting some model parameters (called control variables) to minimize the cost function. At the minimum, the model simulation becomes the "analysis." We have different choices of control variables. They could be model physical parameters that are not well known, the initial state of the simulation, some nudging terms in the model equations that account for the deficiencies of the model, or a combination of all of these. We have been experimenting with the different possibilities.

Initially we will concentrate on the model's physical parameters in order to find the optimum set of parameters

(a) The linear tangent model is the forecast model linearized around the actual trajectory followed by the forecast model in the phase space. for the CART site. Once these parameters are set, we tend to prefer using nudging terms as control variables. Derber (1989) found that this resulted in a better analysis; it also has the advantage of resulting in a continuous description of the atmospheric state, without jumps at the beginning of each assimilation period.

In the minimization process, we need to compute the gradient of the cost function with respect to the control variables. This is used to determine the direction and size of the step in the minimum search. When the model is nonlinear, the gradient cannot be written analytically, but it can be computed by integrating the adjoint model, which, in a discrele model, is the transpose of the linear tangent model (Hoffman ot al. 1992).

\section{The ALFA model}

The model used in this work is an extension of the AER Local Forecast and Assimilation (ALFA) model, which we started developing at Atmospheric and Environmental Research (AER) with the goal of doing local forecasting (Louis et al. 1990). It is a single column model that computes all the physics in the ground and atmosphere and takes the horizontal derivatives needed for the advection terms from a large scale forecast (or analysis). In forecasting mode, the model would be used to compute how the local conditions modulate the large scale flow predicted by a global or regional model. The adjoint technique is used to optimize the model parameters.

Much of the work in the first 2 years of our ARM contract has been to incorporate a sophisticated radiation scheme into the ALFA model. The scheme chosen is the one developed by Toon et al. (1989). It makes use of a generalized two-stream approximation and is designed for vertically inhomogeneous, multiple-scattering atmospheres. We have modified it to allow for fractional cloudiness and have written its adjoint. We have also written the adjoint of the convection scheme (Anthes et al. 1982) and the stratiform precipitation scheme.

An example of the results obtained with the Toon scheme is given in Figures 2 and 3, which show the computed solar and downward IR fluxes for 8-9 July 1992 at Oklahoma City. During, the day, the model computes about $50 \%$ cloudiness; at the end of the second night, it computes $100 \%$ of low clouds. 


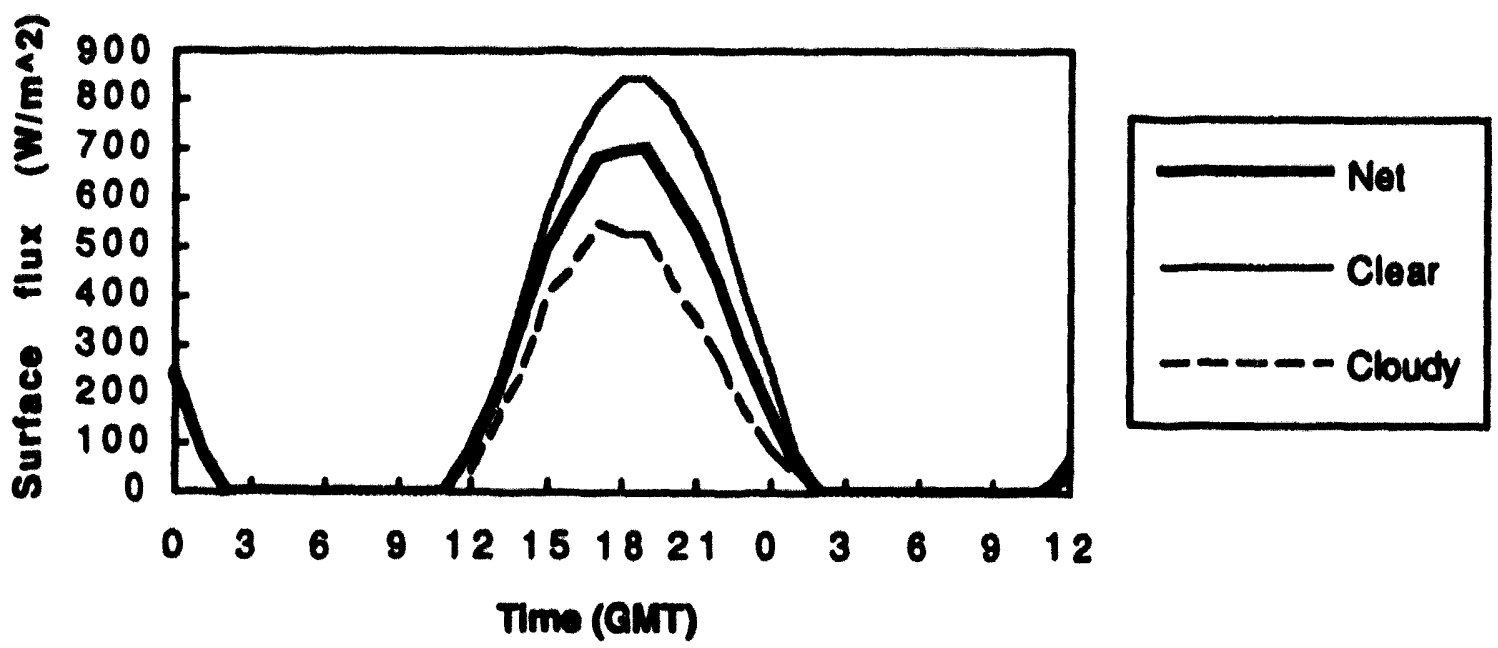

Figure 2. Computed solar fluxes for the OKC stalion, 8-9 July 1992. Clear and cloudy computations are shown separately.
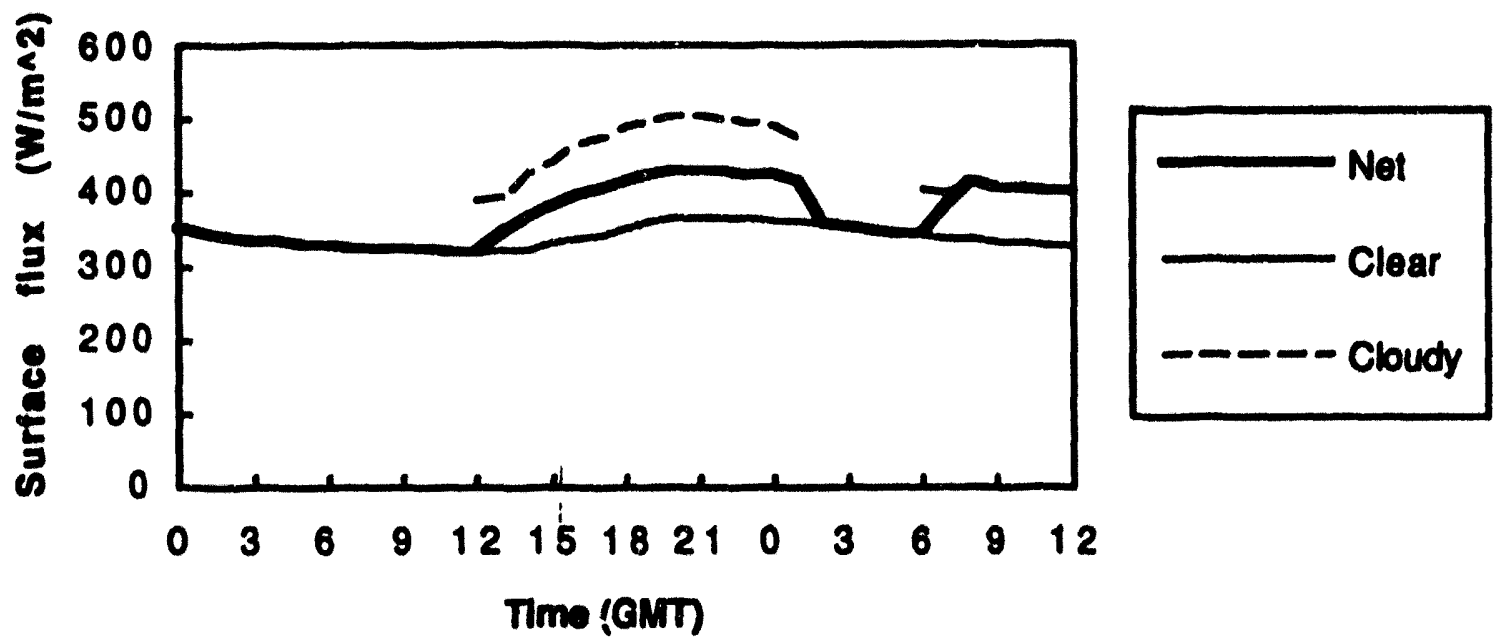

Flgure 3. Computed downward infrared fluxes for the OKC station, 8-9 July 1992. Clear and cloudy computations are shown separately. 
Note that this kind of oulput can be compared to radiometric measurements and can therofore be used in computing the cost function in data aseimilation. That is something that no other data assimilation mothod can do easily. In tect, much effort has been put into developing temperature retrieval techniques for satellite data because the operational data asoimilation schemes could not use the radiances as input data.

Although writing the adjoint of a model is not terribly difficult, and somowhat mechanical once the linear tangent model has been written, dobugging il is quite a challenge. To test the LTM, we estimate the gradients by finite difference (by slightly perturbing the input of the original model) and compare them to the output of the LTM. The adjoint can be tested in two ways: 1) ensure that the scalar product of the input of the LTM by the output of the adjoint is equal to the scalar product of the input of the adjoint by the output of the LTM, or2) compare the gradient computed by the adjoint with that computed by the LTM. These should agree to within machine precision. Unfortunately both of these tests are global in nature; that is, they fest the entire code at once. If a discrepancy is found, there is no indication where the error might be!

\section{Data Assimilation Tests}

We are now doing an extensive sel of tests of both the model optimization and data assimilation. So far, we have only used NWS data for Oklahoma City, but we will soon start using the ARM data as well.

We show here a couple of examples. In both figures we show the surface temperature observations for 2 days; our ALFA forecasts before optimization, which we also call first guess; the ALFA analysis for the first day; and the resulting forecast for the second day. The first guess forecast is performed with what we think may be "reasonable" physical parameters for the Oklahoma site, with the analysis of the NWS as initial condition.

in Figure 4, we do an optimization of the model parameters, using data for the first day. Although the figure shows only surface temperature, all the available NWS data are used, i.e., surface temperature, dewpoint temperature, winds every hour, and sounding profiles (mandatory levels only) -very 12 hours. A24-hour forecast with the now parameters is then pertormed from the state at the end of the optimization poriod.

In Figure 5 wo use the Dorber nudging algorithm to assimilate data during the first day. Wo have used constant nudging: at each time step we add constant terms to all the tendency equations. These terms are different for all the variables and aleo depend on holght. They are our control variables. They are all zero at the start of the assimilation procedure. The liorattve procese is stopped when the forecast orror during the first day is minimum. Again, a 24-hour forecast is performed from the end of the assimilation poriod.

\section{Conclusions}

After atruggling (somowhat longer than expected) with writing the adjoint of the Toon radiation scheme, we are now at the point where we are beginning to get results of model optimization and data assimilation using the adjoint mothod. The first results are very encouraging, as can be seen in the figures shown here. We are confident that the variational data assimilation mothod, using a single-column model, will prove to be a powertul tool for data fusion and data assimilation.

A lot of work remains to be done. Optimization of the model parameters needs to be done with a much longer series of data, to cover different meteorological siluations. The Derber nudging method will require considerable tuning, especially in defining the vertical profiles of the nudging terms. Up to now wo have let them adjust freely, but that creates a problem when observations are avallable at only a fow lovels. A smoothness constraint should probably be enforced.

So far, we have also chosen failly simple situations, avoiding convective cases. It is not known yet whether the kind of thresholds involved in the convection will create convergence problems in the minimization. Finally we need to develop what might be called "observation simulators," l.e., algorithms to create output similar to the observed quantities, for as many of the ARM instruments as possible. 


\section{May 7-8, OKC station}
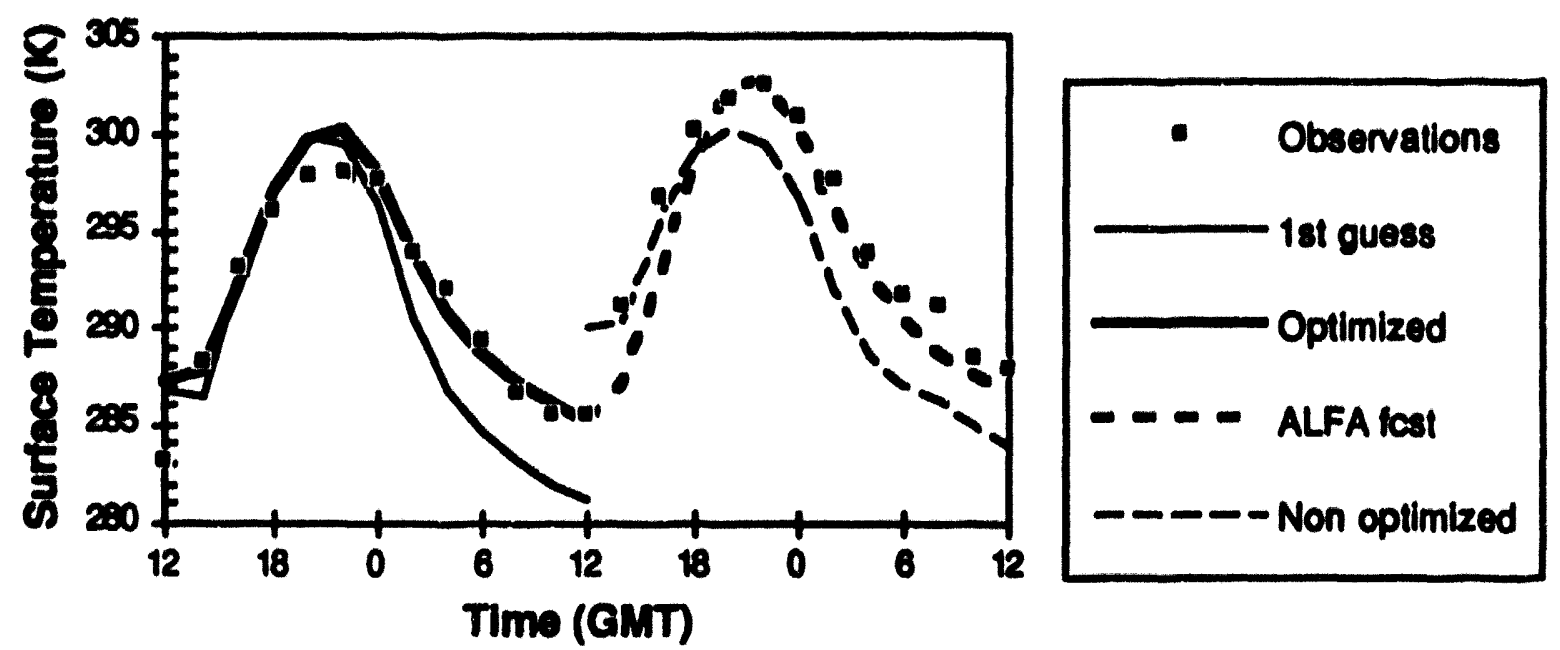

Floure 4. Example of model parameter optimization for Oklahoma City. The optimization is pertormed during the first 24 hours.

\section{July 21-22, OKC station}
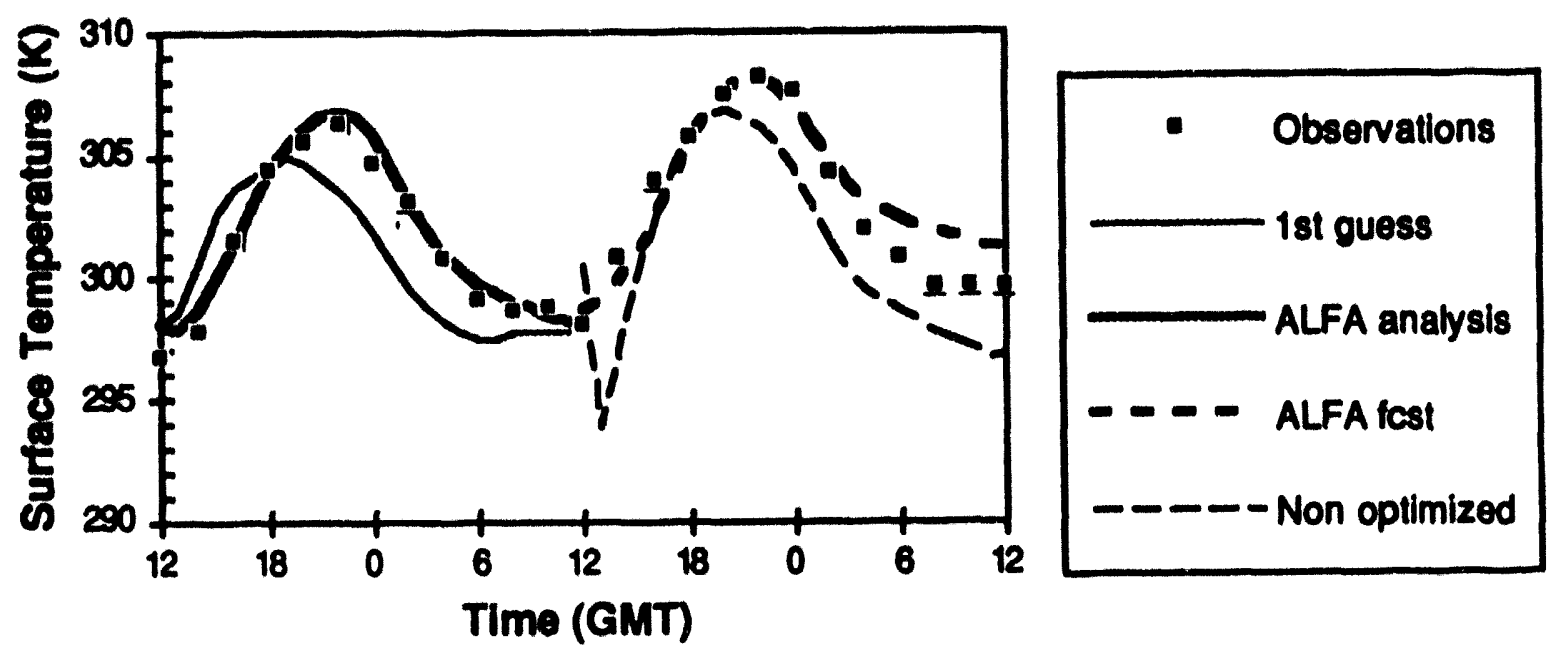

Flgure 5. Example of Derber nudging assimilation at Oklahoma City. The assimilation is performed during the first 24 hours. 


\section{References}

Anthes, R. A., Y.H. Kuo, S. G. Benjamin, and Y.F. LI. 1882. The evolution of the mesoscale environment of eevere local storms: Preliminary modeling results. Mon. Wea. Rov. 110:1187-1213.

Derber, J. C. 1989. A variational continuous aseimilation technique. Mon. Wea. Rev. 117:2437.2446.

Hoffman, R. N., J.-F. Louls, and T. Nohrkorn. 1892. A method for implementing adjoint calculations in the discrete case. Technical Momorandum No. 184. European Centre for Medium-Range Weather Forecasts, Reading, United Kingdom.
Louis, J.F., R. N. Hoffman, and T. Nehrkorn. 1990. Optimizing a local weather forecast model. NSF Contract No. 181-8960592. Avallable trom Atmospheric and Environmental Research, Cambridge, MA 02139.

Toon, O. B., C. P. McKay, T. P. Ackerman, and K. Santhanam. 1989. Rapld calculation of radiative heating rates and photodissociation rates in inhomogeneous multiple scaltering atmospheres. J. Geophys. Res. 94:16287-16301. 


\title{
Interactions Between Aerosols and Clouds
}

\author{
O. B. Toon, A. Ackerman, and E. Jonsen \\ National Aoronautics and Specs Adminibtration \\ Amos Rosearch Conter \\ Moffott Flold, CA 94035
}

The albedo of marine stratus clouds can be altered by the addition of earosols to the marine boundary layer. (Twomey 1977. Chartson of al. 1887, Coakloy of al. 1887). As the number of cloud condencation nucleil incresses, the number of cloud droplets increases, the size of the cloud droplets declines, the drizzle rate declines, and the llquid water inoreseses (Twomey 1877, Radke of al. 1889, Abrecht 1889). The result of these changes is to inopesse the optical depth of the cloud, causing its visible albedo to rise.

A number of studies hav subsequently suggested that anthropogenic increases in aerosol production will altor the earth's climate. The change le due not only to the direct offect of the asrosols on the earth's radiation budget, but also about equaly to the indirect effecte of esrosols on clouds (Penner of al. 1892, Charbon of d. 1892). During the past fow yoars wo have been systomatically oxamining the relations between changes in eorosol sbundance and cloud properties in the hopes of eventually quantifying these interactions. We find that not all types of clouds respond in the same manner to changes in serosol concentrations and that some hypotheses in the literature about the relations between serosols and clouds may be incorrect.

The microphysical studies we have been conducting are besed upon versions of the microphysical model discusesd by Toon ot al. (1988). We have coupled a radiative transfor model to the cloud model (Toon of al. 1889) and have developed a one-dimensional turbulent transport model to representboundary-layer dynamical procesees. The model has been further developed and applied to the marine boundary layer by Ackerman of al. $(1992,1983)$ and to cirrus clouds by Jensen of al. (1993a, 1893b). These two versions primarlly differ by having loe physical proceseses active in the cirrus model. The basic microphysical model has also been embedded in the Penn State/National
Conter for Atmospheric Research (NCAR) mesoscab dynamical model and used to simulated clrus clouds during the Firat 18CCP(4 Regional Experiment (FIRE) project.

Ons of our goals in performing one-dimensional studbe is to develop the microphyslas for these throe-dimenalonal simulations. However, since the microphysics thelf is computationally wy demanding, the one-dimendiond simulatione are often useful for cloud simulations of microphycics in situations in which dynamics either is not important or can be paramoterized. Hore we discuse only ono-dimensional simulations.

Marine stratus are the only clouds for which there are slgnificant data showing offects of aerosols on cloud propertios. We have simulated ship tracks with our model using particle injection rates based upon obeervations (Redke ot al. 1889).

In the simulations, un first model the devalopment of a atratus cloud having properties similar to those obeerved In the region in which ship tracks were studied by Radk of al. (1889). Then we inject particles and follow the subeequent evolution of the cloud albedo, llquid water content, particle size, and so forth for several days.

We find that the modeled cloud responds in a similar manner to that obeerved. The simulated cloud albedo increases from about $50 \%$ to about $60 \%$; the mean dropbt size declines from about 11 to about $9 \mu \mathrm{m}$; and the liquld water content changes from 0.4 to about $0.5 \mathrm{~g} \mathrm{~m}^{-3}$ as the particle concentration Increases from about 50 to $120 \mathrm{~cm}^{-3}$. The longevity of the ship tracks in our simulatione exceeds 24 hours, as is sometimes observed for sctual ship trecks.

(a) Intamationd Satulitis Cloud Climatology Project. 
The simulations show that it is not necessany to hypothesize unusud dynamical processes or gas-to-particle conversion extending over long time periods in order to account for the obeerved properties and longevity of ship tracks. Of course, gas-10-particle conversion almost cortainly does occur at bast near the ship stack, and the alteration of the cloud properties in a confined area probably does induce oynamical motions near the ship track.

Our one-dimensional simulations cannof reproduce the three-dimensional dynamics of the marine boundary layer. However, we believe the presence of ship tracks requires a boundary layer in which relatively little vertical shear is present to avold dispersing the serosols over a wide region. This requirement may account for the relative rarity of ship tracks.

Another bsue regarding marine stratus that wo investigated is the relation between particle production rates and resulting particle concentrations. Baker and Charlson (1980) suggested that this relationship has only two discontinuous solutions: for small production rates, cloud particle numbers are limited to small values (order $50 \mathrm{~cm}^{-1}$ ) by drizzle; for larger production rates, drizzle is suppressed, leading to much higher particle concentrations.

While our aimulations agree that the number of particles is a rapldly increasing function of the particle production rate, we find a continuous relationship between particle concentrations and particle production rate. Our results suggest that the functional form Baker and Charison (1980) essumed for the loss of particles due to coalescence of cloud drope was overty simplified. In addition, we examined the lifetimes of particles in marine stratus clouds. We find that eeveral days can be required for clouds to come to equilibrium with a new particle production rate and that the residence time of the aerosol mass is much longer than the residence time of the particle number. The different llfetimes occur because mass must be removed at the surface, but numbers are also reduced by coalescence. Hence, the distance that particles drift downwind from their source can be overestimated if mass residence times are used.

We have also examined the sensitivity of cirrus cloud properties to serosol concentrations. Changes in cirrus cloud optical depth will have a lesser impact on the earth's radiation budget than equivalent changes in optical depth of a stratus cloud because cirrus clouds impact both solar and infrared radiation in an opposing manner. That is, as cirrus clouds become more optically thick, they reflect more sunlight to space, but they also become better infrared absorbers and thereby prevent intrared loss to epace.

More importantly, cirrus clouds are not very responsive to changes in aerosol concentrations because they require a substantial supersaturation with respect to ice to lead to nucleation. Therefore, once nucleation occurs and 100 crystals begin to grow, they quickly drop the supersaturation below the lovel at which now particles can nucleate. For this reason, the number of cirrus cloud particles is not very sensitive to the number of aerosols. In short, the clouds control the number of ice crystals. The largest impact on cirrus occurs when the number of large or easily activated aerosols changes because then the number that can be activated at a given supersaturation varies (Jensen and Toon 1992). Even then, however, the effects are much less dramatic than for stratus for the reasons mentioned above.

We have not yet examined the sensitivity of continental stratus or of convective clouds to aerosols. Continental stratus usually form where many cloud condensation nuclei are present. Additional aerosols are observed to change cloud albedo by smaller amounts as the aerosol populations increase, so the continental stratus would be expected to be much less sensitive than marine stratus to aerosol concentrations.

In general, the impact of anthropogenic aerosols on clouds needs to be carefully evaluated since the effects of aerosols on clouds depend considerably upon the type of cloud being considered. In addition, for the marine boundary layer, the aerosol residence time by mass considerably exceeds the residence time by number 80 the area downwind of the source affected by aerosols can be incorrectly estimated unless the relevant residence time is used.

\section{References}

Ackerman, A. S., O. B. Toon, and P. V. Hobbs. 1993. A reassessment of the bistability of cloud condensation nucleus concentrations. Nature, submitted. 
Ackerman, A. S., O. B. Toon, and P. V. Hobbs. 1992. Numerical modeling of the stratocumulus-topped marine boundary layer. Proceedings of the International Conference on Clouds and Precipitation, Montreal. Department of Meteorology. McGill University, Montreal, Canada.

Albrecht, B. A. 1989. Aerosols, cloud microphysics and fractional cloudiness, Science 248:1227-1230.

Baker, M. B., and R. J. Charlson. 1990. Bistability of CCN concentrations and thermodynamics in the cloud-topped boundary layer. Nature 142-145.

Charison, R. J., J. E. Lovelock, M. O. Andreas, and S. G. Warren. 1987. Oceanic phytoplankton, atmospheric sulfur, cloud albedo, and climate. Nature 326, m655.

Charlson, R. J., S. E. Schwartz, J. M. Hales, R. D. Cess, J. A. Coakley, Jr., J. E. Hansen, and D. J. Hofmann. 1992. Climate forcing by anthropogenic aerosols. Science 255:423-430.

Coakley, J. A., Jr., R. L. Bernstein, and P. A. Durkee. 1987. Effect of ship-stack effluents on cloud reflectivity. Science 237:1020-1022.

Jensen, E. J., O. B. Toon, D. L. Westphal, and S. Kinne. 1993a. Microphysical modeling of cirrus. Part I: Comparison with 1986 FIRE IFO moasurements. J. Geophys. Res., submitted.
Jensen, E. J., O. B. Toon, D. L. Westphal, and S. Kinne. 1993b. Microphysical modeling of cirrus. Part II: Sensitivity studies. J. Geophys. Res., submitted.

Jensen, E. J., and O. B. Toon. 1992. The potential effects of volcanic earosols on cirrus cloud microphysics. Geophys. Res. Lett. 19:1759-1762.

Penner, J. E., R. E. Dickinson, and C. A. O'Neill. 1982. Effects of aerosols from biomass burning on the global radiation budget. Science 256:1432.1434.

Radke, L. F., J. A. Coakley, Jr., and M. D. King. 1989. Direct and remote sensing observations of the effecte of ships on clouds. Science 246:1146-1149.

Toon, O. B., R. P. Turco, D. Westphal, R. Malone, and M. S. Liu. 1988. A multidimensional model for aerosols: Description of the computational analogs. J. Atmos. Scl. 45:2 123-2, 143.

Toon, O. B., C. P. Mckay, T. P. Ackerman, and K. Santhanam. 1989. Rapid calculation of radiative heating rates and photodissociation rates in inhomogeneous multiple scattering atmospheres. J. Geophys. Ros. 94:16287-16301.

Twomey, S. 1977. The influence of pollution on the shortwave albedo of clouds. J. Atmos. Sci. 34:1149-1152. 


\title{
Are Ground-Based Measurements Sufficient to Quantify Atmospheric Radiative Properties?
}

\author{
S. Kinne and R. Bergstrom \\ NASA-Ames Research Center \\ Moffett Field, CA 94035-1000 \\ T. Ackerman \\ Pennsylvania State University \\ University Park, PA 16802
}

\section{Abstract}

Radiation measurements at the surface and simultaneous ground-based measurements of the atmosphere during the FIRE'91(a) cirrus field experiment provided an opportunity to identify essential measurements and deficiencies in parameterizations of current cloud-radiation models. Comparisons between measured and calculated broadband surface fluxes demonstrate the need for 1) accurate humidity and temperature vertical profiles, 2) data that capture the 3-dimensional structure and vertical extinction of clouds, and 3) additional airborne measurements. In-situ cloud microphysical measurements and radiation measurements near the tropopause (taken simultaneously to those at the surface, to define the radiative properties of the troposphere) are vital to improve radiative model parameterizations (and also satellite retrieval algorithms), which are used in the absence of available measurements.

\section{Introduction}

Tobenefit our understanding of radiative transfer processes in the earth's atmosphere, long-term continuous groundbased measurements at a few selected locations all over the world are planned and are already underway at the first Atmospheric Radiation Measurement (ARM) site, in Oklahoma. One of the major difficulties is the definition of

(a) First ISCCP (International Satellite Cloud Climatology Project) Regional Experiment. the atmospheric radiative properties with measurements from the surface only. Using surface measurements of the atmosphere from the FIRE'91 field experiment and comparing calculated to measured radiative surface fluxes, we seek to identify the most important measurements. This study also points out that many atmospheric properties cannot be measured from the ground and have to rely heavily on model parameterizations, which must be validated and, if necessary, improved.

FIRE' 91 measurements, used in this study, are addressed first. Then, the testing procedure is outlined, including a short description of the radiative transfer model. Next, a few selected results are analyzed and, finally, suggestions for future work are given.

\section{Measurements}

As a precursor to upcoming measurements at ARM sites, data from the FIRE'91 cirrus field experiment provide a unique opportunity to judge the ability of ground-based measurements to describe the radiative properties of the atmosphere.

This study relies only on a data subset, as most of the measurements were not available at the time this study was conducted (an updated study with more data is underway). Vertical profiles of atmospheric variables (e.g., temperature, humidity) are defined by frequent radiosonde launches by the National Oceanic and Atmospheric Administration (NOAA). Model calculations for times between launches are based on a linear time interpolation of these vertical profiles. Cloud properties and cloud 
structural data are based on remote sensing data from the ground, including the Pennsylvania State University 94-GHz radar and 10-channel sun-photometer, the latter providing optical depth values for optically thin clouds. Cloud microphysical properties are based on model parameterizations, as in-situ measurements were not processed at the time this work was done. Downward hemispheric broadband solar and infrared fluxes at the ground are provided by Eppley radiometers of the Pennsylvania State University.

\section{Test}

The "quality test" involves three stages. First, radiosonde data and measurements from the ground (radar and sunphotometer) are used to define the composition of the atmosphere. Then, a radiative transfer model is applied to derive radiative properties for given atmospheric compositions. Finally, calculated surface fluxes of the model are compared to fluxes actually measured at the surface.

The radiative transfer model is based on a four-stream code at eight solar wavelengths and on a two-stream code at twelve infrared wavelengths. Absorption by atmospheric gases in these bands is expressed via exponential sumfitting and based on the HITRAN database. Although the selection of radiative method, spectral resolution, and absorption approximation can notably affect calculated fluxes, the chosen model is found to be sufficiently accurate. Deviations of surface broadband fluxes to values based on more accurate models and/or spectral resolution (less than $4 \%$ ) are found to be small compared with the measurement errors. The model, however, assumes horizontal homogeneity, which is poor for many cloud conditions. To minimize inhomogeneity errors, we use only average flux values for time-periods of at least five minutes.

\section{Results}

Comparisons between measured and calculated fluxes carry a combination of errors related to 1) radiative method, 2) model parameterizations, and 3) measurements for model initialization. As errors of the radiative method are small (see above), any significant lack of agreement must be attributed to inaccurate measurements or bad model parameterizations that must be used if important measurements (e.g., cloud micro- and macrophysical properties) are not available.

Broadband surface flux comparison between measured and modeled values for Coffeyville, Kansas, on December 5 and 6, 1991, are given in Figure 1. Daytime surface flux comparisons have been shown separately for the solar and infrared spectral region. In addition, errors for the modeled solar and infrared fluxes are indicated. Clear sky conditions existed in the morning of December 5 and during the afternoon of December 6. Optically thin cirrus, only, was present the afternoon of December 5 and around noon on December 6. Optically thicker mid-level clouds were observed the morning of December 6.

Clear sky comparisons compare moderately well, with errors generally below $10 \%$. Inaccurate humidity and temperature profiles (at best, only radiosonde data every three hours were available) create errors, especially in the infrared spectral region.

Cloudy sky comparisons show larger errors, especially in the presence of optically thicker clouds for the solar region. This is mainly due to the lack of information on cloud optical depth (the radar was not calibrated). However, the radar positioned the cloud base adequately, as infrared errors remain significantly smaller. Some of the large solar error also may be attributed to the horizontal inhomogeneous cloud structure, despite the use of five-minute flux averages in the comparison. The spatial variability of clouds may contribute to the modeling error, as simultaneous cloud radar observations (sunphotometer measurement) and broadband flux measurements were not completely collocated.

Under cirrus cloud conditions, particularly for the afternoon of December 5, calculations systematically overestimate solar downward fluxes. This may indicate a bad model assumption. Model sensitivity studies with a smaller solar asymmetry-factor of less than 0.8 for cirrus clouds result in much better match between model results and actual measurements. 


\section{DEC}

am. clear

pm. cirrus
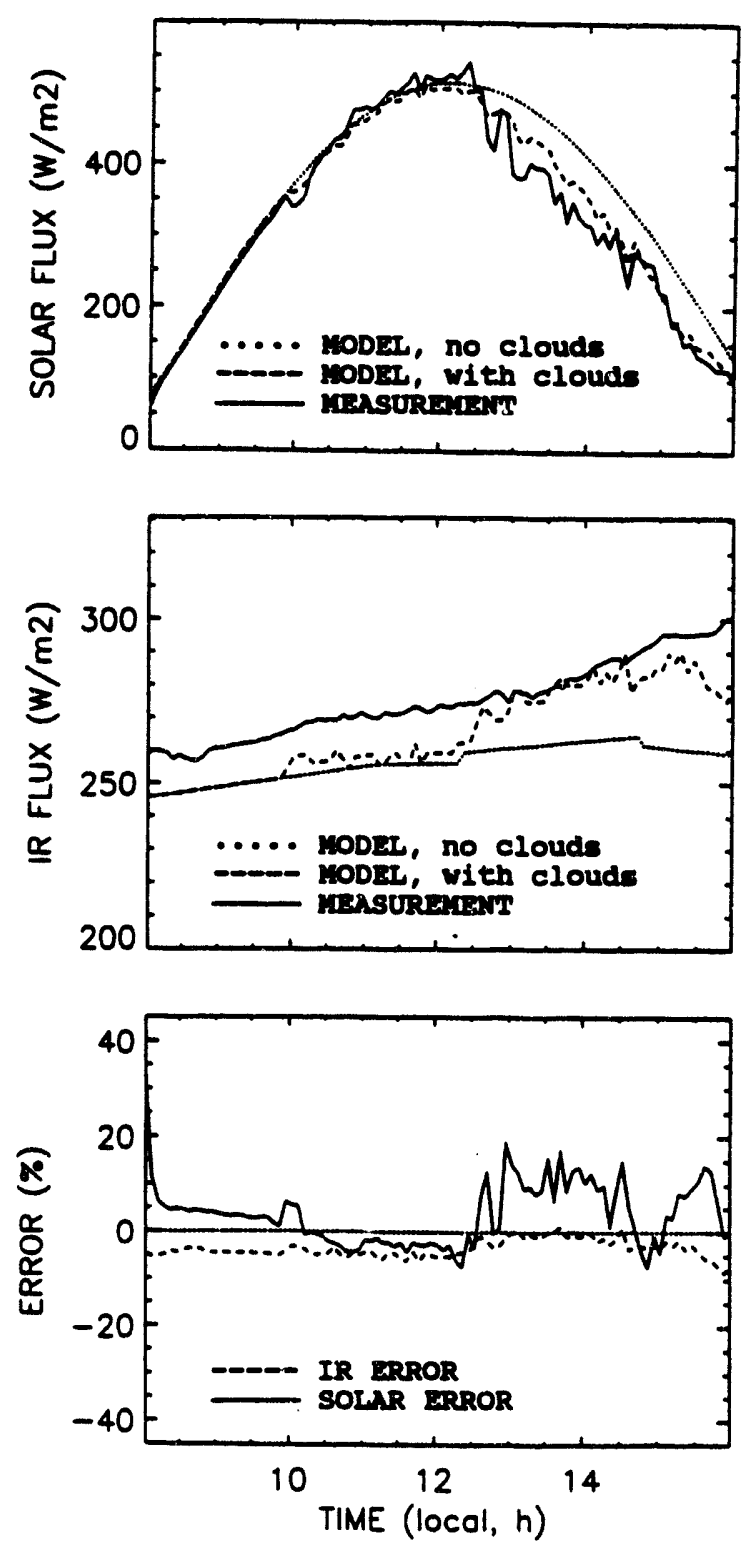

\section{DEC}

am. cirrus and altostratus

pm. clear / cirrus
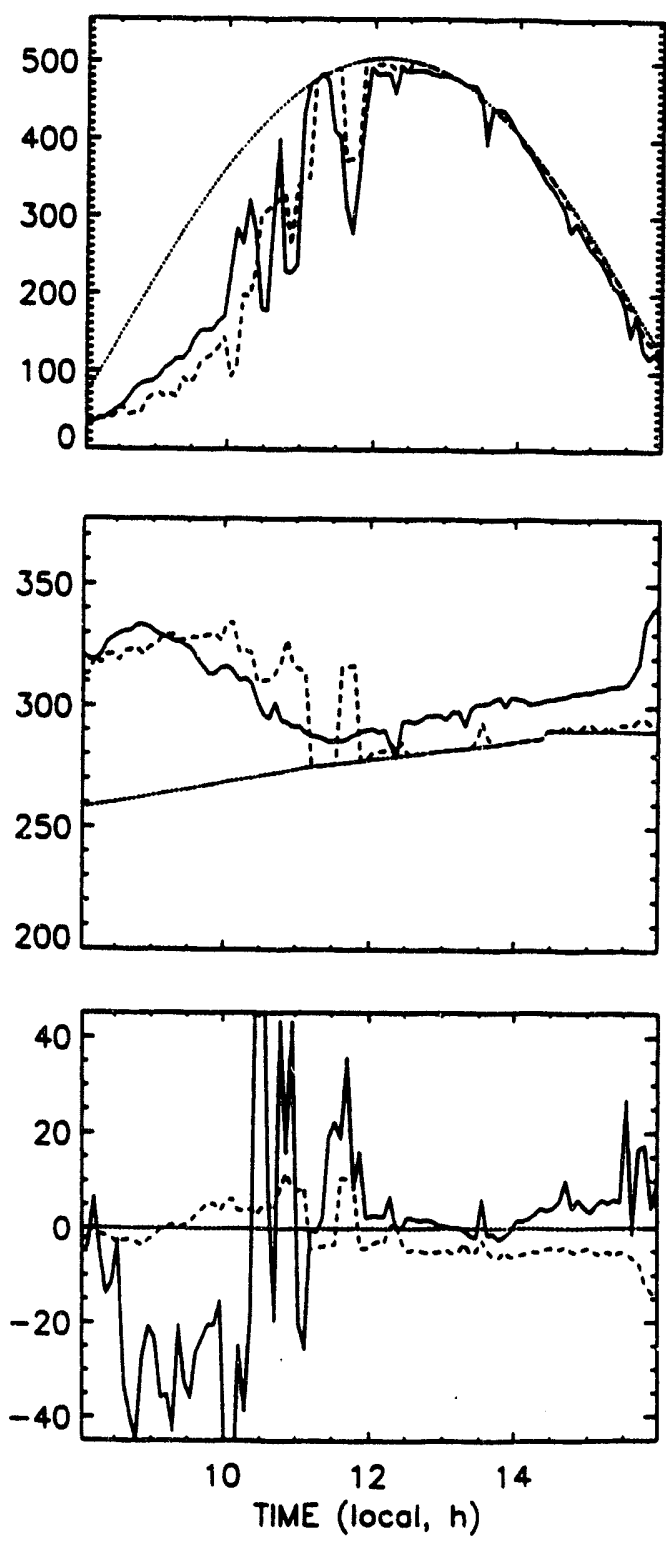

Flgure 1. Comparison between measured (solid line) and modeled (dashed line - clouds included; dotted line - clouds omitted) hemispheric downward broadband solar infrared fluxes for December 5 and 6, 1991, at Coffeyville, Kansas. In addition, corresponding errors for calculated solar fluxes (solid line) and ca!culated infrared fluxes (dashed line) are given. 


\section{Conclusion}

Our results, which are limited to a few ground-based measurements 80 far, indicate that a lack of appropriate model parameterizations make it difficult, if not impossible, to accurately describe radiative properties of the atmosphere with ground-based measurements only. To be successful, the following elements seem vital:

- Ground-based measurements must be improved and new measurements added. For clear sky conditions, in particular, better temperature and humidity profiles are necessary (a few radiosondes are not sufficient). For cloudy conditions, measurements that describe horizontal (radiometer-array) and vertical cloud structure as well as cloud extinction (calibrated radar) are needed.

Airborne measurements are highly desirable. Even as short "field experiments," they should provide in-situ cloud measurements that help us understand cloud microphysics. Even more important are rediation measurements near the tropopause simultaneously to the measurements at the ground, defining the radiative properties of the troposphere in between. Such measurements are vital in order to improve parameterizations in radiative transfer models and also to calibrate satellite retrieval algorithms. 


\title{
Advances in the Development of an Integrated Data Assimilation and Sounding System
}

\author{
W. F. Dabberdt, D. Parsons, Y.-H. Kuo, J. Dudhia, Y.-R. Guo, \\ J. Van Baelen, C. Martin, and S. Oncley \\ National Center for Atmospheric Research(a) \\ Boulder, CO 80307-3000
}

\section{Overview}

The Integrated Data Assimilation and Sounding System (IDASS) provides continuous high-resolution tropospheric profiles. The measurement system (Integrated Sounding System, or ISS) is developed around a suite of in situ and active and passive remote sensors. Individually these sensors satisfy certain needs; together, they function in a synergistic and complementary mode. ISS subsystems may include multiple-frequency UHF wind profiler(s) with radio acoustic sounding (RASS), a NAVAID-based sounding system, a surface meteorological station, a microwave profiler, a high-resolution infrared sounder, and a laser coilometer. The ISS operates as an integrated system. Important aspects of the system are the synergistic interaction of data streams from the individual instruments and the application of real-time and post facto data assimilation, as well as the mechanical integration that provides a transportable central data acquisition, processing, display and communications capability in the field for research studies.

Observations from ISS networks provide a high-resolution description of atmospheric structure on the meso- $B$ scale. Measurements from the in situ and remote sensing instruments are coupled with a state-of-the-art mesoscale modeling system. In the mesoscale data assimilation process, the model solution is relaxed toward the available observations so that it is consistent with the measurements. Over regions where there are no observations, the evolution of the model fields is constrained by the model's dynamic and physical processes. The end product is a highly resolved four-dimensional meteorological data set

(a) NCAR is sponsored by the National Science Foundation. (including three components of wind, temperature, humidity, cloud water, and integrated moisture). The mesoscale data assimilation scheme is the Newtonian nudging technique. During the data assimilation period, observations of wind, temperature, and humidity are used to nudge (or relax) the time-dependent model variables to the observed values.

We are using an enhanced nonhydrostatic version of the Penn State/NCAR mesoscale model which includes parameterizations of surface and planetary boundarylayer processes, convective and nonconvective clouds, and radiation. A comprehensive data analysis system is coupled with the model, allowing real-data experimentation.

Initial evaluations of the measurement and modeling components of the IDASS system were conducted in conjunction with the 1990 and 1991 Winter leing and Storms Programs (WISP) conducted on the Front Range of northeastern Colorado; the spring 1979 Severe Environmental Storms and Mesoscale Experiment (SESAME) study undertaken in the south-central states of Texas, Oklahoma and Kansas; and a November 1992 ISS instrumentation test near the Cloud and Radiation Testbed (CART) central facility.

\section{Integrated Sounding System}

ISS measurement facilities that have been evaluated to date include multiple frequency UHFNHF Doppler wind profilers and RASS; microwave radiometers; a highresolution interferometer spectrometer (HIS); a NAVAID atmospheric sounding system; and a surface meteorological station measuring state variables, winds, trace gases, and all components of the surface energy 
balance. Additionally, we have been developing a 915-MHz boundary-layer radio interferometric wind profiler which offers the potential to measure mean and flux profiles from a single sampling volume (avolding the limitations of beam swinging techniques).

Using assimilation of ISS (and other) data in a mesoscale model, we seek to provide physically consistent vertical profiles of winds, temperature and humidity at each ISS measurement site. Perhaps of even greater value, application of data assimilation to a network of ISS sites can yield highly resolved four-dimensional fields.

\section{Mesoscale Data Assimilation}

The mesoscale data assimilation scheme we are using is the Newtonian nudging technique originally developed by Anthes (1974) and Hoke and Anthes (1976). During the data assimilation period, observations of wind, temperature, and humidity are used to nudge (or relax) time-dependent model variables to the observed values. The dynamical and thermodynamical processes of the model then spread the influence of the observations to nearby points, so that a balanced set of four-dimensional meteorological fields, consistent with the model equations, can be obtained. Specifically, a forcing (or relaxing) term is added to the prognostic equations of the model. The magnitude of the additional forcing term is a function of the difference between the measurement and the model solution and the distance in both time and space between the observations and the specific model grid-point under consideration. Detalls of the mesoscale data assimilation procedure can be found in Kuo and Guo (1989) and Stauffer and Seaman (1990).

The mesoscale model is a very important component of the data assimilation system. We have been using the Penn State/NCAR mesoscale model MM4 (described by Anthes et al. 1987). The model includes parameterizations of surface and planetary boundary-layer processes, convective and nonconvective clouds, and radiation. Because the ISS measurements are "point" measurements, it is desirable to have the highest possible resolution for the assimilation model. Consequently, we have been involved in the development of a nonhydrostatic version of the Penn State/NCAR mesoscale model. The new model (MM5) is capable of real data assimilation with a grid size ranging from $1 \mathrm{~km}$ to $100 \mathrm{~km}$. Preliminary tests on the Valentine's
Day storm during WISP.1990 have shown that the nonhydrostatic mesoscale model is comparable with the hydrostatic model for a grid size of $20-\mathrm{km}$ (which is in the hydrostatic regime).

\section{Recent IDASS Advances}

Recent IDASS developments and advances have occurred over a broad range of technological and modeling/data assimilation areas. Each of these is described in detall in the following papers included elsewhere in these proceedings. The subjects and associated authors include:

- System Design and Implementation of the Integrated Sounding System (Martin and Barrett)

- An Ultra-High Frequency Boundary-Layer Doppler/ Interferometric Profiler (Van Baelen)

- Boundary-Layer Observations Over the Southern Great Plains Cloud and Radiation Testbed Site During the November 1992 Flux-Profiler Test (Oncley and Van Baelen)

- Meso-Beta-Scale Data Assimilation of the Winter Icing and Storms Program/Atmospheric Radiation Measurement 91 Intensive Observing Period Case on 6 March 1991 (Kuo and Guo)

- The Effect of Network Resolution on Data Assimilation in a Mescoscale Model (Dudhia)

- Comparison of Mescoscale Model and Tower Measurements of Surface Fluxes During Winter Icing and Storms Program/Atmospheric Radiation Measurement 91 (Oncley and Dudhia)

- Radiation Studies with a High-Resolution Mesoscale Model (Dudhia).

\section{References}

Anthes, R. A. 1974. Data assimilation and initialization of hurricane prediction models. J. Atmos. Sci. 31:702-719.

Anthes, R. A., E.-Y. Hsie, and Y.-H. Kuo. 1987. Description of the Penn State/NCAR mesoscale model version 4 (MM4). NCAR Tech. Note, NCAR/TN-282+STR, Boulder, Colorado. 
Hoke, J. E., and R. A. Anthes. 1976. The initialization of numerical models by a dynamic initialization technique. Mon. Woa. Rov. 104:1551-1556.

Kuo, Y.H., and Y.-R. Guo. 1989. Dynamic initialization using observations from a hypothetical network of profilers. Mon. Wea. Rev. 117:1975-1998.
Stauffer, D. R., and N. L. Seaman. 1990. Use of fourdimensional data assimilation in a limited-area mesoscale model. Part I: Experiments with synoptic-mesoscale data. Mon. Wea. Rov. 118:1250-1277. 


\title{
Modeling of Clouds and Radiation for Development of Parameterizations for General Circulation Models
}

\author{
D. Westphal, B. Toon, E. Jensen, S. Kinne, A. Ackerman, \\ R. Bergstrom, and A. Walker \\ National Aeronautics and Space Administration \\ Ames Research Center \\ Motfett Field, CA 94035
}

\section{Introduction}

Atmospheric Radiation Measurement (ARM) Program research at NASA Ames Research Center (ARC) includes radiative transfer modeling, cirrus cloud microphysics, and stratus cloud modeling. These efforts are designed to provide the basis for improving cloud and radiation parameterizations in our main stfert: mesoscale cloud modeling. Radiative transfer modeling is described by Kinne et al. (this meetting); stratus and cirrus cloud modeling efforts are described by Toon of al. (this meeting); and mesoscale modeling is described in this abstract.

\section{Cloud Models for ARM}

The range of non-convective cloud models used by the ARM modeling community can be crudely categorized based on the number of predicted hydrometers (Table 1). The simplest model has no predicted hydrometers and diagnoses the presence of clouds based on the predicted relative humidity. This scheme is used in many general circulation models (GCMs) and in numerical weather prediction (NWP) models. Some GCMs now include a single predictive equation for clouds.

The vast majority of cloud models have two or more predictive bulk hydrometers such as cloud water, ice water, rain, snow, graupel, etc. This method provides coarse size resolution by assigning a zero fall velocity to some species (cloud and ice) and non-zero fall velocitles to other species. The assigned fall velocity depends on the density of the category, so that snow and graupel (with low density) fall more slowly than rain. Additionally, some models predict ice number concentration, from which a mean or effective particle radius can be calculated from the ice water concentration. This is valuable for calculating optical properties. The last class of model listed in the table is called size-resolving because each hydrometer category is subdivided into different bins, each with a different size. This subdivision allows the explicit calculation of hydrodynamical processes for each size, such as particle fall speeds or coalescence rates.

The Penn State/NCAR (National Center for Atmospheric Research) mesoscale model has been adapted at ARC to use several of the cloud schemes listed in Table 1. These include the relative humidity (RH), the bulk water (BW), and the size-resolving (SR) schemes. The implementation of the BW scheme uses only two species $(n=2)$ : cloud/ice and rain/snow. The phase of the specie is determined by the grid box temperature. The SR model allows for twenty different sizes ofice nuclei, cloud droplets, and ice crystals, ranging from 0.01 to 600.0 micrometers, equivalent volume radius.

Our modeling approach allows us to intercompare the results of the various cloud schemes within the same dynamical framework, and the use of the PSU/NCAR mesoscale model allows us to compare our results with observations, instead of climate statistics.

The complexity of the BW and SR models is justified by the well-known sensitivity of cloud optical properties to particle size. The RH and simpler BW ( $n=1$ or 2 ) models use prescribed particle size when calculating optical properties. These prescriptions assume some dependence on temperature or altitude or phase. These assumptions may not be valid for both the tropics and mid-latifudes and polar regions, for multi-layer clouds, clouds in both the developing and dissipating stages, or for both the current and the future perturbed climate. The need to develop a general cloud model, valid for all cloud types and for all climates, demands that process models such as the BW (with $n=$ many) or SR be used. 
ARM Sciena Moving

Table 1. Explicit cloud models.

\begin{tabular}{ll}
$n^{(n)}$ & \multicolumn{1}{c}{ Description } \\
& Relativo humidity model \\
$2-8$ & Bulk water cloud model \\
& $\begin{array}{l}\text { Bulk water model of cloud, ice, } \\
\text { snow, graupel, etc. (minimal } \\
\text { size, shape, and density resolution) }\end{array}$ \\
100 & $\begin{array}{l}\text { Size-resolving model: } \\
\text { aerosol }(r), \text { cloud(r), ice(r), otc. }\end{array}$
\end{tabular}

(a) $n$ - number of predicted hydrometer variables.

We suggest that a GCM capable of answering the outstanding questions about cllmato change will be oither an SR type or aBW type with more than eeveral hydrometer classes. Accompanying this increase in cloud resolution, the grid resolution must increase in order to model the large-scale dynamical forcing of the cloud fields. Hence, we suggest that our modeling system using the BW scheme (with n greater than 5) or the SR scheme, on a grid of 100 kilometers or less, is a likely prototype of the GCM required to answer our questions about climate change and, at the same time, to allow direct comparison with Cloud and Radiation Testbed (CART) and other observations.

\section{Initial Conditions}

Using a limited-area modeling system to simulate observations requires accurate initial and boundary conditions. The National Oceanic and Atmospheric Administration (NOAA) Mesoscale Analysis and Prediction System (MAPS) analyses for the United States have been shown to be statistically more accurate than other National Weather Service (NWS) products, such as the Nested Grid Model (NGM), and are available every 3 hours. The analyses are a combination of current synoptic and asynoptic observations and the previous 3-hour forecast. The analysis does not directly include clouds 80 our model must generate its own clouds from the initial cloud-free condition.
For a particular case during NASA's First ISCCP(a) Regional Experiment (FIRE)-Il program, we have found that this spin-up time can be greater than 12 hours. Specifically, by comparing the 11 micrometer blackbody temperature at the initial time with that derived by Minnis (NASALaRC) from satellite data, we find that the observed cloud field is much more widespread.

Later in the simulation, at 12 hours, when clouds were observed by radar and lidar at Coffeyville, the model shows no cloud, despite significant dynamical forcing (vertical velocities in excess of $6 \mathrm{~cm} / \mathrm{s}$ ). Although other explanations exist, one possibility is that the initial conditions of the upper level moleture field were too dry. The quality of the Initial conditions will be investigated using the satellite analyses, lidar, and radar, as well as conventional data.

\section{Cirrus Modeling With the Size-Resolving Model}

Studies by Jensen et al. (1993a, 1993b) show that the onedimensional SR model is capable of reproducing much of the structure of cirrus clouds. The model was used to study the sen sitivity of simulated cirrus microphysical and radiative properties to poorly known model parameters, poorly

(a) International Satellite Cloud Climatology Project. 
underetood physical processes, and environmental conditions. The investigated parameters and procoseses included nucleation rate, mode of nucleation, loe crystal shape, and coagulation. The loading sources of uncertainty in the model were the phese change enorgy barribr, which dominates the homogeneous treezing nucleation rate, and the coagulation sticking efficlency at low temperatures, which controls the production of large loe crystals (radil greater than 100 micrometers). Jensen et al. found that the number of lce crystals that nucleates depended more on the elope of the cloud nuclel distribution at larger sizes than on the total number of cloud nuclel. Observed features such as an increase in lce concentration, a decrease in offective radius, and a decrease in ice water content with increasing cloud hoight were simulated.

While the microphysics of the SR model are capable of producing realistic clouds, the hydrostatic, 60-kilometer version of the PSU/NCAR Mesoscale Model 4 (MM4) does not produce the range of supersaturations that the SR model needs to drive the nucleation processes. For example, in a simulation for FIRE-II, the SR version of the model does not produce clouds over Coffeyville; whereas, the BW model does. The supersaturations were only a fow percent and were too small for homogeneous nucleation to occur, but were sufficient to initiate the simpler BW nucleation scheme. This shortcoming can be corrected by using a finer scale model, such as the non-hydrostatic version of the PSUNCAR model, or by parameterizing the subgrid-scale fluctuations in supersaturation using probability distribution functions related to the large-scale (hydrostatic) variables. We will pursue both approaches.

\section{Cirrus Modeling With the Bulk-Water Model}

The implementation of the BW model in the PSU/NCAR model has two predictive hydrometers: cloud/ice and rain/ snow. The grid box temperature is used to determine whether the hydrometer is in a liquid or a solid state. This form of the BW model does not allow for mixed-phase clouds, a shortcoming that is not too restrictive when studying mid-latitude wintertime cirius. This BW model also lacks a predictive equation for ice number concentration, which prevents a direct calculation of effective or mean particle size from the predicted ice mixing ratio. Hence, this BW model is not as general a model as we expect will be necessary for modeling the diveree range of clouds that influences climate. We plan to add a predictive equation for los number concentration, but in the meantime, must specity the particle radil when calculating the optical depth.

To date, we have used several different values for the cloud, ice, rain, and snow particle radil. We have used 7 to 30 micrometers forwater clouds and 10010300 micrometers for ice clouds. For rain and snow, we integrate over the size distributions implied in the BW scheme to determine effective radil ranging from 30 to 1000 micrometers. (The SR model, of course, explicitly predicts the size distribution 80 that the optical propertles are directly calculated without assumptions as to the effective radius.)

A comparison of optical depths predicted by the model for the FIRE.Il case shows that the larger specified values of cloud and ice effective radil are required to yield reasonable agreement with the optical depths calculated by Minnis et al. Differences may also be due to errors in the predicted mixing ratios of the hydrometers. We are now conducting a more thorough comparison between the BW and SR simulations and Iidar, radar, and satellite data from FIRE-II.

\section{Verification of the Model Simulations With CART Data}

Our modeling must be validated on scales larger than the immediate CART site since small errors in the inilial conditions outside the site could lead to errors over the CART site at the validation time. The need for multidimensional datasets when validating the predictions of mesoscale models cannot be overstressed, although a complete three-dimensional datasetfor validating the model would require measurements beyond the scope of the CART.

More practically, we verify the model outside the CART site using conventional measurements such as sea level pressure, 500 millibar heights, and rainfall amounts. A more thorough validation inside and outside the CART site can now be carried out with the MAPS analyses and satellite analyses of visible and infrared cloud optical depth, cloud top height, blackbody temperature, visible cloud albedo, etc. 
Aircraft measuremente and or vortical profiling technologies such as lldar and radar and combinations of lidar and radar data will be noeded within the CART alte to validate the detalle of the cloud predictions. For example, the NOAA lidar and radar data have been combined to produce timo. and crose-eections of offective radius during FIRE-II, and the Univereity of Wisconsin VIL IIdar is capable of dotormining a two-dimensional time-ecction of the cloud distribution of thin clouds. Some of theses data sources are included in the CART dealgn; wo hopo that others can be added in the future, at loast during intonatve observing periods (IOPs).

\section{References}

Jonsen, E. J., O. B. Toon, D. L. Westphal, S. Kinne, and A. J. Hoymsfiald. 1993a. Microphysical modeling of cirrus. Part I: Comparison with 1986 FIRE IFO measurements. Accepted for publication by J. Geophys. Res.

Jensen, E. J., O. B. Toon, D. L. Westphal, S. Kinne, and A. J. Hoymafield. 1993b. Microphysical modeling of cirrus. Part II: Sensitivity studies. Accepted for publication by J. Goophys. Res. 


\title{
The Role of Atmospheric Radiation in the Generation and Maintenance of Circulations of Different Scales
}

\author{
M. J. Leach and S. Raman \\ Department of Marine, Earth and Atmospheric sciences \\ North Carolina Stato Univorelty \\ Ralolgh, North Carolina 27695-8208
}

\section{Introduction}

Iil is well known that the rediation budget of the at.; osphere is an important component of the earth's climate system. On shorter time scales, radiative transfor affects the ovolution of atmospheric circulation, principally through interaction with cloud and storm systems, and destabilizes the atmosphere continuously. This destabilization is important for subsequent dovelopment of clouds and storm systems. The clouds and storm systems foed back to the radiation budget, as clouds significantly alter both shortwave and longwave radiative transfer. It is important to underatand the role that radiative tranafer plays in the ovolution of these circulation systems to accurately quantify the radiation budget.

The results presented here are from modeling studies designed to isolate the effect of radiative transfer on the generation of circulation systems of different spatial and temporal scales. Two different numerical weather prediction models were used and will be described briefly in the next section. Following that, the radiative transfer model that was used with both circulation models will be described. Finally, results from the modeling studies will be presented, and conclusions and future research efforts will be discussed.

\section{Movel Descriptions}

The numerical model used for the emaller scale simulations has been previously described elsewhere (Huang and Raman 1991). The model is a hydrostatic primitive equation model with TKE- $\varepsilon$ closure. The model simulations are two. dimensional in a horizontal domain of $1200 \mathrm{~km}$ with grid spacing of $5 \mathrm{~km}$. The vertical domain is $12 \mathrm{~km}$ on a stretched oz (sigma-z) coordinate, with increased resolution in the boundary layer. There are 33 grid points in the vertical. The grid is staggered in both the horizontal and vertical. An explicit upstream cublc spline scheme is used for advection, with a quasi-Implicit vertical diffusion scheme (Paegle of al. 1976). Recent improvements to the model include the addition of a five-category explicitcloud physice scheme (Rutledge and Hobbe 1984), a surtace energy balance scheme which includes soll molsture (Louls 1979). and a radiative transfer scheme (Harshvardhan of al. 1987).

The nu. 'rical model used in the larger scale simulations is also a' , drostatic primitive equation model, also described elsewhere (e.9. Holt et al. 1990). The model includes a modified Kuo parameterization for convectlve clouds and calculates large scale precipitation directly. The vertical coordinate is op with 10 evenly spaced levels. The time differencing is split explicit. Lateral boundary conditions are obtained from larger scale models. Two separate case studies are described here: an extratropical and a tropical case study. The extratropical simulation is from January 24 through January 28, 1986, covering a domain from $40^{\circ} \mathrm{W}$ to $140^{\circ} \mathrm{W}$ and $10^{\circ} \mathrm{N}$ to $70^{\circ} \mathrm{N}$ on a $2^{\circ}$ longitude by $1.5^{\circ}$ latitude grid. Lateral boundary conditions are from National Meteorological Center analysis. The tropical case is from July 16 to July 25,1988 . The model domain is from $O^{\circ}$ to $180^{\circ} \mathrm{E}$ and $30^{\circ} \mathrm{S} 1060^{\circ} \mathrm{N}$. The grid spacing is $1.5^{\circ}$ by $1.5^{\circ}$. Boundary conditions are from European Center for Medium Range Weather Forecasting analysis.

The radiation transfer scheme is based on the scheme formulated by Harshvardhan ot al. (1987). The scheme includes radiative transfer in both the shortwave (SW) and infrared (IR) parts of the electromagnetic spectrum. The SW scheme is a 2 stream, delta-Eddington approximation, with absorption by $\mathrm{H}_{2} \mathrm{O}$ and $\mathrm{O}_{3}$. There is both absorption and reflection at the earth's surface. Clouds are assumed 
to have a constant optical depth. In the IR, there is line abserption by $\mathrm{H}_{2} \mathrm{O}, \mathrm{O}_{9}$, and $\mathrm{CO}_{2}$, ea well as absorption in the $\mathrm{H}_{2} \mathrm{O}$ continuum. Clouds are treated as black bodles.

\section{Results}

The explicit cloud physice model is initialized with horizontally homogeneous tomperature and molsture profile with zero ambient wind. A circulation is devoloped by imposing a hoating function in the middle of the domain for the firet $\mathbf{4 0}$ minutes of the simulation. Clouds quickly dovelop after the artificially imposed heating ceases.

The model results shown in Figures 1 and 2 are from a 4-hour simulation. The circulation that develops is as expected, with convergence below cloud base and divergence above cloud top. The appearance of three "colls" of cloud ico and cloud water and the symmetry about the center suggest that gravity waves are starting to propagate away from the heat source. The latent heating in the hydrostatic model is insufficient to continue to organize the circulation.

The same fields are shown in Figures 3 and 4, but with radiative transfer included. The differences in the tomperature and wind fields are evident. The atmosphere is cooler through the entire domain, especially in the lowest layers. This is due to the lack of longwave cooling above the first layer in case 1. The differences in the middle troposphere are less pronounced. The convergence below cloud base for case 2 is much weaker, with shorter wavelength gravity waves in the upper part of the domain. Much less water in both phases forms in case 2. It appears that the radiative forcing helps to maintain the organization of the cloud system, as there is less evidence of the gravity wave activity in the cloud fields. The greater amount of cloud water that forms in case 1 is in response to the greater destabilization of the entire atmosphere through stronger surface heating.

The regional scale model is used with real initial conditions to determine the effect of radiative transfer on larger scale circulation. In the extratropics, the model was initialized with data from Genesis of Atlantic Lows Experiment (GALE) intensive observing period (IOP)2. The results from a 108-hour (4.5-day) simulation are presented in Figures 5 and 6.

The fields shown are the isentropic potential vorticity (IPV) and the $v$ component of the geostrophic wind $\left(v_{g}\right)$. The verification for this case is a very strong north-south jot streak imbedded in a high amplitude mean flow. To isolate the effects of radiative transfer, the surface energy budgot in the model was not included in the simulations. With this severe constraint, neither the simulations with or without atmospheric radiation transfer verify very well. However, rediative transfer maintains the circulation somewhat better, as is evident in both IPV and $v_{p}$.

The same regional scale model was used for a tropical simulation, initialized with data from July 1988. Zonal means for temperature, moisture and the $u$ and $v$ wind components for a 10-day simulation in the Indian monsoon region are shown in Figures 7 and 8 . The model was also run in this case without the surface energy budget included.

The $x$-axis of the figures is latitude from $30^{\circ} \mathrm{S}$ to $60^{\circ} \mathrm{N}$. The effect of radiative transfer is evident in the wind fields. The upper branch of the Hadiey coll is more intense in both hemispheres with radiation, while the equatorial easterly jet is stronger in the case without radiation. This indicates that meridional transport is more effectively maintained by the cloud/radiation interaction. The effect on the precipitation is shown in Figure 8.

Means for a box bounded by $45^{\circ} \mathrm{E}$ to $105^{\circ} \mathrm{E}$ and $5^{\circ} \mathrm{N}$ to $30^{\circ} \mathrm{N}$ are plotted. The precipitation is segregated into grid scale and convective components and also segregated into precipitation over land and over water. Evaporation from sea surface is included. All fields are significantly increased when radiative transfor is included.

\section{Summary and Future Research}

Model results have been presented showing the effects of radiative transfer on mesoscale to regional scale circulations. Radiative transfer appears to be part of the organization mechanism for mesoscale circulation, most likely through interaction with the ice layer. The intensity and activity of a model atmosphere at longer time scales also depends on the radiative transfer.

Results from the mesoscale model will be compared to simple analytic models. More simulations without the surface energy budget will isolate the effect of atmospheric radiative transfer. The effect of explicit cloud physics on the radiation budget will be compared with parameterized clouds. The work on the role of radiative transfer on tropical monsoon and on east coast cyclogenesis will be continued 


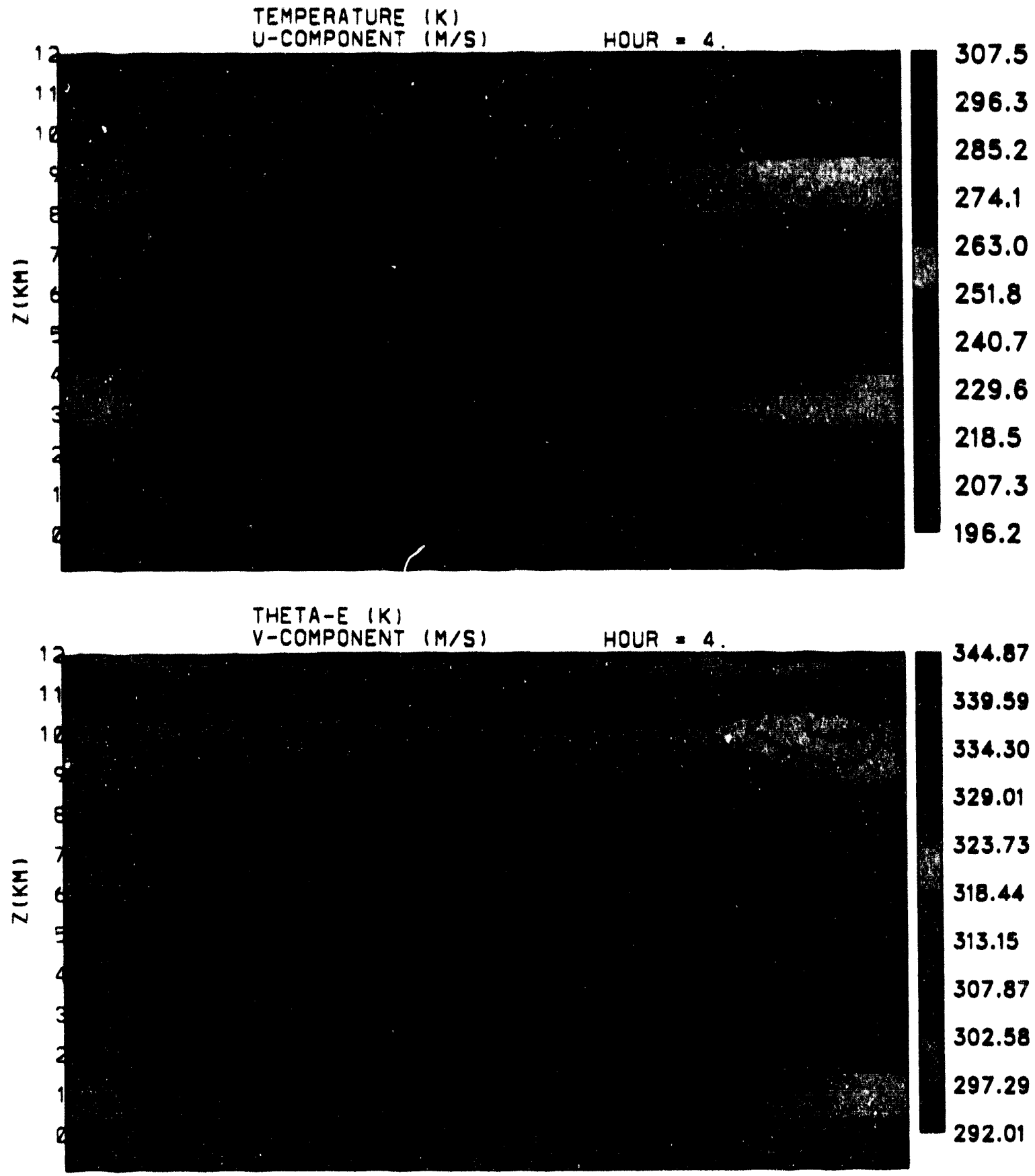

Figure 1. The upper panel is the u component of the wind superimposed on the temperature, and the lower panel is the $\checkmark$ component of the wind with the equivalent potential temperature. $500 \mathrm{~km}$ from the middle of a $1200-\mathrm{km}$ domain are presented. The results are from the explicit cloud physics model 4 hours into the simulation. This is for the case without radiation. 

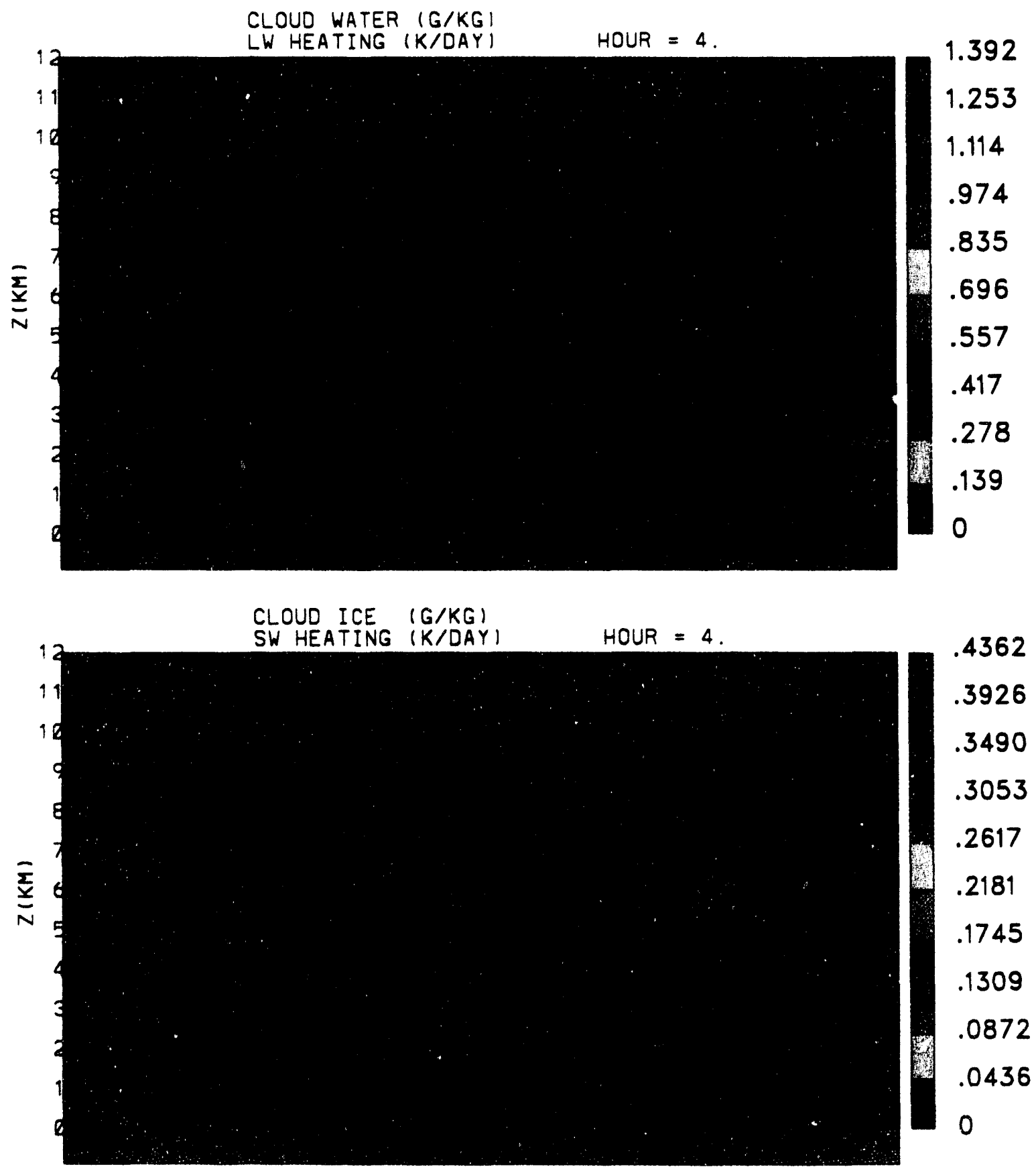

Figure 2. The upper panel is cloud water superimposed on the longwave heating, and the lower panel is cloud ice with shortwave heating. $500 \mathrm{~km}$ from the middle of a $1200-\mathrm{km}$ domain are presented. This is for the case without radiation. 

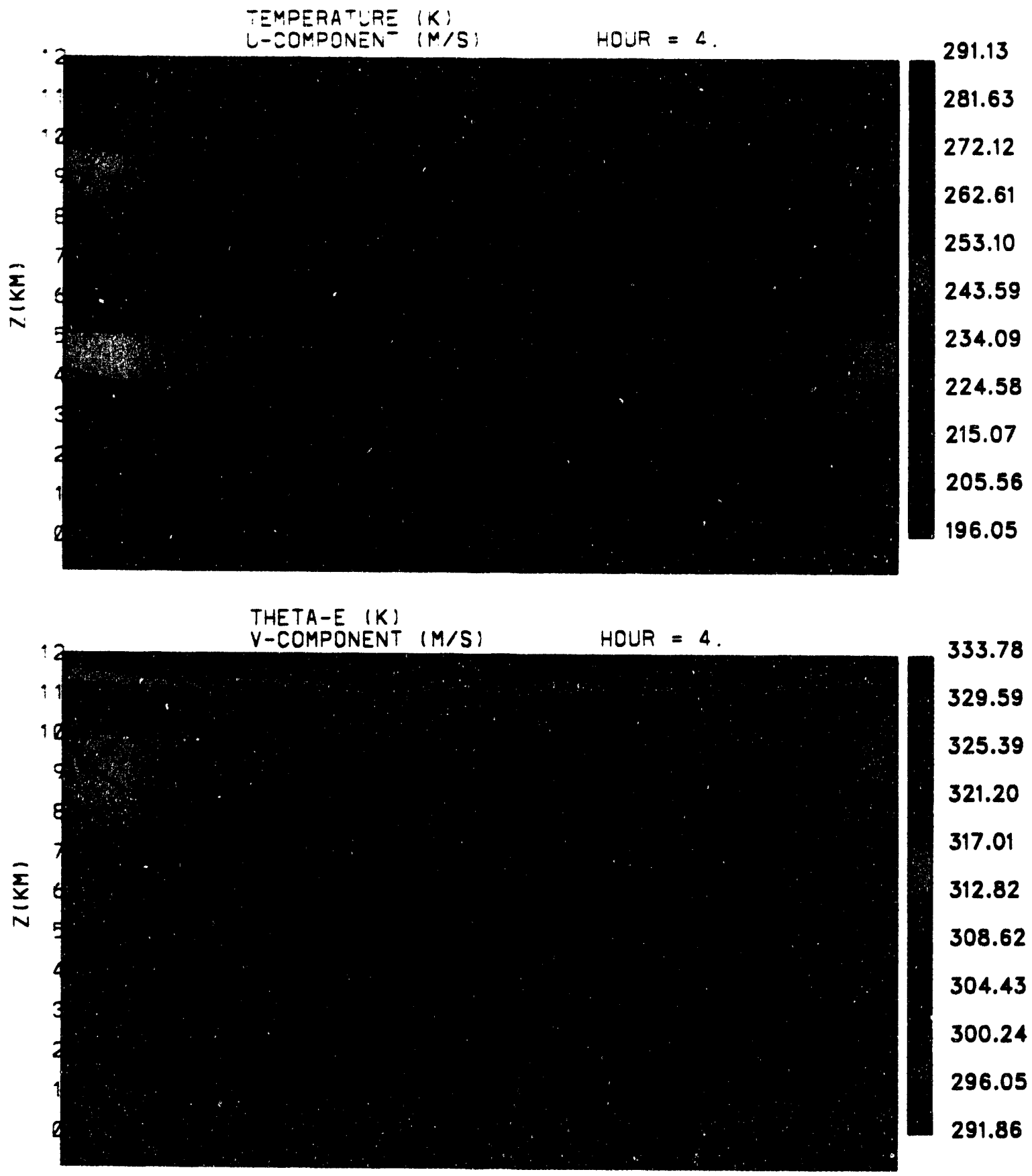

Figure 3. The upper panel is the $u$ component of the wind superimposed on the temperature, and the lower panel is the $\checkmark$ component of the wind with the equivalent potential temperature. $500 \mathrm{~km}$ from the middle of a $1200-\mathrm{km}$ domain are presentad. This is for the case with radiation. 

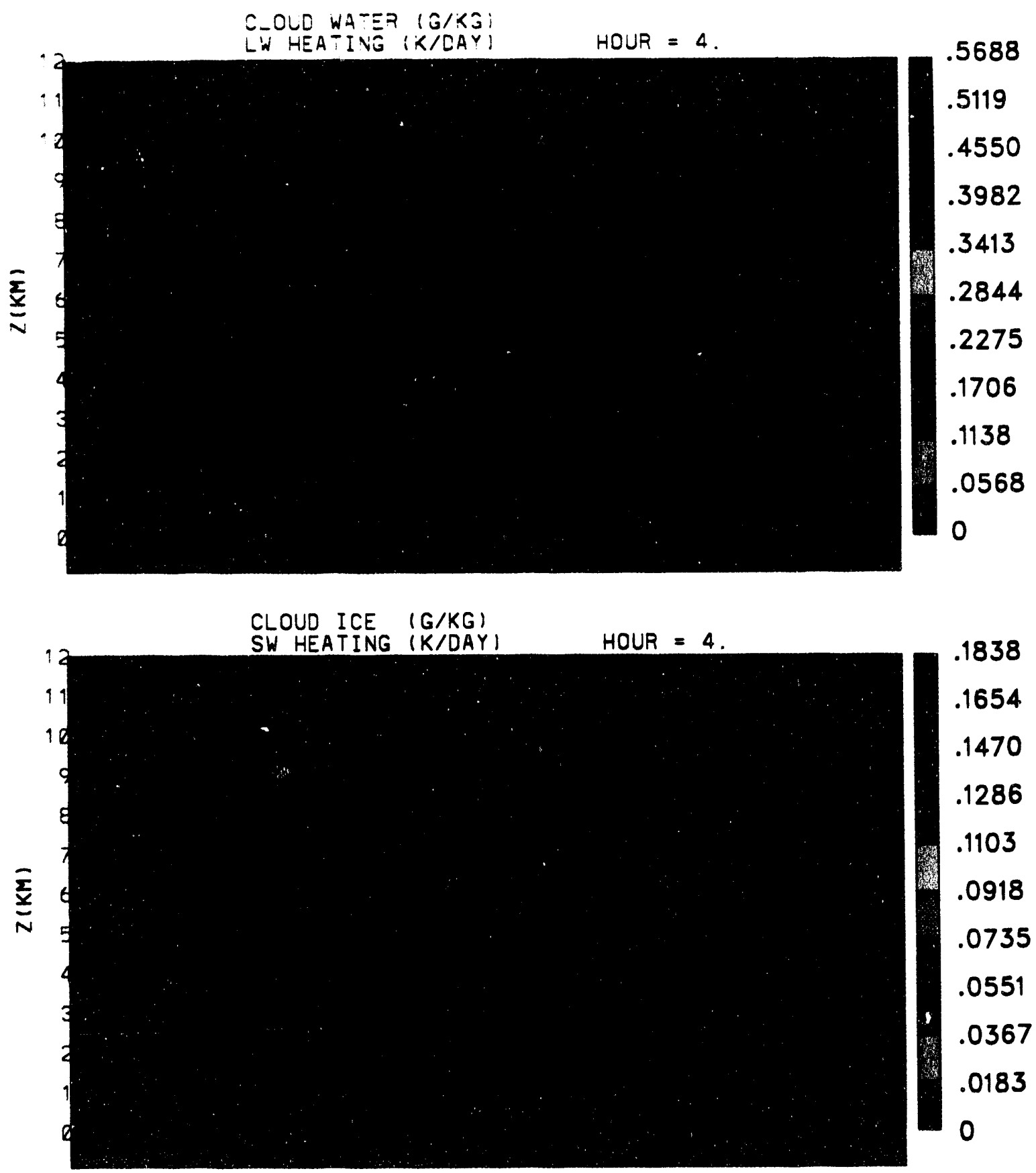

Floure 4. The upper panel is cloud water superimposed on the longwave heating and the lower panel is cloud ice with shortwave heating. $500 \mathrm{~km}$ from the middle of a $1200-\mathrm{km}$ domain are presented. This is for the case with radiation. 


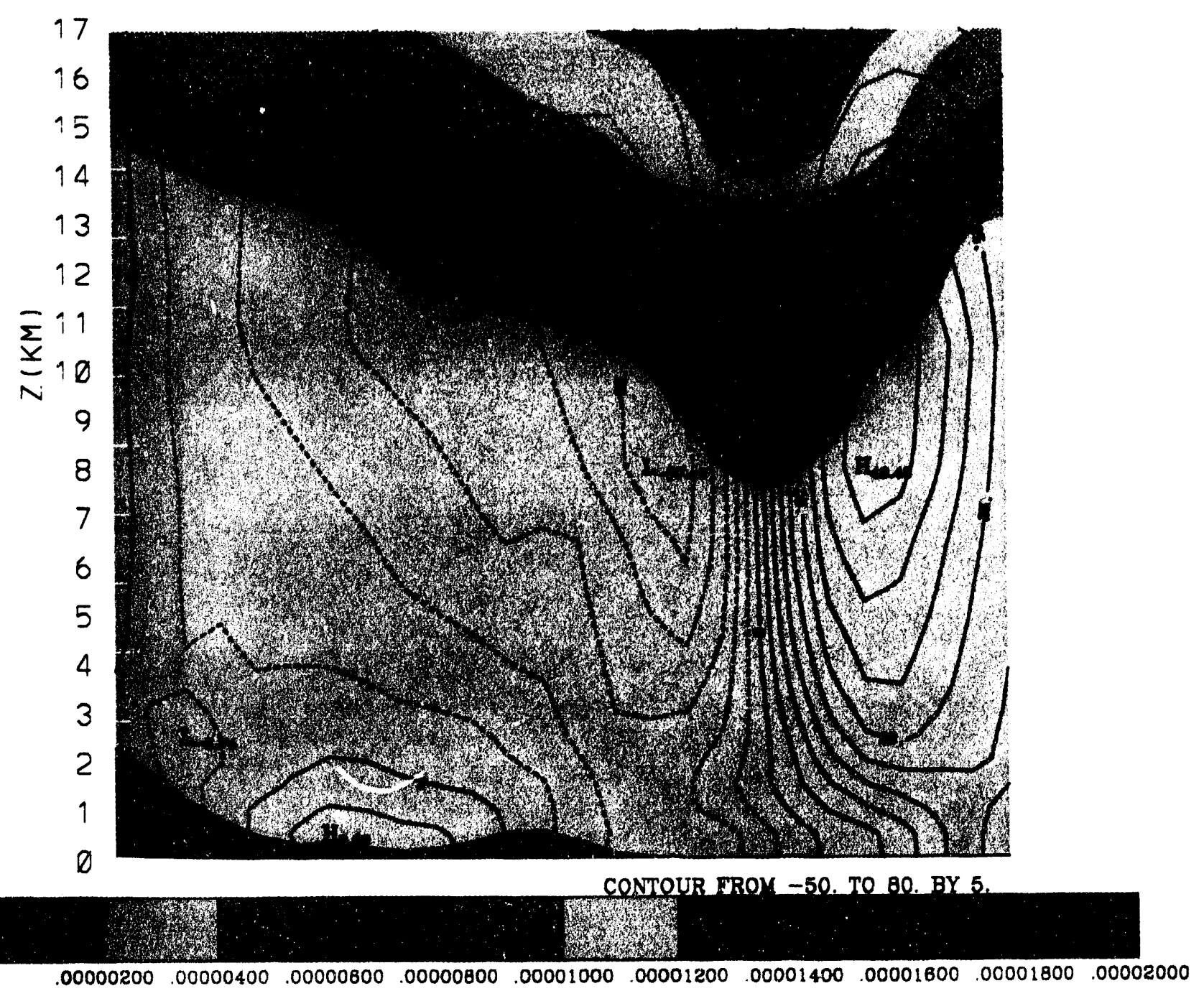

Figure 5. The meridional component of the geostrophic wind is superimposed on the isentropic potential vorticity for 108 hours into a forecast with the regional scale model. This is for the case without radiation. 

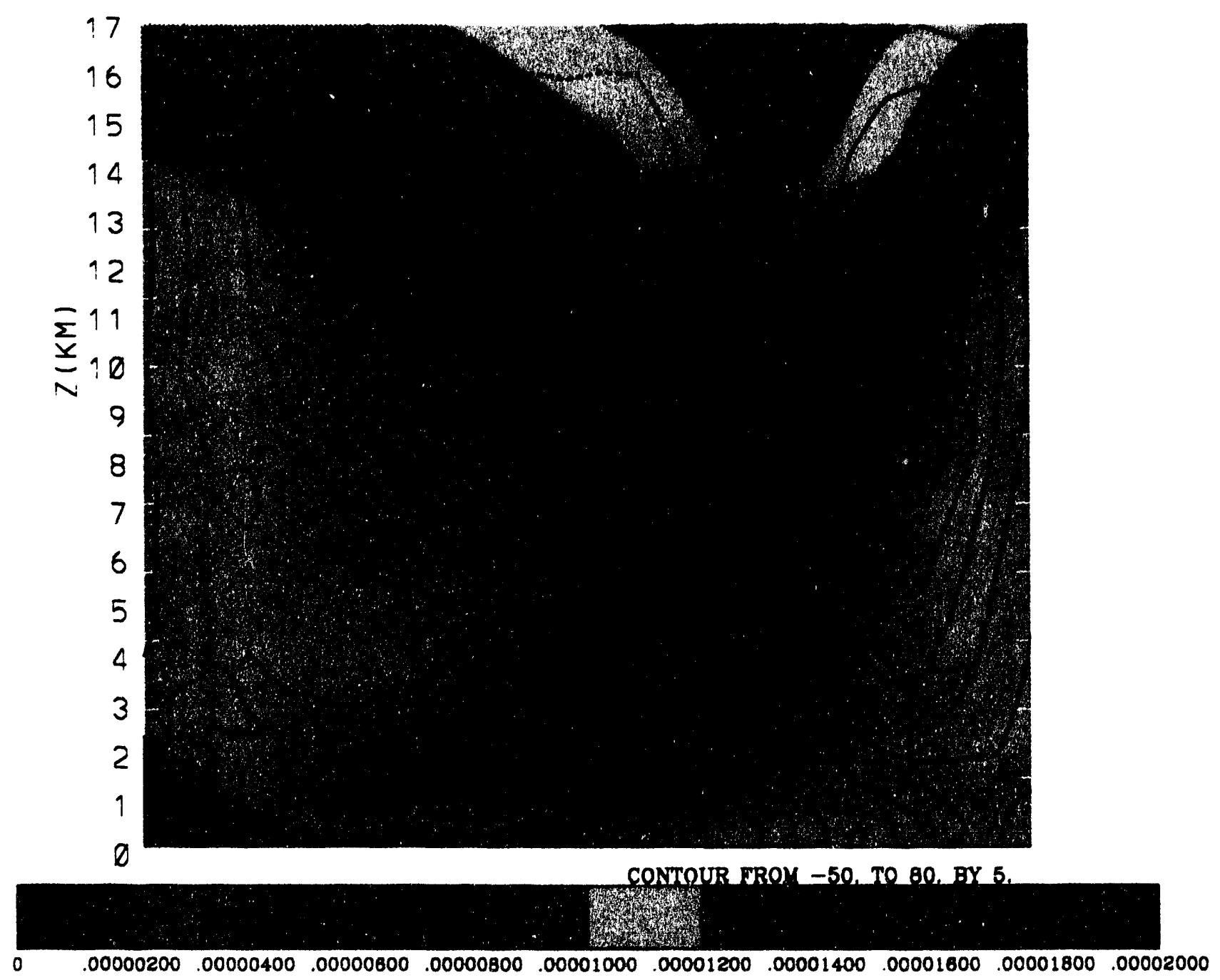

Figure 6. The meridional component of the geostrophic wind is superimposed on the isentropic potential vorticity for 108 hours into a forecast with the regional scale model. This is for the case with radiation. 

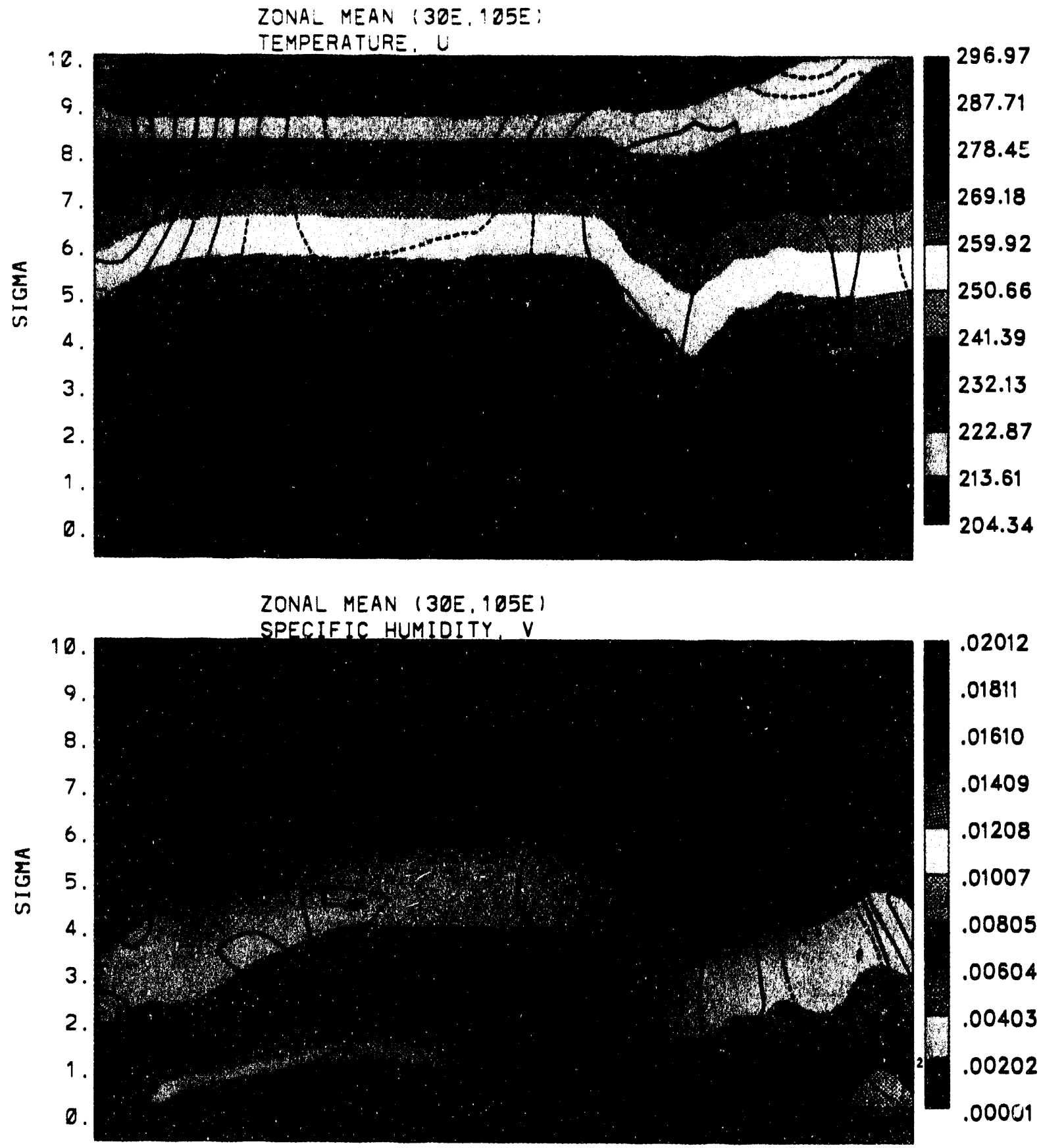

Figure 7. The upper panel is the zonal mean temperature with the zonal mean u component superimposed for a 10-day simulation with the regional scale model. The domain extends from $30^{\circ} \mathrm{S}$ to $60^{\circ} \mathrm{N}$. This is for the case without radiation. The lower panel is the zonal mean specific humidity with the zonal mean v-component superimposed. 


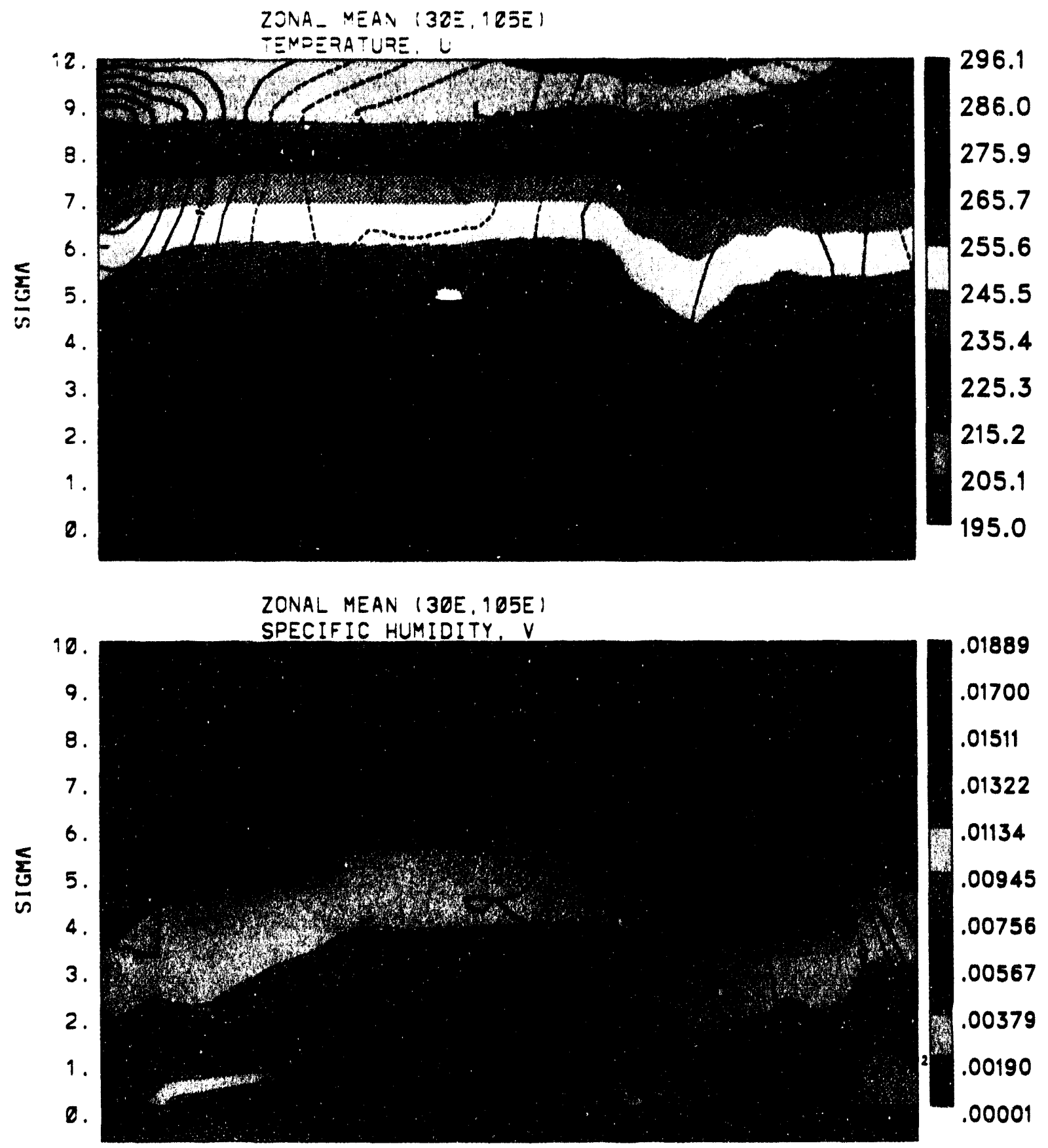

Figure 8. The upper panel is the zonal mean temperature with the zonal mean u component superimposed for a 10-day simulation with the regionai scale model. The domain extends from $30^{\circ} \mathrm{S}$ to $60^{\circ} \mathrm{N}$. This is for the case with radiation. The lower panel is the zonal mean specific humidity with the zonal mean v-component superimposed. 


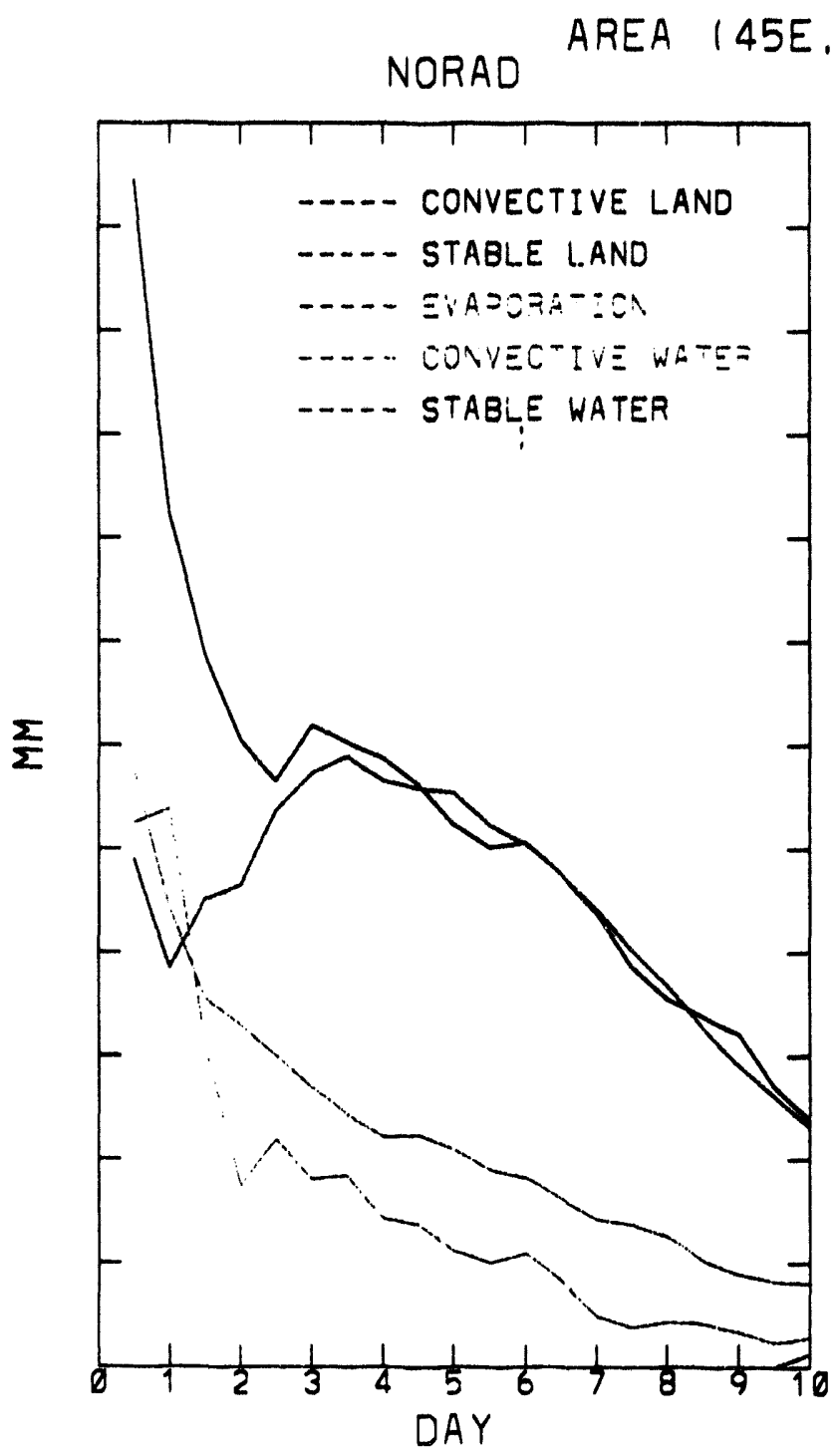

, 105E) (5N,30N)

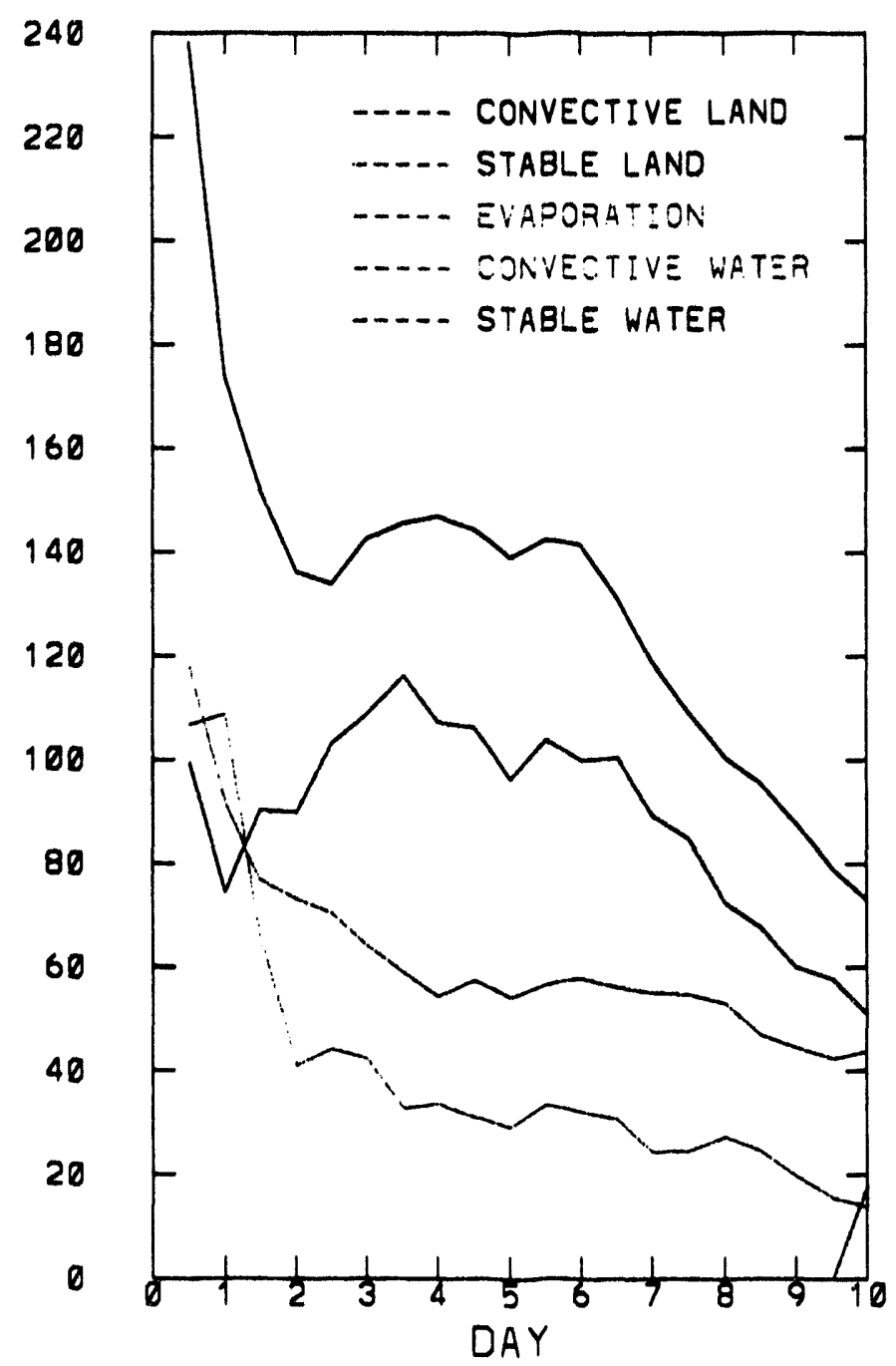

Figure 9. Mean water budget terms for the 10-day' simulation for a subdomain of the simulation. The subdomain is centered ove. the Indian subcontinent and extends from $5^{\circ} \mathrm{N}$ to $30^{\circ} \mathrm{N}$ and from $45^{\circ} \mathrm{E}$ to $105^{\circ} \mathrm{E}$. The labeling scheme is convective land represents convective precipitation from the model over land, convective water represents convective precipitation over water. The same scheme is used for stable precipitation. Evaporation from the sea surface is also displayed. 
and expanded, especially to look at the feedback to specific features. This includes looking at jot streaks in the extratropics and climatological features such as the Somali jot in the tropice.

\section{Acknowledgments}

This work is supported by the U.S. Department of Energy's Atmospheric Radiation Measurement (ARM) Program, under contract number 081575-A-QI with Pacific Northwest Laboratory. Computer time was provided by the DOE at the National Energy Research Supercomputer Center and by the North Carolina Supercomputing Center.

\section{References}

Harshvardhan, R. Davies, D. A. Randall, and T. G. Corsettl. 1987. A fast radiation parameterization for atmospheric circulation models. J. Geophys. Res. 92:1009-1016.
Holt, T., S. Chang, and S. Raman. 1980. A Numerical Study of the Coastal Cyclogenesis in GALE IOP2: Sensitivity to PBL Parameterization. Mon. Wea. Rov. 118:234-257.

Huang, C-Y., and S. Raman. 1991. Numerical Simulation of January 28 Cold Air Outbreak During GALE, Part II: The Mesoscale Circulation and Marine Boundary Layer. Bound.Layer Moteorol. 86:51-81

Louls, J.-F. 1979. A Parametric Model of Vertical Eddy Fluxes in the Atmosphere. Bound.-Layer Meteorol. 17:187. 202.

Paegle, J., W. G. Zdunkowski, and R. M. Welch. 1976. Implicif differencing of predictive equations of the boundary layer. Mon. Wea. Rev. 104:132-1324.

Rutledge, S. A., and P. V. Hobbs. 1984. The mesoscale and microscale structure and organization of clouds and precipltation in mid-latitude clouds. Part XII: A diagnostic modeling study of precipitation development in narrow cold frontal bands. J. Atmos. Sci. 41:2949-2972. 


\title{
Vegetation Forcing and Thermally Induced Generation of Mesoscale Circulation
}

\author{
X. Hong, M. J. Leach, and S. Raman \\ Department of Marine, Earth and Atmoepheric Sciences \\ North Carolina State Univereily \\ Rabigh, NC 27695-8208
}

\begin{abstract}
Surface inhomogeneities, including boundaries between different types of vegetations and land use patterns, have important effects on the structure of the atmospheric boundary layer. Changes in the surface roughness, temperature and wotness make the planetary boundary layer (PBL) nonhomogeneous and produce substantlal horizontal gradlents of boundary layerproperties. Significant differences in the surface thermal energy induce mesoscale circulations.
\end{abstract}

The presence of vegetation modulates the evaporation from the soll and enhances the vertical flux of water vapor into the PBL through transpiration. A realistic canopy formulation must ultimately represent the effects of vegetation on evaporation, energy partitioning, rainfall interception, and soil moisture, as well as albedo and aerodynamic roughness. Inclusion of canopy effects allows the deep-soil moisture (in the root zone) to act as a source for evapotranspiration.

Single-level canopy formulations are most appropriate for use in mesoscale models. We use a single-level canopy model (Deardorf 1978) that contains many parameters with which to evaluate fluxes from the soil beneath the canopy, from open areas between the canopy elements and from the foliage itself. This model can be used with a nonisothermal surface or a canopy for estimating vertical fluxes from the ground foliage system to the atmosphere.

This vegetation parameterization is included in a mesoscale model that has turbulent kinetic energy closure, explicit cloud physics, and a surface energy budget. The model is used to simulate mesoscale circulation between a vegetated area and a bare soil area. We also present the results of numerical experiments testing the sensitivity of mesoscale circulation to different underlying surface characteristics. For understanding the physical processes, the turbulent structure of the PBL is investigated as well.

\section{Model Descriptlons}

We have incorporated a vegetation parameterization into the NCSU mesoscale numerical model using a modified version of Deardortfs (1878) soll-vegetation scheme. Several numerical experiments have been conducted using the NCSU model (Huang and Raman 1991,1992; Boybeyl and Raman 1992) to determine the validity of this model for different meteorological conditions and topographic features.

Deardortfs scheme provides for the heat and water exchanges at the land surface. It includes a representation of a vegetation layer that interacts both with the soll surface and the air temperature. Ground surface temperature and the moisture are obtained by solving simultaneously the energy budget and water budget equations at the soll vegetation-air interface, respectively. The scheme allows for two layers in the soil and a single vegetation canopy. The prognostic equations for the soil temperature and soil water content are described below.

The ground surface temperature $T_{\theta}$ is calculated by a "force-restore" method:

$\frac{\partial T_{Q}}{\partial t}=-C_{1} \frac{H_{A}}{P_{2} C_{8} d_{1}} C_{2} \frac{T_{0}-T_{2}}{\tau_{1}}$

The mean deep soll temperature $T_{2}$ is

$\frac{\partial T_{2}}{\partial t}=-\frac{H_{A}}{\rho_{g} c_{s} d_{2}}$

where $H_{A}$ is the ground surface energy, $P_{g}$ is the soil density and $C_{8}$ is the soil thermal heat capacity. 
The moisture budget equation that describes the vertical diffusion proceseses for the ground surface and the root layer is

$\frac{\partial w_{q}}{\partial}=-C_{1} \frac{E_{q}+0.1 E_{q} \cdot P_{q}}{P_{\omega} d_{1}} \cdot C_{2} \frac{w_{q} \cdot w_{d}}{\tau_{1}}$

Where $w_{g}$ is volumetric concentration of soll moisture at the ground surface, and $w_{d}$ ls soll molsture content within the root layer. The time-dependent equation for $w_{d}$ is

$\frac{\partial w_{d}}{\partial t}=\frac{E_{q}+E_{q}-P_{g}}{P_{\omega} d_{2}}$

where $P_{0}$ is the precipitation rate at ground level.

The conservation equation for mass of liquid water retained by follage is given by

$\frac{\partial w_{\text {daw }}}{\partial t}=\sigma_{p} P\left(E_{f}-E_{t r}\right) 0 \leq w_{\text {dow }} \leq w_{d \text { max }}$

where $w_{\text {dew }}$ is mass of liquid water retained by follage per unit horizontal ground area, $w_{\text {dmax }}$ is maximum value of $w_{\text {dew }}$ beyond which runoff to soll occurs.

\section{Numerical Experiments and Results}

Results of three numerical experiments designed to investigate the effect of different soil types in inducing mesoscale circulations between the vegetated area and the bare soll area are presented in this paper. The three soil types are clay, loam and sand. Different parameters used for the three soil types are shown in Table 1. The vegetation characteristics and initial atmosphericconditions are the same for all three experiments. The initial surface tomperature la $300 \mathrm{~K}$ with a potential temperature lapee rate of $+3.5 \mathrm{Kkm}$. The amblent wind flold is $-1.0 \mathrm{~m} / \mathrm{s}$ The starting time of the experiments is 0800 local time for mid. summer conditions.

Differential soll heat capacity creates differences in the censible heaf flux from the ground to the atmosphere. However, there is a largor latent heat flux from the vegetated area as the vegetation efficiently pumps molsture into the atmosphere from the root zone. All three cases exhiblt the same general characteristics. The strength of the mescecale circulation varies as the bare soll characteristics change. Sandy soil has the lowest heat capacity and the highost albedo of the three soll types.

The mesoscale circulation that develope due to the differences between a vegetated area and sandy soll is the strongest of the three cases and is shown in Flgure 1. The upper frame is the $u$ component of the wind superimposed on the potential temperature field. The u component shown has the initial $-1.0 \mathrm{~m} / \mathrm{s}$ wind subtracted to highlight the induced circulation. The lower frame is the $v$ component of the wind with the actual temperature. The thermal fields show the creation of the baroclinicity in the boundary layer and also well-mixed layers to approximately $2 \mathrm{~km}$. The effect of the stronger surface sensible heat flux is evident as the ground temperature is as much as $15^{\circ} \mathrm{C}$ greater over the sandy soil. The differential heating throughout the boundary layer drives the convergence zone at the intertace between the bare soil and the vegetated area, with return flow at the top of the boundary layer.

The effects on moisture and vertical velocity are shown in Figure 2. The vertical velocity is superimposed on the specific humidity. The vertical velocities are consistent with the horizontal velocities with maximum values where the horizontal convergence is greatest. There is much more moisture through most of the boundary layer as vegetation effectively enhances the latent heat flux due to the transpiration from the root zone.

Table 1. Soil characteristics ( $K_{8}$ is the soll thermal diffusivity, $p_{8}$ is the soll density, and $C_{8}$ is the specific heat of the soil.).

\begin{tabular}{|c|c|c|c|}
\hline Soll Type & Sand & Loam & Clay \\
\hline$K s\left(m^{2}-s^{-1}\right)$ & $0.002 \times 10^{-4}$ & $0.0015 \times 10^{-4}$ & $0.012 \times 10^{-4}$ \\
\hline$P_{8} C_{8}\left(J^{-3} K^{-1}\right)$ & $1.2558 \times 10^{6}$ & $4.186 \times 10^{6}$ & $2.3 \times 10^{6}$ \\
\hline
\end{tabular}




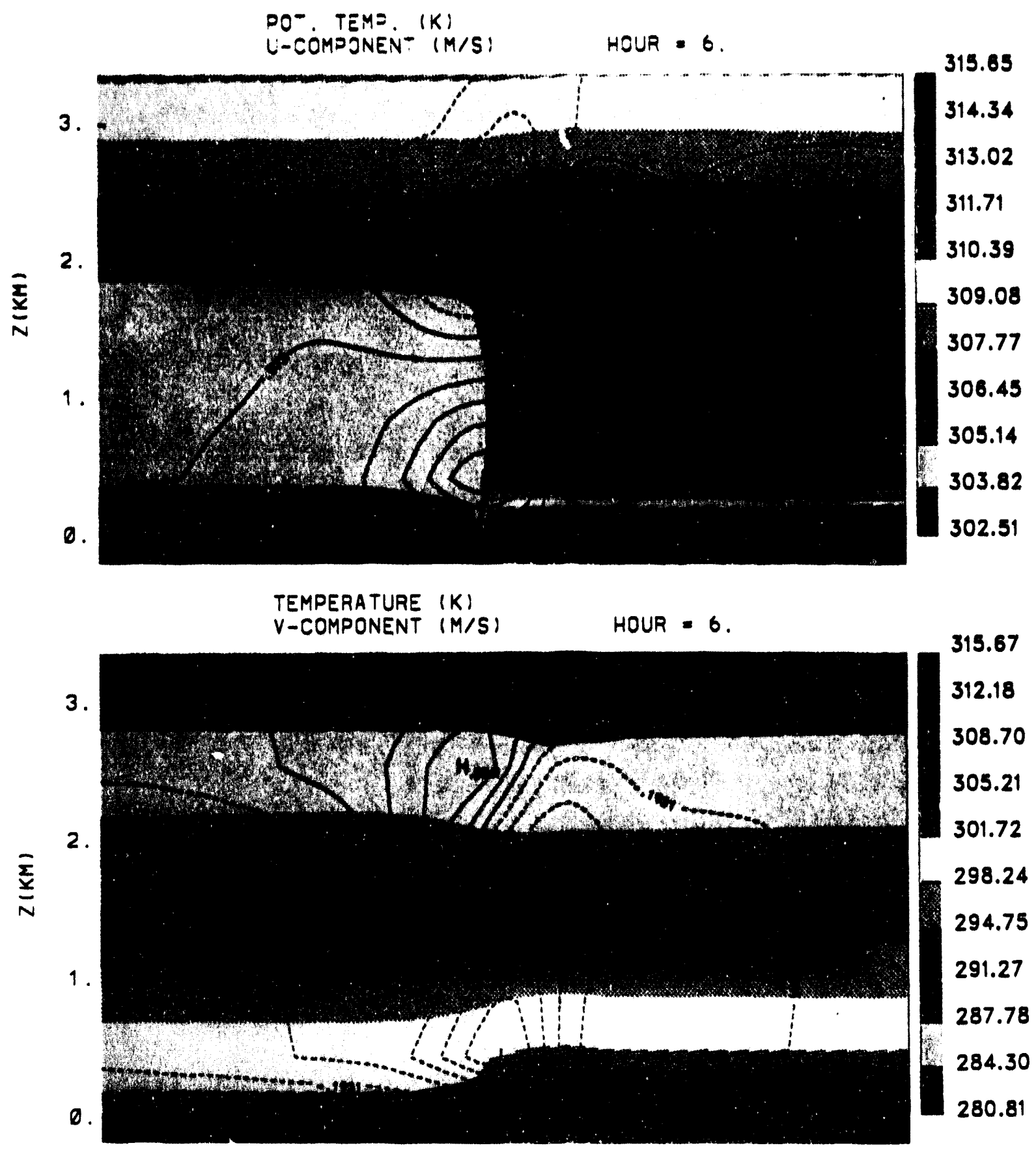

Figure 1. The upper panel is the $u$ wind superimposed on the potential temperature, the lower panel is the $v$ wind with the actual temperature. The results are from a 6 -hour simulation with the mesoscale model. The vegetated area is on the loft, sandy bare soil is on the right. 


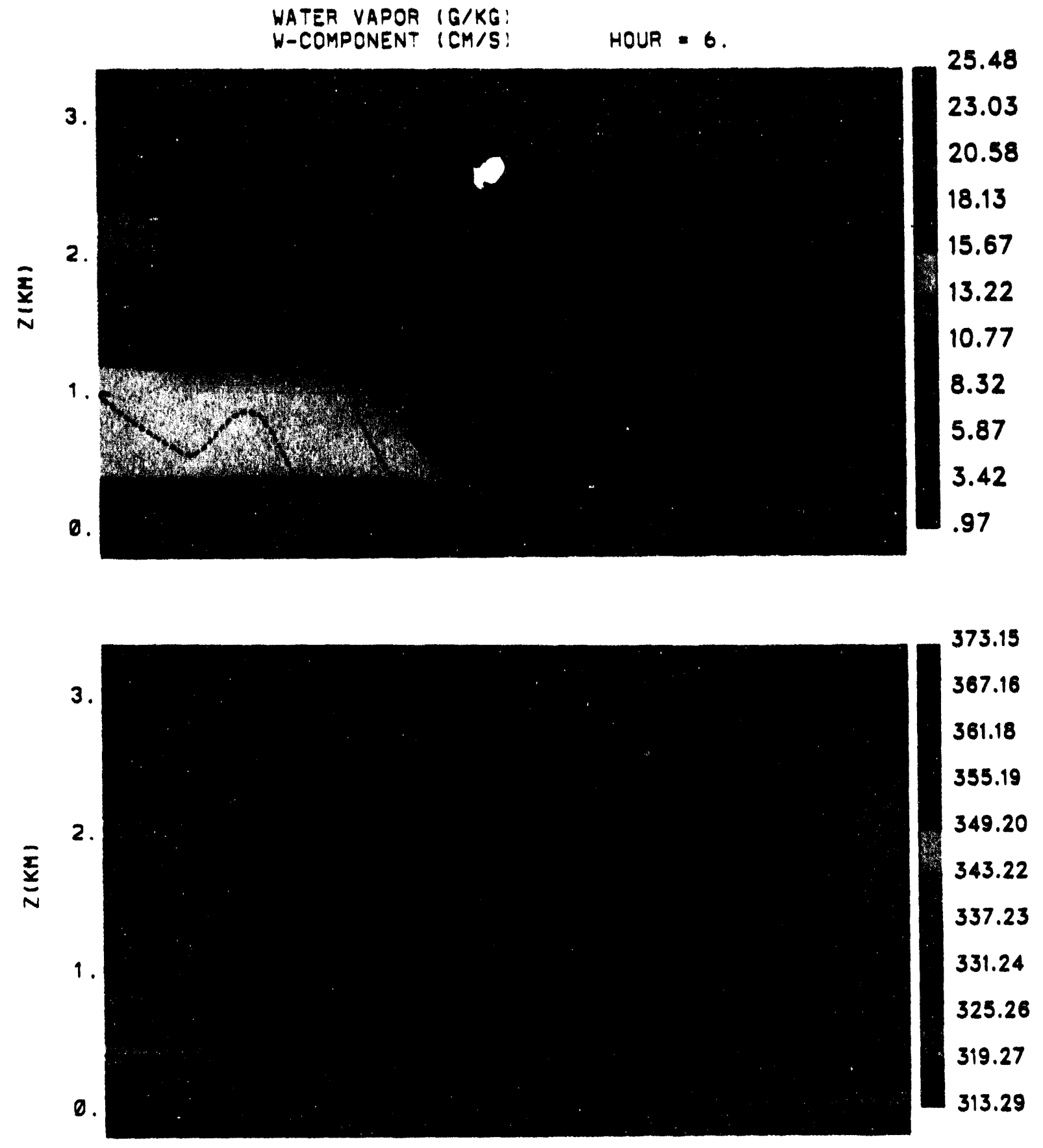

Flgure 2. The upper panel is the $w$ wind superimposed on the potential temperature, the lower panel is the $v$ wind with the actual temperature. The results are from a 6-hour simulation with the mesoscale model. The vegetated area is on the left, sandy bare soil is on the right. 
The effects on turbulence charactelatics are shown Figure 3. The upper frame is the turbulent kinetic energy (TKE) and eddy dittualvity for momentum $\left(K_{M}\right)$, the lower frame is the dissipation ( $\varepsilon$ ) of TKE with the eddy diffusivity for heat $\left(K_{H}\right)$ superimposed. The greater buoyancy production over the bare soll leads to much greater TKE, which in turn loads to greater mixing throughout the boundary layer. One could also infer this from the $K_{M}$ and $K_{H}$ distributions.

As mentioned, all three cases produced similar results. The differences among the cases wore in the intensity of the circulation that developed, the timing of the onsel of the circulation, and the subeequent development of a roturn circulation at the top of the PBL. The features of the three cases are presented in Table 2. The strongest circulation is associatod with the sandy soll, which has the lowest heat capacity but the highest albedo of the three soll types. The weakest circulation is with the clay soll, which has the highest heat capacity.

\section{Summary and Future Work}

The :esults discussed above indicate the effects of different surface characteristics, such as vegetation and bare soll heat capacity, on thermally induced mesoscale circulation. Vegetation increases water vapor flux in the boundary layer but decreases the sensible heat flux. Solls with different heat capacities alier the transfer of heat fluxes to the atmosphere, affecting the PBL structure. Solls with smaller heat capacity transfer more sensible heat, producing more TKE, larger eddy diffuslvity, and greater boundary layer heights. Our results indicate that the same vegetation cover with different bare soil combinations changes the strength and size of the mesoscale circulation.

In the future, we will extend the model to three dimensions, determine the effect of different soil covers and soll moisture on precipltation production, and couple the model with soif hydrology. We will use land use patterns from North
Caroina and Oklahoma. These reglons are two of the locales chosen for the U.S. Department of Enorgy's Atmospherle Radiation Measurement (ARM) Program. Obeervations from the 1991 and 1992 Boardman Regional Flux Exporiment will also be uead to validate the model. Effects of the resulting mesoscale circulation on the wator budgot, hydrology, and radiation budget will then bo investigated.

\section{Acknowledgments}

This work is supported by the U.S. Department of Energy's ARM Program, under contract number 091575-A-Ol with Pacific Northweet Laboratory. Computer time was provided by the DOE at the National Energy Research Suporcomputer Center and by the North Carolina Supercomputing Center.

\section{References}

Boybeyi, Z., and S. Raman. 1992. A three-dimensional numorical sensitivity study of convection over the Florida peninsula. Bound.-Layer Motoorol. 60:325-358.

Deardorti, J.W. 1978. Efficlent prediction of ground eurfeco temperature and molsture, with inclusion of a layer of vegetation. J. Geophys. Res. 20:1889-1803.

Garratt, J. R. 1992. The atmospheric boundary layer. Cambridge University Press.

Huang, C..Y., and S. Raman. 1991. Numerical simulation of January 28 cold air outbreak during GALE, Part l: The model and sensitivity tosts of turbulence closures. Bound.Layer Moterol. 58:381-407, 1991.

Huang, C.-Y., and S. Raman. 1992. A three-dimonsional numerical investigation of a Carolina cosstal front and the Gulf Stream rainband. J. Atmos. Sol. 49(7):560-584. 


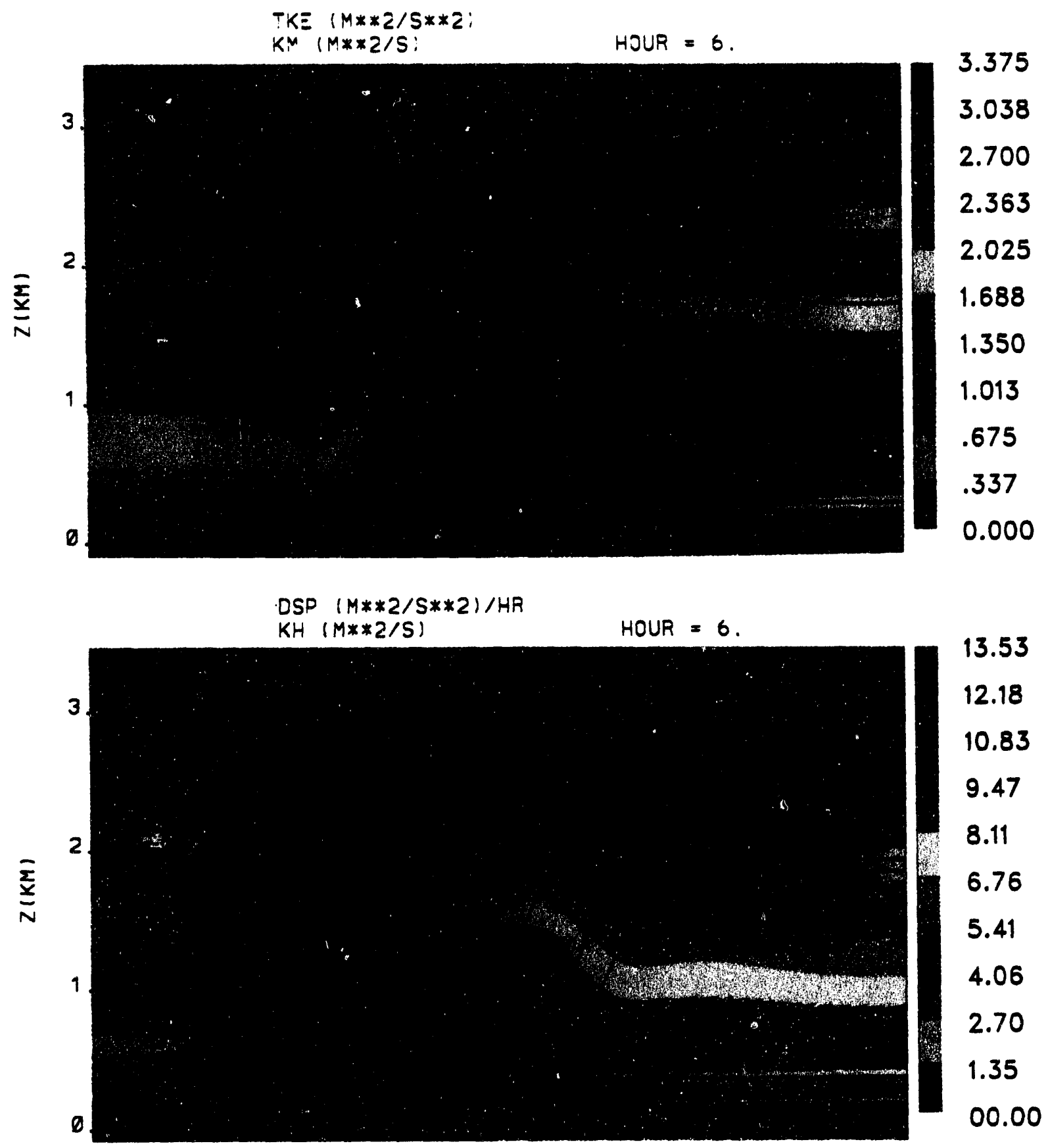

Flgure 3. The upper panel is the momentum eddy diffusivity superimposed on the turbulent kinetic energy (TKE), the lower panel is the scale eddy diffusivity with the dissipation of TKE. The results are from a 6-hour simulation with the mesoscale model. The vegetated area is on the left, sandy bare soil is on the right. 
Tablo 2. Characteristics of the circulation and PBL. in experiments with three soil types.

\begin{tabular}{|c|c|c|c|}
\hline Characteristic & Sand & Loam & Clay \\
\hline$U_{\max }(\mathrm{m} / \mathrm{s})$ & 2.9 & 1.8 & 0.8 (not closed) \\
\hline$U_{\min }$ (return) & -1.7 & -0.9 & -0.4 \\
\hline$V_{\max }$ (return) & 0.6 & 0.2 & 0.1 \\
\hline$V_{\min }$ & -0.9 & -0.3 & -0.3 \\
\hline TKE $\left(m^{* *} 2 / s^{* *} 2\right)$ & 3.4 & 2.7 & 1.7 \\
\hline$K_{M}\left(m^{* *} 2 / s\right)$ & 204.4 & 132.3 & 82.5 \\
\hline$K_{H}$ & 271.8 & 173.6 & 110.5 \\
\hline$T_{\text {onset }}$ (hour) & 1 & 2 & 5 \\
\hline$T_{\text {retum }}$ & 2 & 3 & 6 \\
\hline
\end{tabular}

(a) All results are for 6-hour simulation time, except the last two rows which are the estimated time of onset of the mesoscale circulation and the development of the return flow at the top of the PBL, respectively. 


\title{
Boundary-Layer Observations Over the Southern Great Plains Cloud and Radiation Testbed Site During the November Flux-Profiler Test
}

\author{
S. P. Oncley and J. Van Baelen \\ National Center for Atmospheric Research(a) \\ Boulder, CO 80307-3000
}

\section{Introduction}

In situ flux measurements were made to compare to the momentum flux profiler being designed by the National Center for Atmospheric Research (NCAR) as part of the Instrument Development Program for the Atmospheric Radiation Measurement (ARM) Program.

The NCAR King Air aircraft took about 10 hours of flux data during the period November 12-17, 1992, near the Southern Great Plains (SGP) Cloud and Radiation Testbed (CART) site central facility with the following characteristics:

- Flight legs were centered over the prototype profiler.

- Measurements of $u^{\prime}, v^{\prime}, w^{\prime}, T^{\prime}$, and $q^{\prime}$ were made to allow direct calculation of momentum and sensible and latent heat fluxes by eddy-correlation.

- Legs were $40 \mathrm{~km}$ long to obtain fluxes to $10 \%$.

- Legs were at altitudes corresponding to profiler range gates and covering the bottom to the top of the planetary boundary layer (up to $1 \mathrm{~km}$ ) to observe a large variation of fluxes.

The profiler was operated in Doppler beam swinging (DBS) mode while also processing the signal using interferometry.

Radiosondes were launched from both the central facility and the flux-profiler site to provide measurements of wind, temperature, and humidity above the boundary layer for input to a mesoscale model and to provide an additional comparison for measurements made in the boundary layer.

(a) NCAR is sponsored by the National Science Foundation.

\section{Results}

Figure 1 shows the raw data from two of the ten profile patterns flown during this experiment. Each aircraft profile consisted of a descent to the profiler site followed by 3-5 legs at increasing heights. Note that because of this flight pattern, the downward and upward profiles may differ due to temporal changes during the time of the pattern (about 50 minutes) and due to horizontal gradients.

The profiler was operated in DBS mode alternating between two sets of three beams. Thus, two sets of consensusaveraged winds were available. Finally, several radiosondes were launched during this experiment which may be compared, though they generally were notlaunched exactly when the aircraft was overhead for safety reasons.

The wind profiles agree reasonably well. In both profiles, the two 3-beam DBS estimates are similar, indicating that the air flow was reasonably homogeneous. The temperature and humidity profiles were measured primarily to determine the inversion (and thus boundary layer) height. The nearest radiosonde to the flight pattern starting at 20:18 was launched at 22:34 and shows a deeper, cooler, and drier boundary layer.

Figure 2 shows the fluxes which were computed from the aircraft flight legs using the covariance of horizontal velocity, potential temperature (to remove variations of the aircraft altitude), and humidity with the vertical velocity. The variation of the flux profiles with time may be seen from the two patterns shown. In both cases, the momentum flux profile decreases to nearly zero at the top of the boundary layer as expected. The sensible heat flux profile also shows a decrease with height in both cases. The 18:41 sensible heat flux profile shows a change in sign at the top of the boundary layer due to entrainment of warmer air as the 

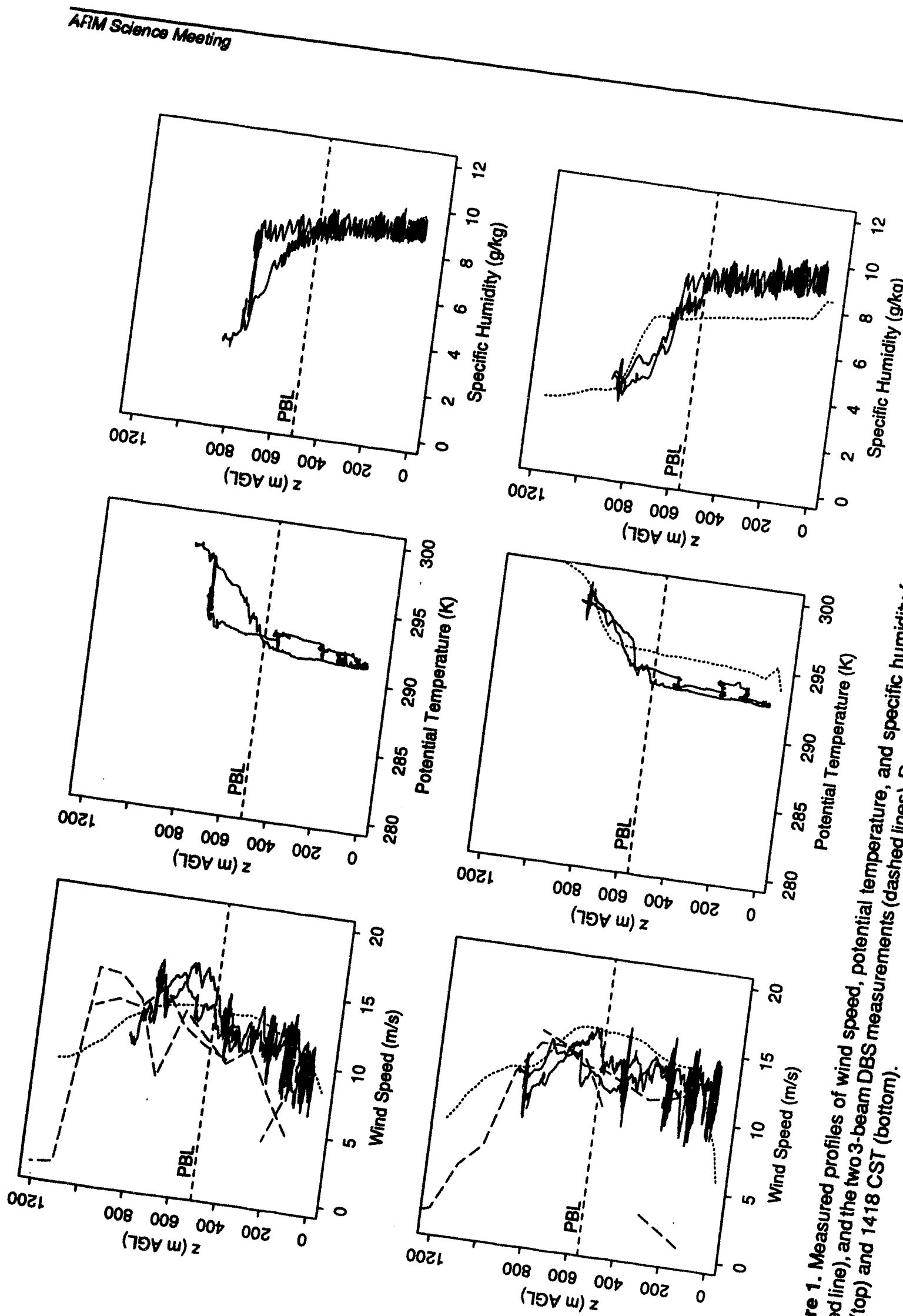

172 

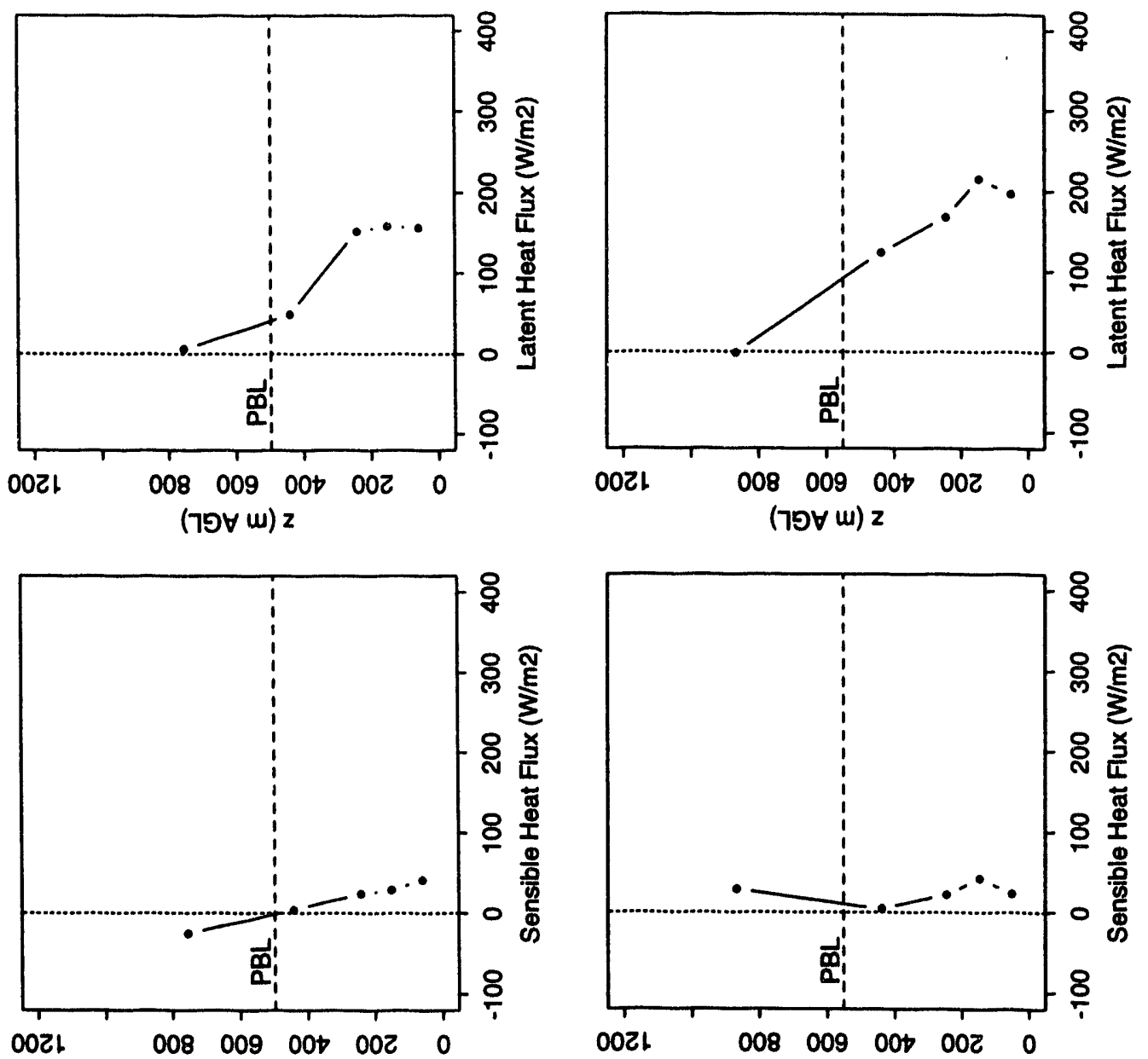

( $(\supset \forall \omega) ~ z$
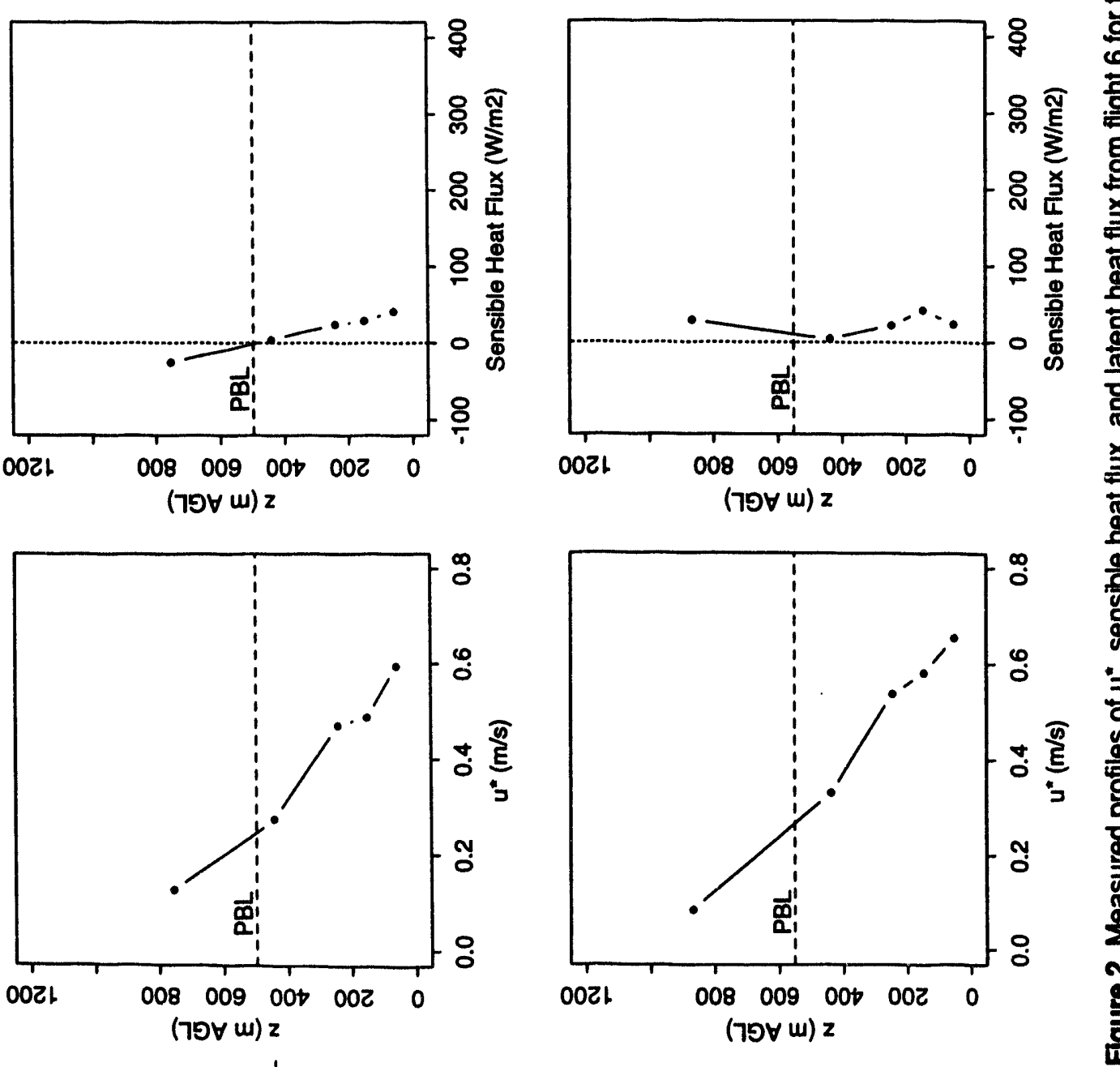
boundary layer grew in the middle of the day. Also, the sensible heat flux decreases in magnitude in the late afternoon. The latent heat flux profiles are somewhat surprising since they do not decrease as rapidly with height. Notice that the latent heat fluxes are larger than the sensible heat, presumably because of strong evaporation after heavy rains in the area just before the experiment.

\section{Future Work}

- Fluxes will be computed from the DBS data. These are expected to be poorer than from the interferometric results since horizontal homogeneity of the fluxes must be assumed for DBS.
- Fluxes will be computed from the interferometric data on each of the 5 beams.

- All profiler flux measurements will be compared with aircraft measurements. 


\title{
Comparison of Mesoscale Model and Tower Measurements of Surface Fluxes During Winter Icing and Storms Program/Atmospheric Radiation Measurement 91
}

\author{
S. P. Oncley and J. Dudhia \\ National Center for Atmospheric Research(a) \\ Boulder, CO 80307-3000
}

\section{Introduction}

This study is an evaluation of the ability of the Pennsylvania State University/National Center for Atmospheric Research (NCAR) mesoscale model (MM4) to determine surface fluxes to see if measured fluxes should be assimilated into model runs.

Fluxes were compared from a high-resolution $(5 \mathrm{~km}$ grid spacing) MM4 run during one day of the Winter lcing and Storms Programs/Atmospheric Radiation Measurement (WISPIARM) experiment (over NE Colorado in winter 1991) with direct flux measurements made from a tower over a representative site by a three-dimensional sonic anemometer and fast response temperature and humidity sensors. This tower was part of the NCAR AtmosphereSurface Turbulent Exchange Research (ASTER) facility. Also, mean values were compared to check whether any differences were due to the model parameterization or model variables.

\section{Model Parameterization}

The "high-resolution" Blackadar parameterization in MM4 was used. This parameterization uses flux-profile relationships applied to values at a single height, z:

$u^{*}=[k U / \ln (z / z 0) \cdot p h I[m]$

$T^{*}=[k(T \cdot T 0)] / \ln (z / 20) \cdot$ phi_h]

$q^{*}=\left\{M k\left[q-q \_s(T 0)\right]\right\} /\left(\ln \left(\left(K u_{-}=2 / K_{-} a\right)+z / z \mid\right) \cdot p h l \_h\right\}$

RI_b $=\left[g^{2}(T \cdot T O)\right] / T U^{\wedge} 2$

(a) NCAR is sponsored by the National Science Foundation.

$$
\begin{aligned}
& \text { phi_m(Ri_b<0) }=-1.86 \text { Ri_b } \ln (z / 20)-1.07\left[R I \_b \ln (z / 20)\right]^{\wedge} 2 \cdot \ldots \\
& \left(R I \_b>0\right)=[-5 \text { RI_b } \ln (z / z 0)] /[1.1 \text {. } 5 \text { RI_b] } \\
& \text { phi_h(RI_b<0) }=-3.23 \text { RI_b } \ln (z / 20)-1.99\left[R I \_b \ln (z / 20)\right]^{\wedge} 2 \cdot . . . \\
& \left(R i \_b>0\right)=\left[-5 R i \_b \ln (z / 20)\right] /\left[1.1-5 R i \_b\right]
\end{aligned}
$$

\begin{tabular}{|c|c|c|}
\hline 20 & Surface roughness height & $10 \mathrm{~cm}$ \\
\hline $\mathbf{z l}$ & Molecular layer depth & $1 \mathrm{~cm}$ \\
\hline M & Moisture availability & 0.3 \\
\hline \multicolumn{3}{|c|}{ Finally, the following values for constants were used: } \\
\hline & Von Karman constant & 0.4 \\
\hline & Prandtl number & 1 \\
\hline -a & Molecular diffusivity & $2.4 \times 10^{\wedge}-5 \mathrm{~m}^{\wedge} 2 / \mathrm{s}$ \\
\hline
\end{tabular}

The ASTER site was assigned the land-use category of "Range-Grassland" for which the iollowing values for parameters are used:

\section{Results}

Variables which are used by the model parameterization were compared to separate the effect of the data and the parameterization in the flux calculation. Since the lowest model level was for a height of $36 \mathrm{~m}$, and the highest ASTER tower was at $10 \mathrm{~m}$, higher data from remote sensors are also shown.

The parameterization for the friction velocity $u^{*}$, which is proportional to the square root of the momentum flux, depends primarily on wind speed. For the WISPIARM experiment, the wind comparison (Figure 1) appears reasonable. The model wind speed generally falls between 

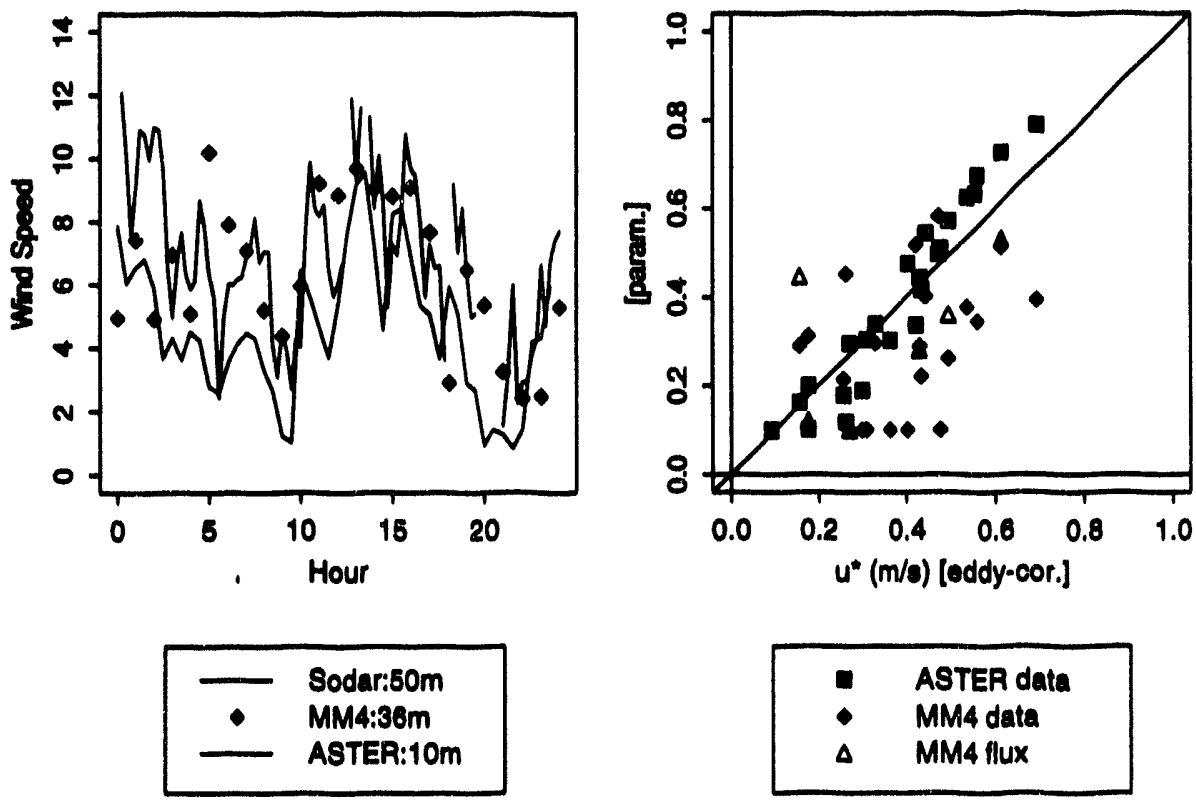

Flgure 1. Time series of wind speed from ASTER tower measurements at $10 \mathrm{~m}, \mathrm{MM} 4$ calculations for $36 \mathrm{~m}$, and sodar measurements at $50 \mathrm{~m}$. Also shown is a scatter plot of u" calculated from the MM4 parameterization by the model, external to the model using MM4 data, and external to the model using ASTER measurements.

the ASTER measurement at $10 \mathrm{~m}$ and a nearby sodar measurement at $50 \mathrm{~m}$. The $\mathrm{u}^{*}$ comparison shows that fluxes calculated by MM4 are within a factor of 2 , however better agreement is expected. The $u^{*}$ comparison also shows values manually computed from the MM4 hourly wind speed data, since values of fluxes computed by the model were only available every 4 hours for these runs. These values again show quite a bit of scatter about a $1: 1$ line. Finally, $u^{*}$ values computed from the wind speed measured by ASTER agree quite well, indicating that the parameterization works when appropriate data are used.

The parameterization for $T^{*}$, which is proportional to the sensible heat flux, depends primarily on the air-ground temperature difference. Figure 2 shows that air temperatures from MM4 were higher at night and lower during the day than ASTER measurements, which is expected since the MM4 values are for a height of $36 \mathrm{~m}$, though the magnitude of this change is too large. Radio acoustic sounding system (RASS) measurements of temperature at $500 \mathrm{~m}$ always are lower, presumably because $500 \mathrm{~m}$ is above the height of the nighttime boundary layer. Ground temperatures from MM4 are much lower during the night and rise approximately to the air temperature measured by ASTER at $1 \mathrm{~m}$ during the day. The infrared surface temperature measured by ASTER does not agree better with the model since it exhibits a much larger diurnal change. A soll temperature measurement made at $3 \mathrm{~cm}$ depth shows a similar diurnal change to the model, but has a lag due to the time required to heat the soil. Thus, the air-ground temperature differences are too large from the model at night, however this is seen to have little effect on $\mathrm{T}^{*}$ (the positive values). During the day, the model values of T* agree well with the ASTER eddy-correlation measurements; however, values calculated by applying the parameterization to the ASTER $1 \mathrm{~m}$ air or surface temperature measurements were quite high and low, respectively. Therefore, MM4 appears to produce ground temperatures which are consistent with its flux parameterization, and these temperatures are representative of a height between the surface and $1 \mathrm{~m}$.

The parameterization for $q^{*}$, which is proportional to the latent heat flux, depends on the air-surface humidity gradient, with the surface value calculated from saturated conditions at the ground temperature. The model humidity 

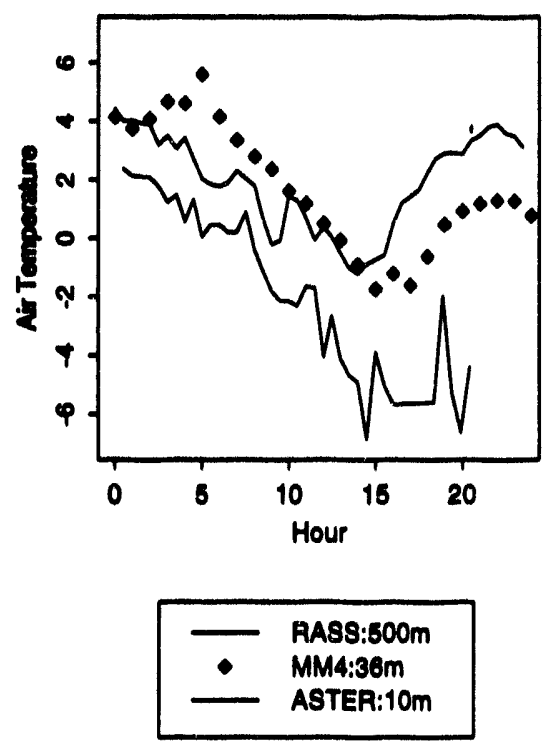
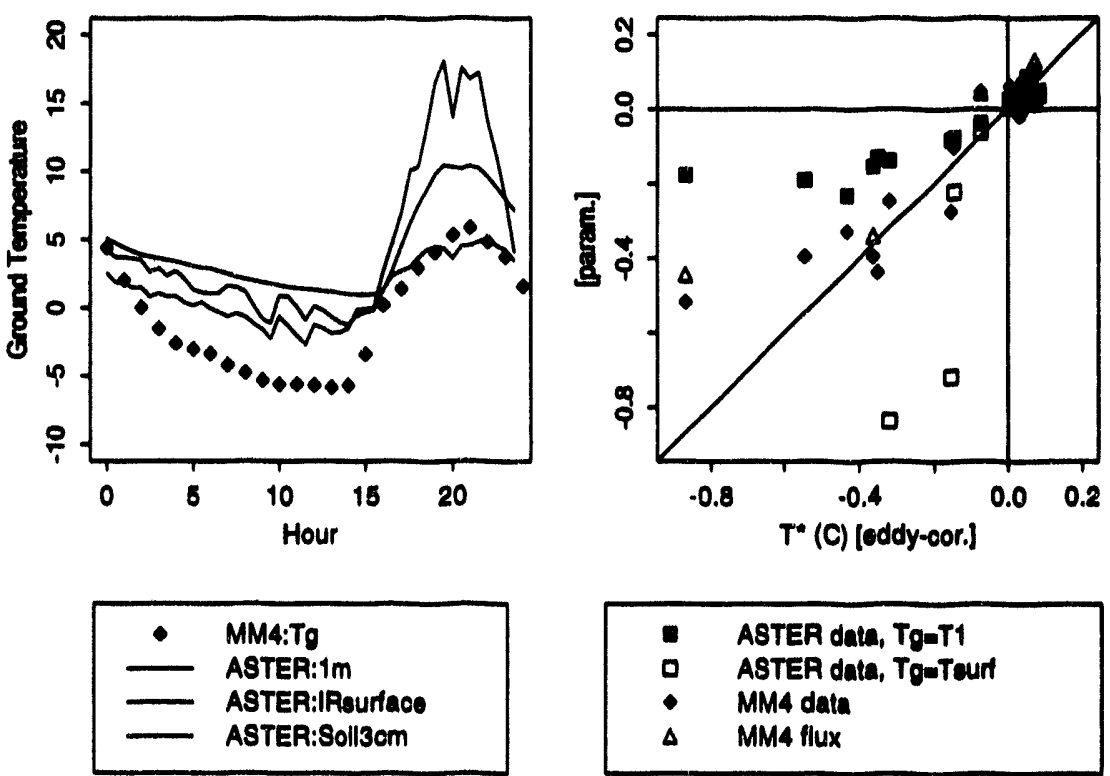

ASTER data, To-T1 ASTER data, TgeTeurt MM4 data MM4 flux

Figure 2. Time series of air and ground temperature from ASTER measurements, MM4 calculations, and RASS measurements. Also shown is a scatter plot of $T^{*}$ calculated from the MM4 parameterization by the model, external to the model using MM4 data, and external to the model using ASTER measurements with Tg set to both the infrared surface temperature and to the lowest measured air temperature.

values (Figure 3) are reasonably close to the only measurement of humidity made by ASTER for much of the period, though a frontal passage was too early by about 5 hours. The $q^{*}$ comparison shows that MM4 underestimates the flux by about $50 \%$. However, using the ASTER $1 \mathrm{~m}$ air or surface temperature measurements for the ground temperature ylelds values for $q^{*}$ which bracket a 1:1 line. Apparently, the conclusion from the $T^{*}$ comparison that the ground temperature should be between the surface and $1 \mathrm{~m}$ remains unchanged. Since the $T^{*}$ comparison from the MM4 data used the same ground temperature as the $q^{*}$ comparison, it must be concluded that good T" agreoment was due to the low daytime MM4 air temperatures.

\section{Conclusions}

- The momentum flux parameterization works well for these data, with the observed differences probably due mostly to areal averaging by the model.

- The sensible heat flux parameterization is very sensitive to the value used for the ground temperature; however, using the model alr and ground temperatures gave fluxes which agreed quite well with direct measurements.

- The latent heat flux also is sensitive to the ground temperature. The model data yielded values which were low by about $50 \%$. 

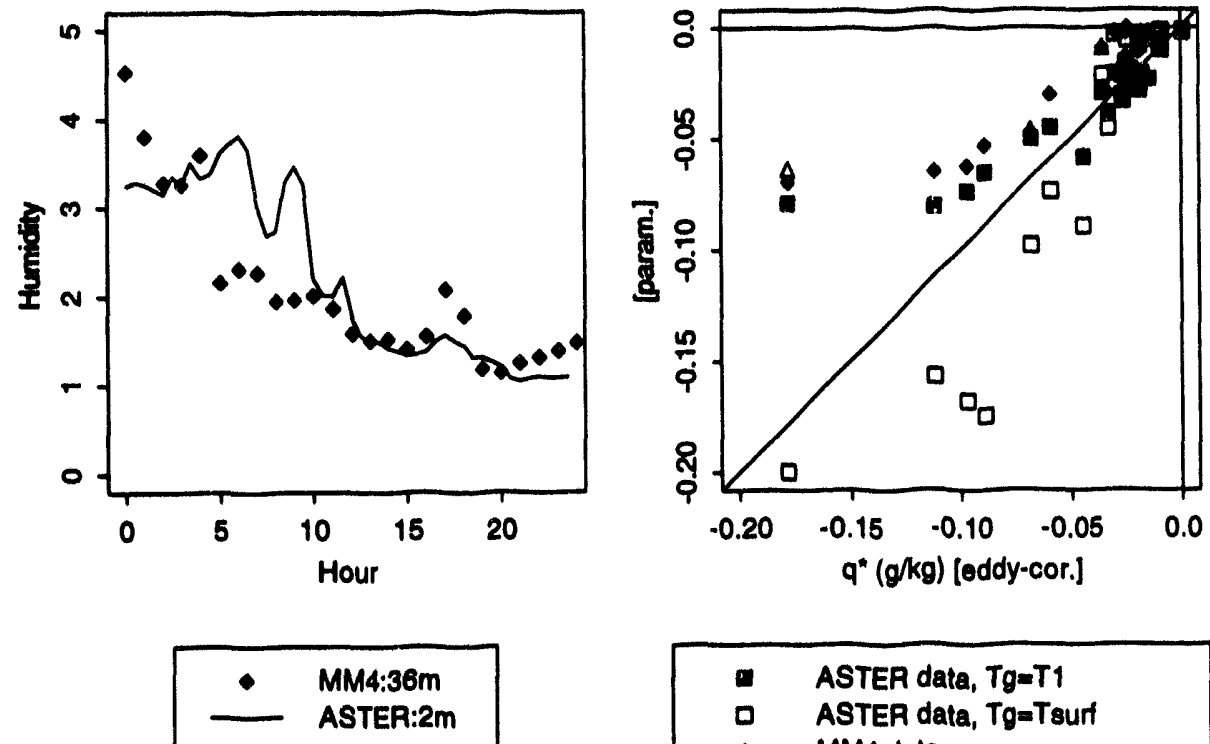

- ASTER data, Tg=T1

- ASTER data, Tg=Tsurf

- MM4 data

$\triangle \quad$ MM4 flux

Figure 3. Time series of specific humidity from ASTER measurements and MM4 calculations. Also shown is a scatter plot of $q^{*}$ calculated from the MM4 parameterization in the same manner as in Figure 2.

- The appropriate ground temperature probably is the air temperature at the height of the roughness length.

\section{Future Work}

Data will be examined from another case (the Storm-Scale Observations Regional Measurement Program.Fronts
Experiment Systems Test [STORM-FEST] experiment in $N$ Kansas during early 1992) to determine if the results shown here are specific to the flow regime during WISP/ ARM. Aircraft measurements will be added to the comparison (available during STORM-FEST) to examine possible differences between tower point sampling, aircraft line sampling, and model area averaging. 


\section{Hierarchical Diagnosis}




\title{
A Hierarchical Approach to Improved Cloud Radiation Parameterization for Climate Models Through the Atmospheric Radiation Measurement Program
}

\author{
J. T. Kiehl, M. W. Moncrieff, J. J. Hack, and W. Grabowski \\ National Center for Atmospheric Research \\ Boulder, CO 80307-3000 \\ V. Ramaswamy \\ Geophysical Fluid Dynamics Laboratory \\ Princeton, NJ
}

\section{Introduction}

Improved parameterization of c'suds for general circulation models will require both ARM observations and the use of more detailed cloud models. We have adopted a parallel implementation approach to improve cloud parameterizations by including identical cloud radiative processes into three models that span the important spatial and temporal scales for cloud research. These models include a one-dimensional detailed ice microphysical model, the Clark cumulus ensemble model, and the Community Climate Model (CCM2) of the National Center for Atmospheric Research (NCAR).

The development of the detailed ice microphysics model is being carried out under National Aeronautics and Space Administration (NASA) Earth Observing System (EOS) funding, but will be of value in validating the bulk ice parameterization used in the cumulus ensemble model. It employs a vertical resolution of $50 \mathrm{~m}$. The Clark cloud model is currently run in two dimensions with a $1-\mathrm{km}$ horizontal resolution and a variable vertical resolution $(\Delta z=200 \mathrm{~m}$ in mid to upper troposphere). The domain extent of the cloud model is $1000 \mathrm{~km}$ in the horizontal and $30 \mathrm{~km}$ in the vertical. The NCAR general circulation model (GCM) is CCM2, the latest version of the CCM. This version of the CCM is a completely new model in both physics implementation and coding structure. Most importantly to the ARM research is the use of a $\delta$. Eddington solar radiation model with 18 spectral intervals (Briegleb 1992), a cumulus mass flux convective parameterization (Hack 1993), and a new cloud prediction scheme, which is an extension of the Slingo (1987) scheme.

\section{Inclusion of Ice Radiative Properties in the Hierarchy of Models}

The first parallel development effort was to implement a column version of the $\mathrm{CCM} 2$ radiation model in the cirrus cloud model and the cumulus ensemble model. This implementation has now been completed. In particular, the cumulus ensemble model now includes a diurnal cycle. Radiation calculations are performed every $2 \mathrm{~min}$, while the dynamical time step is $7 \mathrm{sec}$. The same column model has also been implemented in the cloud microphysics model.

The second effort in parallel implementation was the introduction of explicit ice radiative properties into the shortwave and longwave radiative transfer model. For the general circulation model and the cumulus ensemble model, the radiative properties of liquid, mixed phase and ice clouds are parameterized by using the NCAR CCM2 radiation code in conjunction with the radiative optical properties for liquid droplets from Slingo (1989) and for ico from Ebert and Curry (1992).

Mixed phase optical properties are accounted for through the following relation,

$$
\tau_{c l d}=\left(1-f_{c e}\right) \tau_{c l d}^{\text {liq }}+f_{c o} \tau_{c l d}^{i c e}
$$




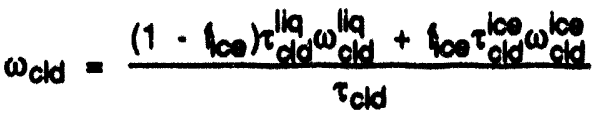

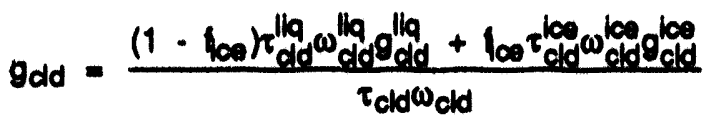

where for the CCM2 $f_{\text {lce }}$ is determined from the following relation,

$$
k_{0 \theta}=\left\{\begin{array}{ccc}
0 & T \geq & -5 C \\
-\frac{T+5}{15} & -5 \leq T \leq-20 C \\
1 & T \leq-20 C
\end{array}\right.
$$

based on the observations of Hobbs ot al. (1974).

The cumulus ensemble model explicitly calculates the ice water concentration and the liquid water concentration, thus $f_{\text {ice }}$ for the cloud model is given by,

$$
t_{c e}=\frac{I W C}{I W C+L W C}
$$

where IWC is the ice water concentration in a given layer and LWC is the liquid water concentration. The optical properties for llquid drops and ice particles are expressed as (Slingo 1989; Ebert and Curry 1992),

$$
\begin{aligned}
& \tau_{\text {cld }}^{i}=\left\{\begin{array}{l}
\text { LWP } \\
\text { IWP }
\end{array}\right\}\left(a^{i}+\frac{b^{i}}{r_{\text {eff }}}\right) \\
& \omega_{\text {cld }}^{\prime}=1 \cdot c^{\prime} \cdot d^{d} r_{\text {eff }} \\
& g_{\text {cld }}^{\prime}=\theta^{\prime}+f_{\text {eff }}^{\prime}
\end{aligned}
$$

where i denotes either liquid (liq) or lce. The ice values derived by Ebert and Curry for the coefficients a through $f$ are based on Takano and Liou's (1989) optical calculations for hexagonal plates. Note that we must specify $r_{\text {off }}$ for both liquid and ice particles. We use $r_{\text {off }}=10 \mu \mathrm{m}$ for liquid drops; for the ice particle size in the GCM we plan to use $20 \mu \mathrm{m}$. This size is based on the recent results of Baum of al. (1992), but this is one of the parameters we hope to obtain more information on from ARM measurements. In the cumulus ensemble model we diagnose $r_{\text {off }}$ from the predicted ice number and the ice water concentration. Hence, in the cumulus ensemble model $r_{\text {eff }}$ is not prescribed as it is in the GCM.

\section{Results from the CCM}

Figure 1a shows the dependence of cirrus cloud radiative heating and longwave cooling on the lce particle size. Figure 1b shows the difference between the ice particle heating/cooling from the standard CCM2 heating cloud heating rates. Note that for $r_{\text {en }}$ greater than $20 \mu \mathrm{m}$, the net effect is to cool the upper troposphere.

Figure 2 indicates the role of ice particle size in the CCM2. Shown is the difference in zonally averaged temperature and zonal wind, differences that are due to a change in ice particle size from 50 to $10 \mu \mathrm{m}$. The use of a $50 \mu \mathrm{m}$ ice particle size results in a cooling of the upper troposphere of order $5 \mathrm{~K}$ (note the interannual variability in this region is around $0.5 \mathrm{~K}$ ). Associated with the upper tropospheric cooling is a weakening of the upper tropospheric zonal wind. These results indicate that the tropical simulated climate is quite sensitive to ice particle size.

\section{Current Investigations}

Currently, the cloud ensemble model is being integrated with the ice optical properties included. We are forcing the model with large scale conditions that are representative of the tropical western Pacific. We intend to integrate this simulation until it comes into an equilibrium balance with the large scale conditions. At this point we will investigate the magnitude of the radiative forcing from the convective and stratiform components of the cloud system. We will then repeat these simulations without the ice optical properties to assess the role of the ice optics on the simulation. 

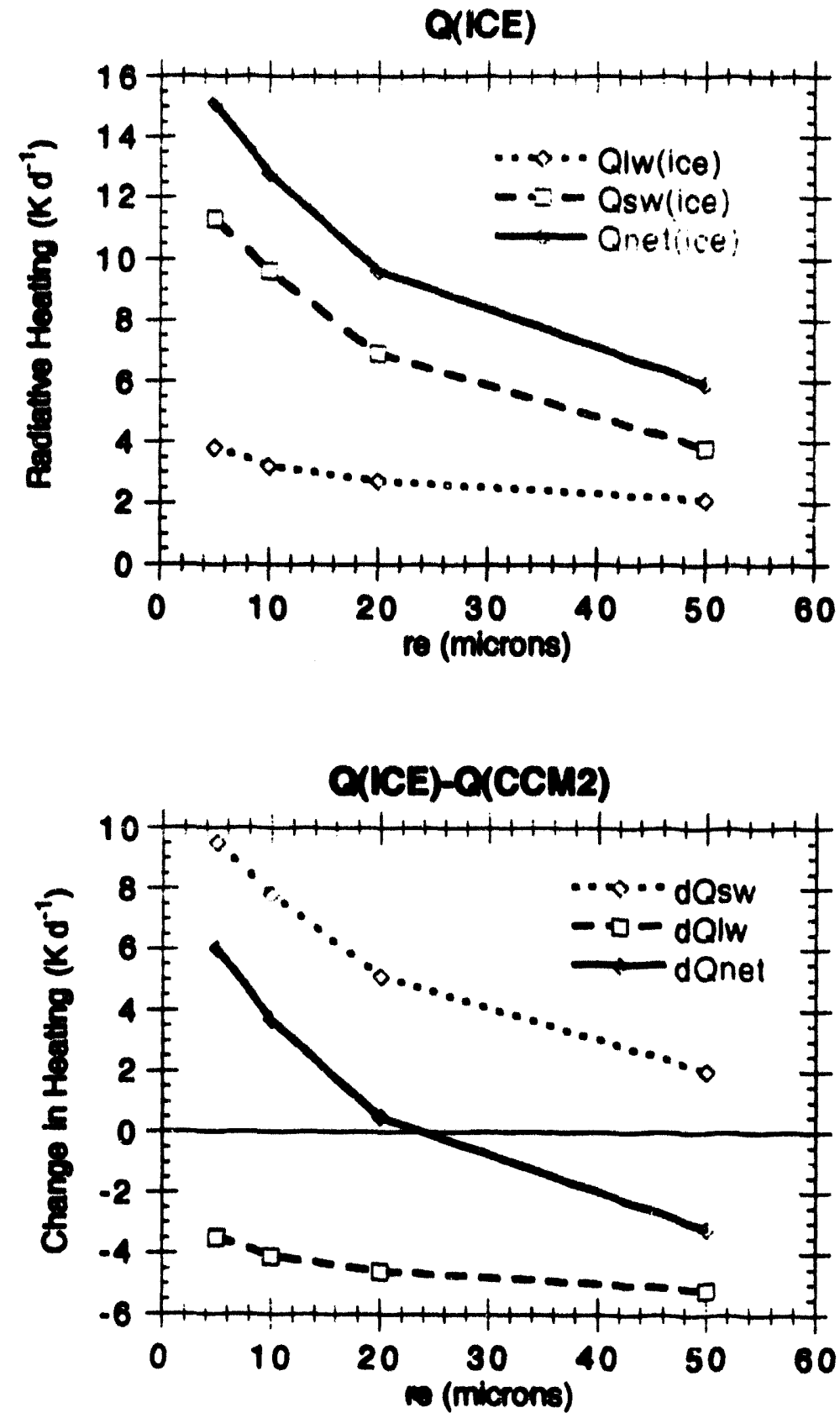

Figure 1. a) Shortwave heating $Q_{e w}$ longwave heating $Q_{w}$ and net heating $Q_{\text {nat }}$ as a function of lce particle size $r_{\text {eff }}$ (microns). b) Difference between results in $1 \mathrm{a}$ and the standard (liquid phase) CCM2 heating rates. 

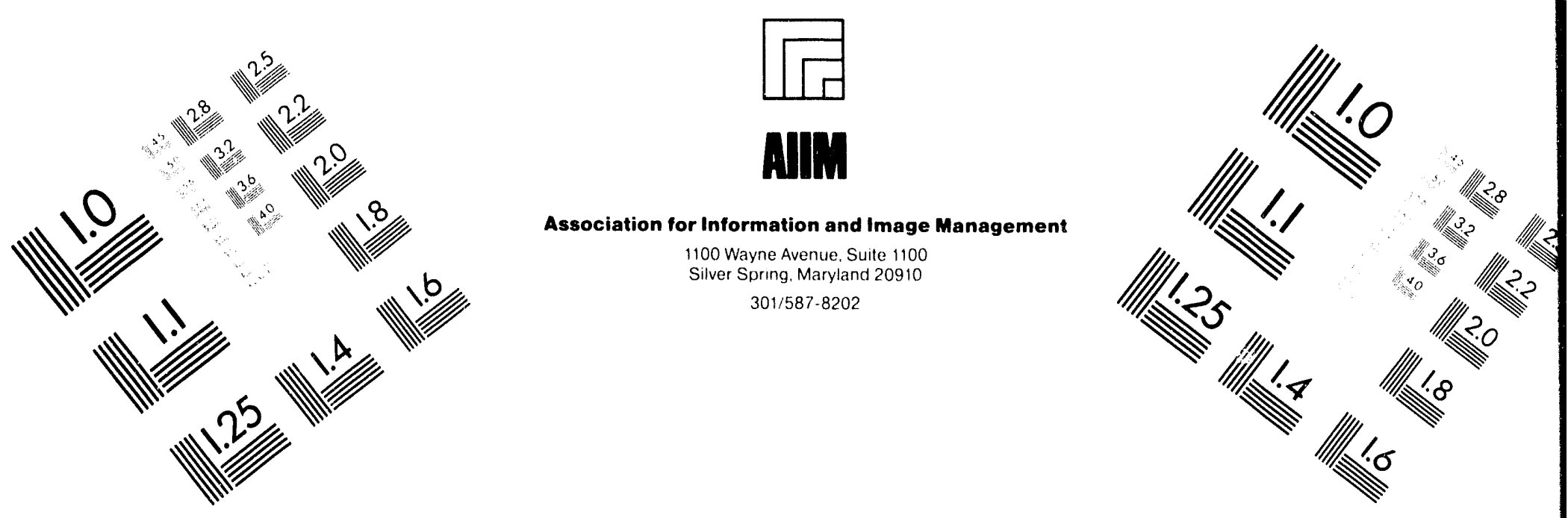

\section{Centimeter}

${ }_{1}$

Inches
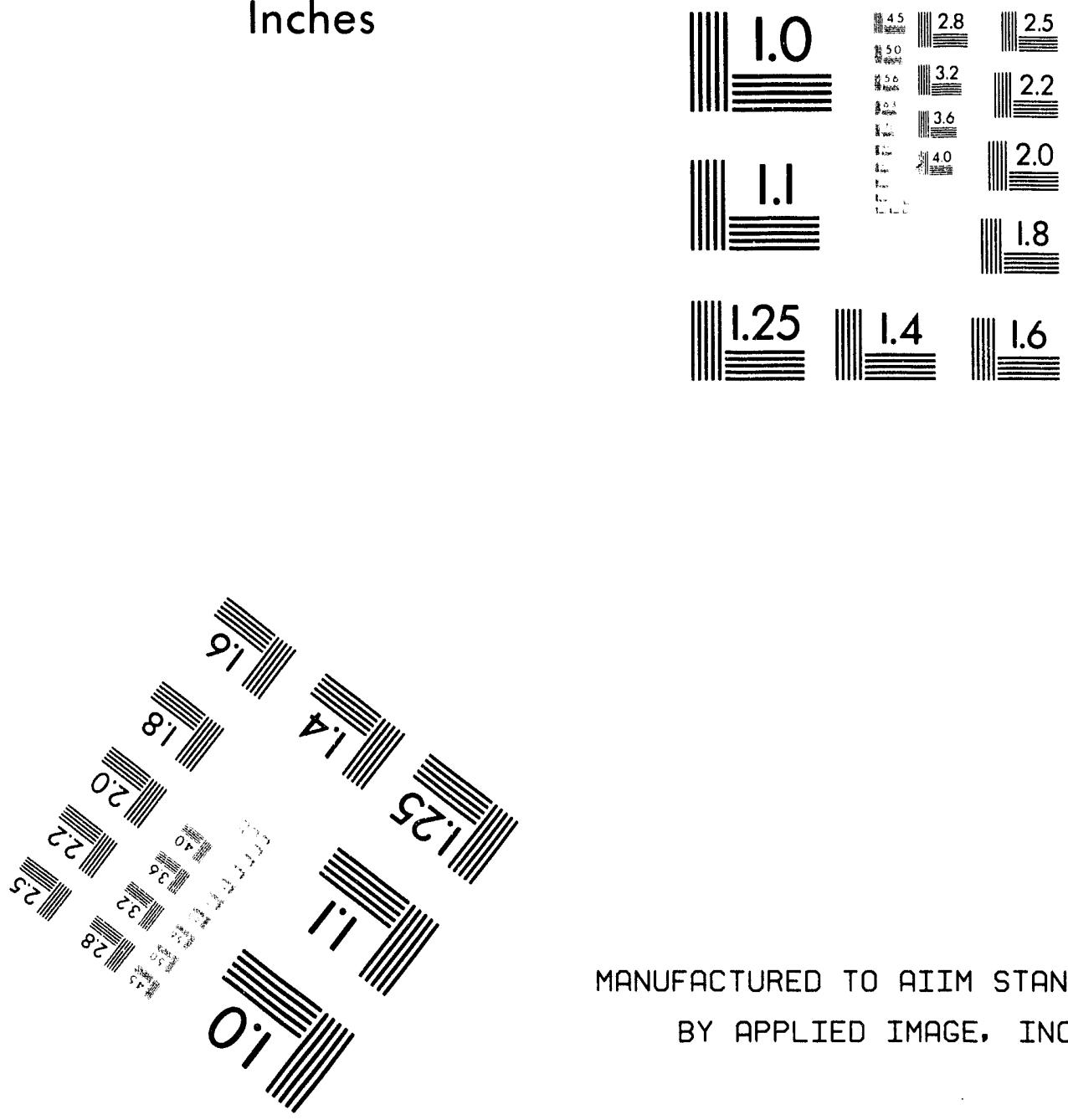

MANUFACTURED TO AIIM STANDARDS

BY APPLIED IMAGE, INC.

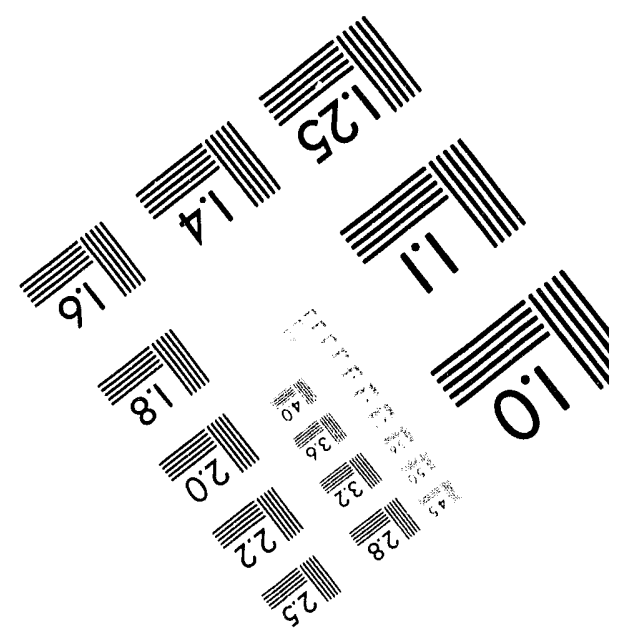



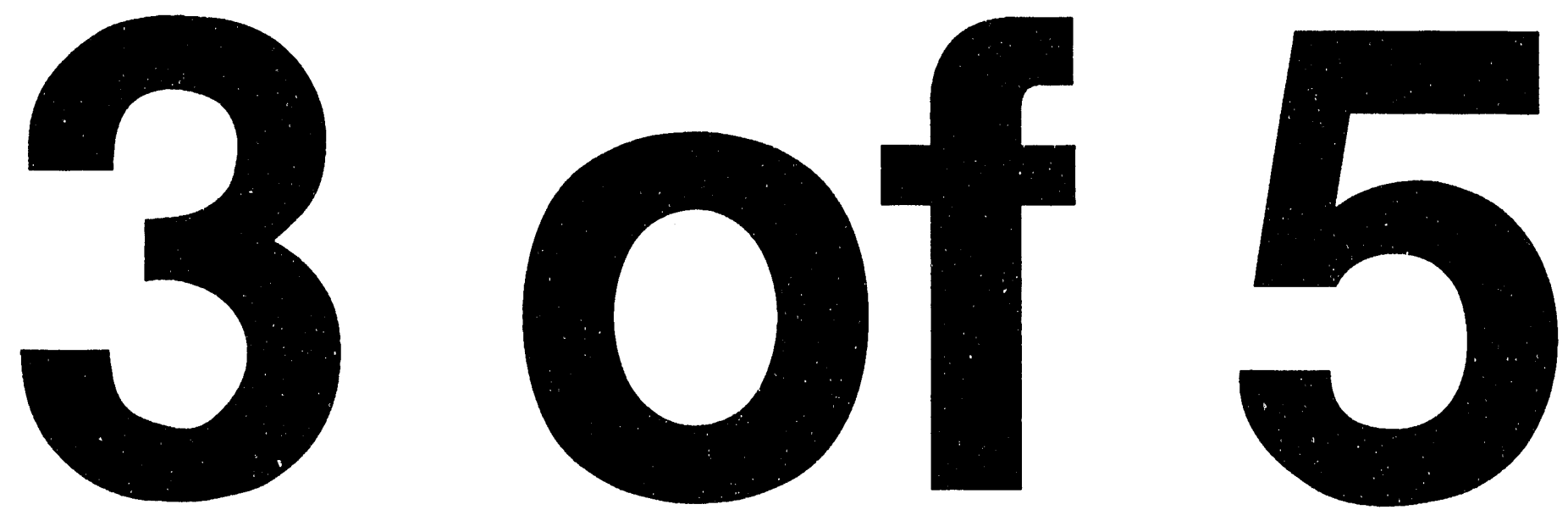

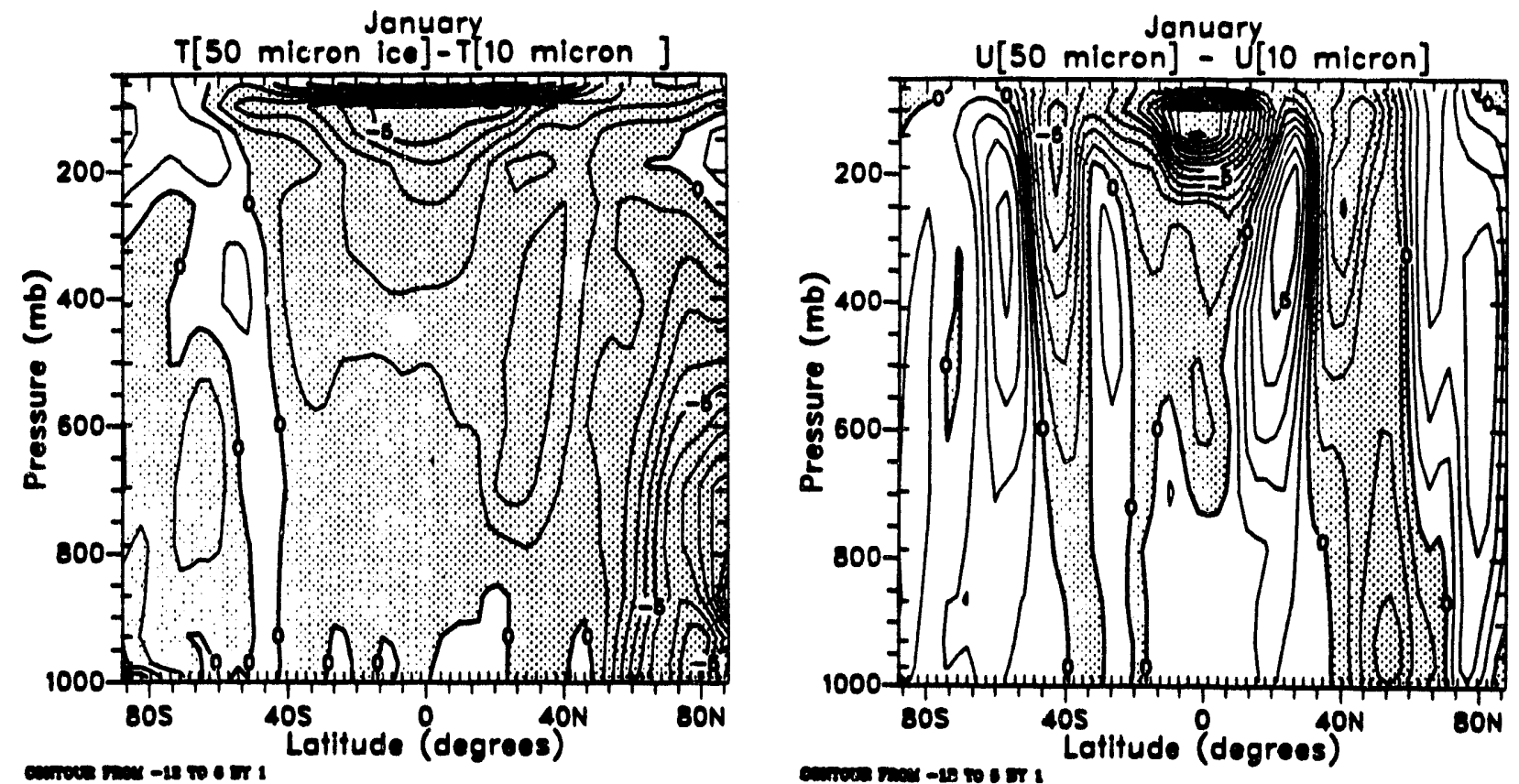

Figure 2. a) Change in CCM2 zonal temperature due to an increase in ice particle size from 10 to 50 microns. b) Change in CCM2 zonal wind due to increase in lice particle size, for January.

\section{References}

Baum, B. A., B. A. Wielickl, and P. Minnis. 1992. Cloudproperty retrieval using merged HIRS and AVHRR data. J. Appl. Meteorol. 31:351-369.

Briegleb, B. P. 1992. Delta-Eddington approximation for solar radiation in the NCAR Community Climate Model. J. Geophys. Res. 97:7603-7612.

Ebert, E. E., and J. A. Curry. 1992. A parameterization of ice cloud optical properties for climate models. J. Geophys. Res. 97:3831.3836.

Hack, J. J. 1993. Parameterization of moist convection in the NCAR Community Climate Model (CCM2). Submitted to J. Geophys. Res.
Hobbs, P. V. et al. 1974. The structure of clouds and precipitation over the Cascade Mountains and their modification by artificial seeding (1972-1973). Research Ruport 8, Dept. Atmospheric Science, U. of Washington, Seattle.

Slingo, J. 1987. The development and verification of a cloud prediction scheme for the ECMWF model. Quart. J. Roy. Met. Soc. 113:899-927.

Slingo. A. S. 1989. A GCM parameterization for the shortwave radiative properties of water clouds. J. Atmos. Scl. 46:1419-1427.

Takano Y., and K.-N. Liou. 1989. Solar radiative transfer in cirrus clouds, I, Single scattering and optical properties of hexagonal ice crystals. J. Atmos. Sci. 46:3-19. 


\title{
Development of a Parameterization Scheme of Mesoscale Convective Systems
}

\author{
W. R. Cotton \\ Colorado State University \\ Department of Atmospheric Science \\ Fort Collins, CO 80523
}

The goal of this research is to develop a parameterization scheme of mesoscale convective systems (MCS) including diabatic heaing, molsture and momentum transports, cloud formation, and precipitation.

The approach is

- Perform explicit cloud-resolving simulation of MCSs.

- Perform statistical analyses (conditional sampling, ensemble averages, term analyses along trajectories) of simulated MCSs to assist in fabricating a parameterization, calibrating coefficients, etc.

- Test the parameterization scheme against independent field data [0.g., Cloud and Radiation Testbed (CART) measurements and in numerical weather prediction (NWP) models emulating general circulation model (GCM) grid resolution].

Thus far we have formulated, calibrated, implemented and tested a deep convective engine against explicit Florida sea breeze convection and in coarse-grid regional simulations of mid-latitude and tropical MCSs.

A "fossil" MCS parameterization scheme has been fabricated (based on a prognostic vertical velocity variance scheme) and is now being generalized, callbrated, and tested.

Several explicit simulations of MCSs have been completed, and several others are in progress. Analysis code is being written and run on the explicitly simulated data. The cases selected are discussed below.

The 10-11 June 1985 squall line that occurred during the PRESTORM field project has been simulated using standard available data to initialize the model. By using the interactive nesting capability of the Regional Atmospheric Modeling System (RAMS), we were able to nest from a coarse grid of $80 \mathrm{~km}$ down to the finest $2.22 \mathrm{~km}$ grid, thus explicitly capturing the system as it transitioned from a convective line only to a system with convective and stratiform regions.

This simulation serves us in two ways. First, it acts as further verification for the Level $2.5 \mathrm{w}$ convective scheme (the "convective engine" for the MCS parameterization). Second, diagnostic analyses guide us in the parameterization of the mesoscale circulations which comprise the fast (and perhaps some slow) manifold processes of an MCS. The diagnostics include conditional sampling of various quantities as well as some Lagrangian tracer analysis to help identify coherent flow branchos within the system. These diagnostics provide further guidance in the fabrication of a parameterization.

Two tropical mesoscale systems are being simulated with RAMS. One of the simulated systems is EMEX 9, so named because it was probed during the ninth aircraft mission of the Equatorial Mesoscale Experiment (2-3 February 1987). The prevailing synoptic feature at the time of EMEX 9 was a deep westerly monsoon trough exiending from northern Australia across into New Guinea. The monsoon trough provided a primary lifting mechanism for the EMEX 9 cluster. Currently, $80-\mathrm{km}$ and $20-\mathrm{km}$ grid spacing runs have been completed. The simulation results support the suspicion that EMEX 9 was originally forced along a land breeze convergence line just north of Australla's Top End peninsula. Explicit cloud-resolving grids will be activated shortly.

Another tropical MCS being simulated is the 5 December 1989 system observed during the Down Under Doppler and Electricity Experiment (DUNDEE). Although, like the EMEX 9 case, the 5 December MCS contains a northward propagating east-west oriented convective line, the 
similarities end there. First, the 5 December system occurs during a break in the westerly monsoon rather than during the monsoon. Second, the system appears to be forced by a sea breeze circulation impinging on the higher terrain just south of Darwin, Australia, rather than being forced by a land breeze over the ocean. Third, the system is quite small in geographic area compared with EMEX 9. Fourth, the lifetime of the system is only about 6 hours, compared with 12 hours for EMEX 9. Although wa have successfully simulated this case in two dimensions, the threedimensional simulation has yet to meet the same measure of success that EMEX 9 has.

A weakly sheared, extra-tropical MCS observed during PRESTORM has been the most challenging case. The simulations have been performed with nested grids from $80-\mathrm{km}$ down to $2.2-\mathrm{km}$ grid spacing. While the runs with the Weissbluth scheme produce an MCS that superficially resembles the observed system, the MCS does not contain the observed organization of the wind fields in the system. Moreover, the explicitly cloud-resolving grids are unable to trigger an MCS without imposing some heat bubbles in the grid. Nonetheless the latest explicit runs are encouraging.

Overall, an MCS parameterization scheme has been proposed which consist of the following components:

1. a deep convective engine (the Weissbluth scheme)

2. a fast manifold (unbalanced) mesoscale response consisting of slantwise ascending and descending
branches-A modified Moncrieff archetypal approach and a new parcel model for specified flow branches derived from model output statistics are being considered.

3. a slow manifold (balanced) mesoscale responseCurrently we are exploring the possibility of using potential vorticity (PV) as a basis for describing the slow manifold processes of MCSs. The beauty of PV is that it contains information on both flow and mass fields; hence, the dynamics and thermodynamics can be traced by predicting one variable. The flow and mass fields themselves can be retrieved by use of the appropriate invertibility principle. Idealized model studies indicate that PV is modified by diabatic heating from MCSs. Other studies imply that a preexisting upper-level PV anomaly destabilizes the atmosphere below and may be a factor in triggering an MCS. We believe that PV may a useful tool to identify the communication between a GCM and the sub-grid mesoscale.

4. A "fossil" MCS component consisting of radiativeconvective cloud responses and precipitation from middle and high clouds (cirrus) remaining after the decay of the active MCS-This component is under development using the Weissbluth small-scale turbulence component of the convective paramererization scheme. 


\title{
Development of a Radiative Cloud Parameterization Scheme of Stratocumulus and Stratus Clouds Which Includes the Impact of Cloud Condensation Nucleus on Cloud Albedo
}

\author{
W. R. Cotton, G. L. Stephens, D. Duda, \\ B. Stevens, and R. L. Walko \\ Colorado State University \\ Department of Atmospheric Science \\ Fort Collins, CO \\ G. Feingold \\ Cooperative Institute for Research in Environmental Sciences \\ University of Colorado, Boulder \\ Boulder, CO 80309-0049
}

\begin{abstract}
A three-dimensional (3-D) model for simulating the effect of enhanced cloud condensation nucleus (CCN) concentrations on stratocumulus clouds is presented. Results of two-dimensional (2-D) tests are discussed for a control run and a sensitivity run where $\mathrm{CCN}$ spectra were taken to be typical of clean marine conditions or mildly continental air, respectively. For a fivefold increase in initial $\mathrm{CCN}$ concentrations, drop concentrations increased about threefold, effective radii $\left(\nabla_{0}\right)$ decreased by $40 \%$, while liquid water contents show less significant variations. These results are consistent with both observations and expectations. Detailed radiative calculations done on previous runs showed similar microphysical structure.
\end{abstract}

Several studies have used methods to simultaneously retrieve the effective radius $\left(R_{e}\right)$ and cloud optical depth from multichannel reflectance measurements (Twomey and Cocks 1982, 1989; Curran and Wu 1982; Nakajima et al. 1991). Results from the Regional Atmospheric Modeling System (RAMS) 2-D simulation were used in a preliminary study of the effects of cloud heterogeneities on the remote sensing of marine stratus cloud properties. The microphysical data from a version of the control run and a run with an increased initial $\mathrm{CCN}$ concentration were used in a series of 2-D radiative transfer simulations. The upwelling radiances from the cloud fields were computed from the Spherical Harmonic Spatial Grid (SHSG) model described in Evans (1993).

Estimates of $R_{\theta}$ and optical depth from the simulated cloud fields were derived by comparing the 2-D model reflectance functions with the reflectance functions obtained from independent pixel (IP) calculations for a range of effective radii and optical depths. The IP-derived estimates of $R_{e}$ and optical depth were often noticeably different from the actual values of the cloud properties directly calculated from the RAMS simulation, a result which suggests that the effects of cloud geometry are important to consider in the remote sensing of these cloud properties. In particular, the maximum differences in the IP-derived versus actual $R_{\theta}$ for the portions of the cloud with an optical depth greater than four ranged from $30 \%$ to $50 \%$ in both simulations. The domain-mean optical depth was underestimated by nearly $10 \%$, while the maximum differences between the retrieved and actual optical depths were over $20 \%$.

All results are preliminary. More definitive results must await 3-D simulations planned for later this spring. 


\section{References}

Curran, R. J., and M. L. C. Wu. 1982. Skylab near-infrared observations of clouds indicating supercooled liquid water droplets. J. Atmos. Sci. 39:635-647.

Cunning, J. B. 1986. The Oklahoma-Kansas Preliminary Regional Experiment for STORM Central. Bull. Am. Met. Soc. 67:1478-1486.

Evans, K. F. 1993. Two dimensional radiative transfer in cloudy atmospheres: The spherical harmonic spatial grid method. J. Atmos. Sci. 50:3111-3124.
Nakajima, T., M. D. King, J. D. Spinhirne, and L. F. Radke. 1991. Determination of the optical thickness and effective particle radius of clouds from reflected solar radiation measurements. Partll: Marine stratocumulus observations. J. Atmos. Sci. 48:728-750.

Twomey, S., and T. Cocks. 1982. Spectral reflectance of clouds in the near-infrared: comparisons of measurements and calculations. J. Meteor. Soc. Japan 60:583-592.

Twomey, S., and T. Cocks. 1989. Remote sensing of cloud parameters from spectral reflectance in the near-infrared. Beitr. Phys. Atmos. 62:172-179. 


\title{
Regional Climatology Sensitivity Studies
}

\author{
J. E. Bossert, C.-Y.J. Kao, and J. L. Winterkamp \\ Earth and Environmental Sciences Division \\ Los Alamos National Laboratory \\ Los Alamos, NM 87545 \\ J. O. Roads and S.-C. Chen \\ Scripps Institution of Oceanography \\ University of California at San Diego \\ La Jolla, CA 92093
}

\section{Introduction}

Recent interest in understanding climate and climate change at regional scales has led to the application of mesoscale models for regional climatology studies. These models can provide an understanding of climate processes in a physically consistent way at much higher resolution than currently offered by general circulation models (GCMs). The methodology and proof of concept for regional climate modeling was initially undertaken by Dickinson of al. (1989) with the Penn State/National Center for Atmospheric Research mesoscale model (MM4). Bossert et al. (1992a) employed the Regional Atmospheric Modeling System (RAMS) mesoscale model for preliminary regional climate simulations in a similar fashion to that of Dickinson et al. Bossert et al. (1992b) discussed the development of a regional-scale climate model for the western United States and compared model-generated surface fields for a monthlong simulation of January 1988 with observed data from over $\mathbf{3 0 0}$ surface cooperative stations.

In the course of our regional climate modeling with RAMS, several questions have arisen which require further investigation. The first involves the model validation procedure. To date, regional climate simulation results have not undergone intense scrutiny and comparison with independent observational data sets. One reason for this is the lack of appropriate mesoscale observations. Particularly in regions of complex terrain, such as the intermountain west, the spatial coverage of existing surface and atmospheric observations is sparse. The second question has to do with grid configurations and physical parameterizations of the regional model and their suitability for long-term simulations. While GCMs are global and were developed to run for extended periods, the evolution of mesoscale modeling has been far different and has focused upon short integration periods on the order of a diurnal cycle, with grid domains covering only a small portion of the globe. Correspondingly, the physical parameterizations within the mesoscale model have not been thoroughly tested for long integration periods. In addition, specification of lateral boundary conditions from high-quality, large-scale data sets or GCM output is of critical importance to the mesoscale simulation.

In recent work, we have tried to address these questions to establish confidence in our modeling procedure. A more rigorous comparison of oui modeling results with various data sets is reported in Roads et al. (1992). In the present paper, we use two simple numerical experiments to examine the impact of grid configuration on the predicted precipitation field from the RAMS model. We intend to demonstrate that the choice of the lateral boundaries and grid configurations can significantly impact the predicted fields of interest.

\section{Approach}

The experiments described herein are based upon a continuous month-long (January 1988) simulation with the RAMS model. A more detailed explanation of the RAMS model is contained in $\mathrm{KaO}$ and Bossert (1992) and elsewhere. The specific model configuration and parameterizations used for the regional climate simulation are described in Bossert et al. (1992a). The regional climate model was initially developed to simulate the 
western United States, in part because the topography of this region is complex and induces a high degree of mesoscale variability which we hoped to capture, and in part because we wanted to test our model with a grid configuration similar to that of Giorgi (1989) for comparison purposes. The actual grid configuration (see Figure 2, Bossert et al. 1992b) was rather arbitrary, the primary requirement being that it include the entire mountain massif of the western United States.

In comparing the simulated monthly precipitation with actual amounts, we found the greatest differences along the Oregon coast, where the model prediction was too dry, and over the highest mountain terrain of the Rockies, where the model prediction was too wet. A 5-day period (days 11-15) from the month-long January simulation, chosen for the sensitivity experiments, provides a prime example of these differences (Figures 1 and 2). Figure 1 shows that observed heavy precipitation as limited to the coastal margin of the Pacific Northwest and the Cascade Range. Other regions which received significant amounts include northern California and northern Idaho. Little precipitation was measured over the Southwest or interior ranges of the Rocky Mountain chain. The simulated 5-day precipitation rate (Figure 2) from the RAMS microphysics scheme shows that the model captured the heavy precipitation over the Cascades in Washington (which extended southward into northern California), as well as the precipitation in Idaho, although the amounts there are excessive. The simulated precipitation field does show large departures from observations in other regions, however. For example, the heavy precipitation amounts observed along the coastal margin of Oregon are missing, while substantial precipitation is simulated over the high mountain terrain of Utah and Colorado. Although none of the cooperative stations are at

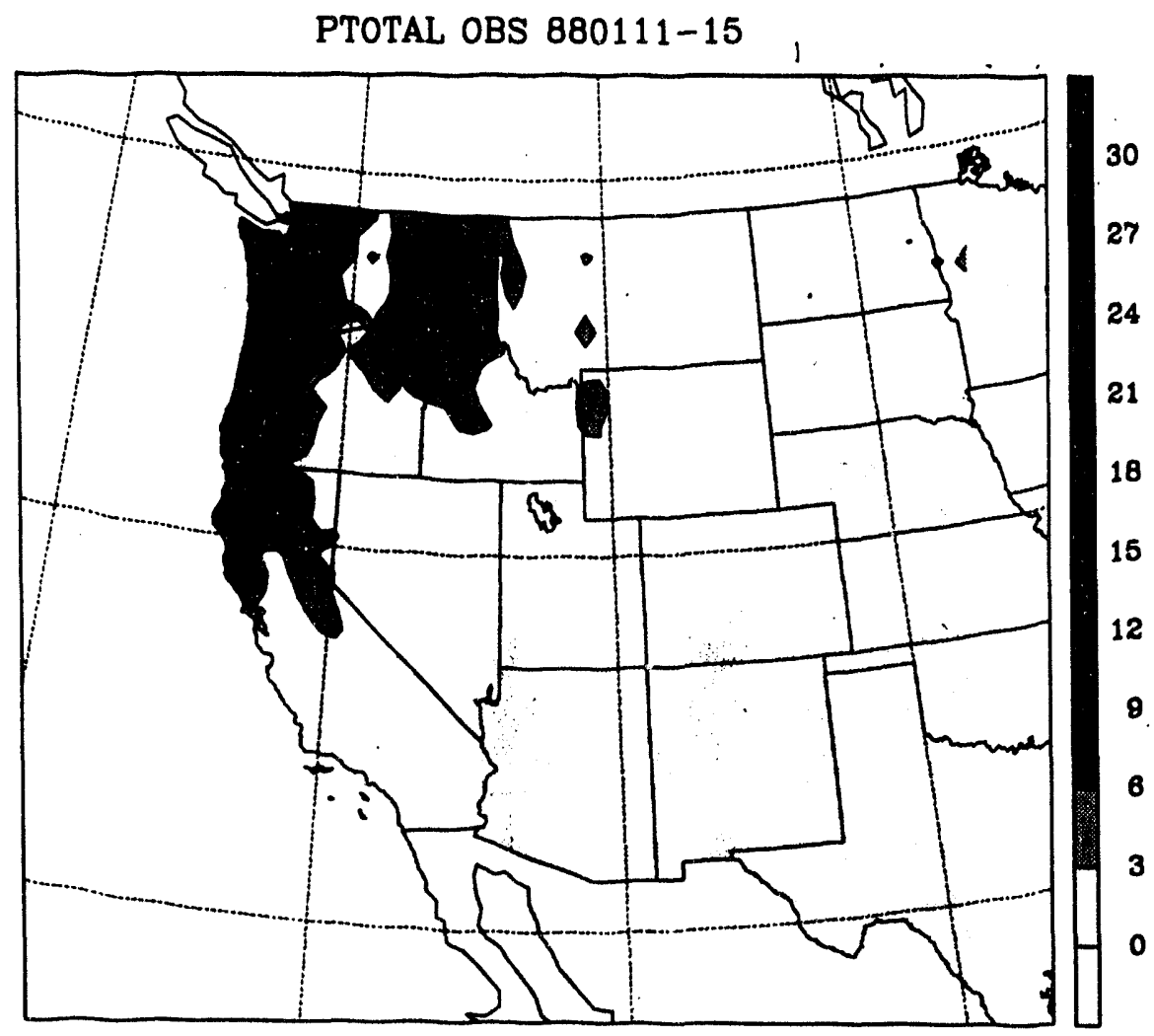

0 TO $\$ 0.16$

Figure 1. Observed precipitation (mm/day) for the period 11 -15 January 1988 , as determined from $~ 300$ surface stations interpolated to RAMS model grid points. 


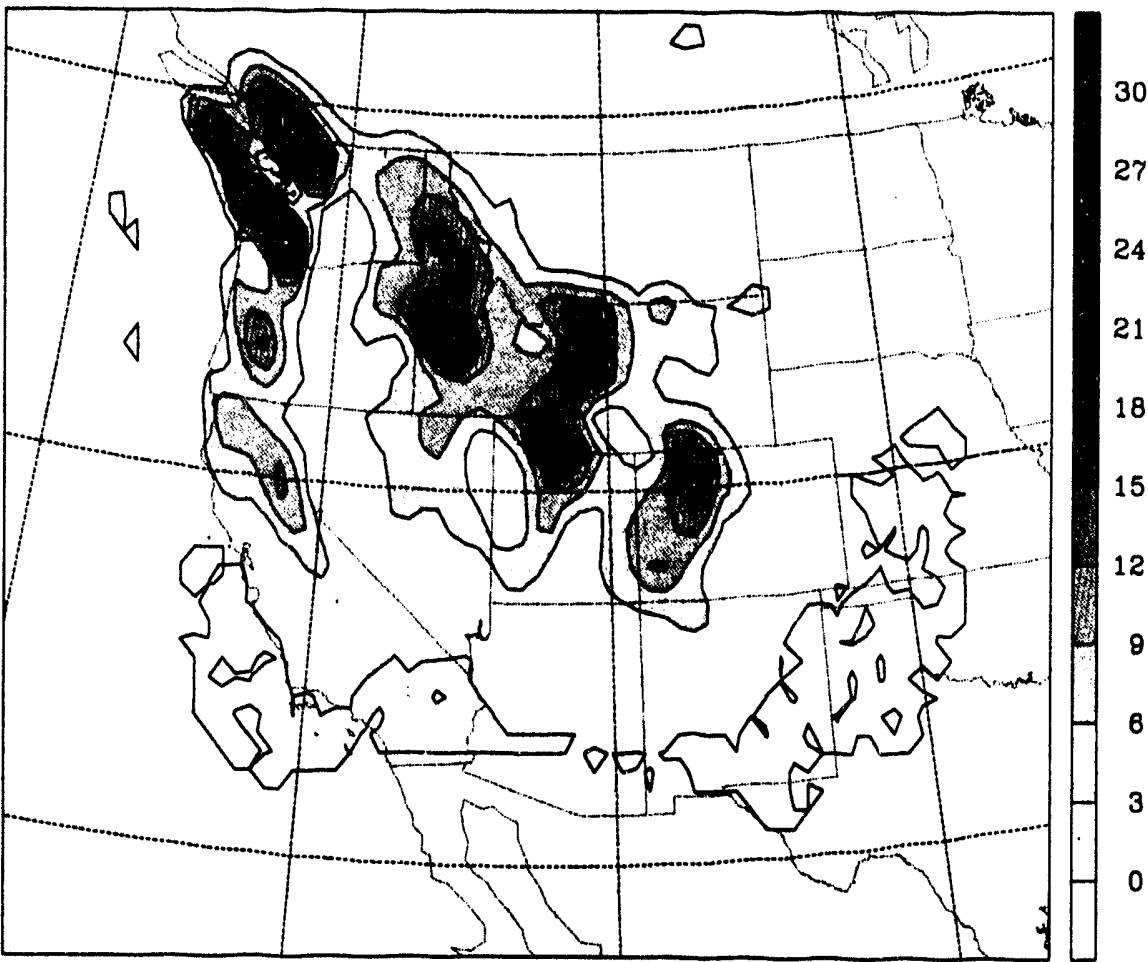

Figure 2. Simulated precipitation (mm/day) for the period 11-15 January 1988 from the RAMS model.

elevations exceeding $2700 \mathrm{~m}$, which prohibits an accurate determination of high mountain precipitation (snowfall) in the central Rockies, the amounts there appear to be too high, based upon the observed storm track over the period.

To try and understand these precipitation differences, we hypothesized that the National Meteorological Center's $2.5^{\circ}$ gridded data, used for model initialization and boundary nudging, may be too dry in the low levels of the troposphere over the eastern Pacific. The drier lower atmosphere combined with the short advective time scale for flow from the model domain boundary to the west coast led us to speculate that the western boundary of the model domain should be located farther out into the Pacific to allow for surface evaporation, thereby increasing the low-level humidity. Consistent with this hypothesis were the high precipitation values over the high Rockies, which suggested that too much moisture was being advected into the Intermountain West at mid-li opospheric levels and not rained out along the coast.

These obvious shortcomings within the climatology simulations provided the motivation for the sensitivity experiments. For the first experiment, we designed a 5 day simulation with a western boundary of the model domain that extended an additional 5 grid points $\left(\sim 2.5^{\circ}\right)$ into the Pacific Ocean. Another consideration for the lack of coastal precipitation concerned the $0.5^{\circ}$ resolution of the model, which, while much higher than present day GCMs, was still rather coarse for a mesoscale simulation and did not adequately resolve the coastal range, especially in Oregon. Thus, for our second sensitivity experiment we included a nested grid with $0.125^{\circ}$ resolution over the Oregon region to better resolve the topography and thereby produce more realistic orographic lifting within the model. 
5-DAY TOTAL PREC. (MM/DAY) EXTENDED - CONTROL

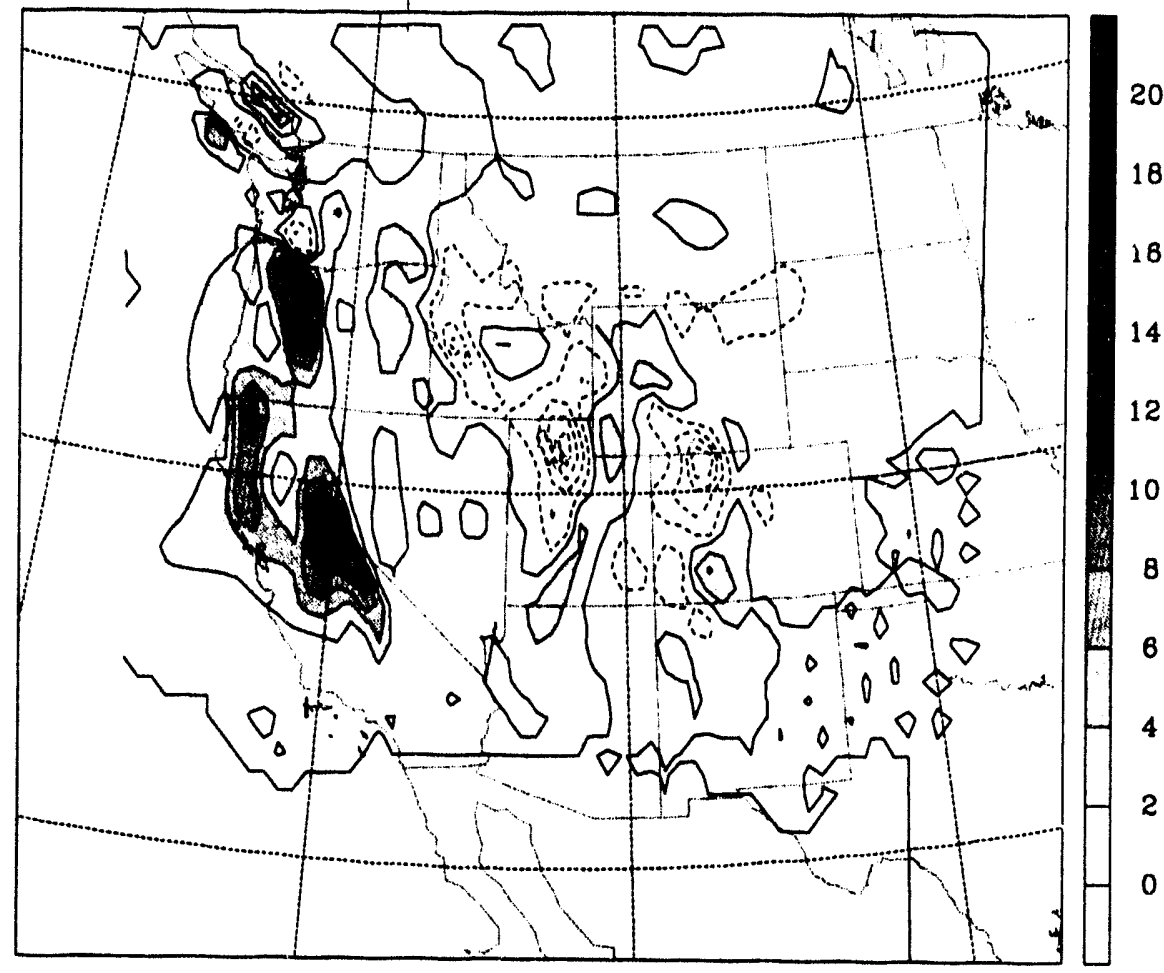

Figure 3. Precipitation differences ( $\mathrm{mm} / \mathrm{day}$ ) between the extended western boundary and control simulations for the period 11-15 January 1988. Shading indicates extended boundary precipitation exceeds control; dashed contours indicate extended boundary precipitation is less than control. Contour interval $2.0 \mathrm{~mm} / \mathrm{day}$.

\section{Results}

The results from the 5-day simulation with the extended western boundary are presented in Figure 3. The figure shows the precipitation difference field between the extended boundary and the control case over the control case domain. The precipitation difference shows that the westward extension does indeed produce the desired effect: dramatically increasing coastal precipitation, while reducing snowfall over the high interior ranges of the Rocky Mountains. The boundary extension increases the precipitation in northern California more than in Oregon, perhaps because this area was closest to the model domain boundary $(\sim 350 \mathrm{~km})$ in the control simulation. As a result, the precipitation over the central Sierras now appears to be excessive. In addition, the increase of precipitation along the Oregon coast is rather minor, leading us to believe that the grid resolution over this region is inadequate to realistically represent the Coast Range which induces the orographic lift necessary for rainout.

In the second sensitivity experiment, we implemented a nested grid over western Oregon to better resolve the coastal mountains. This nested grid was at a $4: 1$ ratio from the coarse grid, having approximately $0.125^{\circ}(\sim 13 \mathrm{~km})$ horizontal resolution. Precipitation results on the nested grid for the 5-day simulation are shown in Figure 4. Total precipitation amounts are heavy along the crest of the Cascades and along the southern Oregon/northern California coast. The rest of the Oregon coast has only minor rainfall. Thus, despite the higher resolution 


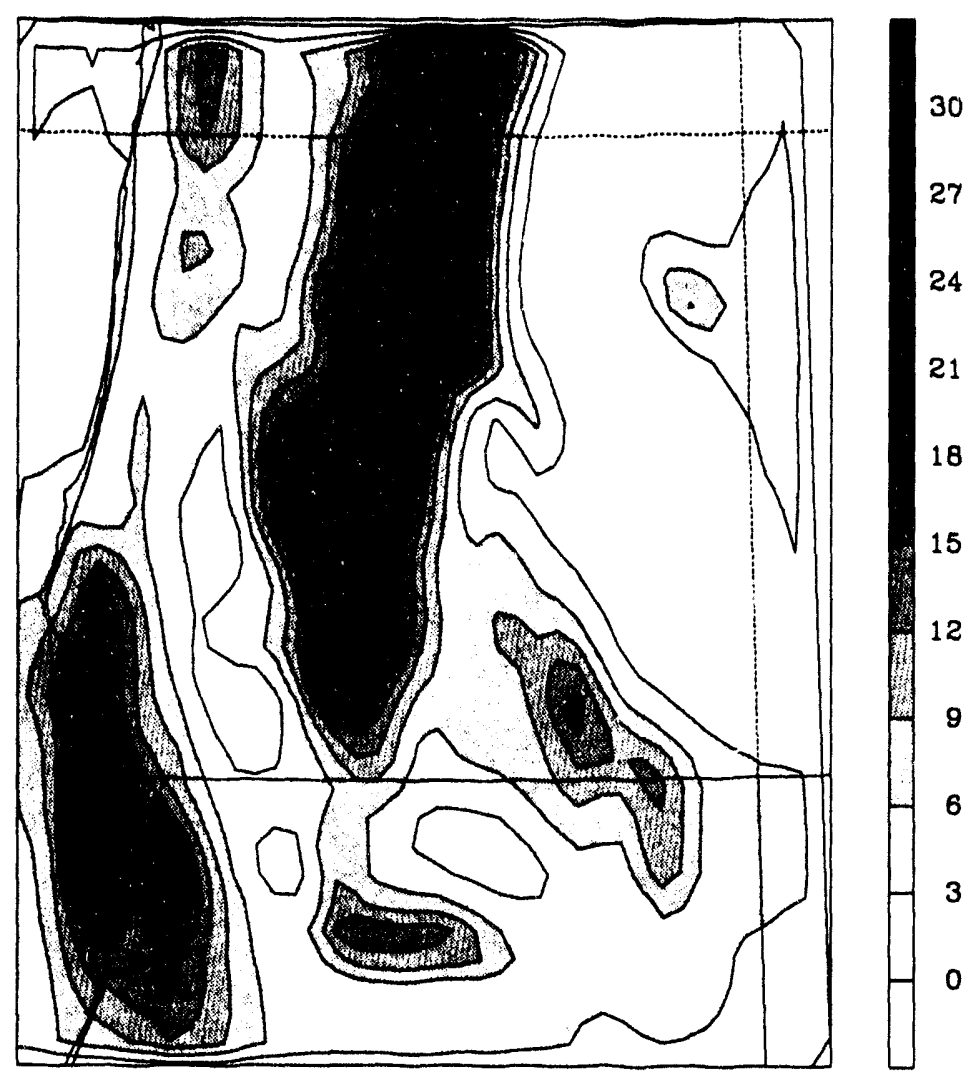

Figure 4. Simulated precipitation (mm/day) on the nested grid for the period 11-15 January 1988 from the RAMS model.

topography with the nested grid, precipitation is still underpredicted along the Oregon coast. In fact, the coastal mountains are still not well represented, even with the increased resolution. Thus, an even smaller grid may be necessary to resolve this topographic feature; achieving such resolution would increase the computational cost enormously. This point raises the question of what is an adequate grid spacing to achieve "reasonable" results in regional climate models.

The nested grid results are averaged back up to the coarse grid, and Figure 5 shows the resulting difference field between the nested grid run and the control run. In general, the precipitation differences appear similar overall to those found in the extended western boundary simulation, with more precipitation over the high terrain and coastal sections of the Pacific Northwest and less within the Intermountain West. Several differences are apparent, however. On the favorable side, the nested grid run concentrates the precipitation difference maxima over the Oregon region and reduces that found over the Sierra Nevada. On the unfavorable side, a huge difference appears along the Washington/Canada border. At present, we have no explanation for this large precipitation increase outside the nested grid region.

\section{Summary}

In this paper we have presented results from two simple regional climate sensitivity experiments designed to test 
5-DAY TOTAL PREC. (MM/DAY) NESTED - CONTROL

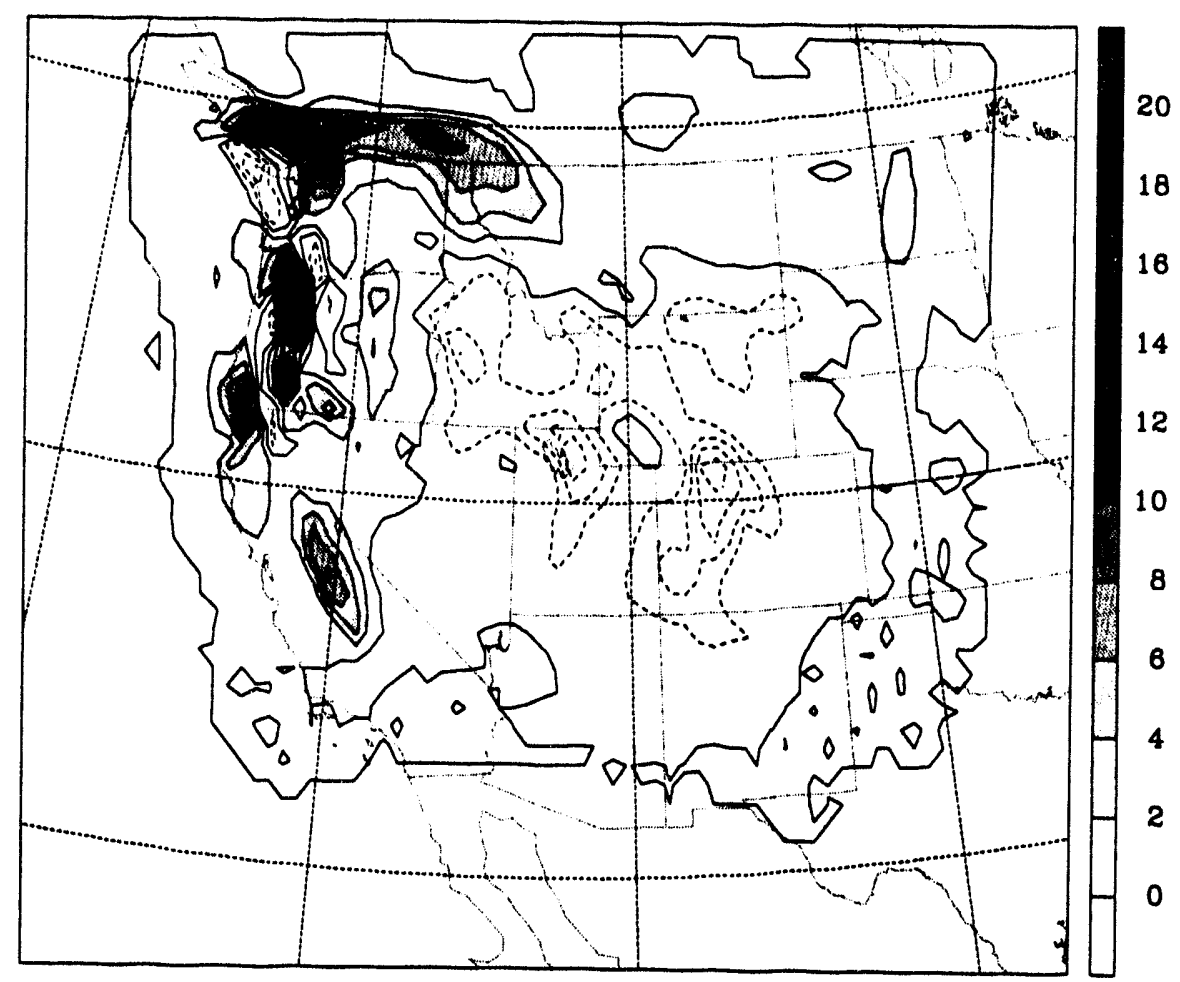

Figure 5. Precipitation differences (mrn/day) between the nested grid and control simulations for the period 11-15 January 1988. Shading indicates extended boundary precipitation exceeds control, dashed contours indicate extended boundary precipitation is less than control. Contour interval $2.0 \mathrm{~mm} /$ day.

the impact of grid configuration on the prediction of precipitation. The simulated precipitation was firstcompared with observed data interpolated to model grid points. Both experiments were found to improve the precipitation field by increasing the amounts in the Pacific Northwest and reducing the amounts within the Intermountain West. However, assessing the accuracy of the modeling results is complicated by the fact that the mesoscale precipitation data set used for model validation is too sparse to provide a rigorous evaluation of the model's performance. This is especially the case over high terrain where most of the winter season precipitation falls. This lack of necessary data is of great concern since validation of the results from regional climate models, especially precipitation, is critical to their future use as a tool for climate change prediction.
We are currently examining precipitation data sets from a variety of additional sources to aid in this validation exercise.

The modeling results show that grid configuration must be given very careful consideration before a regional model can be implemented for climate studies over a particular area of interest. The results presented here suggest that seemingly minor changes in domain boundaries and grid resolution can have a dramatic impact upon predicted results. Looking at the broader picture, our results demonstrate the need for adequate festing of the regional climate model with respect not only to grid conflguration but also to boundary nudging and physical parameterizations before we can establish confidence in its ability to be a useful tool for climate studies. To date, 
little attention has been paid to any of these requirements. We plan to continue to develop a regional model which is ideally suited for climate studies by further examining the questions and problems brought out in this paper. Future experiments will examine the impact of nudging upon the predicted fields and the performance of the surface parameterization during a month-long integration.

\section{Acknowledgments}

This work has been supported by the U.S. Department of Energy's Atmospheric Radiation Measurement (ARM) Program.

\section{References}

Bossent, J. E., J. O. Roads, C.-Y.J. Kao, J. L. Winterkamp, S.-C. Chen, and K. Ueyoshi. 1992a. Regional-scale simulations of the western U.S. climate. Climate Change and Energy Policy, L. Rosen and R. Glasser, eds., pp. 122 133. American Institute of Physics, New York, N.Y.

Bossert, J. E., J. O. Roads, C.-Y.J. Kao, J. L. Winterkamp, S.-C. Chen, and K. Ueyoshi. 1992b. Development of a regional climate modeling system: Simulations with a detailed microphysics parameterization. Proceedings of the Second Atmospheric Radiation Measurement (ARM) Science Team Meeting, October 26-30, 1991, Denver, Colorado, pp. 55-62. CONF 9110336, U.S. Department of Energy, Washington, D.C.

Dickinson, R. E., R. M. Errico, F. Giorgi, and G. T. Bates. 1989. A regional climate model for the western U.S. Clim. Change 15:383-422.

Giorgi, F. 1989. On the simulation of regional climate using a limited area model nested in a general circulation model. J. Clim. 3:941-963.

Kao, C.-Y.J., and J.E. Bossert. 1992. Numerical simulations of an idealized convective system: Comparisons between parameterized and explicitly resolved clouds. Proceedings of the Second Atmospheric Radiation Measurement (ARM) Science Team Meeting, October 26-30, 1991, Denver, Colorado, pp. 51-54. CONF-9110336, U.S. Department of Energy, Washington, D.C.

Roads, J. O., S.-C. Chen, K. Ueyoshi, J. Bossert, and J. Winterkamp. 1992. A preliminary description of the western U.S. climatology. Proceedings of the Seventeenth Climate Diagnostics Workshop, Norman, Oklahoma, pp. 242-247. October 19-23, 1992. U.S. Department of Commerce, Washington, D.C. 


\title{
Simulation of Cloud/Radiation Interaction Using a Second-Order Turbulence Radiative-Convective Model
}

\author{
C.-Y.J. Kao and W. S. Smith \\ Earth and Environmental Sciences Division \\ Los Alamos National Laboratory \\ Los Alamos, NM 87545
}

\section{Introduction}

Extended sheets of low-level stratus and stratocumulus clouds are a persistent feature over the eastern parts of the major ocean basins associated with the quasi-permanent subtropical high-pressure systems. These clouds exert a strong influence on climate through their high albedo, compared with the underlying surface, and their low altitude. The former leads to a reduction of the net shortwave flux entering the atmosphere, and the latter leads to an infrared loss in a way essentially the same as the cloud-free conditions. Randallet al. (1984) estimated that an increase of a few percent of global low-level stratiform clouds may offset the warming caused by a doubling of the atmospheric $\mathrm{CO}_{2}$.

The Atmospheric Radiation Measurement (ARM) Program, sponsored by the U.S. Department of Energy, envisions a locale in the Eastern North Pacific for extensive measurements of stratiform boundary-layer clouds and their interaction with atmospheric radiation. Thus, a physically-based parameterization scheme for marine lowlevel stratiform clouds can be developed for general circulation models (GCMs).

This paper is a modeling study with the current understanding of the important physical processes associated with a cloud-capped boundary layer. The numerical model is a high-resolution one-dimensional version of the second-order turbulence convective/radiative model developed at the Los Alamos National Laboratory (Kao and Yamada 1989; Yamada and Kao 1986). The data collected during the intensive field observations of the First International Satellite Cloud Climatology Project (ISCCP) Regional Experiment (FIRE) (Albrecht et al. 1988) are used to validate the model.
One important finding of the FIRE data is the diurnal variation of cloud behavior (e.g., Blaskovic and Davies 1991; Betts 1990) in terms of cloud liquid water content and cloud depth. To simulate the observed cloud diurnal variability, we incorporated a parameterization of shortwave absorption due to clouds (Slingo and Schrecker 1982) into our model. In this paper, we will focus on the physical mechanisms in modulating the diurnal variation of clouds.

\section{The FIRE Data}

FIRE is a comprehensive field experiment which provides measurements to test theories on the formation. maintenance, and dissipation of marine stratiform clouds. The measurements were obtained off the coast of California, from 29 June to 19 July, 1987, with aircraft, satellite, and surface-based instruments. The fiald site of FIRE was located on San Nicolas Island ( $\left.33.1^{\circ} \mathrm{N}, 119.31^{\circ} \mathrm{W}\right)$. Albrecht et al. (1988) have summarized the overall aspects of the FIRE experiment. Among many observational studies of the FIRE data, Hignett (1991) presented the turbulent structure of a cloud-capped boundary layer during FIRE. Blaskovic and Davies (1991) analyzed the average diurnal variation of cloud properties and related surface variables.

\section{The Model}

The complete model equations have been reported in Yamada and Kao (1986) and Kao and Yamada (1989). Only a brief review is provided here. The basic mean prognostic variables are the horizontal momentum $(U, V)$, the liquid water potential temperature $\left(\theta_{1}\right)$, and the total water mixing ratio $\left(Q_{w}\right)$. Turbulent fluxes in terms of 
momentum, heat, and water substance are obtained from simplified turbulence-closure equations (Yamada 1983). The model also prognostically calculates turbulent kinetic energy $\left(q^{2}\right)$ and turbulence length scale (I). This is an important feature in simulating the transient nature of turbulent mixing within the cloud layer.

The longwave radiation flux is computed according to the method developed by Sasamori (1968), which is based on a chart approach using two-stream numerical approximations to the transfer equations. To reduce computing time, the absorption functions are expressed as analytic functions. It also allows up to three absorbing gases: water vapor, carbon dioxide, and ozone. The fluxes depend on the path length of each absorbing gas. This method is further modified to include cloud effects. Since clouds provide an additional radiation source and their area coverage can be fractional, this method first computes the total flux at each layer by summing the fluxes resulting from each source, which is then attenuated by partial cloudiness.

The shortwave parameterization is designed for the calculation of absorption, reflection, and transmission by stratiform clouds based upon a two-stream approximation for multiple scattering of cloud droplets. In the model, we divide the solar spectrum into six absorption bands and six windows between these bands, according to the water vapor absorption spectrum. Each absorption band is further divided into five sub-bands in which the transmission functions are represented by the exponential function with effective absorption coefficients. The scattering of solar radiation by cloud droplets is represented by a single mean size of cloud droplets according to Slingo and Schrecker (1982).

A bimodal distribution of finer vertical resolution is designed (Kao and Yamada 1989) to resolve detailed variations of thermal stratification near the surface and cloud top. The minimum $\Delta z$ in the simulation is $7 \mathrm{~m}$.

\section{Results}

The initial profile of potential temperature is constructed with a sea surface temperature of $15.5^{\circ} \mathrm{C}$ and a surface pressure of 1012 mbar. A slightly stable lapse rate of 1.5 $\mathrm{K} \cdot \mathrm{km}^{-1}$ is assumed from the surface up to $550 \mathrm{~m}$ (Hignett 1991), followed by a strong inversion layer from $550 \mathrm{~m}$ to
$650 \mathrm{~m}$, with the lapse rate of $50 \mathrm{~K} \cdot \mathrm{km}^{-1}$. Stratification with a lapse rate of $5.0 \mathrm{~K} \cdot \mathrm{km}^{-1}$ is given for the rest of the vertical domain. The initial profile of water vapor mixing ratio is constructed by assuming the relative humidity in the slightly stable layer to be $95 \%$, followed by a relative humidity of $20 \%$ for the rest of the vertical domain. The initial winds are also adopted from Hignett (1991). Since the FIRE observations were associated with surface high-pressure systems, we include large-scale subsidence rates linearly increasing from zero at the surface up to $0.5 \mathrm{~cm} \cdot \mathrm{s}^{-1}$ at $1000 \mathrm{~m}$, and then linearly decreasing to zero at the top of the domain.

Figure 1 shows the time evolution of liquid water potential temperature versus height from day 4 to day 11 of the model simulation. The first three model days were considered as the model adjustment period, which had certain diurnal variations but not as regularly as that shown in Figure 1. The inversion layer is clearly identified with a diurnal cycle in thickness. The potential temperature in the boundary layer also undergoes a distinct diurnal cycle: well-mixed in the nighttime and early mornings versus moderately stratified in the upper part of the boundary layer in the afternoons. Figure 2 shows the time evolution of total water mixing ratio, where its diurnal cycle is in phase with potential temperature shown in Figure 1. In the nighttime and early mornings, the total water is quite well mixed, and, in the afternoons, the total water reaches a minimum in the upper half of the boundary layer. Figure 3 shows the time evolution of cloud liquid water. Again, almost the same diurnal cycle is seen.

To explain the features of diurnal variations in Figures 1 to 3 , it is instructive to show the time evolution of turbulence kinetic energy (Figure 4). Turbulence kinetic energy is more significant in the nighttime and early mornings than in the afternoons, where the depressed turbulence agrees with the stratification shown in Figure 1. Because of this less turbulent mixing, the water supply from the surface is reduced, resulting in a total water minimum as shown in Figure 2, which in turn causes the liquid water minimum shown in Figure 3.

We have postponed the discussion of the reason for the temperature variations shown in Figure 1, especially the distinct stratification in the upper part of the boundary layer in the afternoons. The variation is primarily caused by the cloud radiative heating from absorption of solar radiation. Radiative heating not only heats the air and evaporates the 


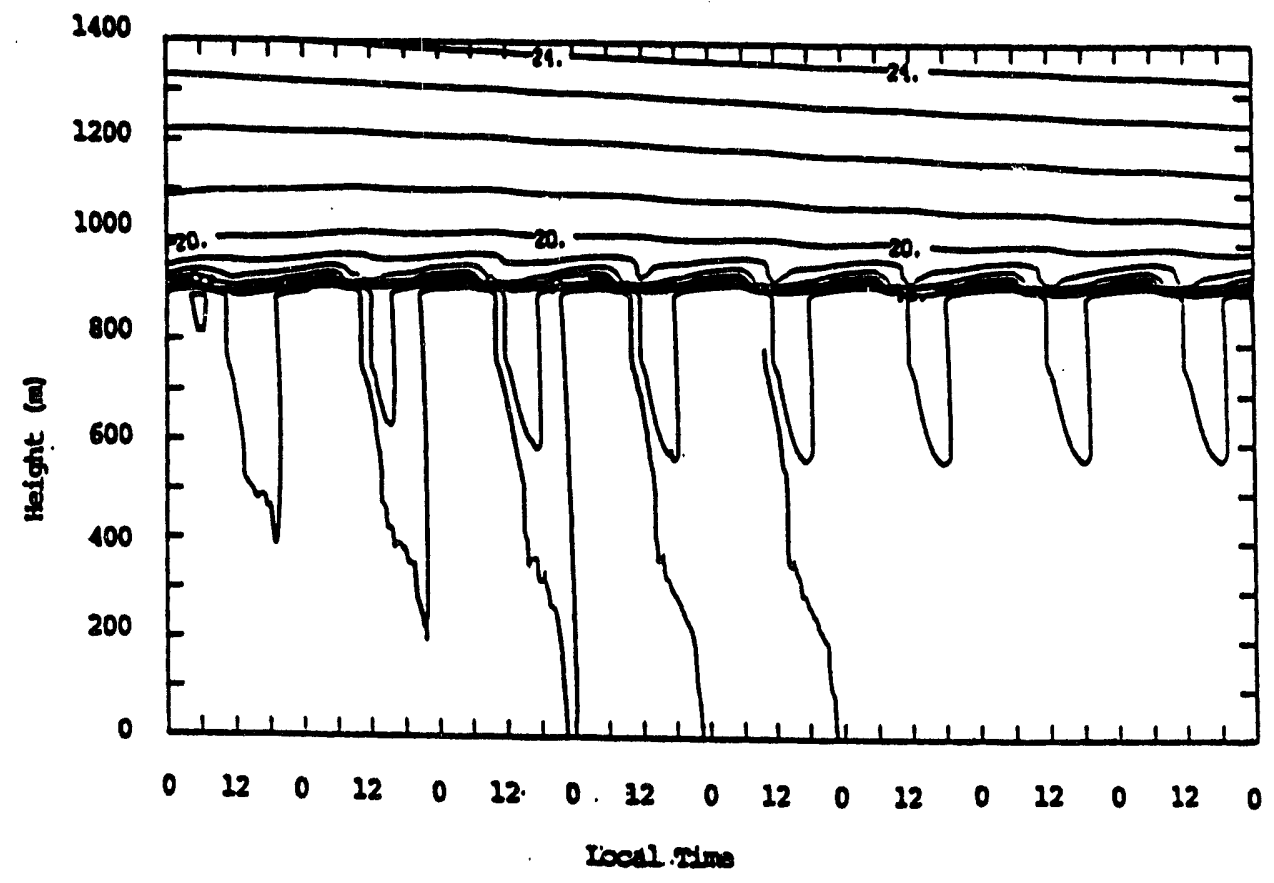

Figure 1. Time evolution of liquid water potential temperature as a function of height.

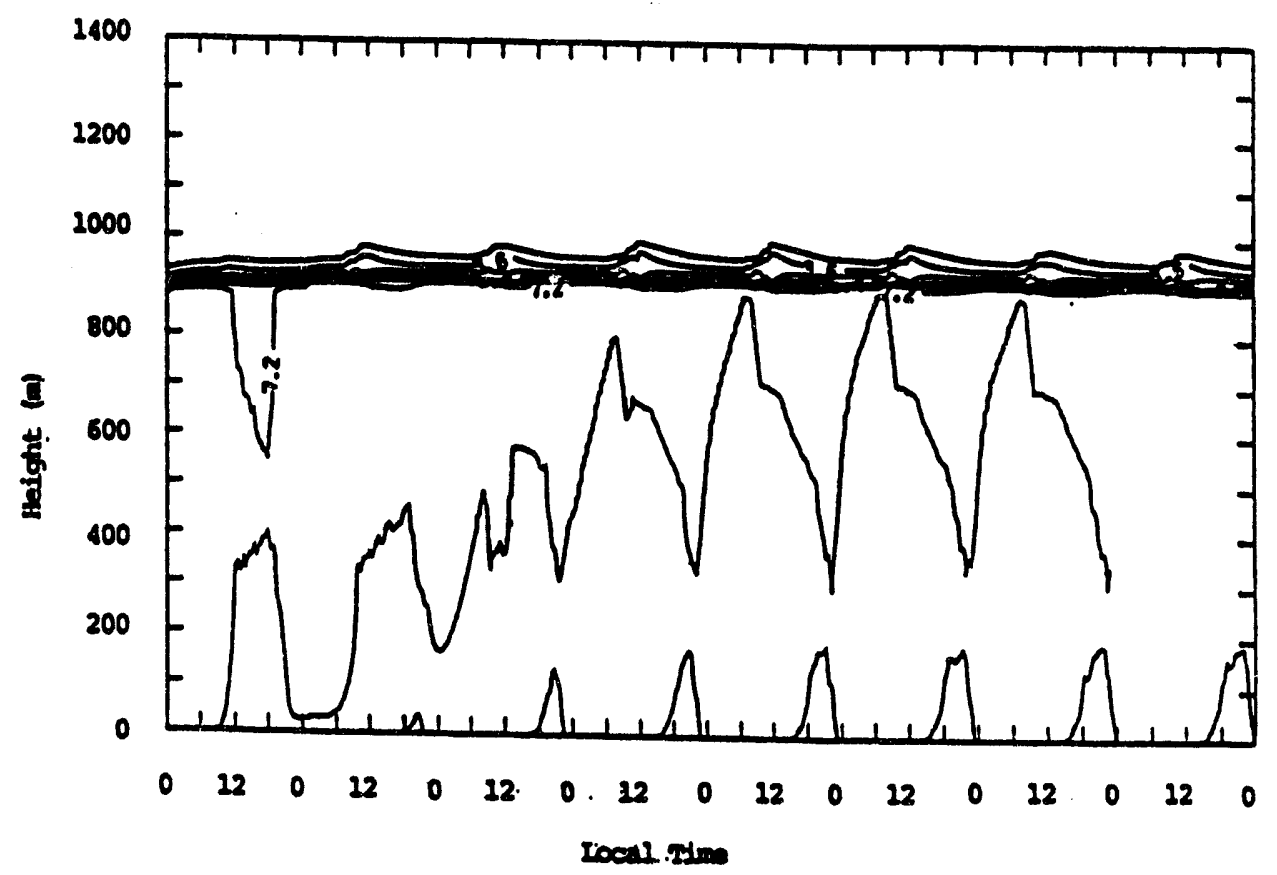

Flgure 2. Time evolution of total water mixing ratio as a function of height. 


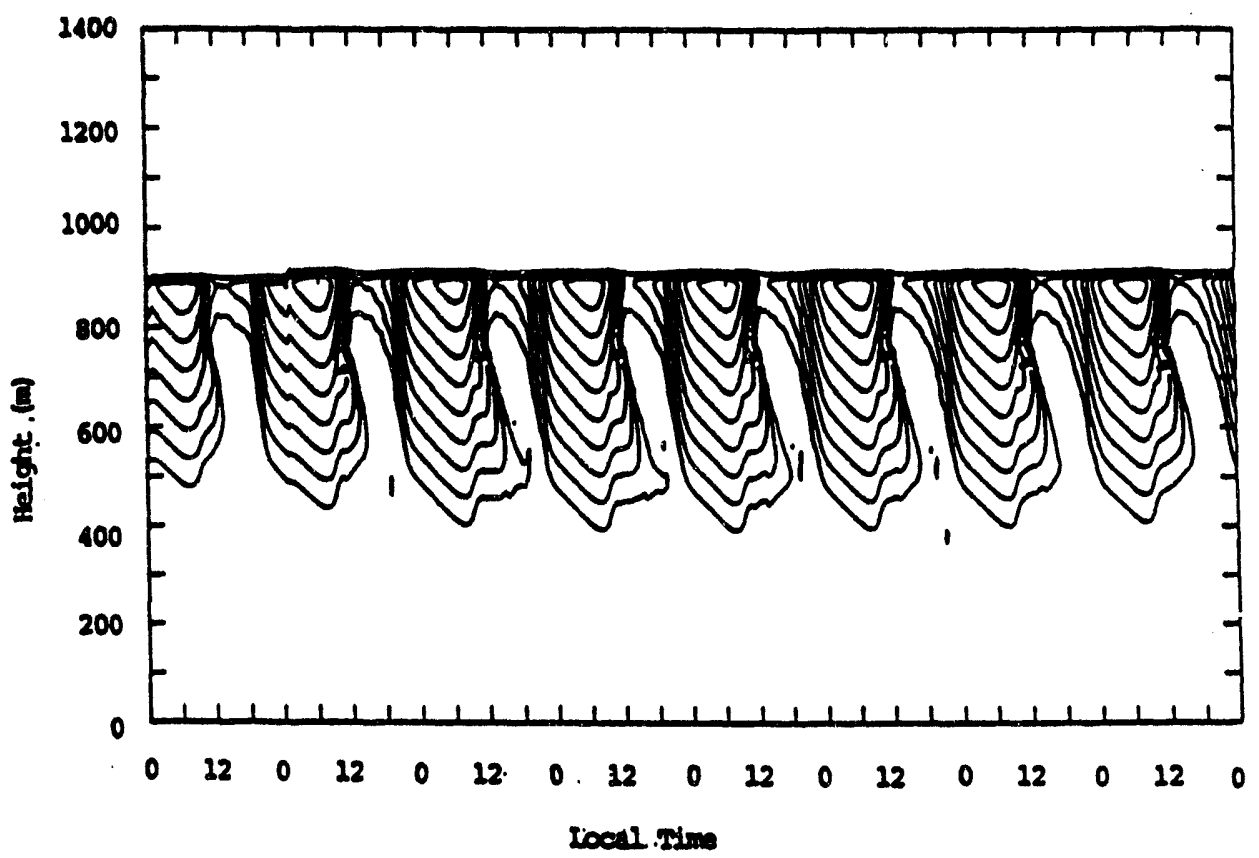

Figure 3. Time evolution of cloud liquid water as a function of height.

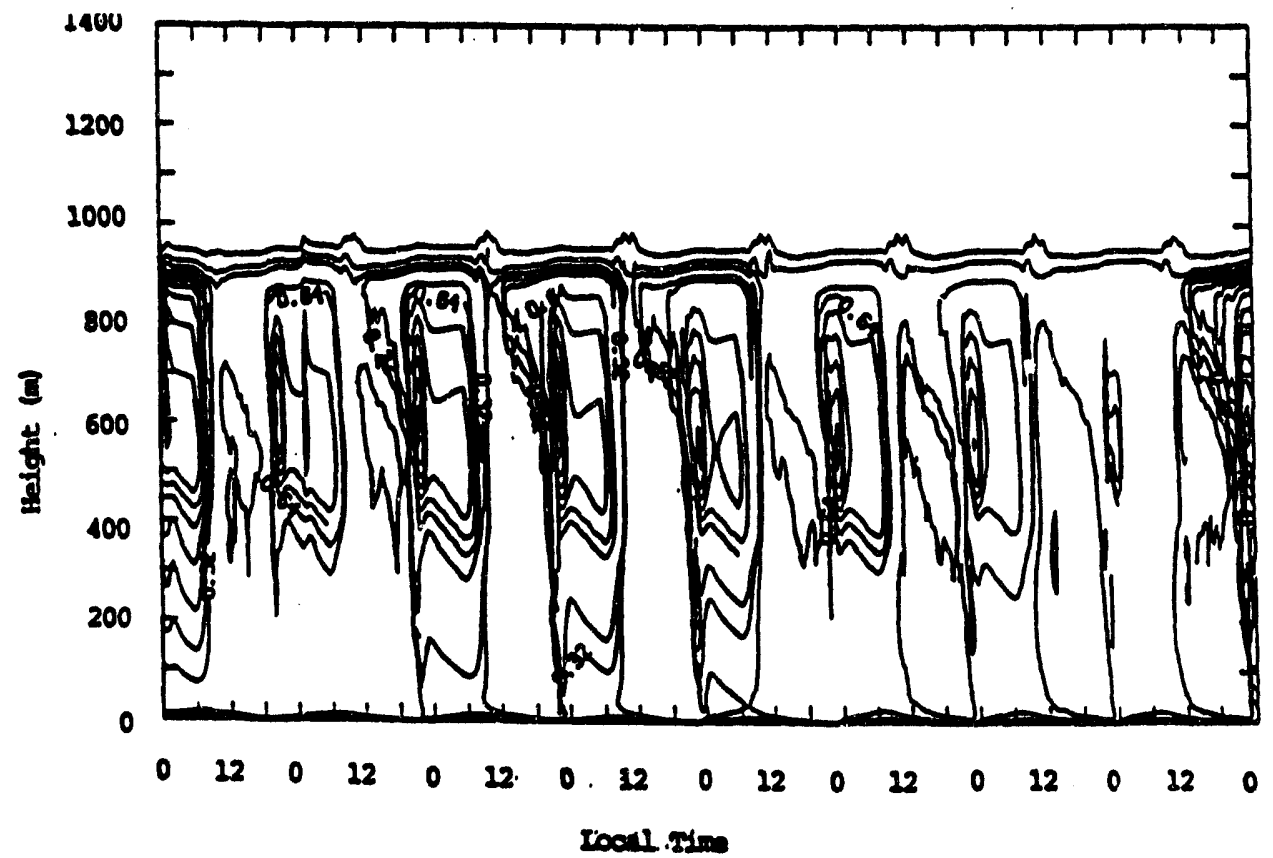

Figure 4. Time evolution of turbulence kinetic energy as a function of height. 
cloud droplet, but also depresses the turbulent mixing by stabilizing the boundary layer. Overall, the physical process described above has been characterized as the "the decoupling of turbulent mixing caused by cloud radiative heating" by several theoretical and numerical approaches (e.g., Turton and Nicholls 1987; Duynkerke and Driedonks 1987) to understand the cloud-capped boundary layers. Our current study here has readdressed this issue with the FIRE data.

\section{Future Work}

Extensive sensitivity tests are required to ascertain the model validity as well as to systematically include all the possible ambient atmospheric and surface conditions. Detailed budget analyses are also useful in categorizing the cloud-capped boundary layers into a few classes. Thus, the parameterization schemes can be designed for GCM or NWP purposes.

\section{Acknowledgments}

This research is supported by the U.S. Department of Energy Atmospheric Radiation Measurement (ARM) Program.

\section{References}

Albrecht, B. A., D. A. Randall, and S. Nicholls. 1988. Observation of Marine Stratocumulus During FIRE. Bull. Am. Met. Soc. 69:618-626.

Betts, A. K. 1990. Diurnal Variation of California Coastal Stratocumulus from Two Days of Boundary Layer Soundings. Tellus 42A:302:304.
Blaskovic, M., and R. Davies. 1991. Dlurnal Variation of Marine Stratocumulus over San Nicolas Island During July 1987. Mon. Wea. Rev. 119:1469-1478.

Duynkerke, P. G., and A.G.M. Driedonks. 1987. A Model for the Turbulent Structure of the Stratocumulus-topped Atmospheric boundary Layer. J. Atmos. Sci. 44:43-64.

Hignett, P. 1991. Observations of Diurnal Variation in a Cloud-capped Marine Boundary Layer. J. Atmos. Sci. 48(12):1474-1482.

Kao, C.-Y.J., and T. Yamada. 1989. Numerical Simulations of a Stratocumulus-capped Boundary Layer Observed Over Land. J Atmos. Sci. 46(6):832-848.

Randall, D. A., J. A. Coakley, Jr., C.W. Fairall, R. A. Kropfli, and D. H. Lenschow. 1984. Outlook for Research on Subtropical Marine Stratiform Clouds. Bull. Am. Met. Soc. 108:103-123.

Sasamori, T. 1968. The Radiative Cooling Calculation for Application to General Circulation Experiments. J. Appl. Meteorol. 7:721-729.

Slingo, A., and H. M. Schrecker. 1982. On the Shortwave Radiative Properties of Stratiform Clouds. Quart. J. Roy. Met. Soc. 108:407-426.

Turton, J. D., and S. Nicholls. 1987. A Study of the Diurnal Variation of Stratocumulus Using a Multiple Mixed Layer Model. Quat. J. Roy. Met. Soc. 113:969-1009.

Yamada, T., and C.-Y.J. Kao. 1986. A Modeling Study on the Fair Weather Marine Boundary Layer of the GATE. J. Atmos. Sci. 43(24):3186-3199. 
Hiararchical Diagnosis

\title{
Prognostic Cloud Water in the Los Alamos General Circulation Model
}

\author{
J. E. Kristjánsson and C.Y.J. Kao \\ Earth and Environmental Sciences Division \\ Los Alamos National Laboratory \\ Los Alamos, NM 87545
}

Most of today's general circulation models (GCMs) have a greatly simplified treatment of condensation and clouds. Recent observational studies of the earth's radiation budget have suggested cloud-related foedback mechanisms to be of tremendous importance for the issue of global change. Thus, an urgent need for improvements in the treatment of clouds in GCMs has arisen, especially as the clouds relate to radiation. In this paper, we investigate the effects of introducing prognostic cloud water into the Los Alamos GCM. The cloud water field, produced by both stratiform and convective condensation, is subject to 3-dimensional advection and vertical diffusion. The cloud water enters the radiation calculations through the longwave emissivity calculations.

Results from several sensitivity simulations show that realistic cloud water and precipitation fields can be obtained with the applied method. Comparisons with observations show that the most realistic results are obtained when more sophisticated schemes for moist convection are introduced at the same time. The model's cold bias is reduced and the zonal winds become stronger because of more realistic tropical convection.

\section{Introduction}

The last decade has seen an increasing demand for better and more reliable models for the general circulation of the atmosphere. As the models have improved, their results have been used as a basis for political documents (IPCC 1990). The models still have many unresolved problems. Perhaps the most notable ones concern the coupling between the atmosphere and ocean and the treatment of clouds. In this paper, we shall address some aspects of the latter problem.
For a long while, cloud treatment was extremely simple in most GCMs, see, e.g., Manabe et al. (1965). The condensation schemes simply dumped out as rain all condensing moisture, meaning that no mass was left in the water/ice phase. The radiation scheme would then assume a certain cloud distribution, which in the earliest models was based on climatology, but was later replaced by some interactive information, e.g., condensation rates. This is still the situation in many of today's models, and there is an urgent need to develop more realistic couplings between condensation and radiation. These couplings are a key feature of the atmosphere's response to changes in extemal forcings, as emphasized by, ө.g., Kiehl and Ramanathan (1990).

One step that can be taken to improve the cloud treatment and its coupling to radiation is to carry cloud waterfice as a prognostic variable in the model. First proposed by Sundqvist (1978), the approach has been subsequently adopted by a few GCM groups, $\theta .9$., Roeckner (1988), Le Treut and Li (1988). But none of these studies have clearly identified the significance of using prognostic cloud water. Furthermore, the cloud water has been computed for stratiform condensation only. In the present model, convection also produces cloud water, which is put together with its stratiform counterpart and subjected to both advection and vertical diffusion.

Since it is well known that both short- and longwave radiative transfer are strongly dependent on liquid (or ice) water path, the cloud water variable enables an improved interaction with radiation. It is perhaps less obvious that the cloud water needs to be prognostic, rather than diagnostic. However, as discussed by Randall (1989), the prognostic feature is expected to be particularly important in areas of deep convection. There, cloud ice, in the form of cirrus 
clouds formed by the deep convection, can be subject to advection over long distances by upper-tropospheric winds, since ice particles have a long lifetime in these environments.

In this paper, the effects of introducing the prognostic cloud water treatment of Sundqvist (1988) in the Los Alamos GCM will be investigated. Results from several annual. cycle sensitivity simulations will be described. The purpose of the simulations was to clarify details concerning the cloud treatment.

\section{The Los Alamos GCM and its Cloud Treatment}

The GCM at Los Alamos National Laboratory (LAGCM) is a modified version of the National Center for Atmospheric Research's (NCAR) Community Climate Model (CCMO) described by Pitcher et al. (1983). The modifications, described in detail by Malone et al. (1986) and Kao et al. (1990) are

- The vertical resolution has been expanded from 9 to 20 levels.

- The advection of moisture has been improved substantially by introducing a fourth-order accurate finite-element scheme in the horizontal and an FCT method in the vertical.

- The vertical diffusion parameterization has been improved by introducing stability-dependent fluxes of heat and moisture between the planetary boundary layer (PBL) and the free atmosphere.

- The mode/ now has prognostic equations for temperature in 6 soil-layers instead of a diagnostic equation.

- There are now prognostic equations for soil moisture based on the model's hydrological cycle, instead of constant soil-moisture conditions.

- Two changes have been made to the condensation scheme, as a consequence of the enhanced vertical resolution in the model. First, the stratiform condensation now requires $100 \%$ relative humidity in the grid box, compared to $80 \%$ earlier. Second, the maximum cloud cover allowed in a stratiform grid box has been changed from $95 \%$ to $80 \%$.
Obvious weaknesses that remain are lack of vegetation and lack of snow cover. Also, the coarse horizontal resolution (R15 spectral truncation) prohibits an accurate positioning of the cyclone tracks.

In the control version of the LAGCM, the cloud treatment is of the greatly simplified type described in the previous section. Convection is treated by the moist-adiabatic adjustment (MAA) scheme of Manabe et al. (1965). Krishnamurti et al. (1980) have shown this scheme to yield unrealistic distributions of precipitation. In both the convective and stratiform treatment, all the condensed moisture is immediately released as precipitation, which falls to the ground in one time-step. In reality, of course, condensation produces clouds, which subsequently may or may not precipitate, depending on their water content, temperature, vertical motions, etc. Furthermore, a portion of the precipitation evaporates on the way down, moistening the air column. In addition, the presence of the ice phase may complicate the picture, $\theta .9$., by causing cooling at the level where precipitation changes from frozen to liquid form. All these modifications to the simplified original picture are taken into account in the alternative condensation treatment introduced in this paper.

The starting point for the new condensation treatment is the scheme of Sundqvist (1988), hereafter termed S88. Cloud water is introduced as a prognostic variable for both stratiform and convective clouds. The cloud water is subject to both 3-dimensional advection and vertical diffusion. As explained by S88, the treatment can be applied to any convection scheme. Because of the inherent weaknesses with the existing convection scheme, we have in this paper applied this cloud treatment to the more sophisticated Kuo (1974) and Arakawa-Schubert (A-S) (1974) schemes, as well as to the MAA scheme. In all three cases the stratiform condensation will be treated in the same way, given by S88. This means that stratiform condensation takes place as long as the relative humidity is above a "threshold value, ${ }^{\text {in }}$ this case, $85 \%$. For humidities between $85 \%$ and $100 \%$, an assumption is made on the partitioning of moisture between the cloudy and cloudfree parts of the grid box.

A fairly detailed parameterization of cloud microphysics is applied to all clouds. Precipitation release is enhanced in those grid points where coalescence is expected to occur, as well as in mixed ice-water clouds (Bergeron-Findeisen 
effect). A noveliy here compared with $\mathbf{S 8 8}$ is the inclusion of the latent heat of freezing and melting. When condensation occurs at temperatures below $273 \mathrm{~K}$, a portion of the condensed water is assured to freeze. This is determined by a function, $P_{f r}$, which increases linearly from zero to unity as the temperature goes from 273 to 233 K. For temperatures below $233 \mathrm{~K}, P_{t}$ is equal to 1 , meaning that spontaneous freezing is expected to occur. Melting is assumed to occur as frozen or mixed precipitation falls through the $275 \mathrm{~K}$ isotherm on its way down. The degree of melting is computed based on the average temperature of the cloud from which the precipitation is falling.

An important advantage of treating cloud water content explicitly is that it can be used in the radiation calculations. Shortwave albedo and absorption as well as longwave emissivity are known to depend strongly on the liquid water path, which is obtained as the vertical integral of the cloud water mixing ratio.

No changes have been made to the model's cloud cover parameterization. The cloud cover is determined as a function of the condensation rate at the actual time-step. There is some empirical "hard-coding", $\theta$. g., a low stratiform cloud will always extend over three model-levels. Also, no clouds are allowed to form above a specific level, which varies with latitude.

\section{Experimental Setup}

So far, four 1-year-cycle simulations using seasonally varying boundary conditions have been conducted, as well as several shorter-term sensitivity experiments. The purpose of the simulations has been to study the performance of the prognostic cloud water scheme, as well as to seek ways to improve the overall treatment of condensation and clouds in the model. In all cases, results were compared with the basic version of the LAGCM, which is termed CONTROL.

The following items were specially investigated:

Sensitivity to cloud water treatment. This was done by comparing runs with prognostic cloud water and diagnostic cloud water (hence no transport of cloud water). These runs will be termed PROG and DIAG, respectively. Both were conducted using the SUNKUO condensation treatment.
Sensittulty to coupling botween condensation and radiation. So far, only the effect of cloud water content on longwave radiation has been investigated. We compared experiments assuming that all clouds are black (BLACK), and conducted two experiments with cloud water dependent emissivities. In the first case, the vertical integral of cloud water through all model levels was taken as a measure of the optical depth (TOTAL); in the other case, the integral was taken only over one model layer at a time (LAYER). All three runs were performed using the SUNKUO condensation treatment.

Sensitivity to cholce of convection scheme. Runs applying the MAA scheme, the Kuo scheme and the A-S scheme for convection were compared. In all cases, the runs were combined with the prognostic cloud water treatment of $S 88$, as explained in the previous section. The runs are termed, respectively SUNMAA, SUNKUO, and SUNAS.

\section{Results}

Comparisons between PROG and DIAG reveal certain changes in precipitation patterns. These changes are not unexpected, since in DIAG all the cloud water produced in a given time step is dumped out as precipitation. The most important difference between the two runs is stronger condensational heating in PROG, especially in the tropics, which has less cirrus than in DIAG. Further investigations are required to fully explain these results, but they seem to suggest that the transport of cloud water may be an important feature in enhancing tropical convection.

When all clouds are treated as "black," cirrus clouds tend to emit unrealistically large amounts of heat, thereby cooling the upper troposphere. Here, BLACK turned out to have more cirrus than LAYER; furthermore the tropical convection was stronger, yielding larger cloud water contents (Table 1). The LAYER run, on the other hand, exhibited a very strong surface inversion at high latitudes, resulting in unrealistically low surface temperature, as well as persistent fog in these areas. This result appears to stem from an insufficient "thermal shielding effect" of the clouds. Precipitation in LAYER is grossly underestimated, possibly because of reduced cloud-top cooling. These results suggest that with 20 vertical levels, it is not 
Table 1. Global averages for selected quantities as given by the different experiments and observations: Cloud cover, vertically integrated cloud water content, precipitation rate, planetary albedo.

\begin{tabular}{|c|c|c|c|c|}
\hline Experiment & $\begin{array}{l}\text { Cloud cover } \\
(\%)\end{array}$ & $\begin{array}{l}\text { Cloud Water } \\
\left(\mathrm{kg} /\left[\mathrm{m}^{*} \mathrm{~m}\right]\right)\end{array}$ & $\begin{array}{c}\text { Procipitation } \\
\text { (mm/day) }\end{array}$ & $\begin{array}{l}\text { Albedo } \\
(\%)\end{array}$ \\
\hline CONTROL & 60.2 & & 2.11 & 29.1 \\
\hline SUNMAA & 75.2 & 0.164 & 2.73 & 34.8 \\
\hline SUNKUO & 52.5 & 0.056 & 2.34 & 26.0 \\
\hline SUNAS & 65.2 & 0.083 & 2.47 & 29.9 \\
\hline PROG/TOTAL & 52.5 & 0.056 & 2.34 & 26.0 \\
\hline DIAG & 63.9 & 0.000 & 2.28 & 27.3 \\
\hline BLACK & 50.9 & 0.064 & 2.68 & 26.0 \\
\hline LAYER & 59.5 & 0.053 & 1.38 & 26.2 \\
\hline Observed & $62(a)$ & $0.072^{(b)}$ & $2.67(c)$ & $31.7^{(c)}$ \\
\hline
\end{tabular}

(a) Hurrell and Campbell 1992.

(b) Njoku and Swanson 1983.

(c) Piexoto and Oort 1992.

appropriate to compute the liquid water path for the emissivity calculations "layer by layer."

The model has been run one full annual cycle with the different convection schemes. We then compared the results for January of the following year (1980). Some comparisons will be made to ECMWF analyses of the Januaries 1979-1886, as well as to the papers referenced in Table 1.

\section{Temperature}

Both SUNKUO and SUNAS significantly reduce the model's cold blas (a well-known feature of this model) in the mid. and upper tropical troposphere and the cold bias in the winter hemisphere (NH).

\section{Zonal Winds}

In all cases there are significant errors. The maximum westerlies in the northern hemisphere (winter) troposphere are shifted poloward compared with observations. The easterlies in the tropical tropopause are too strong. SUNKUO and SUNAS have a larger northern hemispheric (NH) jet maximum than CONTROL, quite close to the observed. The jet in the southern hemisphere (SH) is stronger than observed in both SUNKUO and SUNAS, weaker than observed in CONTROL.

\section{Merldional Winds}

In both CONTROL and SUNMAA, the southerly wind maximum associated with the Hadley cell is displaced 5 to 
$10^{\circ}$ poleward (in NH) and is about twice as large as the observed maximum. SUNKUO and SUNAS, on the other hand, give results quite similar to observations.

\section{Cloud Cover}

In CONTROL, SUNMAA, and SUNAS, the bulk of the cloudiness is between 750 and $950 \mathrm{hPa}$ at all latitudes. At mid-latitudes, SUNMAA has more cloudiness here than does CONTROL because the threshold value in the Sundqvist scheme is lower than in CONTROL. SUNKUO does not exhibit the "excessive" low-level cloudiness, but exhibits more high clouds in the tropics, as well as at high latitudes. The zonally and vertically averaged cloud cover is clearly excessive in the SUNMAA run (Table 1) and too low in SUNKUO. Consequently, the global planetary albedo is too large in SUNMAA, too low in SUNKUO.

\section{Cloud Water Mixing Ratio}

In SUNKUO, SUNMAA, and SUNAS (Figure 1), the vertically integrated cloud water content has maxima in the tropics and in connection with the cyclone tracks in both hemispheres. The zonally averaged values are mainly between 0.1 and $0.01 \mathrm{~kg} \mathrm{~m}^{-2}$ in SUNAS and SUNKUO, which is in good agreement with Njoku and Swanson (1983) (Table 1), whose SMMR-microwave-analysis only applies over ocean areas between $60^{\circ} \mathrm{N}$ and $60^{\circ} \mathrm{S}$. In SUNMAA, on the other hand, the values are generally two to three times larger. This seems to indicate that the "excessive low cloudiness" mentioned above is associated with dense clouds. It has to be kept in mind that, because of the large uncertainty in measurements of cloud water content, the values given by Njoku and Swanson (1983) can only be regarded as an order of magnitude guidance.

\section{Preclpltation}

As seon in Table 1, globally averaged precipitation is larger in the runs with prognostic cloud water than in CONTROL. It is alsocloser to the observed. In all cases, the precipitation is largest in the tropics, with a secondary maximum in the mid-latitude storm tracks.

\section{Discussion}

The MAA scheme does not produce sufficiently deep convection. Another point to note is that when this scheme was used, the number of convective grid columns over the globe was only half of what it is when the Kuo scheme is used. This reduction is probably caused by the abrupt release of the conditional instability in this scheme, rendering a more stable atmosphere than after a corresponding time-step with the Kuo scheme. This stable atmosphere can then undergo stratiform condensation in the next time-step. Hence, one reason for the excessive low cloudiness in the SUNMAA run may be that there is too much stratiform cloud formation. The microphysical parameterization is slightly different for stratiform and convective clouds (S88), respectively, such that the former have longer lifetimes than the latter. This contributes to larger cloud water contents when convective clouds are "replaced" by stratiform clouds.

The excessive cloud coverage in SUNMAA is tied to the model's cloud cover parameterization. The formulation is quite empirical and may not be valid once significant changes are made to other parts of the cloud treatment, as is done in this paper.

\section{Summary and Conclusions}

A sophisticated condensation and cloud package has been incorporated in the LAGCM. The most important features of this package, which is based on the work of Sundqvist (1978, 1988), are prognostic cloud water; sophisticated microphysics, including freezing and melting; and subgrid scale condensation parameterization for stratiform condensation. The package has been coupled to improve convection schemes that also carry cloud water. The cloud water field is subject to transport by both advection and diffusion. Furthermore, clouds are no longer assumed to be "black" emitters in the infrared. Rather, in agreement with observations, their emissivities are assumed to depend on the cloud water content.

The effects of these improvements have been studied by comparing results from sensitivity experiments with the GCM. The main findings so far are 


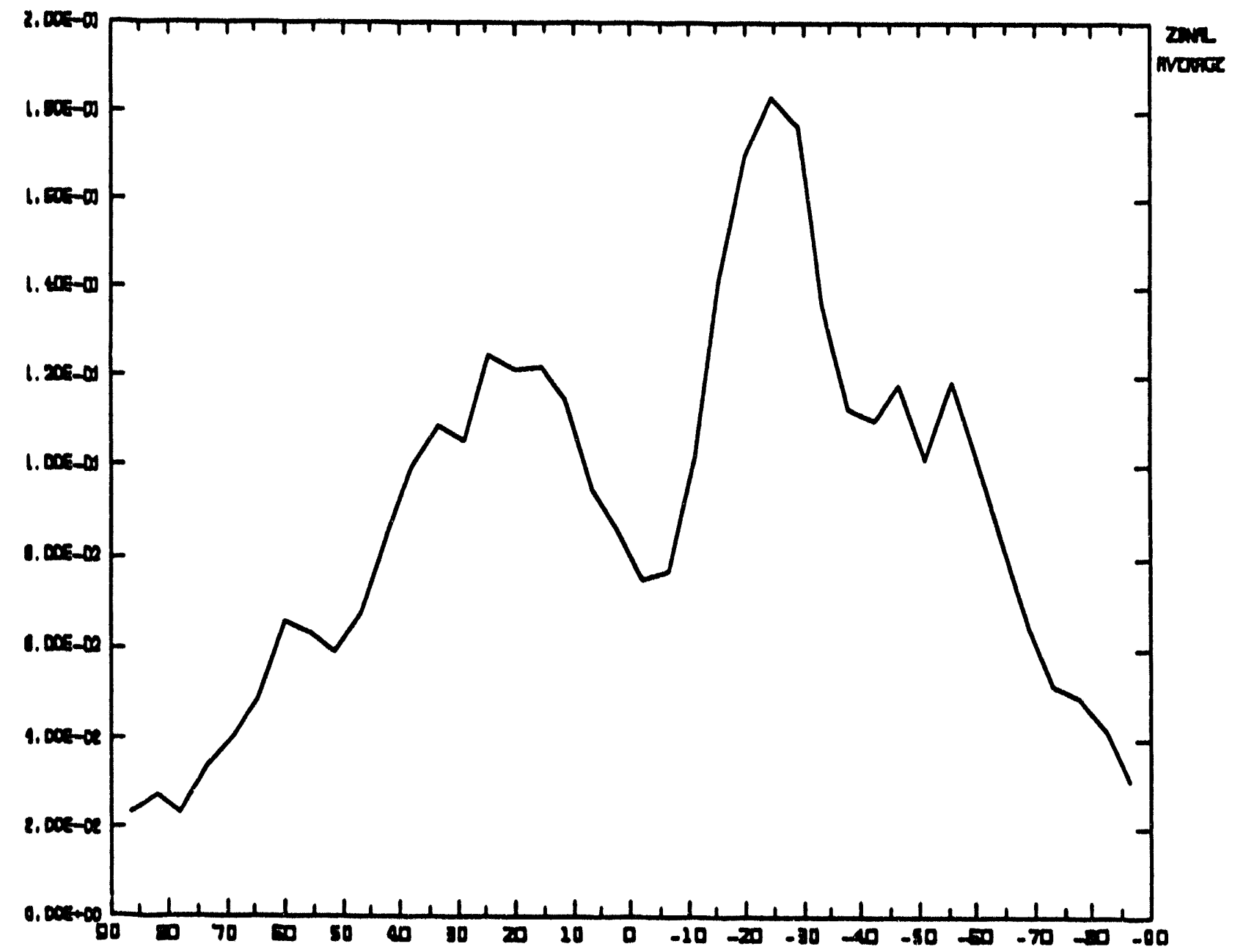

Flgure 1. Vertically integrated zonally averaged cloud water content versus latitude in run SUNAS. Positive latitudes refer to $\mathrm{NH}$, negative to $\mathrm{SH}$. Units are $\mathrm{kg} \mathrm{m}^{-2}$. ,

- The blggest advantage of using prognostc cloud water rather than diagnostic cloud water is that it can be transported by the winds. Hence, a more correct time evolution of the clouds is obtained.

- The results are quite sensitive to the change in the cloud emissivity. When all clouds are black, there is an excessive cooling of the upper troposphere. When the omiseivity la calculated layer by layer, excessive cooling at the ground is found because of an underestimated "shielding" from the clouds.

- All the runs with different convective schemes yield cloud water fields that have many realistlc foatures. However, the integrated cloud water content in the SUNMAA run seems to be overestimated because of very persistent low clouds associated with this scheme.

- The A.S scheme and the Kuo scheme render more realistic latent heating distributions in the tropics, with more deep convection than the MAA scheme. This, togetherwith reduced cloud cover, substantially reduces the model's cold blas and gives stronger zonal winds, which correspond better to observations. 


\section{Future Plans}

The following items will be Investigated further during the next fow months:

- The shortwave albedo will be made dependent on the cloud water content, 800 e.9. . Taylor and Ghan (1992). This will presumably enhance the model's sensitivity to the cloud water treatment.

- The model's cloud forcing and climate sensitivity will be investigated to find out how these important parameters change as the cloud treatment is modified.

- Improvements will be sought to the model's cloud cover parameterization, which is fairly "hard-coded" at the present time.

- Ten-year simulations will be carried out to obtain more confidence in the results. More extensive comparisons will be made to avallable observations.

\section{Acknowledgments}

The authors would like to thank David Langley for assistance regarding computer graphics and model runs. We have benefitted from numerous discussions with Prof. Hilding Sundqvist, Stockholm University, and Dr. Phillip Rasch, National Center for Atmospheric Research.

\section{References}

Arakawa, A., and W. H. Schubert. 1974. Interaction of a cumulus cloud ensemble with the large-scale environment, Part I. J. Atmos. Scl. 31:674-701.

Hurrell, J. W., and G. G. Campbell. 1992. Monthly mean global satellite data sets avallable in CCM history tape format. NCAR Technical Note 371+STR, pp. 33-34.

International Panel on Climate Change (IPCC). 1990. Climate change. The IPCC scientific assessment. Report prepared for Intergovernmental Panel on Climate Change by Working group I. WMONNEP, Cambridge University Press.
Kao, C.-Y.J., G. A. Glatzmaier, R. C. Malone, and R. P. Turco. 1990. Global three-dimensional simulations of ozone depletion under postwar conditions. J. Geophys. Res. 95D:22495-22512.

Klehl, J. T., and V. Ramanathan. 1990. Comparison of cloud forcing derlved from the Earth Radiation Budget Experiment with that simulated by the NCAR Community Climate Model. J. Geophys. Res. 95D:11679-11698.

Krishnamurti, T. N., Y. Ramanathan, H.-L. Pan, R. J. Pasch, and J. Molinari. 1980. Cumulus parameterization and rainfall rates I. Mon. Wea. Rev. 108:465-472.

Kuo, H. L. 1974. Further studies of the parameterization of the influence of cumulus convection on large-scale flow. J. Atmos. Scl. 31:1232-1240.

Le Treut, H., and Z.-X. LI. 1988. Using Meteosat data to validate a prognostic cloud generation scheme. Atmos. Res. 21:273-292.

Malone, R. C., L. H. Auer, G. A. Glatzmaier, M. C. Wood, and O. B. Toon. 1986. Nuclear winter: Three-dimensional simulations including interactive transport, scavenging, and solar heating of smoke. J. Geophys. Res. 91D:1039. 1053.

Manabo, S., J. Smagorinsky, and R. F. Strickler. 1965. Simulated climatology of a general circulation model with a hydrological cycle. Mon. Wea. Rev. 93:769-797.

Njoku, E. G., and L. Swanson. 1983. Global measurements of sea surface temperature, wind speed and atmospheric water content from satellite microwave radiometry. Mon. Wea. Rev. 111:1977-1987.

Peixoto, J. P., and A. H. Oort. 1992. Physics of climate. American Institute of Physics, New York.

Pitcher, E. J., R. C. Malone, V. Ramanathan, M. L. Blackmon, K. Puri, andW. Bourke. 1983. January and July simulations with a spectral general circulation model. J. Atmos. Sci. 40:580-604.

Randall, D. A. 1989. Cloud parameterization for climate modelling: status and prospects. Atmos. Res. 23:245-361.

Roeckner, E. 1988. Cloud-radiation feedbacks in a climate model. Atmos. Res. 21:293-303. 
Sundqvist, H. 1978. A parameterization scheme for non convective condensation including prediction of cloud water content. Quart. J. Roy. Met. Soc. 104:677-690.

Sundqvist, H. 1988. Physically-based modelling and simulation of climate and climatic change, $\theta d$. M. Schlesinger, pp. 433.461. Reidel Publishing, Dordrecht, Holland.
Taylor, K. E., and S. J. Ghan. 1992. An analysis of cloud liquid water feedback and global climate sensitivity in a general circulation model. J. Clim. 5:907-919. 


\title{
Factors Influencing the Parameterization of Anvil Clouds Within General Circulation Models
}

\author{
J. M. Leone, Jr. and H.-N. (Steve) Chin \\ Lawrence Livermore National Laboratory \\ Livermore, California
}

\section{Introduction}

The overall goal of this project is to improve the representation of clouds and their effects within global climate models (GCMs). We have concentrated on a small portion of the overall goal, the evolution of convectively generated cirrus clouds and their effects on the large-scale environment. Because of the large range of time and length scales involved, wo have been using a multi-scale attack. For the early time generation and development of the cirrus anvil, we are using a cloud-scale model with horizontal resolution of 1 to 2 kilometers; for the larger scale transport by the larger scale flow, we are using a mesoscale model with a horizontal resolution of 20 to 60 kilometers. The eventual goal is to use the information obtained from these simulations, together with availative observations, to derive improved cloud parameterizations for use in GCMs. This paper presents results from our cloud-scale studies and describes a new tool, a cirrus generator, that we have developed to aid in our mesoscale studies. (a)

\section{Cloud-Scale Study}

We chose a midlatitude broken-line squall system for our study because it is the predominant spring time convection in Oklahoma, the location of the first Atmospheric Radiation Measurement (ARM) Program site, and because it can be represented as a two-dimensional system, which is less computationally demanding. To facilitate comparisen with

(a) Worked performed under the auspices of the U.S. Department of Energy by Lawrence Livermore National Leborabry under Contract W-7405-Eng-48. published observations, we used a modification of the composite sounding of Bluestein and Jain (1985) to drive our simulations.

The cloud model is an extension of Chin and Ogura's (1989) two-dimensional model, which was used to study a tropical convective rainband. The model is nonhydrostatic and fully compressible; its dynamic framework is similar to that of Klemp and Wilhelmson (1978). Model physics modules include turbulence, a planetary boundary layer (Blackadar 1979), a two-category liquid water scheme (Soong and Ogura 1973), a three-category ice phase scheme (Lin et al. 1983), and long and shortwave radiative transfor (Harshvardhan ot al. 1987).

The ice microphysics, which was developed for convective clouds, has been modified to better replicate widely spreading anvil clouds. The Harshvardhan et al. radiative transfer scheme in the cloud and mesoscale models was simplified in the longwave and shortwave calculations by ignoring partial cloudiness and assuming each grid cell was either completely cloudy or clear. The cloud optical properties wer also modified to distinguish ice clouds from water clouds using the parameterization schemes of Starr and Cox (1985) and Stephens (1978), respectively. Mixed-phase clouds were also considered in the cloud optical properties. Cloud optical properties are thus functions of model-predicted hydrometeor concentrations.

For this study, we conducted a series of simulations using six different combinations of radiation and microphysics complexity, ranging from no radiation and only liquid microphysics to both longwave and shortwave radiation with full liquid and ice microphysics. To validate our results, we compared the simulations with published observations. The general patterns of the dynamic structure, velocity fields, and pressure deviations bore strong similarities to the features reported by Ogura and Liou (1980) and Smull and Houze (1987). The heating $\left(Q_{1}\right)$ and drying $\left(Q_{2}\right)$ 
profiles were similar to those reported in Ogura and Chen (1977) and Gallus and Johnson (1991). Further, the simulations including both ice and longwave radiation produced a simulated radar reflectivity in which both a melting bright band and a realistic transition zone were present.

\section{Results}

An examination of the various runs leads to a number of interesting observations. The first is that the inclusion of $i c e$ phase and radiation has very little influence on the thermodynamics of the cloud ensemble, as evidenced by the similarity of their respective $Q_{1}$ and $Q_{2}$ profiles. On the other hand, a comparison of simulations with and without ice shows that the ice phase has a strong influence on the prosipitating water distribution. When ice is present, the dominant total water maximum moves upward. Further, ice doubles the precipitating water mass in the storm and redistributes the precipitating water between the convective and stratiform portion of the storm, as illustrated in Table 1.

When we examined the simulations which included radiation, we found the longwave radiation increases the precipitating water in both the convective and stratified portinns of the storms. The addition of shortwave radiation further increases the precipitating water in the ice anvil; however, it reduces the precipitating water in the convective region and in the water anvil.
Table 1. Total precipitating water distribution.

\begin{tabular}{llc} 
Radiation & \multicolumn{2}{c}{ None } \\
Microphysics & lce-free & Ice \\
Convective & $87.7 \%$ & 70.25 \\
Stratiform & 12.35 & $29.8 \%$ \\
Total $(g / g)$ & 0.667 & 1.411
\end{tabular}

We observed that the longwave radiative properties are insensitive to the specific representation of the ice phase. In contrast, the shortwave radiative properties depend strongly upon the condensate phase (Figure 1). The optical depth of the simulated water anvil was 138 compared with 4.4 for the simulated ice anvil that contained approximately the same total precipitable water. In addition, the water anvil had an albedo of between 0.8 and 0.9 (depending upon zenith angle) compared with 0.4 to 0.6 for the ice anvil. The water anvil also had a somewhat larger absorption coefficient. Thus the incident radiation on the surface under the water anvil was $1 / 10$ of that under the ice anvil.

In preparation for developing a GCM parameterization for convectively generated cirrus, we completed a detailed water budget analysis for the mature stage of the storm.

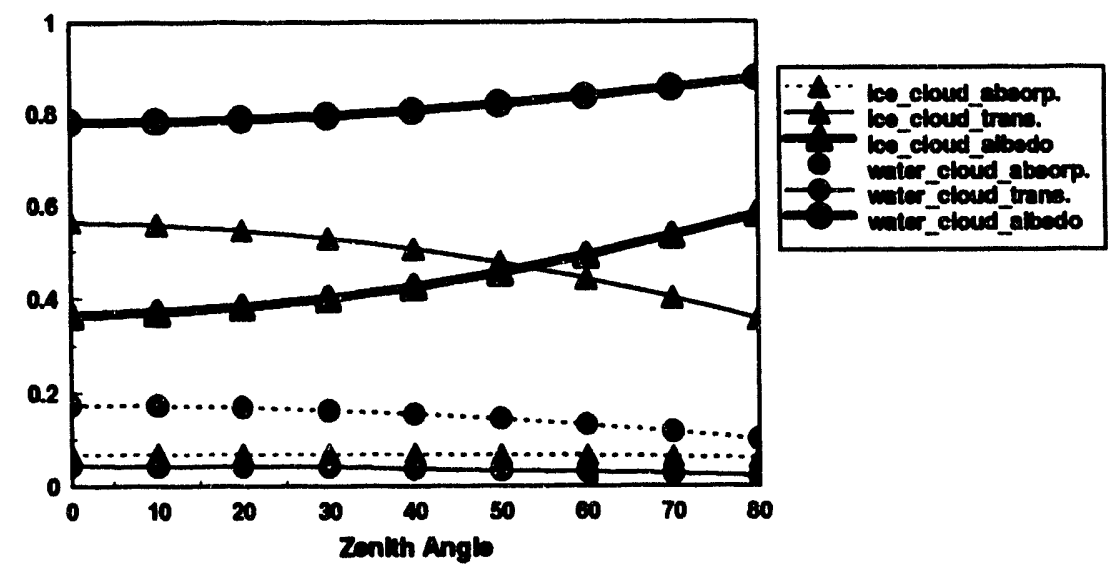

Figure 1. Visible cloud optical properties of temporally and spatially averaged water and ice anvils clouds. 
From this analysis, we found that the mechanisms supporting the leading anvil were quite different from those for the larger rear anvil. For the rear anvil, local microphysics, supported by the induced mesoscale circulation resulting from the interaction between hydrometeors and radiation, is an important contributing mechanism. For an all liquid anvil, the local microphysics contribution is nearly the same size as the transport from the convective core. However, for the ice anvil, the transport from the convective core is nearly twice that in the liquid anvil, while the local microphysics contribution remains nearly the same.

\section{Cirrus Generator}

We are beginning to study the long-range transport of convectively generated cirrus in a mesoscale model. This model, however, cannot resolve the convective parent that injects ice into the upper levels of the atmosphere, so we must introduce cirrus into the domain in some other manner, e.g., via a convective parameterization, through the initial conditions, or by means of the lateral boundary conditions. We have developed a cirrus generator that is included in the mesoscale model for the purpose of developing cirrus within the model domain.

The cirrus generator is a set of tuned forcing functions that represent the mesoscale forcing caused by the unresolved cumulus clouds. It provides the vertical heating profiles and the momentum, moisture, and heat transport by the unresolved clouds. These profiles are derived by spatially and temporally averaging the collective properties of cloud ensembles from either cloud-scale simulations or observations.

Our initial development has been based upon data from the GARP(a) (GATE) and refined by runs of our cloud scale model. The cirrus generator has been successfully used in a model with $20-\mathrm{km}$ horizontal resolution, producing a reasonable mesoscale cloud structure that reproduced the observed mature stage mesoscale vertical velocity maximum in both magnitude and height and reproduced the observed average surface precipitation rate.

(a) Global Atmospheric Research Program's Atlantic Tropical Experiment

\section{Summary}

We have been studying convectively generated cirrus and their effects on the large-scale environment using a midlatitude, broken-line squall system as a test bed. We conducted a series of six simulations using various complexities of radiation and microphysics parameterizations. From these simulations, we found that the inclusion of the ice phase and radiation had little influence on the thermodynamics of the cloud ensemble. However, the inclusion of ice microphysics and longwave radiation significantly increases the water mass and total precipitation in the anvil. We also found that while the longwave radiative properties of the anvil are insensitive to the specific representation of the phase, the shortwave radiative properties depended strongly upon the phase of the condensate. A consequence of this is that by missing the anvil, GCM cumulus parameterization schemes underestimate the cloud albedo and overestimate the surface insolation.

In addition to our cloud-scale studies, we are studying the long-range transport and life cycle of the anvil using a mesoscale model. We have developed a cirrus generator that can be included in a mesoscale model with horizontal resolutions too large to resolve cumulus clouds. The cirrus generator represents the mesoscale forcing caused by the unresolved cumulus clouds and forces cirrus into the mesoscale model, which then interacts with the larger scale flow fields.

In the future, we plan to expand our cloud-scale studies to other convective systems moving initially in tropical Pacific convection. We will begin our mesoscale studies of the life cycle of cirrus anvils using the cirrus generator to develop the cirrus in the mesoscale domain. We will combine the information from our modeling studies with avallable observations to develop more robust GCM parameterizations of cirrus anvils and their effects on the larger scale flow.

\section{References}

Blackadar, A. K. 1979. High resolution models of the planetary boundary layer. Advances in Environmental and Science Engineering, Vol. 1, eds. J. R. Pfafflin and E. N. Ziegler, Gordon and Breach, : 76 pp. 
Bluestein, H. B., and M. H. Jain. 1985. Formation of mesoscale lines of precipitation: Severe squall lines in Oklahoma during the spring. J. Atmos. Scl. 42:1711-1732.

Chin, H.-N.S., and Y. Ogura. 1989. Supplementary modeling sturdy of a tropical convective band. J. Atmos. Sci. 46:1440-1447.

Gallus, W. A., Jr., and R. H. Johnson. 1991. Heat and moisture budget of an intense midlatitude squall line. J. Atmos. Sci. 48:122-146.

Harshvardhan, R. Davies, D. A. Randall, and T. G. Corsetti. 1987. A fast radiation parameterization for atmospheric circulation models. J. Geophys. Res. 92:1009-1016.

Klemp, J. B., and R. B. Wilhelmson. 1978. The simulation of three-dimensional convective storm dynamics. J. Atmos. Sci. 35:1070-1096.

Lin, Y.-L., R. D. Farley, and H. D. Orville. 1983. Bulk parameterization of the snow field in a cloud model. J. Clim. Appl. Meteorol. 22:1065-1092.
Ogura, Y., and Y.-L. Chen. 1977. A life history of an intense mesoscale convective storm in Oklahoma. J. Atmos. Sci. 34:1458-1476.

Ogura, Y., and M.-T. Liou. 1980. The structure of a midlatitude squall line:A case study. J. Atmos. Sci. 37:553567.

Smull, B., and R. A. Houze, Jr. 1987. A midlatitude squall line with a trailing region of stratiform rain: Radar and satellite observations. Mon. Wea. Rev. 113:117-133.

Soong, S. T., and Y. Ogura. 1973. A comparison between axisymmetric and slab symmetric cumulus cloud models. J. Atmos. Sci. 30:879-893.

Starr, D. O'C., and S. K. Cox. 1985. Cirrus clouds. Part I: A cirrus cloud model. J. Atmos. Sci. 42:2663-2681.

Stephens, G. L. 1978. Radiation profiles in extended water clouds. II. Parameterization schemes. J. Atmos. Sci. 35:2123-2132. 


\title{
Modeling Studies of the Indo-Pacific Warm Pool
}

\author{
T. P. Barnett, N. Schneider, M. Tyree, and J. Ritchie \\ Scripps Institute of Oceanography \\ La Jolla, CA 92093-0224 \\ V. Ramanathan, S. Sherwood, G. Zhang, and M. Flatau \\ California Space Institute \\ La Jolla, CA 92093-0221
}

We are conducting a wide variety of modeling ${ }^{(a)}$ studies aimed at understanding the interactions of clouds, radiation, and the ocean in the region of the Indo-Pacific warm pool, the flywheol of the global climate system. These studies are designed to understand the important physical processes operating in the ocean and atmosphere in the region. The modeling studies should help with the design of the Atmospheric Radiation Measurement (ARM) site in this region and also put the eventual measurements from the site into large scale perspective.

A stand-alone Atmosphere General Circulation Model (AGCM), forced by observed sea surface temperature (SST), has been used for several purposes. We evaluated the radiaiive aspects of the code against Earth Radiation BudgetExperiment (ERBE) observations. The comparisons were generally excellent. The most serious disagreement was found with the structure of the low clouds in the model. Members of our group are working to improve this aspect of model performance. For instance, a new convection scheme is being introduced to the AGCM to see if it improves response of the Hadley cell. At the same time, a revised radiation/cloud parameterization for the AGCM is being tested with pre-ARM data-a prelude to more comprehensive testing with the fully operational ARM site in both Oklahoma and the Indo-Pacific warm pool site.

Another study with the AGCM shows the high sensitivity of the tropical circulation to variations in mid- to high-level clouds. Changes in warm pool cloud distribution are not

(a) All models used here have been made available to us through the exceptional generosity of the Max Planck Institute for Meteorology in Hamburg. balanced by (local) convective-radiative adjustments. Rather, the entire Walker and Hadley cells are altered sy the cloud changes. The balances are thus affected by dynamic processes, which emphasizes the need to place warm pool ARM measurement into the context of a good AGCM (or coupled GCM).

A stand-alone ocean general circulation model (OGCM) is being used to study the relative role of shortwave radiation changes in the buoyancy flux forcing of the upper ocean. The shortwave flux, normally ignored by oceanõgraphers, is large and plays a significant role during EI Nino events. Interestingly, it is somewhat balanced by associated changes in precipitation and longwave radiation fluxes, leaving evaporative flux as the dominant process affecting the buoyancy forcing over much of the tropical ocean. These results suggest that many of the encouraging OGCM simulations of tropical ocean variability obtained to date may have appeared correct for the wrong reasons.

Complete studies of the warm pool can only be conducted with a full coupled ocean/atmosphere model (CGCM). With that in mind, we are just bringing on line now the latest version of the Hamburg CGCM. The model produces realistic simulations of the ocean/atmosphere system in the Indo-Pacific without use of a flux correction scheme. We plan to use a spinup of the model and the seasonal cycle of the model to explain the dynamic and thermodynamic processes that maintain the warm pool and that cause it to move seasonally. 


\title{
Cloud Studies in Climate Research Programs with 8-Millimeter Wavelength Doppler Radar
}

\author{
R. A. Kropfll, S. Y. Matrosov, T. Uttal, and B. W. Orr \\ National Oceanic and Atmospheric Administration/Environmental \\ Research Laboratories \\ Wave Propagation Laboratory \\ Boulder, CO 80303
}

\section{Introduction}

The National Ocoanic and Atmospheric Administration's (NOAA) Wave Propagation Laboratory (WPL) operated a sensitive, 8-mm wavelength Doppler radar in three recent studies of clouds: the Cloud Lidar and Radar Exploratory Test (CLARET), the First ISCCP(a) Reglonal Experiment (FIRE II), and the Atlantic Stratocumulus Transition Experiment (ASTEX). Each experiment focused on cloud radiative and microphysical properties in order to improve cloud parameterizations in general circulation models. An overviow of results from these experiments is presented to demonstrate the unique measurement capability of $8-\mathrm{mm}$ wavelength ( $K_{a}$-band) radar.

When Integrated with other remote sensors such as a narrow-band infrared (IR) radiometer or a lidar, an $\mathrm{mm}$-wavelength radar can greatly extend the quantitative microphysical information over that obtainable by any one of the sensors alone. Operating at $8 \mathrm{~mm}$ also has other distinct advantages for groundbased cloud remote sensing; such radars can penetrate optlcally thick and high liquid water content cloud layers. As a result, they can detect cirrus clouds above reglons of light precipitation that attenuate lidar and shorter wavelength radar signals.

Radars at $K_{\mathrm{a}}$-band are generally less sensitive to ground clutter than longer wavelength radars, making them operable at close range. Yot the wavelength is long enough to ensure that size parameters for cirrus cloud particles do notextend into the Mie scattering regime, greatly simplifying interpretation of the data. This paper illustrates the significant cloud observing capability of millimeter wavelength Doppler radar with recent examples from the WPL. Ka-band Doppler radar (Kropfll ot al. 1990).

(a) International Satellite Cloud Climatology Project.

\section{Radar Capabilities}

The WPL 8-mm wavelength radar was designed with good sensitivity and resolution to observe the small-scale structure and microphysical properties of clouds. During ASTEX, for example, it observed, with $37 \cdot \mathrm{m}$ resolution, all marine boundary layer (MBL) stratus and stratocumulus clouds within $5 \mathrm{~km}$ of the radar. More dense nonprecipltating clouds and very light drizzle were routinely observed to ranges exceeding $35 \mathrm{~km}$. Characteristics of the radar are summarized in Table 1.

A new offset Cassegrain antenna with good polarization performance is now being developed for this radar to study ice crystal shapes in cirrus clouds. Its capability for dualpolarization at circular, linear, and elliptical polarization basis states and the microphysical information obtainable from such measurements are described by Matrosov (1991) and by Matrosov and Kropfli (1993). These polarization states are made possible by a rotatable quarterwave plate. This relatively unexplored technology based on Gaussian optics theory (Goldsmith 1991) may be

Table 1. Characteristics of WPL $K_{a}$-band radar.

$\begin{array}{ll}\text { Wavelength (cm) } & 0.866 \\ \text { Peak power (kW) } & 100 \\ \text { Pulse width (m) } & 37 \\ \text { Beam width (deg) } & 0.5 \\ \text { Dual polarization } & \text { yes } \\ \text { Scannable } & \text { yes } \\ \text { Transportable } & \text { yes } \\ \text { Doppler accuracy (4 s dwell) } & <5 \mathrm{~cm} \mathrm{~s}^{-1}\end{array}$


appropriate for other millimeter wavelength radars now being developed for cloud research.

\section{Ice Particle Size and Concentration}

For cirrus clouds that are semitransparent in the IR, Matrosov et al. (1992) suggested a method for estimating median ice particle size and concentration averaged vertically through the cloud. To do this, they invoked a twostream radiation model and assumed a first-order gamma distribution of particle sizes. They showed that cirrus cloud optical thickness, $t$, can be estimated with an IR radiometer sensitive in the $9.9511 .43 \mathrm{pm}$ range, and that $t$ varies as the fourth power of the median particle size, $D_{m}$. Also, radar reflectivity, $Z$, is shown to vary with the sixth power of $D_{m}$. Thus, $D_{m}$, along with the second parameter of the gamma distribution, $N_{0}$, is obtainable from these two relationships.

Recently, a technique for estimating ice particle fallspeed from zenith-pointing Doppler radar (Orr and Kropfli 1993) was incorporated into this two-sensor method to provide profiles of median particle size and concentration rather than vertically integrated estimates only (Matrosov ot al. 1993). The additional relationship needed to achieve this is the power law dependency of fallspeed on particte diameter (Pruppacher and Klett 1978). An example of a profile of $D_{m}$ obtained in this way from FIRE II data is shown in Figure 1.

\section{Ice Mass Path (IMP) and Ice Mass Flux (IMF)}

Sassen (1987) showed that radar reflectlvity can be used to provide an estimate of lce mass content (IMC) in cirrus clouds by means of an empirical power law relationship

IMC $=0.037 \mathrm{Z}, 0.696$

where $Z_{1}$ is radar reflectivity for ice particles. The twosensor method mentioned above is expected to produce better results, however, because it accounts for independent particle size and concentration variations; includes inherently more measurement information; and has a physical basis for its validity. Its limitations are that it can be used only for cirrus clouds that are semitransparent in

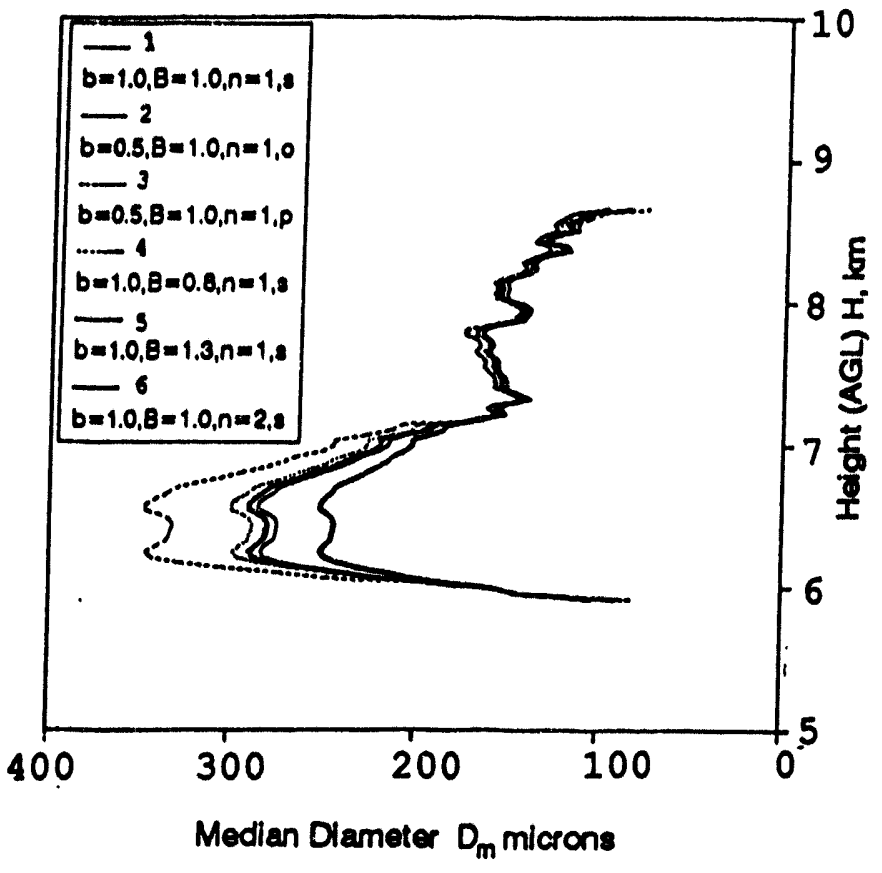

Figure 1. A profile of $D_{m}$ obtained from radar reflectivity and Doppler velocity data along with an IR radiometer for a cloud observed on 26 November 1991 during FIRE II (Uttal et al. 1993). Curves are computed for different values of the particle aspect ratio, b, exponent in the fall velocity-size relationship, $B$, and order of the gamma distribution, $n_{0}$. The letters $s, 0$, and $p$ refer to whether particles are spherical, oblate, or prolate.

the IR, l.e., for optical depths less than about 2.5; and it produces only a pair of values $\left(D_{m}\right.$ and $\left.N_{o}\right)$ representing an average through the cloud depth. The method also cannot be used when liquid water is present. The radar-only method provides ice mass content versus height and is not limited by the cloud optical thickness or amount of cloud liquid present.

Despite the simple empirical approach, the reflectlvityonly method for determining IMP (vertically integrated IMC) produces a result that is well-correlated with downwelling brightness temperature, $T_{b}$, at the surface. It therefore represents a cloud parameter that strongly Influences downwelling IR radiation. Figure 2, from Uttal of al. (1993), shows the relationship between $T_{b}$ measured with a PRT-5 IR radiometer and IMP computed from 
reflectivity when a horizontally homogeneous cirrus cloud with nearly constant base and top heights persisted for about $2.5 \mathrm{~h}$. Also shown on this figure are curves of $T_{b}$ versus IMP computed with a two-stream radiation transfor model for three values of $D_{m}$ as shown by Matrosov of al. (1992). For the fixed $D_{m}$ values indicated in the figure, the concentration of loe particles was allowed to vary to produce the variable IMP along the abecissa in Figure 2. $A D_{m}$ value of $170 \mu \mathrm{m}$ fits these data quite well.

Rough estimates of the vertical flux of ice mase were made by taking the product of the Doppler velocity and the IMC estimated from the Sessen IMC-reflectivity relationship. Averages of this product were obtained for five 25. $\mathrm{min}$ periods on 26 November 1991 during FIRE II. Although there were large variations in the five profiles (not shown), they seemed to have a similar characteristic shape, suggesting that an appropriate normalization might collepee the IMC profiles to a "universal" curve. Figure 3 shows the result of scaling height to the fraction of the distance from cloud base to cloud top and the IMF to the maximum flux value. Such normalization removed much of the profile-toprofile variability.

\section{Hydrometeor Fallspeeds}

A new approach has been taken to investigate relationshipe between reflectivity and the reflectivity-woighted ice particle fallspeed. This approach (Orr and Kropfli 1993) was

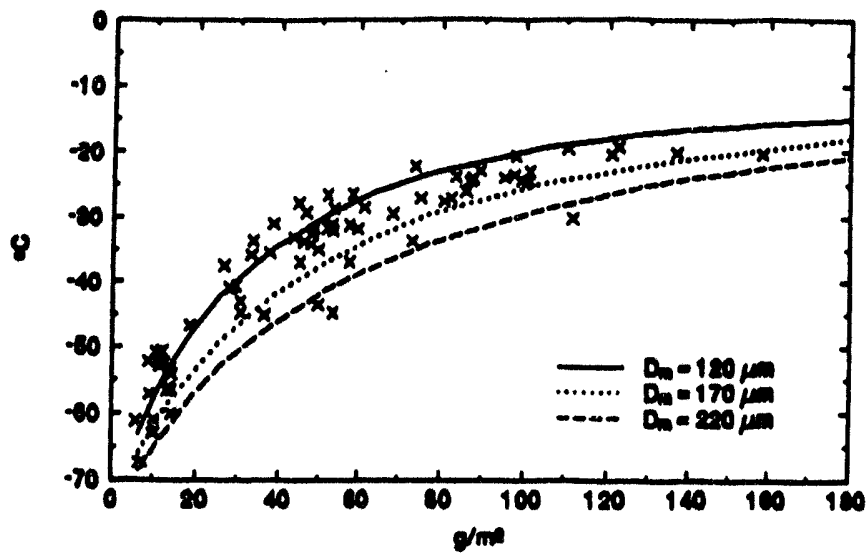

Figure 2. $T_{b}$ versus IMP for a 2.5-h period on 4 October 1989. $T_{b}$ was determined from a PRT -5 IR radiometer and IMP was computed from a vertical integration of IMC (Uttal ot al. 1993).

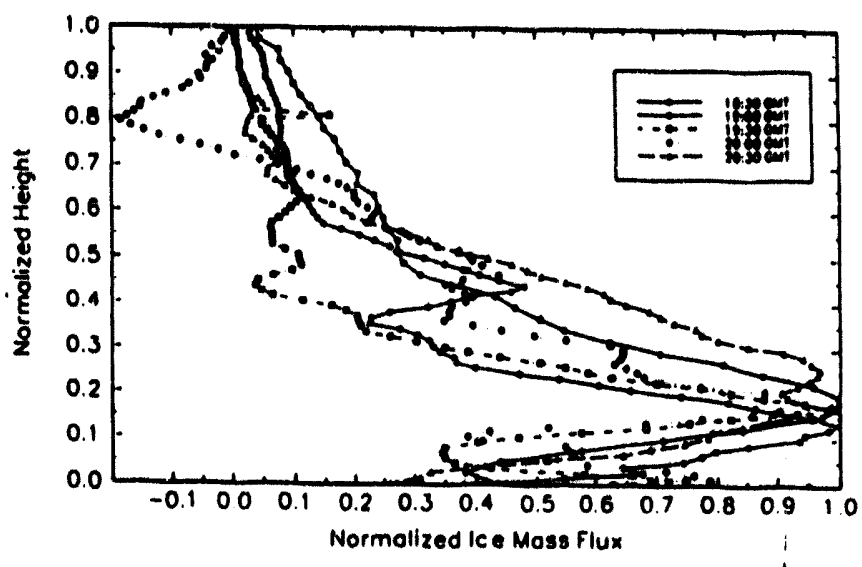

Figure 3. Profiles of IMF with the vertical scale normalized to cloud base and top and IMF normalized to the profile maximum for data obtained on 26 November 1991.

demonstrated with data from the 8-mm radar during FIRE II. Doppler velocities were measured with an uncertainty of lese than about $5 \mathrm{~cm} \mathrm{~s}^{-1}$ (Kropfli of al. 1090), and Doppler velocity profiles were obtained over 3-8 intervals when the antenna was fixed in the vertical for many hours. Such Doppler velocity profiles represent the reflectivity-weighted particle fallspeed plus vertical alr motion.

The vertical air motion in cirrus clouds, when averaged over an hour or longer, is assumed to be small compared with typical ice particle fallspeeds. Thus, a long average of Doppler velocities closely approximates reflectivity. weighted particle fallspeeds; the averaged air motion contribution is negligible compared with typlcal particle fallepeeds of about 0.1 to $1 \mathrm{~m} \mathrm{~s}^{-1}$.

Such averages were performed over $3 \mathrm{~h}$ for reflectivity intervals $1 \mathrm{~dB}$ wide, and the resulting averaged Doppler velocities were plotted against their corresponding reflectivity values (see Figure 4). The figure shows four relationships for different $560-\mathrm{m}$ helght intervals on 25 November 1991 during FIRE II. Shown on the figure are the central heights of each interval and the corresponding mean temperatures. The standard deviations of data about the best-fit curves are lose than $4 \mathrm{~cm} \mathrm{~s}^{-1}$, the trend of fallepeed increasing with reflectivity is clear, and the range of fallspeeds is consistent with expectations for cirrus cloud lice particles (Pruppacher and Klett 1978). 


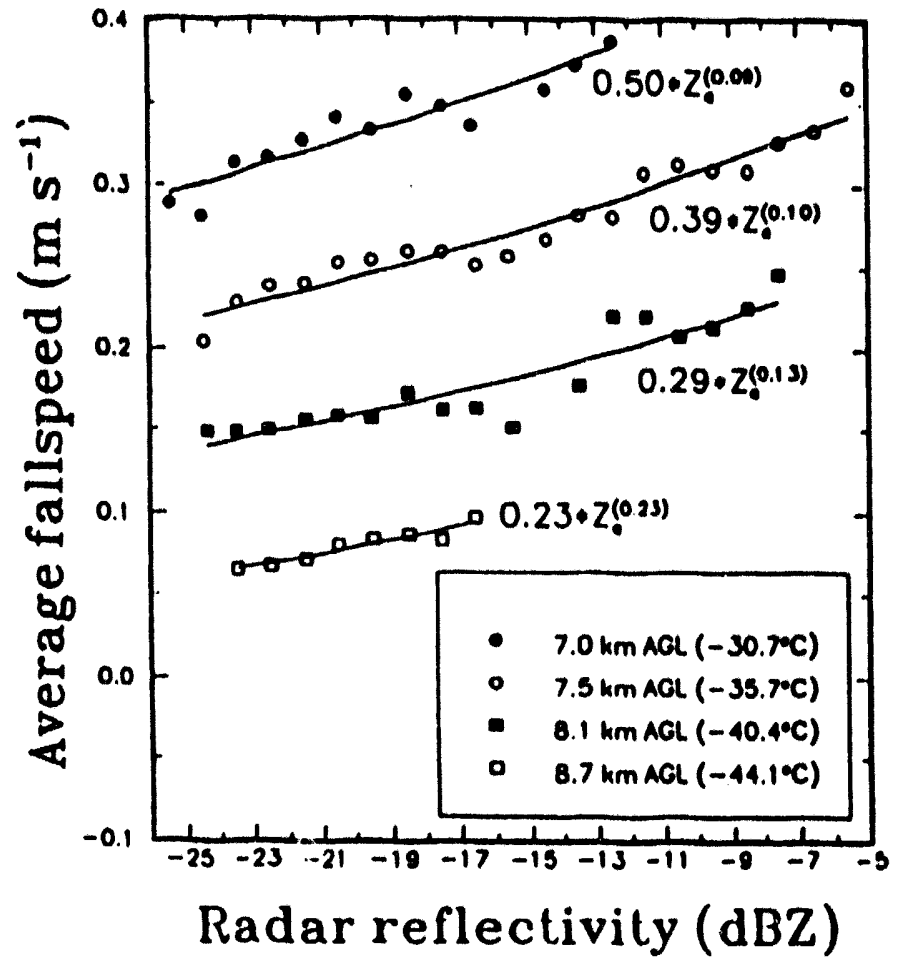

Flgure 4. Fallspeed versus radar reflectivity observed during FIRE II on 25 November 1991.

\section{Cloud Structure}

The 8-mm wavelength radar, with its $37-\mathrm{m}$ range resolution and $0.5^{\circ}$ beam width, is able to document detailed spatial structure of clouds. Small-scale waves having amplitudes from 0.1 to $1 \mathrm{~m} \mathrm{~s}^{-1}$ and periods of 1 or $2 \mathrm{~min}(\lambda \sim 2 \mathrm{~km})$, imbedded generating cells and fallstreaks, and multiple cloud layers were often observed in cirrus clouds during FIRE II. Figure 5 shows an example of a $6-\mathrm{km}$-deep cloud layer with precipitation streamers from cloud base at $2 \mathrm{~km}$ for data obtained on 21 November 1991 during FIRE II. Other examples of multiple cloud layers observed with this radar are found in Martner and Kropfll (1993).

Another notable example observed during ASTEX is that of the "microcell," a long-lived, small-scale cell having a weak reflectivity core (about $10 \mathrm{dBZ}$ ), a diameter less than $2 \mathrm{~km}$, and a distinct vertically stacked convergencedivergence couplet (Kropfll and Orr 1993).

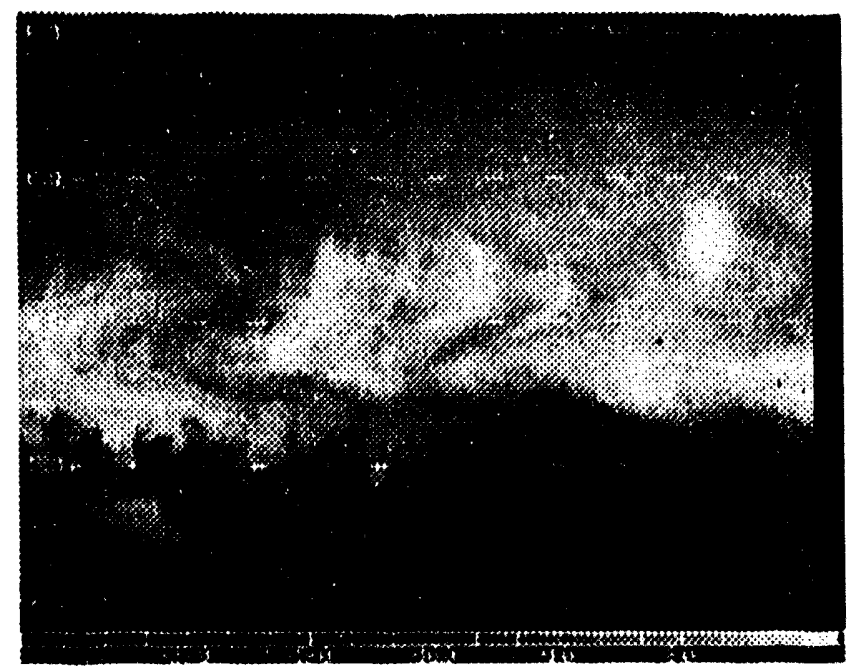

Flgure 5. A6-km-deep cloud with precipitation streamers.

Doppler velocity data from one elevation scan were interpolated to a 2.D Cartesian grid to generate an approximate visualization of the 2-D wind pattern in a microcell. The velocity measurements at the low elevation angles encountered in these observations were assumed to be reasonably good approximations to the horizontal wind projected onto the plane of the scan. Horizontal gradients of the interpolated values were used as estimates of divergence in the plane; the cross-plane contribution was not included. Finally, an upward integration was performed from the surface with a lower boundary condition of $0 \mathrm{~m} \mathrm{~s}^{-1}$ for the surface vertical air motion. The resulting 2-D wind field is shown in Figure 6.

Although the central core of a typical microcell was usually only a few $\mathrm{km}$ in diameter and limited vertically at about $1.5 \mathrm{~km}$ by the MBL inversion, the associated cloud often covered an area 100 times greater than the core. Despite the small size of the core region in which the cloudy air was apparently being generated and the weak reflectivities of the cores, these cells had unexpectedly long lifetimes, often exceeding $2 \mathrm{~h}$.

\section{Conclusions}

We have outlined radar-based methods to estimate various cloud properties from the ground. These methods are 


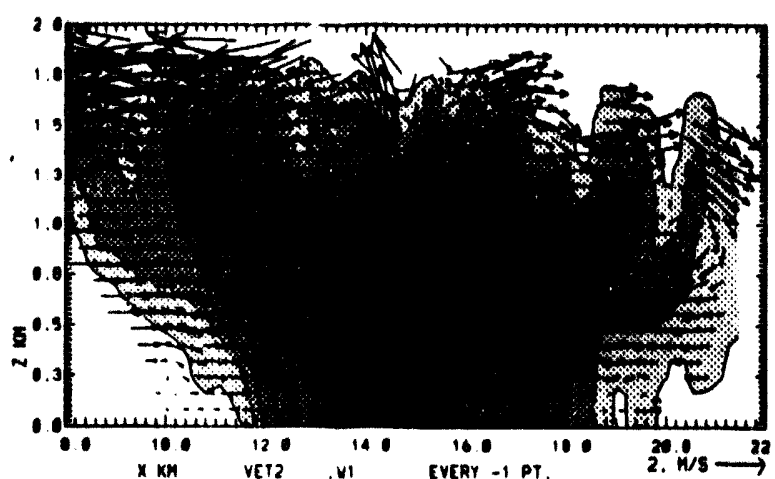

Flgure 6. Two-dimensional wind field computed from an RHl scan through a microcell at 1142 Z on 21 June 1892. The arrow indicates a $5 \mathrm{~m} \mathrm{~s}^{-1}$ wind speed; the vertical axis is the height above the surface in $\mathrm{km}$; and the horizontal axis represents the horizontal distance from the radar. Reflectivity contours are in $5 \mathrm{~dB}$ intervals with the highest contour at $10 \mathrm{dBZ}$.

theoretically plausible and show good consistency with other remote sensing measurements. Novertheless, they are still in need of extensive validation with in situ and other remote sensing observations before they become routinely usable by the general scientific community.

\section{Acknowledgments}

Scott Shaver and Anne Keane contributed greatly to thie work with their extensive help with the data processing. This research was funded in part by the U.S. Department of Energy's Atmospheric Radiation Mossurement Program under contract 164514-A-91, and by the NOAA Climate and Global Change Program.

\section{References}

Goldsmith, P. F. 1991. Quasi-optics in radar systoms. Microwave J. January, p. 79.

Kropfli, R. A., B. W. Bertram, and S. Y. Matrosov. 1990. The upgraded WPL dual-polarization 8-mm wavelength Doppler radar for microphysical and climate research. Proc., 1990 Conference on Cloud Physics, Pp. 341-345. American Meteorological Society, Boston.
Kropfll, R. A., and B. W. Orr. 1993. Observations of microcolls in the marine boundary layer with $8 . \mathrm{mm}$ wavelength Doppler radar. Proc., 26th Conference on Radar Meteorology, Norman, Oklahoma. American Motoorological Society, Boston.

Martner, B. E., and R. A. Kropfll. 1993. Observations of multi-layered clouds using K-band radar. Proc., 31st Aorospece Sciences Mooting and Exhlbit, Reno, Novada, AlAA-93-394, American Institute of Aeronautics and Astronautics, Washington, D.C.

Matrosov, S. Y. 1891. Prospects for the measurements of lce cloud particle shape and orientation with elliptically polarized radar signals. Radlo Scl. 26:847-856.

Matrosov, S. Y., and R. A. Kropfll. 1983. Cirrus cloud studies with elliptically polarized $K_{\mathrm{a}}$-band radar signals: A sugenested approach. J. Ocoan. Atmos. Techn. (submitted).

Matrosov, S. Y., T. Uttal, J. B. Snider, and R. A. Kropfli. 1892. Estimation of lce cloud parameters from groundbased infrared radiometer and radar measurements. J. Geophys. Res. 97:11567-11574.

Matrosov, S. Y., R. A. Kropfll, B. W. Orr, and J. B. Snider, 1993. Retrleval of vertical profiles of cirrus cloud microstructure parameters from Doppler radar and IR radiometer measurements. J. Atmos. Sol. (submitted).

Orr, B. W., and R. A. Kropfli. 1993. Estimation of cirrus cloud particle fallspeeds from vertically pointing Doppler radar. Proc., 26th Conferenco on Radar Meteorology. Norman, Oklahoma. American Moteorological Society, Boston.

Pruppacher, H. R., and J. D. Klett. 1978. Microphysics of Clouds and Precipltation. Reidel Publishing, Dordrecht, Holland.

Sassen, K. 1987. Ice cloud content from radar reflectivity. J. Clim. Appl. Moteorol. 26:1050-1053.

Uttal, T., S. Y. Matrosov, J. B. Snider, and R. A. Kropfll. 1993. Relationships between ice water path and downward long wave radiation for clouds optically thin in the infrared: Obeervations and model calculations. J. Appl. Meteorol. (oubmitted). 


\title{
Parameterization of Cirrus Microphysical and Radiative Properties in Larger-Scale Models
}

\author{
A. J. Heymefield and J. L. Coon \\ National Contor for Atmospheric Reeearch \\ Boulder, CO $80307-3000$
}

This study exploits measurements in clouds sampled during several field programs, Including the Atmospheric Radiation Moasuroment (ARM) Program, to develop and validate parametorizations that represent the physical and radiative propertibs of convectlvely generated cirrus clouds in intormediate and large-scale models (including cumulus ensemble models and general circulation models). Wo focus on cirrus anvils because they occur frequently, cover large areas, and play a large role in the radiation budget. Because of a lack of knowlodge about how anvils develop, ape, and decay, preliminary work focuees on understanding the physical (microphysical, radiative, and dynamical) proceseses that occur in these clouds. Our approach is discussed below.

We have constructed a detalled microphysical package that can bo insertod into exteting Eulerian cloud-ecale models. This microphyalcal peckage is based on the Lagranglan loe crystal trajoctory-growth model developed by Heymsfield (1986) and considers the growth of the following hydrometeor types: water drops, needles, plates, dendrites, columns, bullot rosettes, aggregates, graupel, and hail. Particle growth procesees include diffusional and accretional growth, aggregation, sedimentation, and melting. In light of the new demands by radiative transfor schemes, this package includes and tracks these particle habits. Parameterized ("Kessler-type") microphysical schemes commonly used in cloud-scale models are not appropriate for predicting cloud radiative propertles, which require more detailed knowledge about particle size distributions and shape.

This microphysical package is being implemented in a simple dynamical model that tracks the evolution and disporsion of hydrometeors in a stratiform anvill cloud. Given the momentum, vapor, and lce fluxes into the stratiform region and the temperature and humidity structure in the anvil's environment, this model will suggest anvil properties and structure. We will be using microphysical moasurements from Kwajalein and the Troplcal Ocean Global Atmosphere (TDGA) Coupled Ocoan Atmosphere Rosponse Experiment (COARE) and, as measurements become available, Central Equatorial Pacific Experiment (CEPEX) and ARM, to ovaluato and improve our approach.

The nonhydrostatic, anelastic cloud model described by Clark (1977) and Clark and Hall (1991) will be used to model anvil production and investigate the influence of large-scale effects on the sustenance of anvil clouds.

\section{References}

Clark, T. L. 1977. A small-scale dynamic model using a terrain-following coordinate transformation. J. Comp. Phys. 24:186-215.

Clark, T.L., and W. D. Hall. 1991. Multt-domain simulations of the timedependent Navier-Stokes equations: Benchmark orror analyses of some nesting procedures. J. Comp. Phys. 02:456-481.

Heymafield, A. J. 1986. Ice particle evolution in the anvil of a severe thunderstorm during CCOPE. J. Atmos. Sol. 43:2463-2478. 


\title{
Threo-Dimensional Cloud Characterization from Paired Whole-Sky Imaging Cameras
}

\author{
M. Allmen and W. P. Kegelmeyer, Jr. \\ Sandia National Laboratories \\ Livermore, CA 24551
}

Three-dimenaional (3-D) cloud characterization permits the derivation of important cloud geometry properties such as fractional cloudiness, moan cloud and clear length, aspect ratio, and the morphology of cloud cover. These propertios are needed es in put to the hierarchical diagnosis (HD) and instantaneous radiative transfer (IRF) models, to validate sub-models for cloud occurrence and formation, and to Central Btte radiative flux calculations. A full 3-D characterization will eventually require the integration of disparate Cloud and Radiation Testbed (CART) data sources: whole-sky imagers (WSIs), radar, satellites, cellomotore, volume-imaging lldar, and other sensors. In this paper, wo domonetrate how an initial 3-D cloud property, cloud base holght, can be determined from tusing paired time series of images from two whole-sky imagers.

\section{Background}

\section{GCMs and Clouds}

A major goal for the Department of Energy's (DOE's) global change efforts is to improve the accuracy of general circulation models (GCMs) capable of predicting the timing and magnitude of greenhouse-gas-induced global warming. Research has shown cloud radlative foedback is the single most important feedback effect determining the magnitude of possible climate responses to human activity. Yot, as pointed out by Cess of al. (1989), clouds are not well parameterized in GCMs and are, in fact, currently the greatest factor limiting the eccuracy of atmospheric GCMs. Thus clouds exert the largest influence while at the same time present the largest uncertaintles in predicting global climate change. As a result, cloud studies are critical to understanding global climate change and improving the predictive accuracy of GCMs. In recognition of this problem, a number of important national and international programs
(Rossow of al. 1985) have been initiated to characterize cloud-radiation interactions, including DOE's Atmospheric Radiation Measurement (ARM) Program and the International Satellite Cloud Climatology Project (ISCCP).

In the ARM Program, akey to characterizing cloud-radiation interactions is the effective treatment of cloud formation and cloud properties in GCMs as supported by a field measurements program - "an important feature of the ARM Program Plan is to establish a surfaco-based cloud imaging system at the research sites that will provide appropriate information for parameterizing solar flux over an entire grid coll." The first such Cloud and Radiation Testbed (CART) stte will make measurements, including cloud measurements, over a $30-\mathrm{km}$ diameter region.

\section{Macroscoplc Cloud Properties}

A well-recognized approach to reducing the uncertaintles associated with cloud-radiation interactions involves measuring the macroscopic properties of clouds (shape, size, extent, cloud cover fraction, base hoight, otc.). All these properties can be extracted from a 3-D cloud characterization, and 80 the development of such a characterization is a worthy goal. Accordingly, wo have been studying methods for atmospheric data integration, beginning with fusing WSI imagery to determine cloud base height. This property is a good place to start in that it is a dominant factor in dotermining the infrared radiation from clouds to the lower atmosphere and the earth's surface. Furthermore, as shown by Roseow ot al. (1985), base holghts are essential to measuring the cloud cover fraction at low, medium and high altitude, measurements that, in turn, are needed to establish a cloud-radiation cllmatology. 


\section{Whole Sky Imagers}

Digital whole-sky imagers have a number of advantages for making cloud studles. They are passive and therefore relatively inexpensive and reliable, can be used in unattended operation, and can obtain images of the entire sky dome rapidly. The particular camera which generated our existing data is the Whole Sky Imager developed by the Marine Physical Laboratory (MPL) at the Scripps Institution of Oceancoraphy, as described in Shields ot al. (1990). These Imagers are rugged anc have demonstrated many years of high-rellability field service. Full-resolution (1/3 degrees) digital images can be acquired at one per minute. This is rapld enough to capture most of the cloud dynamics of interest and fully exploit the image motion of the clouds as an aid in 3-D analysis, as described below.

\section{Method}

\section{An Overview}

Unfortunately, the state of the ant in automatic cloud imagery processing was not previously capable of extracting measures as central and important as cloud bottom heights over large regions of the sky dome. The main difficulty is spatially matching up cloud fields from widely separated WSI cameras. However, once correctly registered against each other, computation of cloud bottom heights proceeds in a straightforward fashion from triangulation and knowledge of the camera locations. Our solution to this problem (initially reported in Allmen and Kegelmeyer (1993), on which this report builds) utilizes temporal flow fields from each camera separately. In the following subsections we will review the prior history of this problem, Illustrate its difficulty, and suggest why flow fields provide the additional information necessary to make this problem solvable.

\section{Prlor Work}

Extracting cloud bottom heights via triangulation of registered points has been in the cloud stereoscopy literature for twenty years and is well-understood. The registration liself, however, has not been well addressed. In the earlier literature, e.g., Bradbury and Fujita (1968) and Lyons(1971), the problem was side-stepped though human intervention: the images were registered by hand bofore triangulation. More recently, e.g., Rock (1987), the automatic registration problem has been successfully handled, but only for nearly adjacent views of the sky, which limits to a small fraction of the sky dome the region over which the analysis can be performed. In such a case, the stereoscopic nature of the views permitted simple limited-displacement correlation to suffice as a registration algorithm.

The registration problem facing ARM, however, is considerably harder in that the camera spacing will be on the order of $5 \mathrm{~km}$. This spacing is required to achieve adequate coverage at the required resolution with a small number of cameras. With this baseline spacing, the 3-D nature of clouds generates occlusion and perspective effects that will cause them to image differently at the various cameras. Because of this, correlation-based registration using pixel intensity alone will fail. Further, the visual self-similarity of clouds will defeat token matching (the detection and matching of a small number of visually distinctive regions), which is the only common alternative approach.

As an example, the top of Figure 1 contains an example pair of synthetic simultaneous frames (see the next section for how the data were generated). Careful examination will show that corresponding points appear shifted to the left in the right image. This shift can be difficult to determine by eye, primarily because of the difference in perspective experienced by the widely separated cameras. Finding corresponding points in WSI images of real-world cloud fields is even more challenging, creating the need to also use flow fields to find corresponding points.

\section{Flow Fleld Correlation}

The use of image sequences to identify the correspondence suggests how one can automate the registration process. WSI images are acquired at a rate of one per minute, which provides a comparatively dynamic view of the sky. This provides a means to overcome the registration obstacles mentioned before, which apply only to the attempt to register two static views of the sky.

The temporal sampling rate of the WSI camera is high enough that optical flow fields can be computed using hierarchical correlation methods such as those developed by Burt (1984). With the optical flow fields computed for the 
Images from both WSI cameras, each image pixelbecomes associated with a vector indicating the image motion of that point. Combining this with the intensity value at the pixel, a three-dimensional quantity now represents each image pixel. The core of our approach is to jointly register the flow and intensity fields from the separated WSIs against each other. In this way, the additional constraints provided by the flow field are explotied to make the matching unique.

\section{The Test Data and Results}

We have computed cloud base helghts from various syntheticimagesequences and from real WSI data collected under conditions designed to simulate those of the CART site.

\section{Simulated Data}

The simulated sequences were created with a cloud scene simulation model developed by Cianciolo (1992). The model uses stochastic field generation techniques and knowledge of atmospheric structure and physics to model four-dimensional (3 spatial and 1 temporal) cloud scenes, represented by liquid water content (LWC) values. To this, we attached a cloud density model to derive radiance fields from the LWC volumes. Synthetic images were then generated by projecting a cloud scene using a WSI camera model that is identical to an actual WSI. Since the cloud scene has a temporal component, it can be projected at a sequence of times, creating an image sequence that captures cloud evolution and motion. This allows calculation and, most importantly, verification of cloud base heights on realistic data. Moreover, the simulation can produce a wider range of cloud types than exists in our real imagery.

The top of Figure 1 shows one frame from the left and right image sequence of an altostratus cloud layer. In both cases the images depict a common cloud field as imaged from a horizontally paired set of WSI cameras, where black indicates sky and the mottled dark texture is cloud. The cameras were modeled as being separated by $5 \mathrm{~km}$, and the clouds were set at heights between 3 and $5 \mathrm{~km}$.

Careful examination reveals that the corresponding points appear shifted to the left in the right image. This is difficult to note from simple inspection, primarily because of the difference in perspective experienced by the widely separated cameras.

\section{Simulated Data Results}

The fact that the data are simulated and that we thus know the true cloud height at every point means that we can evaluate the accuracy of the algorithm on a pixel-by-pixel basis.

Some results for the simulated altostratus sequence are presented in the bottom of Figure 1. One of the innovative features of our algorithm is that at each pixel it provides a confidence measure that it has computed the correct height. The bottom left image illustrates, via the middle grey level, the points where the algorithm was confident it had computed the correct height and in fact was correct (within $5 \%$ ). The white points are where the algorithm erroneously computed the heights as being too high, black is where it erroneously computed the heights as being too low, and the rest is background or points where the algorithm evaluated itself as likely to be erroneous and so did not hazard a guess.

Clearly, the number of correct points is far greater than the number of incorrect points. To make this quantitative, the bottom right part of Figure 1 shows the histogram of the errors for the points where the algorithm was confident. The desired result is a sharp peak at zero error; the histogram here has abin size of $200 \mathrm{~m}$ and so demonstrates that $84 \%$ of the confidently computed heights were accurate.

\section{Real Data}

Our real WSI data were taken in May 1992 in White Sands, NM. In an attempt to simulate the eventual CART data, we separated two WSIs by $5.54 \mathrm{~km}$, with a ceilometer located close to the midpoint between them. The intent of the ceilometer was to provide fiduciary points with which to check our algorithm. It was fired once a minute, in time with both WSIs. As a result, when the ceilometer reports the presence of clouds, simple geometry and knowledge of the camera location suffice to compute which pixel on the WSI images corresponds to that ceilometer report. The cloud base height computed at that point can then be compared to the ceilometer measurement.

The top two images in Figure 2 are examples of these real WSI images from mid-day on May 4. The inset white circle indicates the location of the cellometer hit on both images. 
ARM Science Meoting

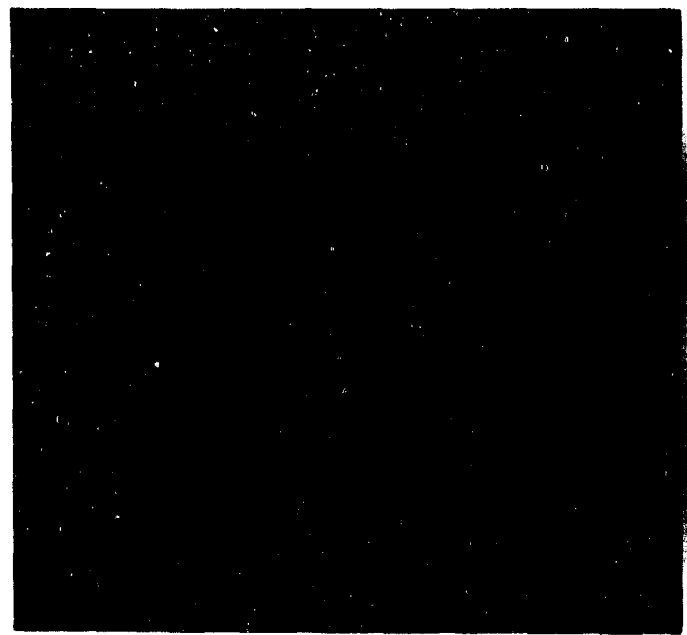

(a) Simulnted A boctrinto, Left Image

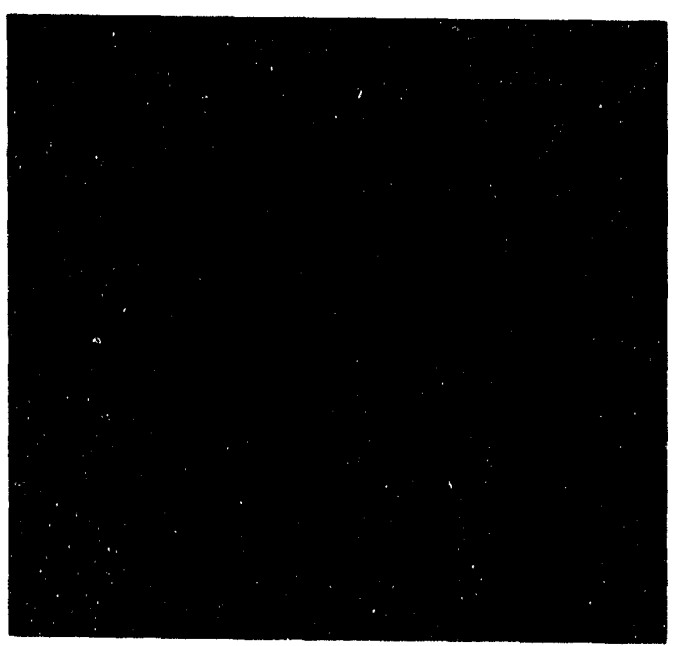

(c) Height Conectuess for Left image

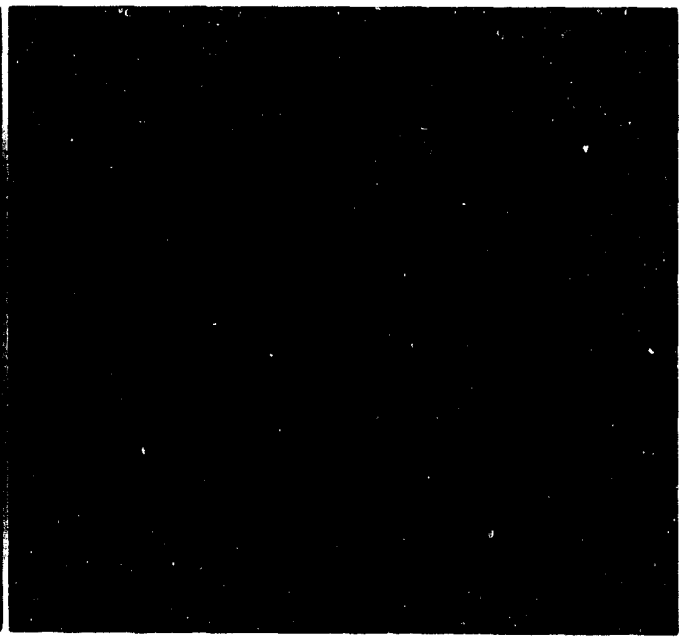

(b) Simulated Altantrutes, Right Image

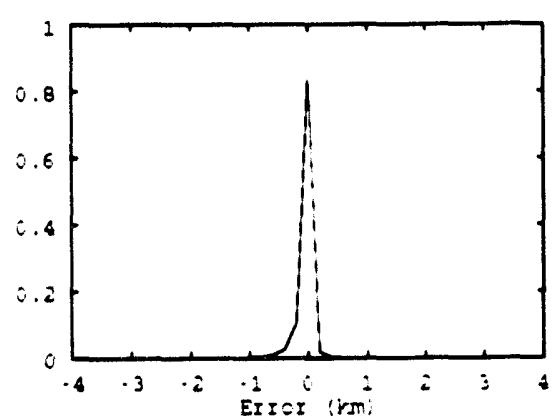

(d) Error Histogrem for Lef Image

Figure 1. One frame of an altostratus cloud scene as viewed from the left (top left image) and right (top right image) cameras. The bottom left image illustrates, via the middle grey level, the points where the algorithm was confident it had computed the correct height and, in fact, was correct (within 5\%). The white points are where the algorithm erroneously computed the heights as being too high, black is where it erroneously computed the heights as being too low, and the rest is background or points where the algorithm evaluated itself as likely to be erroneous and so did not make a report. The bottom right image shows a histogram of the error in height computation. 


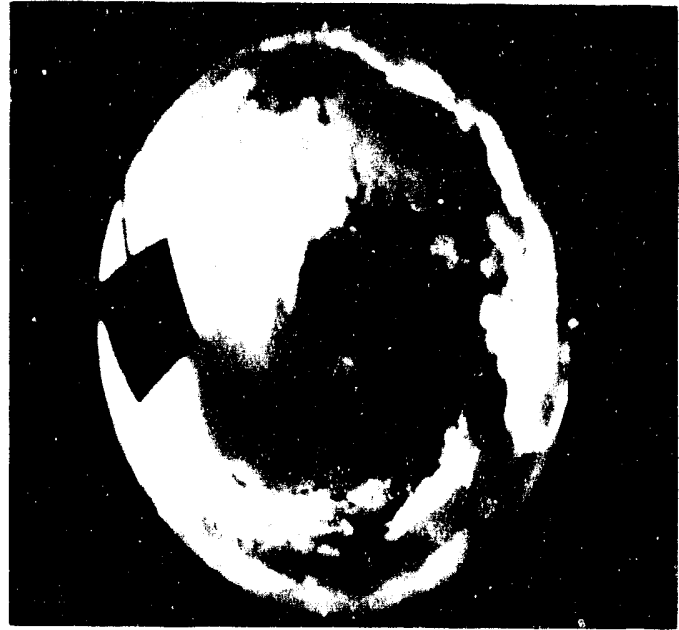

(a) Real WSI, Left Image

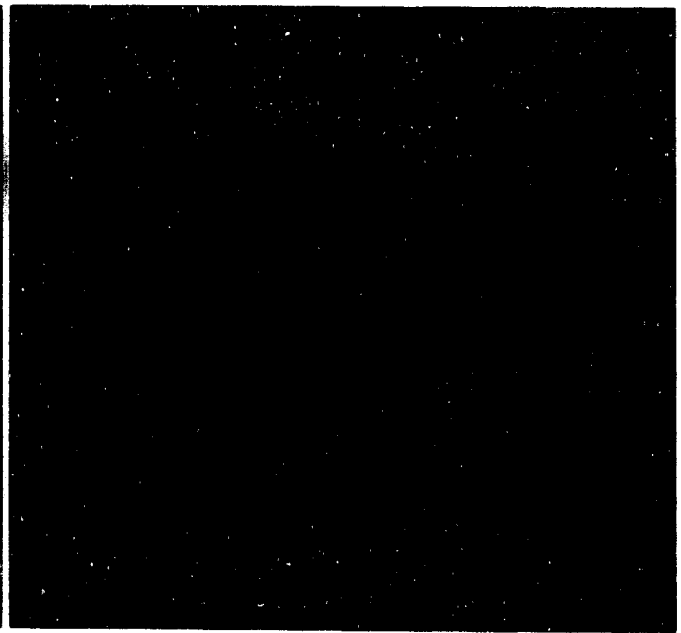

(b) Real WSI, Right Image

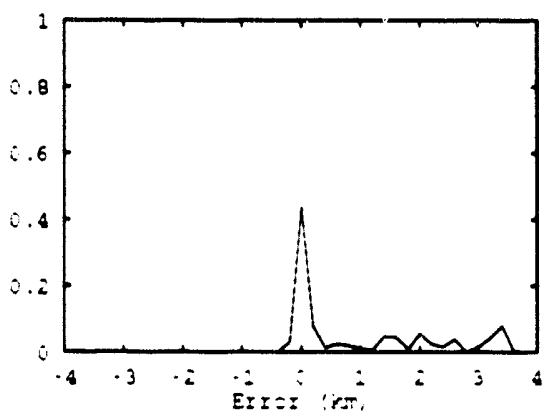

(c) Error Histogram for Entire Dey

Figure 2. One frame of a real cloud scene as viewed from the left (top left image)and right (top right image) cameras. The bottom image shows a histogram of height error as calculated from a ceilometer over the course of a day. 


\section{Real Data Results}

Since we do not know the true cloud base height at every point, a height correctness image such as in the lower left of Figure 1 is not possible. For each frame, wo know the coilometer's height measurement for only a single point, not over the entire field of view. So the error histogram in the bottom of Figure 2 represents statistics extracted from the entire day. The peak of the histogram here is lower than for the simulated data. One straightfonward reason is that the ceilometer had a range of only $4 \mathrm{~km}$. Thus we could gather statistics only on the lower clouds, and low clouds are the worst case scenario for this approach, as the visual disparity of corresponding points is greatest. The algorithm improves in accuracy as the clouds get higher.

More significantly, we are concerned that our ceilometer data are not entirely reliable, as there are many cases where the ceilometer reports the presence or absence of a cloud in direct contradiction of the matching WSI image. We have recently become aware of the possibility of errors in our current camera calibration parameters as well, which would at times induce displacements, making the correct point registration difficult or impossible to find. As a result, we are looking forward to replicating this experiment with firmly documented and high quality CART data, both in order to span a broader range of cloud conditions and to have, from radar and long-range ceilometer, fiduciary points at higher attitudes.

\section{Conclusions and Future Work}

In summary, we began with a discussion of the value of 3-D cloud characterization to ARM's needs and suggested that such a characterization will require the fusion of many data sources from a rich suite that will be available at the CART sites. We have illustrated this principle with a demonsitration of how paired data from widely separated whole-sky imager scan be fused to extract cloud base heights, an important cloud property and one which could not be recovered from either imager alone. An important feature of our approach, one that will help it to generalize to the incorporation of other data sources, is its ability to measure its own confidence in the determined base heights.

Near-term work will be devoted to the understanding of error sources in the cloud base height algorithm, and its subeequentimprovement. We are evaluating the application of epatial filtering to the computed heights, so that the small, isolated regions of incorrect results can be eliminated. We are also investigating how the parameters of the algorithm, the size of the correlation neighborhood around a point for example, can be optimized.

Our future efforts are devoted both to the extraction of further properties of interest (particularly fractional cloud cover and aspect ratio) from paired WSI images, and fusing of WSI images with satellite imagery in order to determine cloud top structure as well.

\section{Acknowledgments}

This research was supported by the U.S. Department of Energy through the Atmospheric Radiation Measurement Program, Sandia National Laboratories, Livermore, contract \#DE-AC04-76DO00789. The Khoros(a) system, developed by Rasure and Williams (1991), was used for code development and digital image visualization. Code for the generation of synthetic cloud scenes was provided by TASC-The Analytic Sciences Corporation, and the code which simulates radiance maps and projects 3-D cloud volumes into simulated WSI imagery was written by ChenHui Sun of Sandia National Laboratories.

(a) A publicly avallable integrabed software developmentenvironment for information processing and visualization. Send e-mail to khoros Qchama.eece.unm.edu for further information. 


\section{References}

Allmen, M., and W. P. Kegelmeyer, Jr. 1993. The computation of cloud base hoight from paired whole sky imaging cameras. In Fourth Symposium on Global Change Studies, pp. 136-141. American Moteorological Society. Boston, Massachusetts.

Bradbury, D. L., and T. Fujita. 1968. Computation of height and velocity of clouds from dual, whole-sky, time-lapse picture sequences. SMRP Research Paper 90, Department of the Geophysical Sciences, University of Chicago, Chicago.

Burt, P. J. 1984. The pyramid as a structure for efficient computation. In Multiresolution Image Processing and Analysis, ed. A. Rosenield, pp. 7-35. Springer, Berlin.

Cess, R., G. L. Potter, J. P. Blanchet, G. J. Boer, S. J. Ghan, J. T. KiehI, H. Le Treut, Z.-X. Li, X.-Z. Liang, J. F. B. Mitchell, J.-J. Morcrette, D. A. Randall, M. R. Riches, E. Roeckler, U. Schlese, A. Slingo, K. E. Taylor, W. M. Washington, R. T. Wetherald, and I. Yagai. 1989. Interpretation of cloud-climate feedback as produced by 14 atmospheric general circulation models. Science 245:513-516.
Clanciolo, M. E. 1992. Cloud scene simulation modeling: The enhanced mode/_Final report. Tech. Rep. TR-60422, TASC, 55 Walkers Brook Drive, Reading, Massachusetts, 01867.

Lyons, R. D. 1971. Computation of height and velocity of clouds over Barbados from a whole-sky camera network. SMRP Research Report 95, Department of Geophysical Sclences, University of Chicago, Chicago.

Rasure, J., and C. Williams. 1991. An integrated visual language and software developmentenvironment. J. Visual Languages and Computing 2:217-246.

Rocks, J. K. 1987. The whole sky sensor: Phase 1 final report. DTIC AD-A179 271.

Rossow, W., F. Mosher, E. Kinsella, A. Arking, M. Desbois, E. Harrison, P. Minnis, E. Ruprecht, G. Seze, C. Simmer, and E. Smith. 1985. ISCCP cloud algorithm intercomparison. J. Clim. Appl. Meteorol. 29(9):877.

Shiolds, J., T. Koehler, and M. Karr. 1990. Automated cloud cover and visibility systems for real time applications. Technical Note 217, Marine Physical Laboratory, Scripps Institution of Oceanography, San Diego, California, 92151 . 6400. 


\title{
Complex Researches of Tropospheric and Stratospheric Ozone Performed at the Institute of Atmospheric Optics as a Basis of a Complete Radiation Experiment
}

\author{
V. V. Zuev \\ Institute of Atmospheric Optice \\ Siberian Branch of the Russian Academy of Sciences \\ Tomsk, Russia
}

Systematic obeervations of the earth's ozone layer over the last ten years indicate a steady decrease of ozone content in the stratospheric maximum and, on the contrary, a increase of ozone concentrations in the troposphere. This trend is illustrated clearly by the results of 20 years' observations of high-altitude czone concentration distribution in the troposphere and stratosphere at Hohenpeissenberg observatory (Figure 1).

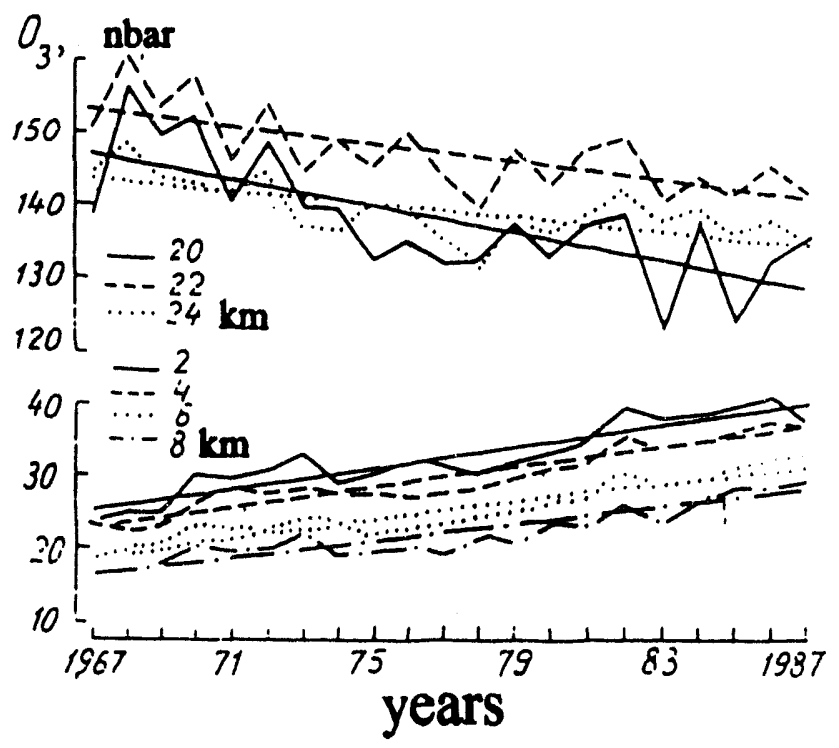

Flgure 1. Twenty years' observations of stratospheric and tropospheric ozone concentrations.
In addition to photochemical and dynamical processes in the stratosphere, the decrease of stratospheric ozone content is also stimulated by high-power aerosol pollutions of the stratosphere after volcanic eruptions. The ozone layer of the stratosphere was subjected to especially strong depression after the Mt. Pinatubo eruption in the Philippine Islands in 1991 (Figure 2).

To describe the ozone cycle in the troposphere and stratosphere as a whole requires simultaneous information on a large number of gas trace species and aerosol and

Total ozone ( D.U.) at January, 28, 1992

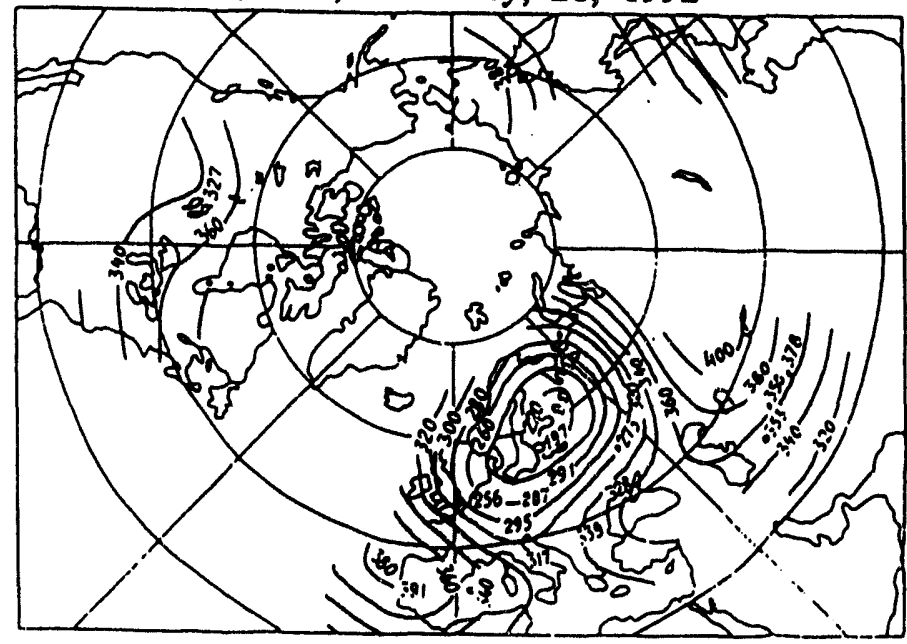

Figure 2. Stratospheric ozone layer after Mt. Pinatubo eruption. 
atmospheric meteorological parameters. Some of these gases and aerosols are $\mathrm{O}_{3}, \mathrm{~N}_{2} \mathrm{O}, \mathrm{NO}_{x}\left(\mathrm{NO}, \mathrm{NO}_{2}\right), \mathrm{CH}_{4}$. nonmethane hydrocarbons $\left(\mathrm{CH}_{2} \cdots N\right), \mathrm{CO}, \mathrm{CO}_{2}$, aldehydes $\left(\mathrm{H}_{2} \mathrm{CO}, \ldots\right)$, ketons, PAN (peroxiacetylenenitrates), $\mathrm{H}_{2} \mathrm{O}$, $\mathrm{H}_{2} \mathrm{O}_{2}$, freons ( $\left.\mathrm{CFCL}_{2}(11), \mathrm{CF}_{2} \mathrm{CL}_{2}(12), \ldots\right), \mathrm{HCL}, \mathrm{HF}, \mathrm{CLO}$, $\mathrm{CLONO}_{2}$, aerosols, meteoparameters.

In 1991 at the Institute of Atmospheric Optics, a long-term complex program on the investigation of stratospheric and tropospheric ozone, "SATOR" (Stratospheric and Tropospheric Ozone Research), was formulated.

The program's primary objective is to perform a long-term complex experiment in the atmosphere by means of a large set of different devices located in one place and to analyze a long series of synchronous atmospheric observations obtained. The structure of the SATOR program is given in Figure 3.

The primary near-term objectives of the SATOR program are the following:

- Investigate the temporal variability of ozone concentration in the lower troposphere as a function of

- variability of the aerosol and gas composition of the atmospheric boundary layer

- the dynamics of the atmospheric boundary layer

- Study the dynamics of the vertical distribution of stratospheric ozone and aerosol over Tomsk as well as investigate the microstructure of stratospheric aerosol and the relation between the variability of ozone and aerosol content in the stratosphere.

To solve the first of the above, we measured aerosol and gaseous composition using optical and laser multiwave long-path meters operating along a specially larranged slightly elevated path between two buildings of the Tomsk Akademic town. (The distance between the buildings is $0.5 \mathrm{~km}$; the meters are at a height from 12 to $18 \mathrm{~m}$ at the ends of the path).

At the beginning of the path at the high-altitude lidar sensing station (HALSS), the aerosol and gaseous composition was measured using contact means, and the air was sampled and subsequently analyzed chemically. Here, at two points at different altitudes, the averaged and pulsed values of temperature, wind velocity and wind direction, air humidity were measured. An acoustic sounder (sodar) and an aerosol lidar were used to determine the altitude of the mixing layer and to study the dynamics of the atmospheric boundary layer.

To solve the second problem, we used the stationary lidars "Aeroson" and "Kolls," which have receiving telescopes with diameters of 1 and $2.2 \mathrm{~m}$. Lidar sensing of the stratosphere was accompanied by launching the sounding balloons with meteo- and ozonosondes. Because of background noise, the photon-counting recording (detecting) regime of sounding laser radiation reflected by stratospheric aerosols and ozone was only at night. Originally in the process of developing the SATOR program, it was proposed to bring it closer to the total radiation experiment.

The potential of the SATOR program can be lllustrated by some results presented in this paper.

Gaseous components are listed in Table 1. These components are detected by means of the laser gas analyzer "Tral." "Tral" is based on tunable $\mathrm{CO}_{2}$ - and $\mathrm{CO}$. lasers equipped with the IR parametric frequency converters made from $\mathrm{ZnGeP}$ and $\mathrm{AgGaSe}_{2}$ along the $1-\mathrm{km}$ path length. The gas analyzer operates on the basis of the method of laser radiation differential absorption at long paths. It is sensitive enough to detect the main gases present at background concentrations. It is of principal importance that the analyzer be able to detect the main greenhouse gases.

Figure 4 gives the time dependencies of the $\mathrm{O}_{3}$ and $\mathrm{CO}_{2}$ concentrations measured with the use of the gas analyzer "Tral." Positive correlation is evident in the behavior of these greenhouse gases, behavior which is due to the final oxidation reaction of $\mathrm{CO}$ and hydroxyl $\mathrm{OH}$ (the main ozone destroyer in the troposphere) in the methane oxidation cycle.

Figures 5 and 6 illustrate lidar capabilities for sounding the vertical distribution of water vapor with detailed structure in the atmospheric boundary layer and up to maximum altitudes of about $17 \mathrm{~km}$.

Since 1985, regular lidar observations of stratospheric aerosol have been carried out at the Institute of Atmospheric Optics, and since 1989, the stratospheric ozone has been monitored. A set of the averaged backscattering coefficients measured at a wavelength of $532 \mathrm{~nm}$ is presented in 


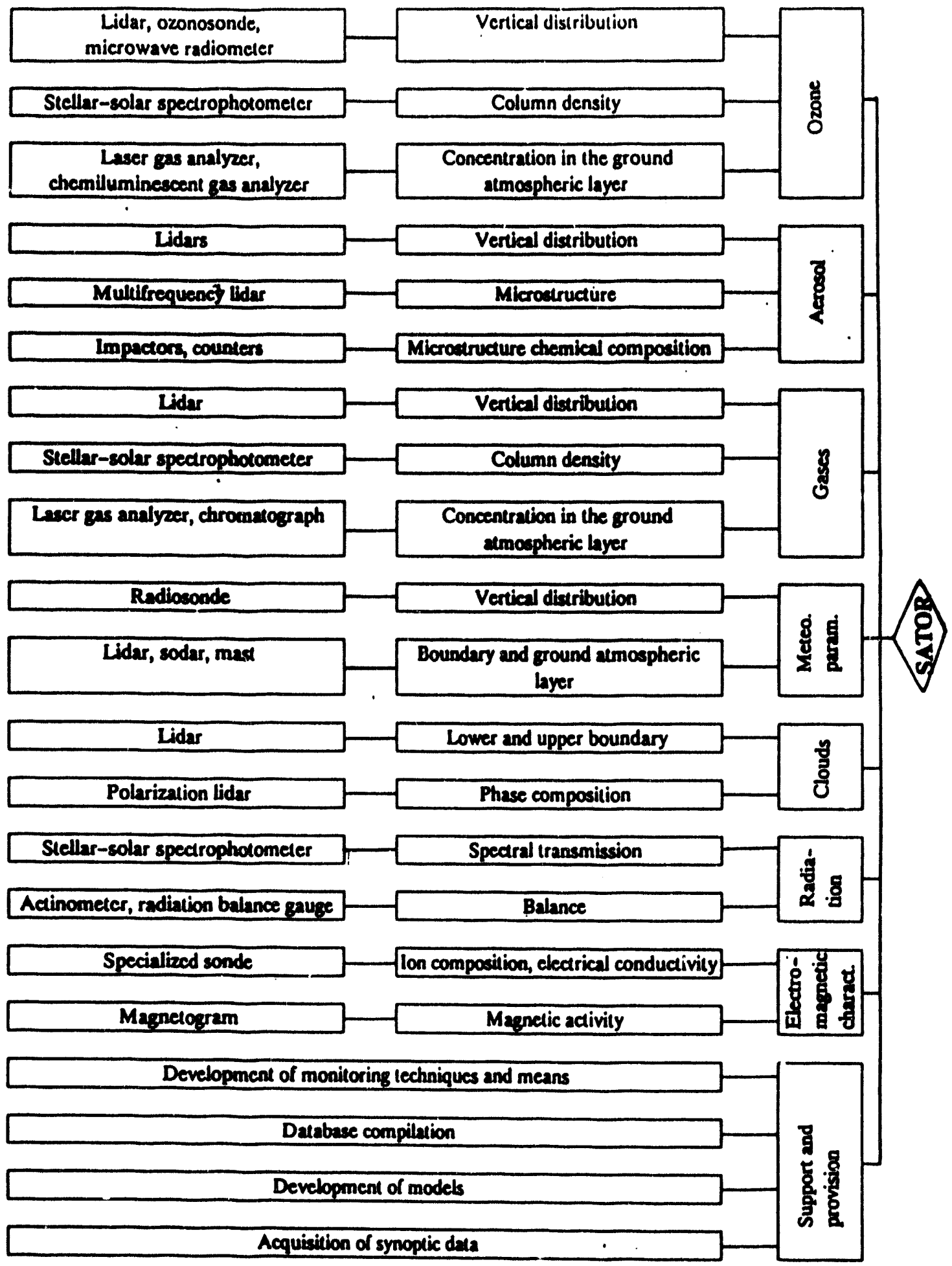

Fgure 3. SATOR program structure. 
Table 1. Gaseous components detected by "Tral."

\begin{tabular}{|c|c|c|c|c|c|c|}
\hline \multirow{2}{*}{\multicolumn{2}{|c|}{ Section }} & \multirow{2}{*}{ Gas } & \multicolumn{2}{|c|}{ Lasing Lines and their Combinations } & \multirow{2}{*}{$\begin{array}{c}\text { CDA } \\
\mathrm{cm}^{-1}-\mathrm{stm}^{-1} \\
\end{array}$} & \multirow{2}{*}{$\begin{array}{c}\text { MDC } \\
\text { pob }\end{array}$} \\
\hline & & & On-Line & Off-Line & & \\
\hline \multirow{16}{*}{1} & 1 & $\mathrm{SF}_{\mathbf{B}}$ & $10 P(16)$ & $10 P(10)$ & 620.0 & $8(-2)$ \\
\hline & 2 & $\mathrm{NH}_{3}$ & $9 R(30)$ & $9 R(28)$ & 75.03 & 1 \\
\hline & 3 & $\mathrm{~N}_{2} \mathrm{H}_{4}$ & 10P(32) & $10 P(34)$ & 3.30 & 15 \\
\hline & 4 & $\mathrm{C}_{6} \mathrm{H}_{8}$ & $9 P(30)$ & $9 P(26)$ & 1.60 & 31 \\
\hline & 5 & $\mathrm{H}_{2} \mathrm{O}$ & $10 \mathrm{R}(20)$ & $10 R(18)$ & 0.001 & 165ppm \\
\hline & 6 & $\mathrm{C}_{2} \mathrm{H}_{4}$ & $10 P(14)$ & $10 P(12)$ & 30.70 & 2 \\
\hline & 7 & $\mathrm{O}_{3}$ & $9 P(14)$ & 9P(22) & 11.10 & 5 \\
\hline & 8 & $\mathrm{C}_{2} \mathrm{~N}_{5} \mathrm{SH}$ & $10 R(26)$ & $10 P(20)$ & 0.38 & 600 \\
\hline & 9 & $\mathrm{C}_{2} \mathrm{H}_{3} \mathrm{Cl}$ & 10P(22) & $9 R(18)$ & 8.75 & 20 \\
\hline & 10 & $\mathrm{C}_{2} \mathrm{HCl}_{3}$ & $10 P(20)$ & $10 R(20)$ & 12.56 & 24 \\
\hline & 11 & $\mathrm{C}_{2} \mathrm{Cl}_{4}$ & $10 P(34)$ & $10 R(24)$ & 4.80 & 60 \\
\hline & 12 & $\mathrm{C}_{2} \mathrm{H}_{5} \mathrm{Cl}$ & $10 \mathrm{R}(16)$ & $10 P(20)$ & 3.24 & 75 \\
\hline & 13 & $\mathrm{C}_{2} \mathrm{H}_{4} \mathrm{Cl}_{2}$ & $10 P(20)$ & $10 R(16)$ & 0.51 & 550 \\
\hline & 14 & $\mathrm{C}_{4} \mathrm{H}_{5} \mathrm{Cl}$ & $10 R(18)$ & $9 \mathrm{P}(22)$ & 9.05 & 45 \\
\hline & 15 & $\mathrm{CF}_{2} \mathrm{Cl}_{2}$ & $10 P(32)$ & $10 P(12)$ & 35.62 & 6 \\
\hline & 16 & $\mathrm{CFCl}_{3}$ & $9 R(22)$ & $9 P(18)$ & 29.10 & 7 \\
\hline & 17 & NO & $2 \times 10 P(24)$ & $2 \times 10 P(26)$ & 1.98 & 41 \\
\hline & 18 & co & $2 \times 9 P(24)$ & $2 \times 9 P(26)$ & 26.59 & 4 \\
\hline \multirow[t]{4}{*}{2} & 19 & ocs & $2 \times 9 P(30)$ & $2 \times 9 P(14)^{1}$ & 113.9 & 1 \\
\hline & 20 & $\mathrm{CO}_{2}$ & $10 R(30)+9 R(14)$ & $2 \times 9 P(40)$ & 0.023 & 4ppm \\
\hline & 21 & $\mathrm{~N}_{2} \mathrm{O}$ & $9 R(40)+9 R(18)^{2}$ & $2 \times 9 P(40)$ & 18.07 & 6 \\
\hline & 22 & $\mathrm{HCl}$ & $P(17)+10 P(32)$ & $P(15)+10 P(16)$ & 34.10 & 3 \\
\hline \multirow[t]{5}{*}{3} & 23 & $\mathrm{HBr}$ & $P(16)+10 P(24)$ & $P(15)+10 P(30)$ & 5.35 & 200 \\
\hline & 24 & $\mathrm{NO}_{2}$ & $P(19)+9 P(20)$ & $P(21)+10 P(26)$ & 3.66 & 300 \\
\hline & 25 & $\mathrm{CH}_{4}$ & $P(21)+9 P(16)$ & $P(19)+9 P(24)$ & 5.94 & 21 \\
\hline & 26 & $\mathrm{H}_{2} \mathrm{CO}$ & $P(17)+10 R(14)$ & $P(18)+9 P(18)$ & 20.95 & 6 \\
\hline & 27 & $\mathrm{HNO}_{3}$ & $P(16)-10 R(20)$ & $P(15)-10 P(12)$ & 9.42 & 110 \\
\hline \multirow[t]{3}{*}{4} & 28 & $\mathrm{PH}_{3}$ & $P(16)-10 P(28)$ & $P(16)-10 P(26)$ & 2.13 & 1ppm \\
\hline & 29 & $\mathrm{C}_{2} \mathrm{H}_{2}$ & $P(21)-9 R(30)$ & $P(18) \cdot 10 R(18)$ & 43.99 & 3 \\
\hline & 30 & $\mathrm{HCN}$ & $P(99)-9 R(16)$ & $P(14)-10 R(22)$ & 10.43 & 110 \\
\hline
\end{tabular}


concentration, ppm 14-15/11/91

$\ldots \ldots+1 E+2$ CQ2 9P2O-9P36
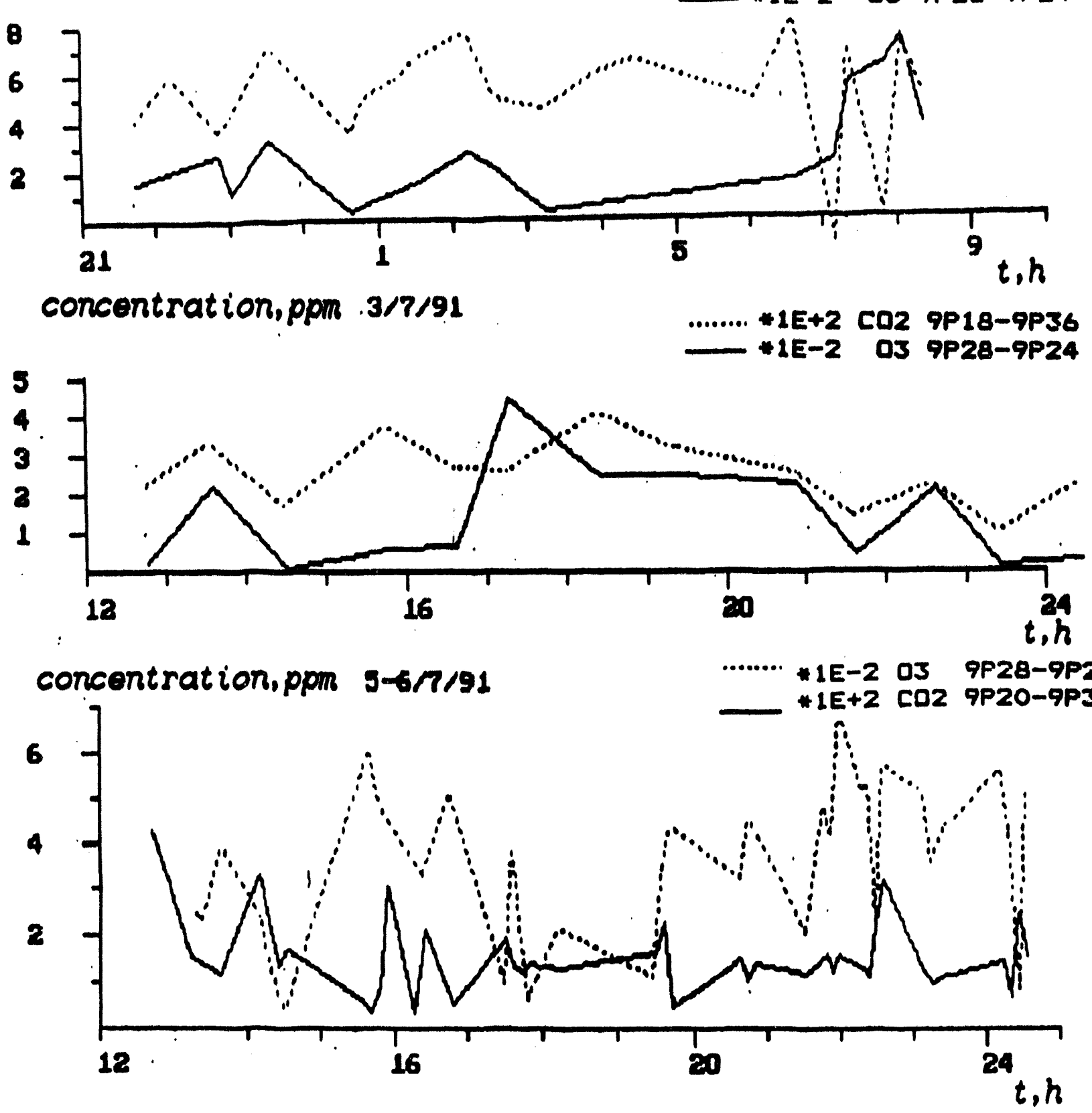

Figure 4. Time dependencies of $\mathrm{O}_{3}$ and $\mathrm{CO}_{2}$ concentrations measured by laser gas analyzer. 

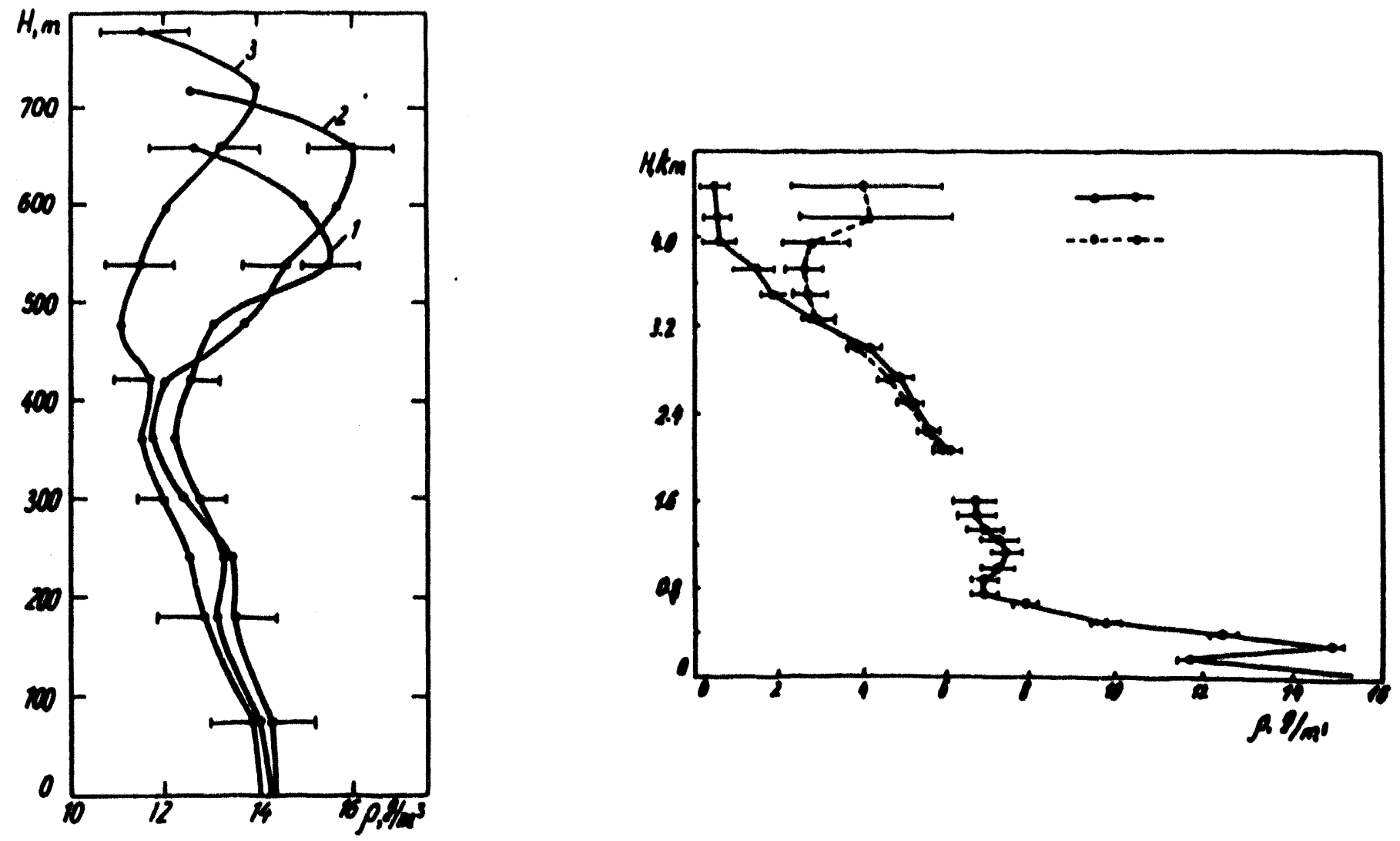

Figure 8. Vertical distribution of water vapor structure in the atmosphoric boundary layer.

Figure 7. The results of simultaneous observations of stratoephorio esrosol and ozone, shortly after the Mt. Pinatubo eruption are shown in Figure 8. As shown in the figure, for 3 days, from July 6 until July 8,1981 , the ozone content in the stratoephoric maximum decreased more than twofold.

Floure 8 showe a soloctive set of lidar obeervations of the volcanic cloud dynamica in the atratosphere over Tomak during a year. Hisevident from the figure that the maximum values of the ecaltoring relationship wore recorded at the ond of January and in February 1992. Over this period the arosol tranemittance of the stratosphere decreased
(Floure 10). Mt. Pinatubo's maximum depreselon of the ozone layer in the stratosphere is obeerved during the eamo period (Figure 11).

In April 1982, the ozone situation stabllized even though acrosol pollution of the stratosphere remained el innilicant. The analysis of the volcanic aerosol microstructure, bacad on the multiwave laser sounding data, indlcaled the tendency for the aernsol to be transformed from a finoly dieporeed fraction to a coaree diepereed one by coring 1982 (Flgure 12). As aerosol particles grow, thoy gredually sottle and separate from the stratosphore, reduding thoir interaction with ozone. 

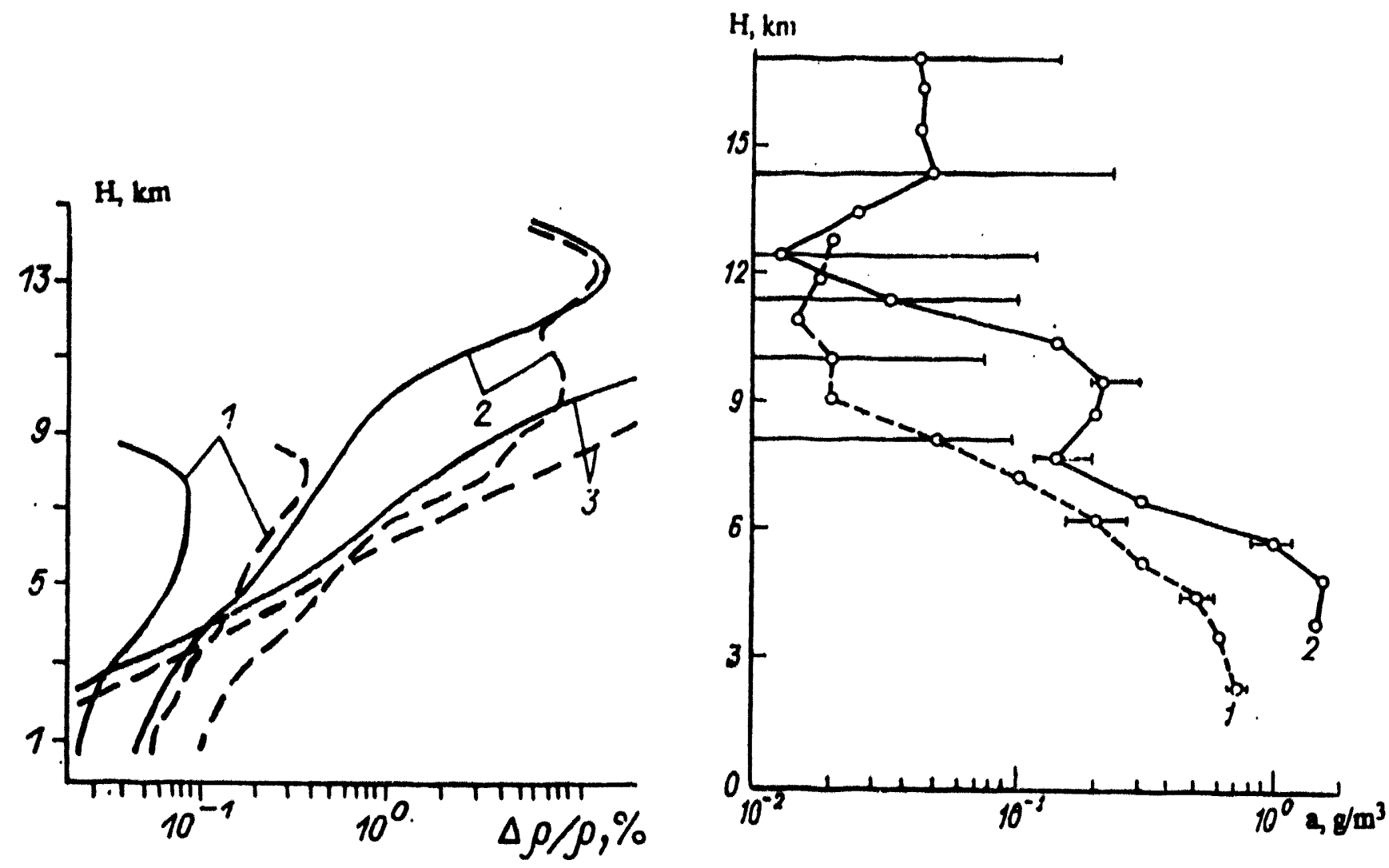

Figure 6. Vertical distribution of water vapor structure at maximum altitudes of about $17 \mathrm{~km}$.

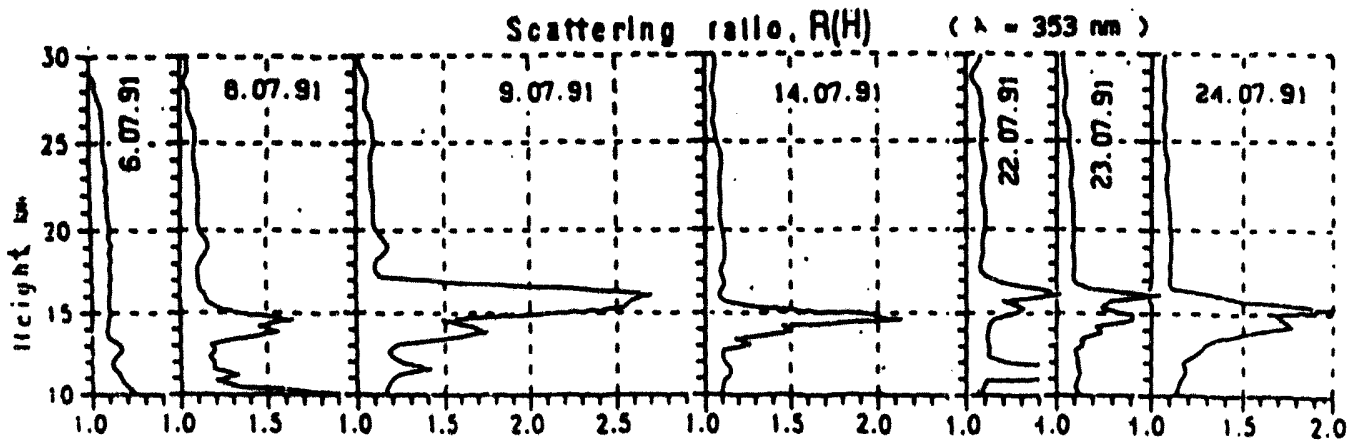

Flgure 7. Averaged back-scattering coefficients measured at $532 \mathrm{~nm}$. 
ARM Sene Months

Sotteplng rollo, $R(H)$ (A. $532 \mathrm{~mm})$

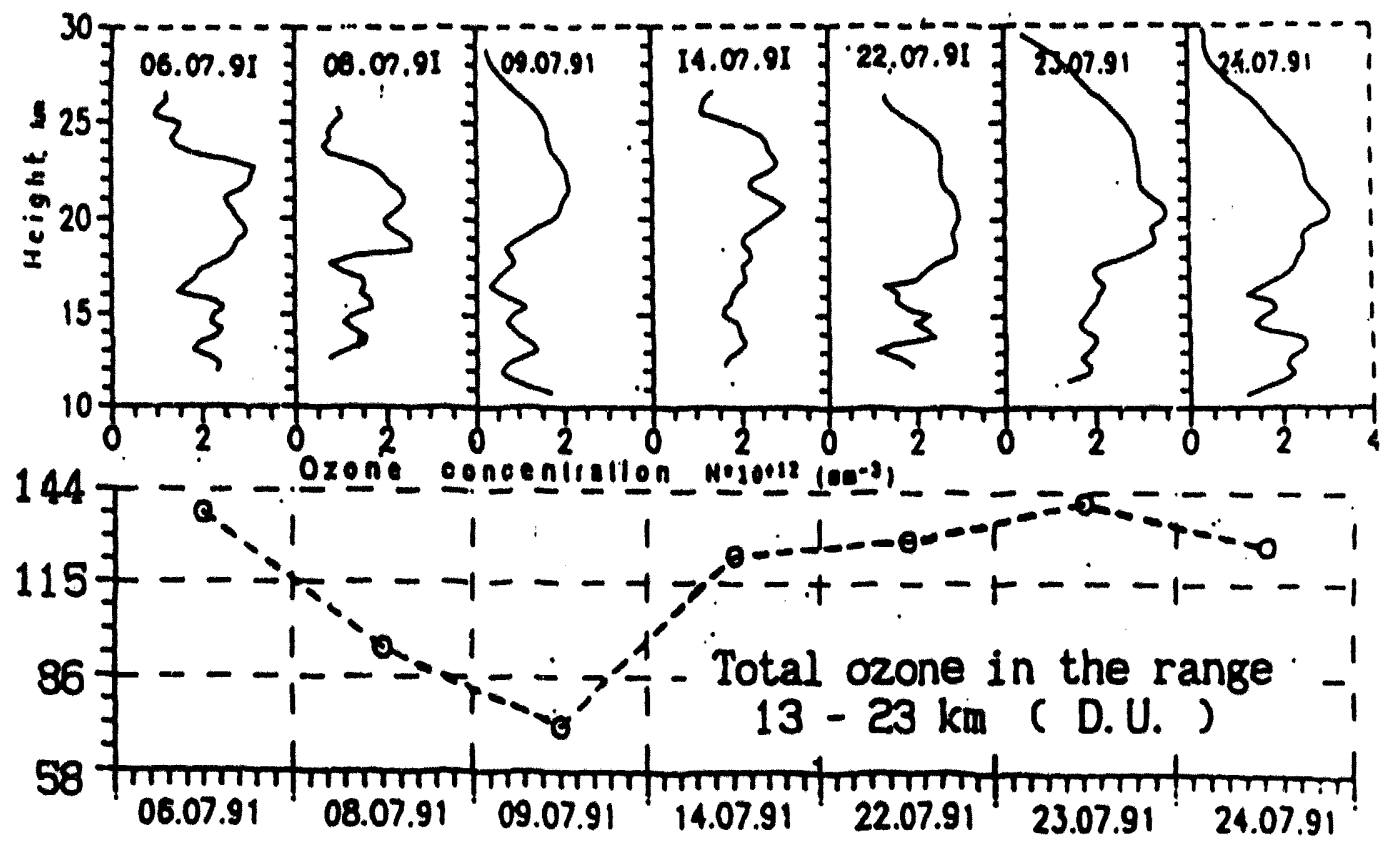

Figure 8. Simultaneous observations of stratospheric aerosol and ozone shortly after the Mt. Pinatubo eruption.

208 


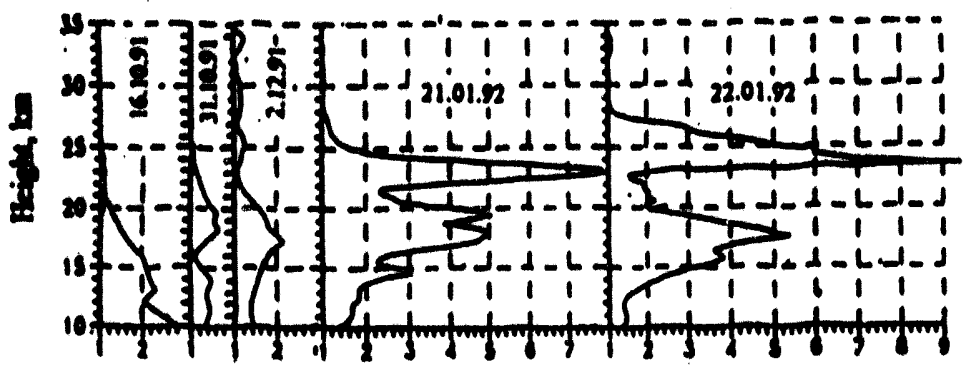

Scutterias ratio, R(I)
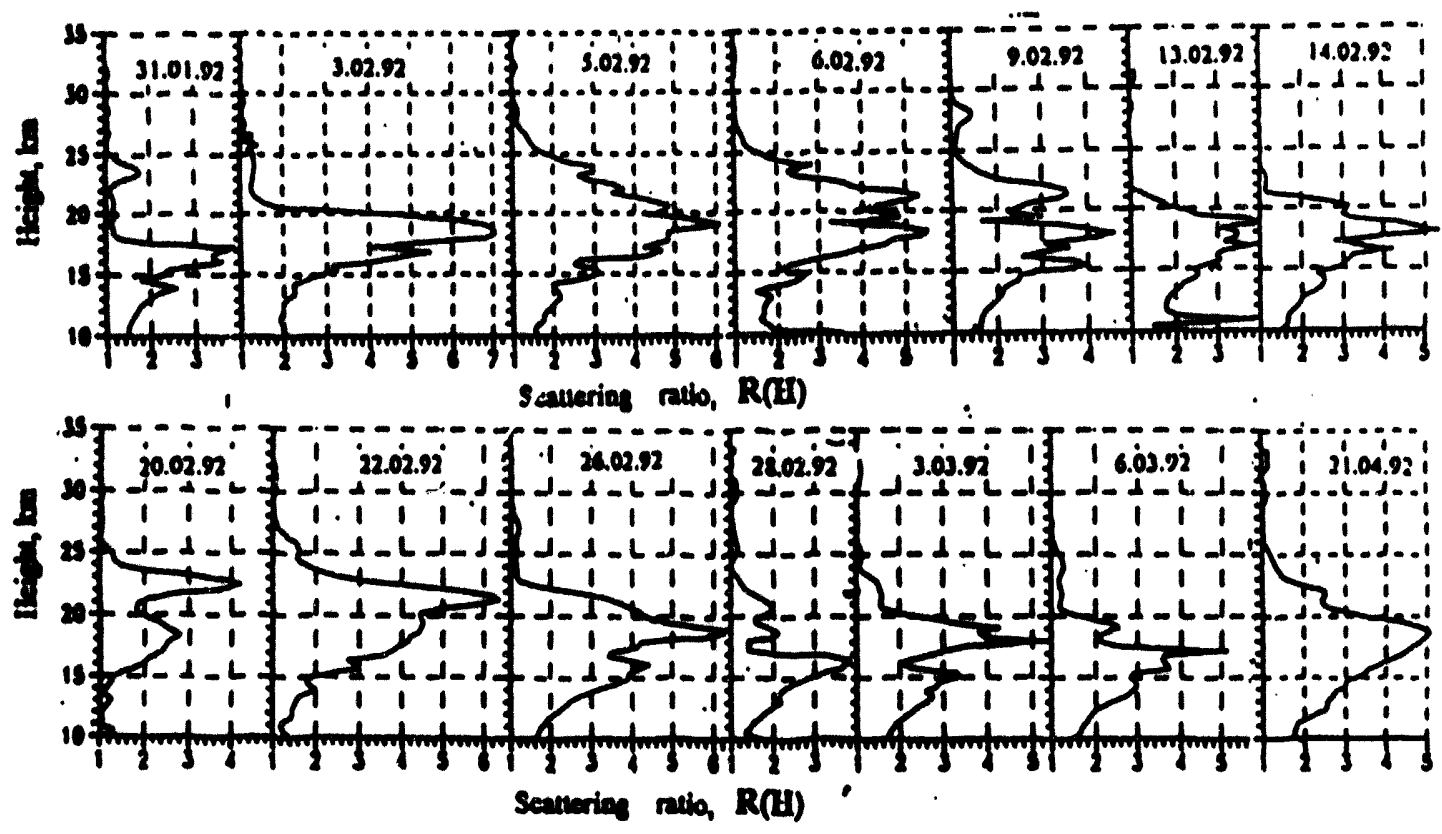

Figure 9. A sebcotive set of lidar obeervations of volcanic cloud dynamics in the stratosphere over Tomak. 
ARil Salines Mooting

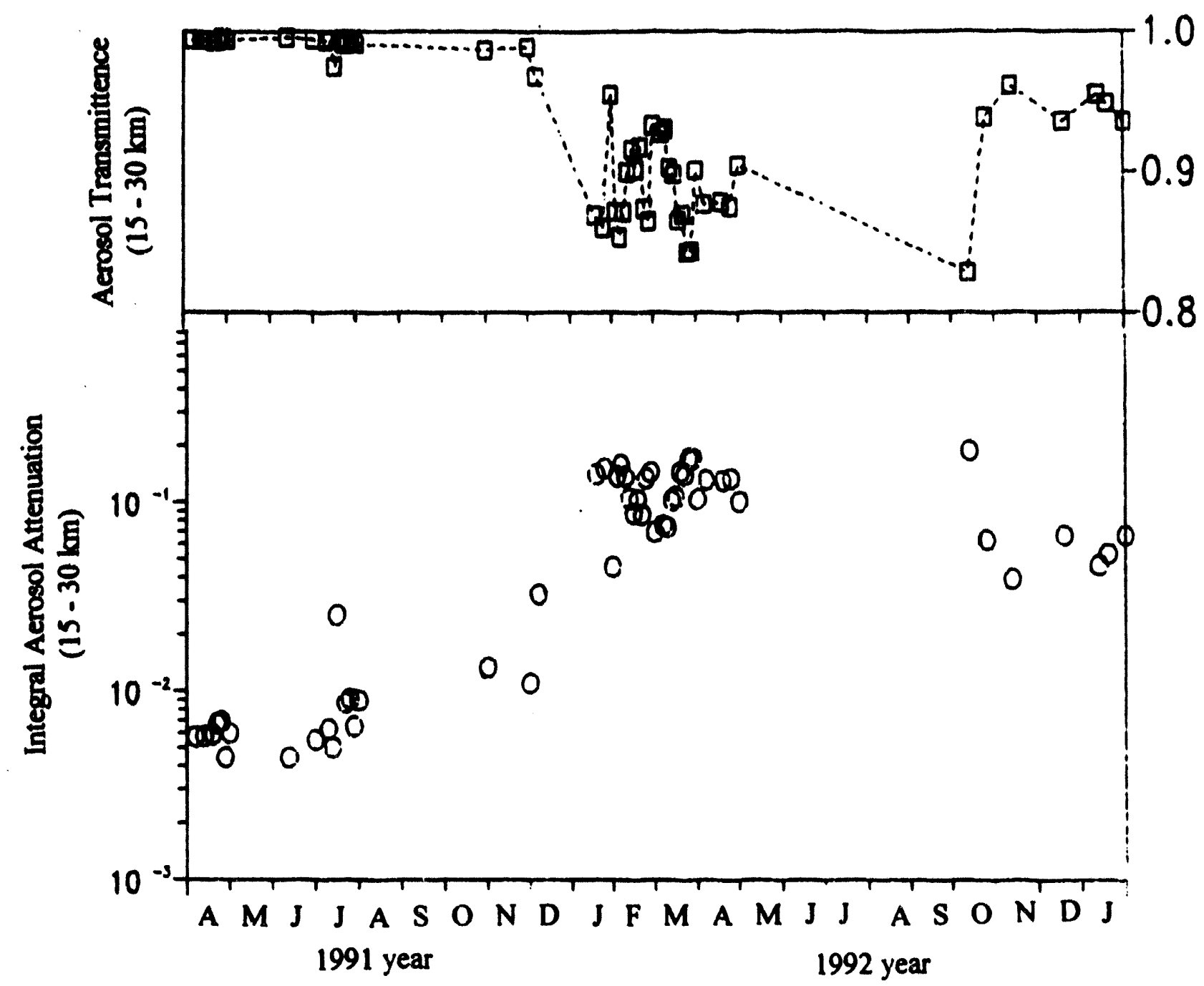

Figure 10. Decreased aerosol transmittance, January and February 1992.

210 

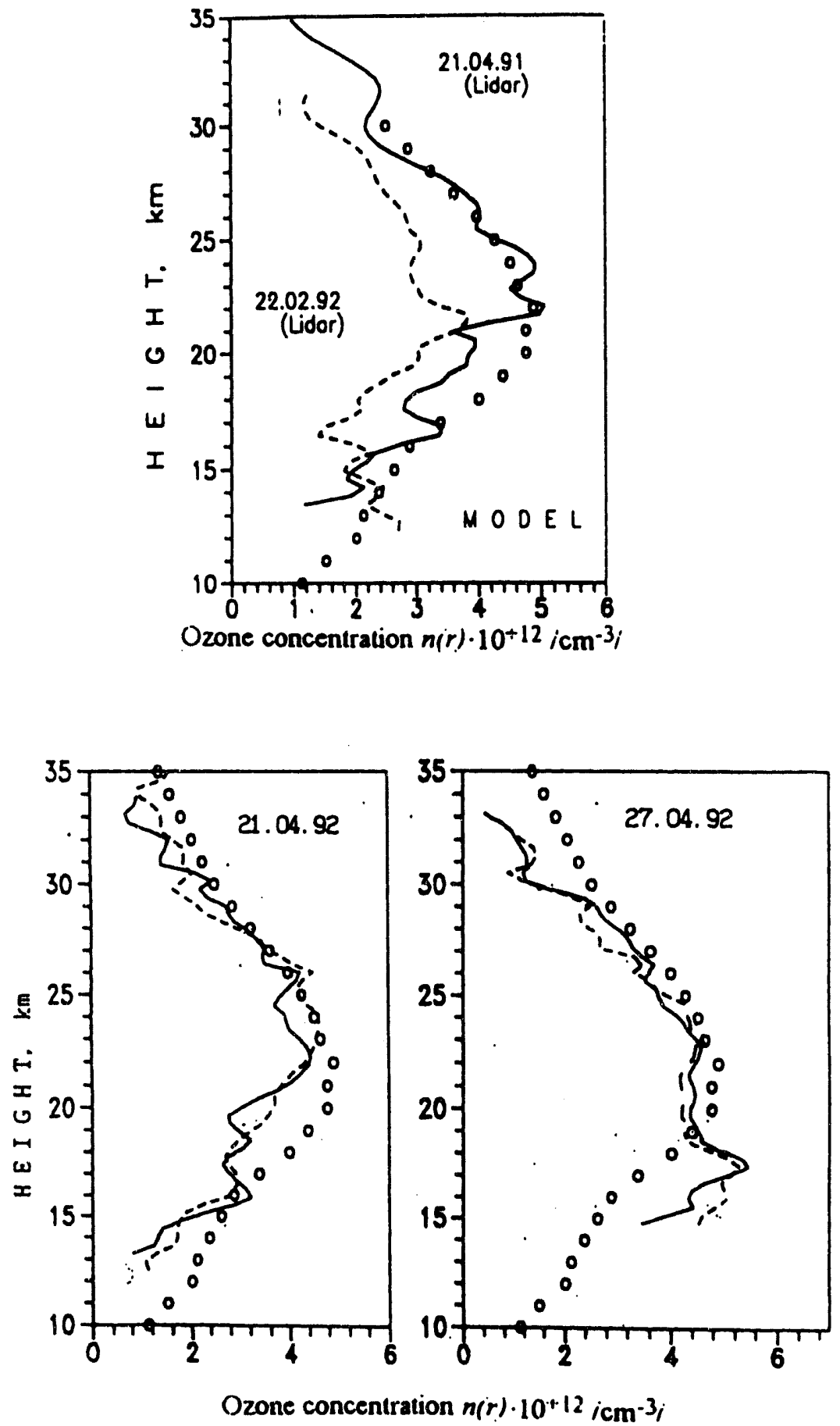

Figure 11. Effect of Mt. Pinatubo eruption on stratospheric ozone over Tomsk. 


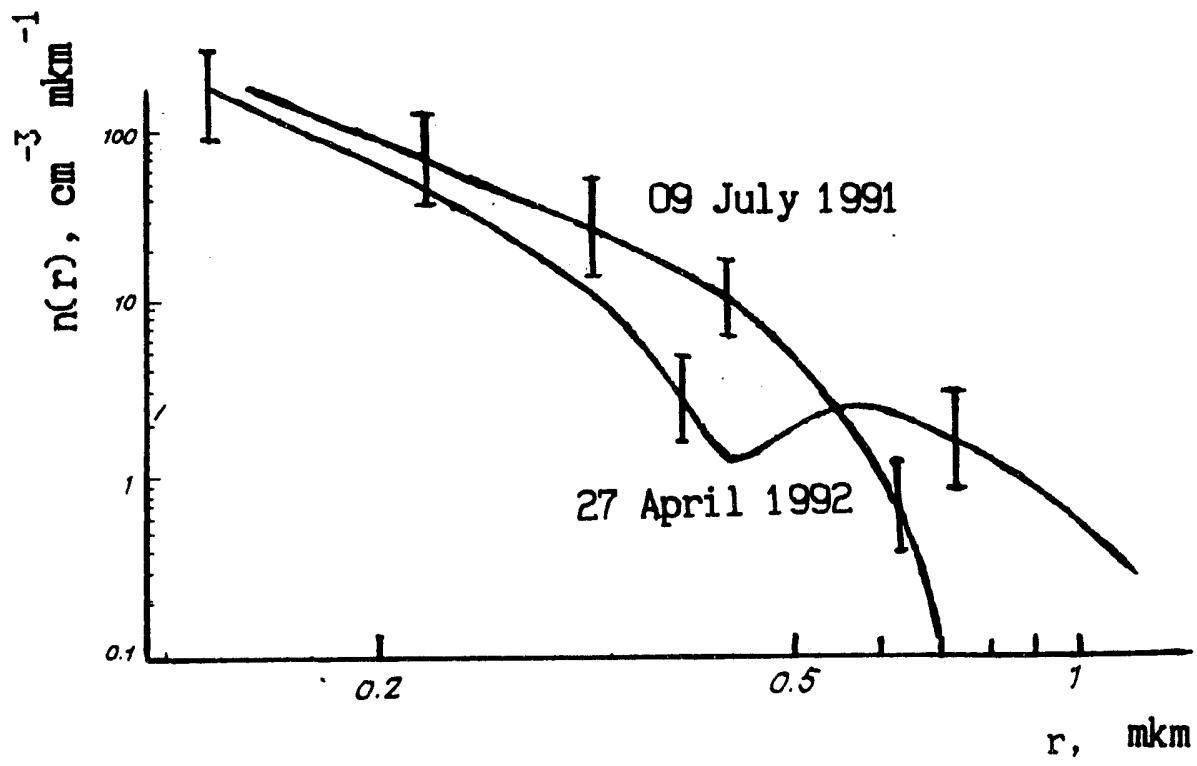

Figure 12. Aerosol transformation. 


\title{
Theoretical Studies of Radiative Properties of Broken Clouds
}

\author{
G. A. Titov \\ Institute of Atmospheric Optics \\ Russian Academy of Science \\ Tomsk, Russia
}

One of the three major goals of the Atmospheric Radiation Measurement (ARM) Program is to improve the quality of radiation models under clear sky, homogeneous cloud, and broken cloud conditions. This report is concerned with the development of the theory of radiation transfer in the broken clouds. Our approach is based on a stochastic description of the interaction between the radiation and cloud field with stochastic geometry; the main results are presented in Titov $(1990,1993)$. In the following, we discuss

- the mean radiation fluxes in the near IR spectral range 2.7 to $3.2 \mu \mathrm{m}$

- the influence of random geometry of individual cumulus clouds on the mean fluxes of visible solar radiation

- the equations of the mean radiance in the statistically inhomogeneous cloud fields.

\section{Mean Radiation}

\section{Fluxes in the 2.7 to $3.2 \mu \mathrm{m}$ Wavelength Range}

In radiation models, as components of general circulation models (GCMs), one should calculate very accurately the net fluxes of upwelling and downwelling radiation at chosen levels in the atmosphere. These fluxes can be calculated by integrating the spectral fluxes; and, in turn, in calculating latter characteristics, one should take accurately into account the wavelength dependences of optical parameters of clouds and aerosols and of the absorbing properties of atmospheric gases.

In the wavelength range 2.7 to $3.2 \mu \mathrm{m}$, the real and imaginary parts of the water refractive index and, hence, the single scattering albedo and scattering phase function are strong functions of wavelength (Figure 1).

To the extent that the single scattering albedo is small $(\sim 0.5-0.6)$, the mean spectral albedo will result mainly from the radiation having undergone only several first orders of scattering. For this reason, the mean spectral albedo will be strongly influenced by the scattering phase function. The use of only one scattering phase function for calculating the mean spectral albedo, which is the commonly used approximation, may lead to large errors (Figure 2).

The dependence of the mean spectral albedo on the wavelength and cloud type (cumulus or stratus) is shown in Figure 3. Calculations were performed both with and without regard to absorption by water vapor and carbon dioxide. In the presence of absorption by water droplets, the mean spectral albedo $<\mathrm{R}_{\mathrm{Cu}}>$ of a cumulus cloud field is larger than the mean spectral albedo $<R_{S P}>$ for stratus clouds even at the zero solar zenith angles $\zeta_{0}^{\circ}$; whereas, in the visible spectral range, where the scattering can be treated as conservative (for which the single scattering albedo is equal to 1), the opposite is true.

Figure 4 illustrates the dependence of the value of $\Delta R=$ $\left\langle R_{\mathrm{St}}\right\rangle-\left\langle\mathrm{R}_{\mathrm{Cu}_{\mathrm{u}}}\right\rangle$ on the single scattering albedo $\mathrm{W}$ : for a certain value of $W_{0}$, the value of $\Delta R$ alters its sign. In interpreting this effect, one must keep in mind that the significant contributor to the mean spectral albedo of cumulus clouds field is that radiation exiting the sides of a large number of cumulus clouds undergoes, on the average, fewer scattering orders than the radiation exiting the tops and bottoms of stratus clouds. From this fact and from the possibility to represent the mean albedo in terms of scattering order series, it follows that the lower scattering orders will be more of a factor to the mean albedo of cumulus clouds than of stratus clouds; for higher scattering orders, the opposite may be valid. As the single scattering 

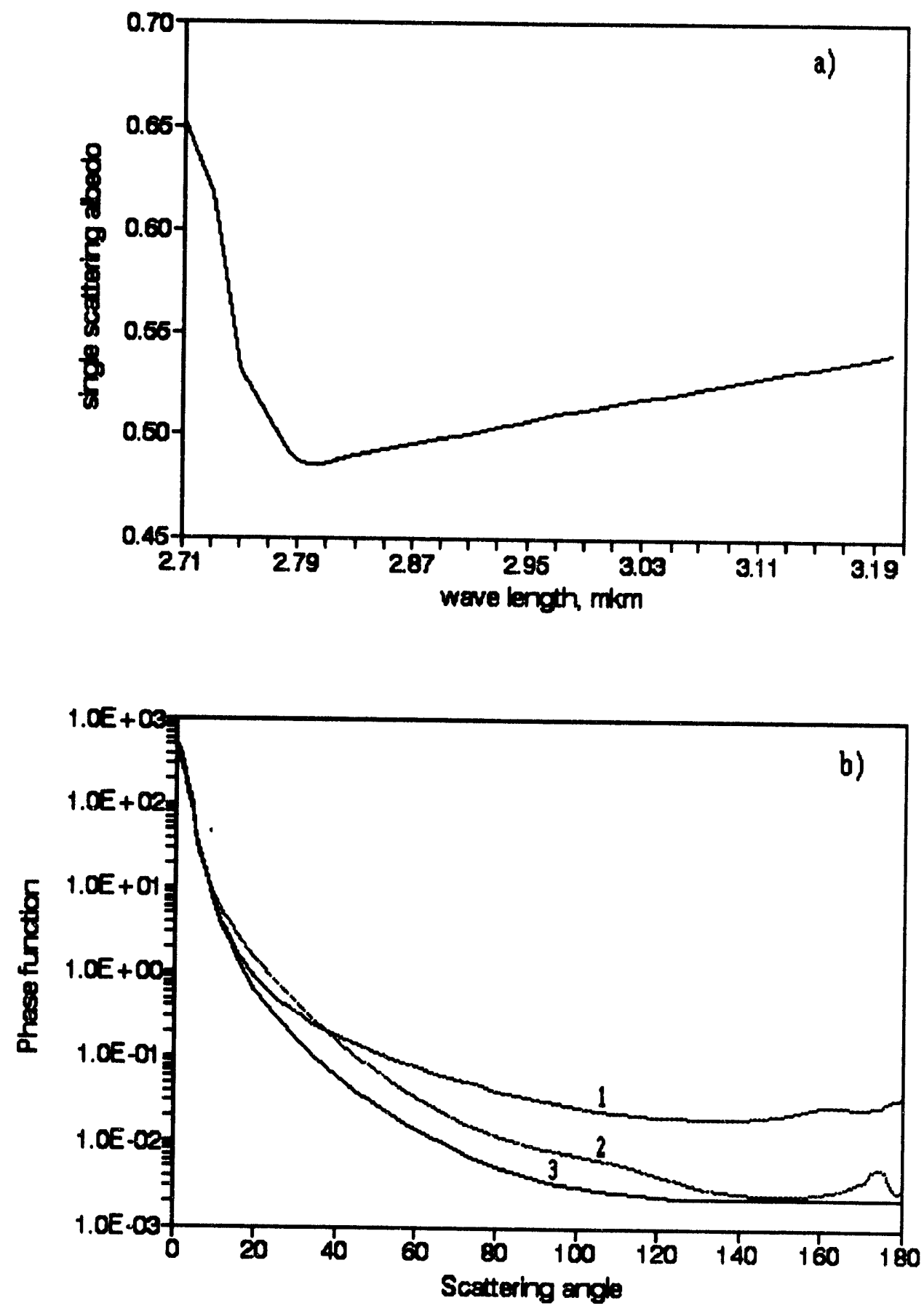

Figure 1. Single scattering albedo as a function of wavelength (a) and the phase functions for different wavelengths (b): $\lambda=2.73 \mu \mathrm{m}(1) ; \lambda=2.79 \mu \mathrm{m}(2) ; \lambda=3.2 \mu \mathrm{m}(3)$. 


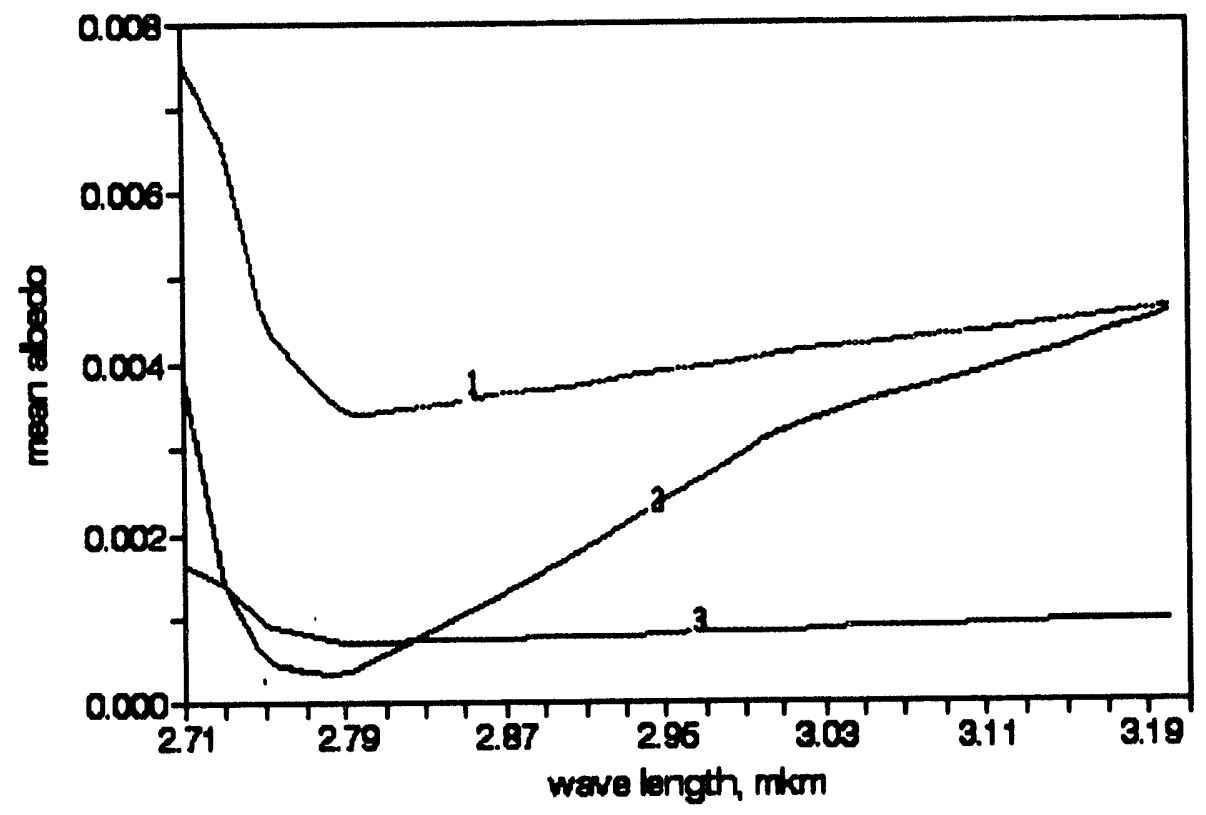

Figure 2. The influence of scattering phase functions $g_{\lambda}(\mu)$ on mean albedo where $\sigma=30 \mathrm{~km}^{-1} ; H=D=0.5 \mathrm{~km}, N=0.5$, $\zeta_{0}=1^{\circ}:$ standard calculations (2); $\lambda=2.73 \mu \mathrm{m}(3) ; \lambda=3.2 \mu \mathrm{m}(1)$.

albedo decreases, the relative role of high scattering orders becomes less important, which provides the explanation for the effect given in Figures 3 and 4.

\section{Radiation Transfer in Statistically Inhomogeneous Cloud Fields}

The equations for mean radiance were obtained in statistically homogeneous cloud fields (Titov 1990 and 1993). In reality, cloud cover is statistically inhomogeneous if, for no other reason, than the physically distinguishable vertical direction in the atmosphere. At present, numerical simulation of brightness and radiation fields (Titov 1990, 1993) is practically the only technique for calculating the mean fluxes and brightness fields of radiation modulated by statistically inhomogeneous broken clouds. In this method, one of the input parameters is the cloud shape, which is difficult to determine experimentally and to describe accurately by mathematical facilities, especially considering the highly irregular and "wild" shapes of real clouds.
For model calculations, individual clouds are approximated by truncated paraboloids of rotation. The cloud is divided into $m$ layers and in each layer the true cloud shape is approximated by a cylinder. This approximation can be made with as high a resolution as desirable. The unconditional and conditional probabilities of cloud occurrence are specified as constant functions and within each layer the cloud field is statistically homogeneous. As a result, in each layer the equations for mean radiance hold with the corresponding changes in boundary conditions. The final result can be easily obtained if one successively uses the equations for mean radiance with the corresponding unconditional and conditional probabilities of cloud occurrence.

Presented in Table 1 are the mean fluxes of direct $<S>$ and diffuse $\left\langle Q_{s}\right\rangle$ transmitted radiations, as well as the mean albedo $\langle R>$ calculated with the method of numerical simulation (MNS) and with the equations for mean radiance (EMR). As the table shows, the calculational results are in good agreement. 


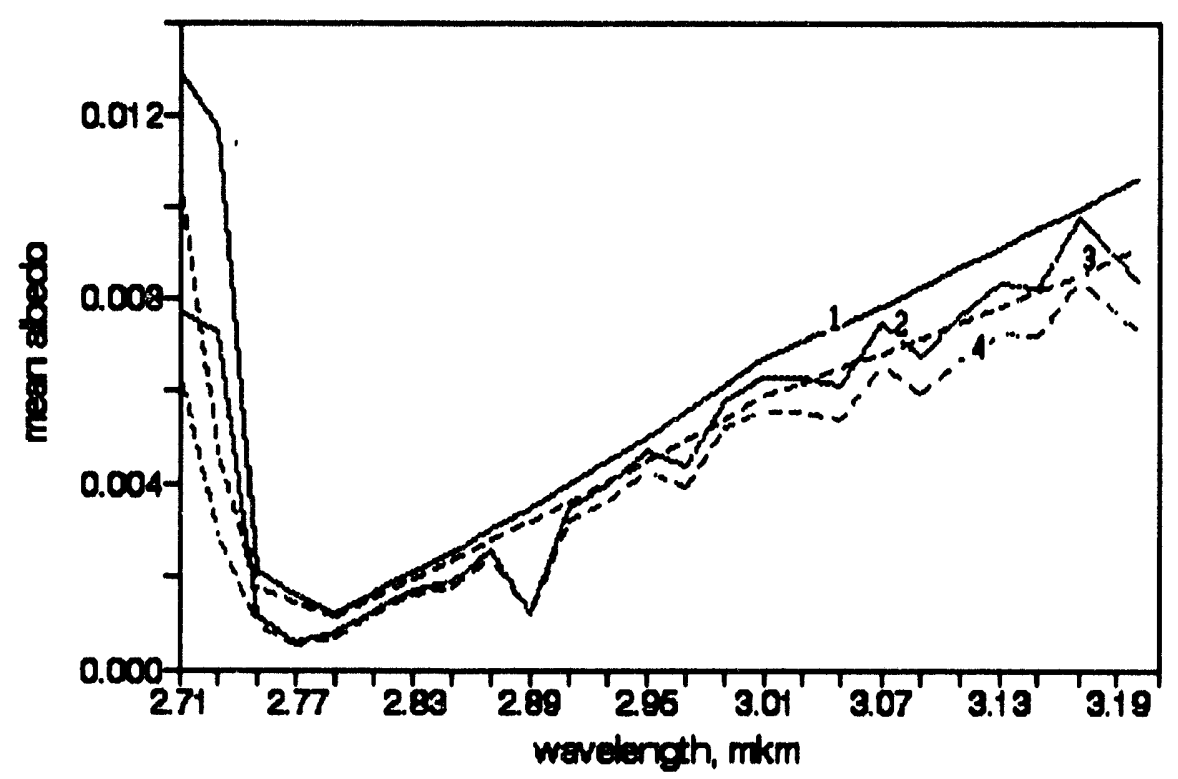

Figure 3. The mean albedos with $\sigma=30 \mathrm{~km}^{-1}, H=D=0.5 \mathrm{~km}, N=0.5, \zeta_{0}=0^{\circ}$ for different cloud types: $C u$, without taking the gas absorption into account (1); $\mathrm{Cu}$, taking into account the absorption by water vapor (2); St, without taking the gas absorption into account (3); St, taking into account the absorption by water vapor (4).

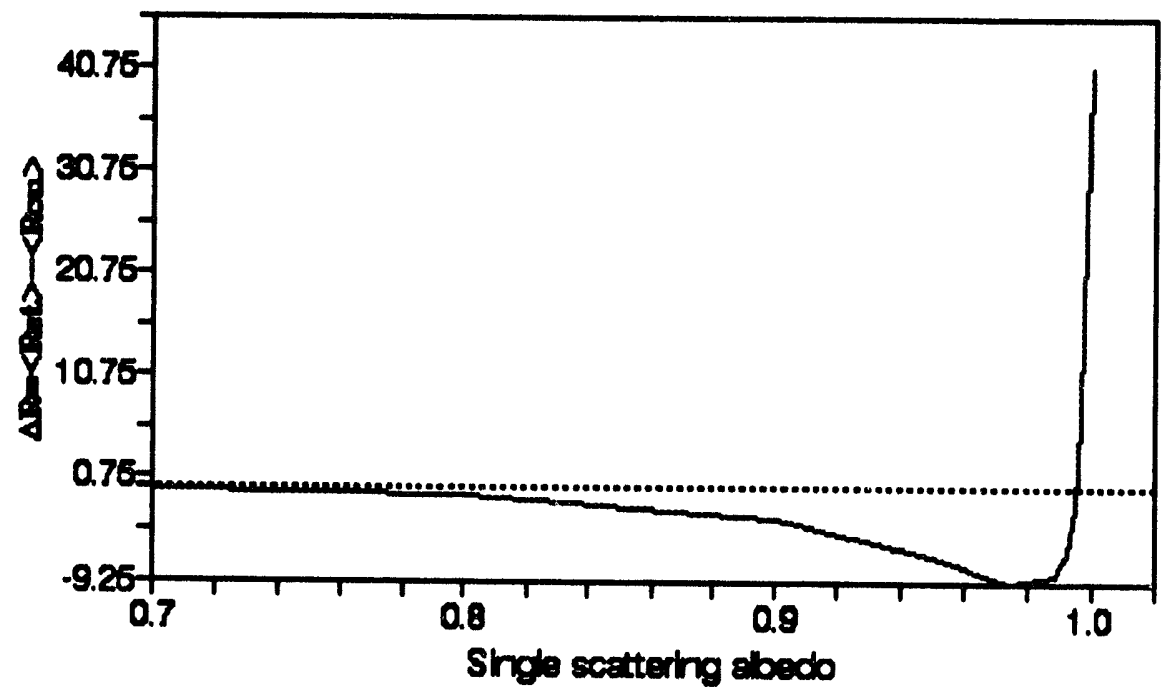

Figure 4. The influence of the single scattering albedo on the value of $\Delta R=\left\langle R_{S t}\right\rangle-\left\langle R_{C u}\right\rangle\left(\Delta R^{*} 10^{3}\right)$ with $\zeta_{0}=0^{\circ}, N=$ 0.5 , surface albedo $A_{8}=0, \gamma=H / D=2, \tau=60$. 
Table 1. The mean fluxes calculated by two different mothods with $D=1 \mathrm{~km}, \sigma=30 \mathrm{~km}^{-1}, H=0.5 \mathrm{~km}, \zeta_{0}=60^{\circ}$

\begin{tabular}{|c|c|c|c|c|c|c|}
\hline \multirow[b]{2}{*}{$\mathbf{N}$} & \multicolumn{2}{|c|}{$\langle S\rangle$} & \multicolumn{2}{|c|}{$\left\langle Q_{1}\right\rangle$} & \multicolumn{2}{|c|}{$\langle R\rangle$} \\
\hline & MNS & ERM & MNS & ERM & MNS & ERM \\
\hline 0.10 & 0.878 & 0.857 & 0.069 & 0.086 & 0.053 & 0.053 \\
\hline 0.30 & 0.629 & 0.615 & 0.199 & 0.218 & 0.171 & 0.168 \\
\hline 0.50 & 0.419 & 0.404 & 0.316 & 0.324 & 0.265 & 0.272 \\
\hline 0.70 & 0.211 & 0.213 & 0.389 & 0.395 & 0.401 & 0.392 \\
\hline
\end{tabular}

The main advantages of the method, which is based on solving the equations for mean radiance, are as follows:

- Whereas in MNS the input parameter is the shape of individual clouds, in solving the EMR, the input parameters are the conditional and unconditional probabilities of clourt occurrence which have clear physical and mattiematical meaning and can be determined experimentally.

- For intermediate and large cloud fractions the efficiency of the method based on EMR is much higher than that of the MNS.

- Equations for mean radiance can be solved not only with Monte Carlo techniques, but also with other numerical and approximate methods.

We hope that this approach to the problem of radiation transfer in statistically homogeneous cloud fields will be useful when the individual clouds have random geometry.

\section{Models of Broken Clouds with Random Geometry of Individual Clouds}

The models of broken cloudiness would be constructed by coupling the Poisson indicator field (P-model) and the sum of Gaussian fields $\left(G_{n}\right.$-model) with decreasing variances and correlation radil ( $P G_{n}$-model) (Bablch and Titov 1992). This procedure is essentially very close to the generalized Brownian movement that is used to simulate fractal clouds.

The problem of numerical construction of sampling realizations in the $P G_{n}$-model is reduced to simulation of two types of independent random fields. The algorithms for simulating the Polsson indicator field and homogeneous lsotroplc Gaussian field with known spectral density can be found in Titov $(1990,1993)$ and Mikhailov (1978), while the basic idea of the algorithm is illustrated in Figure 5. The vertical cross sections of individual cumulus clouds in the $P G_{n}$-model are presented in Figure 6. As the figure shows, the obtained pictures are very close to realistic cloud images.

Figure 7 shows the mean fluxes of direct $\langle S>$ and scattered transmitted $\left\langle Q_{8}>\right.$ radiations, as well as the mean albedo $\langle R\rangle$, which were calculated within appropriately coordinated $P$ - and $P G_{1}-$ models. The differences between the corresponding fluxes can reach essential magnitudes. The use of the $P G_{n}$ models with $n \sim 5-6$ may result in random geometric cloud shapes that differ from the paraboloids (see Figures 5 and 6), and these differences may increase.

The results of comparison allows us to draw the preliminary conclusion that because of the nonlinear dependence of the radiation field upon the cloud characteristics, the random geometric shape of individual clouds can have a significant effect on the transfer of solar radiation.

\section{References}

Babich, E. A., and G. A. Titov. 1992. Mathematical Models of a Broken Cloud Field with a Random Geometry of Individual Clouds. J. Atmos. Ocean. Optics. 5(7):757-765.

Mikhailov, G. A. 1978. Dokl. Akad. Nauk SSSR, 238(4):793. 795.

Titov, G. A. 1990. Statistical Description of Radiation Transfor in Clouds. J. Atmos. Sci. 47(1):24-38.

Titov, G. A. 1993. Rad ative Transfer in Cloud Field with Random Geometry. Tronds in Geophysical Researchs, India (in press). 


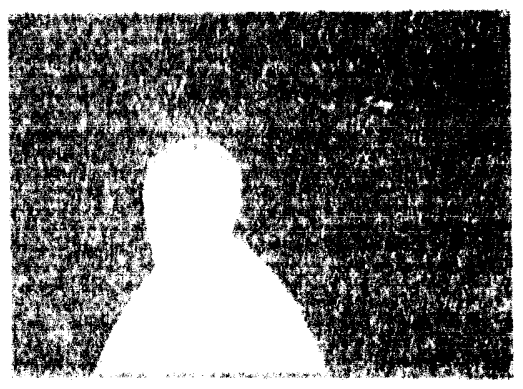

$M=1$

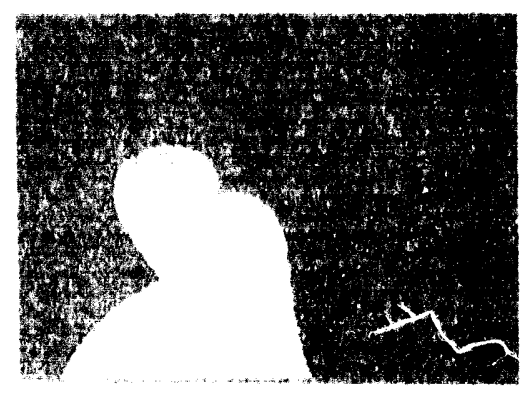

$n \geq 2$

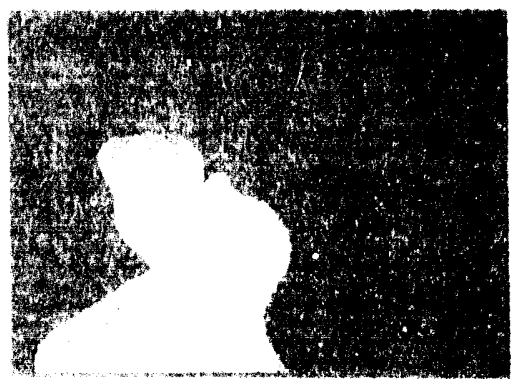

$n=4$

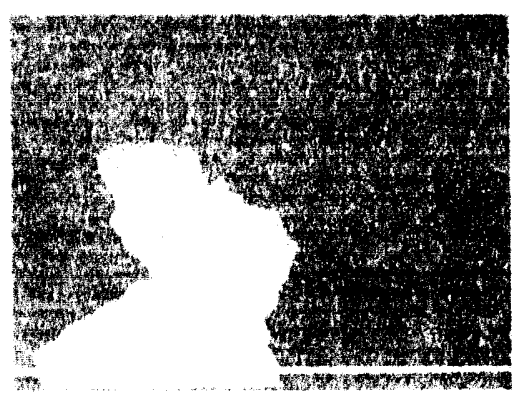

$12=6$

Figure 5. The basic idea of the algorithm for simulating the Poisson indicator field and homogeneous isotropic Gaussian field with known spectral density.
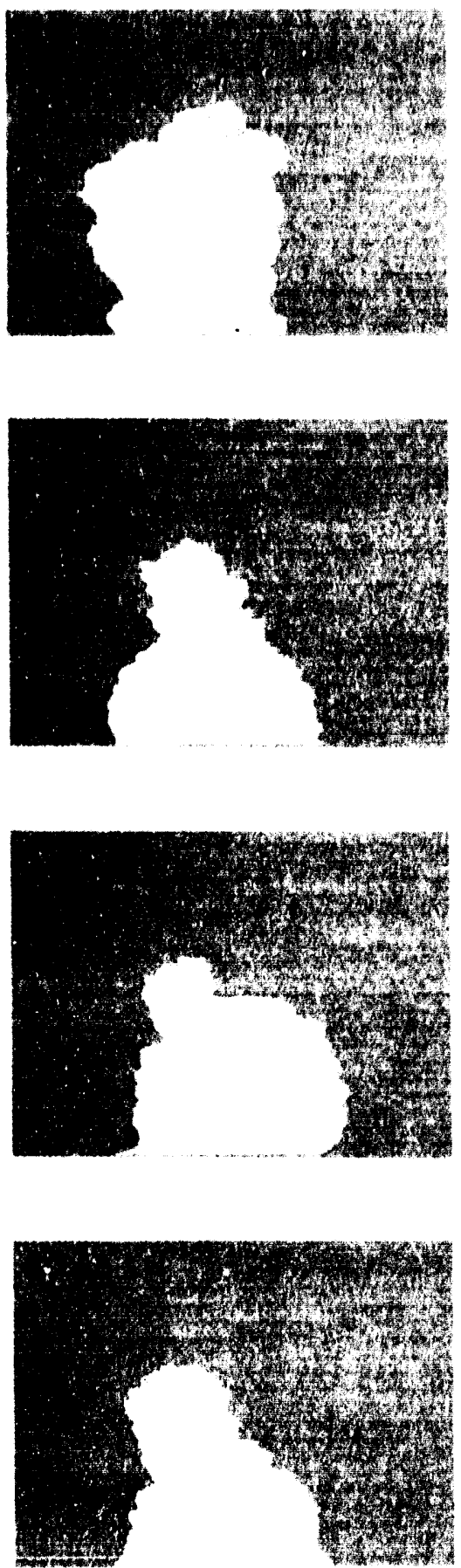

Figure 6. Vertical cross sections of individual cumulus clouds in the PG -model. 

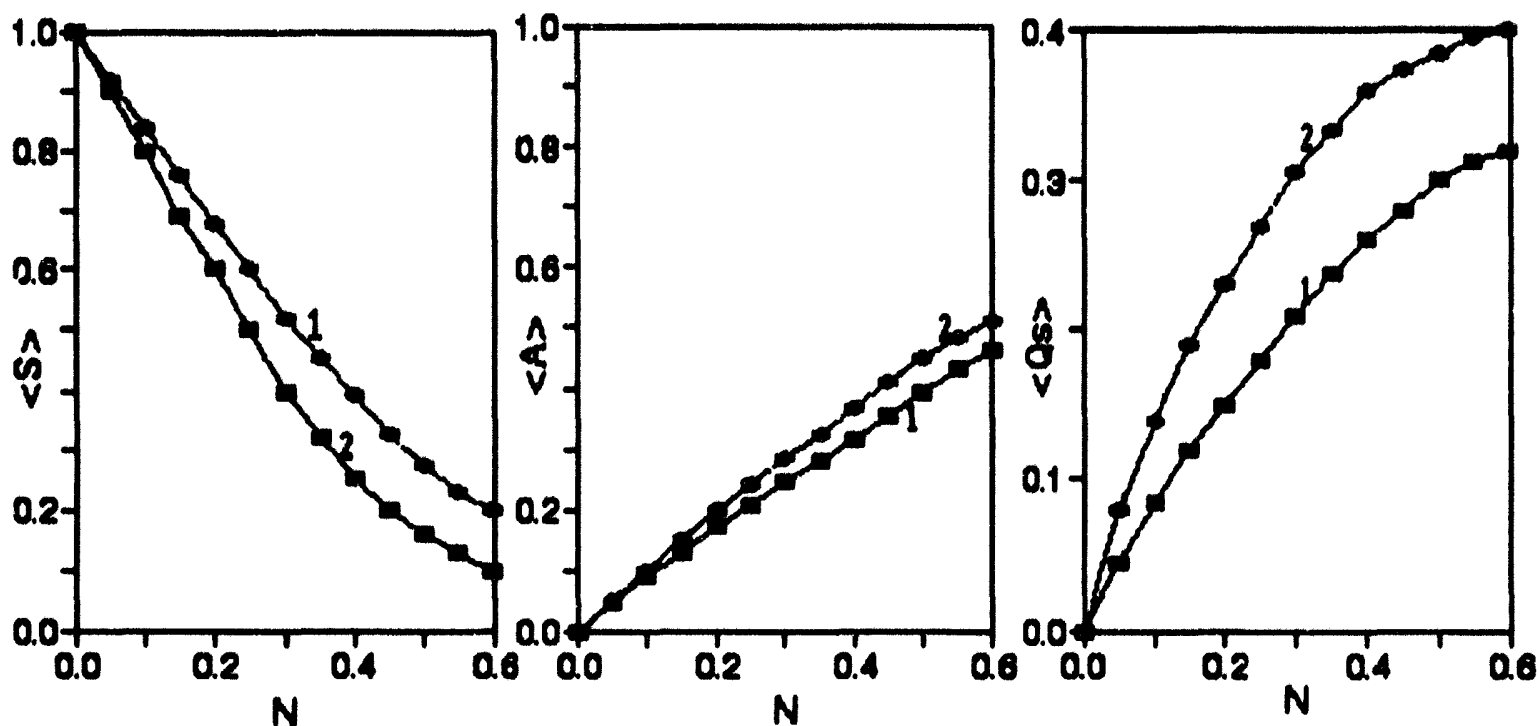

Figure 7. Dependence of the mean fluxes $\langle S\rangle,\langle A\rangle$, and $\left\langle Q_{8}>\right.$ on the random geometry of individual clouds with $\zeta_{0}=$ $60^{\circ}, \sigma=30 \mathrm{~km}^{-1}, H=1.08 \mathrm{~km}$, and $\angle D^{2}>1 / 2=1.143 \mathrm{~km}$ (curvo 1 refers to the calculations for a P-model, curvo 2 refiore to $P G_{1}-$ model). 


\title{
Radiation Experiment in Support of the Atmospheric Radiation Measurement Program
}

\author{
V. E. Zuev, V. V. Zuev, and G. A. Titov \\ Inatitute of Almospheric Optice \\ Siberian Branch of the Russian Academy of Sciences \\ Tomak, Aussia
}

The objective of this work is to test and improve the radiation models under clear sky, homogeneous, and broken cloud conditions. The effort will produce data of field measurements of the atmospheric radiation taken simultaneously with measurements of the atmospheric parameters which determine the radiative transfer under three meteorological conditions mentioned above. These measurements provide a baels for the detaled testing and verification of the radiation models in GCMs and are applicable in various scientific centers.

Experimental investigations of the dependence of radiation fields on the parameters describing the physical state of the atmosphere will be carried out as part of this program. The experiment will include measurements of the following characteristics of the radiation field and the parameters of the "atmosphere-underlying surface" system:

- meteorological measurements-pressure and temperature as functions of the altitude and the three. dimensional field of wind velocity

- atmospheric composition-concentration and phase composition of water; content of the greenhouse gases; size distribution and optical parameters of aerosol and cloud particles

- surface properties-albedo and effective radiative temperature

- geometric structure of clouds-cloud fraction, location, shape, vertical and horizontal size

- radiation measuremente-epectral radiance, broe Jband and net fluxes, relative fluxes, and polarization state.

A unique instrumentation complex, which is a rellable base for carrying out the radiation experiment, has been developed at the Institute of Almospheric Optics of the Siberian Branch of the Russian Acadomy of Sciences. A number of the atmospheric parameters are measured by several methods by means of devices. This approach is important for intercalibration and increasing the rellability and the accuracy of the data obtained. Figure 1 illustrates the principal instrumentation of the conceplual project. The capabilities of the instrumentation are briefly described Dolow.

The meteorological and aerological stations situated in the vicinity of Tomsk are also presented in Figure 1. The data from these stations will be used for planning and carrying out the experiment and for interpreting the results.

\section{Atmospheric Parameter Measurements}

The detailed study of various physical effects associated with laser radiation propagation in the scattering and absorbing media provides a methodological basis for lidars. The lidars are capable of obtaining the quantitative information about the vertical profiles with high spatial resolution, and in some cases about the spatial distribution of the atmospheric parameters.

The multitrequency lldar is used for measuring the ozone and aerosol concentration profiles and for retrieving the aerosol size distribution function. At present, the multifrequency lidar operates better at night, when it can cover the 5-30 km height range; in the day, the height range is 5 to $15 \mathrm{~km}$. The principal parameters of the multifrequency lidar are given in Table 1. 


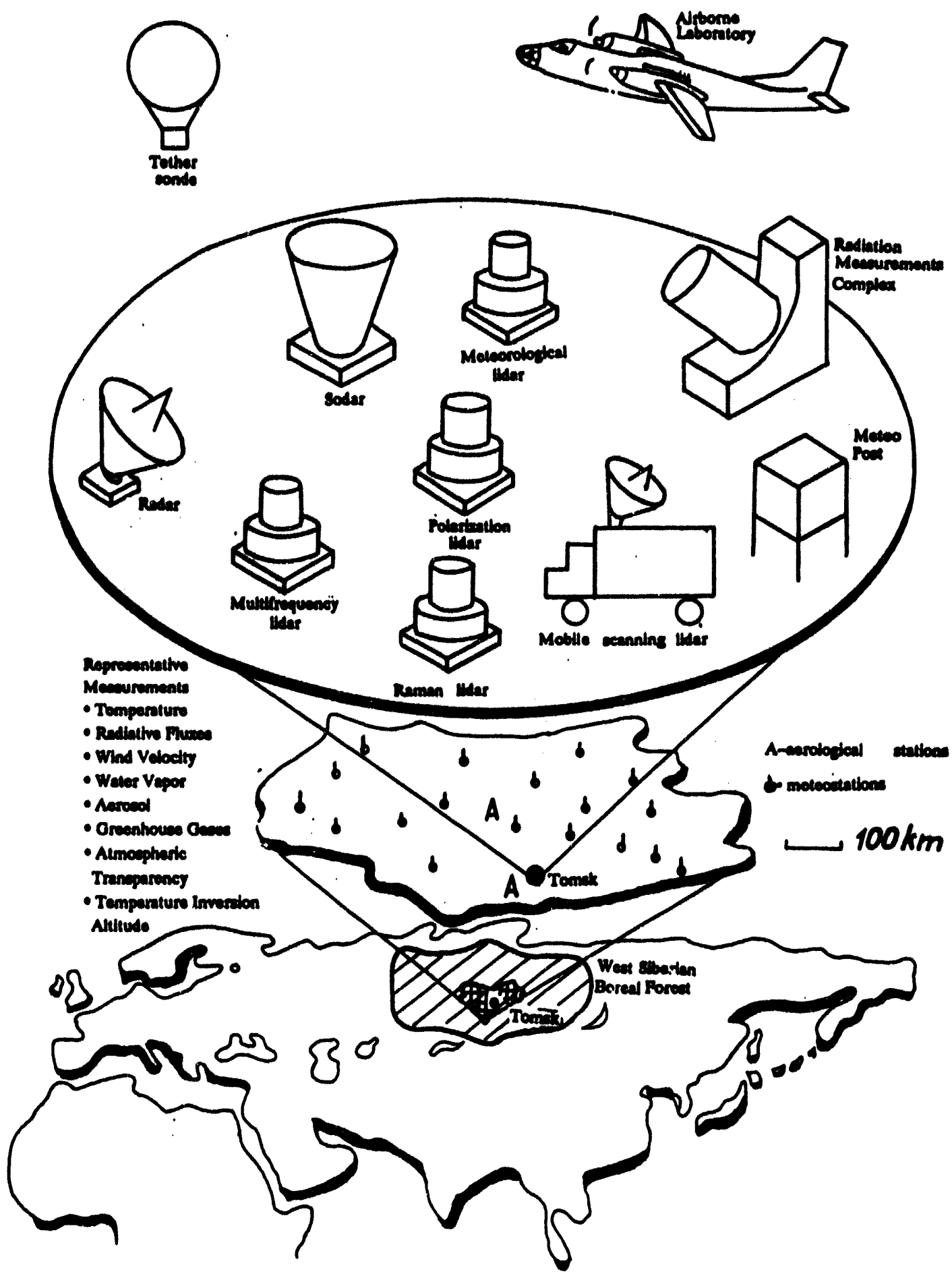

Figure 1. Conceptual design for the Complex Rediative Exporimont. 
Table 1. Paramotors of the multifrequency Ildar.

Paramotore of sounding

Range of sounding heights

Temporal resolution

Spatial rosolution

Lasor

Cu(578 mn)+trequency doubling

$\mathrm{XoCl}$

XoCl+Raman-coll $\left(\mathrm{H}_{2}\right)$

Nd:YAG

Nd:YAG+frequency doubiling

Nd:YAG(532 nm)+Raman-cull $\left(\mathrm{H}_{2}\right)$

$\mathrm{Sr}+$

Cu

Au

Pb

Tolescope diameter, $m$

Tolescope focal length, $m$

Tolescope viewing fiold, mrad

Wavelengths, $\mathrm{nm}$

Detector-photomultiplier

Number of gates

Computer

\author{
Ozone, Arosol, Temporature \\ $5.40 \mathrm{~km}$ \\ $15-30 \mathrm{~min}$ \\ $50-500 \mathrm{~m}$
}

Transmituer

\begin{tabular}{|c|c|c|c|}
\hline$\lambda, n m$ & $E, \mathrm{~mJ}$ & $P_{\text {man }}, W$ & $\mathrm{t}_{1} \mathrm{~Hz}$ \\
\hline 289 & & 0.2 & $5 \cdot 10^{3}$ \\
\hline 308 & 50 & & $50-100$ \\
\hline 353 & 30 & & 50.100 \\
\hline 1084 & 150 & & 10 \\
\hline 528 & 50 & & 10 \\
\hline 683 & 30 & & 10 \\
\hline 431 & & 1 & $2.5 \cdot 10^{3}$ \\
\hline 511 & & 2 & $2.5 \cdot 10^{3}$ \\
\hline 628 & & 2 & $2.5 \cdot 10^{s}$ \\
\hline 723 & & 1 & $2.5 \cdot 10^{3}$ \\
\hline
\end{tabular}

Recelver

$2.2 \quad 0.35$

$10 \quad 1$

0.5

$308,353,1064,532 \quad 289,308$

$683,431,511,628,723$

Acegletration

PMT-79, PMT-130, PMT-142

512

IBM PC AT
The "LOZA-3" moblle aorosol IIdar measures the following atmospheric parameters:

- the sce tering coefficients and estimated aerosol mass concent' "lon along the vertical, horizontal, and slant paths

- spatial and temporal variations of the lower boundary and the optical depth of clouds

- wind velocity in the boundary layer
- helght of the mixing layer, the size and velocity of the thermal plumes under various convective conditions, and the temperature inversion height.

The lidar can operate in the scanning regime (Table 2); thus, it can obtain quantitative data on the spatial distribution of the enumerated parameters. In addition, the lidar can be situated at the basic site of the experiment, can be placed at the necessary distance, and can operate in an independent regime. 
Table 2. The "LOZA-3" mobile sorosol lidar.

Paramotore of sounding

Range of eounding diatancose

Cloar sky

Cloud fielde

Temporal resolution

Spatial resolution

Angular resolution

Operation regime optlcal-phyaical parametore

of arosol and cloud formations

$3-4 \mathrm{~km}$

$7.10 \mathrm{~km}$

1.38

$7.5 \mathrm{~m}$

$20^{\circ}$

round-the-clock, cycle

Laser

Diameter, $m$

0.3

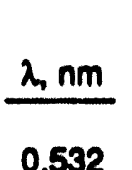

0.532

Lens tolescopo
Tranemilier

E,mJ

30
$P_{\text {mand }} W$

1.25
$\mathrm{H}, \mathrm{Hz}$

15
Recolver

Focus, $m$

1
Fiold of viow

$0.5-5$

\begin{tabular}{|c|c|c|c|}
\hline & & adumn & \\
\hline & Azimuth & Elovation & Scannine rate ranos \\
\hline Two-coordinate platform & $0.340^{\circ}$ & $.10-50^{\circ}$ & $0.5-10 \mathrm{grad} / \mathrm{s}$ \\
\hline $\begin{array}{l}\text { Computer } \\
\text { Photoreceiver } \\
\text { Analog-digital convertor } \\
\text { Number of gates }\end{array}$ & Syet & $\begin{array}{l}\text { and regls } \\
\text { IBM PC/ } \\
\text { photomu } \\
8 \text { digits; } \\
1024\end{array}$ & \\
\hline
\end{tabular}

The "MAKPEL-2" alrborno lldar measures the horizontal size and spatial variations of the upper boundary of the cloud fieids, the optical depth and the phase composition of clouds, and the statistical parameters describing the vertical geometrical structure of cumulus clouds. The lidar
Is used onboard the "OPTIK-E" AN-30 Alrcratt Laboratory. It can operate over the basic site or fly along the selected routes. The principal parameters of the lider are given in Table 3. 
Tuble 3. Paramotors of the aliborne polarization lldar.

Paramotore of sounding

Sounding diatance

Tomporal resolution

Vertical resolution

Horizontal resolution

Aireratt

Lesor

Lacar

Bd:YAG+frequency

doubling

Telescope diameter, $m$

Focal longth, $m$

Fiold of viow, mrad

Polarization

Analyzer

Detector

Analog-digital converter

Computer
Cloud upper boundary altitude; phase

state of clouds; atmospheric aorosol

$0.2 .7 \mathrm{~km}$

0.18

$1.5 .30 \mathrm{~m}$

$10.100 \mathrm{~m}$

AN-30; IL-18
Tranamitier

\begin{tabular}{|c|c|c|}
\hline$\lambda, \mathrm{nm}$ & $E, \mathrm{~mJ}$ & $\mathrm{t}, \mathrm{Hz}$ \\
\hline $\begin{array}{l}532 \\
(1064)\end{array}$ & $\begin{array}{l}100 \\
60\end{array}$ & $\begin{array}{l}1.15 \\
1.15\end{array}$ \\
\hline
\end{tabular}

Recelver

0.15

0.75

$1-20$

linear

Wollaston priam

Reglatration

Photomultipliers PMT-84, PMT-141, PMT-83

4 channels, $10 \mathrm{~ns}, 7$ digits

Data proceseing onboard the aircraft and

on the ground
The "gTRATOSFERA-1 M" polarization lldar is designed to measure the profiles of the backscattering coefficient, Stokes paramoters, scattering matrix of cirrus clouds, and aorosol and molecular components of the atmosphere (Table 4a). The lidar operates at night. The relative error in measuring the beckscattering coofficient is $15 \%$ to $20 \%$. The scattering matrix elements are measured with an absolute error of \pm 0.04 .
The additional lidar channel (Table 4b) measures the scattoring ratio

$$
R=\frac{\sigma_{m}}{\sigma_{m}+\sigma_{a}}
$$

where $\sigma_{m}$ and $\sigma_{a}$ are the molecular and aerosol beckecattering coefficients, respectively. The lidar aleo measures the temperature and humidity profiles. 
Tablo 4a. Parameters of the "STRATOSFERA-1M" polarization lidar.

Parameters of sounding

Range of sounding heights:

for measuring the backscattering coefficient only for measuring the scattering matrix elements Spatial resolution (depends on the height)

Lasor

Nd:YAG

Antenna diameter, $m$

Detector-photomultiplier

Dark current

Maximum photon counter rate

Computer backscattering coefficients, Stokes parameters; scattering matrices of cirrus clouds; aerosol and molecular atmospheric components

$5-30 \mathrm{~km}$

$5-20 \mathrm{~km}$

24-480 m

Tranamitter

$\lambda, \mathrm{nm}$

$\underline{E, m J}$

$\underline{P, W}$

$\mathrm{f}, \mathrm{Hz}$

532

50

$--$

25

Recelver

0.5

PMT-130

$102 \mathrm{~Hz}$

Registration

$100 \mathrm{MHz}$

IBM PC/AT

Table 4 b. Parameters of the lidar channel of temperature and humidity measurements.

Parameters of sounding

Range of sounding heights

Spatial resolution

(depends on the height)

Laser

Nd:YAG

Antenna diameter, $m$

Focal length, $m$

Field of view, mrad

Spectral width of the

momochromator, $\mathrm{nm}$

Detector-photomultiplier

Dark current

Maximum photon counter rate

Computer

\begin{tabular}{lll} 
Temperature & & Humidity \\
\cline { 1 - 1 } $1.5-10 \mathrm{~km}$ & & $1.5-8 \mathrm{~km}$ \\
$48-480$ & $48-480$
\end{tabular}

Transmitter

Anm EmJ P.W

532

80

14

\section{Scattering ratio \\ $1.5-12 \mathrm{~km}$ \\ 48-480}

Recelver

2.2

10.07

0.4

0.6

PMT-104

$10^{2} \mathrm{~Hz}$

Regletration

$100 \mathrm{~Hz}$

IBM PC/AT 
The Moteorological lidar employs the correlation method to measure the wind parameters and the differential absorption method to measure temperature, humidity, and pressure. The lidar measures the following parameters (Table 5):

- the profiles of wind velocity in the lower troposphere up to the altitudes of 1 to $2 \mathrm{~km}$. The error in measuring the wind speed is $\pm 7 \%$, the error in measuring the wind direction is $\pm 7^{\circ}$

- the profiles of temperature $\left( \pm 5^{\circ}\right)$, humidity, $( \pm 10 \%)$, and pressure ( \pm 7 mbar) up to a height of $1 \mathrm{~km}$
- the height of the lower cloud boundary and the cloud fraction

- the height and the thickness of temperature inversions

- the statistical characteristics of aerosol inhomogeneities.

The Raman lldar measures the profiles of temperature, humidity, and the extinction coefficient in the 0.05-1.5 km height range with a spatial resolution of $0.015 \mathrm{~km}$ (Table 6).

Table 5. Parameters of the meteorological lidar.

Parameters of sounding

Range of sounding heights

Temporal resolution

Spatial resolution

Laser
Nd:YAG
Nd:YAG+frequency doubling
Nd:YAG(532 nm) +A1 ${ }_{2} \mathrm{O}_{3}: \mathrm{Ti}^{3}+$

Telescope diameter, $m$

Telescope focal length, $m$

Telescope viewing field, mrad

Wavelengths, $\mathrm{nm}$

Detector-photomultiplier

Number of gates

Computer wind velocity, temperature,

humidity, aerosol

$1.2 \mathrm{~km}$

3-10 min

$50-200 \mathrm{~m}$

Transmitier

$\lambda, \mathrm{nm}$

1064

532

$725 \pm 3$

\begin{tabular}{l}
$E, \mathrm{~mJ}$ \\
\hline 250 \\
100 \\
20
\end{tabular}

$\begin{array}{lll}P, W & & f, H z \\ 2 & & 5-30 \\ 2 & & 5-30 \\ 0.3 & & 5-30\end{array}$

Recelver

0.3

0.64

1

$1064,532,725 \pm 3$

Registration

PMT-83, PMT-84

128

IBM PC/AT 
Table 6. Parameters of the raman lidar.

Parameters of sounding

Range of sounding heights

Spatial resolution

Laser

$\mathrm{Cu}$

Antenna diameter, $m$

Detector-photomultiplier

Dark current, $\mathrm{Hz}$

Spectral width of the

monochromator, $\mathrm{nm}$

\section{Maximum photon counter rate}

Computer temperature, humidity, extinction coefficient.

$0.05-1.5 \mathrm{~km}$

$15 \mathrm{~m}$

Transmitter

$\lambda, \mathrm{nm}$

$\underline{E, m J}$

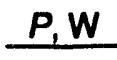

f, Hz

511

2

$5 \cdot 10^{3}$

\section{Recelver \\ 0.4 \\ PMT-104 \\ $10^{2}$ \\ 1.2}

The meteorologlcal acoustlc sensor identifies the stratification type (class of atmospheric stability) and determines the height of the mixing layer, the structural characteristics of the temperature field, the height and thickness of temperature inversions, and the wind velocity up to a height of $1 \mathrm{~km}$.

The sensor's specifications are as follows:

Altitude range
Currier frequency
Electric power
Antenna (diam)
Beam width
Noise-cancelling shield
Height
Pulse repetition period
Pulse duration
Vertical resolution
Recording system
Power supply
Power consumption,
Electronic equipment
Antenna heater

35-300 m

$1650-1850 \mathrm{~Hz}$

$65 \mathrm{~W}$

paraboloid, $1.0 \mathrm{~m}$

$<15^{\circ}$

frustum

$1.55 \mathrm{~m}$

$4.0 \mathrm{~s}$

$150 \mathrm{~ms}$

$25 \mathrm{~m}$

echo sounder, PC

$220 \mathrm{~V}, 50 \mathrm{~Hz}$

$300 \mathrm{~W}$

$1000 \mathrm{~W}$
The "OPTIK-E" AN-30 Aircraft Laboratory is equipped with the instrumentation for acquiring data on the meteorological parameters, gas composition, and aerosol content of the atmosphere at the altitudes of flights, as well as for measuring characteristics of the radiation field and underlying surface. The maximum altitude of flights is $8000 \mathrm{~m}$; the speed of flights varies from 250 to $400 \mathrm{~km} / \mathrm{h}$; the maximum range of flights is about $2400 \mathrm{~km}$; sufficient runway length is $1300 \mathrm{~m}$.

The aircraft carries the following instrumentation:

- meteorological complex

- aerosol complex

- photoelectric counter

- diffusion battery

- nephelometer supplied by thermo- and hydrooptical devices

- filters

- gas analyzing complex 
- "MAKREL-2" lidar

- spectro-photo-radiometric complex

- TV.03 thermal imaging camera

- onboard recording system

- navigation complex.

The instrumentation measures the following parameters:

- air temperature and its fluctuations

- humidity

- pressure

- wind velocity and its fluctuations,

- cloud microphysics

- number density and/or mass concentration of aerosol

- aerosol particle size distribution in the range from 0.005 to $10.0 \mu \mathrm{m}$

- chemical composition of aerosol ions:

- $F$

- $\mathrm{SO}_{4}^{2-}$

- NA+

- $\mathrm{NH}_{4}^{+}$

- $\mathrm{As}^{5+}$

- $\mathrm{K}^{+}$

- $\mathrm{NO}_{3}$

- $\mathrm{Cl}^{-}$

- $\mathrm{Cd}^{2+}$

- elements

- Al

- $M g$

- Si

- $\mathrm{Cr}$

- Ga

- Ba

- Mo

- Ca

- Cu

- Ti

- Sb

- Sn

- B

- Bo

- Mn

- $\mathrm{Ag}$

- Co

- Fo

- $\mathrm{Br}$

- Mo

- W

- Pb

- Ni

- $\mathrm{Hg}$

- V

- Zn

- In

- aerosol scattering coefficient (at the scattering angle of $45^{\circ}$, at $\lambda=0.42,0.52$, and $0.61 \mu \mathrm{m}$ ) and the polarization degree of scattered light at these wavelengths

- content of aerosol volatile components at temperatures $10^{\circ}$ to $400^{\circ} \mathrm{C}$
- gases:

- ammonium

- acetylene

- acetone

- benzine

- benzene

- xylene

- ozone

- nitric oxide

- nitrogen peroxide

- carbon monoxide

- sulfur anhydride

- hydrogen sulfide

- toluol

- carbon dioxide

- extinction coefficient of water (at depths down to $25 \mathrm{~m}$ )

- extinction coefficient of clouds $(\lambda=0.53 \mu \mathrm{m})$

- vertical profile of the aerosol scattering coefficient $(\lambda=$ $0.53 \mu \mathrm{m}$ )

- intensity of upwelling and downwelling radiation in the atmosphere-underlying surface system at wavelengths $\lambda=0.44,0.63,0.67,1.05,1.20,1.60$, and 8 to $15.0 \mu \mathrm{m}$

- upwelling and downwelling fluxes of solar and thermal radiations

- temperature of the underlying surface in the range from $-40^{\circ}$ to $60^{\circ} \mathrm{C}$

- onboard recording system (based on IBMPC/AT with a streamer) records the flight course, the air and actual speed of flight, drift angles, barometric altitude, bank and pitching angles, overload, presence of thunderstorms (radar indicator).

The aircraft-laboratory has been used in the DUNA USAUSSRExperiment (Dushanbe), ODAEX (Odessa), "Vertical 86 and $87^{\prime \prime}$ (Tomsk), and the SATOR Experiment (SUbprogramme of TOREUREKA). In addition, this aircraft laboratory has been used to inspect the air quality over the cities of Alma-Ata, Balkhash, Pavlodar, Ust'-Kamenogorsk, Khabarovsk, Komsomol'sk-on-Amur, Ulan-Udo, Nizhnevartovsk, Nizhnii Tagil, and PetropavlovskKamchatskii.

Otherpossible applications for the aircraft/laboratory include

- investigating the transboundary transfer

- studying air pollution over urban areas with indication of the emission sources 
- measuring water turbidity in the upper $25 \mathrm{~m}$ layer of the coeans

- determining chlorophyll and hydrosol content in water

- detecting oll films on the water surface

- measuring height of plants and their spectral albedo.

The radar will determine the vertical and horizontal cloud size, cloud water amount and phase composition. Its specifications are

$\begin{array}{ll}\text { Wavelength } & 0.8 \text { and } 3.2 \mathrm{~cm} \\ \text { Field of view } & 44^{\prime} \\ \text { Diameter of the recelving antenna } & 3 \mathrm{~m} \\ \text { Pulse duration } & 1 \text { and } 2 \mathrm{~ms} \\ \text { Pulse repetition rate } & 600 \text { and } 30 \mathrm{~Hz} \\ \text { Range of operation } & 300 \mathrm{~km} .\end{array}$

The radlosonde measures the profiles of temperature, humidity, pressure, and wind velocity up to $30 \mathrm{~km}$ with spatial resolution of 50 to $150 \mathrm{~m}$. In addition, special sensors installed on the radiosonde can measure the ozone profiles and upwelling and downwelling fluxes of the longwave radiation.

The standard meteorological parameters (temperature, relative and absolute humidity, wind velocity) are measured at several heights in the 0.5 to $10 \mathrm{~m}$ range. The fluxes of heat, humidity and momentum are determined from data on gradient measurements.

The serosol ground-based complex of instruments measures the particle size distribution function in the range $d=-0.006-10 \mu \mathrm{m}$ and collects the aerosol samples for subsequent chemical analysis. (The list of the elements and ions to be determined is the same as for the aircraft laboratory.)

Optical properties of the aerosol particles are studied by the following devices:

1. The aurecle nephelometer $(\lambda=0.63 \mu \mathrm{m})$ measures the scattering phase function within the angle $20^{\prime}-10^{\circ}$ and simultaneously with the inverse problem solution provides data on the concentration of particles with the size $d=2-20 \mathrm{~mm}$.

2. The nepholomoter with local scattering volume measures the scattering coefficient at three wavelongths in the visible spectral range $(\lambda=0.42,0.52$, and $0.62 \mu \mathrm{m})$. Thermo- and hydro-optical devices provide for the measurement of the aerosol parameter of condensation activity $\lambda$ and estimation of the volatile components in the aerosol composition (by heating up to $300^{\circ} \mathrm{C}$ ). If necessary, the local volume nephelometer can be additionally equipped with a device for estimating the soot content in aerosol particles.

3. The multwave measurer of transparency determines atmospheric transmission at extended horizontal paths in 22 spectral intervals in the range $\lambda=0.44-12 \mu \mathrm{m}$. Solution of the inverse problem makes it possible to estimate the size distribution function of the aerosol particles in the range $d=0.1-10 \mu \mathrm{m}$, and statistical correction of the data makes it possible to determine the extinction caused by the continuous absorption by water vapor in the transparency window at $\lambda=8-12 \mu \mathrm{m}$.

4. The acrosed complex (analogous to the one for the airborne laboratory) is also used to determine the gaseous composition. The complex includes chromatographic and hemiluminscent devices for determining the content of greenhouse gases and of the ozone cycle gases (see the description of the gas measured by the aircraft laboratory).

5. The all-eky camera system is used to study the geometrical structure of the cloud fields.

\section{Radiation Measurements}

The complex of standard dovices will measure the integral and broadband fluxes of the incident, scattered, and total solar radiation, as well as the integral and broadband fluxes of the longwave radiation at the ground surface.

The solar spectral photometer is designed for measuring the radiation intensity in the sun's direction and for determining the aerosol spectral optical thickness (in the transparency windows $\lambda=0.3-12 \mu \mathrm{m}$ ) and the total content of some greenhouse gases $\left(\mathrm{H}_{2} \mathrm{O}, \mathrm{CO}_{2}, \mathrm{CO}, \mathrm{O}_{3}, \mathrm{CH}_{4}, \mathrm{~N}_{2} \mathrm{O}\right.$, CFC) by the solar spectrophotometric method with low resolution $(\Delta \lambda=10-40 \mu \mathrm{m})$.

Specifications for the photometer are

- angular resolution $0.3^{\circ}$

- wavelength range 0.3-12 $\mathrm{mm}$ 
- the number of the spectral channels optical depth rel. units

- error in following the sun $\pm 0.1^{\circ}$

- time of recording the individual spectrum

- data storage on IBM PCIAT.

The stellar apectral photometers with analogous functions is used instead of the solar photometer at night.

The scanning filter spectrophotometer of the daytime sky measures the spatial-angular structure of the sky and underlying surface brightness in the wavelength range 0.4-1.06 $\mu \mathrm{m}$. Scanning is controlled by a special program. The instrument's specifications are

- angular resolution

$1^{\circ}$

- the number of the spectral channels (filters) 6

- error in photometering $1-3 \%$

- scanning range of the elevation angle $\pm 90^{\circ}$ of the azimuth $\pm 180^{\circ}$

- wavelength resolution $5-20 \mathrm{~nm}$

- time recording the individual $0.1-1 \mathrm{~s}$

- data storage on IBM PC/AT.

The filters can be installed centered on the absorption bands of atmospheric gases.

The scanning fitter radlometer measures the spatialangular structure of brightness (radiative temperature) of the sky, clouds and the surface in the transparency windows at $\lambda=4-12 \mu \mathrm{m}$. Scanning is controlled by a special program. Specifications for the radiometer are

- angular resolution

$0.5-1^{\circ}$

- number of spectral channels (filters)

6

- error in absolute measuring the radiative temperature

$0.1^{\circ}$
- scanning range of the elevation angle $\pm 90^{\circ}$ of the azimuth $\pm 180^{\circ}$

- wavelength resolution

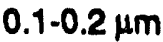

- radiative temperature resolution

$$
(\lambda=10 \mu \mathrm{m})
$$

The filters can be matched to the absomption bands of atmospheric gases.

The epectrophotometer recording the weak fluxes of the atmospheric radiation is designed to measure the cloud optical depth. Its specifications are

- field of view

$20^{\prime}$

- the number of the spectral channels

5

- wavelengths

$0.63 \mu \mathrm{m}, 0.91 \mu \mathrm{m}, 3.7 \mu \mathrm{m}$, $10.8 \mu \mathrm{m}, 12 \mu \mathrm{m}$

- wavelength resolution: in the range 0.63-0.91 $\mu \mathrm{m} \quad 0.1 \mu \mathrm{m}$ in the range 3.7-12 $\mu \mathrm{m} \quad 0.2 \mu \mathrm{m}$

The photometer is calibrated in absolute units and is compatible with any kind of computers.

At present, monitoring atmospheric parameters and radiation fields to solve the problems of global nature and climate change is one of the principal scientific directions of the institute of Atmospheric Optics. The geophysically significant series of observations of some atmospheric parameters (for example, aerosol optical and microphysical parameters, including the antropogeneous aerosol, ozone, etc.) have been obtained by means of the instrumentation complex described above.

These observational series can be used to improve the available regional radiation models and to create new models. The latter is especially important for modeling regional climatic change. The available instrumentation complex is permanently modernized, as are the methods of retrieving the atmospheric parameters from the observational data. However, the Institute of Atmospheric Optics cannot carry out the radiative experiment without financial support. 


\section{Radiometry}




\title{
Technical Progress in the Atmospheric Radiation Measurement Instrument Development Program
}

\author{
J. W. Griffin, Technical Monitor \\ ARM Instrument Development Program \\ Pacific Northwest Laboratory \\ Richland, Washington
}

\section{Goals of the Instrument Development Program}

The primary goal of the Atmospheric Radiation Moasurement (ARM) Instrument Development Program (IDP) is to develop fieldable atmospheric sensing systems which 1) provide a needed atmospheric observation/ measurement requisite to the success of the ARMProgram and 2) are adaptable for sustained deployment at one or more of the ARM Cloud and Radiation Testbeds (CART). In this sense, the ARM IDP more closely resembles an engineering development activity than a basic sensor system research and development effort. In fact, instruments selected for IDP funding support were purposely chosen for 1) their relatively high degree of maturity and 2) the likelihood that they could actually bo fabricated, installed, and maintained at one of the ARM CART sites at a reasonable cost. Inherent in the second criterion is the requirement that the instrument will be operable and maintainable by CART Site Operations staff following a minimal amount of training (i.e., "principal investigator instruments and/or those requiring a covey of graduate students for operation are excluded from consideration). Without exception, instrument projects that were terminated before completion of the first 3-year funding cycle did not demonstrate the potential to meet the "fieldability" criteria within the tirst two years.

\section{Summary of Projects}

A summary of ARM IDP project status including project titles, principal investigators, institutions, and current instrument status appears in Table 1. Projects that have been terminated or had their work scope modified before the end of FY93 are noted. Fiscal Year 1993 is the third and final year of the initial (3-year) funding cycle for ARMfunded instrument development projects. That is, IDP principal investigators will be required to submit a new proposal in order to be considered for funding beyond September 30, 1993. As for the first funding cycle, continuation proposals will be peer-reviewed and funding awarded on a competitive basis.

\section{Deployment of IDP Instruments}

Over the past 30 months, six IDP-developed instruments have emerged as likely near-term contributors to the ARM CART instrument suite. These include the Multi-Filter Rotating Shadowband Radiometer (MFRSR), the Atmospheric Emitted Radianco Interferometer (AERI), the Polarization Diversity Lidar (PDL), the Raman Water Vepor Lidar (RWVL), the Micro-Pulse Lidar (MPL), and the Cloud Profiling Radar System (CPRS). Status of these instruments is summarized bolow.

\section{MFRSR}

The first MFRSR prototype was deployed at the Southern Great Plains (SGP) CART in June 1992. A brief description of this instrument, its measurement capabilities, and representative data are summarized later in this document

\author{
AERI \\ Plans are currently being made to deploy and test the first \\ AERI prototype (designated AERIO0) at the SGP CART in
}




\begin{tabular}{|c|c|c|c|}
\hline IDP Project Title & Principal Investigator(s) & Institution(s) & 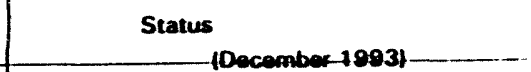 \\
\hline $\begin{array}{l}\text { "Uevelopment of Rotating Shadowband Spectral Radiometer in } \\
\text { Support of the ARM Program" (MFASR) }\end{array}$ & Michalsky/Harrison & SUNY Albany & Presently deployed at SGP CART \\
\hline $\begin{array}{l}\text { "High Spectral Resolution Measurements for the ARM } \\
\text { Program" (AERII, AERI-X, and SORTI) }\end{array}$ & Revercomb/Murcray & $\begin{array}{l}\text { Univ of Wisconsin and Univ of } \\
\text { Denver }\end{array}$ & $\begin{array}{l}\text { AERIOO and SORTI prototypes presently } \\
\text { deployed at SGP CART }\end{array}$ \\
\hline -Micro-Pulse Lidar * (MPL) & Spinhirne & NASA Goddard & Prototype presently deployed at SGP CART \\
\hline $\begin{array}{l}\text { "Laser Remote Sensing of Water Vapor" } \\
\text { (also denoted Raman Water Vapor Lidar or RWVLI }\end{array}$ & Goldsmith/Melfi & $\begin{array}{l}\text { Sandia National Laboratory (SNL) } \\
\text { and NASA Goddard }\end{array}$ & $\begin{array}{l}\text { Hybrid bidar design and fabrication presently in } \\
\text { progress. Delivery of system to SGP CART } \\
\text { anticipated in Fall, } 1994\end{array}$ \\
\hline "Polarization Diversity Lidar" (PDL) & Sassen & University of Utah & $\begin{array}{l}\text { Prototype PDL presently operational at Univ of } \\
\text { Utah }\end{array}$ \\
\hline $\begin{array}{l}\text { "Ground-Based Millimeter-Wave Cloud Profiling Radar System" } \\
\text { (CPRS) }\end{array}$ & Mcintosh & University of Massachusetts & $\begin{array}{l}\text { Prototype CPAS tested at SGP CART in hudy } \\
\text { 1993. Prototype is currenty operationat at } \\
\text { Univ of Mass. }\end{array}$ \\
\hline $\begin{array}{l}\text { "Development of a Short-Wave infrared Spectral Radiometer } \\
\text { for the ARM Program" }\end{array}$ & Murcray & University of Denver & Dovelopment in progress at Univ of Denver \\
\hline $\begin{array}{l}\text { "Passive Cloud Dynamics Measurement - A Flow Field } \\
\text { Registration Approach" }\end{array}$ & Thorne and Kegelmeyer & Sandia National Laboratory (SNL) & $\begin{array}{l}\text { Some cloud image enarysis products to be } \\
\text { implemented at SGP CART using Scripps } \\
\text { Whole-Sky-tmagers }\end{array}$ \\
\hline $\begin{array}{l}\text { "Tethered Balloon Sounding System for Vertical Radiation } \\
\text { Profolles" }\end{array}$ & Whiteman & $\begin{array}{l}\text { Pacific Northwest Laboratory } \\
\text { (PNA1) }\end{array}$ & Prototype presenty undergoing testing at PNIL \\
\hline $\begin{array}{l}\text { 11 "Widely-Deployable Low-Cost Radiometer for the ARM } \\
\text { Extended Observing Stations" and } \\
\text { 2) "Pounting Shortwave / Near-Infrared Radiometer for ARM" }\end{array}$ & Kryter and Simpson & $\begin{array}{l}\text { Oak Ridge National Laboratory } \\
\text { (ORNL) }\end{array}$ & $\begin{array}{l}\text { 1) Project redirected during FY92 to topic (2) } \\
\text { 2) Instrument presently resides at ORML (less } \\
\text { tracker) }\end{array}$ \\
\hline 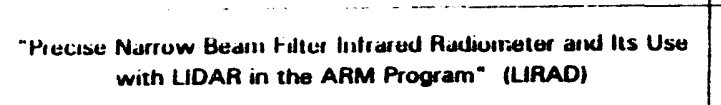 & Platt & $\begin{array}{l}\text { CSIRO Division of Atmospheric } \\
\text { Reseurch Meltourne. } \\
\text { Austratia }\end{array}$ & $\begin{array}{l}\text { Radioaneter dumonstrated in Tropical Wusturn } \\
\text { Pacific dering TOGA COARE }\end{array}$ \\
\hline
\end{tabular}


Table 1. (contd)

\begin{tabular}{|c|c|c|c|}
\hline $\begin{array}{c}\text { "Development of an Integrated Data Assimilation and Sounding } \\
\text { System" }\end{array}$ & Dabberdt / Gage and Westwater & $\begin{array}{l}\text { NCAR Applied Tochnology } \\
\text { Laboratory and NOAA Wave } \\
\text { Propagation (WPL) and } \\
\text { Aeronomy (AL) Laboratories }\end{array}$ & $\begin{array}{l}\text { Integrated Sounding Systems (ISS) deployed } \\
\text { and tested in Tropical Westeen Pacific during } \\
\text { TOGA COARE. Non-hydrostatic version of } \\
\text { four-dimensional data assimilation code ready } \\
\text { for deployment at SGP CART }\end{array}$ \\
\hline $\begin{array}{l}\text { 1) "Plotometric Studies of Clouds from an ARM Site" and } \\
\text { 2) "Aerosol Characterization Using Stellar Imagery" } \\
\text { "Spectral Inversion of Solar Radiation Measurements to } \\
\text { Recover High-Resolution Spectral information, Constituent } \\
\text { Concentrations, and Particle Sizes }\end{array}$ & $\begin{array}{c}\text { Buchwold (11) and Weber (2) } \\
\text { Sprattin }\end{array}$ & $\begin{array}{l}\text { Los Alamos National Laboratory } \\
\text { (LANU) } \\
\text { Oak Ridge National Laboratory } \\
\text { (ORNL) }\end{array}$ & $\begin{array}{l}\text { 1) Terminated at the end of FY92 } \\
\text { 2) Re-scoped project topic } \\
\text { Work performed in collaboration with Harrison } \\
\text { at SUNY Albany to support analysis of MFASR } \\
\text { data }\end{array}$ \\
\hline $\begin{array}{l}\text { "Development of High Spectral Resolution LIDAR Technology } \\
\text { (or the DOE ARM Program" (HSRL) }\end{array}$ & Eloranta & University of Wisconsin & Project terminated at end of FY92 \\
\hline $\begin{array}{l}\text { "Develop a Radiation Measurement System (RAMS) for the } \\
\text { Atmospheric Radiation Measurement Program (ARM)" }\end{array}$ & Valero & NASA Ames & Project terminated at and of FY92 \\
\hline
\end{tabular}


March 1893. A briff description of this instrument, its mossurement capabilitios, and representative data are eummarized later in thle document.

\section{PDL and RWVL}

The deslons for theee two instruments, scheduled for field domonstratione in 1983, will likely be combined to form an ARM "hybrid Ildar declon" capable of measuring/detecting aerosol extinction/backscatter coefficients, cloud boundaries, cloud phase, and vertical water vapor profiles. Pending succeseful completion of these two IDP projects in FY93, design and procurement of the ARM hybrid lidar is anticipated early in FY94. A brief description of the RWVL instrument and its measurement capabilities are summarized later in this document.

\section{MPL}

This instrument development effort, funded for two years beginning in FY92, will likely result in an eye-safe, fieldable laser cellemeter capable of detecting thin cirrus cloud layers at high altitudes. Such measuremente are not currently possible with commercially-available laser collometers. Initial testing of the MPL will occur during the Tropical Ocean Global Atmosphere/Coupled Ocoan Atmosphere Response Experiment (TOGACOARE) fild campalgn in the Tropical Western Pacific during the firstquarter of 1983. Assuming initlal MPL fiold pertormancs is satiofactory, tosting at the ARM SGP CART is anticipated carly in FY94.

\section{CPRS}

This instrument is scheduled for initial field testing af the SGP CART in the summer of 1993. Because of the anticipatod high capital cost of this remote sensing system, the CPAS is expected (pending successful field trials) to be deployed at the SGP CART only during intentive obeervation periods. A brief description of this instrument, its measurement capabilities, and representathe data are summarized later in this document. 


\title{
High Spectral Resolution Fourler Transform Infrared Instruments for the Atmospheric Radiation Measurement Program
}

\author{
H. E. Rovercomb, W. L. Smith, R. O. Knuteson, F. A. Best, \\ R. G. Dedecker, T. P. Dirkx, R. A. Herbeleb, and J. F. Short \\ Univarsity of Wisconsin \\ Madison, Wisconsin \\ H. B. Howell \\ National Ocoanic and Atmospheric Administration \\ Systems Design and Applications Branch \\ Madison, Wisconsin \\ D. Murcray and F. Murcray \\ University of Denver \\ Denver, Colorado
}

\begin{abstract}
Accurate and spectrally detailed observations of the thermal omiseion from radiatively important atmospheric gases, aerosols, and clouds have been identified as crucial for realizing the overall objectives of the Atmospheric Radiation Moasurement (ARM) Program to improve the treatment of radiation and clouds in climate models. The observed spectra will be used for many diverse functions, including identifying and eliminating absolute orrors in calculated spectra for known atmospherlc stutes; evaluating and improving cloud radiation calculations; characterizing the distribution and evolution of effective cloud radiative properties; and studying the state parameter changes associated with cloud formation, evolution, and dissipation.
\end{abstract}

The deployment of several high spectral resolution instruments at each of the geographically distributed ARM Cloud and Radiation Testing (CART) sites will characterize climatologically diverse conditions, including the temporal and spatial properties of atmospheric weather systems. As part of the Instrument Development Program (IDP), the University of Wisconsin (UW) and the University of Denver (UD) are developing three types of Fourier transform infrared (FTIR) instruments: the Atmospheric Emitted Radlance Interferometer (AERI), the AERI-X and the Solar Radiance Transmission Interferometer. The spectral characteristics of these instruments are defined in Table 1.

The AERI prototype, bullt by the UW, will be deployed at the Southern Great Plains CART site in March and is the primary subject of this paper. Operational versions of the AERI will be installed both at the central site and at four extended boundary locations to sample horizontal inhomogeneities for radiometric studies and for remote sensing. The ultra-high-resolution Solar Radiance Transmission Interferometer (SORTI) (built by the UD) is operating successfully at UD and will be tested at a CART this summer. The radiometric performance of the $0.1 \mathrm{~cm}^{-1}$ AERI-X interferometer subsystem being developed by the UD will be demonstrated this year. The complete AERI-X system will make use of the AERI calibration, operational control, and analysis subsystems. More detalls on the SORTI and the AERI- $X$ will be provided at a later date.

\section{AERI Measurement Characteristics}

The spectral measurement characteristics of the AERI are illustrated in Figure 1 by the sample clear sky spectra collected during the DOE Spectral Radiance Experiment (SPECTRE) conducted in Coffeyville, Kansas, in the fall of 1991.

When the sirst operational version of AERI is installed at a CART this ear, spectra of this type will soon be collected 
Table 1. System Configurations Summary.

\begin{tabular}{|c|c|c|c|}
\hline & AERI & AERI-X & SORTI \\
\hline Function & $\begin{array}{l}\text { Zenith Vlewing } \\
\text { Atmospheric Emission }\end{array}$ & $\begin{array}{l}\text { Extra High-Rusolution } \\
\text { Zenith Viewing } \\
\text { Atmospheric Emission }\end{array}$ & $\begin{array}{l}\text { Ultra-High-Resolution } \\
\text { by Solar Tracking for } \\
\text { Atmospheric Transmission }\end{array}$ \\
\hline Spectral Coverage & $3.19 \mu \mathrm{m}$ & $7.14 \mu \mathrm{m}$ & $3.13 \mu \mathrm{m}$ \\
\hline Spectral Resolution & $1 \mathrm{~cm}^{-1}$ & $0.1 \mathrm{~cm}^{-1}$ & $0.003 \mathrm{~cm}^{-1}$ \\
\hline Calibration Sourcos & $\begin{array}{l}\text { 2-3 high omissivity } \\
\text { blackbodies }\end{array}$ & $\begin{array}{l}2 \text { high omiselvity } \\
\text { blackbodies }\end{array}$ & $\begin{array}{l}\text { Uses airmass variation, } \\
\text { to derive transmission }\end{array}$ \\
\hline
\end{tabular}
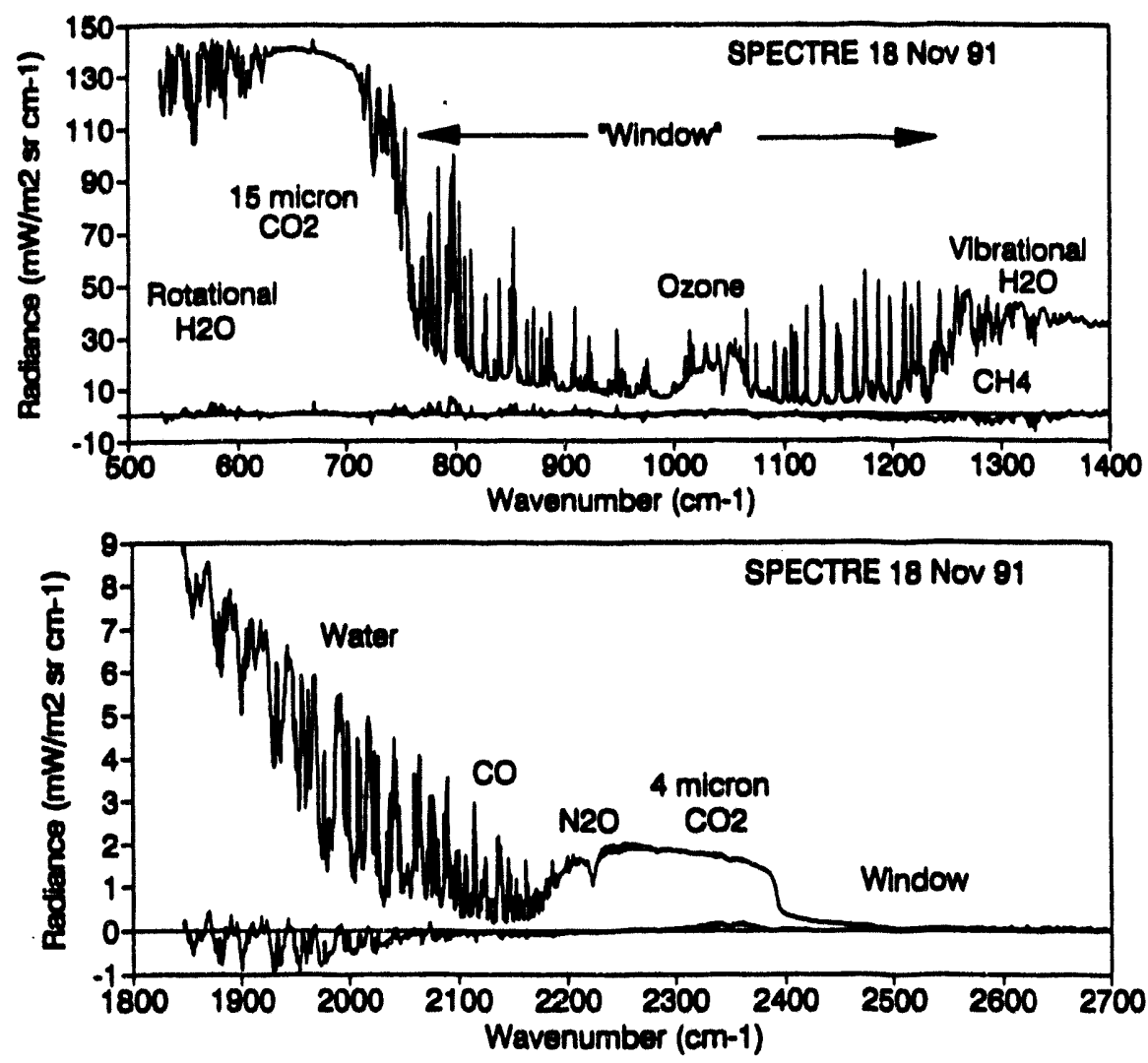

Figure 1. Sample spectra from the AERI longwave and shortwave bands. The difference of the measurements from spectra calculated with the Air Force Phillips Lab line-by-line program (FASCOD3) is also shown. 
overy 10 minutes, 24 hours a day. The spectral coverage is esesentially continuous from 3 to 19 microns, although reglone with little spectral intorest, such as the opeque portions of the 6.3 micron water band, are not shown. The epectra shown are apodked to a reselution of $1 \mathrm{~cm}^{-1}$, high onough to generally separate foatures caused by broed continua or cloud rofisctance from those of abeorption lines. In addition to sky radiances, the AERI instrument generates spectra of the standard deviation during the 4-minute sky viow, which allow the stability of the sky to bo asecesed. To eatisty the requirements for verifying rediative transtor modela and for remoto sensing, the AERI incorporabestab-of-the-art rediometric pertormance. The absolute calbration accuracy is botter than $1 \%$ of the ambiont radiance, and the callbration reproduclbility and noles levels are bes than a fow tenths of a percont (Revercomb ot al. 1993).

\section{AERI System Design}

The AERl instrument is an advanced version of the "Baby HIS" (high spectral resolution intorferometor sounder), a prototype dosigned and fabricated at UW in 1989 as part of the HIS Program (Smith ot al. 1987/89; Rovercomb ot al. 1888a). AERI employs a commercially avallable interforometer (Michelson Sories MB100 from Bomem, Inc. of Quebec, Canada), with cornercube Michelson mirrors mounted on a common rocking arm supported by flex pivots. It has proven to be very rugged and dependable. The interferometor data are digitized in the MB100 module, transferred to an IBM personal computer in the electronics module. Hore it is Fourier transformed by a DSP card and stored, then linked to another more powertul IBM personal computer (AERI Front End Processor or AERI-FEP) for data analysis, product generation, quality control, and ARM network tranaters.

A pair of detectors in a "sandwich" configuration is used to give the broad epectral coverage desired without the need for two cooling systems. The sandwich configuration consists of a shortwave InSb detector stacked in front of a photoconductive $\mathrm{HgCdTe}$ detector, which views the longwave by transmiselon through the InSb. Currently, the detectors are cooled to $77 \mathrm{~K}$ using a dewar equipped with a liquid nitrogen (- $\mathrm{N}_{2}$ ) auto-fill system doveloped during the IDP. In the future, we plan to incorporate a mechanical Stirling cooler to eliminate the need for routine use of $\mathrm{LN}_{2}$.
The AERI radiometric calibration subeystom uses a now approsch developed under the ARM program IDP. To avold the problems associated with operating cold reforencese in humid environmente, one hot reference source $\left(60^{\circ} \mathrm{C}\right)$ and one ambient reference source are used instead of the amblent and liquild nitrogen sourcas used in earliorHIS ground-besed work. The hovamblent approsch also has the advantage of greatly simplifying oporational requirement in many environmente whore providing lares amciunts of liquid nitrogen ls difficult. The new approsch was verlified by thorough testing, in which rediance epectra callbrated with both hot/ambient and cold/amblent calbrations were intercompared. The calibration schome involves periodle cycling through 2-minute viows of the reference blackbodies and 4-minute zenith sky views to yield a calibrated sky spectrum approximatoly overy 10 minutes.

The blackbody reference sources are high emlasivity cavities (about 0.995 ) carefully designed, fabricated, and teoted at the UW to provide the extremely woll-characterized sources required for the hotamblent calibration (Beot ot al. 1992). The hot and ambient sources are of identical design, although the temperature of the hot source is controlled to $60^{\circ} \mathrm{C}$, while the ambient source is allowed to float. Cavity temperature monitors have been carefully callbrated and reforenced to National Institute for Standarda and Technology (NIST) standards using a Guideline digital Platinum Resistance Thermometer. Thermal models show that the temperature gradients in the hot reference cavity are less than $0.3^{\circ} \mathrm{C}$.

Extensive real-time analysis is performed in the AERIFEP, ylelding accurately callbratod radiances on a standard wavenumber scale (to simplify spectral calculations when more than one AERI is being used). The baslc analysls functions include longwave channel nonlinearity correction, complex radiometric calibration, finite field of viow correction, and wavelength calibration and scale standardization. The radiometric and wavelength callibration implement techniques developed under the HIS program at UW (Revercomb ot al. 1987/89; 1988b; 1989a) have been verified under the IDP by intercomparison tests with the HIS aircraft instrument. The technique used to correct for HgCdTodetectornonlinearity is new and was developed during the IDP. In addition, a technique for accurately defining the spectral resolution function from self. apodization was also developed. 
Because of the operational aspects of the CART deployment, a major effort has been devoted to developments required for automated operations. Significant sottware development has been performed to accomplish reliable operation:

- analysis computer resource allocation

- ARM notwork interface

- intra-AERI notwork for data transfer to AERI-FEP

- power failure recovery sottware

- daily reset for data set management

- AERI status interrogation

- QC displays of spectra and housekeeping

- remote modification of operating configuration

- $\mathrm{LN}_{2}$ auto-fill system for detector dewar

Relatively minor modifications will make most of this software directly applicable to support the task of making the AERI-X operational, as proposed here.

\section{AERI Field Experience}

The AERI prototype has been extensively tested in the field. It operated very successfully in three campaigns, including the Spectral Radiance Experiment (SPECTRE) at Coffeyvilie, Kansas, conducted in conjunction with the
National Aeronautics and Space Administration's First ISCPP Regional Experiment cirrus cloud study (11 November - 7 December 1991); the joint agency STORM Fronte Experiment Systems Test (STORM-FEST) program at the boundary layer site near Seneca, Kansas, (1 February - 15 March 1992); and aboard the research ship Point Sur off Monterey, California, for Nayy-sponsored atmospheric refractivity observations (8-11 May 1992). We present a small sampling of results to illustrate the high quality of the AERI data for ARM Program applications.

An example of cloudy brightness temperature spectra from SPECTRE is shown in Figure 2. As found with HIS aircraft observations, clouds often do not behave as pure "blackbodies" for which the brightness temperature would be constant in the regions between absorption lines in the atmospheric window between 8 and 13 microns (770. $1250 \mathrm{~cm}^{-1}$ ). The low cloud spectrum in the figure is close to that of a pure blackbody cloud, but the middle cloud shows major derivations from that simple behavior. The deviations from blackbody behavior are being used to derive cloud microphysical properties (Smith et al. 1993).

Examples of differences between observed and calculated spectra are shown for selected water vapor features in Figure 3. These are both regions where verification for the long paths and variable temperatures of the actual atmosphere is important.

The two STORM-FEST spectra on the top section of Figure 3 are in excellent agreement with calculations for the weak lines from 1100 to $1225 \mathrm{~cm}^{-1}$, a region which

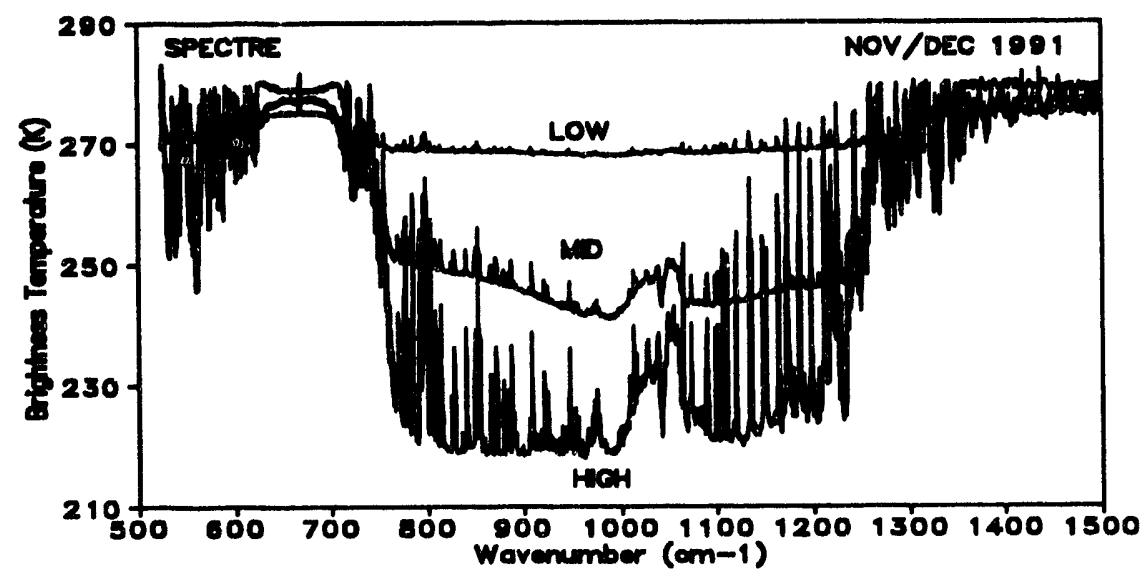

Figure 2. AERI measurements of the downwelling spectra of clouds showing deviations from pure blackbody emission. 


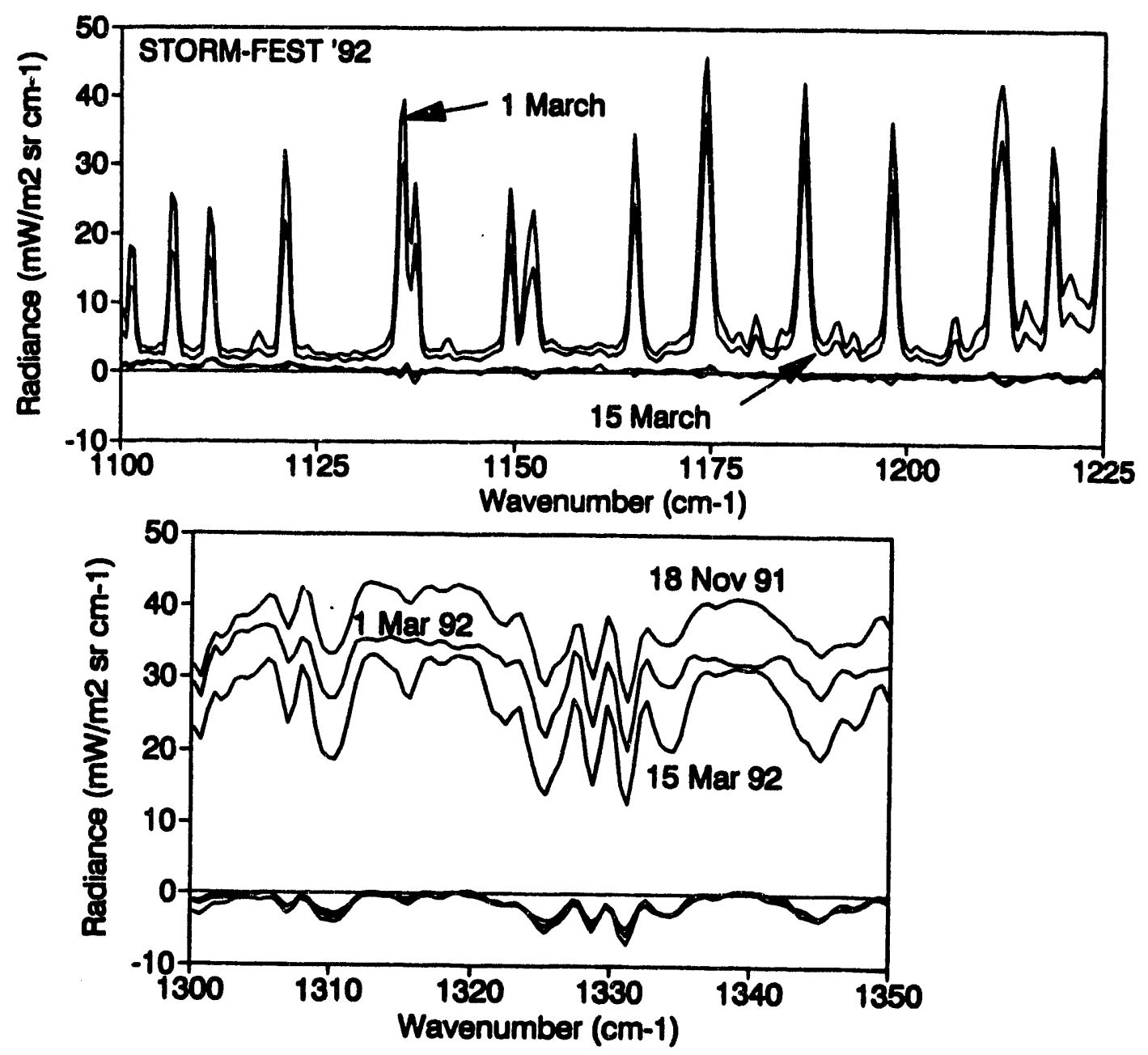

Figure 3. Examples from STORM-FEST of water vapor spectroscopy for which recent improvements have been made (upper) and for which improvements are still needed (lower). The curves plotted about the zero line are differences between AERI observations and FASCODE calculations.

consistently showed large differences before the recent update of the HITRAN line parameter database (Revercomb et al. 1989b; 1990; 1991). The bottom section of Figure 3 illustrates substantial, consistent differences in a region where the water vapor continuum from foreign broadening needs substantial improvement (Revercomb et al. 1989b). The region around $2000 \mathrm{~cm}^{-1}$, which shows large differences between AERI and FASCOD3, is also believed to be due to deficiencies in the water continuum (Knuteson et al. 1993; Theriault et al. 1991).
The influence of atmospheric temperature structure on the downwelling radiance spectrum is illustrated in Figure 4, which shows a close-up of the 15 micron $\mathrm{CO}_{2}$ band. The positive curvature of the general radiance trend between 625 and $710 \mathrm{~cm}^{-1}$ is indicative of a tenperature inversion in the atmospheric boundary layer, as was present on 1 March 1992 during this observation. The negative curvature of the same region for the 15 March spectrum shows that the temperature decreased with altitude in the boundary layer. The excellent correspondence of the detailed features of 


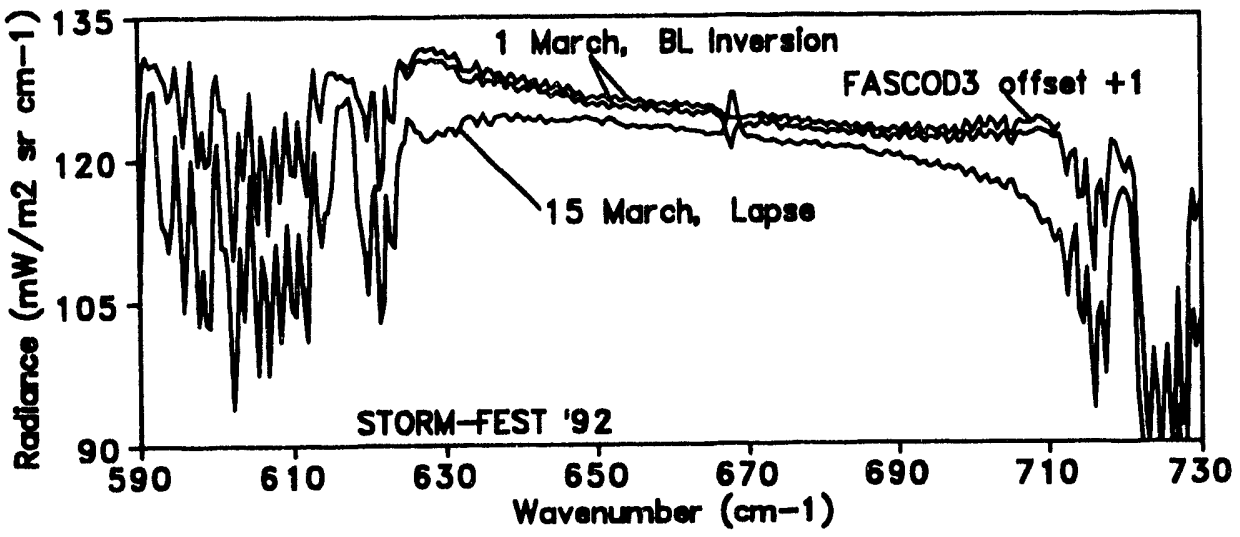

Figure 4. Spectra illustrating the strong sensitivity of high resolution downwelling radiances to the temperature lapse rate.

the calculated and observed spectra also shows that the individual $\mathrm{CO}_{2}$ lines contribute toward characterizing the vertical temperature structure.

\section{Summary}

Major accomplishments of the AERI IDP effort have been to 1) develop and extensively test a new radiometric calibration subsystem with improved accuracy and robustness; 2 ) interact with Bomem, Inc., leading to the development of a two-channel interferometer with the required software characteristics; 3) develop new operational control software and network interfaces; 4) develop new analysis techniques to handle the complete calibration, including a detector nonlinearity correction, wavelength scale standardization, and a finite field-ofview correction; 5) integrate the required hardware, operational control software, and analysis software into a complete system which interfaces to the CART data system and operates remotely without the attendance of expert operators; and 6) perform extensive field testing of the AERI system prototype.

The AERI prototype is operating at the Southern Great Plains CART site, and the first operational AERI has had extensive field testing in preparation for deployment at CART. We expect the SORTI and the AERI-X to follow the same implementation scheme, with prototype testing at CART followed by installation of an operational version. The AERI-X transition to operations should be rapid, because it will make use of systems for real-time processing and automatic operations developed for the AERI.

\section{References}

Best, F. A., H. E. Revercomb, R. O. Knuteson. 1992. Calibration Issues for the Atmospheric Emitted Radiance Interferometer (AERI). Utah State University, Logan, Utah, September 14-16, 1992.

Knuteson, R. O., H. E. Revercomb, W. L. Smith. 1993. Fonward Model Comparisons with the High-resolution Interferometer Sounder (HIS). Proceedings of the Optical Remote Sensing of the Atmosphere Sixth Topical Meeting, Salt Lake City, Utah, March 8-12, 1993. Optical Society of America, Washington, D.C.

Revercomb, H. E., H. Buijs, H. B. Howell, R. O. Knuteson, D. D. LaPorte, W. L. Smith, L. A. Sromovsky, and H. W. Woolf. 1987/1989. Radiometric Calibration of IR Interferometers: Experience from the High-resolution Interferometer Sounder (HIS) Aircraft Instrument. RSRM '87: Advances in Remote Sensing Retrieval Methoots, eds., A. Deepak, H. Fleming, J. Theon. A. Deepak Publishing, Hampton, Virginia. 
Revercomb, H. E., D. D. LaPorte, W. L. Smith, H. Buijs, D. G. Murcray, F. J. Murcray, and L. A. Sromovsky. 1988 a. High-Altitude Aircraft Measurements of Upwelling IR Radiance:Prelude to FTIR from Geosynchronous Satellite. Mikrochimica Acta [Wien] 11:439-444.

Revercomb, H. E., H. Buijs, H. B. Howell, D. D. LaPorte, W. L. Smith, and L. A. Sromovsky. 1988b. Radiometric Calibration of IR Fourier Transform Spectrometers: Solution to a Problem with the High Resolution Interferometer Sounder. Appl. Opt. 27:3210-3218.

Revercomb, H. E., W. L. Smith, L. A. Sromovsky, R. O. Knuteson, H. Buijs, D. D. LaPorte, and H. B. Howell. 1989a. Radiometrically Accurate FTS for Atmospheric Emission Observations. Proceedings 7th International Conference on Fourier Transform Spectroscopy, ed., D. G. Cameron. SPIE Vol 1145.

Revercomb, H. E., W. L. Smith, R. O. Knuteson, H. M. Woolf, and H. B. Howell. 1989b. Comparisons of FASCODE Spectra with HIS Observations. Proceedings of the 12th Annual Reviow Conference on Atmospheric Transmission Models, 5-7 June, eds., E. P. Shettle and F. X. Kneizys, Optical/Infrared Technology Division, Geophysical Laboratory, Hanscom Air Force Base, Massachusetts.

Revercomb, H. E., R. O. Knuteson, W. L. Smith, H. M. Woolf, and H. B. Howell. 1990. Spectroscopic Inferences from HIS Measurements of Atmospheric Thermal Emission. Optical Remote Sensing of the Atmosphere, 1990 Technical Digest Series, Vol 4. Optical Society of America, Washington, D.C.

Revercomb, H. E., R. O. Knuteson, W. L. Smith. 1991. High-resolution Spectral Measurements of Upwelling and Downwelling Atmospheric Infrared Emission with Michelson
Interferometers. Proceedings of the 14th Annual Review Conference on Atmospheric Transmission Models, eds., L.W.Abreu and F. X. Kneizys, Phillips Laboratory, Hanscom Air Force Base, Massachusetts. Report \#PL-TR-92-2059 SR, No. 267. Hanscom Air Force Base, June 11-12, 1991.

Revercomb, H. E., F. A. Best, R. G. Dedecker, T. P. Dirkx, R. A. Herbsleb, R. O. Knuteson, J. F. Short, and W. L. Smith. 1993. Atmospheric Emitted Radiance Interferometer (AERI) for ARM. Fourth Symposium on Global Change Studies. 73rd Annual Meeting of the American Meteorological Society, Anaheim, California, Jan 17-22, 1993. American Meteorological Society, Boston, Massachusetts.

Smith, W. L., H. M. Woolf, H. B. Howell, H.-L. Huang, and H.E. Revercomb. 1987/1989. The Simultaneous Retrieval of Atmospheric Temperature and Water Vapor Profiles Application to Measurements with the High-resolution Interferometer Sounder (HIS). RSRM '87: Advances in Remote Sensing Retrieval Methods, eds., A. Deepak, H. Fleming, J. Theon. A. Deepak Publishing, Hampton, Virginia.

Smith, W. L., X. L. Ma, S. A. Ackerman, H. E. Revercomb, and R. O. Knuteson. 1993. Remote Sensing Cloud Properties from High Spectral Resolution Infrared Observations. J. Appl. Meleorol., in press.

Theriult, J.-M., H. E. Revercomb, R. O. Knuteson, and H.-L. Huang. 1991. Intercomparison of FIT and HIS spectral measurements with FASCODE calculations in the 7-11 $\mu \mathrm{m}$ region. Optical Remote Sensing of the Atmosphere, Williamsburg, Virginia, November, 1991. Optical Society of America, Washington, D.C. 


\title{
Development of Rotating Shadowband Spectral Radiometers and General Circulation Model Radiation Code Test Data Sets in Support of the Atmospheric Radiation Measurement Program
}

\author{
J. Michalsky, L. Harrison, \\ M. Beik, W. Berkheiser III, and J. Schlemmer \\ Atmospheric Sciences Research Center \\ University at Albany \\ State University of New York \\ Albany, NY 12205
}

\section{Introduction}

This program focuses on the development of spectral versions of the rotating shadowband radiometer (RSR). The RSR is a tool for the simultaneous measurement of total horizontal, diffuse horizontal, and direct normal irradiance. The Atmospheric Radiation Measurement (ARM) Program supports the development of a sevenchannel RSR consisting of individual filtered detectors that may be selected (at time of manufacture) in the 350- to $1700-\mathrm{nm}$ range with bandwidths of either 10 or $20 \mathrm{~nm}$, depending on wavelength. Another spectral variant of the RSR is a spectrometer version that contains a 256channel diode array. This instrument is designed to yield $0.6-\mathrm{nm}$ resolution at $350 \mathrm{~nm}$ and $8-\mathrm{nm}$ resolution at $1050 \mathrm{~nm}$.

Besides instrument development and calibration activities, we are collecting a data set at our Albany facility. This data set consists of high quality shortwave and longwave radiation measurements and meteorological variables from the Albany County airport, including upper air observations. The data are for use in testing radiation codes used in global climate models and are provided to those requesting them for their model tests.

In this paper, we discuss the main points of five papers that have been submitted to journals or are conference proceedings within the last year. These include papers describing 1) the seven-channel multifilter rotating shadowband radiometer (MFRSR) (Harrison ot al., in press); 2) an objective algorithm for obtaining optical depths without the necessity of human intervention (Harrison and Michalsky 1993); 3) the cosine bench for measuring the cosine response functions of the RSRs and other radiometers (Michalsky et al. 1992); 4) rotating shadowband spectroradiometer progress (Harrison et al., in press); and 5) the effects of Mount Pinatubo on the aerosol above the Oklahoma-Kansas Cloud and Radiation Testbed (CART) site (Michalsky and Larson 1993).

\section{Multi-Filter Rotating Shadowband Radiometer}

The MFRSR (see Figure 1) is a ground-based instrument for simultaneously measuring total horizontal, diffuse horizontal, and direct normal irradiance in seven wavelength passbands. At each wavelength, each of the three components of solar shortwave irradiance is measured with the same filter-detector using the shadowband technique.

In this technique, total horizontal and diffuse horizontal irradiance are measured by a pyranometer sensor (one collecting radiation over $2 \pi$ steradians with a Lambertian response) that is alternately shielded and unshielded from direct solar beam radiation by a metal band. From these two measurements, direct normal radiation is calculated. The band is controlled and the data are acquired and stored by our own data acquisition system. Data are retrieved through either a direct serial connection or a telephone modem. 


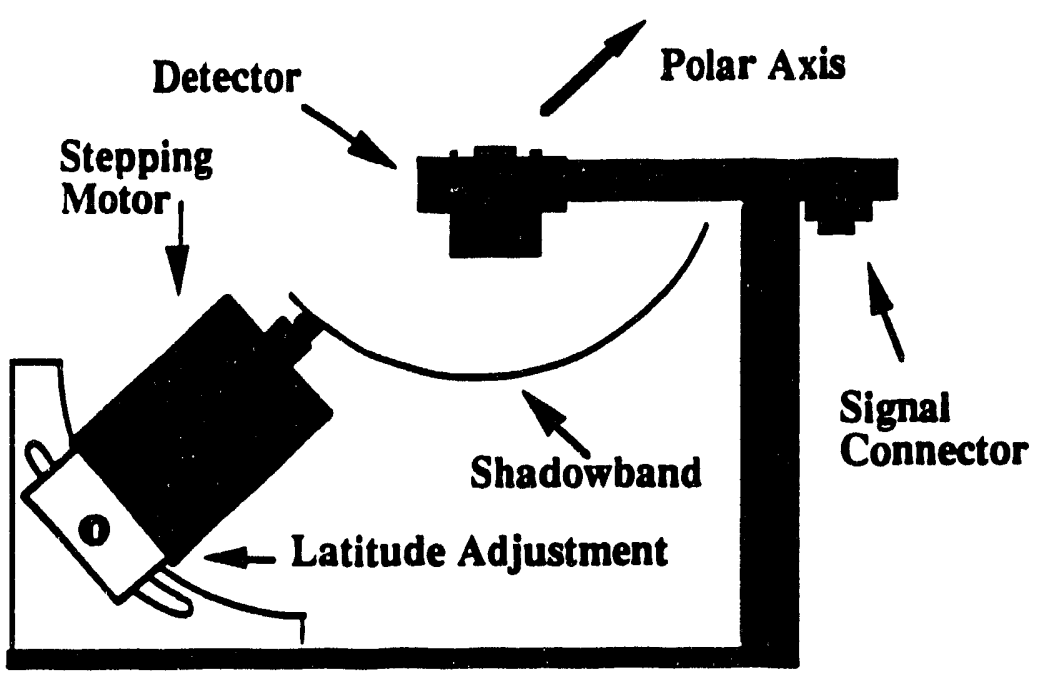

Figure 1. Multi-filter rotating shadowband radiometer.

The key points of Harrison et al. (in press) are

- All three components at each wavelength have the same calibration, thus reducing the errors associated with intercomparing results from independent instruments.

- Direct normal measurements can be used for Langley analysis, which produces a determination of the solar constant and, therefore, calibration of all three components. This can be used to track detector stability and provide a comparison to the calibration using the standard light.

- The instrument achieves an accuracy of the direct normal component comparable to a tracking instrument and an accuracy of the total and diffuse components that exceeds commercial pyranometers. These higher levels of accuracy are the result of the improvement in cosine response and because the measured direct component can be corrected for the remaining error in cosine response. Figure 2 illustrates the comparability of the direct. In this figure, the optical depths obtained simultaneously by a tracking MFRSR detector and an MFRSR operated normally are compared. Optical depth is very sensitive to tracking errors, and the results are generally indistinguishable with errors comparable to that obtained when comparing two tracking devices.

\section{Objective Algorithm for Optical Depth Retrieval}

The MFRSR instrument is expected to be deployed in large numbers. Each instrument is capable of producing seven directirradiance plots each day. Each clear morning or afternoon may be subjected to Langley analysis for optical depth and extraterrestrial irradiance calculations. If all 27 MFRSRs are deployed at each CART site, human analysis of the data will be impossible or, at best, costly. Furthermore, Langley analysis is somewhat subjective in that the handling of marginal data may be inconsistent. The objective algorithm developed by Lee Harrison sequentially removes data outside the 2-6 air mass range, data during cloud passages, and data whose residuals are beyond 1.5 standard deviations of a least squares fit to the points that remain. Furthermore, if fewer than one third of the points in the 2-6 air mass range remain or if the standard deviation of the remaining points exceeds a limit, the analysis is terminated.

The key points of Harrison and Michalsky (1993) are

- Such an algorithm is needed to reduce large amounts of data objectively. The algorithm works not only for MFRSRs, but for any tracking sunradiometer. 


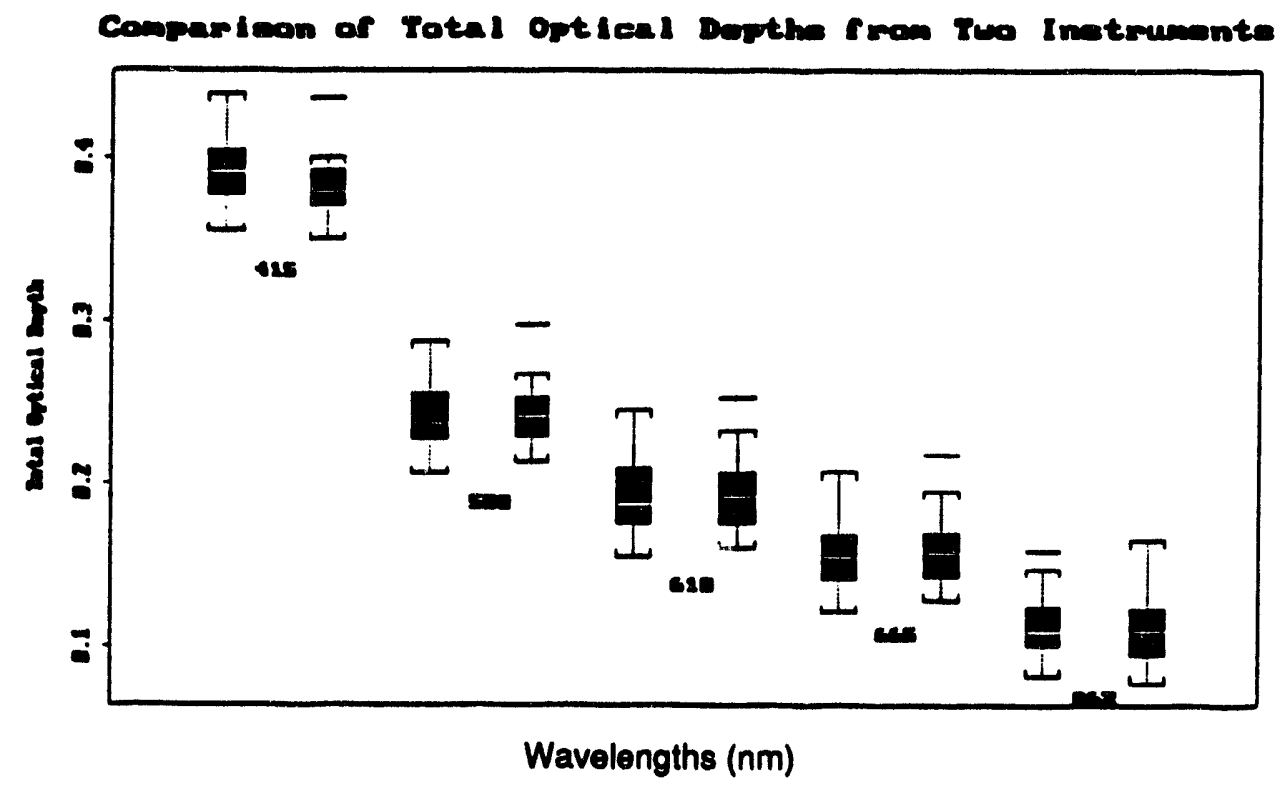

Figure 2. MFRSR and tracking MFRSR detector head optical depth comparison.

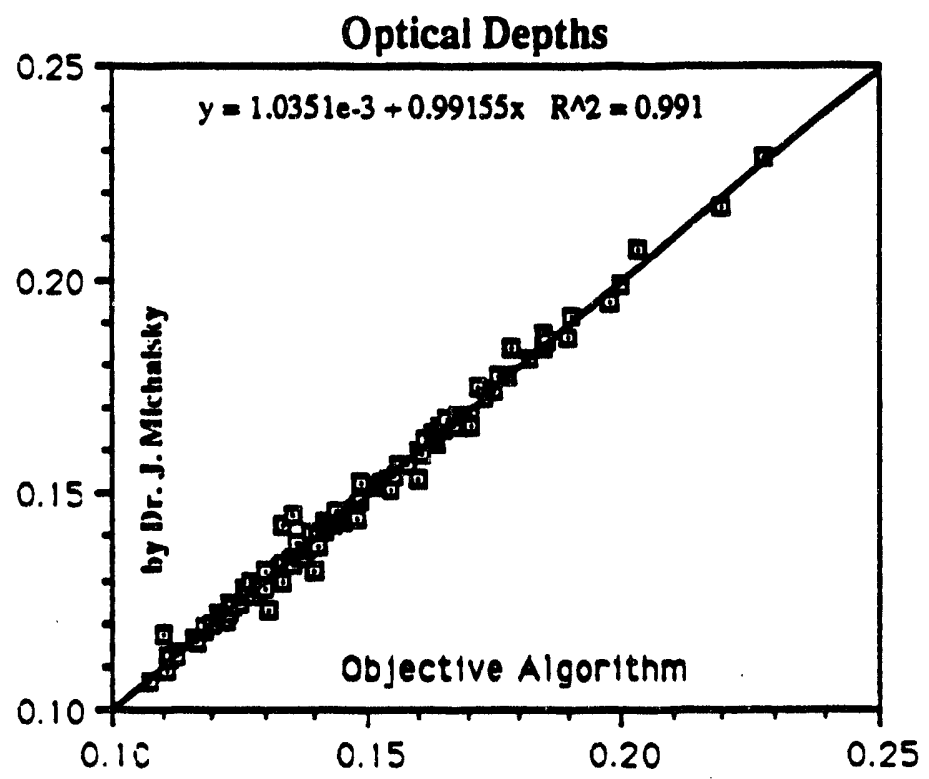

Figure 3. Scatter plot of optical depths determined by the objective algorithm versus subjective analysis. 
- Comparisons of optical depths retrieved by this algorithm and those derived subjectively compare very closely (seo Figure 3).

- Extrapolation of Langley regressions to zero air mass (extraterrestrial irradiance) can be used to track calibration (800 Figure 4).

\section{Cosine Response Measurement Bench}

Pyranometers receive radiation from $2 \pi$ steradians with a response that is Lambertian, i.e., its response to unit flux varies as the cosine of the angle of incidence. All pyranometers fail this true cosine response criterion to some degree. The measurement of cosine response is normally difficult and time-consuming and is performed only at a few angles. A bench to test standard radiometers and the MFRSRs has been constructed to automate these tests. Responses of three Eppley PSP pyranometers used in the CART faclities appear in Figure 5.
The key points of Michalsky et al. (1992) are

- The cosine bench automates an otherwise difficult measurement.

- Assumption of true cosine response is never quite true for any device.

- Pyranometers used at the Oklahoma CART site have been tested.

- Knowing cosine response is critical, especially for the MFRSR where direct is calculated and for high accuracy measurements such as those for ARM.

\section{Rotating Shadowband Spectroradiometer}

A second spectral version of the RSR is the 256-channel spectrometer version that we call the rotating shadowband spectroradiometer (RSS). The receiver optics of this version of the instrument are shown schematically in Figure 6.

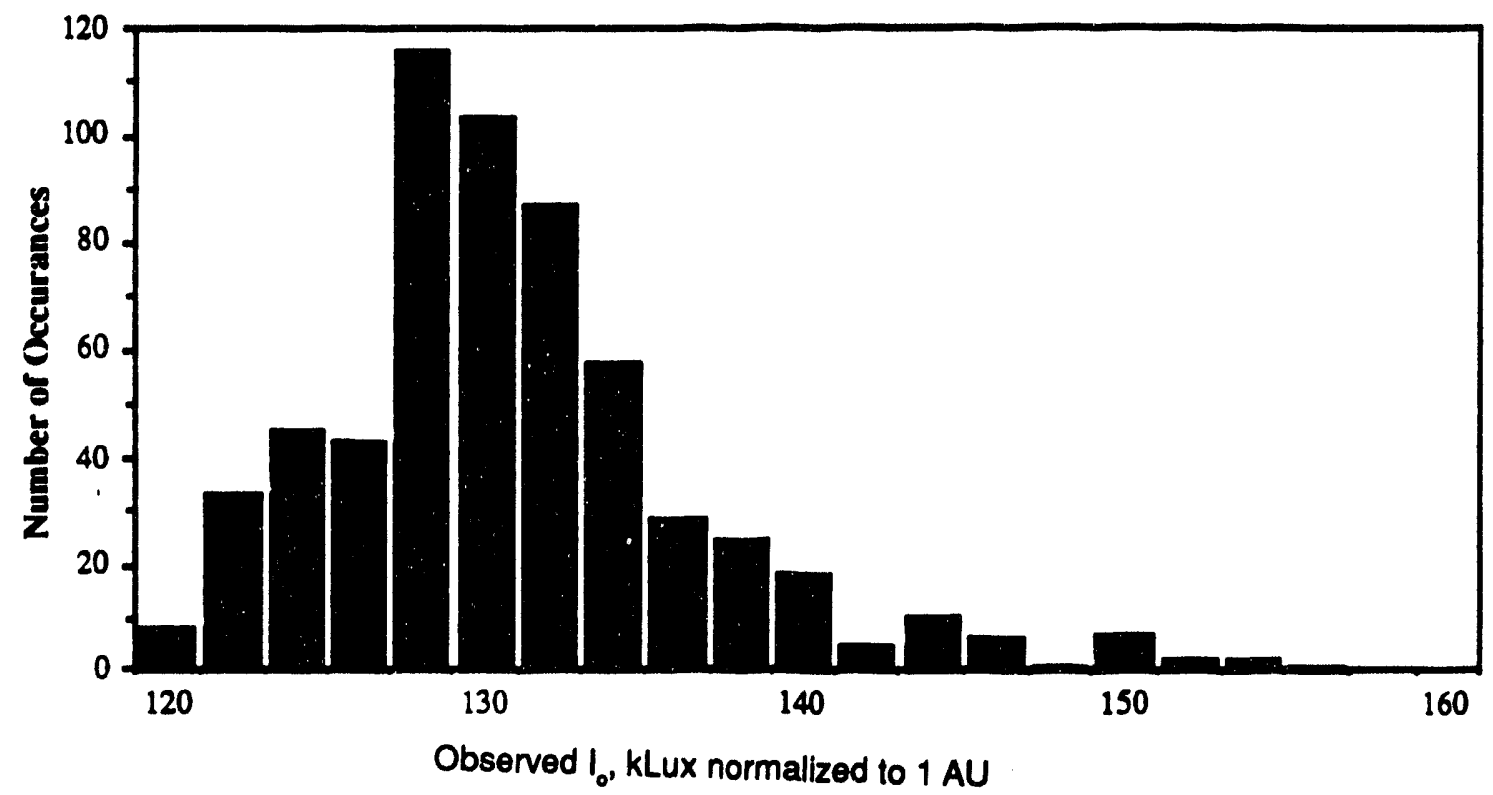

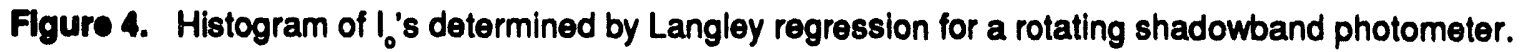




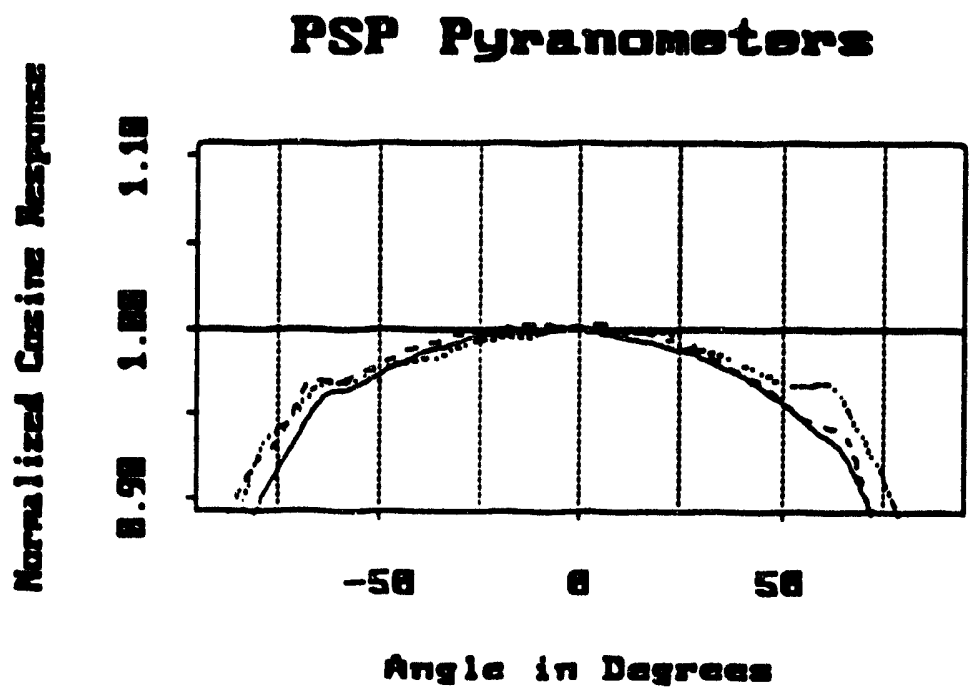

Figure 5. Cosine responses of three different Eppley PSP8.

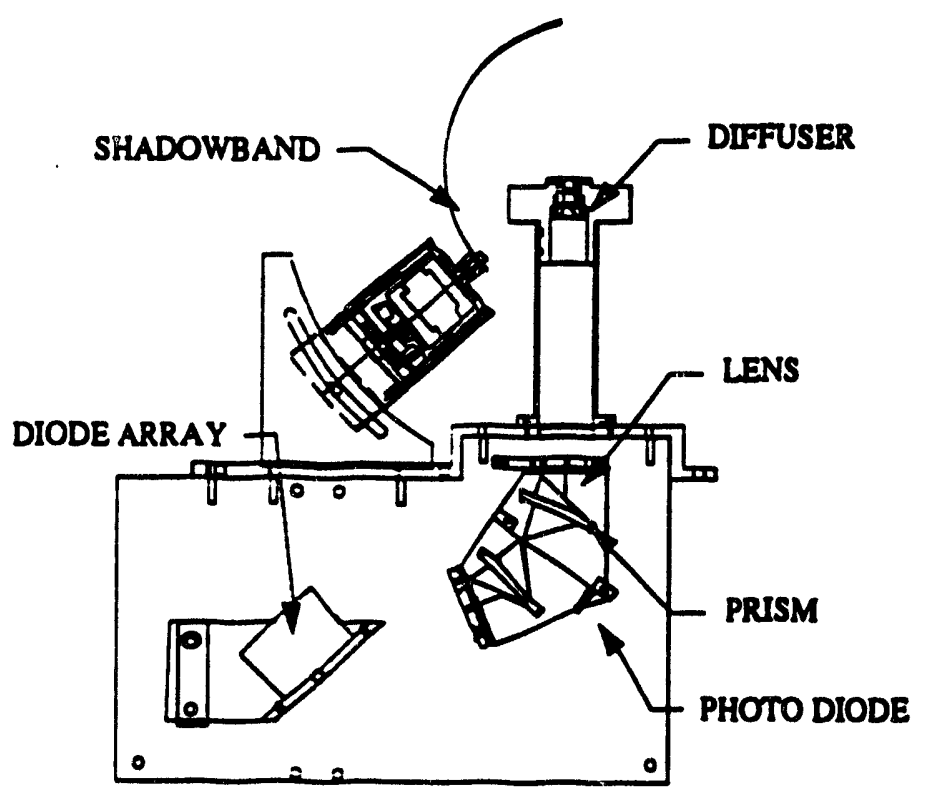

Figure 6. Rotating shadowband spectroradiometer. 
Solar radiation is incident on the Lambertian receiver modeled after the MFRSR receiver. The exit port of this diffuser-integrating cavity is a slit. The light from the slit is collimated by the first lens, passes through two dispersing quartz prisms, and is focused by a second lens onto the diode array. The system design minimizes spherical aberration and corrects for chromatic aberration with a tilt of the detector.

The key points of Harrison et al. (in press) are

- The prototype has 256 channels covering the 350- to 1050-rim spectrum with variable resolution between 0.6 and $8 \mathrm{~nm}$, respectively. Total horizontal, diffuse horizontal, and direct normal irradiance are measured.

- CAD drawings exist for the hardware and electronics.

- All subsystems are performing as expected.

- Much software and hardware integration is needed.

- Substantial effort is needed to achieve $1 \%$ accurate calibrations, which are now at $5 \%$ to $10 \%$.

\section{Mount Pinatubo Aerosol Perturbation of the Stratosphere}

Mount Pinatubo erupted in June 1991, introducing an estimated 20 million tons of $\mathrm{SO}_{2}$ into the stratosphere (Bluth et al. 1992). This amount was about three times larger than the El Chichon eruption of 1982. The dispersion of the plumes from the two volcanoes differed: Mount Pinatubo effluent was more eventy dispersed between hemispheres, while El Chichon was confined mostly to the northern hemisphere. The Pinatubo aerosol will affect the stratosphere for the first two or three years of operation of the first CART site. Since stratospheric aerosol can be rather uniformly distributed in latitude zones, we may use other measurements in the mid-northern latitudes to estimate the effect over Kansas and Oklahoma. Figure 7 is a plot of stratospheric aerosol above Boulder, Colorado, resulting from the Mount Pinatubo eruption.

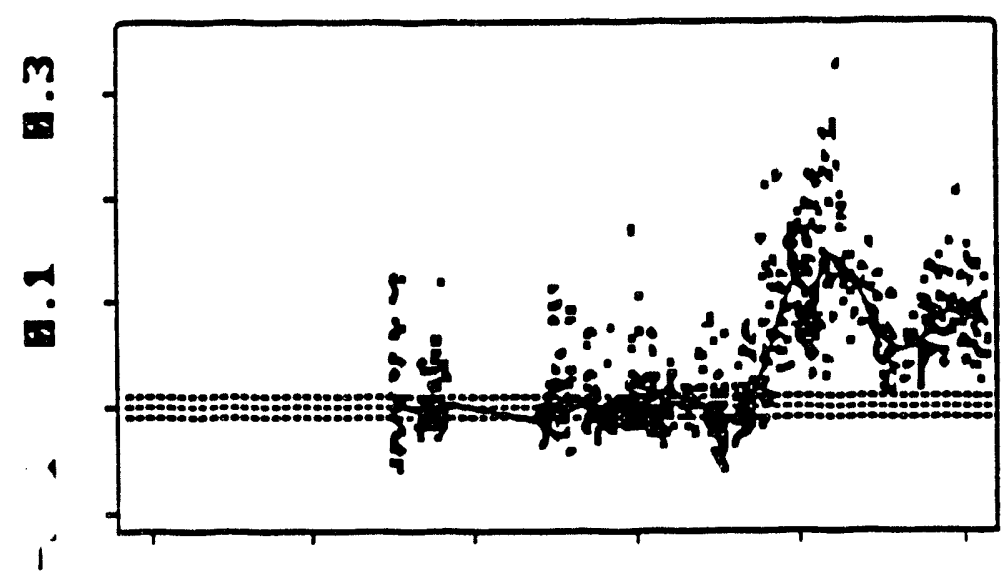

$1980 \quad 1989 \quad 1998 \quad 1991 \quad 1992 \quad 1993$

Years

Figure 7. Mount Pinatubo aerosol optical depth as a function of time for Boulder, Colorado, at $555 \mathrm{~nm}$. 
The key points of Bluth et al. (1992) and Michalsky and Larson (1993) are

- The stratosphericand troposphericaerosol contributions may be separated in a ground-based measurement.

- Mount Pinatubo produced about $50 \%$ greater optical depth at mid-northern latitudes than El Chichon.

- Spectral data obtained with the MFRSR will allow us to determine some size information through mathematical inversion methods.

- The stratospheric data from other sites will allow us specify the monthly average tropospheric aerosol contribution until Mount Pinatubo aerosol decreases to background levels.

\section{References}

Bluth, G.J.S., S. D. Doiron, C. C. Schnetzler, A. J. Kruger, and L. S. Walter. 1992. Global Tracking of the $\mathrm{SO}_{2}$ Clouds from the June 1991 Mount Pinatubo Eruptions. Geophy. Res. Lett. 19:151-154.
Harrison, L., and J. Michalsky. 1993. Objectlve Algorithm for the Retrieval of Optical Depths from Ground-Based Measurements. (Appl. Opt., in press).

Harrison, L., M. Beik, and J. Michalsky. 1993. The Automated Rotating Shadowband Spectroradiometer. Optical Remote Sensing of the Atmosphere: Technical Digest 5:448-451.

Harrison, L., J. Michalsky, and J. Berndt. 1993. An Automated Multi-Filter Rotating Shadowband Radiomoter. An Instrument for Optical Depth and Radiation Measurements. (Appl. Opt., in press).

Michalsky, J. J., L. C. Harrison, and W. E. Berkheiser III. 1992. Cosine Response Characteristics of Radiometric and Photometric Sensors. Proceedings of the 1992 Annual Conference of the American Solar Energy Society, ASES, Boulder, Colorado, June 1992, pp. 335-339.

Michalsky, J. J., and N. Larson. 1993. Time-Dependent Bohavior of Mount Pinatubo Aerosol. Optical Remole Sensing of the Atmosphere: Technical Digest 5:298-301. 


\title{
Development of a Balloon-Borne Stabilized Platform for Measuring Radiative Flux Profiles in the Atmospheric Boundary Layer
}

\author{
C. D. Whiteman, J. M. Alzheimer, G. A. Anderson, and W. J. Shaw \\ Pacific Northwest Laboratory \\ Richland, WA 99352
}

\section{Introduction}

A stabilized platform has been developed to carry broadband short-wave and long-wave radiometric sensors on the tether line of a small tethered balloon that ascends through atmospheric depths of up to $1.5 \mathrm{~km}$. The resulting vertical profiles of radiative flux and flux divergence should prove useful in evaluating atmospheric radiative transfer models, especially for cloudy conditions where the radiative transfer codes are in an early state of development.

Our stabilized platform, called the Sky Platform, was designed to keep the radiometers level despite unpredictable movements of the balloon and tether line occasioned by turbulence and wind shear. The sky platform has a triangular frame that is carried within a three-line harness inserted into the tether line 30 mor more below the balloon. Level sensors detect when the platform is offlevel. The automatic control loop drives motors, gears, and pulleys located on two of the vertices of the triangular frame to "climb" the harness lines to keep the platform level. Radiometricsensors, an electronic compass, and an on-board data acquisition system make up the remainder of the Sky Platform.

Because knowledge of the dynamic response of the tether line-platform system is essential to properly close the automatic control loop on the Sky Platform, a MotionSensing Platform (MSP) was developed to fly on the tether Iine in place of the Sky Platform to characterize the Sky Platform's operating environment. This unstabilized platform uses an array of nine solid-siate linear accelerometers to measure the lateral and angular accelerations, velocities, and displacements that the Sky Platform will experience. The MSP aiso carries a flux gate magnetometer to measure platform heading; a data acquisition system; and, during special callbration experiments, two single-axis rate gyroscopes.

A recent paper by Alzheimer ot al. (1993) describes the Sky and Motion-Sensing platforms, and a paper by Shaw and Whiteman (1993) uses a numerical radiation model to assess the engineering performance requirements of the Sky Platform stabilization system. The model, which performs angular integrations of a variety of published sky radiance distributions, estimates the irradiance measurement errors that would be produced on tited radiometers. It also assesses the effect of oscillatory motions of the radiometer on measured irradiances.

The model shows that the most stringent requirement on radiometer leveling is encountered during clear daytime conditions. In these conditions, the mean tilt of the radiometer must be less than $1^{\circ}$ with oscillations less than about $2^{\circ}$ in order for the measured radiation to be accurate to 10 watts per square meter. During nighttime, or under daytime conditions : vhen the radiation field is more isotropic, these conditions may be relaxed significantly.

This paper presents field performance tests of the Sky and Motion-Sensing platforms, as conducted at Richland, Washington, on February 17, 1993. The tests were pertormed primarily to characterize the stabilization system on the Sky Platform. Test flights were performed on this cold winter day from 1400 to 1800 Pacific Standard Time (PST). During this period, temperature profiles were near the dry adiabatic lapse rate. Flights were made through a jet wind speed profile having peak wind speeds of $7 \mathrm{~m} / \mathrm{s}$ at a height of $100 \mathrm{~m}$ above ground level (AGL). Wind directions were from the northwest. All flights were continuous ascents, rather than in discrete steps with halts at set altitudes. 


\section{Sky Platform}

The overall performance of the prototype Sky Plattorm stabilization system is presented in Figure 1 by comparing Sky Platform tilt angles on the two nominally horizontal axes from a stabilized and an unstabllized flight. The stabilized flight was conducted as a 5-minute ascont starting at 1704 PST, while the unstabilized flight was a 5-minute ascent starting at 1714 PST. The stabilization system was deactivated during the unstablilzed flight, and the motors were pinned so that the platform would be horizontal if the harness system were oriented vertically. Since only small changes in atmospheric turbulence lovele are expected between the stabilized and unstabilized flights, comparisons of mean and standard deviations of the tilt anglos can be used to assess overall stabllization system pertormance.

For Sky Platform axis 1, the unstabilized performance was $19.48 \pm 2.25^{\circ}$ and the stabilized porformance was $0.74 \pm$ $1.60^{\circ}$. For axis 2 , the unstabilized performance was 20.07 $\pm 1.93^{\circ}$ and the stabilized performance was $0.82 \pm 0.80^{\circ}$. The gain on the control loop was set somewhat too high on axis 1 during this test, resulting in higher amplitude oscillations than for axis 2, but the results show that the stabilization system is effective in keeping the platform level for accurate radiation measurements.

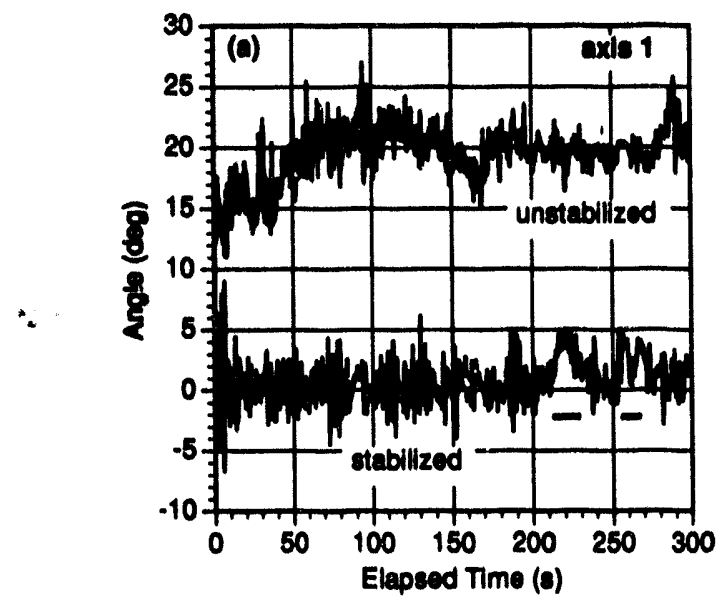

During two periods in the stabilized flight, Indicated by horizontal lines on Figure 1, the stabllization system was unable to compeneate for very high tother line tilt angles. The range of tilt angle compensation can be increaced, however, by simply increasing the number of wrape of harness line around the control system pulleys. This will be done for future flights.

\section{Motion-Sensing Platform}

The performance of the Sky Platform can be improved in the future by tuning the automatic control loop's response to various tether line motions. The goal is to decrease the control system's sensitivity to high-frequency lateral accolerations while increasing its responso to low-frequency angular accelerations that tilt the platform. A MotionSensing Platform has thus been developed to make the measurements required to design filters for the automatic control system on the Sky Platform.

An example of the performance of the MSP in measuring angular velocitios is shown in Figure 2 by comparing the performance of the 9-accelerometer array on the MSP with that of a calibrated rate gyroscope (actually, a rate s9nsor assembly). MSP angular volocity values were obtained by integrating angular accelerations determined from

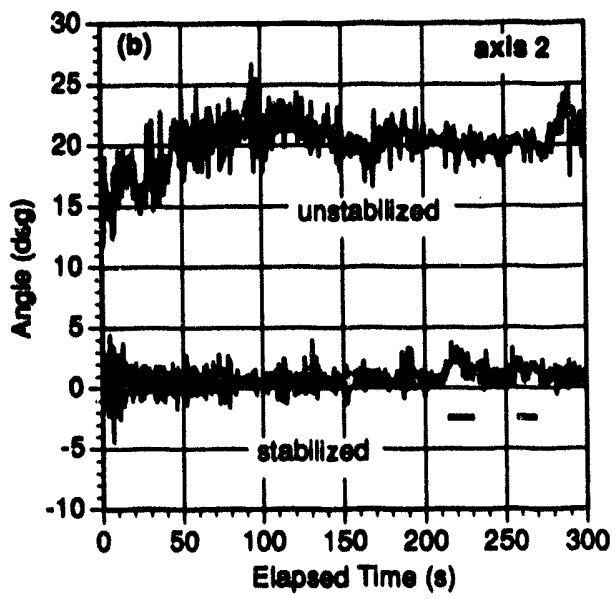

Figure 1. (a) Axis 1 and (b) axis 2 Sky Platform tilt angles for unstabilized and stabilized 5-minute ascents through depths of about $140 \mathrm{~m}$ AGL on February 17, 1993. See text for explanation of horizontal lines. 


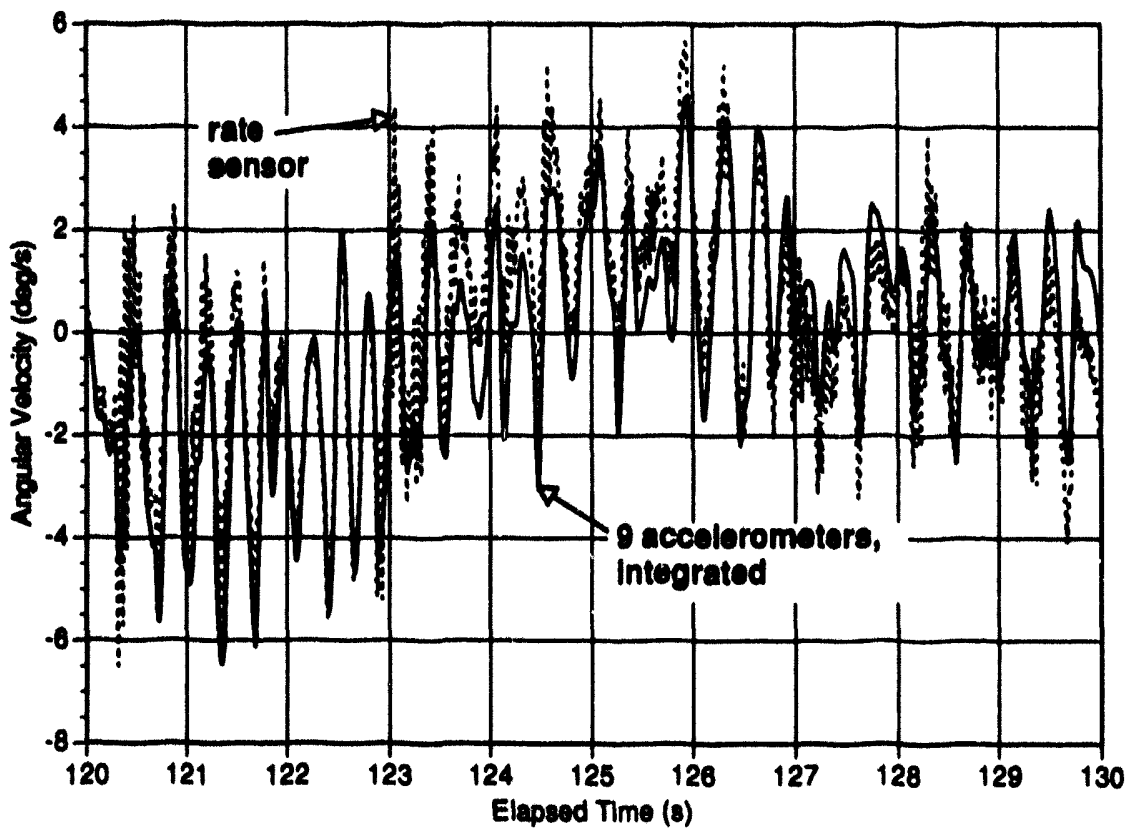

Figure 2. Angular rate measurements about the $x$ axis of the Motion-Sensing Platform, as measured independently by a rate gyro and by an integration of angular accolerations determined with the MSP 9-accelerometer array. The 10-s time series corresponds to MSP ascent through the height range from 56 to $64 \mathrm{~m} \mathrm{AGL}$.

the 9-accelerometer array. A third-order Buttenworth filter was used to pre-filter the angular acceleration data to reduce aliasing.

The conclusion from Figure 2 is that the MSP' is able to measure angular accolerations reliably compared with the rate gyro for frequencies that are typical of the tether line. The amplitude spectrum of frequencles present on the tether line for the entire 5-min flight is shown in Figure 3, as obtained from the rate gyro. The spectrum shows a rapld falloff in the amplitude of tether line motions as frequency increases. A peak is apparent in the spectrum at $3 \mathrm{~Hz}$. Other low-amplitude peaks (not shown) are present at 21 and $37 \mathrm{~Hz}$, and efforts are under way to determine if these peaks are produced by the winch drive chain, vibrational modes of the platform frame, or other non-meteorological causes.

\section{Conclusions}

Initial tests of the prototype Sky Platform show that it is effectively stabilized to an average tilt angle of less than $1^{\circ}$ with standard deviations of less than $2^{\circ}$ under wintertime conditions with moderate shear in a 200-m-deep wellmixed boundary layer. The Motion-Sensing Platform appears to be capable of characterizing the dynamice of the balloon-Sky Platform-tether-line system and should be able to provide the information necessary to improve the control loop stablization system on the Sky Platform. Further development of the Sky and Motion-Sensing platforms should result in a radiometric sounding system capable of measuring long-and short-wave irradiance and radiative flux divergence profiles in the atmospheric boundary layer; 

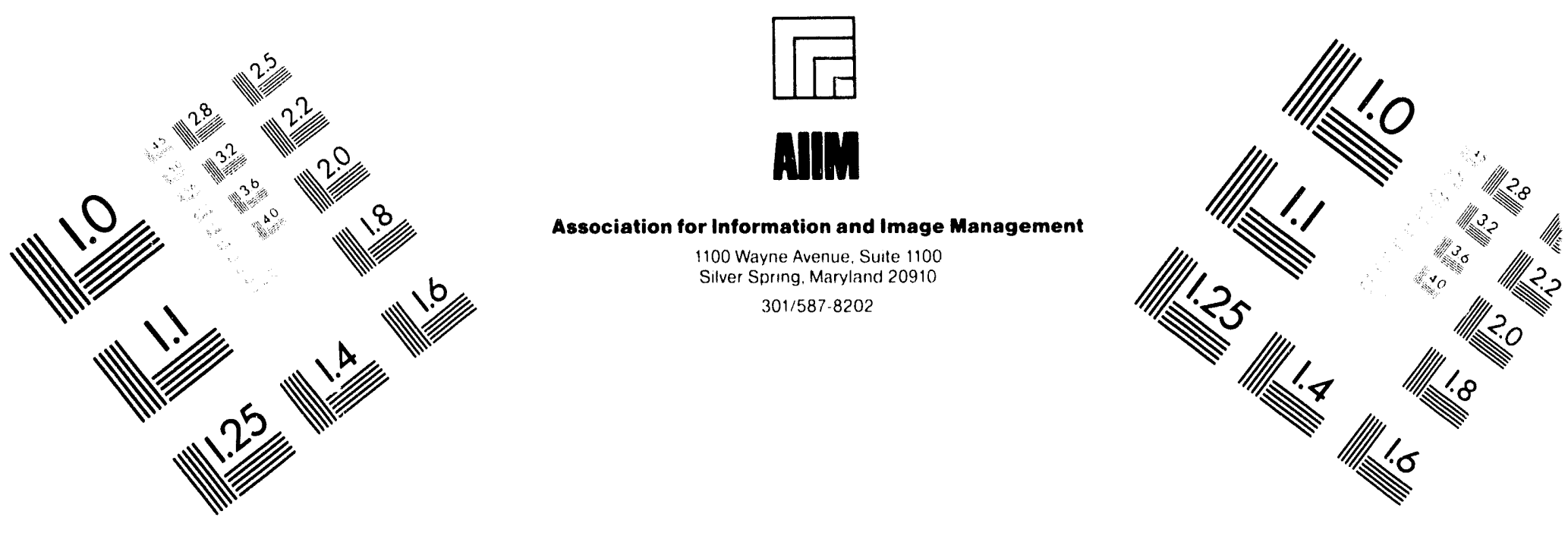

\section{Centimeter}

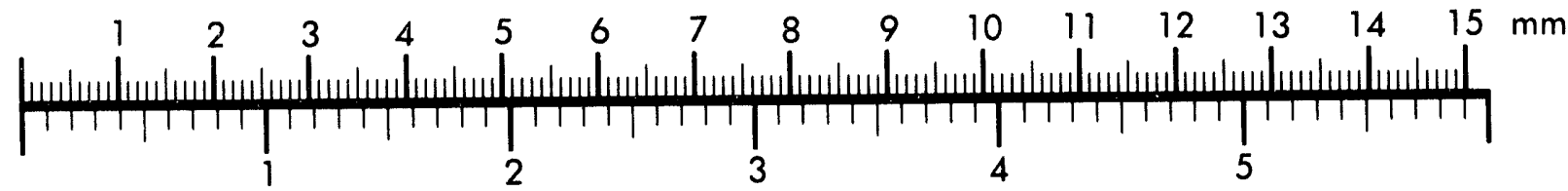
Inches
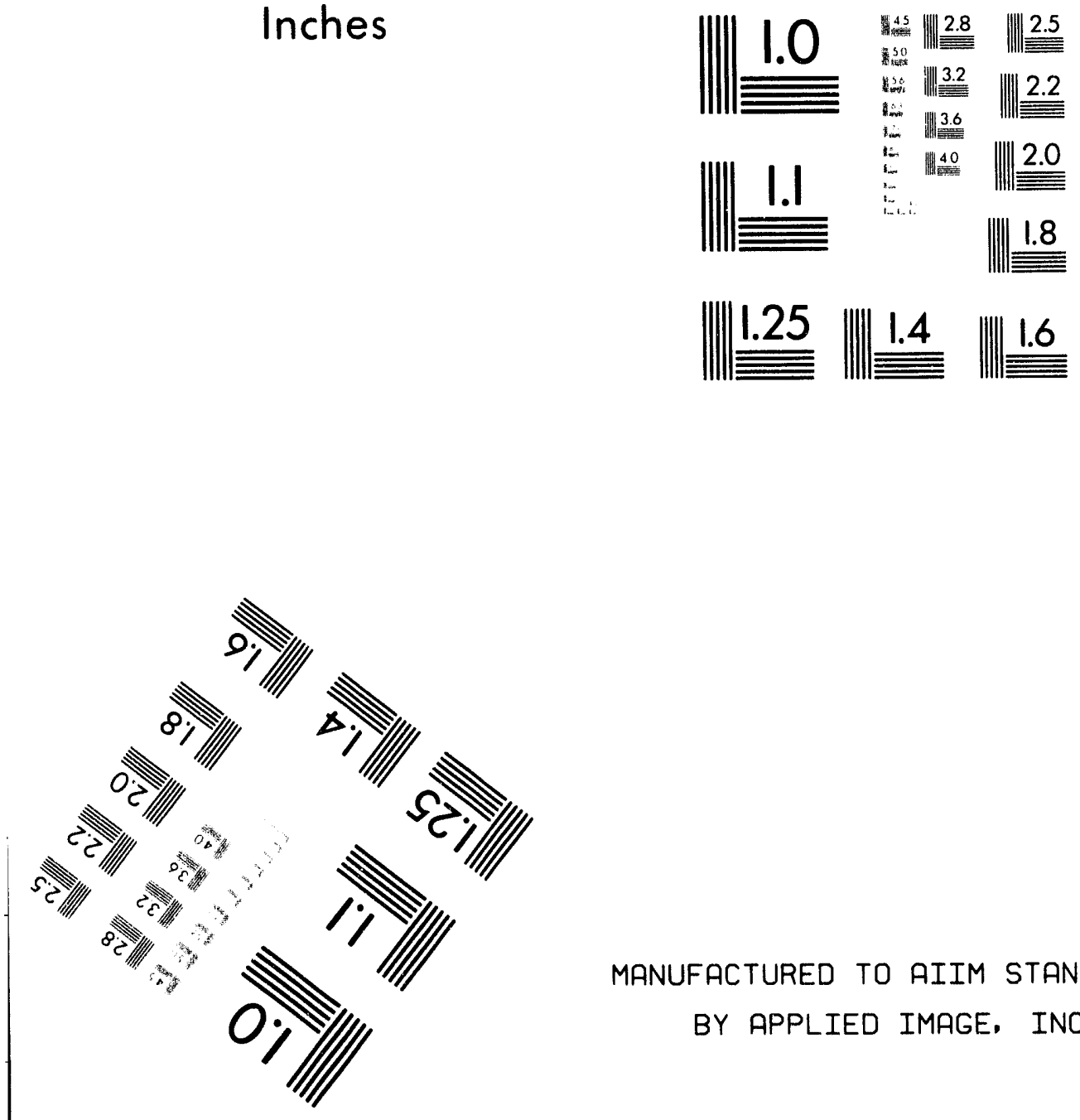

MANUFACTURED TO AIIM STANDARDS BY APPLIED IMAGE, INC.

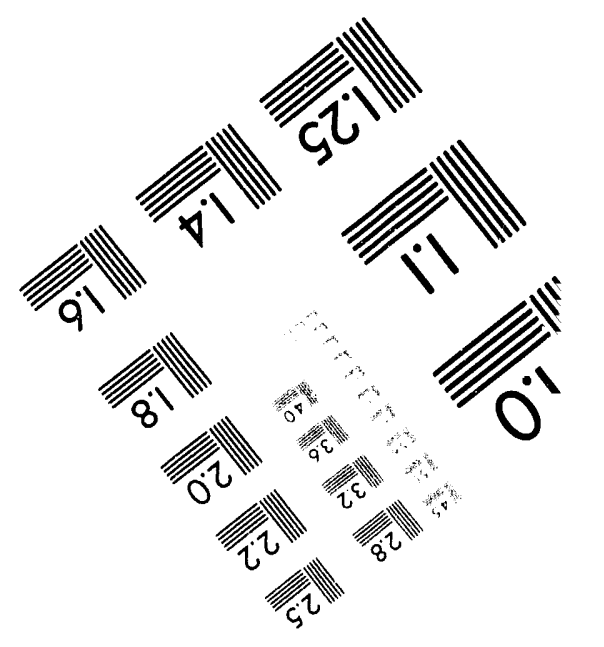


1
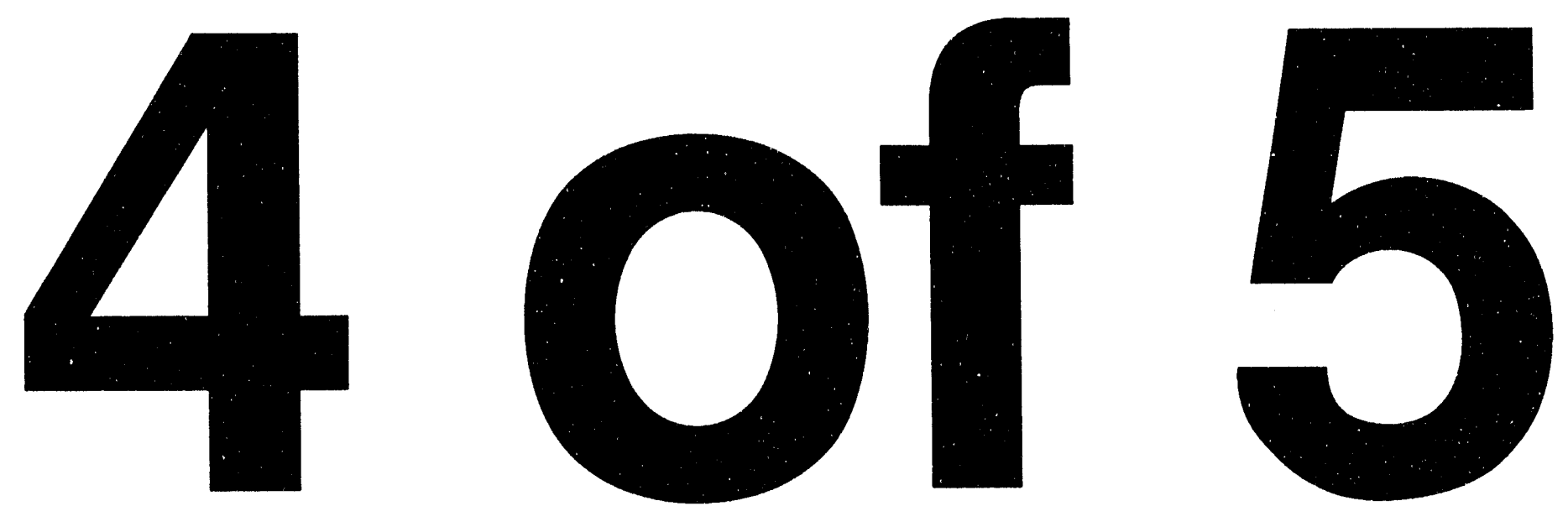


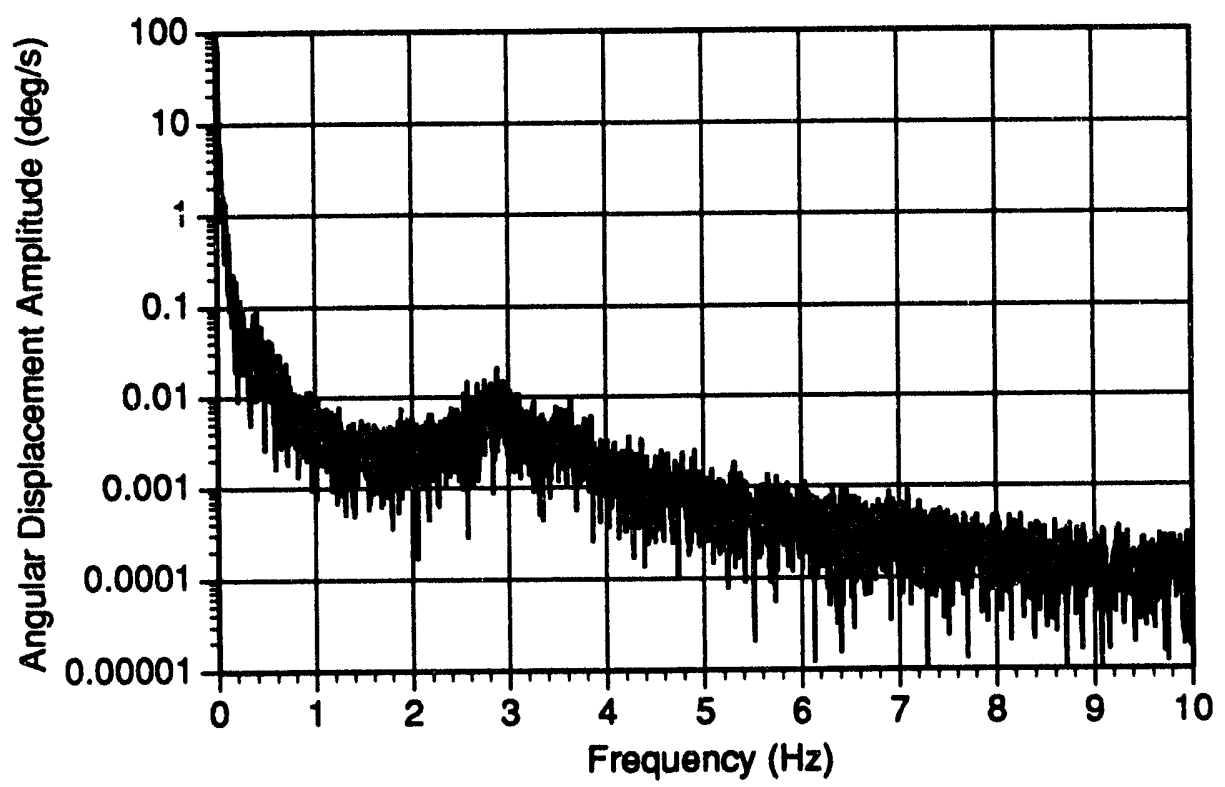

Figure 3. Spectrum of angular displacements measured by a rate gyro about the $x$ axis of an unstabilized Motion-Sensing Platform during a complete 5-minute ascent through a depth of about $140 \mathrm{~m}$.

the prescribed error bars will depend on radiometer calibration and Sky Platform stabilization performance.

\section{Acknowledgments}

Research was supported by the U.S. Department of Energy under Contract DE-AC06-76RLO 1830. Pacific Northwest Laboratory is operated by Battelle Memorial Institute for the U.S. Department of Energy.

\section{References}

Alzheimer, J. M., G. A. Anderson, and C. D. Whiteman. 1993. Stabilized Platform for Tethered Balloon Soundings of Broadband Long-and Short-Wave Radiation. Preprints, Eighth Symposium on Meteorological Observations and Instrumentation, January 17-22, 1993, Anaheim, California, pp. 175-180. American Meteorological Society, Boston, Massachusetts.

Shaw, W. J., and C. D. Whiteman. 1993. Accuracy of Radiation Profiles from a Self-Leveling Balloon-Borne Platform. Preprints, Eighth Symposium on Moteorological Observations and Instrumentation, January 17-22, 1993, Anaheim, California, pp. 121-126. American Meteorological Society, Boston, Massachusetts. 


\title{
Boundary Layer Heat Budgets from Wind Profiler/Radio Acoustic Sounding Systems Data: A Feasibility Study
}

\author{
M. Furger(a) and C. D. Whiteman \\ Pacific Northwest Laboratory \\ Richland, Washington
}

Radar wind profilers (WP) and radio-acoustic sounding systems (RASS) are now available commercially and offer the promise of improving our knowledge of processes in the lower atmosphere on scales that were until now unresolved spatially and/or temporally. Plans to install a network of boundary layer WP/RASS within two or three years are now under discussion in the U.S. Department of Energy's ARM (Atmospheric Radiation Measurement) Program and in other national programs. The new devices raise the prospect of gaining better insight into planetary boundary layer processes. With this background in mind, we re-evaluate some classical methods for the calculation of kinematic and dynamic quantities using measurements from these new remote sensing devices.

In this paper, we restrict our study to the evaluation of the heat budget equation (e.g., Pielke 1984) for the atmospheric volume dV defined by a triangular array of vertically pointing WP/RASS:

$\iint \underbrace{\bar{\rho} c_{p} \nabla \cdot\left(\overline{\left.v^{\prime} \theta v^{\prime}\right)}\right.}_{A} d V=-\iiint[\underbrace{\bar{\rho} c_{p} \frac{\partial \bar{\theta}}{\partial t}}_{B}+\underbrace{\bar{\rho} c_{p} \nabla \cdot(\overline{v \theta v})+\nabla \cdot \bar{A}}_{C}] d V$

where $\rho=$ air density

$c_{p}=$ specific heat at constant pressure

$v=$ vector wind

$\theta v=$ virtual potential temperature

$\mathbf{R}=$ vector net radiation

(a) On leave from Paul Scherrer Institute (PSI), Villigen, Switzerland. and the overbars and primes indicate a Reynolds decomposition of the individual terms over a suitable averaging period. The four terms of the heat budget equation are A: turbulent virtual potential temperature flux divergence, B: time rate of change of heat storage, C: mean virtual potential temperature flux divergence, and $D$ : net all-wave radiative flux divergence.

Our goal is to determine the sensitivity of the heat budget calculations to WP/RASS wind and temperature measurement uncertainties. This can be done, in a first step, with a Monte Carlo-type model. For this purpose we define a hypothetical triangular array of WP/RASS with a distance between stations on the order of $30 \mathrm{~km}$, and a height range of $2 \mathrm{~km}$ resolved into 100 -m-deep layers. Mean profiles of wind and virtual temperature are assumed, and the WP/RASS measurement errors are added as perturbations to these mean profiles. These errors are simulated by randomly sampling from Gaussian distributions with standard deviations corresponding to measurement precisions obtained from the scientific literature. Evaluation of the heat budget terms for a large number of randomly perturbed profiles yields a distribution of values from which basic statistical quantities such as standard deviations can be calculated. These standard deviations are regarded as confidence limits and are used to estimate the effect of the measurement uncertainties on heat budget terms for individual height layers.

The results presented here are based on the assumptions of 1) ideally flat terrain; 2) horizontally homogeneous meteorological conditions, i.e. no horizontal gradients in wind, temperature, and radiation, no time change in temperature; and 3) dry atmosphere with nophase changes or clouds. The radiation profiles have been extracted from climatological data of Grand Junction, Colorado (McKeo 
and Whiteman 1977). Values varied lin- arly from $90 \mathrm{~W} / \mathrm{m}^{2}$ at the surface to $116.7 \mathrm{~W} / \mathrm{m}^{2}$ at the top. The turbulent sensible heat flux term in the equation was calculated as a residual of all other terms. We assumed a wind speed of $10 \mathrm{~ms}^{-1}$ for all lovels and stations, thus having a nondivergent wind situation. Temperature was assumed to decrease linearly with height with a lapse rate of $0.005 \mathrm{Km}^{-1}$. Surface temperature was $19.1^{\circ} \mathrm{C}$.

The precisions of $915 \mathrm{MHz}$ WP/RASS have been taken from values published by Neff and Wilczak (1992) and Martner et al. (1993). Wind errors can be considered to be less than $1 \mathrm{~ms}^{-1}$ under ideal conditions, while temperature measurement errors are less than $1 \mathrm{~K}$. These values are valid for 1 -hour averages. To account for errors in the net radiation profiles, we used a standard deviation of $15 \mathrm{~W} / \mathrm{m}^{2}$, which corresponds to a relative error of approximately $15 \%$.

Figure 1 shows the mean values and standard deviations of the turbulent sensible heat flux divergence term as a function of height for three different temperature measurement standard deviations. We selected this term because it shows the largest standard deviation of all terms, a fact that clearly emerges from the calculation of the term as a residual. The mean values are distributed around zero, as expected for a horizontally homogeneous situation. The peaks in the curves are a result of the random number generator. The resulting standard deviations increase with increasing measuremont standard deviations. To evaluate this result, a comparison with typical observed values proves useful. For convective situations, maximum daily values reach 0.2 to $0.25 \mathrm{~W} / \mathrm{m}^{3}$, which is about half the standard deviation for a temperature error of $1 \mathrm{~K}$.

The situation for wind measurement uncertainties is presented in Figure 2. The effect of wind measurement uncertainties differs strongly from the effects of temperature measurement uncertainties. The standard deviations increase with height for values larger than $1 \mathrm{~ms}^{-1}$. This behavior reflects the accumulation of errors that are due to the integration of the vertical velocities computed from mass conservation. Thus an error in the mass balance is always propagated to the next level, where another error can be added, and so on. The $0.2 \mathrm{~ms}^{-1}$ curve suggests that only little can be gained by improving wind measurements, while a great deal could be gained by improving temperature measurements.
Simulations for different averaging times suggest that a minimum averaging time of $6 \mathrm{~h}$ is required to get standard deviations less than $0.2 \mathrm{~W} / \mathrm{m}^{3}$ for quasi-stationary conditions. The disadvantage of time averaging is to lose resolution of diurnal variations (if we average over six consecutive hours) or resolution of synoptic weather events (if we average over the same hour of six consecutive days). The size of the triangular area affects the standard deviations inversely. Larger triangle sizes give smaller standard deviations and vice versa. This beneficial effect of larger triangle size is countered, however, by expected deviations from our assumption of linear variation in the meteorological fields as triangle size is increased.

We conclude that atmospheric heat budget terms are rather strongly affected by present-day measurement uncertainties even under favorable weather conditions, although time and space averaging brings some improvement. The calculation method itself still has important shortcomings, however, which may prove unresolvable in practice. On the other hand, some of the terms in the budget equation, such as the time rate of change of heat storage, can be calculated with acceptably small error bars, thus opening the perspective of obtaining longer term, climatological statistics of quasi-continuous temperature and wind measurements in the atmospheric boundary layer.

In the future the, method will be applied to data gathered during the WISP(a)/ARM/ASCOT(b) 91 experiment in Colorado's Denver/Boulder area, as this dataset is the only one currently available with three non-collinear 915 . MHz WP/RASS stations.

\section{Acknowledgments}

Research was supported by the U.S. Department of Energy under Contract DE-AC06-76RLO 1830. Pacific Northwest Laboratory is operated by Battelle Memorial Institute for the U.S. Department of Energy.

(a) Winter lcing and Storm Program.

(b) Atmospheric Studies in Complex Terrain Program. 


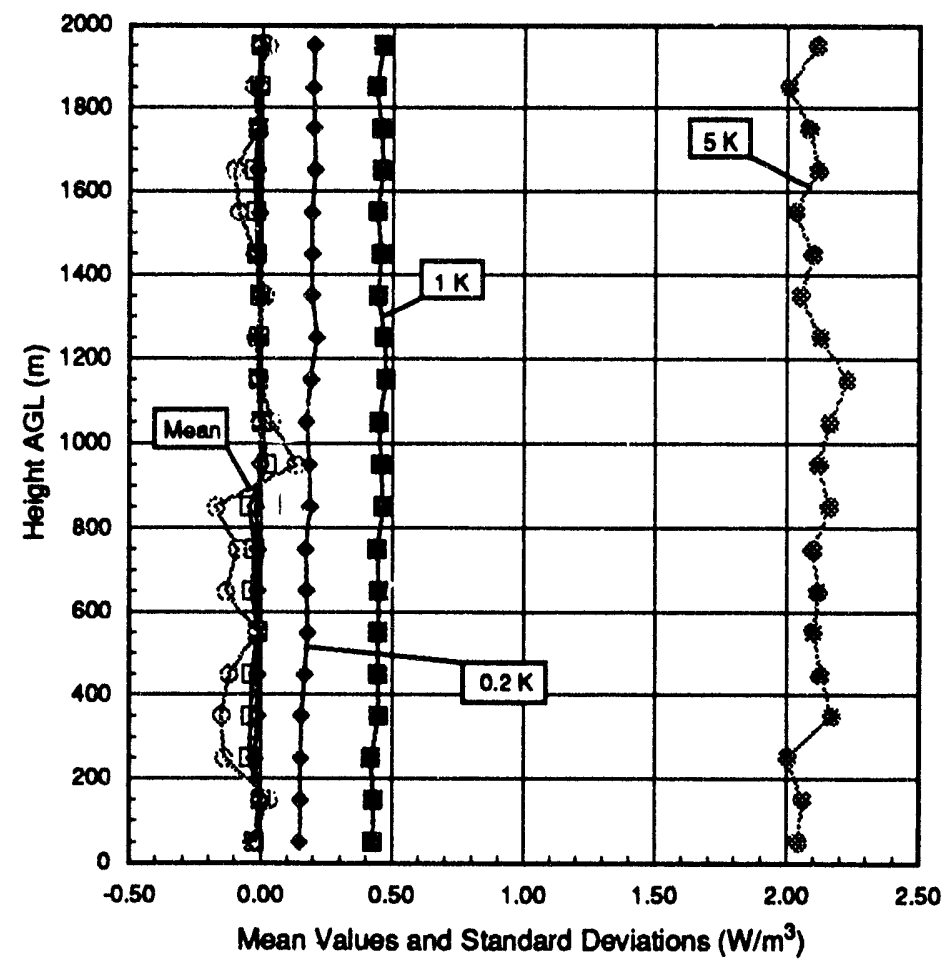

Figure 1. Monte Carlo simulations of the effects of temperature measurement errors on the standard deviations of turbulent sensible heat flux divergence calculated as a residual in the atmospheric heat budget equation. Solid symbols: standard deviations; outline symbols: mean values. 


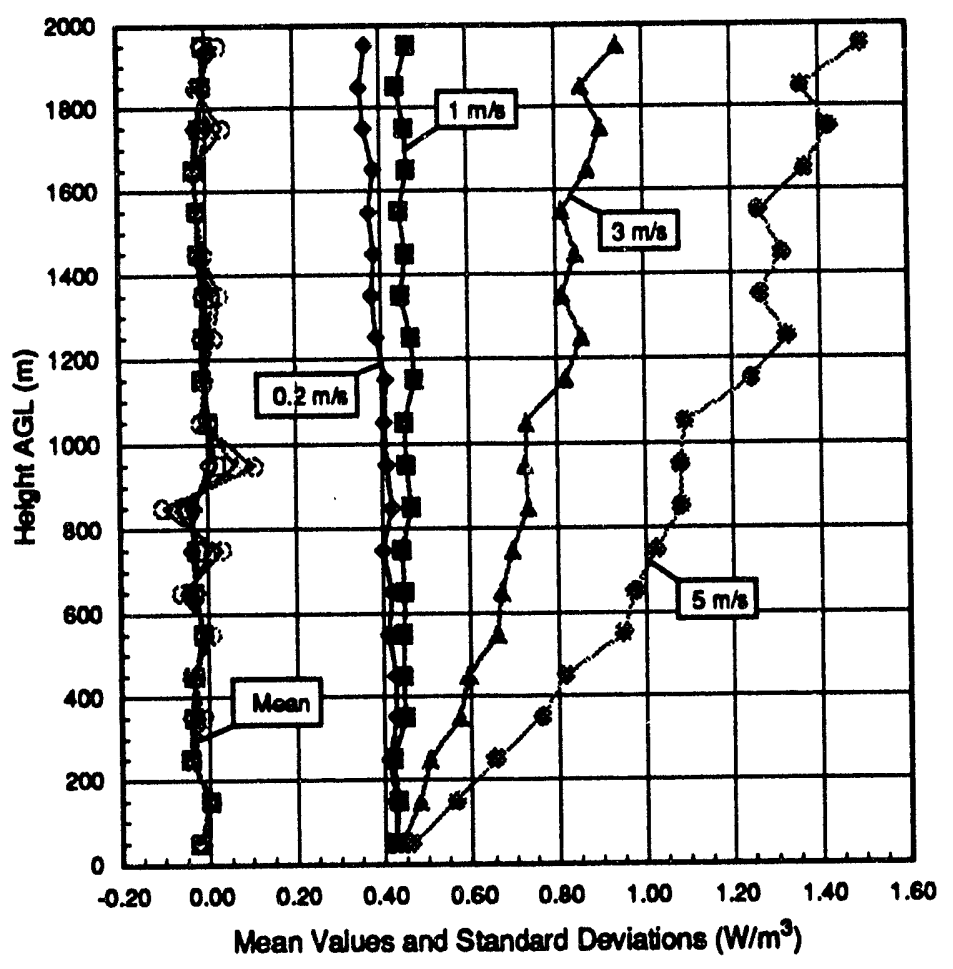

Figure 2. Monte Carlo simulations of the effects of wind measurement errors on the standard deviations of turbulpnt sensible heat flux divergence calculated as a residual in the atmospheric heat budget equation. Solid symbols: standard deviations; outline symbols: mean values.

\section{References}

Martner, B. E., D. B. Wuertz, B. B. Stankov, R. G. Strauch, E. R. Westwater, K. S. Gage, W. L. Ecklund, C. L. Martin, and W. F. Dabberdt. 1993. An evaluation of wind profiler, RASS, and microwave radiometer performanco. Bull. Am. Met. Soc. (submitted).

McKee, T. B., and C. D. Whiteman. 1977. Components of infrared net radiation in a mountain valley. Atmospheric Science Paper No. 279, Colorado State University, Fort Collins, Colorado.
Neff, W. D., and J. M. Wilczak. 1992. The application of 915-MHz radar wind profilers to the study of transport in the atmospheric boundary layer. Proc. 10th Symp. Turbulence and Diffusion, 29 Sept-2 Oct 1992, Portland, Oregon, pp. 276-280. American Meteorological Society, Boston, Massachusetts.

Pielke, R. A. 1984. Mesoscale Moteorological Modeling. Academic Press. 


\title{
Accuracy of Net Radiation Profiles from a Self-Leveling Balloon-Borne Platform
}

\author{
W. J' Shaw and C. D. Whiteman \\ Pacific Northwest Laboratory(a) \\ Richland, WA 99352
}

Net radiation profiles near the earth's surface provide the means to assess the importance of radiative heating in the lower atmosphere and the effectiveness of lower atmospheric radiation parameterizations in global climate models. Pacific Northwest Laboratory has developed a self-leveling platform that carries short- and long-wave radiometers aloft using a tethered balloon. While the leveling mechanism is effective, it cannot be perfect. It is therefore necessary to determine likely errors that residual motions of the platform may introduce into the measured radiation field.

This poster describes tests of a model used to establish the likely errors from residual platform motion. The model uses a standard numerical integration scheme to calculate the irradiance measured by an ideal but tipped sensor in an arbitrary radiance field. Sky and surface radiances are determined using published parameterizations for shortand long-wave radiation. The model is useful both for determining the degree to which platform motion must be stabilized to reduce measurement errors to acceptable levels and for calculating errors due to actual platform motion. Its application is easily extended to ships, aircraft, or other moving platforms.

(a) Research was supported by the U.S. Department of Energy under Contract DE-AC06-76RLO 1830. Pacific Northwest Laboratory is operated by Battelle Memorial Institute for the U.S. Department of Energy.
Field measurements to evaluate the parameterizations used in this model were carried out in May 1992 under clear skies using short- and long-wave radiometers mounted on a computer-driven platform. The platform repeatedly stepped through 360 degrees of azimuth and 20 degrees of elevation about the horizontal over a 24-h period. Radiosondes were released to account for the dependence of downwelling long-wave radiance on atmospheric water vapor.

These observations compare satisfactorily with model integrations. Using the parameterized radiances and Gaussian tipping in one dimension, we have shown that the balloon-borne radiometer platform will work satisfactorily if oscillations about level are on the order of a degree or two. However, the constraints on the mean departure from level are much more severe. To achieve errors less than a $10 \mathrm{~W} / \mathrm{m}^{2}$ under clear daytime skies, mean deviations from level must be on the order of a degree or less. In practice, mean deviations are those that persist for periods that are long compared to the time over which the radiance field changes significantly. Oscillations are departures from level whose durations are short compared with the time for significant radiance field changes. 


\title{
Narrow-Beam Fast Filter Radiometry and the Use of the Lidar/Radiometer Method in the Atmospheric Radiation Measurement Program
}

\author{
C.M.R. Platt, J. W. Bennett, S. A. Young, \\ M. D. Fenwick, P. J. Manson, G. R. Patterson, and B. Petraitis \\ CSIRO Division of Atmospheric Research \\ Station Streot \\ Aspendale, Victoria, Australia
}

\section{Introduction}

A central goal of the Atmospheric Radiation Measurement (ARM) Program is to characterize the cloud properties in columns of atmosphere above the centres of the ARM Cloud and Radiation Testbed (CART) sites. Techniques developed at the CSIRO Division of Atmospheric Research over the past decade or two are very relevant to this ARM concept. The basis of the techniques, which have come to be known collectively as the Lidar/Radiometer (LIRAD) method, is to use two independent groundbased observations of clouds. A laser radar, or lidar, measures the cloud height, structure and particle phase. A narrow-beam filter radiometer measures the cloud infrared (IR) radiance. By combining the lidar and radiometer observations with a radiosounding of temperature, accurate information on cloud infrared emittance can be obtained. If the cloud is semi-transparent, which is often the case for high cirrus ice clouds, then the cloud IR optical depth and profile of IR absorption coefficient can also be obtained.

The secret of success in the technique is to use a radiometer with a narrow field-of-view compatible with that of the lidar ( $10 \mathrm{mR}$ or 0.5 degree) and with a response time fast enough to follow the observed rapid fluctuations in cloud emission, which can often occur as different clouds come into view. The radiometer axis should also be aligned with the lidar and the two axes placed as close together as possible so that lidar and radiometer are looking effectively at the same volume of cloud.

An infrared narrow beam filter radiometer is ideal for this purpose. For a filter width of about $1 \mu \mathrm{m}$ wavelength and a suitable detector, such a radiometer can yield a response time of about one second, which will follow most cloud fluctuations with minimum lag.

\section{The LIRAD Method}

The use of narrow beam filter radiometers with lidars goes back some considerable time. The CSIRO Mark I radiometer was designed and constructed in 1970 (Platt 1971). Since then, improved versions (Mark II and Mark 111) have been constructed (Platt et al. 1987). Using the LIRAD method, much information has been obtained on the optical properties of cirrus (e.g., Platt et al. 1987, Platt and Harshvardhan 1988), as well on midlevel clouds (e.g., Platt and Bartusek 1974, Platt et al. 1978).

Basically the LIRAD method is a means of obtaining two independent pieces of information on clouds, one in the visible spectrum and one in the infrared. In principle, using the theoretical relationship between visible and infrared optical properties, one can then determine the total spectral radiative properties of the clouds, to an accuracy which is quite acceptable at present, as LIRAD has proven to be the only reliable method of obtaining such data.

In LIRAD, the infrared radiometer measures the cloud radiance continuously in a narrow region of the spectrum (10.84 $\pm 1 \mu \mathrm{m}$ in the Mark I to Mark III radiometers. Mark II and III also had capability for 8-13 $\mu \mathrm{m}$ measurements). Using suitable spectral data combined with radiosonde ascents, the cloud radiance is corrected for atmospheric transmittance and emission from water vapour, ozone, 
and carbon dioxide. The cloud infrared emittance is then calculated from the lidar profile using coincident radiosonde data. The cloud infrared absorption coefficient is assumed to be proportional to the lidar backscatter coefficient and, using a radiative transfer equation, the constant of proportionality is adjusted until the observed and computed radiances are equal. This process produces both averages and profiles of absorption coefficient and emittance.

A plot of the integrated attenuated lidar backscatter coefficient versus infrared emittance also gives a numerical value of the isotropic backscatter to extinction ratio, modified by multiple scattering, which can then be used to retrieve the true profile of cloud backscatter coefficient. The process is then iterated to retrieve optimum values of infrared emittance. The method also gives a figure for the ratio between visible extinction and infrared absorption coefficients. This can be compared with theoretical predictions using different particle sizes (e.g., Platt 1979). A plot of infrared absorption coefficient against mid-cloud temperature is shown in Figure 1, the result of many observations on cirrus clouds taken over one year.

A polarization diversity lidar also gives data on depolarization ratios, which in turn give information on the extent of cloud glaciation. A comparison of past data on mid-latitude and tropical clouds forming at low temperatures (Platt et al. 1987) indicates consistent differences between

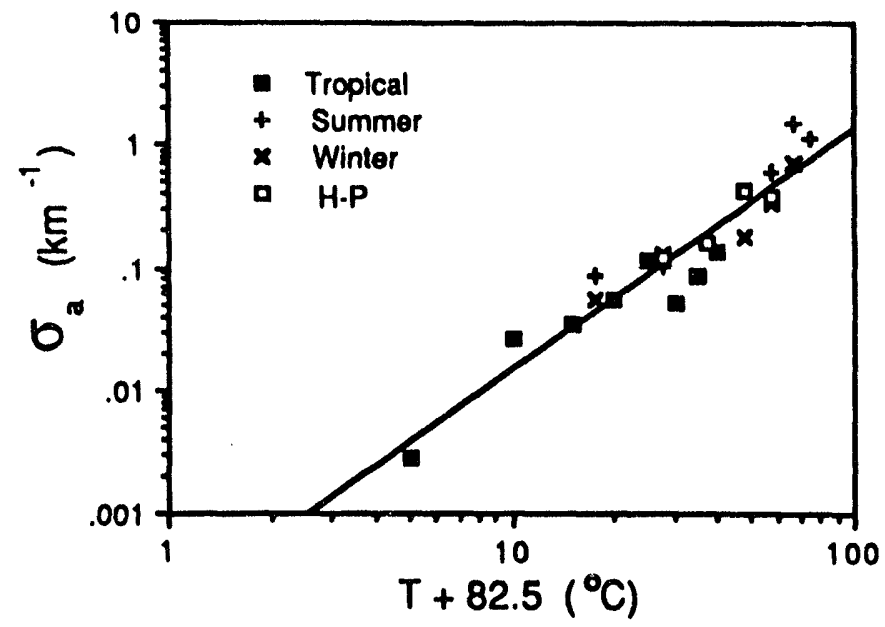

Flgure 1. The variation in average infrared absorption coefficient $\left(\sigma_{a}\right)$ at $10.84 \mu \mathrm{m}$ wavelength with midcloud temperature $(T)$ for a year's data on mid-latitude cirrus (after Platt and Harshvardhan 1988). values of depolarization ratio $\Delta$, with tropical clouds giving the lower values $\left(\Delta=0.3\right.$ at $\left.-60^{\circ} \mathrm{C}\right)$ compared with midlatitude clouds $\left(\Delta=0.4\right.$ at $\left.-60^{\circ} \mathrm{C}\right)$. This aspect requires further investigation.

\section{The Use of LIRAD in ECLIPS}

The use of LIRAD is illustrated briefly in terms of some data obtained recently in the Experimental Cloud Lidar Pilot Study (ECLIPS) project. (See WCRP 1988).

Figures 2 and 3 show, respectively, the cloud boundaries and plot of integrated lidar backscatter versus infrared emittance for an altocumulus cloud observed during ECLIPS2. Such data are being analyzed further at present to obtain the optical properties of different cloud types observed.

\section{Improved Filter Radiometer}

An improved narrow-beam fast filter radiometer has been developed for use with the LIRAD method, or similar observations, in the ARM Program. The characteristics of this radiometer are shown in Table 1.

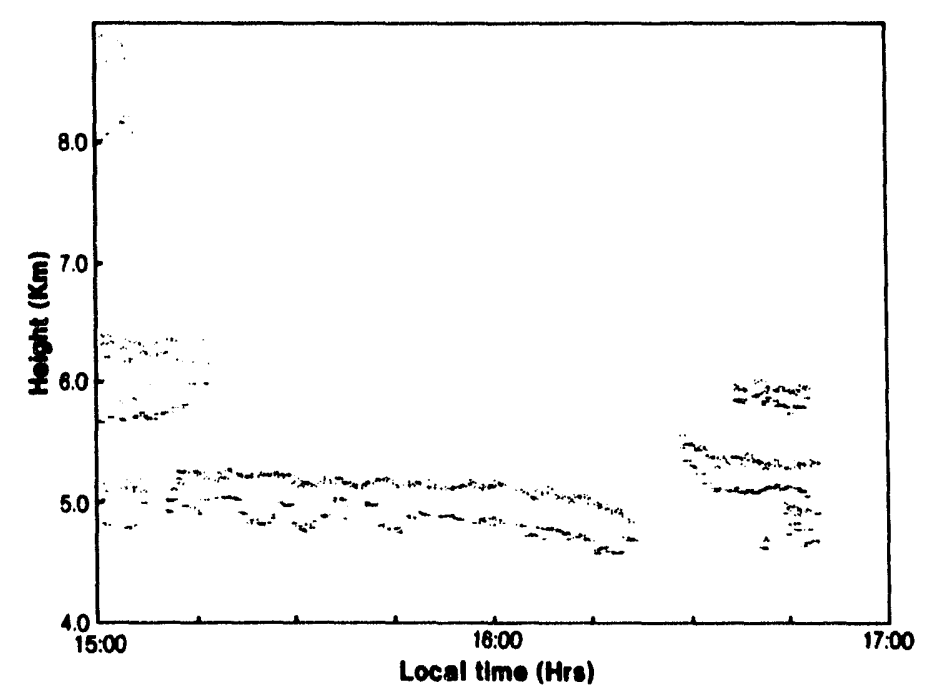

Flgure 2. Cloud boundaries retrieved from lidar data for an altocumulus cloud on 20 June 1991, during ECLIPS. 


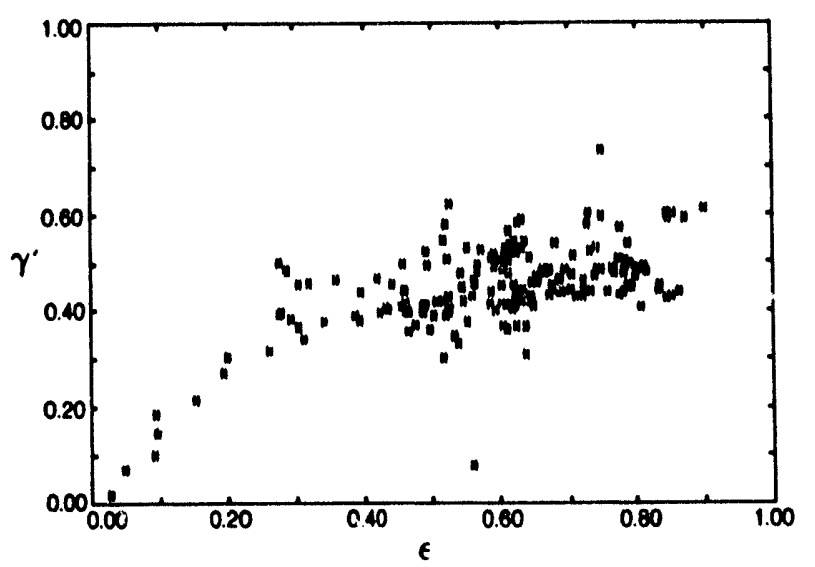

Figure 3. Integrated lidar backscatter $\left(\gamma^{1}\right)$ plotted against infrared emittanco $(\varepsilon)$ at $10.84 \mu \mathrm{m}$ for the same cloud as Figure 2.

A schematic of the optical lay-out of the instrument is shown in Figure 4. The optics comprises a simple Newtonian system with a primary mirror diameter of $5 \mathrm{~cm}$ and a primary focal length of $25 \mathrm{~cm}$. After the field aperture, the radiation is collimated by an off-axis paraboloid, passes through a filter, and is focused finally on the $\mathrm{HgCdT} \theta$ detector. In the primary aperture, a chopper at $200 \mathrm{~Hz}$ chops the input radiation against a blackbody which occupies half the aperture and is controlled at $40^{\circ} \mathrm{C}$. This method of chopping is inherently very stable, as the chopper blades give only an unchopped contribution to the

Table 1. Characteristics of the ARM narrow-beam radiometer.

Focal length

$250 \mathrm{~mm}$

Primary mirror diameter

$50 \mathrm{~mm}$

Effective aperture

$9.05 \times 10^{2} \mathrm{~mm}^{2}$

Detector

Field aperture

$\mathrm{HgCdTe} 1 \mathrm{~mm}$ sensor

3 to $30 \mathrm{mR}$
Spectral channels
(1) $8.62 \pm 0.40 \mu \mathrm{m}$
(2) $10.86 \pm 0.50 \mu \mathrm{m}$
(3) $12.04 \pm 0.55 \mu \mathrm{m}$

Minimum detectable radiance $2 \times 10^{-3} \mathrm{Wm}^{-2} \mathrm{sr}^{-1} \mathrm{hz}^{-1 / 2}$ radiation at the detector. The aperture consists of a variable iris which effectively varies the field aperture from 3 to $30 \mathrm{mR}$.

A 45 degree gold-plated mirror at the input allows vertical or slant-path viewing and also rotates through 180 degrees to view a liquid nitrogen blackbody source or through 90 degrees to view an ambient temperature blackbody. The radiometer is fully computer-controlled, and data are recorded automatically on disk.

The use of three spectral regions across the 8-13 $\mu \mathrm{m}$ atmospheric window region allows investigation of the cloud characteristics over a region where the refractive indices of ice and water both vary, but in a different manner, thus allowing additional information on cloud phase and particle size, at least when the cloud is semitransparent.

\section{The ARM Pilot \\ Radiation Observation Experiment (PROBE)}

The Division of Atmospheric Research took part in ARM's TOGA COARE(a) PROBE experiment at Kavieng, Now Guinea $\left(2.5^{\circ} \mathrm{S}, 150.8^{\circ} \mathrm{W}\right)$ in January - February, 1993. This afforded an opportunity to use the new radiometeralongside the CSIRO Mark II radiometer in a direct comparison. The Division's $0.532 \mu \mathrm{m}$ lidar was also used, and the data obtained on cirrus clouds, as well as some altocumulus, will be analyzed with the LIRAD method. The PROBE will also provide excellent radiosonde data every six hours, together with continuous microwave data of water vapour column and cloud liquid water column observations from the National Oceanic and Atmospheric Administrations's (NOAA) Wave Propagation Laboratory (WPL). The water vapour column data will be invaluable in allowing for any variations in water vapour radiance and transmittance at times between radiosonde observations.

A preliminary analysis of the data indicates the variability of the cirrus and its considerable geometrical depth at times and also the persistence of the cirrus cover.

(a) Troplcal Ocoan Global Atmosphere/Coupled Ocean Atmosphere Response Experiment. 


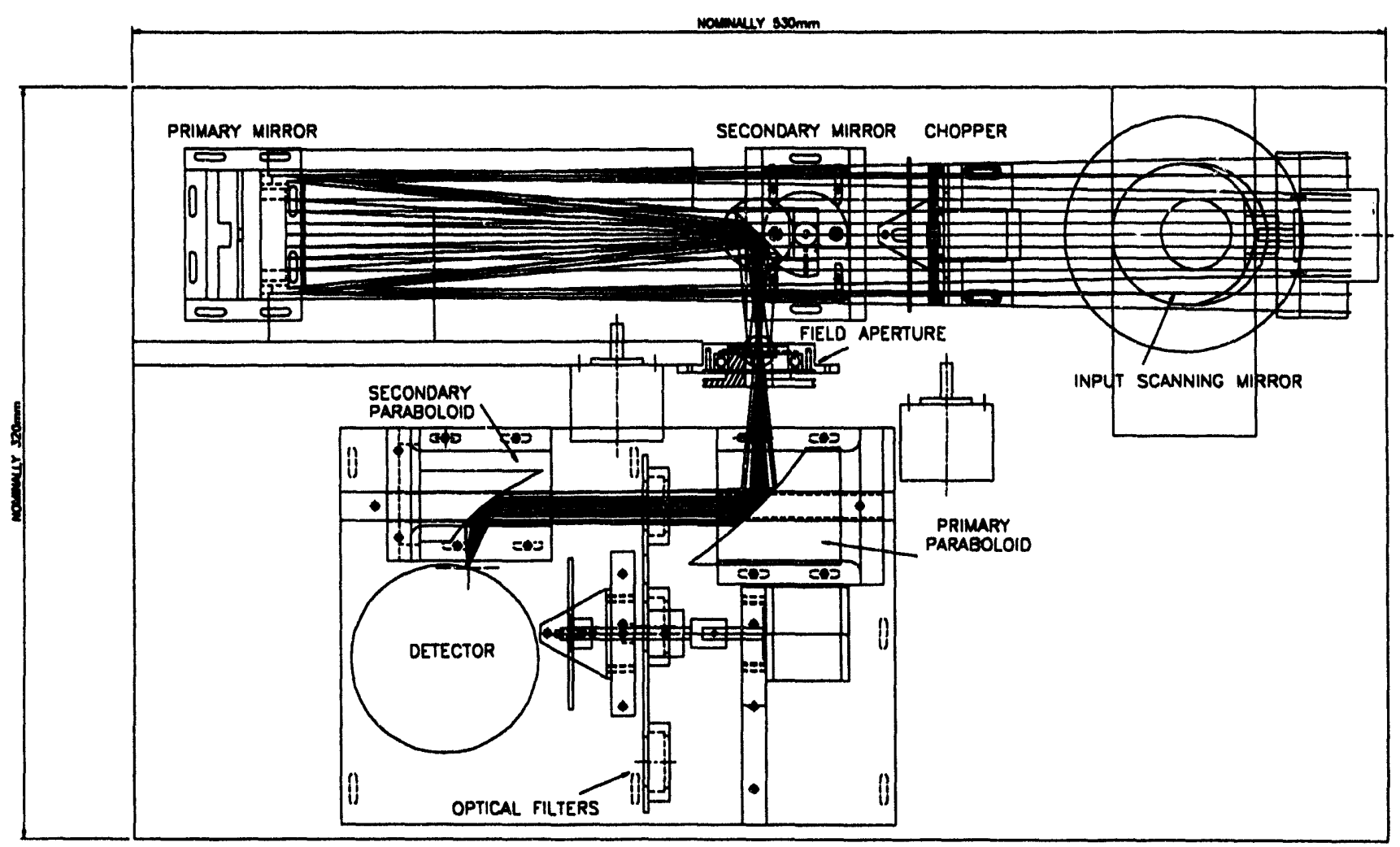

Figure 4. Schematic of the CSIRO/ARM filter radiometer optical system.

Particularly interesting was an apparent diurnal variation in both the cirrus cover and the optical depth with a maximum at about midday.

The ARM filter radiometer was run for about $70 \%$ of the time on the $8.62 \mu \mathrm{m}$ filter; however, for some periods, the radiometer was run with the $10.86 \mu \mathrm{m}$ filter enabling a direct comparison with the Mark if radiometer which used a $10.84 \mu \mathrm{m}$ filter. Such a period is shown in Figure 5. As the input radiance is chopped against a $40^{\circ} \mathrm{C}$ blackbody, the zero radiance when viewing liquid nitrogen actually gives a large negative signal; whereas, the zero voltage occurs when the input radiance is from a $40^{\circ} \mathrm{C}$ blackbody. The responses of the two radiometers to various clouds are quite evident. The water vapour radiance is large, which is typical for the tropics. Periodically, there are either cirrus radiances or larger cumulus radiances superposed.
Also evident is the superior behaviour of the new ARM radiometer. The two radiometer apertures were equal in the comparison; however, the ARM and Mark II timeconstants were 1 second and 5 seconds, respectively. By looking at the signal and noise levels during the calibration episodes in more detail, we calculate the minimum detectable radiances (MDR) of the two instruments as 4.9 $\times 10^{-3} \mathrm{Wm}^{-2} \mathrm{sr}^{-1} \mathrm{hz}^{-1 / 2}$ (ARM) and $6.8 \times 10^{-2} \mathrm{Wm}^{-2} \mathrm{sr}^{-1} \mathrm{hz}^{-1 / 2}$ (CSIRO Mark II). The former is about twice the predicted value in Table 1.

A lidar profile of tropical cirrus is shown in Figure 6. This shows a typical deep layer extending through $6 \mathrm{~km}$ altitude.

Analysis of the data will provide a valuable comparison data set for future ARM CART observations in the tropical west Pacific region. 

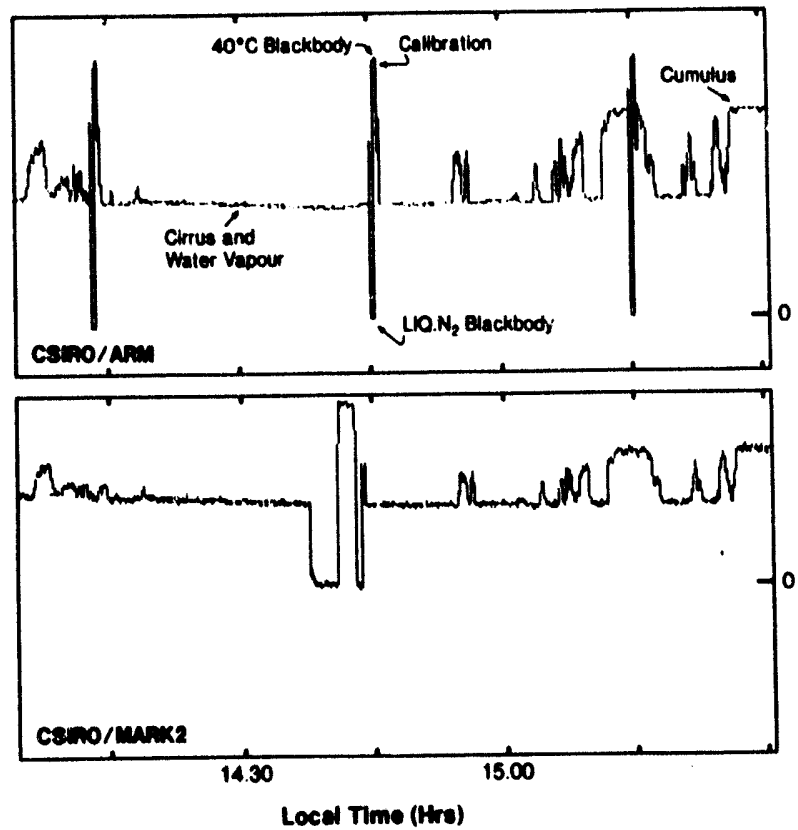

Figure 5. Comparison of the signal and noise output from the CSIRO Mark II and ARM radiometers for an observation period during PROBE. As the radiance is chopped against a $40^{\circ} \mathrm{C}$ blackbody, maximum negative signal is obtained when viewing a liquid nitrogen blackbody.

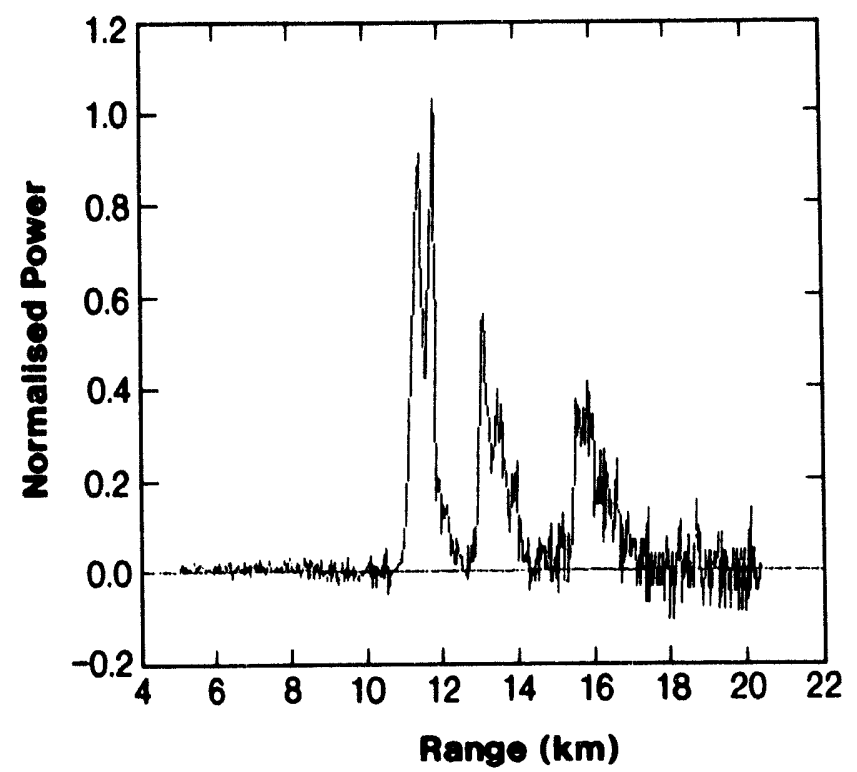

Figure 6. Lidar backscatter profile of tropical cirrus during PROBE.

\section{References}

Platt, C.M.R. 1971. A narrow-beam radiometer for atmospheric radiation studies. J. Appl. Moteorol. 10:1307. 1313.

Platt, C.M.R., and K. Bartusek. 1974. Structure and optical properties of middle-level clouds. J. Atmos. Scl. 31:10791088.

Platt, C.M.R., N.L. Abshire, and G. T. NcNice. 1978. Some micro-physical properties of an ice cloud from lidar observation of horizontally oriented crystals. J. Appl. Meteorol. 17:1220-1224.

Platt, C.M.R. 1979. Remote sounding of high clouds: 1: Calculation of visible and infrared optical properties from lidar and radiometer measurements. J. Appl. Moteorol. 18:1130-1143.

Platt, C.M.R., J. C. Scott, and A. C. Dilley. 1987. Remote sounding of high clouds. Part VI: Optical properties of midlatitude and tropical cirrus. J. Atmos. Sci. 44:729-747.

Platt, C.M.R., and Harshvardhan. 1988. Temperature dependence of cirrus extinction: Implications for climate foedback. J. Geophys. Res. 93:11051-11058.

World Climate Research Programme (WCRP). 1988. An experimental cloud lidar pilot study. Report of the WCRP/ CSIRO cloud base measurement workshop. CSIRO, Mordialloc, Australia. Feb 28 - Mar 3, 1988. WCRP-15 WMO/TD - No. 254, CSIRO, Division of Atmospheric Research, Private Bag No. 1, Mordialloc, Victoria, Australia. 


\title{
Aerosol Characterization Using Stellar Imagery
}

\author{
P. G. Weber \\ Los Alamos National Laboratory \\ SST-9, MS D436 \\ Los Alamos, NM 87545
}

\section{Introduction}

The objective of this project is to svaluate the feasibility of using perturbed stellar images (moditied by atmospheric scattering and absorption) for characterizing atmospheric solid and liquid aerosols (particles and clouds). The beseline boundary conditions for the study included the potential use of one or more solid-state camera arrays for nighttime, multi-spectral observations.

We begin by describing the rationale for aerosol measurements, including nighttime applications. We then describe a versatile Monte Carlo code that we have used to compute scattering by atmospheric aerosols. Several examples of applications of interest to the Atmospheric Measurement Radiation (ARM) Program will be briefly described. Finally, we present our conclusion: our computations indicate a limited applicability for stellar imagery in aorosol studies, requiring only relatively simple instrumentation.(a)

\section{Aerosol Scattering and Its Relevance to ARM}

A major goal of the ARM Program (DOE 1990) is to improve the understanding and treatment of radiative forcing and feedbacks in global circulation models (GCMs). Focal points of these efforts are the spectral dependence of the radiation budget and the radiative properties of clouds.

Aerosols contribute to these issues both through their direct modification of the radiation balance and through

(a) This project is funded by the DOE Atmospheric Radiation Measurement instrument Devolopment Program (ARMMP), Dr. Jeff Griffin, program monitor. This report serves to document progress from funding covering May to September 1992. their effects on the formation and dynamics of clouds. The ARM Instrument Development Program (IDP) has recognized the importance of these issues, and several instrument development activities address measurement of direct and diffuse sunlight, solar irradiance attenuation, solar aureole measurements, all-sky imaging, and cloud dynamics. The interpretation of data from these and other instruments will yield some of the atmospheric radiative transport data needed by ARM.

The (daytime) solar intensity and solar aureole have been measured for decades and have been used to extract aerosol information. Frequently, the aerosol size distribution is described by a simple parameterization to allow inversion of the data. Detailed computations of aerosol scattering also date back many years.

Direct combinations of detailed experiments with sophisticated modeling are less common; this program is thus expected to make significant contributions to the state of the art. We have looked in some detail at the solar aureole measurements of Green et al. (1971), who provided absolutely calibrated data. These data can be simulated by our code, thus providing a consistency test. We further use these data to check scaling of stellar and lunar irradiances.

The nighttime aerosol distributions are also important. First, nighttime aerosol data provide continuity of measurement and improve the understanding of aerosol dynamics. Second, the formation and dynamics of clouds continue through the night and are affected by aerosol distributions. Third, stellar measurements allow the simultaneous use of a multitude of sources and, thus, allow better spatial distribution measurements.

Astronomers are very familiar with the effects of "seoing" induced by atmospheric aerosols. In clear sky conditions, the naked eye may not be able to discern an aureole about a star; however, photographs of stars do show noticeable 
aureoles. Quantitative estimates of the effects of haze on stellar images were provided many years ago by van de Hulst (1949). Hestates that ecattering by aerosols dominates images from a 36-inch diametor telescope from at least four minutes of are through fifteen degrees from the star.

Potential meteorological messurements of stars in the 1990 s operate in a somewhat different regime than the photographic imaging performed in the 1940s. We would use solid state detectors with linear intensity response, excellent wavelength response, and digital processing to make quantitative measurements in timescales of order minutes at the receiver plane of a relatively simple optical system.

\section{The MIE-2 and FLASH Computer Codes}

We have chosen to use the FLASH Monte Carlo code to solve the light-scattering problem. This code is adescendant of the program written by Collins et al. (1972). The modern version runs on a Cray and on the Space, Science, and Technology (SST) Division Convex computers and has more than sufficient accuracy for our applications.

The version we have adopted incorporates the curvature of the earth and allows up to fifty aerosol layers and insertion of model clouds if desired. The code is designed to allow computations of twilight situations, where the illuminating source is below the horizon, but still is seen in the atmosphere. FLASH includes measured Rayleigh scattering coefficients as a function of wavelength and temperature, molecular absorption, and a set of Stokes parameters generated through a separate Mie code, which uses a single complex index of refraction at each wavelength. The Mie theory gives accurate solutions to scattering from dielectric spheres and has been well tested against laboratory measurements. Aerosol collections from aircraft show nonspherical particles, particularly near urban areas. However, simulations using assumed spherical particles have been successful in the past. Furthermore, the condensation of water on aerosols leads to the formation of quite spherical scatterers.

Our standard inputs to the code to date include the standard rural aerosol size distributions given by Shettle and Fenn (1979), and the continental, winter, 30 degrees latitude atmospheric density, temperature and humidity profiles from the Air Force Geophysics Laboratory handbook (1985). The vertical aerosol distribution takes the form suggested by Eltermann (1968), with the loweot layer chosen from the surface visibility at a wavelength of $0.55 \mu \mathrm{m}$. Surface reflectivity is assumed to be angularly uniform.

\section{Some Applications of the Codes to ARM- Relevant Situations}

We have, to date, generated 72 sets of angular contours in spectral brightness and percent polarization at four wavelengths $(0.4,0.55,0.86$, and $1.06 \mu \mathrm{m})$, six humiditios (relative humidity of $0,0.5,0.9,0.95,0.98$ and 0.99 ) and three ground-lovel visibilities $(5,23$ and $50 \mathrm{~km})$ with a surface albedo of 0.2 .

In addition, we have successfully compared the code output for an aerosol-free atmosphere (Rayleigh scattering and absorption only) to the exact results of Coulson of al. (1970). We have also investigated the use of the almucantar contour of sky brightness towards extraction of the ecattering function (the intensities are independent of vertical aerosol distribution for this case). These calculated skies do not represent all the aerosol conditions that would be encountered; however, the tested visibilities likely cover much of both the visibility and humidity ranges that would be expected.

Some general observations on these results are as follows. The direct solar, lunar or stellar transmission is measurable with reasonable instrumentation and shows good sensithity to higher humidities. We also see a reverse trend of transmission with humidities at two selected wavelengths $(0.4$ and $0.86 \mu \mathrm{m})$, suggesting the efficacy of a ratio measurement. For the aureole measurements, we 890 that useful data may be obtained between 0.3 degrees (the extent of the solar [and lunar] discs) and 3 degrees, where the data for various conditions coalesce; better discrimination appears at smaller angles. For polarizations, the major variability occurs at high humidities; polarization differences are generally too small for reasonable instrumentation to measure. Clearly, one gains an advantage from making multiple measurements (e.g., direct intensity, aureole intensity, and polarization at a number of well-chosen wavelengths). 
Our computations have allowed us to quantify possible stellar measuremonts. The low light levels of stars limit our messurement capabilities to direct transmission and intograbed aureole data. This loads us to suggest a relatively straightforwand cotical system with simple solid state detectors; budgets permitting, we would like to consider bullding a prototype.

Other applications to ARM are suggested. Oak Ridge National Laboratory (ORNL) and Los Alamos National Laboratory (LANL) would like to use LANL modeling support to interpret data obtained by the ORNL pointing shortwave/near-IR radiometer. Taking the actual specifications for the radiometer, we can run a set of representative serosol scenarios to cover the range of the instruments applicability. We could, if desired, construct a look-up table to relate instrument signals to the most likely aerosol distributions.

Further, we have working versions of the high spectral resolution HITRAN database, as well as LOWTRAN and MODTRAN. These can be used to determine spectral regions of interest. Using these codes in conjunction with the inversion techniques described by Travis Spratlin (ORNL) should lead to definition of an excellent set of wavelengths. Interpretations of daytime data from the ARMCART all-sky camera(s) could proceed along lines very similar to the above.

With regard to investigations of the "Umkaehr" problem, Lee Harrison (State University of Albany) has suggested obtaining vertical distributions of aerosols at twilight. This is a natural application for the codes; indeed, FLASH was written with precisely this capability. The possibility of obtaining vertical aerosol profiles (even only maximally twice a day), but with reasonably simple instrumentation, should be of interest. Dr. Harrison has also suggested that a study of multiple scattering in clouds would be useful.

Computing the relative importance of multiple scattering compared with single scattering would shed light on the quality of the single-scattering assumption made in some analyses and candefine the range of reasonable application of this simplification.

Other computational and science support can be provided to ARM through the Aerosols Group or other mechanisms. The Aerosols Group's 1992 report delineates the desire for many measurements of aerosols: our codes can be applied to modeling some cases of interest. Finally, ARMCART is now implementing some serosol measurement capabilities. We have contacted Robert Leifer to initiate discussions on possible collaborations.

\section{Conclusions}

We have brought a new modeling system to the ARM Program, namely, a Monte Carlo code for atmospheric aerosol scattering calculations. A number of applications of the programs are immediately obvious and can be pursued, depending on funding levels. We continue to work with ARM Science Team members to define and pursue problems of interest.

Our first sets of calculations also point to a potential now instrument for ARM/IDP, namely, a simple stellar observation system. The proposed instrument may be as simple as a collection of well-baffled tubes, with spectral filters and reasonable photodiode detectors. The instrument would measure direct irradiance reductions by the atmosphere, as well as the integrated aureole light over a range of angles. While probably not definitive, such an instrument would provide accessible nighttime aerosol data.

\section{References}

Airforce Geophysics Laboratory Handbook of Geophysics and the Space Environment. 1985. NTIS AD/A167, National Technical Information Service, 5285 Port Royal Rd., Springfield, Virginia 22168.

Collins, D. G., W. G. Blattner, M. B. Wells, and H. G. Horak. 1972. Backward Monte Carlo Calculations of the Polarization Characteristics of the Radiation Emerging from Spherical Shell Atmospheres. Appl. Opt. 11:2684.

Coulson, K. L., J. V. Dave, and Z. Sekera. 1970. Tables Related to Radiation Emerging from a Planetary Atmosphere with Rayleigh Scattering. University of California Press, Berkeley.

Elterman, L. 1968. UV, Visible and IR Attenuation for Altitudes to $50 \mathrm{~km}$. AFCRL-68-0153. ADA671933, Office of Aerospace Research, Hanscomb Airforce Base, Bedford, Massachusetts. 
Green, A.E.S., A. Deopak, and B. J. Lipostsky. 1971. Interpretation of the Sun's Aureole Based on Atmospheric Aureole Models. Appl. Opt. 10:1263.

Shettle, E. P., and R. E. Fenn. 1979. Models of the Atmospheric Aerosols and the Effects of Humidity Variation on Their Optical Properties. AFGL TR-79-0214, ADA 085951, Air Force Geophysics Laboratory. Hanscomb Aifforce Base, Bedford, Massachusetts.
U.S. Department of Energy (DOE). 1990. Atmospheric Radiation Moasurement Program Plan. DOE/ER-0441, Washington, D.C.

van de Hulst, H. C. 1949. Scattering in the Atmospheres of the Earth and Planets. Chap. III of The Atmospheres of the Earth and Planets, ed. G. P. Kuiper. University of Chicago Press, Chicago. 
Remote Sensing 


\title{
Laser Remote Sensing of Water Vapor: Raman Lidar Development
}

\author{
J.E.M. Goldsmith, M. Lapp, and S. E. Bisson \\ Sandia National Laboratorles \\ Livermore, CA 94551 \\ S. H. Molfi and D. N. Whiteman \\ NASA Goddard Space Flight Center \\ Greenbelt, MD 20771
}
R. A. Ferrare and K. D. Evans
Hughes STX
Lanham, MD 20706

\section{Introduction}

The goal of this research program is the development of a critical design for a Raman lidar system optimized to match Atmospheric Radiation Measurement (ARM) Program needs for profiling atmospheric water vapor at Cloud and Radiation Testbed (CART) sites. This work has emphasized the development of enhanced daytime capabilities using Raman lidar techniques, but the evolution of the ARM Program has led us to investigate other characteristics as well, in order to provide a range of options for implementing a Raman lidar system at CART sites.

Raman lidar systems detect selected species by monitoring the wavelength-shifted molecular return produced by Raman scattering from the chosen molecule or molecules. For water-vapor measurements, the nitrogen Ramansignal is observed simultaneously with the water-vapor Raman signal; proper ratioing of the signals yields the water-vapor mixing ratio. Raman lidar is used currently to perform meteorologically important, sustained, reliable nighttime profiling of water vapor. Daytime measurements present added challenges because of the difficulties inherent in detecting Raman signals against solar backgrounds. One approach to overcome this problem is to operate in the solar blind region of the spectrum (wavelengths shorter than $\sim 285 \mathrm{~nm}$ ). This approach effectivoly reduces the solar background, but attenuation of the laser beam and the backscattered Raman radiation (primarily due to absorption by tropospheric ozone) greatly reduces the signal also. Alternatively, the background skylight reaching the detector can be reduced by using a narrow field-of-view receiver and narrowband spectral detection. We have pursued both of these approaches in our studies.

This abstract touches briefly on the main components of our research program, summarizing the results of these efforts, and provides references for more detailed information.

\section{Nighttime Measurements}

Two advanced lidar systems have been used to quantify the "real-world" capabilities of Raman lidar systems for profiling atmospheric water vapor in a variety of field campaigns. Two such campaigns are especially relevant to the ARM Program. Shortly after the second ARM Science Team Meoting in October 1991, the Raman lidar system developed at the NASA Goddard Space Flight Conter participated in the FIRE/SPECTRE(a) Fiold Campaign at Coffeyville, Kansas, from November 12 until

(a) First ISCPP (International Satellite Cloud Climatology Project) Regional ExperimentSpectral Radiance Experiment. 
December 7, 1991 (Melfi et al. 1992). The high-quality measurements produced by this system, and the reliability of its operation, provide a striking demonstration of its utility in this "mini-CART" environment.

Detailed evaluation of the performance of Raman lidar systems (or lidar systems in general) is complicated by the lack of an appropriate instrument that can provide accurate measurements for intercomparison purposes. Several studies have demonstrated the limitations of radiosondes for such evaluations. To provide a more rigorous test of Raman lidar capabilities, the system developed at Sandia National Laboratories was collocated with the Goddard system at the Goddard Space Flight Center in Greenbelt, Maryland; a number of intercomparisons were performed from October 26 through November 20, 1992. These intercomparisons, using lidar systems based upon different excitation wavelengths and two different formats of detection packages, resulted in water-vapor profiles of remarkable similarity. They provide a striking confirmation of the precision of Raman lidar systems for profiling atmospheric water vapor (Goldsmith ot al. 1993a; Goldsmith of al. submitted).

\section{Performance Modeling}

We have developed a detailed Raman lidar instrument model to predict the daytime and nighttime performance capabilities of Raman lidar systems. The model simulates key characteristics of the lidar system, using realistic atmospheric profiles, modeled background sky radiance, and lidar system parameters based on current instrument capabilities. The model operates by tracking photons through the atmosphere and the instrument, incorporating atmospheric attenuation (due to Rayleigh and aerosol scattering and absorption by ozone and oxygen) at all of the wavelengths of the Raman lidar process.

We have used this model to guide our development of lidar systems based on both the solar-blind concept and the narrowband, narrow field-of-view concept for daytime optimization. The model, assumed lidar parameters, and representative results are presented in Goldsmith and Ferrare (1992) and Goldsmith et al. (1993b). Briefly, ranges of $3-4 \mathrm{~km}$ with $75-\mathrm{m}$ range resolution and $\sim 10$-minute counting times are predicted for systems based on both concepts using large (but commercially available) laser systems and telescopes.

\section{Laboratory Studies}

Our model calculations indicate that the optimum excitation wavelength for the solar-blind concept is in the range 260. $266 \mathrm{~nm}$ (Goldsmith and Ferrare 1992; Goldsmith of al. 1993b). Unfortunately, this wavelength range lles between the two wavelengths most readily obtained from highaverage-power laser systems, namely $248 \mathrm{~nm}$ from KrF lasers and $308 \mathrm{~nm}$ from XeCl lasers. Although 266-nm radiation can be produced by frequency-quadrupling the output of Nd:YAG lasers, current (flashlamp-pump) technology limits the output power of these systems to substantially lower values.

Our laboratory research has therefore emphasized the development of the Raman-shifting technology necessary to wavelength-shift the output of KrF lasers into the 260- to 266-nm range. In particular, we have performed a comprehensive study of a KrF pumped, nitrogen Raman shifter with emphasis on optimizing the first Stokes conversion efficiency at $263 \mathrm{~nm}$ (Bisson et al., 1992; Bisson, submitted). First Stokes conversion efficiencies as high as $12 \%$ were achieved in nitrogen:hellum mixtures. Further improvements were achieved by seeding the Raman coll with the backward first Stokes radiation. Experiments were also conducted with a frequency-doubled Nd:YAG laser as the pump source. These experiments showed that conversion efficiencies as high as $20 \%$ might be obtainable with a KrF laser if the beam quality were improved.

\section{Daytime Capabilities}

We have pursued two concepts for extending the daytime capabilities of Raman lidar. As described above, for the solar-blind approach, model calculations suggest an optimum excitation wavelength in the 260- to 266-nm range. To explore solar-blind operation concurrent with our wavelength-shifting studios, we performed measurements using the 248-nm output of a KrF excimer laser (Whiteman et al. 1993). Measurements to a range of 2.5-3.0 km were obtained using ozone profiles provided by electrochemical cell radiosondes (ozone profiles are needed to correct the experimentally observed water vapor and nitrogen signals for the different absorption coefficients at the two wavelengths). The range we are able to obtain and our ability to adequately derive ozone 
profiles directly from the lidar data both appear to be limited primarily by afterpulsing in our photomultipliers; we are currently investigating methods to avoid this difficulty.

We have also investigated the narrowband, narrow fieldof-view approach using $308-\mathrm{nm} \mathrm{XeCl}$ laser excitation (Bisson and Goldsmith 1993). Preliminary measurements have demonstrated measurements to a range of $\sim 3 \mathrm{~km}$ even under relatively dry conditions during mid-afternoon with a clear sky. Recent modifications to the detection system should enable a further reduction in the field-ofview, providing further enhancement in the daytime capabilities of the system.

\section{Conclusions}

We are currently acquiring an additional set of daytime measurements using the modified narrowband, narrow field-of-view system, and comparing our measurements (both daytime and nighttime) to our computer model. We have also started the final phase of the program, in which we integrate all of our results into a design plan that displays a range of options for implementing a Raman lidar system at CART sites. In these design options, we include the capabilities of Raman lidar systems for measuring other physical parameters, such as aerosol characteristics (Ferrare et al. 1992; Ferrare et al. 1993) and temperature (Ferrare et al. 1990, 1993; Evans et al. 1993). Finally, our designs will incorporate possibilities for measuring additional physical parameters such as cloud phase information from polarization studies, cloud structure from physical scans, and aerosol size information from multiple excitation wavelengths.

\section{References}

Bisson, S. E., J.E.M. Goldsmith, M. Lapp, D. N. Whiteman, R. A. Ferrare, and S. H. Melfi. 1992. A Parametric Study of an Excimor Pumpod Raman Shifter for Lidar Applications. Conference on Lasers and Electro-Optics, 1992, pp. $462-$ 464. OSA Technical Digest Series, Optical Society of America, Washington, D.C.

Bisson, S. E. A Parametric Study of an Excimer-Pumped, Nitrogen Raman Shifter for Lidar Applications. Submitted to Appl. Opt.
Bisson, S. E., and J.E.M. Goldsmith. 1993. Daytime Tropospheric Water Vapor Profile Measurements with a Raman Lidar. Optical Remote Sensing of the Atmosphere, Technical Digest 1993 5:19-22. Optical Society of America, Washington, D.C.

Evans, K. D., S. H. Melfi, R. A. Ferrare, andD. N. Whiteman. 1993. Tropospheric Temperature Measurements Using a Raman Lidar. Optical Remote Sensing of the Atmosphere, Technical Digest 1993 5:169-172. Optical Society of America, Washington, D.C.

Ferrare, R. A., D. Whiteman, and S. H. Melfi. 1990. Raman Lidar Measurements of Temperature in the Troposphere and Lower Stratosphere. Technical Digest on Topical Meeting on Optical Remote Sensing of the Atmosphere, 1990, Vol. 4, pp. 508-511. Optical Society of America, Washington, D.C.

Ferrare, R. A., S. H. Melfi, D. N. Whiteman, and K. D. Evans. 1992. Raman Lidar Measurements of Pinatubo Aerosols over Southeastern Kansas during NovemberDecember 1991. 16 $16^{\text {th }}$ International Laser Radar Conference, Part 1, pp. 13-16. NASA Conference Publication 3158, National Aeronautics and Space Administration, Washington, D.C.

Ferrare, R. A., S. H. Melfi, D. N. Whiteman, and K. D. Evans. 1993. Coincident Measurements of Atmospheric AerosolProperties and Water Vaporby a Scanning Raman Lidar. Optical Remote Sensing of the Atmosphere, Technical Digest 1993 5:11-14. Optical Society of America, Washington, D.C.

Goldsmith, J.E.M., and R. A. Ferrare. 1992. Performance Modeling of Daytime Raman Lidar Systems for Profiling Atmospheric Water Vapor, $16^{\text {th }}$ International Laser Radar Conference, pp. 667-670. NASA Conference Publication 3158, Part 2, National Aeronautics and Space Administration, Washington, D.C.

Goldsmith, J.E.M., S. E. Bisson, D. N. Whiteman, S. H. Melfi, R. A. Ferrare, and K. D. Evans. Raman Lidar Profiling of Atmospheric Water Vapor: Simultaneous Measurements with Two Advanced Systems. Bull. Am. Met. Soc., submitted.

Goldsmith, J.E.M., S. E. Bisson, D. N. Whiteman, S. H. Melfi, R. A. Ferrare, and K. D. Evans. 1993a. Evaluation of 
ARM Solence Mroting

Raman Lidar Profiling of Atmosphoric Wator Vapor: Simultaneous Measurements with Two Advanced Systoms. Optical Romoto Sonsing of the Atmosphoro, Technical Digest 1993 5:15-18. Optical Society of America, Washington, D.C.

Goldsmith, J.E.M., S. E. Blsson, M. Lapp, S. H. Molff, D. Whiteman, R. Forrare, and K. Evans. 1983b. Raman Lidar Measurements of Atmospheric Water Vapor: Devolopment of a Daytime-Optimized System for the Atmospheric Radiation Measurement Program. Fourth Conterence of Global Change Studies, p. 50-52. American Meterological Society, Boston, Massachusetts.
Molfi, 8. H., D. Whitoman, R. Forrare, K. Evans, J.E.M. Goldemith, M. Lapp, and S. E. Bisson. 1982. Raman Lidar Mesaurements of Wator Vapor and Aorosol/Clouds During the FIRE/SPECTRE Fiold Campaign. 16 $6^{\text {th }}$ International Laser Radar Contorence, Part 2, pp. 663-666. NASA Conforence Publication 3158, National Aoronautics and Spaco Administration, Washington, D.C.

Whitoman, D. N., R. A. Ferrare, S. H. Molfi, and K. D. Evans. 1993. Solar Blind Raman Scattering Measuremonts of Water Vaporusing a KrF Excimer Laser. Optical Remoto Sonsing of the Atmosphere, Technical Digest 19935:165168. Optical Society of America, Washington, D.C. 


\title{
Cloud and Aerosol Characterization for the Atmospheric Radiation Measurement Central Facility: Multiple Remote Sensor Techniques Development
}

\author{
K. Sassen \\ Department of Meteorology \\ University of Utah \\ Salt Lake City, UT 84112
}

\begin{abstract}
Determining the means to characterize the microphysical and radiative properties of the cloudy atmosphere from a Cloud and Radiation Testbed (CART) site has been the central theme of this Atmospheric Radiation Measurement (ARM) Instrument Development Program (IDP) research project. Previous studies have clearly indicated that only a varied combination of remote sensing instruments can provide this sort of information on an extended basis. However, the makeup of the remote sensor ensemble appropriate for a CART site obviously depends on the specific scientific research objectives at a particular locale, as well as logistical ( $\theta .9$. , instrument reliability and safety) concerns.
\end{abstract}

We have taken an approach that specifies two levels of instrument observational capabilities. Using a basic combination of remote sensors, the first level provides for the measurement of the three-dimensional distribution of clouds and aerosols, including their boundaries, type, and phase. In addition to this fundamental characterization of the state of the cloudy atmosphere, the second level addresses more specific measurement categories, such as effective cloud particle size, to help provide fundamental radiative cloud quantities. Although the latter measurements are currently more feasible to obtain from comprehensive remote and in situ field campaigns, we intend to develop, test, and verify these multiple remote sensor techniques 80 that they can be applied to increasing the information content of CART operations (though, we hope, relatively minor component or system additions and improved data analysis algorithms).

To accomplish this process, the University of Utah and the National Oceanic and Atmospheric Administration's (NOAA) Wave Propagation Laboratory (WPL) (see companion papers) participated in major field projects to obtain detailed cloud datasets, along with complementary cloud modeling and empirical studies. We describe our progress to date in our main research areas below.

\section{Polarization Diversity Lidar (PDL) Development}

As reviewed at the start of the ARM Program (Sassen 1991), polarization lidar techniques have unique cloudsensing capabilities, particularly with regard to the unambiguous discrimination of cloud phase and accurate determination of cloud boundaries. Various polarization techniques had not been systematically evaluated in the field, but such evaluations could now be faclitated by modern lidar designs based on high-speed microcomputers for system control and multistream data handling (Sassen 1991).

The design of the state-of-the-art PDL system accommodates the testing of these hybrid techniques, which include simultaneous two-color linear depolarization, variable field-of-view (FOV), and rapid scanning operations. The current PDL specifications are listed in Table 1, which, along with the schematic system diagram of Figure 1, illustrates the main features and advanced capabilities of this versatile instrument. A $33 / 486$ microcomputer system tracks four polarization datachannels at spatial resolutions down to $1.5 \mathrm{~m}$ and at the 10- $\mathrm{Hz}$ pulse repetition frequency (PRF) of the dual-wavelength laser transmitter. This capability not only provides for unequalled resolution of cloud features in space and time, but also permits rapld scanning and variable FOV operations. 
ARM Scienco Mooting

Table 1. Specifications of the two-color PDL system.

\begin{tabular}{|c|c|}
\hline \multicolumn{2}{|l|}{ Operational: } \\
\hline Wavelength (Nd:YAG) & $0.532+1.06 \mu \mathrm{m}$ (simultaneous \\
\hline Peak Energy & $0.45 \mathrm{~J}$ each color \\
\hline Maximum PRF=- & $10 \mathrm{~Hz}$ \\
\hline Pulse Width & $9 \mathrm{~ns}$ \\
\hline Beamwidths - Transmitter & $0.45 \mathrm{mr}$ \\
\hline Recoiver & 0.2-3.8 mr, high-spoed shutter \\
\hline Recoiver Diameter & $30 \mathrm{~cm}$ (2 telescopes) \\
\hline Detectors - Visible & 2, Gated PMTs \\
\hline IR & 2, APDs \\
\hline Maximum Scan Rate & $5^{\circ} \mathrm{s}^{-1}$ \\
\hline \multicolumn{2}{|l|}{ Data Handling: } \\
\hline Number of Channels & 4 (simultaneous) \\
\hline Sample Width (resolution) & $1.5 \mathrm{~m}$ maximum \\
\hline Range Gates & $8 \mathrm{k}$ maximum \\
\hline Pulses Averaged & $1-10+$ \\
\hline Maximum Throughput & $164 \mathrm{k}$ samples/second \\
\hline Digitizer Resolution & 8 bits \\
\hline Storage & $8 \mathrm{~mm}$ video tape \\
\hline \multicolumn{2}{|l|}{ Polarization Properties: } \\
\hline Transmitter & Vert. (Vis) + Horiz. (IR) \\
\hline Receiver & Vert. + Horiz. (Vis + IR) \\
\hline
\end{tabular}

In Figure 2, we provide high-resolution dual-wavolength displays of a cirrus cloud recently probed at our Facility for Atmospheric Remote Sensing (FARS) on the University of Utah campus. Although little difference in returned laser power as a function of wavelength is apparent (aside from signal noise effects), the cirrus depolarization data are unusual in that much lower linear depolarization ratios (8) occur in the upper cirrus layer (at left at $\sim 9.4 \mathrm{~km}$ ) at the $1.06 \mu \mathrm{m}$ wavelength. Recent studies of cumulus and altocumulus show wavelength-dependencies in backscattering resulting from small-particle effects, so it is possible that the upper cirrus layer was composed of quite small $(\sim 1 \mu \mathrm{m})$ ice crystals.

The PDL unit, which is permanently truck-mounted, and supporting passive remote-sensing instruments participated

in the November-December 1991 First ISCCP(a) Regional Experiment (FIRE) intensive field observation (IFO) II field campaign in Coffeyville, Kansas. Here the unique highresolution multichannel PDL data were used to research fundamental cloud processes.

For example, the depolarization data collected over the 5-6 December period revealed highly unusual cirrus cloud properties, including spherical scatterers between $-40^{\circ}$ to $-50^{\circ} \mathrm{C}$, which we concluded was a result of large cloud condensation nuclei of stratospheric volcanic origin, as reported in Sassen (1992). (A variety of supporting in situ and ground-based data have since supported the

(a) Intemational Satellite Cloud Climatology Project. 


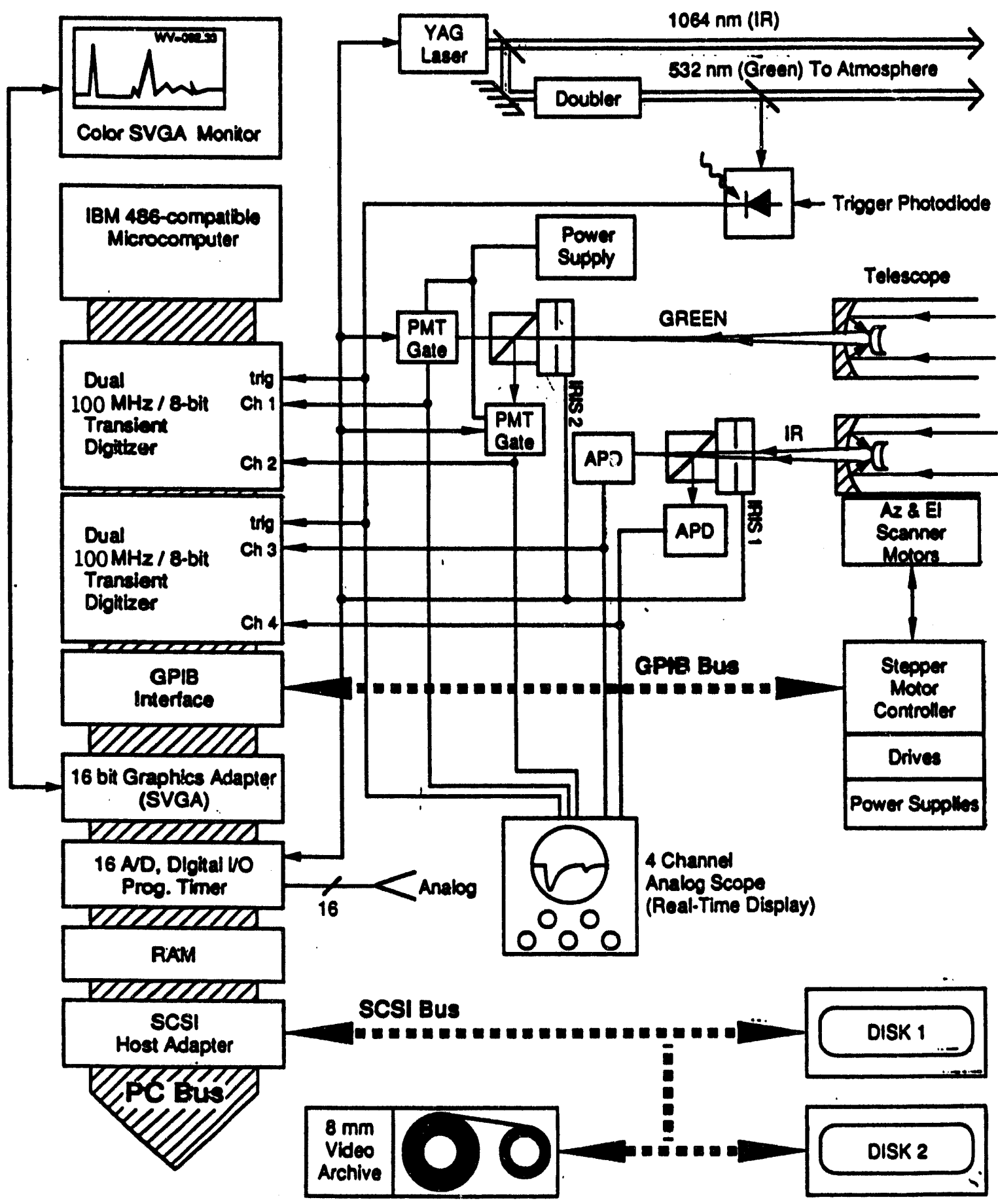

Figure 1. PDL LIDAR control and data ecquisition system. 


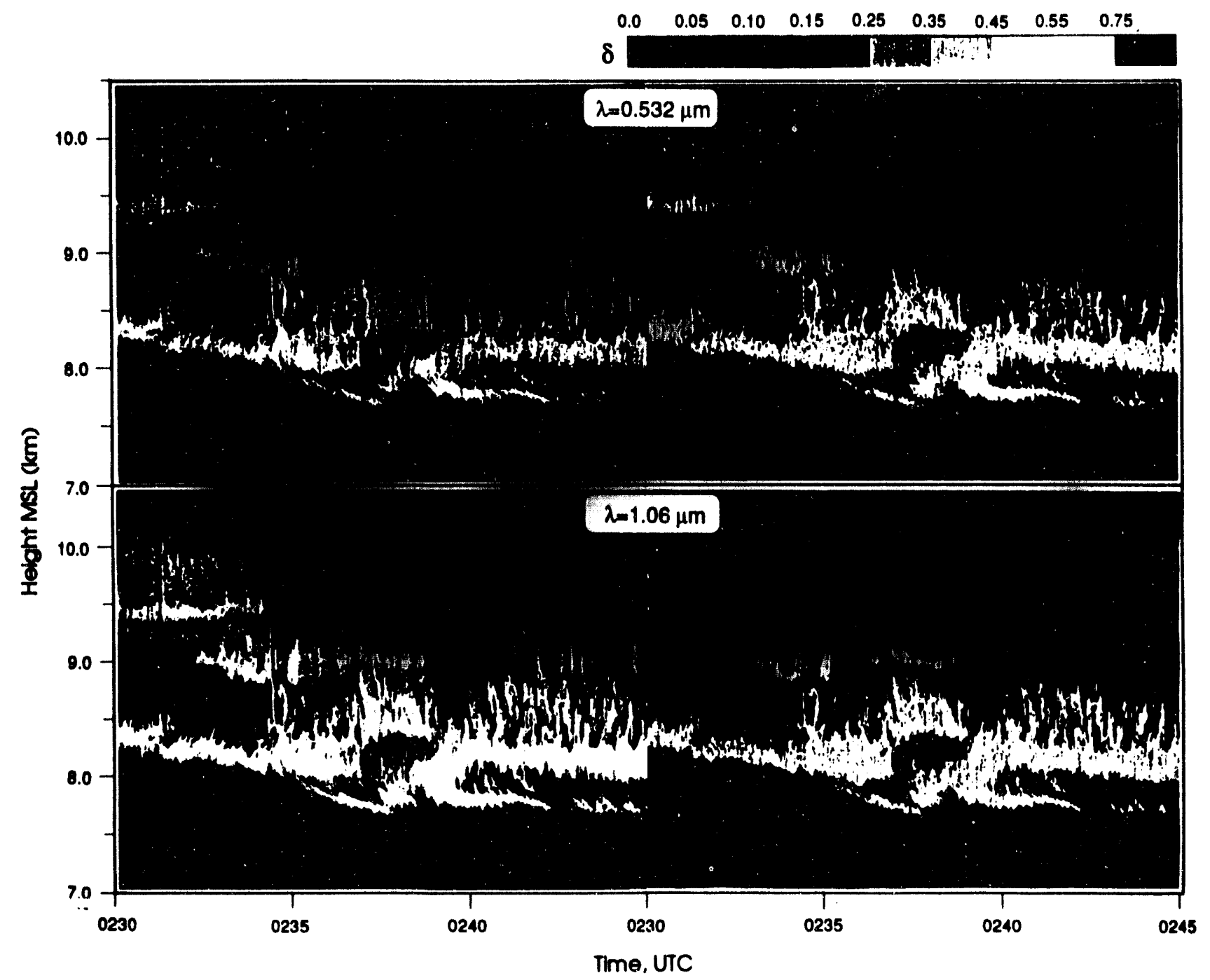

Figure 2. Height-time PDL displays of cirrus clouds sampled from FARS on 9 April 1993, showing the log of returned energy (left) and linear depolarization ratios (right, note $\delta$ scale at top right), at both the $0.532 \mu \mathrm{m}$ (top) and $1.06 \mu \mathrm{m}$ (bottom) wavelengths. Weak noisy signals are removed before $\delta$ values are calculated.

hypothesis that tropopause folding activities associated with a subtropical jet stream introduced stratospheric aerosol into the cirrus clouds.)

NOAA WPL instrumentation participated not only in this field campaign but in two other cloud/radiation field experiments as well: CLARET II (Cloud Lidar And Radar Exploratory Test), and ASTEX (Atlantic Stratocumulus Transition Experiment). In all three experiments, our measurements were augmented by many other instruments including in situ cloud microphysics data from aircraft, 
surface radiation instruments, radiosondes, radar wind profilers, surface meteorological stations, and infrared radiometers and spectrometers. These experiments have provided an excellent opportunity for gathering data and comparing retrieved parameters with other methods to meet our ARM objectives.

\section{Cloud Modeling and Empirical Studies}

We have applied a sophisticated adiabatic cloud growth model, developed previously to study cirrus cloud particle nucleation processes, to simulate remote sensor returns from water, mixed water and ice, and ice phase clouds. This approach not only holds promise for interpretating field data, but also is requisite to evaluating the information contents of various remote sensing techniques.

The model uses detailed microphysical and dynamical equations to generate cloud contents that evolve with height in updrafts, beginning with a specified size distribution of dry cloud condensation nuclei (CCN). These verticallyresolved cloud contents represent a significant advantage over using height-invariant cloud compositions based on limited in situ data, although for some simulation purposes, the use of empirical temperature-dependent cirrus cloud contents are currently justified. We have improved the model so that cloud particle size distributions generated during diffusional growth are now converted to backscatter and extinction coefficients, and returned signal profiles for essentially any lidar or radar system, using subroutines that select Mie or Rayleigh theory for spherical or small (relative to the incidentwavelength) nonspherical particles, or approximations based on geometrical optics ray tracing, conjugate gradient-fast Fourier transform, or empirical methods for ice crystals.

These numerical methods have been used to simulate polarization diversity lidar returns (with a first-order multiple scattering approximation) from water and mixed-phase clouds (Sassen et al. 1992), to develop an autonomous liquid cloud base detection algorithm for analyzing a winter mountain storm polarization lidar dataset (Sassen and Zhao 1993), and to obtain water and ice cloud mass content relationships using radar refleclivity factors (Llao and Sassen, in press). We have also initiated numerical studies aimed at evaluating the special PDL data-gathering capabilities such as the dual-wavelength and variable FOV techniques.

\section{Future Plans}

We will continue to apply cloud modeling and empirical analysis methods to evaluate the multiple remote sensor approach for characterizing the cloudy atmosphere. Recommendations for CART instrumentation to satisfy this objective will include an appropriate combination of microwave radar and lidar polarization techniques, preferably on a single scannable platform to obtain the three-dimensional cloud distribution. We look fonward to taking full advantage of CART facilities through PDL participation in focused intensive observation period studies of important cloud properties.

\section{Publications}

Liao, L., and K. Sassen. In press. Investigation of relationships between $\mathrm{K}_{\mathrm{a}}$-band radar reflectivity and $\mathrm{ice}$ and liquid water contents. Atmos. Res.

Sassen, K. 1991. The polarization lidar technique for cloud research: A review and current assessment. Bull. Am. Met. Soc. 72:1848-1866.

Sassen, K. 1992. Evidence for liquid-phase cirrus cloud formation from volcanic aerosols: Climate implications. Science 257:516-519.

Sassen, K., H. Zhao, and G. C. Dodd. 1992. Simulated polarization diversity lidar returns from water and precipitating mixed phase clouds. Appl. Opt. 31:29142923.

Sassen, K., and H. Zhao. 1993. Supercooled liquid water clouds in Utah winter mountain storms: Cloud seoding implications of a remote sensing dataset. J. Appl. Meteorol. 32:1548-1558. 


\title{
Development of a Dual-Frequency, Millimeter-Wave Cloud Profiling Radar System
}

\author{
R. E. Mclntosh \\ University of Massachusetts \\ Amherst, MA 01003
}

\section{Introduction}

The Microwave Remote Sensing Laboratory (MIRSL) is developing a mobile, scanning millimeter-wave redar for the Atmospheric Radiation Measurement (ARM) Program. This project is intended to fill the void for instrumentation that can remotely measure the physical boundaries and phase of cloud particles in three dimensions. At present, there is no routine method for gathering such data. Imaging lidars cannot penotrate into thick cloud cover; thus, they give limited vertical inforination about clouds. In situ measurements from aircraft can provide accurate microphysical data, but aircraft are expensive to operate and the measurements cover only a limited region of the sky. A ground-based redar is the only practical way to routinely provide three-dimensional characterizations of cloud properties on the scale and in the detail required for ARM.

In this paper, we show preliminary data, which demonstrato that polarimetric millmeter-wave radars can distinguish cloud water droplets from ice particles. We also describe the ground-based Cloud Profiling Radar System (CPRS), which will be able to simultaneousty obtain threedimensional images of the polarimetric response of clouds at 33 and $95 \mathrm{GHz}$ (9 and $3.2 \mathrm{~mm}$ ). This systom should be complete and ready to participate in an intensive operation period (IOP) planned for June 1993.

\section{Millimeter-Wave Advantages}

Millimeter-wave radars offer significant practical advantages over microwave frequency radars because they are more sensitive to cloud particles and because they are more compact and portable. For spherical targets such as small liquid cloud droplets, the backscatter coefficient is proportional to $\lambda^{4}$. Table 1 shows the relative magnitude of this backscatter at various frequencies and the antenna diameter required to achieve a 1.0 degree beamwirth. These data demonstrate why millimeter-wave radars can use smaller antennas and lower peak power transmitters.

Millimeter-wave systems can also scan fading targets more quickly than lower frequency radars. Cloud structures can change rapidly, so accurate cloud pameterization depends on the measurement speed. Since the time needed to make accurate reflectivity and Doppler measurements is proportional to the radar's wavelength, millimeter-wave systems can obtain three-dimensional images of the clouds in reasonable times.

Table 1. Comparison of microwave and inillimeter-wave radars.

$\begin{array}{llllll} & \begin{array}{l}\text { S-band } \\ (2 \mathrm{GHz})\end{array} & \begin{array}{l}\text { C-band } \\ (4 \mathrm{GHz})\end{array} & \begin{array}{l}\text { X-band } \\ (10 \mathrm{GHz})\end{array} & \begin{array}{l}\text { Ka-band } \\ (35 \mathrm{GHz})\end{array} & \begin{array}{l}\text { W-band } \\ (95 \mathrm{GHz})\end{array} \\ \text { Relative Sensitivity } & 0.0 \mathrm{~dB} & 12.0 \mathrm{~dB} & 28.0 \mathrm{~dB} & 49.7 \mathrm{~dB} & 67.1 \mathrm{~dB} \\ \begin{array}{l}\text { Antenna Diameter } \\ \text { for } 1.0^{\circ} \text { beamwidth }\end{array} & 8.6 \mathrm{~m} & 4.3 \mathrm{~m} & 1.7 \mathrm{~m} & 0.5 \mathrm{~m} & 0.18 \mathrm{~m}\end{array}$




\section{Success of Polarimetry In Detecting Ice Particles}

Recent experiments with 95-GHz polarimetric radars show that high polarization purity radars can discriminate cloud particle phase. Over the past three years, MIRSL has participated in joint ground-and air-based experiments with the University of Wyoming's Atmospheric Science Department. The purpose of this collaborative program is to characterize polarimetric backscatter from ice particles and to demonstrate that polarimetric millimeter-wave radars can distinguish between cloud water and ice particles.

The initial ground-based measurements were held at the University's Elk Mountain Observatory, which is equipped with a variety of ground truth equipment, including a cloud sampling wind tunnel and a two-dimensional imaging probe. The mountain, whose geometry induces lingering cap clouds in the winter, provided a variety of ice cloud types from which data were collected. The radar and ground truth data clearly related the polarimetric backscatter seen by the radar to actual particle shape and orientation.

Our collaboration with the University of Wyoming continued in a series of experiments in which a $95-\mathrm{GHz}$ polarimeter was flown on the University's King Air aircraft. The radar measured two-dimensional cloud profiles and the aircraft collected air truth data. Polarimetric data from these flights, shown in Figure 1, illustrate depolarization in the melting layer of a cloud.

\section{Development of the CPRS}

The CPRS will be mounted on a mobile laboratory which can be transported to the first Cloud and Radiation Testbed (CART) site. The major components of the CPRS (depicted in Figure 2) are

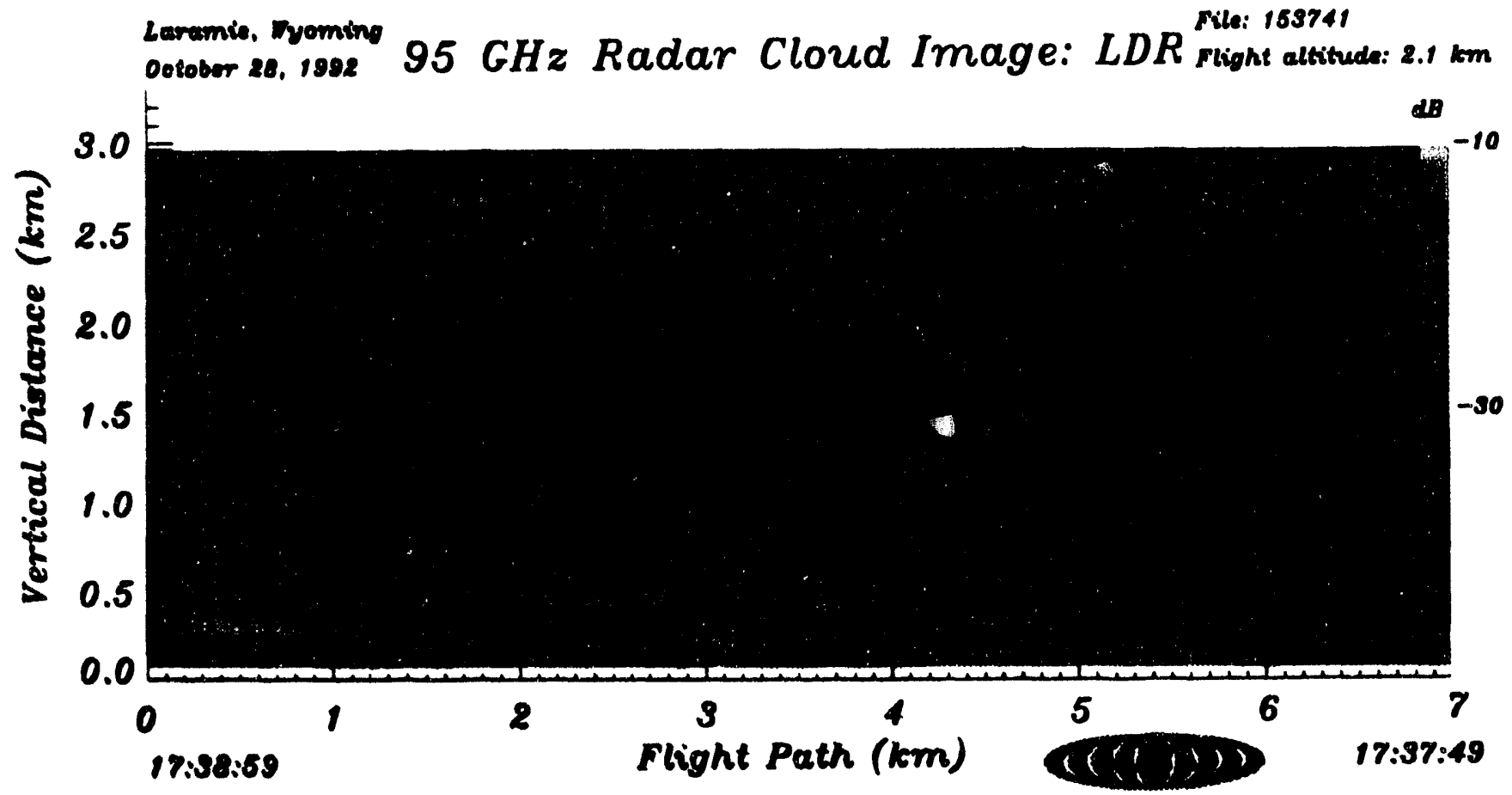

Figure 1. Recent $95-\mathrm{GHz}$ image illustrating detection of the melting layer. 
33 GHz/93 GHz Radar

on EI-over-Az Mount

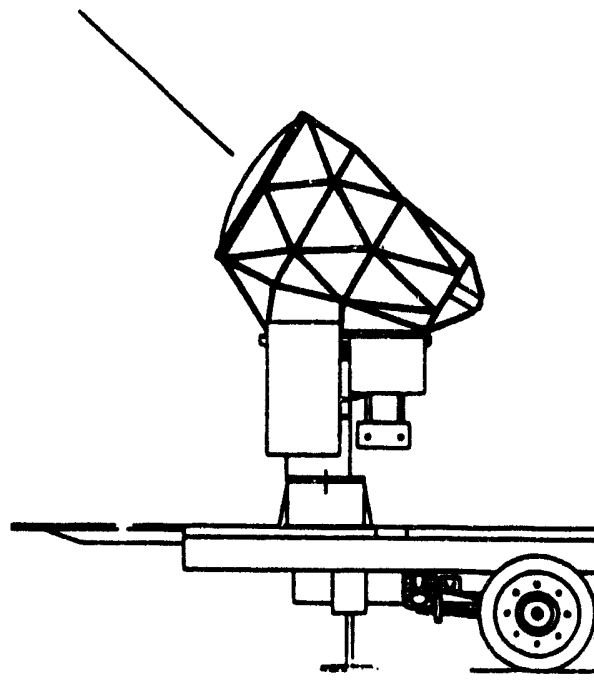

Air-conditioned Cube:

VXI Data Acquisition System

Callbration Equipment

Radar Power Supplies

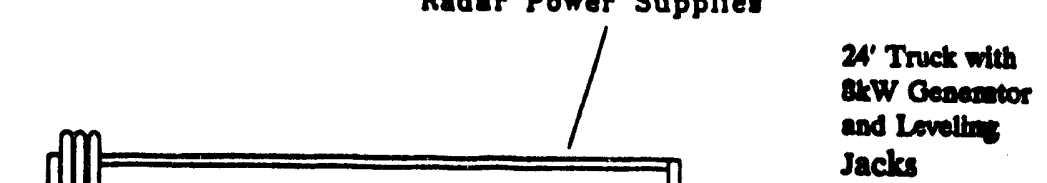

24' Truck with

Jacks

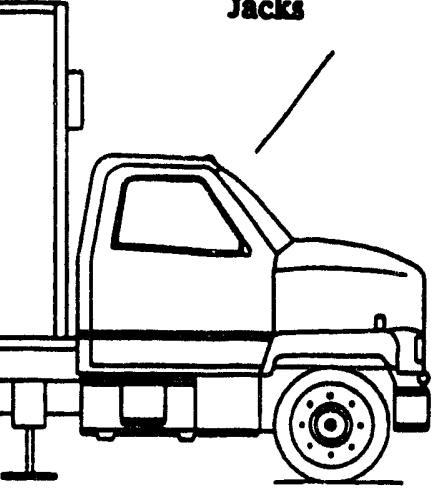

Figure 2. The Cloud Profiling Radar Syatom.

- a polarimotric 95-GHz Doppler radar (1.5 kW poak power)

- a polarimotric 33-GHz Doppler redar (120 kW paak power)

- a 1-m diamotor, low crose-polarization dielectric lons antonna

- a high-speed VXI-based data acquiatilon and digltal signal procesesing (DSP) systom.

The dual-frequency antenna feed and lons was deaigned at the Univereity of Masesechusetts' Antenna Laboratory. It produces collocated radiation patterns with a $0.5^{\circ}$ boamwidth and a $0.18^{\circ}$ boamwidth at $33 \mathrm{GHz}$ and $95 \mathrm{GHz}$, respectively. Each radar is independently controlled and has its own data acquisition and DSP oystem. The CPRS can transmit V or H polarized energy simultaneously at both frequencies, switch polarizations from pulee to pules, and simultaneously recolve orthogonal $V$ and $H$ polarized components of beckecatter.

\section{Unique Measurement Capabilitles of the CPRS}

The CPRS and its mobile platform offer unique capabilities for the scientific study of clouds. The truck-mounted pedestal can ecan oither the entire sky or a particular sector at a maximum rate of $9 \% / 80 c 0 n d$. Horizontal (vertical) profiles can be scanned and stacked vertically (horizontally) to form 3-D images. Images of roflectivity, radial volocity, and polarimotric paramotors can be derived from the redar data. These radar obeervations will be compared with data from other remote in situ sensors to obtain empirical relationshipe between water and ice contents of the clouds.

Bacause such relationships are not yot known, we intend to oporate the CPRS as often as possible to observe a wide variaty of cloud types. The database formed from these messurements should improve our ability to identify cloud particle phase via remote sensing instruments. 


\section{Instrumentation for Southern Great Plains}




\title{
Implementation of Instruments and Facilities at the Southern Great Plains Cloud and Radiation Testbed Site
}

\author{
D. L. Sisterson and M. L. Wesely \\ Environmental Research Division \\ Argonne National Laboratory \\ Argonne, IL 60439
}

Considerable progress has been made in installing instruments and trailers at the Southern Great Plains (SGP) Clouds and Radiation Testbed (CART) site (Figure 1). While initial activity concentrated on the central facility, planning proceeded for installation of extended facilities, which will begin operations in 1993. Observations at the central facility were started in June 1992, and a site operator began work in August 1992. Members of the site operations team assisted in the implementation during the first year, helping to develop the site; coordinate installation of instrumentation, and provide safety procedures. In addition, the representatives of the site scientist, the instrument team, and the data management team were frequently on site.

To reduce the number of prob: Ims associated with installing and operating instruments at the SGPCART site, a number of procedures were developed for CART instruments, Instrument Development Program (IDP) systems, and instruments of opportunity. A key role is held by the on-site instrument coordinator, who conducts a checklist review before an instrument is brought to the site.

The purpose of the checklist is to identify installation and operational requirements of the instrument. This prereadiness review generates work orders at the site based on requirements for electrical, trailers, the site data system, operation, safety, etc. Instrument representatives or "mentors" are informed of any deficiencies.

When the checklist is sufficiently complete, an operational readiness review is conducted by site operations. This review uses a checklist to determine requirements for training, documentation, operational support, safety, etc. Again, deficiencies are identified.

On the basis of the number and severity of the deficiencies, site operations either accepts the instrument, does not accept the instrument but operates it in a degraded mode until the problems are fixed, or does not operate the instrument. Similar procedures are followed for other types of equipment and some aspects of the site data system.

\section{Central Facility}

Table 1 shows the progress to date on implementation of instruments, facilities, and general aspects of the site data system at the central facility. The 160 acres of the central facility, which is located between the towns of Lamont and Billings, Oklahoma, contain the greatest concentration of SGP CART instrumentation. The site data system and the base of operations for the site operations are also located at the central facility. Instrumentation at the central facility includes systems that address all of the Atmospheric Radiation Measurement (ARM) Program's measurement strategles: instantaneous radiative transfer (IRF), singlecolumn modeling (SCM), four-dimensional data assimilation (4DDA), and hierarchical diagnosis (HD). During initial phases of implementation, instrumentation for IRF studies has been emphasized.

In the near future, several instrument systems in addition to those listed in Table 1 will be installed. The atmospherically emitted radiance interferometer (AERI) from the University of Wisconsin will undergo initial testing as an IDP instrument in March 1993, followed by occasional operation to produce data for the Science Team. A commercially produced laser ceilometer will be installed in summer 1993, and a more powerful micropulse lidar from the Goddard Space Flight Center of the National Aeronautics and Space Administration will undergo IDP testing later in the year. A $50-\mathrm{MHz}$ radar wind profiler and radio acoustic sounding system (RASS) is expected to be 


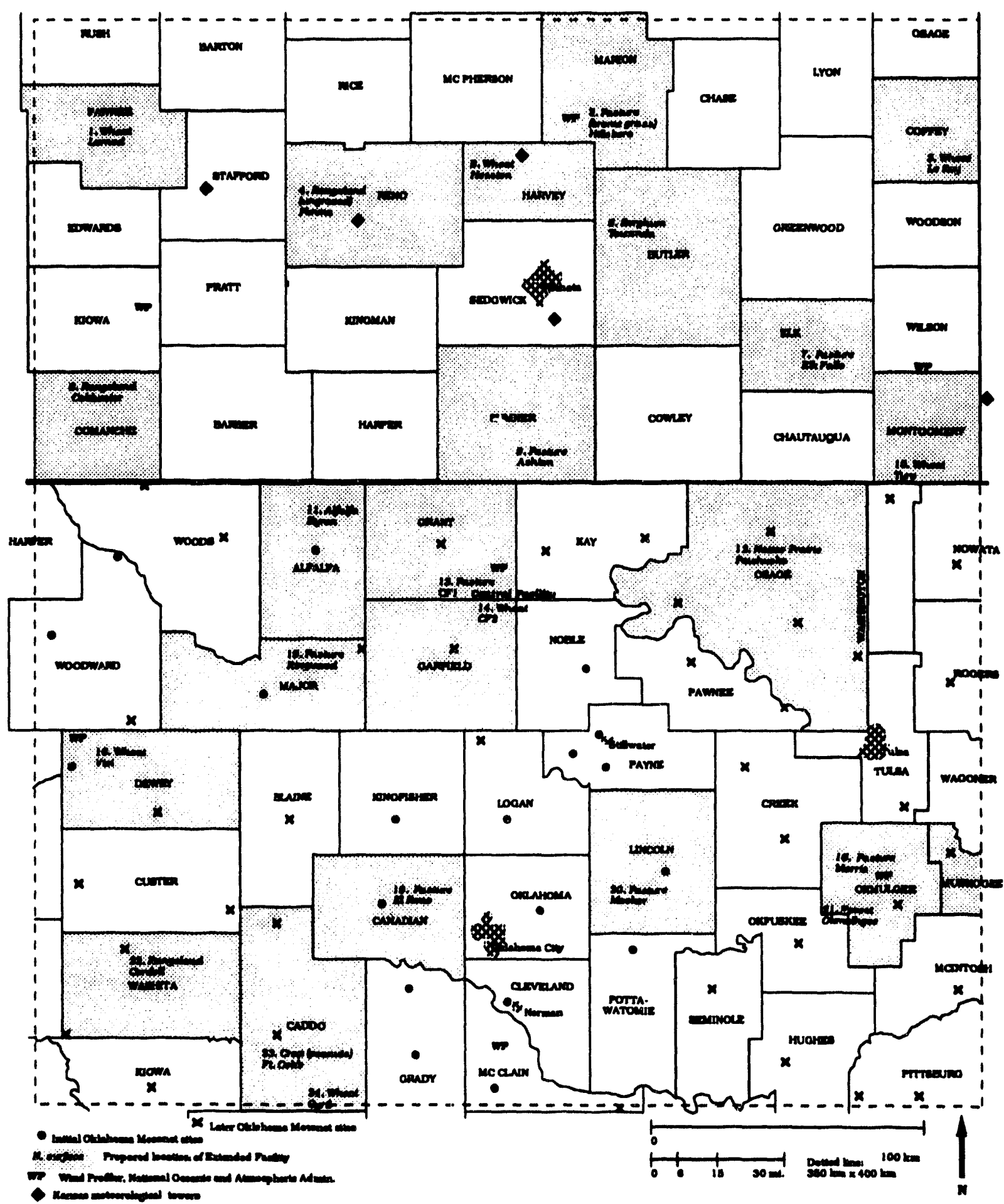

Figure 1. Overall viow of the SGP CART site in Oklahoma and Kansas. Individual counties are outlined. 
Table 1. Dates of installations of instrumentation, side data system versions, and facilities at the SGP CART central facility.

Date

Equipment or Qualifier

Observation or Function

\begin{tabular}{|c|c|c|}
\hline 6/12/92 & $\begin{array}{l}\text { Multifilter rotating } \\
\text { shadowband } \\
\text { radiometer (MFRSR) }\end{array}$ & $\begin{array}{l}\text { Total, direct, and diffuse solar } \\
\text { irradiances in selected wavebands }\end{array}$ \\
\hline $\begin{array}{l}6 / 13 / 92 \\
6 / 13 / 92\end{array}$ & $\begin{array}{l}\text { Pyranometer (PSP-1) } \\
\text { Pyranometer with solar } \\
\text { occulting disk (PSP-2) }\end{array}$ & $\begin{array}{l}\text { Total downwelling solar irradiance } \\
\text { Diffuse solar irradiance }\end{array}$ \\
\hline 6/13/92 & $\begin{array}{l}\text { Pyrhollometer on solar } \\
\text { tracking mount (NIP) }\end{array}$ & Direct-beam solar irradiance \\
\hline $6 / 13 / 92$ & $\begin{array}{l}\text { Fyrgeometer with solar } \\
\text { occulting disk (PIR) }\end{array}$ & $\begin{array}{l}\text { Downwelling diffuse infrared } \\
\text { irradiance }\end{array}$ \\
\hline \multicolumn{3}{|c|}{ Portable Automated Mesonet II (PAM-1I) System(n) } \\
\hline 5/16/92 & $\begin{array}{l}\text { Primary observations } \\
\text { Primary observations } \\
\text { Primary observations } \\
\text { Primary observations }\end{array}$ & $\begin{array}{l}\text { Wind speed and direction at } 10 \mathrm{~m} \\
\text { Temperature and relative humidity at } \\
\text { Barometric pressure } \\
\text { Precipitation rate }\end{array}$ \\
\hline \multicolumn{3}{|c|}{ Energy Balance Bowen Ratio (EBBR) Surface Flux Station } \\
\hline 9/15/92 & $\begin{array}{l}\text { Primary observations } \\
\text { Supporting observations } \\
\text { Supporting observations } \\
\text { Supporting observations } \\
\text { Supporting observations } \\
\text { Supporting observations } \\
\text { Supporting observations }\end{array}$ & $\begin{array}{l}\text { Latent and sensible heat fluxes } \\
\text { Net radiation } \\
\text { Soil heat flux } \\
\text { Air temperature and relative humidity } \\
\text { Soil moisture content (top } 5 \mathrm{~cm} \text { ) } \\
\text { Soil temperature (top } 5 \mathrm{~cm} \text { ) } \\
\text { Wind speed and direction at } 3 \mathrm{~m}\end{array}$ \\
\hline
\end{tabular}

Microwave Radlometer (MWR)(b)

6/11/92 Primary observations

Vertically integrated water vapor and liquid

915-MHz Wind Profiler and Radlo Acoustic Sounding System (RASS)

10/6/92

Primary observations

Primary observations

Vertical profiles of wind speed and direction

Vertical profiles of virtual temperature

Balloon Borne Sounding System (BBSS)

$5 / 27 / 92$

Primary observations

Vertical profiles of wind speed and

direction

Primary observations

Vertical profiles of air and dewpoint temperatures

Primary observations

Vertical profiles of pressure and altitude 
ARM Soience Mroting

Table 1. (contd)

60-Noter Tower Platiorm
$12 / 10 / 92$
$2 / 12 / 93$
81t0 Data syotem (8D8)
$5 / 21 / 92$
$9 / 17 / 92$
$1 / 31 / 93$

Tower

Sensors

Version 1, development system

Version 2, development and production systems; T-1 lines

Version 3, development and production systems, upgraded plotting capabilitios

\author{
Telephone service \\ Electrical service \\ Water service \\ Improved roadway to site \\ Tornado shelter \\ Septic field \\ Grounding fleld for all tralers \\ All trailers \\ Fiber and telephone communications plant
}

Support for instruments

Alr and dowpoint temperature at $60-\mathrm{m}$ lovel

$8 / 92$

$8 / 92$

2/93

(a) PAM-ll systoms are to be replaced by surface meteorological observation systems (SMOS) in late March 1993. SMOS also include a measure of snow depth.

(b) SIROS instruments and the MWR were initially located near the calibration trailer but were moved to the central cluster location on 10/17/92.

Installed in mid-summer. For whole-sky imaging, a system on loan from the Scrippe Marine Physical Laboratory will become operational in fall 1993, then will be replaced with a dedicated CART unit from the same source in 1994. Instrumentation for observing aerosol properties and ozone concentration near the surface will be installed in early 1894. An infrared radiometer to view the sky temperature directly above the microwave radiometer will come on line during fall 1983. Finally, a cloud profiler radar system being developed by the University of Massachusetts for the IDP will undergo tests in 1993 at the central facility.

\section{Extended Facllitles}

At extended facilities, surface meteorological observation stations (SMOS) measure surface meteorological conditions; solar and infrared observation stations (SIROS) deloct broadband and selected narrowband irradiances; and elther energy balance Bowen ratio (EBBR) or eddy correlation (EC) stations evaluate the air-surface exchange rates of heat, molsture, and momentum (Figure 2).

Between 20 and 25 locations, Including the central facility, will be used for extended facilities (Figure 1). These observations are necessary for SCM experiments but are alone inadequate to provide a complete sampling of the overall CART area. As Table 2 shows, sevoral sources of external data will be used, providing information to the Science Team to properly estimate the surface boundary conditions representative of large portions of the SQP CART sito.

Three extended facilities now contain EBBR stations and SMOS. This number will gradually increase to the numbers indicated in Table 2 by July 1993. Ton SMOS and EBBA stations will be operational by early 1994, and installation of EC stations will begin in early 1994. 


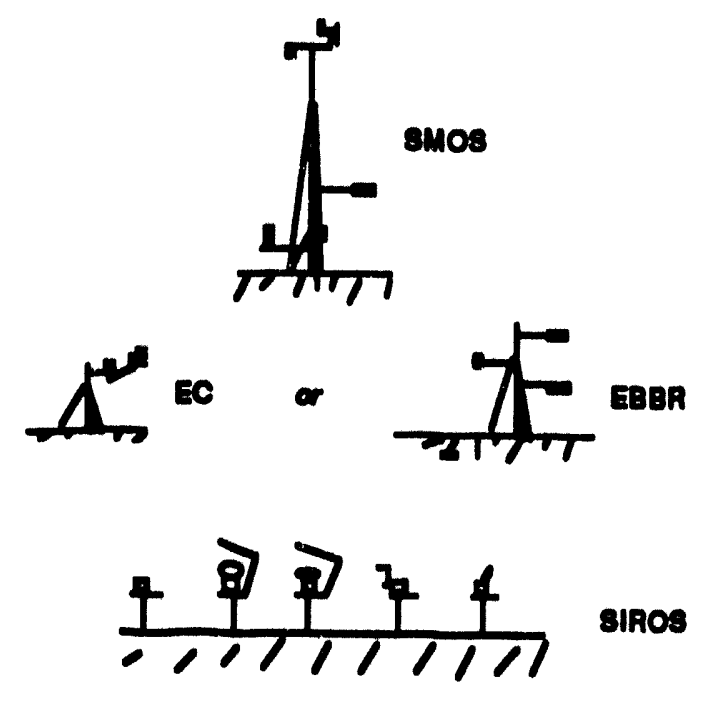

Flgure 2. A schematic view of extended-facillty componente.

\section{Boundary and Auxillary Facillties}

The CART boundary facilities are designed to measure winds, temperature, and humidity in vertical columns, providing information necossary for SCM and other investigations. The boundary facilities will generally be located near one of the six sites indicated by "WP" in Floure 1, where 404-MHz wind profilers are operated by the National Ocoanic and Atmospheric Administration/ National Weather Sorvice. Three areas near the six sites have been chosen for installation of CART boundary facllity inatruments in 1993: Hilleboro, on the northern odge; Vicl, on the southwest edge; and Morris (formerly Heckell), on the southeastorn odge. Extended facllitbe will be collocated or stationed within $10 \mathrm{~km}$ of these three initial boundary feclitites.

The three selected boundary facilities will be equipped in lato fall 1883 with CART balloon-borne sounding systoms (BBSS) to obeerve vertical profiles of wind, tomperature, and humidity, and with microwave radiometers to senses the total integrated column of water liquild and vapor. In addition, AERls may be deployed there lator to obtaln eatimatos of vertical profilos of temporature and humidity on a more continuous basis than is possible with the BBSS. Continuous molsture profiling in the presencs of clouds remains an unmet measurement need.

Auxillary facilities are needed to map cloud characteristics as seen from the surface with whole-sky imagers (WSI). With a WSI at the central facility, several auxillary fecllitios located approximately $5 \mathrm{~km}$ away will provide the needed cloud obeervations. Installation of the firet auxiliary facility is expected in 1994, simultaneously with implementation of a WSI at the contral facility. After evaluation of the syatoms and the aseociatod algorithms, soveral other statlons may be installed in 1994 and 1995.

\section{Acknowledgments}

This work was supported by the U.S. Department of Energy, Office of Energy Research, Office of Health and Environmental Research, via purchase order 105734A.01 from Pacific Northwoat Laboratory. 
Table 2. Routine network obeervation stations in the SGP CART domain. Each CART extended facility consists of one SIROS; one SMOS if a KSU, NOAWNWS, or OK Mosonot station is not located within $10 \mathrm{~km}$; and an EBBR station for untilled fields or an EC station for tilled fields. Data are usually reported as hourly or half-hourly averages.

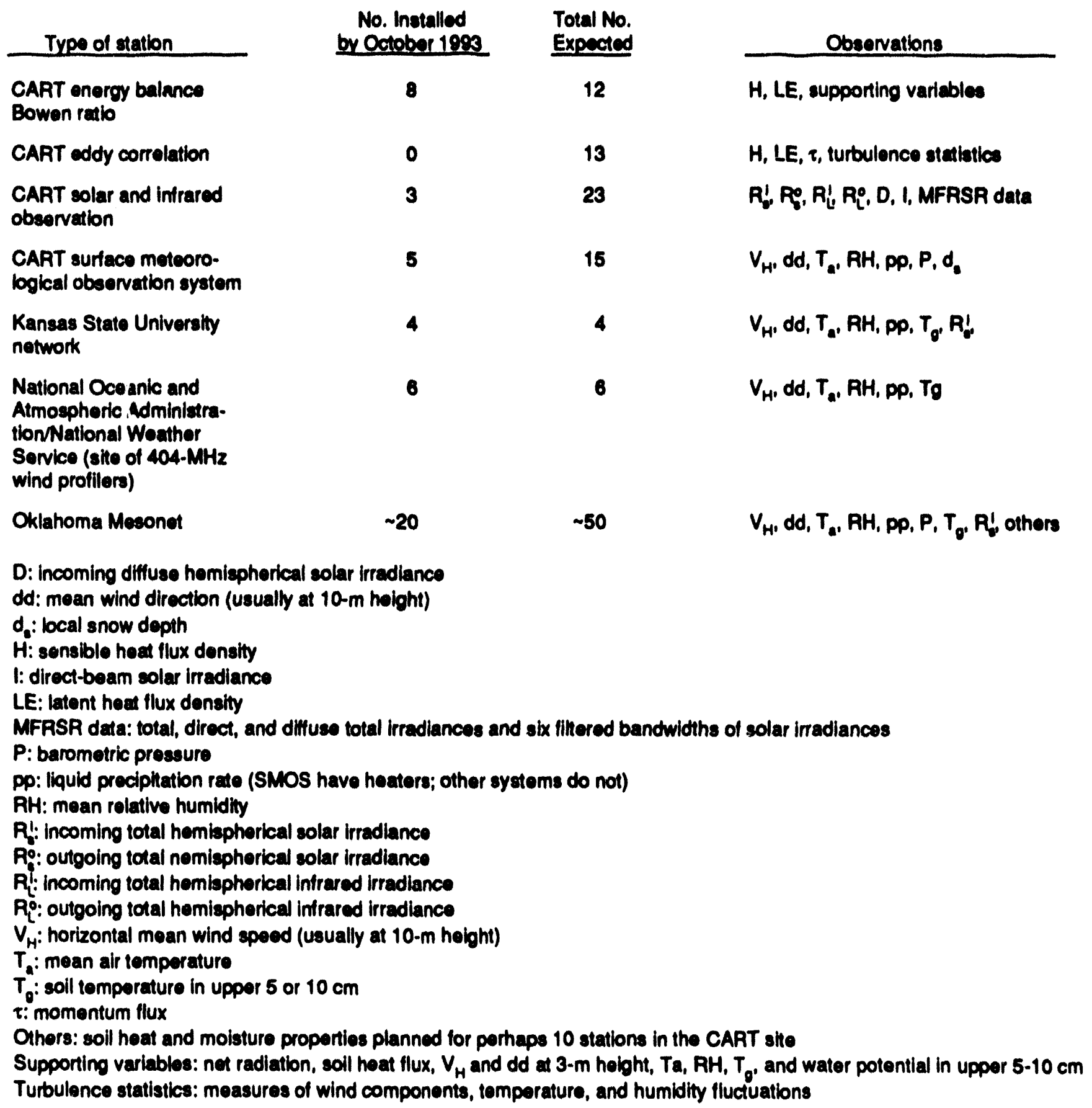


Tropical Western Pacific 


\title{
The Troplcal Western Pacific: Status Report
}

\author{
T. Ackerman \\ Ponnoylvania Sato Univeraity \\ Univereity Park, PA 16802 \\ F. J. Barnes \\ Los Alamos National Laboratory \\ Los Alamos, NM 87545 \\ W. Clemente \\ Los Alamos National Laboratory \\ Los Alamos, NM 87545 \\ D. Renns \\ National Ronowable Enorgy Laboratory \\ Golden, CO 80401.3393 \\ E. R. Westwator \\ National Oceanic and Atmospheric Adminiatration \\ Wave Propagation Laboratory \\ Boulder, CO 80303 \\ T. Barnot \\ Scripps Institute of Ocsanography \\ Lavolla, CA 92093
}

\section{Introduction}

The Atmospheric Radiation Measurement (ARM) Program's Tropical Westem Pacific (TWP) locale is the eccond of the five primary Cloud and Radiation Testbeds (CART) to be established. Phased implementation is cumently planned to begin in 1994.

The TWP locale encompasese an immense region of the tropical weatern Pecific bounded roughly by $10^{\circ} \mathrm{N}$ to $10^{\circ} \mathrm{S}$ extending eastward from Indoneala to somewhat east of the international datoline. Climatologically, it is charactorized by warm sea surface temperatures, deep and frequent atmosphoric convection, high rain rates, strong coupling botween the atmosphere and ocean, and subotantial variability associated with the EINIho-Southem Osclllation (ENSO) phonomenon. Amy number of diegnostic studies can be cited that show the relationship botween climatic variability in this region, particularly ENSO, and variabllity in other parts of the planet.

Geographically, this localo is characterized by the martime continent area in the southwest portion and cesentially open ocean in the northeast portion. Dotals on the locals and the scientific challenges are presented in the procesedings of the second ARM Science Team meoting held in Denver in October 1991 (Clements of al. 1992). The purpose of this paper is to report on activities since the 1991 Science Team meeting and discuss future plans. 


\section{Overvlew of Program Actlvitles}

In this ecotion, TWP cottutties sincs the last Sclence Toam meoting are bribliy summarked. Additional information on any ectivity may bo obtained from the Troploal Woatom Pactice Program Oricos (BIII Cloments: 505-687-2537; Falirioy Bamee: 505-687-4033)

\section{Pllot Radiation Obeervation Exporiment}

As the ARM Program's firet fiold campaign, wo conducted a Pilot Rediation Observation Exporiment (PROBE) from Novembor 1902 through Fobruary 1903 at aslib in Kaviong. Now Iroland Provinos, Papua Now Guinoa $\left(2^{\circ} 34^{\prime} 8\right.$, 150 46'E). PAOBE was conducted in colleboration with tho Tropleal Ocwan Clobal Atmosphore (TOCA) Program's Coupled Ocsan Atmosphere Rospones Exporiment (COARE). This ambitious and aucociastul offort consumad much of the TWP varn's time during the past two years.

\section{Tho Experiment}

PROBE was conducted to inveatigats the rob of troplcal clouds on the eolar and terreatrial rediation budgets. A molecolological, colar and infrared radiation, mobture, and cloud monliforing station wases co up at the National Woather 8ervios observing etation at the alrport in Kaviong (2³4'8, 150\%48'E), Now Ireland Province, Pepua Now Guinea. The PROBE station consiated of thres sescontainers which housed inatruments and data-logging equipment.

Data more collectad in two phases: November-Decomber 1992 and January-February 1993. The third sescontainer and other instruments wore added in Phase II. All data more archived at the sits, and somo have already been hand-carried beck to inveatigators in tho United States. A emall sample of the brosd-band radiation data was tranemitted beck dally via the Geostationary Oporational Environmontal Sablilis (COE8) dong with the data from the rawinseonds. A peper describing the details of PROBE and precenting soms sample data is being propared for publication.

\section{A Noto On Loglatica}

During Phase II (January 6 to Fobruary 28, 1993) of PROBE, tho NOMAWave Propagation Laboratory oporated adual-frequenoy Microwave Wator Subatanos Radiomolar (MWSA) and a Fouribr Transform Infrared Radiomotor (FTIR). Hore, we summarize some of our logletical exporiencose during PROBE.

Tho MWSR and FTIR wore shipped in aseacontainor from Boulder, Colorado, to Kavieng. Although minor difilcultibe were encountered in plecoment of the sescontalner at the PROBE sito, all equipment and computers arrived intect.

The only major problem we encountered was obtaining the liquid nitrogen necoseary to calibrate the FTIR. Atthough wo started working on this problem six months bofore the start of the experiment, we experienced numerous unenticipated and fruatrating dolays in shipping. In addition, a provlous shipment of the 1500-liter container for Phase I of PROBE was vandalized enroute with the result that only a small frection of the original amount reached the fiold sith. Howover, because of special security precautions arranged by the manufacturer, wo wore succeseful in obtaining two complate shipments of liquild nitrogen during Phase II. The MWSR operated continuouely through PROBE and, except for poriods of rain, the data epposar to bo good. Bacause of the problems in obtaining llquid nitrogen, only twenty days of high-quality cellibrated epectra were obtained. For more detalle on this oporation $h$ PROBE, ece the paper by Westwator of al. In this volums.

\section{Modelling Studies of the Indo-Pacifle Warm Pool}

Scrippe Institute of Ocsanography is conducting a varibly of modeling studies aimed at understanding the initoractions of clouds, rediation, and the ccoan in the region of the indo. Pacific warm pool. (8oo paper by Bamott of al. In this volume.) These studies are dosigned to understand the important phyaical proceseses oporating in the occan and atmoephere in the region. The modeling studies should holp with the design of the ARM CART in this region and also put the eventual mesaurements from the sito into a 
large-scale perepective. These tools are avallable as resources permittio ARM investigator in torected in studying the TWP.

\section{TWP Sclonco Workshop '}

A workshop was hold in Santa Fo, Now Moxico, on 18.19 May 1992 to botter define important science drivere for the TWP. Twenty scientiats presented ecientific hypotheses that could bo tooted in the TWP. These hypotheses were discusesed and working groups made recommendations following the formal presentations. A report is being prepared.

\section{Ocean Measuroments Working Group}

An Ocean-based Measurements Working Group (OMWOG) was formed in the apring of 1992 to provide an interface botween the ARM functional teams and oceanbased operations. OMWOG will serve all of ARM's cocan operation activitios, although it is intially focusing on the TWP project. An ocean platforms catalog that will provide information on platforms for ocean-based operations is boing prepared.

\section{Early Awarenose and Notworking}

The importance of making wostern Pacific nations aware of the ARM Program and its plans for the TWP far in advance of any actual negotiations was identified as a critical task earty on. It was also evident that we needed to cetcblish good working relations with regional organizations that might want to collaborate or could be holptul in identifying and obtaining sites. In addition, a number of organizations and programs have conducted scientific research in the TWP area. Wo have taken every opportunity to interact with as many of these organizations as possible.

The collaboration with TOGA COARE in conducting PROBE was the basis for establishing many of these contacts. The experience gained by the PROBE is a valuable resource to the TWP project. All of the organizations with whom wo have interected, and others, could play a role as wo procoed with implementing the TWP. Some of the agencies wo have established working relations with are the South Pacific Applied Geosciences Commission (SOPAC); the
South Pacific Reglonal Environmental Programme (SPREP); tho United Nations Educationial, Scientific, and Cultural Organization's (UNESCO) Office of the South Pecific; the Univerelity of the South Pacific; the Australian Bureau of Moteorological Rosearch Center (BMRC); the Division of Atmoephoric Rosearch (DAR), within Australia's Commonwoalth Scientific and Industrial Pasearch Organization; the Now Zealand Moteorologica: Servico; and the Now Zoaland Inattute of Wator and Atmospheric Resesarch.

We are continuing to keep our relationshipe active with these organizations and will be establishing now ones where appropriate. These activities will be critical to implementation of the TWP CART sites and in their continuing operation. There is a gonuine potential for cooperation and collaboration within the TWP locals, but we must proceed in an educated and careful manner.

\section{NEPA Activitios}

Wo are determining the lovel of activities required for compliance with the National Environmental Folicy Act (NEPA) for the TWP project. The completion of a LOs Alamos National Laborafory ESsH questionnairo began this process. A TWP project description, a DOE environmental checklist, and a momo of intent to comply with extra-territorial regulations were prepared and submitted to DOE Albuquerque Operations Office.

\section{Program Offico Audits}

The Program Office at the Department of Energy and at the Pacific Northwest Laboratory audited the PROBE and TWP projects during May and June 1982, respectively. Past ectivities, current status, future plans, and anticipated budgets were reviowed. These audits helped to better focus on ascience and siting strategy for the TWP program.

\section{JASON Rovlow}

In August 1982, the TWP status, its ovolving science and siting strategy, and PROBE wore presented to tho JASON committee as part of their annual review of the ARM Program. 


\section{Proposed Sclence and Stting Strategy}

As a result of the TWP Science Workehop, the Program Olinos Audits, resctions from the JASON committeo reviow. our experience with PROBE, and the anticipatod budget situation, wo are formulating a now proposed strategic plan for the scionos and siting of the Troplcal Westorn Pacific.

\section{Sclentific Considerations}

The overall goal of APM is to improwe doud and rediation paramotorization in goneral circulation models (GCMs). The components of the current plan that are aimed at achioving this goal inctude 1) dotalled obeorvations of radiation and cloud propertics and aseociated data analyeis, 2) studies of robvant atmoephoric proceseses using highresolution models with explicit phyaics, and 3) simulations with mesoscale models and single column versions of GCMs. The deaign and implementation of the Southern Great Plains (SGP) CART has been predicated on these components.

As ARM moves on to the implementation of subeequent sites, the conceptual plan and CART design may need to be modified in ordor to addrese the same goal in a different locale. As we move to consider the proposed TWP site, an examination of the principal science issues and relevant logistical considerations is required. Because of the large area, rolative inaccessibility, and predominance of cosan, this locale is also charactorized by a critical lack of climatological data from the ocean, the atmosphere, and the interface.

The major aress of scientific importance for the TWP localo have been identified as

- rediation budget and cloud forcing

- water and energy budgots

- ocean-atmoephere interactions.

Over the course of the past year, a workshop and several meetings have been hold expressly to consiver science lesuses and moasurement needs in the TWP. These moetings have provided stimulating discussions and interesting idess. From these, wo have begun to distill an implementation strategy that flows from an amalgam of science issues and logistical and financial considerations.
At a minimum, the TWP science iseves require

1. continuous, basic obeorvations of radiation and cloud propenties over a broad spatial domain

2. detailed, but not necessarily continuous, obeervations of atmospheric structure and properties at soms location(s)

3. observations of surface fluxes, boundary layor properties, and doud structure in a puroly cosanic environmont

4. observations of radiative fluxes at the top of the atmosphere (TOA).

The first requirement comes from the need to cetablish the magnitudes and variabilities of the surface radiation budpot and cloud forcing across the Pacific basin, the escond from the need to understand how tropical convection impacts wator and enorgy budgets in the tropics, and tho third from the need to understand the coupling of the occan and atmosphere and how it affects convective organization over the water. The fourth requirement provides the rediation source and sink terms from space.

\section{Othor besues}

The logistical and financial constraints inctude factors such as

1. Infrastructure support throughout much of the area of interest is extremely limited or non-existent.

2. Potential political problems and instabilities mandate against fixed, long-term plans in some areas; funding for capital equipment will be phased over several years.

3. Installing and maintaining instrumentation is 20 costly that cooperation with other agencies with mutual intoreats is crucial.

4. The envisaged ocean site has not been tried bofore and will require considerable development work to draft an acceptable plan.

Our experience gained with PROBE highlights the need to design and build systems that are largely self-contained and can operate with only a minimal amount of attention. The high cost and slowness of shipping replacement parts requires that systems be rugged, reliable, and redundant. 
Standardization of instrument and computer parts, whonoverposelble, is highly dosirable, and some inventory of epare parts will have to be kept on site or at a contral location.

The financial iseuse are driven by the amount of monoy avallable to the ARM Program and the need io continue inotrumenting the SCP site. It is unlikely that the ARM budget can eupport the combined dovelopment of two major sitea simultaneousty. Thus, a siting strategy for the TWP that can bogin with modest exponditures and expand to fit yearty funding profiles is highly dosirablo.

Neo, the ARM Program extends beyond the first two sites to inctude the North Slope of Alaska. Eastorn Ocsan Margins, and the Gulf Stream locales. The siting strategy for the TWP should provide for common design and dovolopment of instruments and a common deployment schome which could be used at these future sites. Thus, a straboy that is floxible and, to some extent, portable from one remote bcale to another is highly desirable. This strateoy is particularty important for the ocean-based component of the program, which is likely to be quite expensive. Fachioning a common and economical design for ocoan-based instrumentation will take considerable time and offort.

Given the high cost of operating in a remote environment and the current funding situation, it is critical that ARM management seize overy opportunity for collaboration with appropriate existing scientific programs and multination organizations in the region. A fow installations are being operated in the islands, but these are rather modest in scope and instrumentation. The most active site in the area is maintained by the Australian research community in Darwin. This site has state-of-the-art instrumentation such as Doppler radar and wind profilers and is currently being upgraded to support TRMM validation research. Collocation and cooperation with this activity should be a high priority for ARM.

\section{Evolving Science and Sting Plan}

The combination of science issues with logistical and financial considerations has lad to the proposal of a phased, floxible deployment scheme. The scheme is decigned to begin the acquisitior, of data in the TWP soon (currently summer 1994), augment that acquisition consistently over the next fow years, and culminate with the deployment of an oceanic facility. The proposed scheme has four distinct components:

1. deploying three to five Atmospheric Radiation and Cloud Stations (ARCS) across the western Pacific

2. adding an augmented ARCS to the Australian Bureau of Moteorology research faclitios in Darwin

3. developing and deploying an enhanced oceanic facility incorporating some combination of moorings, ocean platforms, and island-based remote sensors

4. deploying upward-looking solar radiometers on a number of the 65 to 70 established ATLAS moorings in the TOGA TAO array, which extends across the Pacific basin from $10^{\circ} \mathrm{N}$ to $10^{\circ} \mathrm{S}$.

Details of this science and siting strategy are given in ovolving document "Science and Siting Strategy for the Tropical Westem Pacific CART Locale," which is available from the TWP Program Office (Ackerman et al. 1993).

This strategy will rely on the use of satellite data, and unmanned aerospace vehicles (UAVs) when they become available. ARM has been exploring the possible use of UAVs in support of the CART. The development of simple sounding packages that could be flown on small UAVs would be immensely valuable to this oceanic site. It would provide the means to do atmospheric sampling over a broad spatial area, a capability that is currently missing. The additional development of simple radiation and cloud sampling packages would also be of great utility to this site. Close coordination between the TWP program planning and ARM's UAV and satellite programs will be maintained.

\section{Meetings}

We participated in the following professional meetings:

Twenty-First Annual Session of the South Pacific Applied Geoscience Commission, 26-29 September 1992, Nuku'alofa, Tongatapu, Tonga. Peter Lunn and Clements made joint presentations on the ARM Program and the TWP Projoct.

1992 Western Pacific Geophysics Meeting of the American Geophysical Union, 17-21 1992, Hong Kong. Clements presented a paper entitled 'The USDOE ARM Program: Tropical Western Pacific and Southern Great Plains CART Projocts." 
73nd Annual Meeting of the American Meteorological Society, 17-22 January 1993, Anaheim, California. Ackerman's paper was presented; Barnes hold a poster session; Clements and Renné also participated.

GOALS (Global Ocean Atmosphere Land System) Conference/Workshop, 1-2 March 1992, Honolulu, Hawaii. Clements presented ARMTWP overview.

\section{References}

Ackerman, T., W. Clements, and D. Renné. 1993. Science and Siting Strategy for the ARM CART Locale. Working report, available from the Tropical Westem Pacific Program Office, P.O. Box 1663, MS F665, Los Alamos National Laboratory, Los Alamos, NM 87545, or from the Tropical Western Pacific Site Scientist Office, Department of Moteorology, Pennsylvania State University, 503 Waker Bldg., University Park, PA 160802-5013.

Clements, W., T. Ackerman, and D. Rennó. 1992. Tropical Western Pacific Project: Status. Proceedings of the Second Atmospheric Radiation Measurement (ARM) Scienco Team Meeting, October 26-20, 1991, Denver, Colorado. CONF. 9110336, U.S. Department of Energy, Washington, D.C. 


\section{Gulf Stream Locale}




\title{
VAS-Derived Cloud Observations over the Gulf Stream Locale During the Winter Months
}

\author{
R. J. Alliss and S. Raman \\ Department of Marine, Earth and Atmospheric Sciences \\ North Carolina State University \\ Raleigh, NC 27695-8208
}

\section{Introduction}

Clouds have long been recognized as having a major impact on the radiation budget in the earth's climate system. One of the preferred areas for the production of clouds is off the east coast of the United States. The formation of clouds in this region, particularly during the winter months, is caused predominately by the presence of the Gulf Stream, which flows northeastward just off the southeast coast. The geography of the region is such that the cold North American continent lies adjacent to the relatively warm shelf waters $\left(10^{\circ} \mathrm{C}\right)$, which in turn are bounded by the much warmer Gulf Stream $\left(25^{\circ} \mathrm{C}\right)$. The Sargasso Sea to the east of the Gulf Stream consists of waters slightly cooler than those observed in the Gulf Stream. This unique setup provides the surface forcing necessary for the frequent oocurrence of cyclogenesis, coastal frontogenesis, and cold air outbreaks, all of which are major cloud producers. Figure 1, obtained from NOAA-9 Advanced Very High Resolution Radiometer (AVHRR), shows the position and sea surface temperature of the Gulf Stream area on January 28, 1993.

Recently, attempts have been made to characterize the frequency of cloud occurrence and associated cloud properties via inferences from satellite radiance measurements. The $\mathrm{CO}_{2}$ technique for deriving cloud top pressures (Chahine 1974; Smith ot al. 1974) was first applied by Menzel et al. (1983). The technique takes advantage of the differing partial $\mathrm{CO}_{2}$ absorption in three of the infrared channels, making each channel sensitive to a different level in the atmosphere. Clouds appear in the $\mathrm{CO}_{2}$ channel images according to their level in the atmosphere. Low clouds will not appear in the high-level channels, while high clouds appear in all channels. By measuring the upwelling infrared radiation from the earth. atmosphere system in several of the VISSR(a) Atmospheric Sounder (VAS) $\mathrm{CO}_{2}$ channels simultaneously, we are able to distinguish thin cirrus clouds due to the transmission of terrestrial radiation through the cirrus.

The objective of this study is to quantitatively evaluate the type and amount of cloudiness most present during the winter months (December-February). The study makes use of 7 years (1985-1993) of multispectral observations using the $\mathrm{CO}_{2}$ technique from the GOES VAS. Statistics of cloud cover during this period are also presented. Additionally, VAS observations of seven cold air outbreak (CAO) events occurring during January 1993 are presented. Cold air outbreaks are one of the most dynamic events to occur over the Gulf Stream Locale (GSL). This surface energy exchange process occurs when cold dry continental air moves over the warmer Gulf Stream waters, allowing the airmass to destabilize and produce clouds. Since this area is dominated by air-sea interactions such as the CAO, the importance of these processes is essential to understanding cloud genesis.

In the following section of this paper, the methodology and technique for deriving the cloud parameters are described. Current results, including the cloud climatology and cloud distribution during seven cold air outbreak events, are addressed next. The paper concludes with a discussion of future research.

(a) Visible and Infrared Spin-Scan Radiometer. 


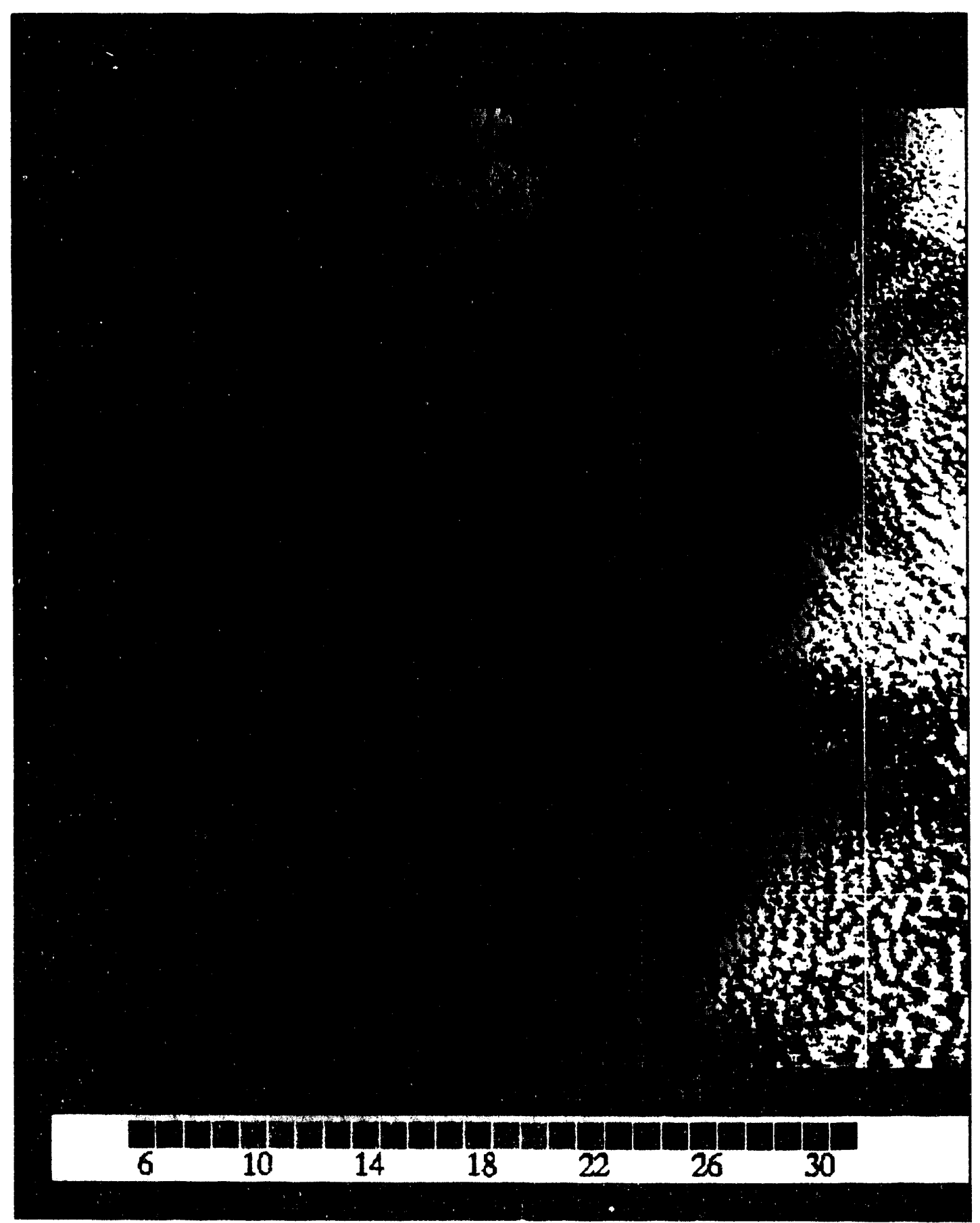

Flgure 1. Sea surface temperature of the Gulf Stream Locale obtained from the NOAA9 AVHRR during the cold air outbreak of 28 Jan. 93. 


\section{Methodology and Description of $\mathrm{CO}_{2}$ Technique}

The VAS rediometer detects infrared radiation in 12 spectral bands that lio between 3.9 and $15 \mu \mathrm{m}$ with 7. or $14-\mathrm{km}$ resolution. The $15-\mu \mathrm{m} \mathrm{CO} 2$ band channels provide good sensitivity to the temperature of relatively cold regions of the atmosphere. An example of the vertical resolution of the three $\mathrm{CO}_{2}$ channels used in this study is given by the temperature profile woighting functions shown in Figure 2. Each curve in the figure shows the senstivity to variations in atmospheric temperature of the rediance observed by the indicated channel. Only clouds above the 350-mblevel contribute significantly to the radiance to space obeerved

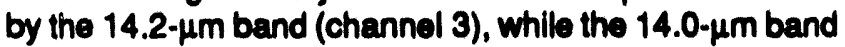
(channel 4) senses down to $700 \mathrm{mb}$ and the 13.3- $\mu \mathrm{m}$ band (channel 5) senses down near the surface of the earth.

To assign a cloud top pressure to a given cloud element, the $\mathrm{CO}_{2}$ technique is used. As shown by Smith and Platt

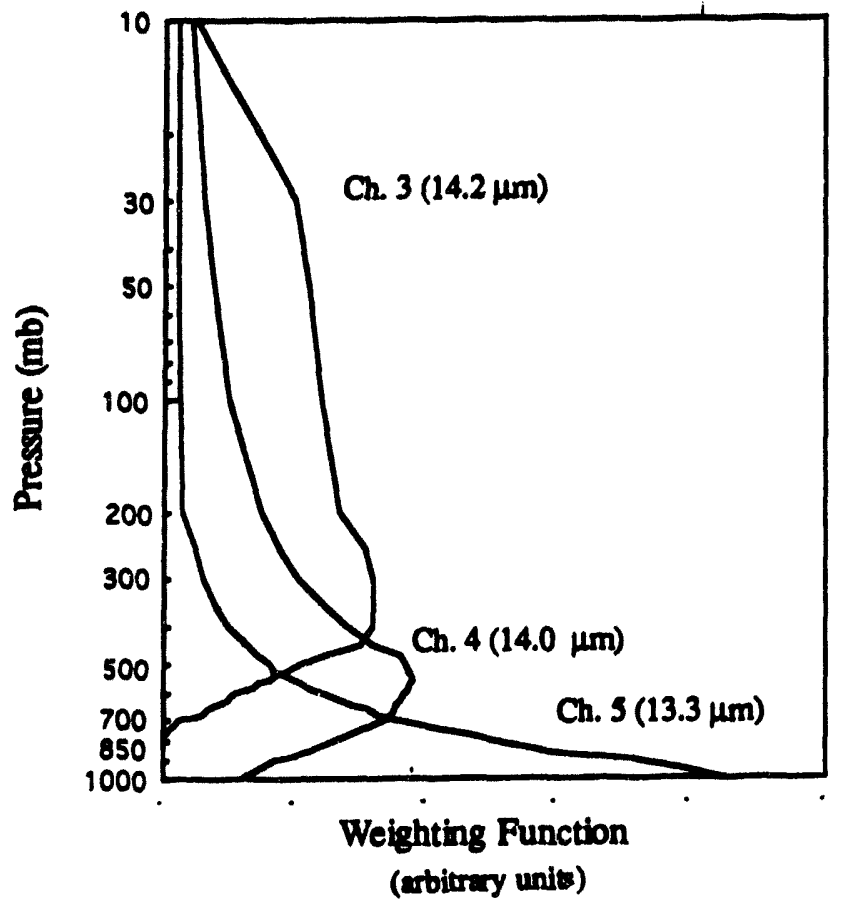

Flgure 2. The temperature profile weighting function for the VAS $\mathrm{CO}_{2}$ spectral bands centered at 14.2, 14.0 and $13.3 \mu \mathrm{m}$.
(1978), the ratio of the deviations in cloud-produced radiances, $I(v)$, and the corresponding clear air radiances, $I_{c}(v)$, for two spectral channels of frequency $v_{1}$ and $v_{2}$ viewing the same field-of-view can be written as

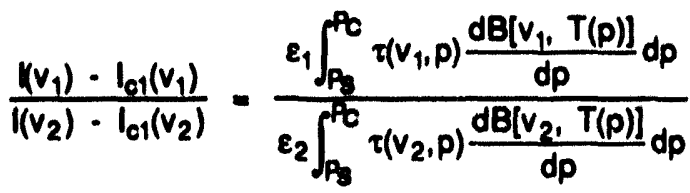

where $\varepsilon=$ the cloud emissivity

$P_{.}$- the surface pressure

$P_{c}=$ the cloud pressure

$\tau(n, p)=$ the fractional transmittance of radiation of frequency $v$ emitted from the atmospheric pressure level (p) arriving at the top of the atmosphere $(p=0)$

$B[v, T(p)]=$ the Planck radiance of frequency $v$ for temperature $T(p)$.

If the frequencies are close together, then $\varepsilon_{1}$ approximates $\varepsilon_{2}$, and one has an expression by which the pressure of the cloud within the field-of-view (FOV) can be specified.

The left side of Equation (1) is inferred from the observed radiances from VAS in a given FOV, and the clear air radiances are inferred from spatial analyses of VAS clear radiance observations in neighboring FOVs. The right side of Equation (1) is calculated from a temperature profile and the profiles of atmospheric transmittance for the spectral channels as a function of $P_{c}$, the cloud top pressure (1000 to $100 \mathrm{mb}$ is spanned by discrete values at 50 -mb intervals). Global analyses of temperature and moisture profiles from the National Moteorological Center (NMC) are used.

The optimum cloud top pressure is found when the absolute difference of the left side $\left(v_{1}, v_{2}\right)$ minus the right side $\left(v_{1}, v_{2}, P_{c}\right)$ is a minimum. Using the ratios of radlances of the three $\mathrm{CO}_{2}$ spectral channels, three separate cloud top pressures can be determined (14.2/14.0, 14.0/13.3, and 14.2/13.3). If $\left(1-I_{\mathrm{c}}\right)$ is within the noise resporse of the instrument (roughly $1 \mathrm{~mW} / \mathrm{m}^{2} / \mathrm{st} / \mathrm{cm}^{-1}$ ), the resulting $P_{c}$ is rejected. As described by Menzel et al. (1983), the most representative cloud height and amount are those that best satisfy the radiative transfer equation for the three $\mathrm{CO}_{2}$ channels.

Once cloud top pressure and height have been determined, an effective cloud amount (also referred to as effective 
emiselvity) can be ovaluated from the infrared window channol data uaing the following relation:

$N_{2}=\frac{1(w) \cdot b_{b_{1}}(w)}{B\left[w, T\left(P_{c}\right)\right] \cdot I_{b_{1}}(w)}$

The effective cloud amount is the ratio of the radiance difference the obeerved cloud producese to the radiances difference an opeque cloud at the same lovel would produce in the infrared window. Hore

$\begin{aligned} N & =\text { the fractional cloud cover within the FOV } \\ N \varepsilon & =\text { the effective cloud amount } \\ w & =\text { the window channel frequency } \\ \left.\left(P_{e}\right)\right] & =\text { the opaque cloud radiance. }\end{aligned}$
$B\left[W, T\left(P_{c}\right)\right]=$ the opaque cloud radiance.

Uaing the infrared window channol and the three cloud top preseures, three effective cloud amount dotorminations are mado.

If no ratio of radiancese can be rellably calculated because I- I f within the inatrument nolso bvel, then a cloud top preasure is calculated directly from the VAS-obeerved $11.2 \mu \mathrm{m}$ infrared-window channel brightness tomporature comparison with the temperature profile, and the effective omiseivity is aseumed to be unity. This occurs for most low clouds below $700 \mathrm{mb}$; here, the $\mathrm{CO}_{2}$ technique cannot find a pair of woighting functions where both have adequate sensitivity. Thus, all clouds are assigned a cloud top pressure either by the $\mathrm{CO}_{2}$ ratios or the infrared window calculations.

Fielde of view are determined to be cloar or cloudy through inspection of the $11.2 \mu \mathrm{m}$ brightness temperature with a correction for moisture abeorption. If the moisture-corrected $11.2 \mu \mathrm{m}$ brightness temperature is within $2 \mathrm{~K}$ of the known surface temperature (taken from the $1000 \mathrm{mb}$ NMC model analysis adjusted with hourly observations), then the FOV is aseumed to be clear and no cloud parametere are calculated.

The VAS $\mathrm{CO}_{2}$ technique is independent of the fractional cloud cover. Cloud heights and effective cloud emissivities can be determined for partially cloudy FOVs. However, there are a few assumptions. The effectlve cloud emiseivity is aseumed to be independent of wavelength. The cloud is assumed to be of infinitesimal thickness; Smith and Platt (1978) have indicated that this assumption introduces errors approaching one-half (one-quarter) the thickness of the cloud for optically thin (thlek) cloude whore the integrated emittunces is bes than (oreator) 0.6.

The $\mathrm{CO}_{2}$ abortithm dotermines the holght of the radiative contor of the doud. For optladly thick clouds, this is near the doud top; for optically thin clouds, it is noar the cloud middls.

The VAs $\mathrm{CO}_{2}$ technique sesumes the presence of only ono cloud layor; when multiple layore are seneed, it derives acloud altitude in botween the aftitudes of the two separate layere. Multilayer cloud sttuations whore an opeque cloud underibe a tranemiselve cloud cause errore of about 100 $\mathrm{mb}$ in the hoight of the tranamiselve cloud; for most cases the cloud is found to be too low in the atmosphore. The orror in tranemicaive cloud hoight is largest whon the undertying opeque layer is in the middle troposphere $(\mathbf{4 0 0}$ $-700 \mathrm{mb}$ ) and emall to negligible whon the opeque layer is near the surface or cloes to the transmiealve layer.

Because the VAS FOV resolution is coaree $(7 \mathrm{~km})$, wry emall eloment clouds are difficult to detect. Aleo, because the woighting functions for the VAS channols are broad, the vertical resolution is limited. Instrument noise causes the $\mathrm{CO}_{2}$ algortithm to mise $50 \%$ of the thin cimus with offective emiseivity lose than 0.10; this reprecents about $5 \%$ of all observations. The algorithm, however, overeatimates the frequency of eomitransparent clouds by about $5 \%$ because of the large observation area used in the cloud proceseing. Errors introduced by instrument noise and large observational area, howevor, tend to cancel.

\section{Discussion of Results}

\section{Cloud Climatology}

A statistical summary of all cloud obeervations over the GSL between December 1 and February 28 (1985-1993, except for the 86-87 season) is shown in Table 1. Ton points were chosen and averaged to represent the GSL. The cloud top pressures are divided into 10 vertical lovels from $100 \mathrm{mb}$ to $1000 \mathrm{mb}$, and the effective cloud amounts are subdivided into five intervals from 0 to 1.0.

As indicated in the preceding section, the effective cloud amount is defined as the product of the fractional cloud cover $N$ and the emissivity of the cloud $\varepsilon$ for each 
Table 1. Cloud statistice (\%) for the Gulf Stream Locale during the 7 wintore (Dec. - Fob.) botween 1985 and 1893, not including 1886-1887.

\begin{tabular}{|c|c|c|c|c|c|}
\hline \multirow{2}{*}{$\begin{array}{l}\text { Lovel } \\
\text { (mb) }\end{array}$} & \multicolumn{5}{|c|}{ Effective Emiselvity (\%) } \\
\hline & $0.0 \cdot 0.2$ & $0.2 \cdot 0.4$ & $0.4 \cdot 0.6$ & $0.6-0.95$ & $0.05 \cdot 1.0$ \\
\hline $100-199$ & 0.0 & 0.0 & 0.0 & 0.0 & 0.2 \\
\hline $200-299$ & 1.1 & 2.8 & 1.8 & 6.1 & 3.2 \\
\hline $300-399$ & 0.5 & 2.3 & 2.8 & 7.8 & 2.7 \\
\hline $400-499$ & 0.1 & 0.8 & 1.5 & 3.0 & 2.1 \\
\hline 500.599 & 0.0 & 0.1 & 0.3 & 0.9 & 2.3 \\
\hline 600.699 & 0.0 & 0.0 & 0.0 & 0.1 & 7.4 \\
\hline $700-799$ & 0.0 & 0.0 & 0.0 & 0.0 & 6.8 \\
\hline $800-899$ & 0.0 & 0.0 & 0.0 & 0.0 & 6.9 \\
\hline $800-998$ & 0.0 & 0.0 & 0.0 & 0.0 & 5.4 \\
\hline 1000 & 30.8 & 0.0 & 0.0 & 0.0 & 0.0 \\
\hline
\end{tabular}

$30.9 \%$ clear

obeervational area. When Ne is lese than unity, VAS may be obeerving broken opaque cloud, overcast tranamisaive cloud, orbroken transmissive cloud. All of these possiblities are labeled as "cirrus." It is not poselble to distinguish between them with the $\mathrm{CO}_{2}$ technique. Here "cirrus" refers to an observation where the VAS radiometer detects radlation both above and below a cloud layer.

Effective emisesivity observations less than 0.85 are labeled as cirrus ranging from the thin low emissivity clouds on the loft to the thick high emissivity clouds on the right. Those greater than 0.95 are considered to be opaque clouds, since the cloud top height derived from Equation (1) is very close to the height derived from the window channel by itself. Most cloud heights below $700 \mathrm{mb}$ were determined from the infrared wind ow channel and, thus, were assumed to have an effective emissivity of 1 . This assumption prevents the misinterpretation of low broken cloud as cirrus.

Table 1 reveals that clouds were detected on average 69\% of the time during this period, with $32 \%$ of those observations identified as cirrus and $37 \%$ identified as opaque cloud. Independent observations by Warren ot al. (1986) found
$32 \%$ cirrus $\quad 37 \%$ opaque

that the GSL is covered by clouds over $70 \%$ of the time. Cirrus obeervations ranged from 100 to $600 \mathrm{mb}$ and varbd in thickness. Opaque clouds were detected at all bevels but most commonly found between 600 and $850 \mathrm{mb}$. High clouds $\left(\boldsymbol{P}_{\mathrm{c}}<400 \mathrm{mb}\right)$ were observed $31 \%$ of the time, while clouds between 400 and $700 \mathrm{mb}$ were obeerved $19 \%$ of the time. Low clouds having cloud top pressures no loss than $700 \mathrm{mb}$ were found approximately $19 \%$ of the timo. Clear sky observations were found on average nearly $33 \%$ of the time during the winter months.

Figure 3 shows the geographical distribution of percent cloud amount (or effective emissivity) and frequency of clear sky during the same period. The area covered by this region is from $30^{\circ}$ to $40^{\circ} \mathrm{N}$ latitude and $60^{\circ}$ to $85^{\circ} \mathrm{W}$ longitude. During this seven-year climatology, clouds occurred most frequently east of the Gulf Stream region $(70 \%)$ and consisted mainly of opaque clouds ranging in height from $800 \mathrm{mb}$ to $400 \mathrm{mb}$. Over the axis of the Gulf Stream, cloudiness was detected no more than $50 \%$ of the time, consisting mainly of high transparent clouds. In the lower panel, VAS observations where the $\mathrm{CO}_{2}$ technique derives a cloud top pressure of $1000 \mathrm{mb}$ are shown. These 

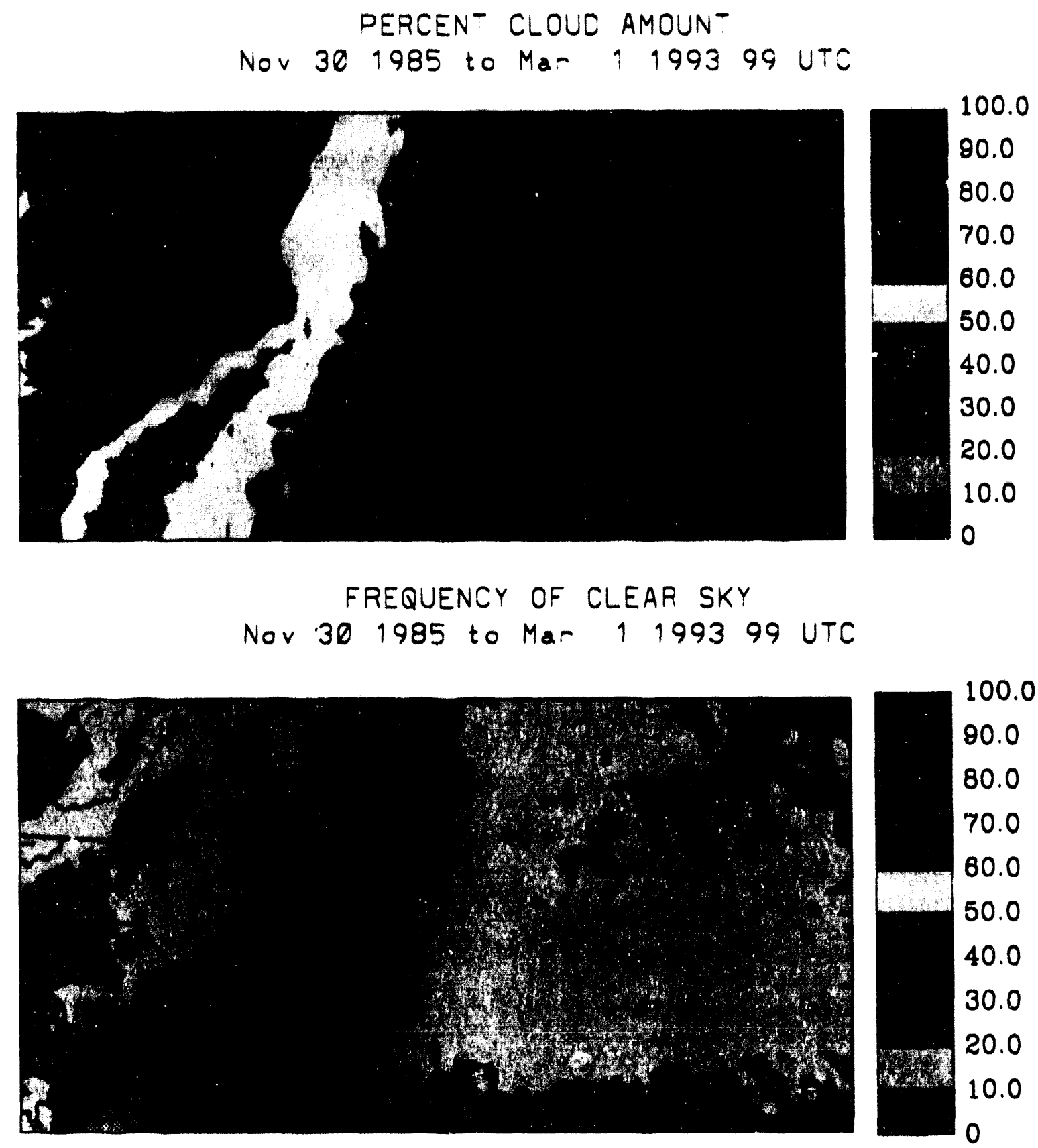

Figure 3. Percent cloud amount and frequency of clear sky during the winter months (Dec.-Feb.) 1985-1993, not including 1986-1987. 
obeervations are laboled es cloar cky. Cloar eky conditions wore found moot often along the axis of the Culf Stroam whit a marked decrease to the oavel.

\section{Cloud Conorition Proceseses Assoclated with CAOS}

About 15 to 20 CAO ovents affect the mid.Attantio region annually botweon Decombor and March, with about fivo of thoes boing intones (air temporature < $0^{\circ} \mathrm{C}$ ) (Croseman 1988). During January 1983, eoven CAO ovents occurred, with the moet intonse obeerved on January 28.

Figure 4 is a vialble image from GOES-7 at 17 UTC 4 Jan 23. Cloud atreots are obeorved east of the QSL. During the eoven CAOS, VAS cloar sky obeorvations over the C8L wore obeerved noarty $50 \%$ of the time. Compared with the climatology, clear shy observations during thees CAOs were found nearty $20 \%$ more often; howover, high thin cirrus clouds wors obeerved much bees frequently (18\%). Low opeque doude wore obeerved $18 \%$ of the time, sbout the samo frequency as the climatology. Figure 5 shows the percent cloud amount and frequency of clear sky during the soven CAO ovents. The $25 \%$ of cloudinese obeerved over the Gult Stream to the east of Cape Hattoras is in response to the sharp gredient in sea surfece temperature (8ST) in the region.

During CAOs, cold dry airmases is modified as it moves acrose the shelf waters. Over this region the air is not saturated, thus cloud genesis is inhibltod. Deppending on the air temperature, the trajectory and intenaity of the wind, clouds typically develop on the eastern side of the Gulf Stream where the airmase has become most unstablo. This conditioning period is most responalble for the cloud minimum seen over the GSL (Figure 4). The SBT image shown in Figure 1 shows this process. No clouda are observed over the shelf waters and the wostom Gulf Stream, as indicated by the unobatructed viow of the eea surface. Howover, on the eastern side of the Gulf Stream, the SST field becomes obecured as attenuation due to low clouds increases. The aftenuation effects become more dominant further offshore. Over the Sargaseo Sea, to the east of the Gulf Stream, low clouds were identified $36 \%$ of the timo, epproximately $10 \%$ more than in the cllmatology. These conslated of oumulus and stratocumulus which doveloped in the boundary layer as the cloud top preseures wore estimated to bo around $800 \mathrm{mb}$.

The other 24 days of the month were charectorized by an above-normal amount of cloudinees. The winde in the reglon wore predominably from the southwed, transporting warm moist air from the Gulf of Moxico northosetwerd. Cloude were obcerved epproximatoly $70 \%$ of the time over the G8L and over the entire domain. Of the $70 \%$ of cloud obeervations, $50 \%$ wore lidentilied by VAS as cimus cloud. VAS cloar shy obeorvatione were obeerved only $5 \%$ to $10 \%$ of the timo.

\section{Conclusions and Future Work}

The principal finding is that the occurrence of clouds over the Gulf Stream Locale during the winter months is dependent on atmospherio procoseses and their likely intoraction with the Gulf Btrearn. A cloud climatology conaibting of eaven wintere indicates that clouds occur epproximatoly $70 \%$ of the timo. However, during periods of cold air outbreaks, cloudineas cocure much bes frequently. From the limiled number of analyzed CAO ovente, VAS obeervations indicato that the Gulf Stream Localo plays an important rob in the formation and maintenances of clouds, particularty shallow, opeque clouds downwind of the Gulf Stroam Localo.

Future work will focus on leolating other atmospheric procoseses and their relationehip to cloud production over the Gulf Stroam Localo. In addition, diurnal effects of cloud distribution and the land, alr-ece interaction processe will be inveatigated and reported on in the future.

\section{Acknowledgments}

This work was supported by the U.S. Departinent of Energy Atmospheric Radiation Measurement Program under contract 091575-A-Q1 with Pacific Northwest Laboratory. 


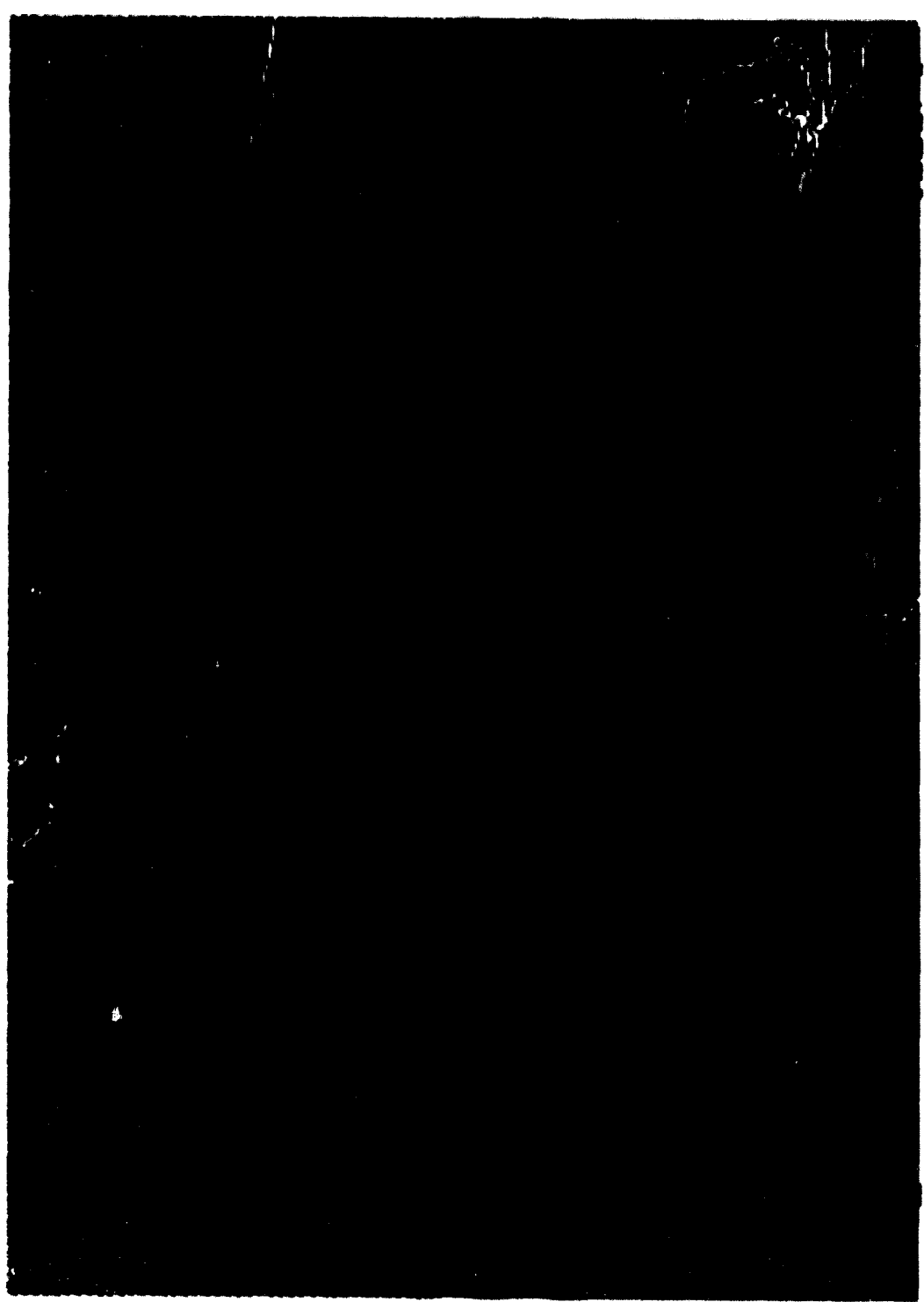

Floure 4. COES-7 visible imagery (17 UTC) during the CAO event on 28 Jan. 1993. Cloud streets are readily vialble as the cold dry continental air moves over the warmer Gulf Stream. 


$$
\text { DERCEN- C_OUC AMOLN' }
$$
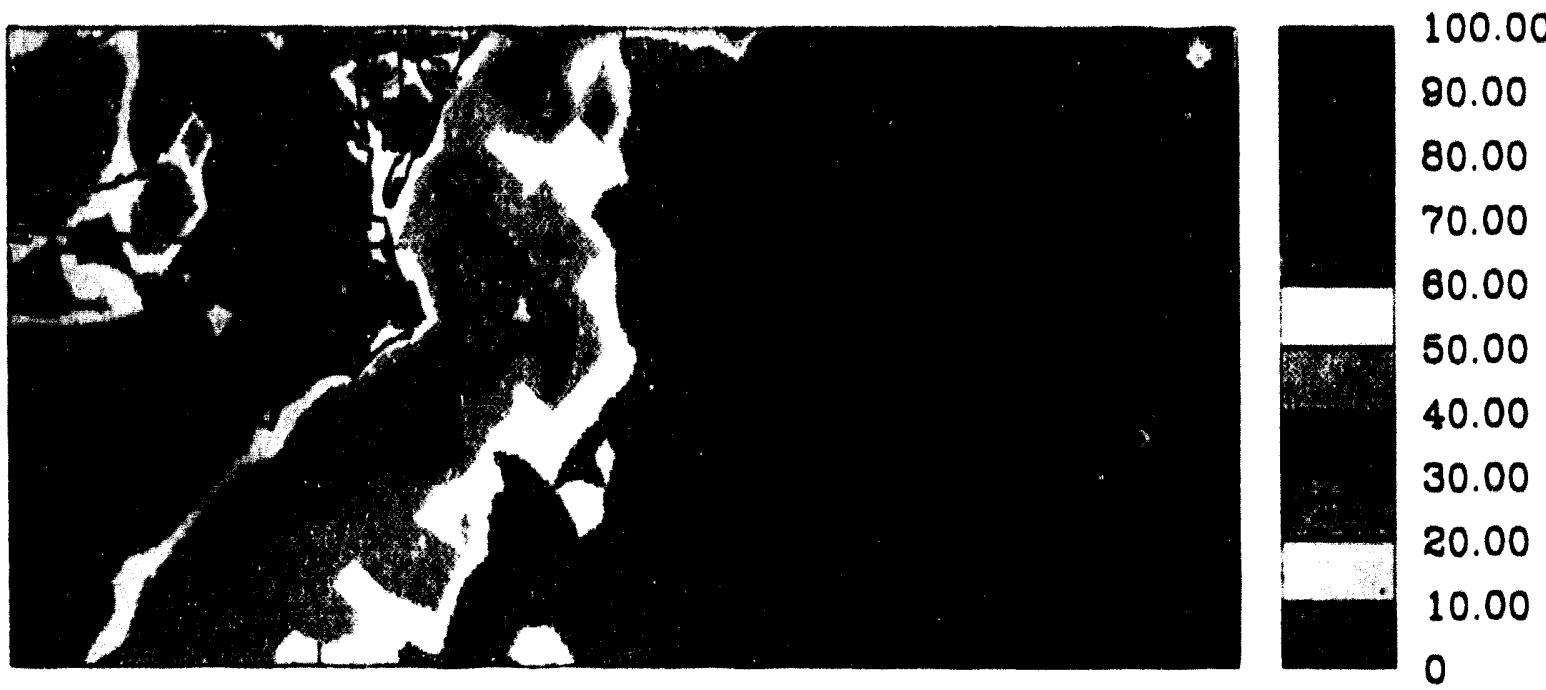

FREQUENCV OF CLEAR SKY
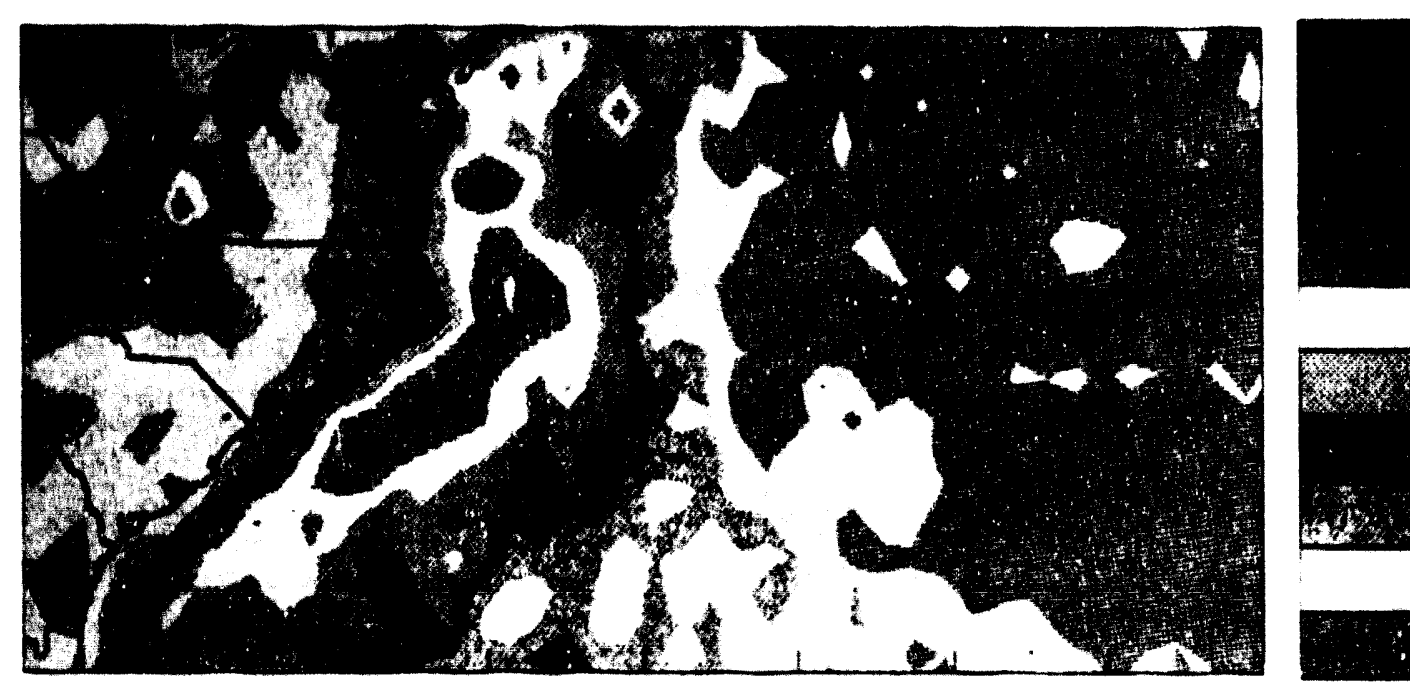

100.0

80.00

80.00

70.00

60.00

50.00

40.00

30.00

20.00

10.00

0

Figure 5. Percent cloud amount and frequency of clear sky during seven cold air outbreak events, January 1993. 


\section{References}

Chahine, M. T. 1974. Romote sounding of cloudy atmoephores. 1: The single cloud layer. J. Atmos. Sol. 31:233-243.

Groseman, A. L. 18e8. Boundary Layor Warming by Condeneation: Ar esa interaction during an extroms cold alr outbreak from the esstom cosat of the United States. Procesedinge, Sswenth Conference on Ocwan-Atmoephers interaction. Amorican Motoorological Socibly, Booton, Masesachusotts.

Gruber, A., and T. 8. Chen. 1988. Diumal vertation of outgoing longwave rediation. J. Clim. Appl. Moteorol. 0:1. 16.

Monzel, W. P., W. L. Smith, and T. R. stowart. 1983. Improved cloud motion wind wector and dittude aselonment using VAS. J. Cllm. Appl. Motworol. 22:377-384.
Rocesow, W. B., and A. A. Lecis. 1980. Globel, seasonal oloud varlations from satellite rediance moseuremonts. Part II: Cloud propertibe and radiative offects. J. Cllm. 3:1204-1253.

Smith, W. L., and C.M.R. Platt. 1978. Intercomparieon of radiosonde, ground beased laser, and eatollite deduced cloud hoights. J. Appl. Moteorol. 17:1786-1802.

Smith, W. L., H. M. Wooli, P. G. Abol, C. M. Hayden, M. Chalfant, and N. Grody. 1974. Nimbus 5 sounder data procseaing ayotom. Part 1: Moasuremont charectoriatloa and data reduction procedures. NOM Tech. Momo. NESS 57, 90 pp.

Warron, 8. G., C. J. Hahn, J. London, R. M. Chorvin, and R. L. Jenno. 1888. Global diatribution of total cloud cover and cloud type amounte over land. NCAR Tech. Nots NCARTN-273+8TR, 228 pp. 


\title{
Scientific Issues in the Gulf Stream Locale(a)
}

\author{
P. Michael and M. L. Daum \\ Brookhaven National Laboratory \\ Upton, NY 11973 \\ S. Raman and M. Leach \\ North Carolina State University \\ Raloigh, NC 27695-8028
}

\section{Locale Characteristics}

Cloud and Radiation Testbed (CART) sites have been selected so that, taken collectively, they span the range of attributes needed to realize the goals of the Atmospheric Radiation Measurement (ARM) Program. Thus, the selection of a locale as a primary CART site was based on its ability to contribute uniquely and significantly to the ARM scientific mission. The Gulf Stream locale has four characteristics of significance to climate research and to the ARM program:

1. The Gulf Stream locale exhibits extreme ranges in magnitude of surface heat fluxes; no other locale exhibits such a range in air-water temperature differentials and latent heat fluxes. These large fluxes provide a rigorous test of surface exchange and convection schemes in general circulation models.

2. This locale provides the best opportunity among the candidate locales to observe mature synoptic storms. These systems, which are characterized by extensive stratiform clouds, are the only such cloud systems that might be resolved by a general circulation model. Hence, they offer the possibility of testing process models and parameterizations, including microphysics parameterizations, without the usual difficulties associated with sub-grid variability.

3. The inhomogeneity that exists in the GulfStream Locale provides a severe test for cloud generation modules of

(a) A draft report with more dotail on this extended abstract is availeble from Paul Micheol, Building 318, Brookhaven National Laboratory, Upton NY 11973. Telephone: 516-282-2264, FAX: 516282-3264. Intomet: PMICHAELOBNL.GOV climate models. These modules usually assume uniformity of surface conditions over domains of several hundreds of kilometers.

4. The anthropogenic aerosol loading off the east coast of the United States is highly variable; thus, the locale provides a testbed for hypotheses on the effects of changes in cloud condensation nuclei (CCN) concentrations upon cloud optical properties, structure and life cycle.

Of the five primary CART sites, the Gulf Stream locale, a western boundary current location, uniquely experiences positive sensible and latent heat fluxes that span wide ranges and are likely the largest on the earth (item 1 above).

It should be noted that while the locale characteristics derive from the properties of the Gulf Stream, the locale itself is best defined as those places where the effects of concern manifest themselves; for example, the greatest air-sea temperature differences during cold air outbreaks occur not over the core of the Gulf Stream but over its landward edge, and the large-scale storms reach their maturity beyond the northern wall of the Gulf Stream. Therefore, implementation of ARM program measurements is not to be limited to locations within the Gulf Stream proper.

\section{Science Issues}

The science issues, posed as questions, are grouped below under the two classes of ARM objectives as initially outlined in the ARM program plan and restated in the ARM report on the recommendation of potential locales for ARM sites. 


\section{Class 1 ARM Objectivo}

Rolale obeerved instantaneous rediative properties of the atmosphere (epectrally resolved) to the then-present atmoeshoric ismporature and composition (epecifically including wator vapor and clouds) and surfeco rediative proporties, both as a function of position, and dovelop paramotorizations for thece rolationships.

1. Cloud claselification: What modifications are needed in the cloud cleselfication sechemes ueed in climate modele in ordor to charectorize rediation edequately?

2. Wator vapor distribution: How does spatial and tomporature diatribution of wator vapor affect radiation?

3. Aorosol effects: How do the number donsity and size distribution of aprosols and CCN affect radiative tranofion?

4. Surfece roughness: What is the effect of surface roughnese upon radiation?

\section{Ciass 2 ARM Objective}

Dovelop paramotorizations to doscribe atmospheric composition (egain, spectically including water vepor and clouda) and surfece proporties governing atmoepheric radiation in torme of relovant prognoetlc variables, with the objective of incorporating these parametortzations into conoral circulation and related modols.

1. Surfeco flux parameterizations: What is the range of applicability of the parametorizations of censible and latont heat fluxes?
2. Surfece fluxes and cloud formation: What is the effect of variations of latent and sensible hoat flux upon cloud formation?

3. Cloud life cycle: What improvements are required in the mothods of predicting the formation, maintenancs, and diselpation of large-scale cloud systeme?

4. Surteco hotorogenoity: What is the effect of eurfeces heterogenolty upon cloud formation?

5. Arroeol affects on clouds: How do the number donalty and size distribution of sorosols and CCN affect cloud size, structure, and lifotime?

6. Sub-grid processes: What is the effoct of surface hotorogenelty upon model paramoterization?

\section{Phased Implementation}

Tho ultimate targot is a cories of measurements, aimed at investigating epecific scientific lesues, conducted over a porlod of time, and with sufficient continulty that a full range of conditions can bo spanned. Before full occupation, which is some yoars in the future, it is anticipated that activitios can be undertaken both to further ARM objectives and to asalat in the desion of the CART program. The expectation is that considerable omphasis will be put upon developing a preoccupation design for using and incorporating existing data into the ARM data stream. In addition, opportunities for limited augmentation will bo inveetigated. Any preoccupation activities would, of couree, be dependent upon the avallability of resources. 


\section{Summary of Breakout Sessions}




\title{
Summary of Combined Heiarchical Diagnosis and Single-Column Model Breakout Session
}

\author{
D. A. Randall \\ National Center for Atmospheric Research \\ Boulder CO 80307-3000 \\ J. T. Kiohl \\ Department of Atmospheric Science \\ Colorado State University \\ Ft. Collins, CO 80523 \\ M. Bradley \\ Lawrence Livermore National Laboratory \\ Livermore, CA 94550
}

\section{Discussion of Intensive Operation Periods (IOPs)}

The group made several recommendations for LPs in general. One of the main points was that members of the Atmospheric Radiation Moasurement (ARM) Science Team should be given as much advance notification as possible of planned or potential 1OPs. The group also recommended that a load scientist be appointed for each IOP. This scientist should have expertise in the topic of primary focus of the IOP. The lead scientist's main responsibility would be to make sure that the operations carried out in the IOP address the relevant scientific issues to the fullest possible extent. This would be done in coordination with both other Science Team members and the EST, as well as the Site Scientist and Site Manager. These same recommendations apply to campaigns. (Note: The specific information discussed during the breakout session and summarized here was current during the first week of March 1993, but may have been superseded.)

\section{June 1993}

This IOP was requested by the National Center for Atmospheric Research (NCAR) to test the five-beam profiler and to evaluate warm season data assimilation. It is a dataassimilation-oriented IOP. Although it was discussed during the November Single Column Model (SCM)/DA workshop, several Science Team members were not aware of this
1OP, hence, the general consensus was that there should be more advance notice for future 1OPs. Science Team members need information about this IOP as soon as possible. Chris Walcok stated that ARM should provide rainfall data over the site for this IOP and agreed to work with Ric Cedenwall (EST Contact for this IOP) to define the specific rainfall data requirements.

Dave Parsons made a brief presentation on this LP. (He had given a presentation on this IOP at the SCM workshop in Richland, Washington in November 1992). Following are the main points of his presentation in Norman:

What: Warm season data assimilation and Integrated Sounding System (ISS) test, fourdimensional data assimilation (4DDA) using standard Cloud and Radiation Testbed (CART) data and comparing with LAPS or Mesoscale Analysis and Prediction System (MAPS)

When: June 93 for 10 days, exact dales depend on weather conditions

Observations planned:

4 radiosonde launches per day from each of the following National Weather Service stations: Oklahoma City, Dodge City, and Topeka

3 NCAR CLASS soundings, 8 per day 
Additional radiation observations at Crosschain Loran Atmospheric Sounding System (CLASS) sites-downwelling solar and IR

2additional 915-MHz profilers (central facility 915 , plus 2 others to make a small triangle to get $u, v$, and $w$ and to get vertical vorticity component and divergence)

End product: $5-\mathrm{km}$ horizontal grid of balanced fields for SCM and hierarchical diagnosis (HD)

Other points: Would like to get Andy Heymsfield to include iormvar sounding. Bob Kropfli, Bob Mcintosh, and Ken Sassen are planning to participate.

\section{Fall 1993}

There will be an IOP sometime during the September November 93 time period for the purpose of conducting a series of prototype unmanned aerospace vehicle (UAV) flights. (On the last day of the workshop, several presentations were given on the UAV program, including one on the plans for the prototype UAV flights.) The SCMHD group requested that ARM make a general announcement of this IOP and schedule a planning meeting very soon.

\section{Spring 1994}

As a result of a request from three Instrument Development Program (IDP) team members attending the breakout session, there will be an IDP-oriented IOP in March or April 1994. The primary focus of this IOP will be to validate and calibrate new instruments. A two-day workshop should be held soon to plar, for this IOP. A lead scientist with appropriate specialization should be selected to coordinate this IOP.

\section{Science Team Guidance for Specific CART Sites}

The group recommended that small committees of Science Team members be formed to focus on scientific issues for specific sites. Jeff Kiehl is interested in forming a group of about ten people who would meet regularly to discuss the tropical western Pacific CART site.

\section{Discussions of HD “Key Questions"}

This discussion resulted from a suggestion by Jeff Klghl at the November 92 HD Workshop that the HD team compile a list of a few key scientific questions regarding the role of small-scale processes in climate processes. The goal was to bring the group together; the key questions could be the "glue" that would make the HD team a true working group.

Jeff received 17 suggestions (an additional one was submitted after the meeting). Although Jeff did not anticipate it, the call for questions caused some concern among the HD Science Team members that the establishment of such a list would appear to imply a prioritization of the HD research projects, i.e., that some were more important than others. Although there were mixed reactions to the key question concept a majority seemed to agree that, if handled properly, the exercise could be helpful.

Steve Ghan suggested that the questions could be divided into five categories:

- upper tropospheric water vapor

- issues involving cirrus clouds (their formation and radiative properties)

- effects of convective clouds

- effects of aerosols-direct and indirect (e.g., cloudaerosol interactions)

- subgrid-scale cloud radiative properties.

The group selected five HD Science Team members to refine the key questions list: Bill Cotton, Tony Del Genio, Steve Ghan, Jeff Kiehl, and Ken Sassen.

The participants will decide soon if they need to reconvene in fall of 1993. 


\section{ARM/Unmanned Air Vehicle Satellites}




\title{
Unmanned Aerospace Vehicle System Concept
}

\author{
W. R. Bolion \\ Sandia National Laboratories \\ Livermore, CA 94550
}

\section{Introduction}

The Atmospheric Radiation Measurement/Unmanned Aerospace Vehicle (ARMUAV) Program has as a major mission to support the ARM Cloud and Radiation Testbed (CART) sites with an airborne measurement capability. The UAV capability will complement piloted aircraft and supplement the capabilities of ground-based CART instruments. The ARMUAV Program strategy emphasizes meaningful scientific activity embedded in the development activities and has three phases of increasing system capability:

- Demonstration flights in the first year emphasize early scientific results and initial operational experience with UAVs in an atmospheric research role. Because of the desire for an early demonstration, this activity will be conducted with existing UAVs and instruments.

- An interim measurement capability in the second and third years will make use of improved UAV and instrument capabilities to provide sustained operations at a location in the continental United States; for example, the Southern Great Plains (SGP) CART site. The system used for interim operations will consist of near-term UAVs assumed to be capable of greater than $14-\mathrm{km}$ altitude and endurance in excess of 24 hours, and existing and near-term instruments.

- The full-capability ARMUAV system will follow in the third and fourth years, capable of sustained and autonomous operation in remote locations, for example, in the Tropical Western Pacific. The UAVs will be capable of $20 \mathrm{~km}$ altitude and multi-day endurance and will carry instruments tailored for UAV application.

\section{Elements of the UAV System}

In addition to the ground-based instruments, the ARW UAV system consists of the UAV, its instrument suite, and the instrument interface and telemetry system. The UAVborne instruments and supporting system provide a capability to obtain measurements at selected locations in the atmospheric column and transmit the data to the ground for recording and subsequent processing. The elements of this system and their functional relationships are shown schematically in Figure 1.

\section{Demonstration Flights}

The first ARMUAV development phase is the UAV demonstration flight (UDF) series. UDF will consist of three to five flights conducted at the SGP CART site over approximately a 2-week period. The primary scientific objective of UDFs is a radiative flux divergence measurement, accomplished by measuring flux at two altitudes and subtracting to obtain the flux divergence. The lower altitude measurement will be made from the SGP tower, a tethersonde, piloted aircraft, or UAV. The higher altitude measurement will be made from a UAV, piloted aircraft, or satellite. The details of this experiment are being developed in consultation with the ARM Interim Science Team.

In addition to the scientific objectives, UDF has programmatic objectives which include obtaining operational experience with UAVs and addressing airspace and safety approval issues that must be resolved for later phases of the program. 


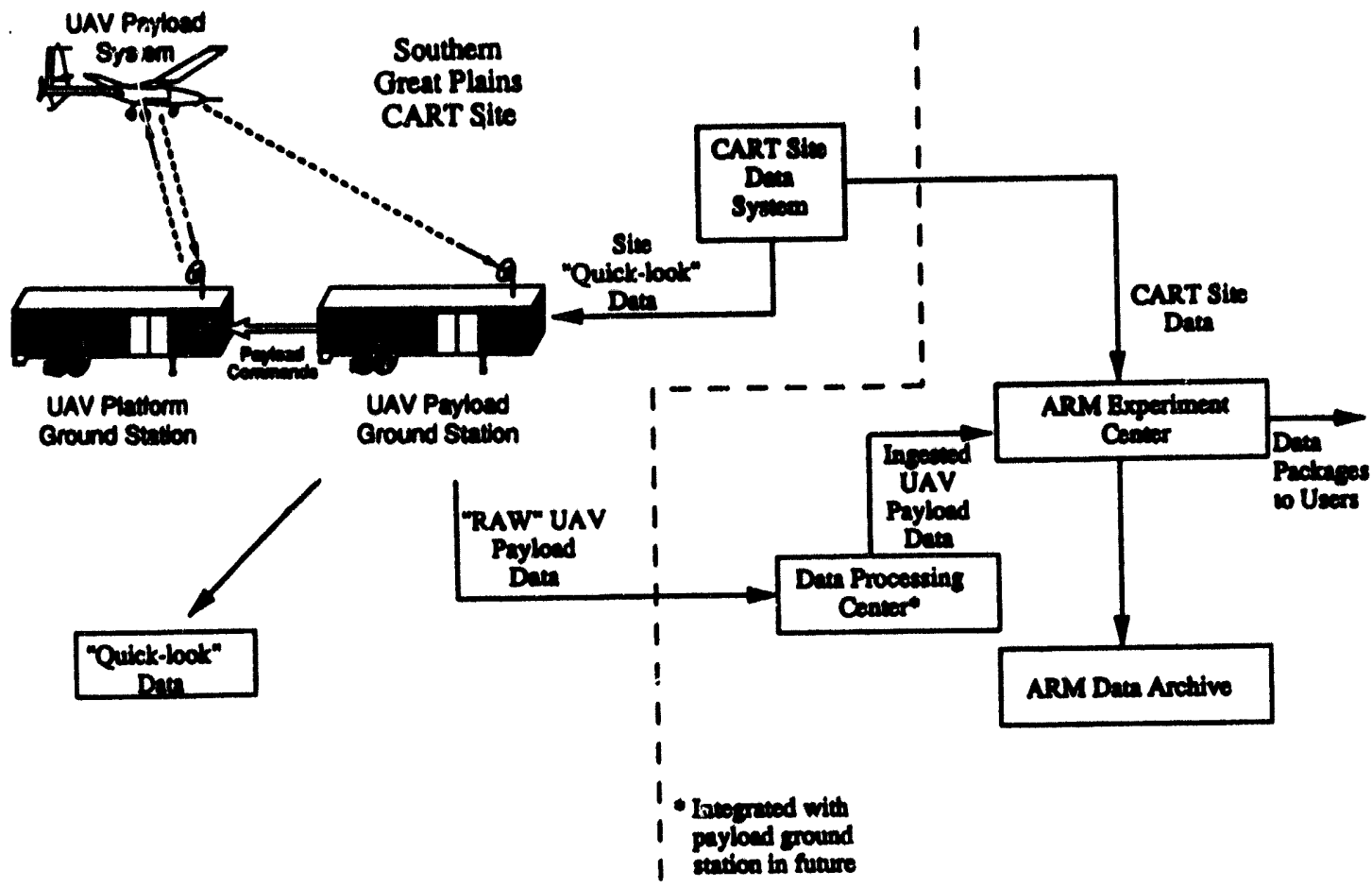

Foure 1. Functional elements of the ARMMAY system.

The preliminary instrument list for UDF was selected to maich the rediative flux divergence objective. Preliminary packaging studies indicate that usoful instrument payloads drawn from the preliminary instrument list can be carried in candidate UAVs. Primary instruments include hemispherical field-of-view broadband solar and IR radiometers, and a total diffuse direct radiometer. Supporting instruments include a meteorological package (1emperature, pressure, water vapor), a narrowband radiometer (solar and IR radiance), and perhaps a video camera.

UAVs suitable for UDF should have endurance in excess of $6 \mathrm{~h}$; altitude of $7 \mathrm{~km}$ or greater; a payload capacity of at least $60 \mathrm{~kg}$; and, by virtue of configuration and technology, be on the "development path" toward a full-capability UAV. Candidate UAVs that appear to be suitable include the General Atomics "Gnat 750-45" (arid enhancements) and the Aurora Flight Sciences "Perseus B."
The UDF payload system will consist of the instruments, the instrument interface and signal conditioning, and redio frequency transmission to the ground station. The payload ground station will be equipped to receive and record all data, and scale and display "quick look" data. Formatting the data (ingest) will be accomplished at an existing data processing center for UDF but will be integrated into the payload ground station in the future.

\section{Summary}

UDF will provide valuable scientific data and will be an important step along the development path leading to the full-capability ARMVAV system. Subsequent steps will develop improved instruments and fully capable ground support equipment suitable for remote deployment, as well as take advantage of improved UAV capabilities. 


\title{
The Atmospheric Radlation Measurement Unmanned Aerospace Vehicle Program: An Overvlew
}

\author{
P. A. Crowloy \\ Environmental Sciencos Division \\ U.8. Department of Enorgy \\ Washington, D.C. \\ J. Vitko, Jr. \\ Sandia National Laboratories \\ Livermore, CA 84550
}

\section{Introduction}

This paper and the one that follows describe the start-up of the ARM-Unmanned Aerospace Vehiclo (UAV) program. This program has its origins in the Atmospheric Radiation Measurement (ARM) Program's long recognized needs for in-situ airborne measurements of radiative fluxes, flux divergences, selected cloud properties, and upper tropospheric water-vapor profiles. While manned alrcraft have and will continue to provide many useful measurements, the need to fly above the tropopause (18 $\mathrm{km}$ in the tropical Pacific) for multiple diumal cycies (48 to $72 \mathrm{~h}$ ) and at a sufficiently low cost to permit sustained coverage (over $1000 \mathrm{~h}$ per year) appears to be met best by anemerging new generation of UAV specifically designed for climate studies. Thus in 1992, the Department of Energy (DOE) proposed the ARM-UAV program to complementmanned aircraft measurements and to provide an observational system for sustained high-altitude measurements over the Cloud and Radiation Testbed (CART) sites.

Two factors are now enabling us to start the ARM-UAV program. The first is that the DOE has recelved initial funding from the Strategic Environmental Research and Development Program (SERDP) to develop improved atmospheric remote sensing tectniques that are compatible with both UAVs and small satellites. While the SERDP program emphasizes instrument development, it also provides for measurement flights for calibration/validation and initial data gathering and evaluation. The second enabling factor is that there are now several possibilities for leased UAV operation over the next year. Examples include, but are not limited to, the existing Gnat 750-45, with its $7.8 \mathrm{~km}$ colling, as well as the planned FY93 demonatration of two $20 \mathrm{~km}$ capable UAVs - the Pereous$B$ and the Raptor.

Thus the funding of some initial filghts and the avallability of leased UAVs will enable us to start up the ARM-UAV program. Additional funding will be required to continue this program.

\section{Interim Science Team}

These ARM-UAV activities will ultimately be gulded by a science team selected through a peer-reviowed competitive process. Howover, to ensure a smooth and efficient startup, an Interim Science Team (IST) has been formed to provide initial scientific guldance. The IST members, drawn primarily from the existing ARM community, mot in December to formulate a near-ferm strategy for the first year's activitles and to identify key scientific questions underlying the longtorm ARM-UAV program.

The meeting identified two broad classes of missions for ARM-UAV: "quasi-continuous missions," which emphasize consistent long-term observations of key radiation-cloud parameters as part of a continuous ARM data stream, and "investigative missions," which change with time and focus on testing specific hypotheses. The basic quasi-continuous mission is the continuous/near-continuous measurement of radiative fluxes along with both in situ and remote 
conaing meacurements of wator vepor and cloud propertice. Reprecentativo inveetlgative micsions include tosting of specific hypotheeses on the rob of deep convection in the tropical Pacticic, the orying/molotening of the uppor troposphere, the source of the asymmotry in water vepor concentrations in the northom and southern hemiephores, ozone chemiatiy noar the tropopause, olc.

To eddrese these miesions, one ultimatbly needs a UAV cepable of autonomous operations sbove the tropical tropopause $(20+\mathrm{km})$ for multiplo diurnal cycles $(48-72 \mathrm{~h})$ with payloads of $150-200 \mathrm{~kg}$ at eufficiently low cost to allow extended operations (over $1000 \mathrm{~h}$ per year). Howover, wo 800 two koy phases preceding this "full capabllity" phase. The first is a "demonstration phase" meant to establish our presence quickly by using "oxisting" instruments and basing the most capable UAV to get some scientifically important data by the end of FY93 or earty FY94. The second or "intorim capability" phase would provide an operational capablity at the Southern Great Plains (SGP) CART site, i.e., $14+\mathrm{km}$ for a minimum of $24 \mathrm{~h}$ with oither line-of-sight or autonomous control. The third and final phase is fullcapability operations and would allow for autonomous operation over the tropopause in the troplcal Pacific for multiple days.

\section{UAV Demonstration Flights}

Particular attention has been given to defining the first or demonstration phase. The demonstration mission mustbe scientifically important and along the path to the long-term miscions; at the same time, It must make use of "oxisting" cquipment and bo relativaly simplo so as to bo flown within a yoar with a good likelihood of succoses. Following the recommendation of the Intorim Scionco Team, thees UAV domonstration filohts (UDF) will emphadze fux divorences mosauremonts, a koy bullding block for many future misclons.

Tho badoldoals to make up- and down-looking broectbend hemisphorical fiux moceurements from a UAV and combins these with almilar moasurements made from the ground (actually tower and tothorsondo) and from sabilitios to obtain the flux divergence. These measurements would later be expanded to include multiplo UAVs to provide flux divergence measurements between different almosphoric bvels.

These flux divergence measurements are key to ARMUAV interim and long-range goals which focus on the relationship botween atmospheric heating and cloud and water- vapor distributions. When completed, the UDF will be the first demonstration of climate measurements from a UAV and will provide accurate measurements of flux divergence and selected radiative properties for testing models.

The succeeding papers provide additional descriptions of the UDF ecience mission, strawman payload, and syetems operation concepts. Though we have made much progrees UDF is still in an evolutionary state and subjoct to change. Wo wolcome your feedback! 


\title{
Strawman Payload for the Unmanned Aerospace Vehicle Demonstration Flights
}

\author{
J. Vikko, Jr. \\ Sandia National Laboratorias \\ Livormoro, CA 84650
}

\section{Introduction}

The firet phase of the Atmosphoric Redlation MoseurementUnmanned Aorospace Vehich (ARM-UAV) program is a demonstration phase meant to provide both scientific reoulte and operational experience within the first year of the program. These UAV demonstration fights (UDF) will emphasize flux divergence measurements, a koy bullding block in the longer term goals of ARM-UAV. The basic ldea is to make up- and down-looking broadband flux messurements from a UAV and combine these with similar moseurements made from the ground, other alroratWAVs, and satelities to obtain the flux divergence. Of necessity, the UDF will uee "existing" equipment-both in terms of inatrumentation and UAVs. A strewman UAV payload for this misalon is houriatically shown in Figure 1.

This payload consists of three main groupings of instrumonts: emall, accurato, fux rediometore which provide the critical flux measurements; an ancillary packa oe which provides epectral radiances and vialble imagery to aid in the interpretation of the flux divergence; and a movorological package which charactorizes tho bulk stato of the atmoephere. Selected portions of this payloed are deacribed briofly bolow.

\section{Flux Radiometers}

Francisco Valero of National Aeronautice and Space Administration (NASA) Ames has soveral flux radiometers that appear to be woll sulted to near-term UAV applications. Thoee include both broadband hemispherical solar and IR flux rediometers '9BHSR and BBHIR), as woll as the totel direct diffuse radiometer (TDDR), which measures both the direct and diffuse solar radiation in each of seven narrow spectral channels. All three types of instruments haw been flown extensively on aircraft and, in some cases, on balloons; typically wolgh lese than $5 \mathrm{~kg}$; and can operate unattended and over wide tomporature ranges (as low as $-20^{\circ} \mathrm{C}$ ).

From the onset, these instruments have been dedined with calloration in mind. They have estimated cccurecles as high as $1 \%$ in the solar and several percent $(-3 \%)$ in the IR and appear to be well accepted in the climatecommunity.

The current plan is to fis two each-one up-looking and one down-looking - of the BBHSR and BBHIRInstruments. In addition, we will fly a single up-looking TDDR. While the TDDA does not measure the broadband flux, it does measure the direct and diffuse components of the solar rediallon. This information is needed to attain the maximum accuracy in analyzing the solar brosoband fiuxes from the BBHSR.

\section{Ancillary Package}

The ancillary packege provides spectral radiances and vielble imagery to holp interprot the flux dwergence data. Spectral radiances holp in two ways. First, thoy provide information on the epectral signature of the scene and therefore can be usoful in rolating the enoroy dotected by a broedband radiomoter to the actual enorgy incident on the instrument. Second, they can bo used to initor cloud, aerosol and water vapor properties and, hence, can bo used in conjunction with flux divergence measurements to teat various radiative modele.

Two epectral rediomotors are under consideration. The first is the Scanning Spectral Polarimotor (SSP) now boing devoloped by Greame Stephens and co-workers at Colorado Stato Univoraity and first scheduled to fit this apring. The SSP is a nadi-looking spectral rediombior for measuring daytime cloud, serosol and surfece propertises. Hcovers the epectral range from 0.4 to 2.4 microns in cbout 


\section{We have identified a strawman payload for thefirst UAV mission}

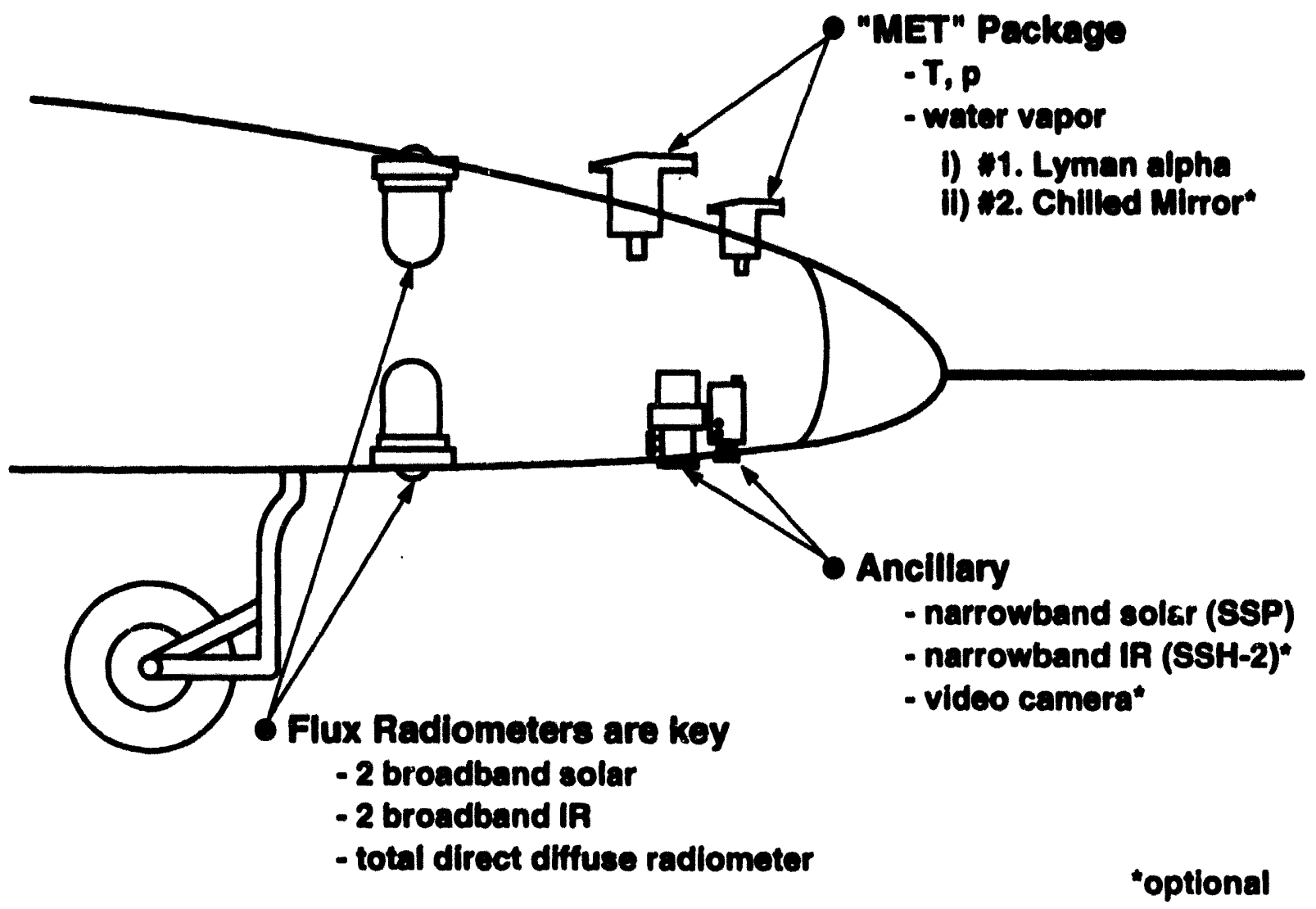

Figure 1. A stylized strawrman payload for the UAV Demonstration Fight (UDF). (Instruments marked with an astorisk are optional and will be included only if schedule and budget allow.)

90 bands and includes polarization capability. The second is an IR epectral radiomoter, the SSH-2, that was originally designed as a Dofense Moteorologlcal Support Program instrument to obtain temperature and water vapor profiles. The SSH-2 has recently been used for aircraft-based measurements in the First ISCCP(n) Regional Experiment (FIRE) intensive field observations in 1992.

\section{Meteorological Package}

The moteorological package measures such bulk atmospheric parvmeters as the temperature, pressure

(a) Imbrnational Sabelitio Cloud Climatology Project. and water-vapor profiles. Commercially avallable instruments with appropriate characteristics are readily available for measuring the temperature and preseure profiles, though their configuration will need to be engineered. The mesesurement of upper tropospheric wabr vapor is less straightforward. We recommend two instruments if resources and time allow. Our first priority is a Lyman-alpha hygrometor. These instruments can measure dewpoints from $-80^{\circ} \mathrm{C}$ to $50^{\circ} \mathrm{C}$ with an accuracy of about $0.5^{\circ} \mathrm{C}$ (or $2 \%$ in relative humidity) with millisecond response times. Our second priority is a chilled mirror hygrometer typically used in the community. These instruments provide relatively good accuracies in a small package, but do exhibit degraded response times and accuracies at low dew points. 
suo!̣SOS delsOd 


\title{
Surface Aerosol Measurements at Lamont, Oklahoma
}

\author{
R. Lolfor, R. Knuth, and H.N. Leo \\ Environmental Moseurements Leboratory \\ U.S. Department of Eneroy \\ 376 Hudeon $8 t$. \\ Now York, NY 10014-3621
}

Climate research at the Environmental Moacurements Leboratory (EML) is now primarly directed to the first Atmoephoric Radiation Moasurement (ARM) Cloud and Radiation Testbed (CART) site in Lamont, Oklahoma. The U.S. Department of Energy (DOE) has established the CART site in order to charectorize ompirically radiative proceseses in the earth's atmosphere with improved resolution and accuracy. This information will improve the predictive capability of global climato models.

The scientific community recognizes that atmospheric aerosols can play an important role in climato change. The eoroesol oftect could bo comparablo in magnitude, but opposite (0.9., cooling) to the hoating caused by the incrosaing atmosphoric concentration of carbon dioxide.

In December of 1902 the ARM Aerosel Working Group prepared the document Prlority Recommendations for Acrosel Measurements in the ARM program." A sories of experiments was described to anower queations on the direct and indirect radiative effects of aerosols. The objectives of each of the "Direct Radiative Forcing" priority experiments are reproduced here.

\section{- Priority 1 Experiment}

"Compare direct and diffuse spectral shortwave surface rediative fluxes to those predicted by a model such as LOWTRAN to establish the errors and uncertainties in using a "climatologically-averaged" description of aerosols by air mass type (rural, urban, marine, desert) included in such models."

\section{- Priority 2 Exporiment}

Delermine the errors and uncertainties in predicting surfece radiative fluxes by a radiative transfor code that takes explicticognizance of the aerosol scattering and abeorptive properties."

\section{- Priority 3 Exporiment}

Dotermine the error and uncertainties in the exposel optical propertice computed from maasured chemical compositions and microphyalcal properties of eoroeobs."

By the end of winter 1994, the earosol equipmont should be operational at the site and provide data that can be ueed in the above three priority experiments.

Aorosol redlattwe models, such as "LOWTRAN 7," require charectorization of eurfecs esrosols and air meases to choose the moet representative conditions for rediation calculations. To proporty epecily the correct input to this model, EML b using instruments at the CART elib, housed in a dedicalad trailer, to meacure the following exrocel charectoriatica:

1. particie concentration (TSI, Inc. condenaation nuciel countor)

2. optical scattoring coofficiont (MRI integrating nephelomoter, on loan from PNL, to be replecad by a Radiance Research nepholomoter in late sporing 1993)

\section{3. optical abeorption cosficiont (filtor bechniqua)}

4. corosol eize diatribution (PMS Inc. Paseive cavily cerosed spactromeler probe Model PCASP-X).

In addition, the ozone concentration will be measured using an ultra-violet photomotric analyzer (Dasibi Environmental Corp. Model 1008 RS). All instruments except the Radiance Research nephelomoter and the optical absorption instrument are at EML for teating and ovaluation bofore installation in Lamont, Oklahoma.

A special aorosol sampling manlfold (Figure 1) has beon designed at EML and will be built at the site bofore the equipment is installed in the aerosol trailor. The manitold 


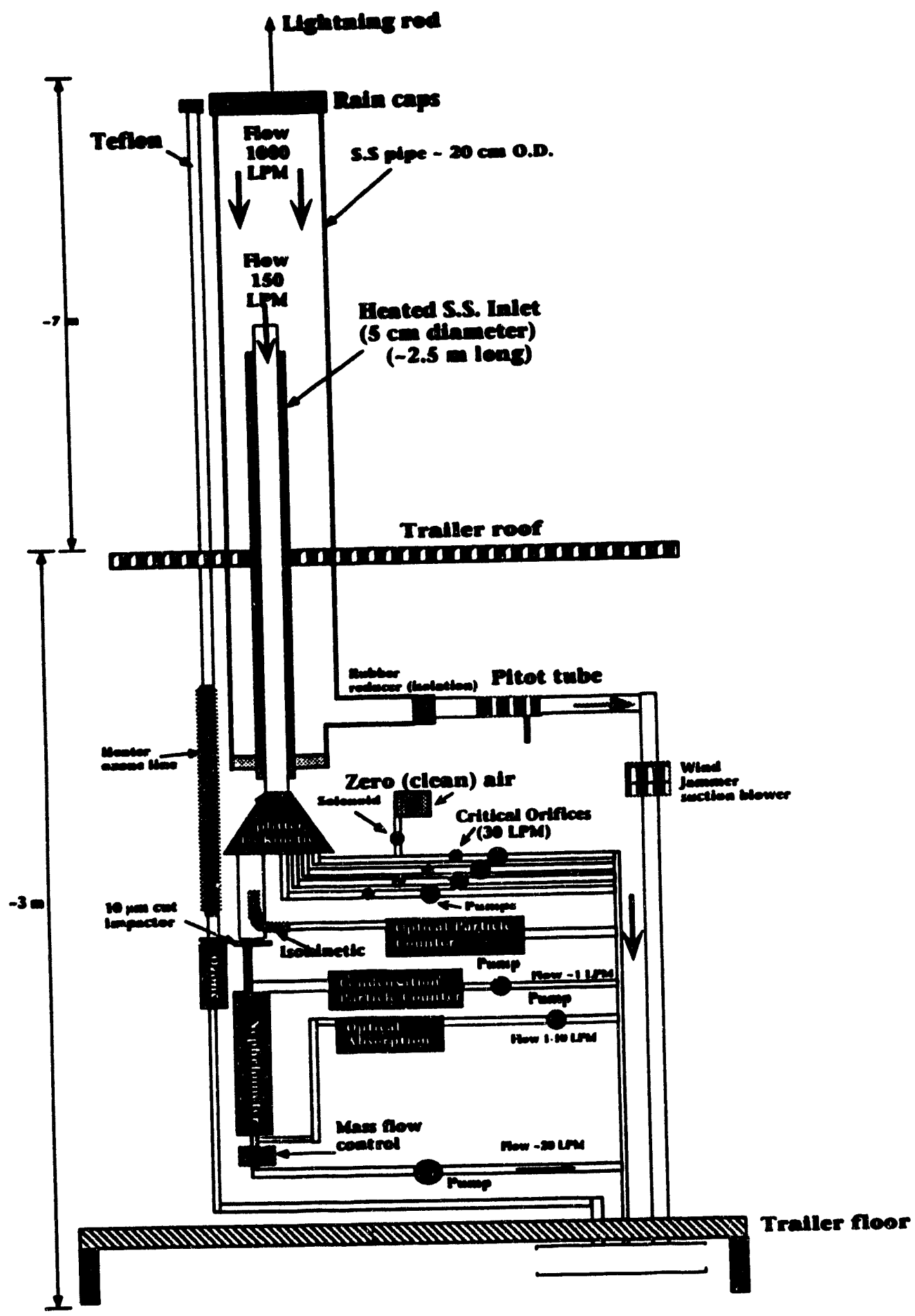

Figure 1. Preliminary design of the aerosol trailer at Lamont, Oklahoma. 
will provide dried aerosol to the instruments and will be limitad to sizes below $10 \mu \mathrm{m}$ using an impactor designed at the University of Washington.

The interfacing of these instruments (hardware and software) to the main computer is being done by Richard Eagan of Argonne National Laboratory. A field data ingestor (FDI), located in the aerosol trailer, will interrogate and control instruments via RS-232 serial interface ports. In addition, the FDI will sample analog signals and perform control functions.

The size distribution and composition of aerosols measured at a specific location depend on the origin and the trajectory of the air mass passing over the site. Backward air mass trajectory analysis can be used to help characterize an air mass, but is not sufficient to qualitatively identify the aerosol composition of the air mass at the site. Additional analyses must be performed along the trajectory to understand how the original air mass has or has not been modified. Hoight analysis, precipitation events, the vertical temperature structure of the atmosphere, the relative humidity field, and other physical parameters are important to classity the air mass after it has traveled a fow days. To this end, EML has developed a three-dimensional air mass trajectory model for the CART site. This model is discussed in the paper given by Hsi-Na Leo and Robert Leifer (this Proceedings). 


\title{
Retrieval of Three-Dimensional Cloud Structure for Estimating Cloud-Radiative Forcing
}

\author{
L. Shi, J. del Corral, V. Ramanathan, and E. Boer \\ Scripps Institution of Oceanography \\ University of California at San Diego \\ La Jolla, CA 92039
}

The vertical structure of cloud-radiative heating plays an important role on the energy conversion and the dynamic structure of the atmosphere. To gain abetter understanding of the three-dimensional cloud-radiative forcing field, Boer and Ramanathan (1992) have developed a threedimensional imaging algorithm to reconstruct the threedimensional structure of clouds from satellite advanced very high resolution radiometer (AVHRR) measurements. The algorithm combines a spatial coherence technique with spectral variations in reflectivities to identify clear-sky and adopts a delta-Eddington technique to retrieve cloud optical depths.

The algorithm is further refined in our current study. A discrete-ordinate radiative transfer model is used in this study to provide criteria for clear-sky detection and to obtain optical thickness from measured radiances. A prototype Atmospheric Radiation MeasurementCloud and Rediation Testbed (ARMCART) data set is employed to validate the cloud parameterization in the model. These data are provided by the Cloud Lidar And Radar Exploratory Test (CLARET) conducted in Boulder, Colorado, during September-October 1989 (Eberhard et al. 1992). The data setcontains surface radiation measurements, atmospheric sounding, and cloud observations.

\section{Comparison of Model Results with Surface Measurements}

Three CLARET cases have been selected in our study: 1) stratus clouds on Day 276, 2) cirrus clouds on Day 271, and 3) mixed-phase clouds on Day 277. For the stratus cloud case, the depolarization signature of the ruby lidar showed only a water phase in the clouds. The cloud base altitude was between 700 and 950 meters above ground level. The cirrus clouds of Day 271 were situated within the altitude of 6-8 km. The microwave radiometer obeerved no detectable water, indicating that these clouds were composed mostly of ice. For the mixed cloud case on Day 277, visual observations revealed a variety of cloud types, including altocumulus, altocumulus undulatus, altostratus, cirrostratus, and wave clouds. The cloud base altitudes were at $3-5 \mathrm{~km}$ above ground level. The bulk structure of the clouds during the observation period was very complex, and the details changed considerably as the clouds advected over the site. Mixed liquid/ice water phase of clouds was detected.

Figure 1 shows the comparison of the model-calculated downward solar fluxes with the surface observations for Case 1. The data sets used by the model consist of vertical profiles of temperature and dew-point temperature, cloud base and penetration heights measured from a ruby lidar,

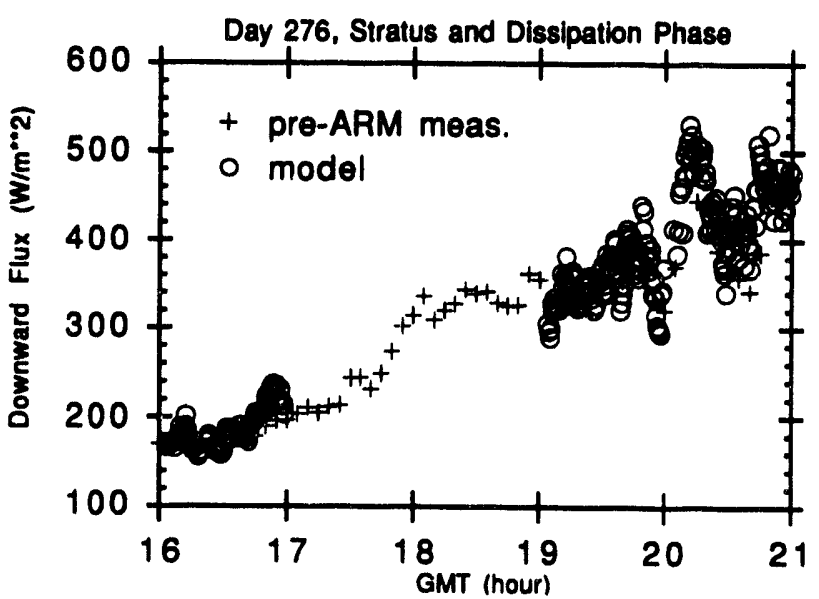

Flgure 1. Time series of observed (cross signs) and modeled (circles) shortwave downward fluxes at the surface for Case 1 (stratus clouds). 
and integrated liquild water path dotermined from a microwave rediometer. The cloud measurements have been averaged at every 30 seconds. In the figure, the cincles represent the results calculated from the radiative trancfer model; the cross signs represent the surface mosaurements (averaged at every 5 minutes) from a Pyranometer. The cloud effect in the model cakculation is paramoterzed in terms of liquid water content and effective radius of the cloud drops, using the parameterization scheme devoloped by Ackerman and Stephens (1987). The values of effective radius are assumed to be $8 \mu \mathrm{m}$ for the stratus cloud phase and $5 \mu \mathrm{m}$ for the dissipation phase. The integrated liquid water path of the clouds during 16 17 GMT is 0.1-0.15 mm; the value decreases steadily to $0.01 \mathrm{~mm}$ during the dissipation phase of the clouts. Because of the absorption and reflection of the solar rediation by the stratus clouds, the downward shortwave flux resching the surface is small $\left(-200 \mathrm{~W} \cdot \mathrm{m}^{-2}\right)$ in the stratus clouds phase, increasing to over $450 \mathrm{~W} \cdot \mathrm{m}^{-2}$ as the clouds thin out.

The general behavior of the modeled downward flux series is cimilar to that of the obeervational series, with smaller values in the stratus cloud phase and increasing values in the diseipation phase. Plane-parallel assumption has been ueed in the model calculation. The general agreement botween the model results and measurements indicates that the plane-parallel assumption can simulate the stratifom cloud condition well.

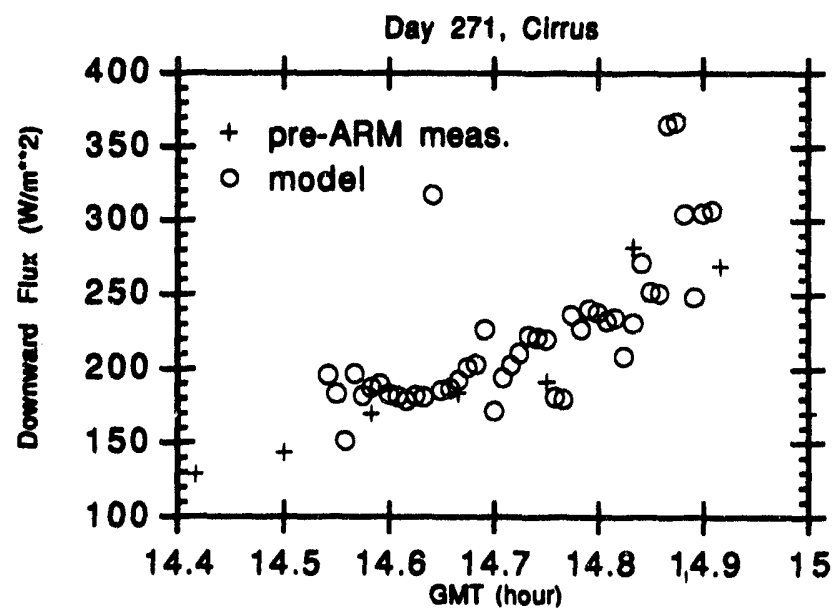

Fiouro 2. Time series of observed (cross signs) and modeled (circles) shortwave downward fluxes at the surface for Case 2 (cirrus clouds).
The intercomparison for Case 2 is shown in Figure 2. The cirnus parameterization scheme from the study of Takano and Liou (1889) has been ueed in the model calculation. In their study, the extinction coeficient, single scattoring albedo and asymmetry factor in the shortwaw epectrum were computed based on the observed lce crystal size distributions for four typlcal cirrus clouds, l.e., cirrostratus, cirrus uncinus, warm cirrus, and cold cirrus. The optloal properties of warm cirrus and cirrus uncinus haw boen used in our model calculations for the clouds with thlckneses larger than $1 \mathrm{~km}$ and less than $1 \mathrm{~km}$, respectivoly. Thore is a 7-hour difference between the Greenwich Nean TIme (GMT) and the local solar time (LST) of the slte. Thus, the local solar time corresponding to the GMT chown in Figure 2 is 7.5-8 LST. The solar flux incroasing with time is partly due to the increasing solar radiation and partly due to the thinning clouds.

In Figure 2, three modeled results have much higher values than the observations; one is found between 14.6 and $14.7 \mathrm{GMT}$ and the other two are found botmeen 14.8 and $14.9 \mathrm{GMT}$. For these three points, the CLARET dath set reports no clouds detected by the radar. Thus clear-aky condition has been used in the model calculation. Wo suspect that very thin clouds were present at the timo; however, the signal was too woak to be detcoted by the radar.

For the mixed-phase cloud (Case 3) depicted in Figure 3 , the intercomparison shows more deviation of the model

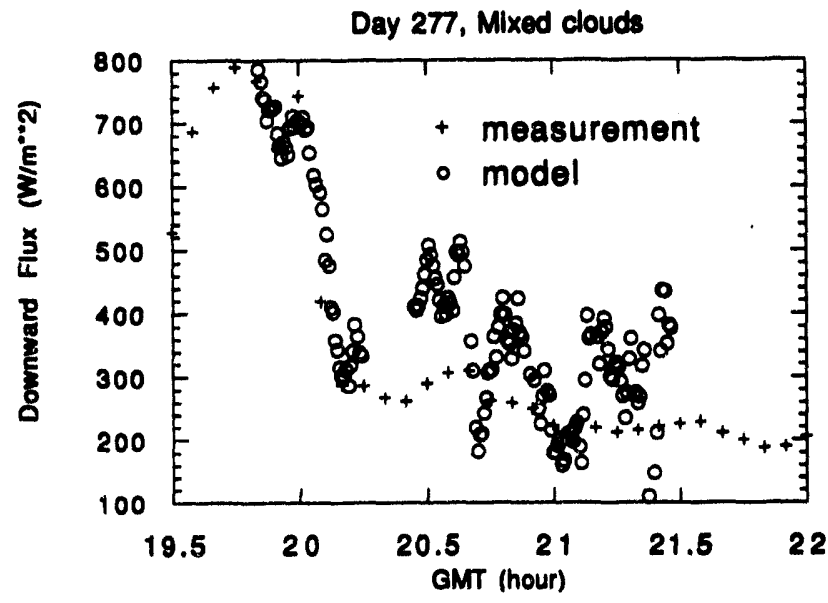

Figure 3. Time series of observed (cross signs) and modeled (circles) shortwave downward fluxes at the surtece for Caso 3 (mixed-phased clouds). 
results from the surface measurements. At the beginning of the cloud series when both liquid and $i c e$ water paths of the clouds are small, the model results agree well with the surface observation. However, as more clouds move into the region, the differences increase.

These differences result from a combination of factors. First, plane-paraliel assumption is used in the model calculation. This assumption might not work as woll as for stratiform clouds; some of the deviation might be caused by cloud side effects. Second, the cloud-microphysics changes considerably with time. iHowever, a constant value of $5 \mu \mathrm{m}$ is assumed for the effective radius of clouds in the model calculation. Third, the surface radiation measurement represents the integrated cloud effects from the whole sky; whereas, the lidar measures the clouds only at the zenith. This explains the much larger variation among cloud measurements than among surface radiation measurements.

\section{Improvement of the 3-D Cloud Retrleval Algorithm}

Coakley and Bretherton (1982) have developed a technique for determining clear and cloudy sky radlances using the spatial coherence method. The method uses the local spatial structure of the radiance field to determine the radiances associated with cloud-free and completely cloudcovered fields of viow. This approach is used in the present study as the first criterion for detecting a clear-sky pixel.

The escond criterion for determining a clear-sky pixel is based on threshold values calculated by the discreteordinate radiative transfer model. We have found that the clear-sky reflectivities of channels 1 and 2 can vary significantly, not only with respect to the solar zenith angle but also with respect to the satellite zenith angle. With this consideration, the threshold values for upper-bond of clear-sky reflectivities are calculated at the full satellite span angle as a function oi solar zenith angle.

Color information from the visible channels is employed in setting the third criterion for clear sky detection. As the spectral band widths of channels 1 and 2 are approximatoly 0.58-0.68 $\mu \mathrm{m}$ and $0.725-1.10 \mu \mathrm{m}$, respectively, the reflectivity value of the shorter wavelength channel (channel 1) should be higher than that of the longer wavelength channel (channel2) for a clear-sky pixel; and the reflectivity values of the two channels should be very close 10 each other if the pixel is completely filled with a thick cloud. A pixel is considered to be cloudy if the reflectivity ratio of channel 1 to channel 2 is smaller than the threshold value at the corresponding solar zenith angle.

The pixels which fail to meet one or more of the clear-sky criteria are classified as cloudy pixels. Cloud-top altitude of each pixel is calculated according to cloud-top temperature. Cloud optical thickness $(t)$ is obtained by comparing the observed pixel reflectivity value in channel 1 to the roflectivity value calculated from the radiative transfer model. Because a cloudy pixel can have different reflectivity values when $t$ is observed at different satellite zenith angles, the reflectivities with respect to different zenith angles have been calculated by the model. The cloud-base altitude is derived from cloud optical thickness and extinction coefficient. A visualization algorithm is applied to display the 3-Dclouds for verifying visually the cloud reconstruction scheme. Further work will be done to improve the cloud reconstruction scheme by collocating the satellite and surface measurements of clouds from the Southern Great Plains site in Oklahoma.

\section{Future Work}

The satellite and surface measurements of clouds and radiation fields from the ARM Western Pacific site will be used in our study to estimate the three-dimensional structure of the atmospheric cloud-radiative forcing. The estimation will help to understand the relationship between the largescale molsture and radiative heating fields. The analyees will also provide a basis to examine the parameterizability of clouds in general circulation model studies and to investigate the role of cloud-radiative feedbacks on the sea surface temperature. 


\section{References}

Ackerman, S. A., and G. L. Stephens'. 1987. The absorption of solar radiation by cloud droplets: An application of anomalous diffraction theory.J. Atmos. Sci. 44:1574-1588.

Boer, E., and V. Ramanathan. 1992. Three dimensional Imaging of cloud structure using satellite radiances: A now tool for cloud studies. 1992 AGU Spring Moeting (abstract).

Coakley, J. A., Jr., and F. P. Bretherton. 1982. Cloud cover from high-resolution scanner data: Detecting and allowing for partially filled fields of view. J. Geophy. Res. 87:4917-4932.
Eberhard, W. L., T. Uttal, K. A. Clark, R. E. Cupp, E. G. Dutton, L. S. Fedor, J. M. Intrieri, S. Y. Matrosov, J. B. Snider, and R. J. Willis. 1992. Remote sensing data from CLARET: A prototype CART data set. NOAA Technical Momorandum ERL WPL-223,1-58.

Takano, Y., and K.-N. Llou. 1989. Solar radiative tranator in cirrus clouds. Part 1: Single-scattoring and optical properties of hexagonal ice crystals. J. Atmos. Scl. 46:3-18. 


\title{
Backward Air Mass Trajectory Analysis for the First Cloud and Radiation Testbed Site at Lamont, Oklahoma
}

\author{
H.-N. Leo and R. Leifer \\ Environmental Measurements Laboratory \\ U.S. Department of Energy \\ 376 Hudson St. \\ Now York, NY 10014-3621
}

The size distribution and composition of aerosols measured at a specific location depend on the origin and the trajectory of the air mass passing over it. Backward air mass trajectory analysis can be used to 1) identify the characteristics of an air mass and 2) help to determine which aerosol model should be used in LOWTRAN 7 for radiation calculations.

Examining the trajectory is not sufficient to characterize the aerosol composition of the air mass at the site. Additional analyses must be performed along the trajectory to understand how the original air mass has or has not been modified. Height analyses, precipitation events, the vertical temperature structure of the atmosphere, and the relative humidity field, as well as other physical parameters, are important for classifying an air mass after it has traveled for a fow days.

The primary objective of this research is to characterize a climatology of air masses that pass over the Atmospheric Radiation Measurement (ARM) Program's Oloud and Rediation Testbed (CART) site at Lamont, Oklahoma. A three-dimensional air mass trajectory model and a method of analysis were developed at the Environmental Measurements Laboratory (EML). The classification of the air mass types (rural, marine, urban or desert) is used to decide which aerosol model is appropriate for calculations in a radiation model, such as LOWTRAN 7.

\section{Trajectory Computations}

Input data consisting of the analyses of the wind field, temperature, relative humidity, and precipitation at 12-hr intervals for the 1000-, 925-, 850-, 700- and 500-mb pressure level are used in EML's trajectory computer program. The data are recelved daily from the global circulation model of the U.S. Navy's Fleet Numerical Oceanography Center, Monterey, California. In this model, the horizontal grid interval is 2.5 degrees and covers a 33 by 19 horizontal grid matrix of the United States. The trajectories are computed backwards twice a day from the CART site, and the results are stered for further analyees. Trajectories reaching to the lateral boundaries of the matrix or $500-\mathrm{mb}$ height are terminated.

\section{Analyses}

Horizontal and vertical cross sections of the trajectories are plotted to identify the origin and the path of the air mass. If an air mass travels over rural areas for a long period of time, it is designated as a "rural" air mass.

Vertical profiles of temperature and relative humidity along the trajectory are also plotted. These profiles provide information on the atmospheric stability, above or below the inversion height and the moisture content of the air mass and help to determine whether the air mass along the trajectory was modified. If an air mass travels below the inversion height and passes over a polluted clity, it may be modified by urban emissions. In this case, the air mass arriving at the CART site would be designated as "urban."

A moist air mass coming from the ocean and traveling above the inversion height before descending to the CART site would be designated as "marine." In some cases, the air mass may be accompanied by rain before ariving at the site. The rain may modily the air mass by removing pollutants, therefore, the air mass is designated as "rural." 


\section{Future work}

We will incorporate source emission inventory data $\left(\mathrm{SO}_{2}\right.$, $\mathrm{NO}_{x}$ and VOC, etc.) from the Environmental Protection
Agency into our analyses. When the instrumentation to characterize the aerosol at the site is operational, the resulting data will help to improve air mass identification. 


\title{
The Effect of Network Resolution on Data Assimilation in a Mesoscale Model
}

\author{
J. Dudhia \\ Mesoscale and Microscale Meteorology Division \\ National Center for Atmospheric Research \\ Boulder, CO CO307-3000
}

\section{Introduction}

One goal of the Atmospheric Radiation Measurement (ARM) Program is to characterize meteorological fields over wide areas (200-km square) in order to better paramelerize sub-grid-scale variability in general circulation models used for climate studies. Such adetailed knowledge over these areas is impossible with current observational methods alone, but the synthesis of a dataset by combining observations with a mesoscale numerical model is foasible. Current data assimilation techniques allow observed data to be incorporated while a model is running, thus constraining the model to fit the data as well as the data to be dynamically consistent with the model atmosphere. This interaction may therefore be regarded as a dynamical analysis technique.

The technique used for data assimilation here will be the nudging method (Stauffer and Seaman 1990, Kuo and Guo 1989). Specifically, observational nudging where data at observational sites are gradually forced in the model without the need for a gridded analysis. This method is particularly appropriate for asynoptic data covering meso-B-scales, such as will be available at the Cloud and Radiation Testbed (CART) sites. The method makes it possible to incorporate the wide variety of data coming from these sites.

A question that arises in the program's planning is, What are the minimum data necessary for a good characterization of the meteorological fields over a typical CART site? It is important to have a cost-effective balance that provides enough data for a four-dimensional data assimilation (FDDA) system to be accurate. This requires a unified view of data collection and assimilation.
The concept of an Integrated Data Assimilation and Sounding System (IDASS) ensures that the needs of data collection are partly determined by the requirements of an assimilating mesoscale model. Hence, the sounding strategy is geared towards allowing the model to do the best possible job in representing the atmosphere over CART sites, for example.

It is not clear a priori what density of coverage or types of data are required for a good simulation. In this work, we address the problem of determining the impact of varying the density of coverage of an ideal network by purely numerical experimentation. We use one model run to provide data and another independent run to assimilate it. The results of such tests are important to the design of the CART site observational network and the IDASS.

\section{Method}

The model used was the Penn State/National Center for Atmospheric Research (NCAR) Mesoscale Model, which has been applied in both hydrostatic and nonhydrostatic modes for this study. The nonhydrostatic version has been recently developed (Dudhia, in press) and requires little change to the data assimilation techniques developed for the hydrostatic model, except that it is now preferable to have the data at specified heights rather than pressures, as used formerly.

Numerical modeling studies were carried out to investlgate the effect of data resolution on the accuracy of model predictions. The method of four-dimensional data assimilation was used to incorporate data from one model 
simulation into an independent simulation of the same ovent with degraded initial and boundary conditions.

These observation system simulation experiments (OSsES) wore carried out on 1) a strong winter-time cold front cace over Colorado (13 February 1980), and 2) a epring-time sovere convective outbreak in Oklahoma (10 April 1979). Hore we will concentrate mostly on presenting results from the spring-time case.

In both the OkJahoma and Colorado cases, nonhydrostatic 5-km grid simulatione were run to generate the "true" data that were assimilated into $20-\mathrm{km}$ simulations by the observation nudging technique. This method spreads obeervational influences over an area and time period surrounding each observation, typically with a 100- to $200-\mathrm{km}$ radius of influence. The data were in the form of profiles of temperature, humidity and wind taken hourly at eoveral individual columns of the $5-\mathrm{km}$ simulation. The influence of changing network density was studied by using unlform square grids of 4, 9, 16 and 25 profiles covering the $500-\mathrm{km}$ square domain of the $5-\mathrm{km}$ simulation. A control simulation was also run without data assimilation. Figure 1 shows the $3 \times 3$ ideal network in the Oklahoma case's domain.

For the Oklahoma case, the 5-km simulation was $18 \mathrm{~h}$ and the $20-\mathrm{km}$ simulations were $24 \mathrm{~h}$ long starting $6 \mathrm{~h}$ before

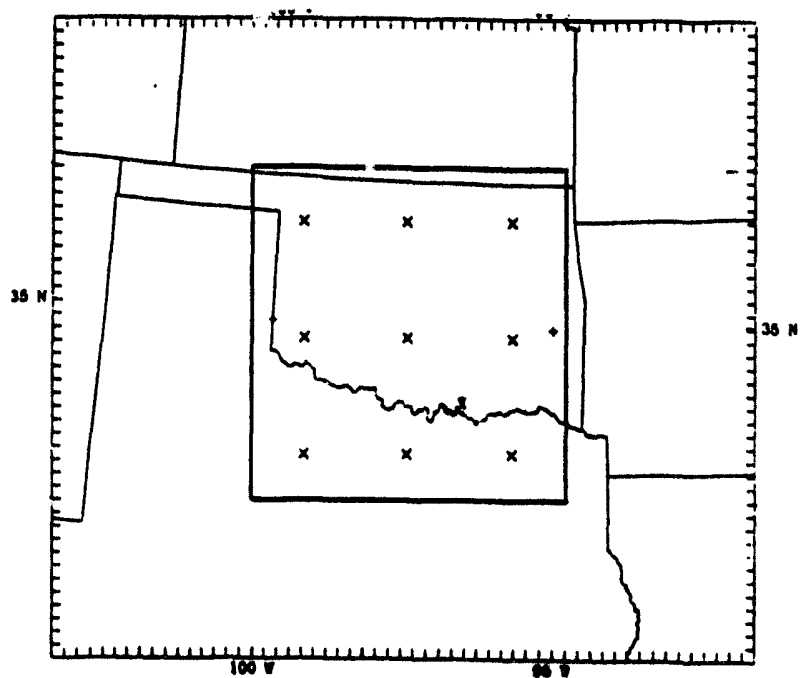

Figure 1. The 20-km domain with the $5-\mathrm{km}$ domain outlined. The $3 \times 3$ network positions are marked by crosses. the $5 . \mathrm{km}$ run that provided the data. Both wore nonhydrostatic. For the Colorado case, the 5-km and $20-\mathrm{km}$ simulations were $12 \mathrm{~h}$ long, the latter boing hydrostatic.

\section{Results}

The root mean square (r.m.s.) errors in the fiolds at eoveral chosen pressure levels and threat scores for the rainfall pattern wore used to verity the simulations. Deapite the meteorological difference between the two cases studied, conclusions for both were quite similar. Figure 2 demonstrates an example of the results for the temperature r.m.s. at 200 (top), 500 (middle) and 800 (bottom) mb in the Oklahoma case.

These revealed that the gain in accuracy over the control simulation, which had no four-dimensional data assimilation, was significant for even only 4 profile sites in a $2 \times 2$ notwork. There was a further but much bae significant gain with a $3 \times 3$ network, but beyond that the gain was small for $4 \times 4$ and $5 \times 5$ networks. The r.m.s. errors were typically half that of the control for these highresolution assimilation runs.
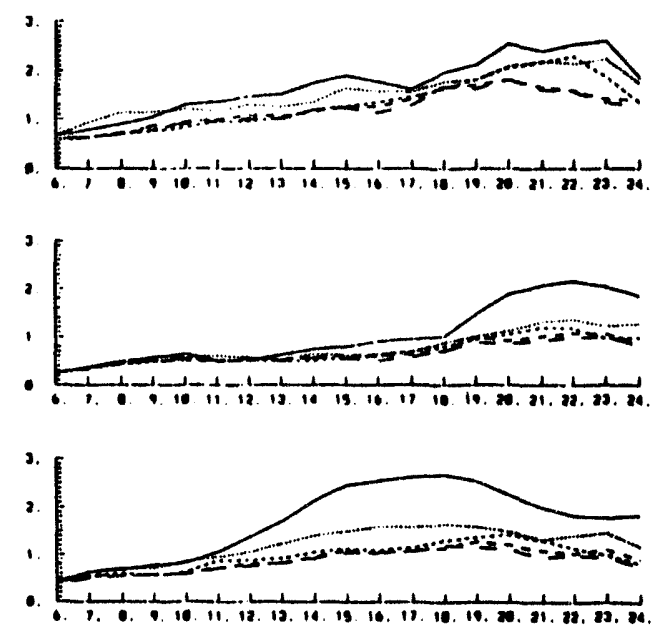

Figure 2. Root mean square error versus time in the $5-\mathrm{km}$ Oklahoma domain of temperature at 200,500 and $800 \mathrm{mb}$ for five experiments. Continuous line is the Control, shortest dash to longest dash are $2 \times 2$ to $5 \times 5$ FDDA experiments, respectively. Vertical axis is in $\mathrm{K}$, horizontal in hours. 
For the wintertime case, the frontal position was very simllar irrespective of data assimilation, 80 r.m.s. 800 res wore the beat measure of the gain from data assimilation.

The results from r.m.s. scores are supported by the rainfall patterns for the Oklahoma storm case shown in Figures 3a-d. These display a definite improvement with

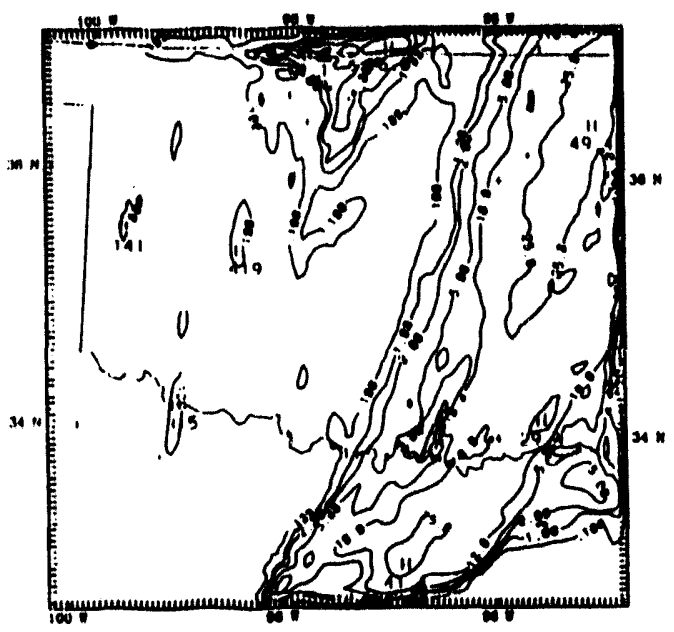

Figure 3a. 3-hourly rainfall total ending 12211 April 1979 for the "ruth" simulation. Contours 0.1, 1, 2, 5, 10, $25 \mathrm{~mm}$.

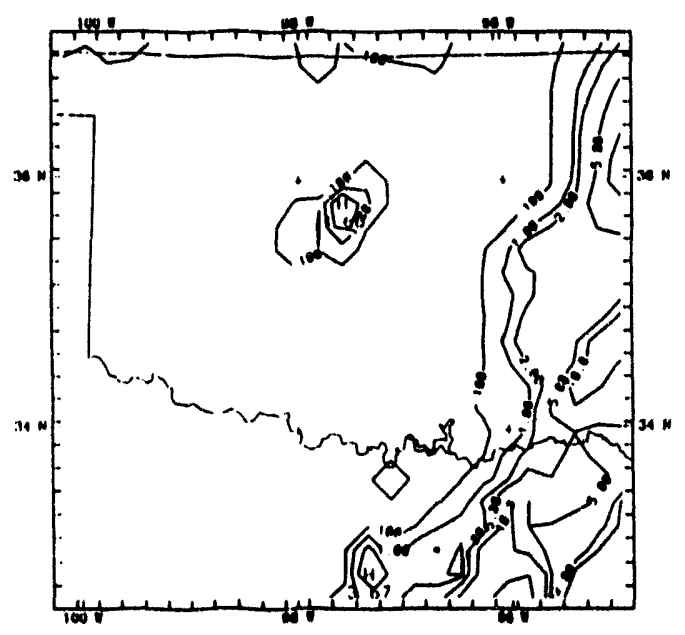

Figure 3b. As Figure 3a but for no-FDDA run. increasing assimilated data. While the control run (Figure 3b) produces weak rainfall at $21-24 \mathrm{~h}$, the assimilation with 25 sites (Flgure 3d) produces much stronger precipitation in approximately the correct position. However the amount is still underestimated compared with the "ruth" run (Figure 3a).

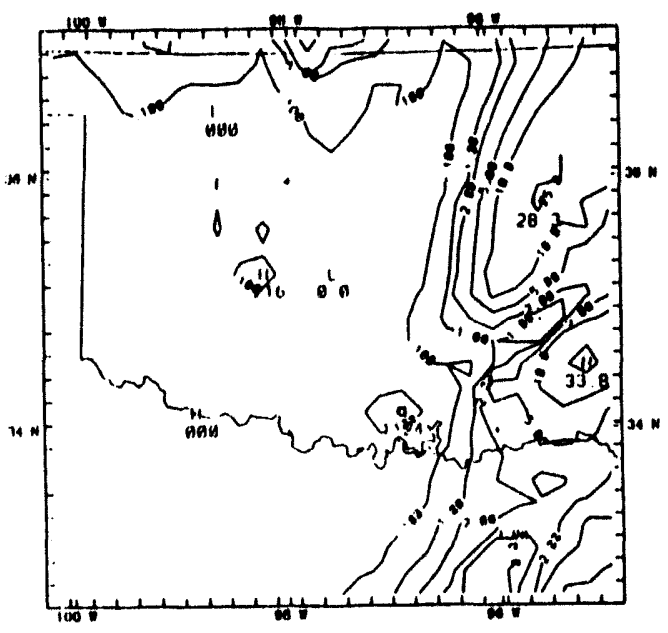

Figure 3c. As Figure 3a but for FDDA $3 \times 3$ run.

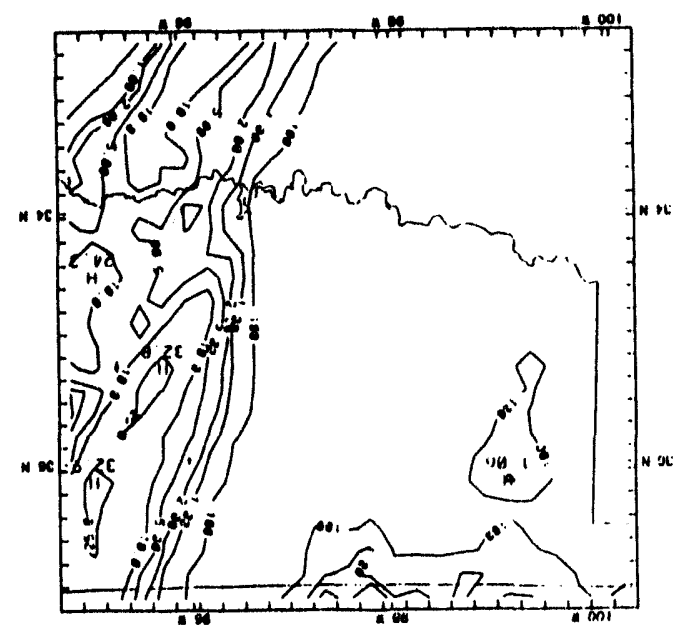

Figure 3d. As Figure 3a but for FDDA 5 × 5 run. 


\section{Dlecussion and Further Work}

This implise that much of the benofit of assimilation ls gained by own coarse notworke probably because most of the moleorologloal filde' variabilities are at large scales oven in strong meso-recale ovents. This tact can be verified by Fourler analysis of the longth scales associated with the "true" simulation where largor scales have larger amplitudes.

Hownver the r.m.s. error does not converge to zero as the notwork epecing is reduced, and it ls necseseary to dotormino whothor this represents the influence of the advection of imporfuct data through the boundaries. Studles carried out on caelmilating data into a $5-\mathrm{km}$ grid model show that the raintall still is doficiont with oven 25 aseimilation sitos, 80 the reculte appear not to be very dependent on model recolution botween 20 and $5 \mathrm{~km}$. Further studbe of boundary offects are to be carried out in these $5-\mathrm{km}$ simulations. Theses should show the importance of characterizing the exterlor flow sccuratoly when the domain of interestis only of order hundreds of kilometers in scale.

Prollminary results have also demonstrated that data overy hour are proferable to data every 3 hours, but more teste are needed on temporal resolution. It is, however, unlikely that incroasing beyond hourly resolution helpe becaues the time scale of typlcal data aasimilation nudging constants is of this order.

Further, studies would be required also to show how usetul wind data alone are, as is easily achievable by profilers, or whether thermal data such as from RASS or balloons are cesential. Previous data assimilation studies have shown that the mass field adjusts to the whd on large scales so that tomperature errors can be corrected just by using wind assimilation. This result is scalo-dopendent, and its appllcablity to CART site notworks will need investigation.

At this stage, a tentative conclusion would be that the primary goal in data collection for input to mesoscale models is to sample the boundaries of the area of interest and the exterlor upstream conditions well in order to get an sccurate representation of the events within the area. In some respects, by the time the data are collected in the site, it is too late to force the model except to Improve the scouracy downstream of the obeervation or to verify the model againat. A prospective uppor-air network for CART sites probably should not be concentrated within the atte, but should be spread arcund its boundarios to give mesoscale models the best chance to represent the meteorology within the site accurately. As shown in this study, even a fow profiling sites are ueoful becaues of the inherently large ecales of meteorological fields (with the excoption, perhape, of molature).

With a sultable network of upper-air and surface obeervations, the data sesimilation techniquecan be applied at CART eltes to produco four-dimensional moteorologloal fields that are representative of the conditions over the whole site. Wo hope that future applications of the model will include near-real-time running, possibly on a work station at the site, to provide continuous analyese over long poriods.

\section{Acknowledgments}

This work has been funded by the Department of Enorgy ARM Project under grant DEA105-90ER61070. Computing was carried out on the NCAR CRAY-YMP supported by the National Science Foundation.

\section{References}

Dudhia, J. In press. A nonhydrostatic version of the Penn State/NCAR mesoscale model. Mon. Wea. Rov. 121.

Kuo, Y.-H., and Y.-R. Guo. 1989. Dynamical initialization using observations from a hypothetlcal network of profilors: Impact on short range numerical weather prediction. Mon. Woa. Rov. 117:1975-1998.

Stauffer, D. R., and N. L. Seaman. 1990. Use of fourdimensional data assimilation in a limited area mesoscale model. Part 1: Experiments with synoptlc-scale data. Mon. Woa. Rov. 118:1250-1277. 


\title{
Radlation Studles with a High-Resolution Mesoscale Model
}

\author{
J. Dudhla \\ Mososcale and Microscalo Moteorology Divlaion \\ National Conter for Atmosphoric Research \\ Boulder, CO 80307.3000
}

\section{Introduction}

The paramotorization of radiation in general circulation models (GCM) relies strongly on correctly representing the moan radiative proportios over a typical grid size $(200 \mathrm{~km})$. The quality of the model's feedbeck botween clouds and radiation would depend on the accuracy of this representation; thls foedback is a koy fector in dotormining the climato's behavior under various future sconarios.

For CCM, cloud-rediation effects are often parametorized aimply in torms of cloud fractions at various bvels and some epectified degrees of overlep between clouds at difiorent bvels. Theee paramoterizations are difficult to ovaluate obeervationally uniese a dotalled knowledge of the three-dimensional radiative and cloud fields can be obtained over a wide area typical of a grid scale. A parallel approsech to this massive obeervational effort would be to represent these fiolds in a mesoscalo model.

The use of a mesoscale model allows both clouds and radiation to recolve a high-resolution treatment that can be tres of the asaumptions of overlapping and cloud fraction. To this end, a version of MMS, making use of the fully compreselble nonhydrostatic primitive equations, bo being applied to determine typical rediative properties of the atmoephore.

Hore the model will be introduced and preliminary results of teats with radiation will be shown. Of particular interest for this study are the domain-averaged properties of a cloud systom as it evolves during one diurnal cycle. The case presented here is one of deep convection over Oklahoma on 10-11 April 1979 during the Sovere Environmental Storms and Mososcalo Experiment (SESAME) program.

\section{The Model}

The nowest veralon of the Penn State/National Conter for Atmospheric Research (NCAR) Mososcalo Model (Mins) is upgraded to include a radiation peckeos. The modol's physics is quite complete with respect to the requirements of mesoecale simulations, including reprecentations of the boundary layer; a molature scheme with cloud and loo processes; convective paramotorizations for coares (>10 km) grid scales; and asurfeco heat budgot dependent on rediative, sensible and latent heat fluxes.

Preprocesesing peckages allow the model to bo Inltialized with data analyzed from synoptlo observations. Boundary conditions also may be provided by lator analyees or a numerical weather prediction model run on alareordomain. This Initialization allows a reallotic ro-creation of cbeerved mesoscalo systoms that intoract with largor-scalo testures, a critical requirement for a true four-dimensional representation of the meborological parameters in general and the cloud fields in particular.

For the purposes of the Atmospheric Radiation Measurement (ARM) Program, a simplo longwawe and shortwave package based on broadband embelvity and a single-stream integration, respectively, (Dudhla 1889, Chen and Cotton 1883) has been implemented in this model. It ls epplled to three-dimensional simulations of an OKlahoma severe weather event (10-11 April 1979). The model is being run on 20- and 5-km grids for 24 and 18 houre, respectivaly, with the latter domain nested inaide and taking hourly boundary conditions from the former.

The radiation scheme fully interects with the clouds in the model (Stephens 1978) and the surface energy budgot 
contributing to ground temperature tendencies. It aleo allows for ios, precipitation, and carbon dioxide offects in the longwave echems.

Thus bonowaw cooling at cloud top, heating at cloud bace, and shortwave cloud heating are all represented. The longwave fluxes are aleo intliuenced by wator vepor in the atmoephore and ground omiaslvity. Shortwave cloud absorption and albedo and clear-air scattoring and absorption caueed by wator vepor are considored with zenith angle depondencies. Typloally rediativo heating and surfecos flux caloulations are updated overy 30 minutes. Duchla (1809) has shown tests of thisecheme with ldealized cloud layere to produce realistic heating rates.

\section{Results}

The results for the 20-km simulation are deecribed here. The model domain covers an area $980 \times 1080 \mathrm{~km}$. Simulations wore run with and without the rediative scheme. For this case, atmoepheric rediative hoating was found to have littibeffect on the convective development and rainfall pattem. The case was one in which severe convection with high updrafts formed as a result of strong instability that dowloped when a capping inversion was removed. A wideapread low stratiform cloud east of a sharp dry line persisted through much of the simulation.

Comparison of domain-averaged temperatures in simulations with and without the radiative scheme (Figure 1a) showed differences in the boundary layer as the new schome produced more daytimo surfece heating than tho old surtace radiation scheme that allows for clouds in an integrated sense. Also an upper nighttime dipole of cooling above warming of about $0.5 \mathrm{~K}$ amplitude reveals the longwave radiative influence of widespread upper clouds, and low-lovel daytime warming above the boundary layer is shortwave heating of low stratiform clouds. As shown by the difference field in Figure 16, cloud amounte wore also affected by radiation, low-lovel clouds being decreased during the day and high clouds being increased at night.

The moan rate of radiative heating shown in Flgure 2 varies from a fow degrees of cooling at night to no not cooling during the day in clear alr. The cloud effects

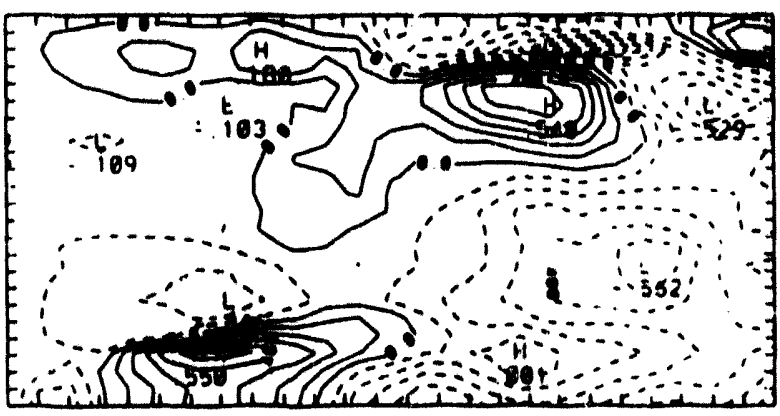

Figure 1a. Domain-averaged tomporature with radiation minus without radiation. Vertical axis is model bwel, horizontal is time (24 h starting at $0800 \mathrm{C8T}$ ). Contour interval $0.1 \mathrm{~K}$.

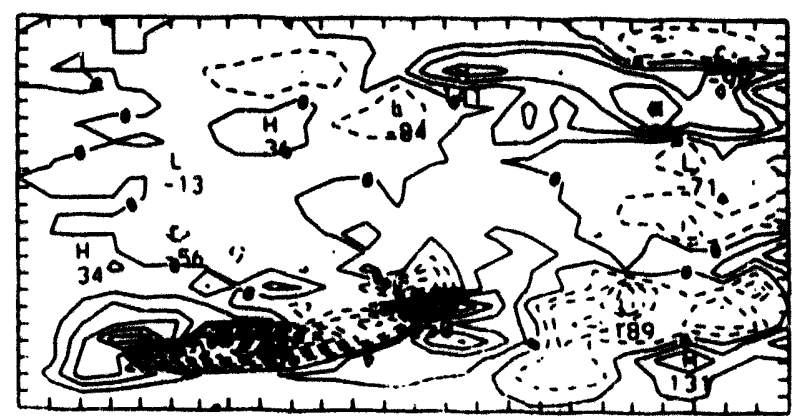

Figure 1b. Domain-averaged cloud with radiation minus without radiation. Axes as in Figure 1a. Contour interval 0.004 gkg.

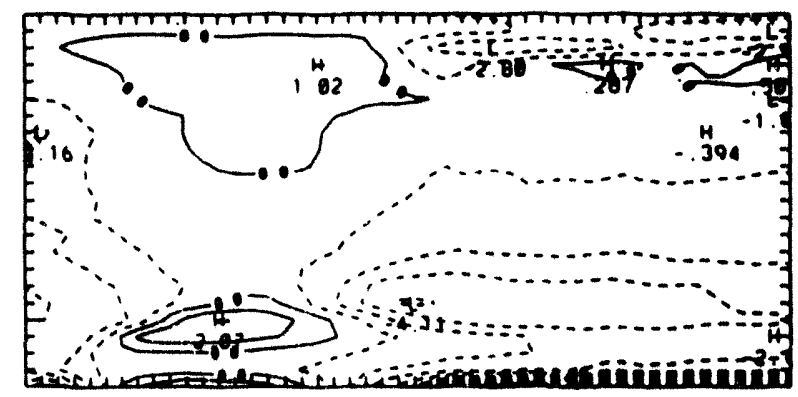

Figure 2. Domain-averaged rediativo hoating rats. Axes as in Figure 1a. Contour interval $1 \mathrm{~K} / d a y$. 
mentioned earlier and additional cooling of low-lovel cloud tope at night all contribut to the domain-scale averece. In this cases, the degree of overtep of low-lovel and high-lowl clouds would affect the amount of low-lowl cloud-top cooling. Lees overlap would result in more cooling.

The model reaults at aselocied timb, $00 Z$ (1800 CST), both at cloud bowls $(0.9 ., 300 \mathrm{mb}$ ) and at the eurface, show mescecalo veriablity in rediative cooling hoeding particularty caused by clouds (Figure3). The figure shows local cloudtop cooling rates of 10-20 $\mathrm{K} \mathrm{day}^{-1}$ and similar cloud-base warming rates in contrast to the 2-3 $\mathrm{Kday}^{-1}$ in char air. The heating maxima occur where the cloud bease intersects the 300-mb surface.

The influence of low cloud cover upon surface longwave rediative fluxes is 70-100 $\mathrm{W} \mathrm{m}^{-2}$, while clear drier air gives $50 \mathrm{~W} \mathrm{~m}^{-2}$ less dowmward IR than neighboring clear moister air east of the dry line (bold dashed line) as seen in Figure 4a. Shortwave hoating (Figure 4b) bolow cloud cover is negligible at the low solar angle near suncet, but in the clear air the effect of changing solar elevation with longitude is seen.

\section{Conclusions and Further Work}

An atmospheric rediative schemo has been incorporated into a mesoscale model. The above results are part of the

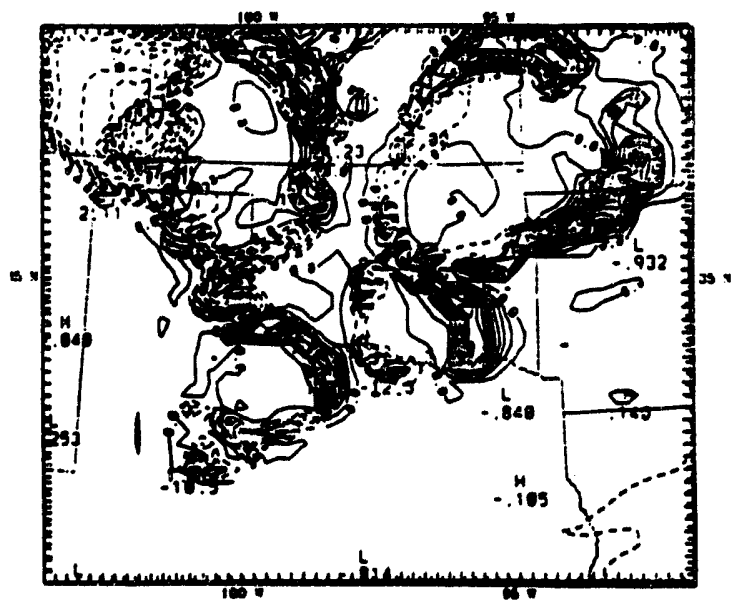

Foure 3. Not radiative heating at $300 \mathrm{mb}, 12-\mathrm{h}$ forecast time. Contour interval $1 \mathrm{~K} / \mathrm{day}$.

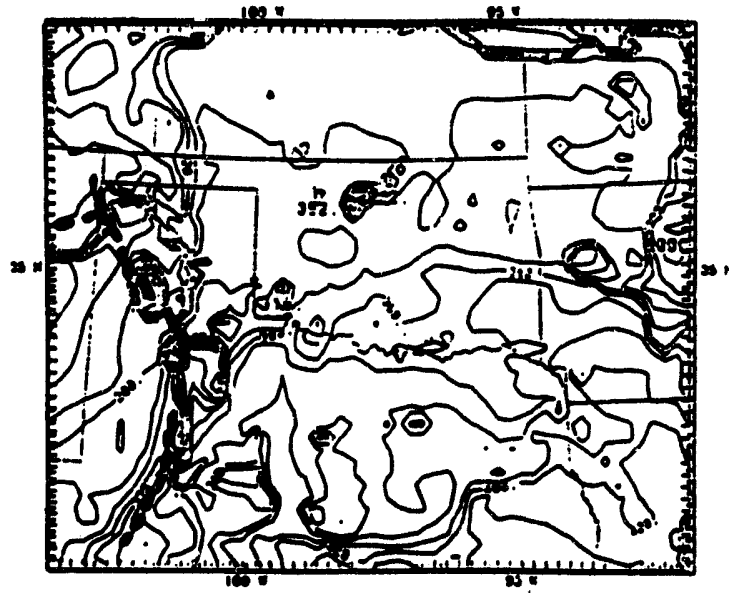

Figure 4a. Downwerd IR flux at surfeco, 12-h foreceat time. Contour interval $10 \mathrm{~W} \mathrm{~m}^{-2}$.

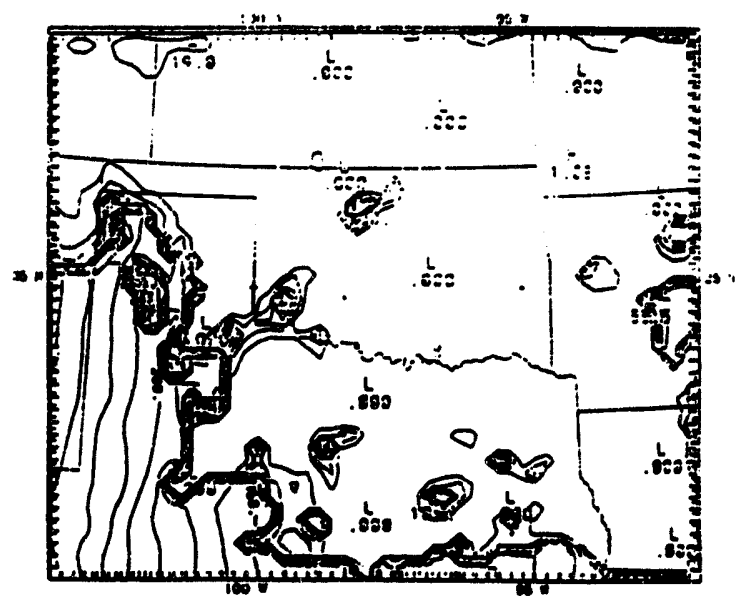

Figure 4b. Downward solar flux at surface, 12-h forecast timo. Contour interval $10 \mathrm{~W} \mathrm{~m}^{2}$.

tosting for this now scheme and demonetrate that fl glves results in keeping with generally accopled rediativo influences.

The domain-averaged radiative heating is distincthy for the $20-\mathrm{km}$ grid influenced by two cloud layers, one of hioh cirrus and one of low stratus, and the diumal cycle of heating in these layers.

By resolving cloudy and clear columns and cloud layere, the model can give some indication of the true atmoephoric 
radiative fluxes. These may then be compared with averages over areas typical of GCM grid boxes to check the GCM assumptions about partially cloud-covered grid boxes in their radiative packages.

Use of a mesoscale model also affords the possibility of verifying the predicted radiative fielde against in situ observations, for instance of long-and short-wave fluxes at the ground, and against other available measurements such as from aircraft. The scheme's treatment of clouds is based currently on theory and may be tuned if systematic errors are encountered in these intercomparisons.

There are limitations to be considered, however. The mesoscale model is likely to use a 5-km grid size in future applications related to the Cloud and Radiation Testbed (CART) sites, where there are still sub-grid scale fluctuations in the cloud fields, particularly in the case of small cumulus. This limitation can be overcome with selected very-highresolution runs, but at these scales, the radiation scheme breaks down because of the three-dimensionality of the fluxes.

Another practical limit is the ability of a $5-\mathrm{km}$ mesoscale model to represent the true clouds accurately. Data assimilation techniques allow the model to incorporate mesoscale observations during the run, but it is clear that the cloud field depends heavily on the water vapor field in which there is often high mesoscale variability. To compound this problem, remote sensing techniques cannot provide water vapor profiles, so balloon data are necessary-but they are limited in their temporal and spatial coverage. In convectively unstable situations, there is also the problem of unpredictability where differences in the location of, for instance, the first convective tower may lead to widely different results.
Thus, in general, the data assimilation model output cannot be compared cloud-by-cloud with observations. The model's cloud/radiative interaction may sometimes have to be verified by comparing similar clouds in the model with observations requiring aircraft measurements of cloud properties. The improvements made to the model's radiation scheme and also possibly to the cloud scheme through such comparisons will lead to a model simulation that can closely resemble realistic meteorological environments. The output would be a full dataset sufficient for representing GCM grid areas and could therefore be used as a testbed for GCM parameterizations. This dataset complements the CART observations that are limited in coverage.

\section{Acknowledgments}

This work has been funded by the Department of Energy ARM Project under grant DEA 105-90ER61070. Computing was carried out on the NCAR CRAY-YMP supported by the National Science Foundation.

\section{References}

Chen, C., and W. R. Cotton. 1983. A one-dimensional simulation of the stratocumulus capped mixed layer. Bound.-Layer Meteorol. 25:289-321.

Dudhia, J. 1989. Numerical study of convection observed during the Winter Monsoon Experiment using a mesoscale two-dimensional model. J. Atmos. Sci. 46:3077-3107.

Stephens, G. L. 1978. Radiation profiles in extended water clouds. Part II: Parameterization schemes. J. Atmos. Sci. 35:2123-2132. 


\title{
Meso-Beta-Scale Data Assimilation of the Winter Icing and Storms Program/Atmospheric Radiation Measurement Program 91 Intensive Observing Period Case on 6 March 1991
}

\author{
Y.-H. Kuo and Y.-R, Guo \\ Mesoscale and Microscalo Motoorology Division \\ National Center for Atmospheric Research \\ Boulder, CO $80307-3000$
}

\section{Introduction}

Tosupport the Atmospheric Radiation Measurement (ARM) Program, we have proposed to develop an Integrated Data Assimilation and Sounding System (IDASS) (Dabberdt et al. 1991). The IDASS is composed of a measurement component and amodeling component. The measurement component (Integrated Sounding System, or ISS) is developed around a suite of in situ and active and passive remote sensors. Individually, these sensors satisfy cortain needs; together, they function in a synergistic and complementary mode. The modeling component is an enhanced nonhydrostatic version of the Penn State/NCAR mesoscale model with data assimilation capability. The goal of IDASS is to provide continuous high-resolution tropospheric profiles of basic meteorological variables by continuous assimilation of measurements taken by ISS. A prototype of ISS was used in the WISP(a)/ARM-91 field exercise over the front range of Colorado. The objectives of this study are 1) to assess the impact of mesoscale data assimilation on the model results and 2) to evaluate the basic concept of IDASS. The case chosen for this study is the March 6-7, 1991, case.

\section{The Model and Experiment Design}

The domain of the PSU/NCAR model used in this study (see Figure 1) has a horizontal mesh of $65 \times 73$ and grid

(a) Winler loing and Storms Program.

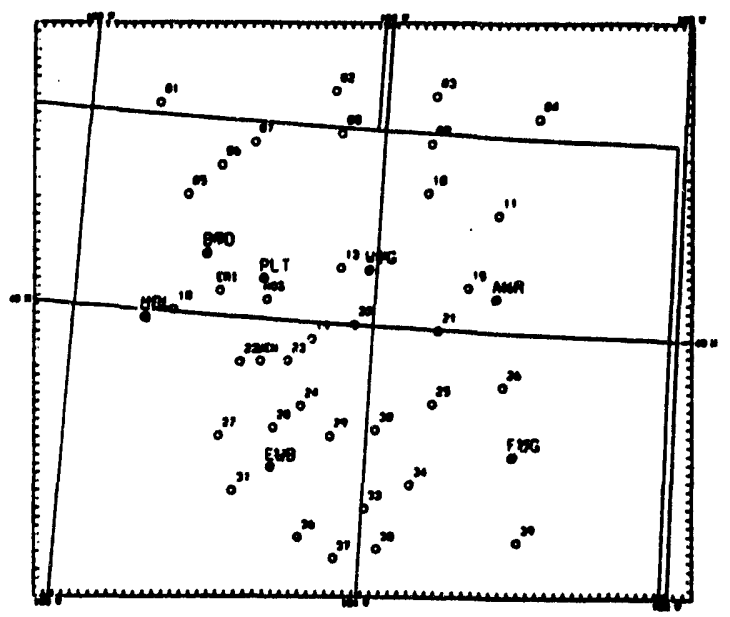

Figure 1. Obeerving sites for WISPIARM-91 experiment: solid dots are Cross-chain Loran Atmospheric Sounding System (CLASS) sites, and open circles are Portable Automated Mesonet (PAM) data sites. The map indicates the model domain used in this study.

distance of $5 \mathrm{~km}$. There are 23 layers in the vertical. The precipitation physics include explicit prediction of cloud water, rain water, snow, and ice. The planotary boundary layer (PBL) formulation was originally doveloped by Blackadar (1979), which includes surface fluxes of sensiblo heat, latent heat, and momentum. The hourty latoral boundary conditions are provided by a $20-\mathrm{km}$ veraion of the PSUNCAR model, which covers the contiguous 48 states of the United States. 
Three experiments were conducted:

- Exp. MM5: standard 24-h forecast, without data assimilation, starting from 0000 UTC 6 March 1991

- Exp.CLS: assimilation of wind, temperature and specific humidity from all 7 CLASS soundings during the 24-h period, from 0000 UTC 6 to 0000 UTC 7 March 1991

- Exp. WPR: assimilation of wind, virtual temperature and precipitable water from the 405-MHz wind profiler, radio acoustic sounding system (RASS), and microwave radiometer at Platteville during the above same 24-h poriod.

\section{The Data Available for IOP \#4}

Table 1 shows the availability of special observations from 7 CLASS sites for this case. Forty-eight CLASS soundings were available during the 24-h period from 0000 UTC 6 March to 0000 UTC 7 March. As an example, Figure 2 shows the time-height section of wind observatians from the Platteville CLASS site. In addition to soundings and surface observations, a suite of remote sensing platforms is available at the Platteville site, including wind profiler, RASS, ground-based microwave radiometer, and Doppler SODAR. The availability of remote sensing observations from these platforms provides us an opportunity to evaluaie the basic design concept of IDASS. Figure 3 shows the time-height section of the profiler wind observations at the Platteville site.

Table 1. CLASS sounding data during IOP *4.

\begin{tabular}{|c|c|c|c|c|c|c|c|c|c|}
\hline Station & $\underline{00 z}$ & $\underline{03 z}$ & $\underline{06 Z}$ & $\underline{09 Z}$ & $12 Z$ & $\underline{152}$ & $\underline{18 Z}$ & $21 Z$ & 242 \\
\hline Platteville (PLT) & Yes & Yes & Yes & Yes & Yes & Yes & Yes & Yes & Yes \\
\hline Berthoud (BTD) & Yes & Yes & Yes & Yes & Yes & Yes & Yes & Yes & Yes \\
\hline Mobile (MBL) & Yes & No & Yes & Yes & No & No & No & No & No \\
\hline Akron (AKR) & Yes & No & Yes & Yes & Yes & Yes & Yes & Yes & Yos \\
\hline Elbert (ELB) & Yes & No & Yes & Yes & Yes & Yes & Yes & Yes & Yes \\
\hline Fiagler (FLG) & Yes & Yes & No & Yes & Yes & Yes & Yes & Yes & Yes \\
\hline Wiggens (WIG) & Yes & Yes & Yes & No & No & No & No & No & No \\
\hline
\end{tabular}

Figure2. Wind data from CLASS sounding at Platteville. Solid lines show the wind speed.

\section{Results}

Figures 4 and 5 show the surface temperature errore at the end of the 24-h forecast period, 0000 UTC 7 March 1991, for Exps. MM5 and CLS. These errors are calculated by interpolating the model results to the 39 PAM stations and comparing directly with the surface reports. Since the surface observations were not used in the data assimilation procedure, they can be used as independent verifications for model prediction. A comparison of Figures 4 and 5 


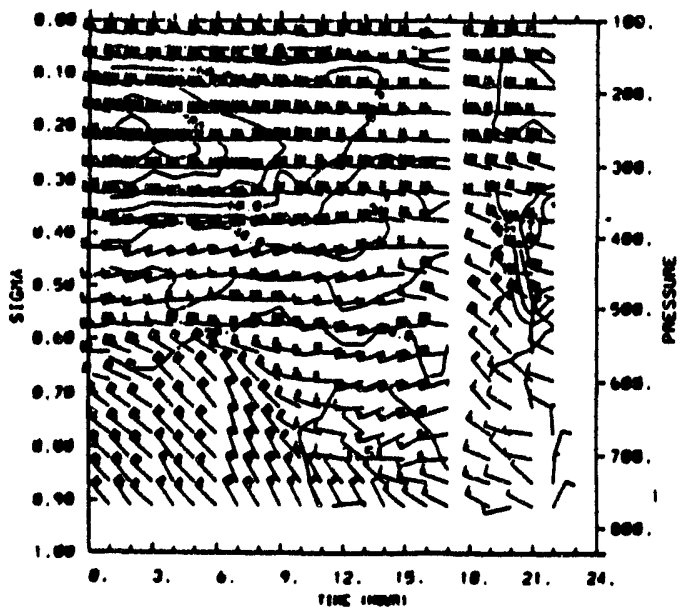

Firuse 3. Same as Figure 2, but from a $405-\mathrm{MHz}$ wind profiler at Plattovillo.

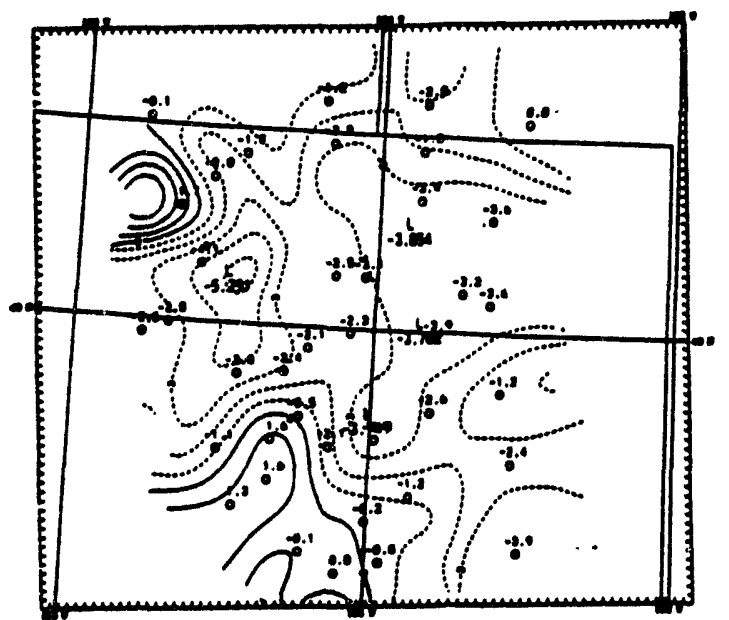

Froure 4. The lowest model bevel temperature errors to compare with PAM Data for 24-h Forecast Valid at 0000 UTC 7 March 1993 for MM5. The negative values mean that the model forecast is too cold, and positive values moan too warm. The contour interval is $1 \mathrm{~K}$.

charly shows that the assimilation of wind, temperature and moisture data from the 7 CLASS sites significantly reduced the model forecast errors. The station-averaged RMS orror for Exp. MMS was $2.46 \mathrm{~K}$, while that for Exp. CL8 was $1.67 \mathrm{~K}$.

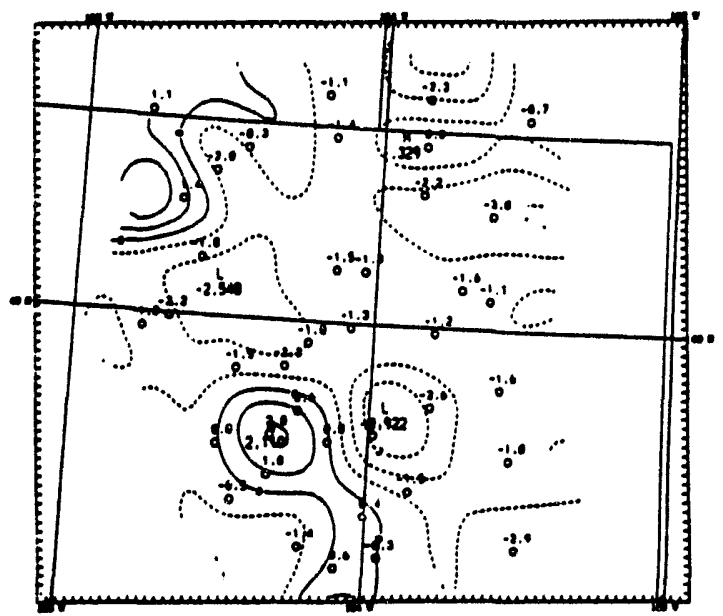

Figure 5. Same as Figure 4 but for CLS.

Figures 6, 7, and 8 show the RMS errors of wind, temperature and specific humidity at Plattovillo for Exps. MM5, CLS, and WPR averaged over the 8 sounding times. The CLASS soundings at Platteville serve as the verification. With the assimilation of wind profiler data, the vertically averaged wind error was reduced from $8.7 \mathrm{~m} \mathrm{~s}^{-1}$ to $6.8 \mathrm{~m} \mathrm{~s}^{-1}$ (comparing the results of Exp. MM5 and WPR). Aso, the assimilation of ground-based radiometer data also led to improvement in the moisture fields, which the precipitable water error reduced from $0.33 \mathrm{~mm}$ to $0.27 \mathrm{~mm}$. Interestingly, the assimilation of profiler wind, RASS and microwave radiometer observations did not lead to significant improvement in the temperature fields.

\section{Conclusions}

1. Assimilation of all available observations during the WISPIARM-91 experiment improved the accuracy of the model data.

2. The concept of "Integrated Data Assimilation and Sounding System" is valid. The assimilation of remote sensing data from a wind profiler and a ground-based microwave radiometer improved the model wind and moisture fields. 


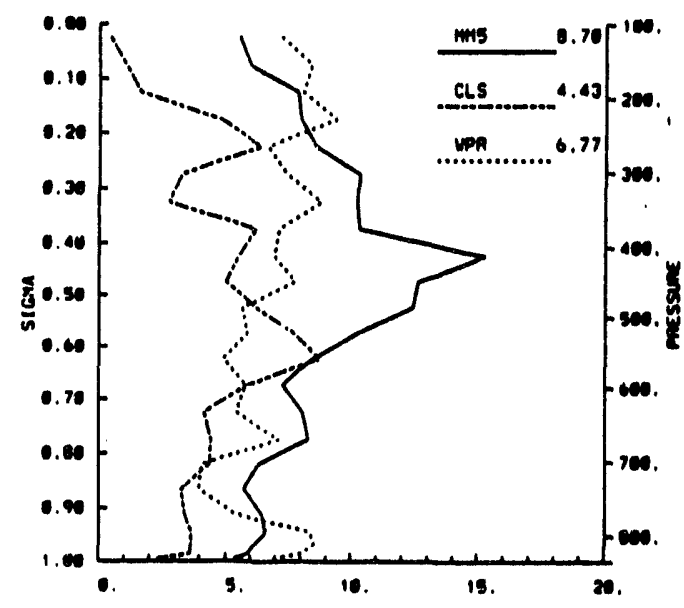

Figure 6. Time-averaged RMS errors of wind $\left(m 8^{-1}\right)$ to compare with CLASS sounding data at Plattoville during the 24-h period from 0000 UTC 6 March to 0000 UTC 7 March 1991. The numbers on the right hand of the key are the vertically integrated errors for each of the experiments.

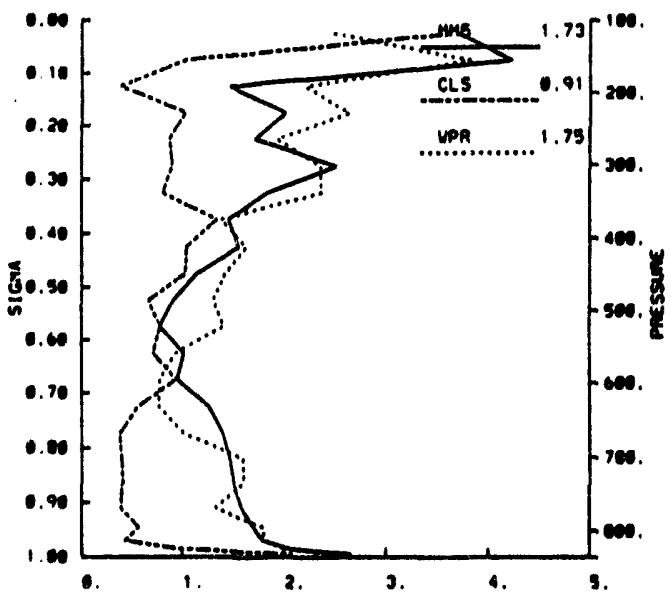

Flgure 7. Same as Figure 6 but the errors of temperature (K).

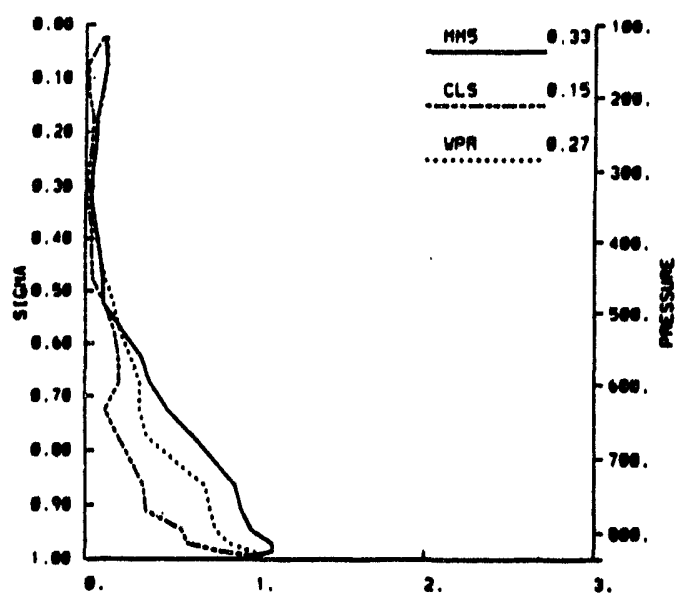

Flgure 8. Same as Figure 6 but the errore of specific humidity $(\mathbf{g} / \mathrm{kg})$.

3. The use of temperature information from CLASS soundings is necessary for the design of IDASS. Assimilation of wind, virtual temperature and moisture data from a wind profiler, RASS, and a radiomoter does not lead to immediate improvement in temperature field.

\section{References}

Blackadar, A. K. 1979. High resolution models of the planetary boundary layer. Advances in Environmental Science and Engineering, No. 1, eds., Plaffilin and Zelgler. Gordon and Breach, Science Publishers, Cooper Station, NY.

Dabberdt, W. F., C. Martin, H. L. Cole, J. Dudhia, T. Horet, Y.H. Kuo, S. Oncley, J.van Baolen, K. S. Gage, W. Ecklund, D. Carter, R. Strauch, E. Westwater, H. Revercomb, and W. L. Smith. 1991. An integrated data assimilation and hybrid sounding system. Preprints, Internatlonal Conference on Mesoscale Moteorology and TAMEX. pp. 385-392. Dec. 3-6, 1991, Talpel, Taiwan. 


\title{
Combined Ground- and Satellite-Based Profiling of Temperature and Water Vapor
}

\author{
B. B. Stankov, E. R. Westwater, J. B. Snider, and J. H. Churnside \\ National Oceanic and Atmospheric Administration \\ Environmental Research Laboratories \\ Environmental Technology(a) Laboratory \\ Boulder, CO 80303
}

Fundamental studies on clouds and radiative transfer require representative measurements of temperature: and water vapor profiles. To supplement radiosonde balloon launches, information on vertical profiles of temperature and water vapor at Cloud and Radiation Testbed (CART) sites will be provided by remote sensors.

Instruments planned for deployment include wind profilers and Radio Acoustic Sounding Systems (RASS) at 915and $50-\mathrm{MHz}$, dual-channel microwave radiometers, and cloud lidars providing cloud base height (Wesely 1992). Future CART instruments may include millimeter wavelength radars and Raman Ildars. In addition to DOE oper. ated instruments, data from nearby National Weather Service (NWS) wind profilers/RASS at 404-MHz and synoptic radiosonde launches will also be available. Finally, image and radiance data from thermal sounding channels from geostationary and polar-orbiting satellites will be available.

The fusion or integration of these data into a representative plcture of temperature, water vapor, and clouds over a CART domain will be achallenging task for four-dimensional data assimilation models (Dabberdt et al. 1992). In the work reported here, we have summarized work supported by DOE's algorithm development program, for which computer codes will be supplied to DOE/ARM.

\section{Combined RASS and TIROS(b) Operational Vertical Sounder (TOVS) Temperature Sensing}

Neither thermal soundings from polar-orbiting satellites (e.g., from TOVS) nor from RASS are completely adequate

(a) Formerty the Wave Propegation Laboratory

(b) Television and infrared observation satellite. for operational needs. TOVS soundings typically lac $k$ the vertical resolution needed for many applications, while RASS is lacking in coverage above about $500 \mathrm{mb}$. We are developing techniques to combine TOVS and RASS data to take advantage of each system's separate strengths.

Wind profilers are Doppler radars that measure winds by measuring signals backscattered from refractive index perturbations at the scale of one-half the radar wavelength. These perturbations drift with the mean wind, and measuring their translational velocity provides a direct measure of the mean wind. The RASS combines acoustic sources with wind profilers to obtain measurements of the profile of virtual temperature (May et al. 1988). The profilers measure the speed of refractive index perturbations induced by acoustic waves (approximately matched with the radar's half-wavelength) as they ascend at the local spoed of sound, which is directly related to the virtual temperature at each height.

Currently, wind profiler and RASS combinations operate at three electromagnetic frequencies: 915,404 , and $50 \mathrm{MHz}$. If corrections for vertical winds are introduced, all RASS systems measure virtual temperature with an RMS accuracy of about $0.5 \mathrm{~K}$. The height range of RASS is a function of acoustic and electromagnetic transmitter power, as well as the areas of the receiving antenna. Atmospheric limitations include acoustic attenuation, which is a strong function of frequency, and horizontal wind, which advects the acoustic pulse out of the electromagnetic pulse. For representative RASS systems, height ranges reached $50 \%$ of the time are 0.7,3.2, and 5.5 km for the 915-, 404- and 50-MHz systems (Martner et al. 1993). Temporal resolutions of 20-60 minutes are commonly avallable. To provide complete profiles, both the upper and lower altitude range gates require supplementary information.

Another source of information is from fllight-level temperature and wind data from commercial carriers, available 
from Alrcraft Communication and Recording System (ACARS) data (Benjamin et al. 1991). Depending on the carrier, the soundings could have a vertical resolution of $2000 \mathrm{ft}$ (about $700 \mathrm{~m}$ ), could originate every $7.5 \mathrm{~min}$, and could have an irregular distribution in space and time because of flight patterns and schedules. For flight-level data, temperature and winds are available as much as every $2000 \mathrm{tt}$ from 31,000 to $43,000 \mathrm{th}$.

Data from RASS and ACARS may be combined with radiance observations from NOAA polar orbiting satellites made by the TOVS and are available approximately four times each day. To illustrate the potential for combining data from these instruments, Figure 1 shows soundings without (a) and with (b) TOVS data. The physical retrieval algorithm used for the results in Figure 1 is based on statistically extrapolating RASS and ACARS data to lovels extending to $0.1 \mathrm{mb}$ and inserting this first guess profile into the International TOVS Processing Package (ITPP). These algorithms are among those that will be supplied to DOE for implementation at the Oklahoma CART site.

\section{Water Vapor Profiles from Dual-Channel Radiometers}

Until advanced moisture sensing instruments such as Raman lidar are implemented at CART sites, only twicedally radiosonde soundings are available for direst measurement of moisture profiles. We are investigating several techniques for deriving water vapor profiles between radiosunde launches, some of which depend on the direct assimilation of precipitable water vapor (PWV) into a fourdimensional data assimilation model (Dabberdt etal. 1992).

Until such models are fully operational, we have provided DOE/ARM with a statistical method of deriving profiles from dual-channel microwave radiometric measurements of PWV and surface meteorological measurements. We have found that the accuracy of the derived profiles can be improved if the information on presence or abeence of clouds is known. For example, this information can be derived from lidar ceilometer measurements or a precision radiation thermometer (PRT) infrared radiometer. An example of a dual-channel retrieval using both clear and cloudy coefficients is shown in Figure 2.

Another very important factor in determining the accuracy of profile retrievals is the accuracy and representativeness
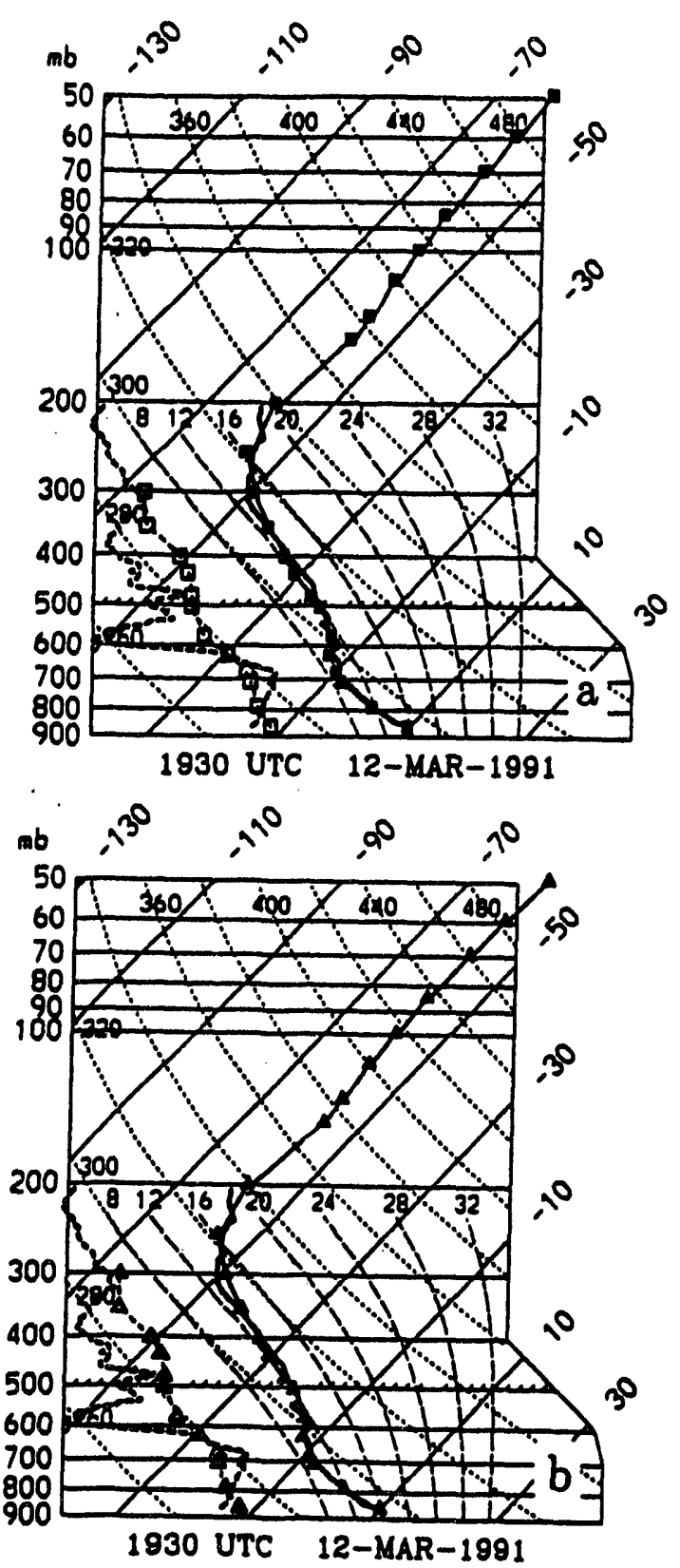

Flgure 1. Skew T-log P diagrams of temperature and humidity profiles during clear conditions obtained at Plattoville, Coloredo, on 12 March 1991, 1930 UTC for (a) regression-derived first-guess temperature (solid squares) and dewpoint temperature (open squares); and (b) ITPP. retrieved profiles using regression-derived, first-guess temperature (solid triangles) and dewpoint temperature (open triangles). 


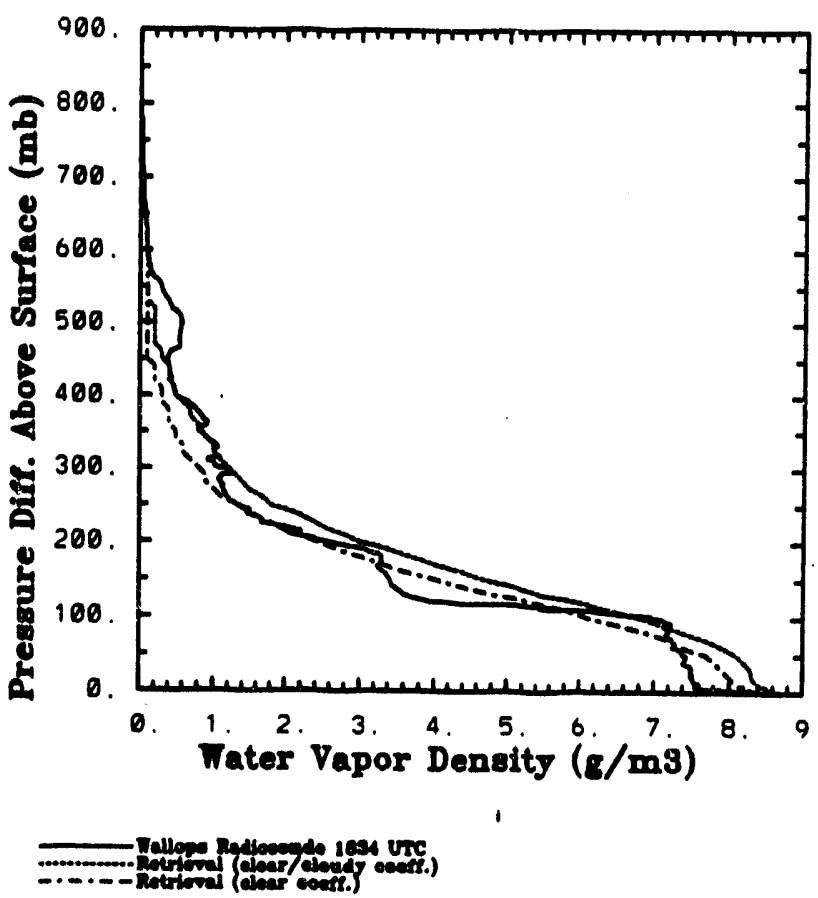

Figure 2. Dual-channel microwave radiometric retrieval of water vapor profile at Coffeyville, Kansas, 18 November 1991, 1834 UTC.

of surface meteorological measurements. Wo have found that if measurements of surface parameters depart substantially from those measured by a radiosonde, the resultant retrievals are poor. Accurate surface measurements can also be used for quality control of rediometrically derived PWV. Finally, when simultaneous and collocated measurements of cloud-base helght, RASS, and microwave radiance are available, additional improvements to retrieval accuracy can be obtained (Stankov et al. 1993).

\section{Neutral Network Radiometric Temperature Retrlevals}

Another approach that could have promise for obtaining profiles from combinations of sensors is artificial intelligence, in particular artificial neural networks. In an artificial neural network, we start with an input data vector that consists of radiometric data, RASS data, satellite data, and any other available data. The input data are used to generate a profile of temperature and/or humidity through a one- or wo-step process. In the one-stepversion, each temperature value is a nonlinear woighted sum of all input data. In the two-step version, intermediate values are calculated as nonlinear weighted sums of all input data, and each of the output values is a nonlinear woighted sum of all of these intermediate values. The nonlinear weights are obtained by training the network with an historical data set. This process is similar to a statistical inversion in its dependence on an historical data set.

We have conducted a preliminary investigation into the utility of artificlal neural networks (Churnside ot al. 1994). Calculated temperatum profiles using 6-channel microwave radiometer data from Stapleton Airport in Denver as the input data were compared with profiles obtained using a statistical inversion based on the same data (Schroeder 1990). We conclude that the overall performance of the neural network was comparable to that of the statistical inversion. This conclusion is based on an overall RMS error over the test data set of $4.3^{\circ}$ for the neural network compared with $4.0^{\circ}$ for the statistical inversion.

Qualitatively, the neural network method is better than the statistical inversion at reproducing strong inversions near the ground. This can be seen in Figure 3, where we have plotted the true profile, the statistical inversion, and the neural network retrieval for an extreme temperature inversion. The neural network comes closer to reproducing the inversion, although the retrieval is worse than the statistical inversion at high altitudes. This promising technique can also be applied to RASS and TOVS temperature retrievals, as well as to multi-sensor retrievals of water vapor.

\section{Plans}

Future work on retrieval algorithm development will focus on three main aspects: 1) incorporating cellometer and RASS measurements into real-time molsture retrievals; 2) using the twice-a-day radiosonde releases as further constraints on molsture (and temperature) retrievals; and 3) comparing neural network, a priori statistlcal and nonstatistical methods for moisture profile retrieval. 


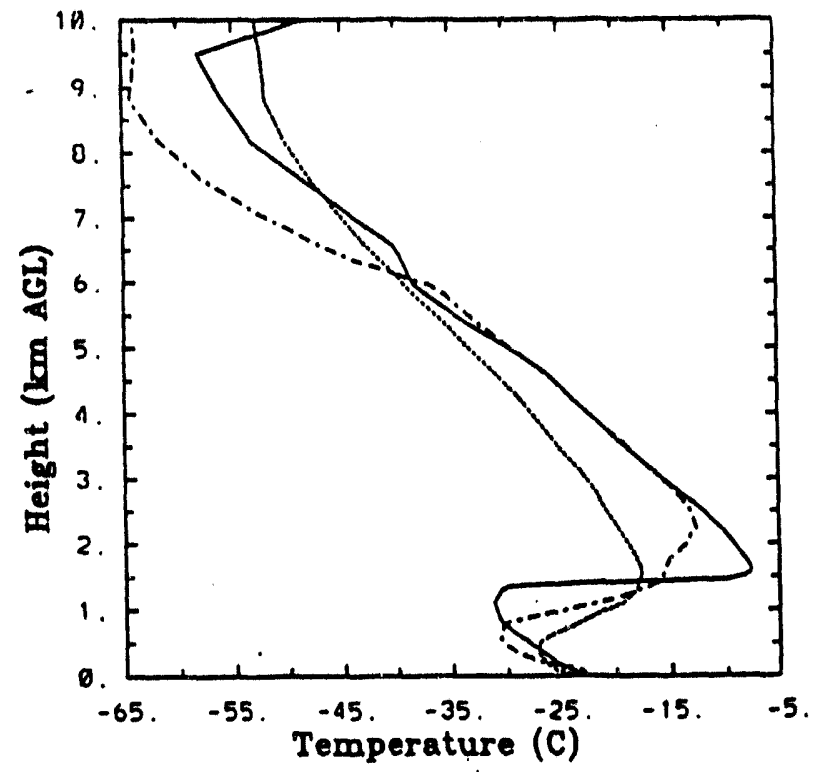

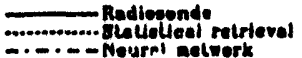

Floure 3. Temperature as a function of hoight for an extreme case from the radiosonde (solid line), the statistical retrieval (dotted line), and the neural network with 20 hidden-layer neurons (dashed line).

\section{References}

Benjamin, S. G., K. A. Brewster, R. Brömmer, B. F. Jewett, T. W. Schlatter, T. L. Smith, and P. A. Stamus. 1991. An isentroplc three-hourly data assimilation system using ACARS aircraft observations. Mon. Wea. Rev.119:888-906.

Chumside, J.H., T. A. Stermitz, and J. A. Schroeder. 1994. Temperature profiling with neural network inversion of microwave radiometer data. J. Atmos. Tech. Ocoan. 11:105-109.
Dabberdt, W. F., C. Martin, H. L. Cole, J. Dudhla, T. Horat, Y.H. Kuo, S. Oncley, J. van Baelen, K. S. Gage, W. Ecklund, D. Carter, R. Strauch, E. R. Westwater, H. Revercomb, and W. L. Smith. 1992. An integrated data assimilation and sounding system. Proceedings of the Second Atmospheric Radlation Measurement (ARM) Sclenco Team Mooting, pp. 77-80. October 26-30, Denver, Colorado. CONF. 9110336, U.S. Department of Energy, Washington D.C.

Martner, B. E., D. B. Wuertz, B. B. Stankov, R. G. Strauch, E. R. Westwater, K. S. Gage, W. L. Ecklund, C. L. Martin, and W. F. Dabberdt. 1993. An evaluation of wind profiler, RASS, and microwave radiometer pertormance. Bull. Am. Mot. Soc. 74:599-613.

May, P. T., K. P. Moran, and R. G. Strauch. 1988. The altitude coverage of temperature measurements using RASS with wind profiler radars. Geophys. Res. Left. 15:1381-1384.

Schroeder, J. A. 1990. A comparison of temperature soundings obtained from simultaneous radiometrle, radioacoustic, and rawinsonde measurements. J. Atmos. Ocean. Techn. 7:495-503.

Stankov, B. B., E. R. Westwater, D. Kim, and J. $\mathbf{S}$. Schroeder. 1993. "Toward Obtaining a Real-Time Integrated Temperature and Humidity Profile from the Ground and Space-Based Remote Sensors Using the ITPP." Proceedings 7th TIROS-N Operational Vertical Sounder(TOVS) Study Conference, 2/8-16/93, Igls, Austria

Wesely, M. L. 1992. Status of Instrumentation for the Southern Great Plains Clouds and Radiation Testbed. Proceedings of the Second Atmospheric Radiation Moasurement (ARM) Science Team Mooting. pp. 137. 140. October 26-30, Denver, Colorado. CONF-9110336, U.S. Department of Energy, Washington D.C. 


\title{
Microwave and Infrared Radiometric Observations of Water Vapor and Clouds During the Pilot Observation Experiment
}

\author{
E. R. Westwater, J. H. Churnside, and J. B. Snider \\ National Oceanic and Atmospheric Administration \\ Environmental Research Laboratories \\ Environmental Technology Laboratory(a) \\ Boulder, CO 80303
}

During Phase Two of the Pilot Radiation OBservation Experiment (PROBE) held in Kavieng, Papua Now Guinea, (January 6 - February 28, 1993) the NOAA Wave Propagation Laboratory operated a dual-frequency microwave water substance radiometer (MWSR) and a Fourier transform infrared radiometer (FTIR). A complete description of the goals of PROBE and of the sulte of instruments deployed at the Kavieng site is given by Clements et al. in a separate article in this proceedings and in Clements ot al. (1992). In this report, we summarize some of our experiences during PROBE, as well as present some preliminary data that were obtained during this experiment.

\section{Logistics}

The MWSR and FTIR were housed in a seatainer shipped from Boulder, Colorado, to Kavieng. Although minor difficulties were encountered in placing the seatainer at the PROBE site, all equipment and computers arrived intact. After a day of unpacking and set-up, the instruments were operating. Of course, we benefitted from the previous work done in setting up the PROBE site for power, telephones, and security. The only major problem that we encountered was in obtaining the liquid nitrogen $\left(\mathrm{LN}_{2}\right)$ that was necessary for calibrating the FTIR. Although we started working on the $\mathrm{LN}_{2}$ problem at least six months before th start of the experiment, numerous unanticipated and frus ung delays

(a) On October 1, 1993, the Wave Propagation Laboratory (WPL) was renamed the Environmental Technology Laboratory. Because the work reported here was done before October 1 , the text contains references to the WPL. in round trip shipping from Kavieng to Lae resulted in the arrival of $L N_{2}$ on January 22. In addition, a previous shipment of the 1500-liter container for Phase One of PROBE was vandalized en route with the end result that only a fraction of the amountreached the field sito. However, due to special security precautions arranged by the $\mathrm{LN}_{2}$ manufacturer, we were successful in obtaining two complote shipments during the Phase Two operation.

\section{Dual-Frequency Micro- wave Water Substance Radiometer (MWSR)}

The dual-frequency MWSR deployed was designed, constructed, and field-tested by WPL; a complete description of the previous system is given by Hogg ot al. (1983). In addition to electronics upgrades, this instrument differed fromearlier versions by operating the lowerchannel at $23.87 \mathrm{GHz}$ rather than $20.6 \mathrm{GHz}$. Both generations of instruments were designed to run continuously, to provide unattended operations, and to operate in almost all weather conditions.

The characteristics of the new instrument are shown in Table 1. The internal gain calibration of the radiometers is done by switching between the antenna and two temperature-controlled blackbody loads; if weather permitted, external calibration was done approximately every day using the "tip cal" method (Hogg ot al. 1983).

The observed radiance at the two frequencies is sensitive to column amounts of water vapor and cloud liquid. Wo 
Table 1. Characteristics of the WPL dual-channal MWSR used in PROBE.

$$
\text { Operating frequencles }
$$

Vlewing

Antenna half-power beam width

Bandwidth (double side band)

Integration time

Sonsitivity (for $30 \mathrm{sec}$ )

Absolute accuracy
23.87 and $31.65 \mathrm{GHz}$

Zonith

$5.0^{\circ}$

$1 \mathrm{GHz}$

30800

$0.1 \mathrm{Krms}$

$0.75 K$ derive precipltable water vapor (PWV) and integrated cloud liquid (ICL) every 30 seconds (or in some cases, overy two minutes) using a parameter retrieval tochnique known as "linear statistical inversion" (Westwater and Strand 1968). Our a priori data set was developed from Cross-chain Loran Atmospheric Sounding System (CLASS) radiosonde observations that were obtained during Phase One of PROBE. Previously, the statistical accuracies of the derived PWV were evaluated by comparison with National Weather Service (NWS) radiosonde data and with CLASS observations with resultant RMS differences of 1.7 and $1.1 \mathrm{~mm}$ RMS, respectively. The accuracy of derived ICL is estimated to be $10 \%$ to $20 \%$. As discussed in the "Plans" section below, we believe that the MWSR can be combined with FTIR, a Wind Profiler/Radio Acoustic Sounding System (RASS), a cloud lidar, and a CLASS balloon facility to provide valuable data for a tropical site.

\section{Fourler Transform Infrared Radiometer (FTIR)}

The FTIR developed by WPL (Shaw et al. 1991) is a ground-based Fourier transform interferometer that is similar to the ground-based instrument being developed for the ARM Program (Revercomb et al. 1992). The FTIR is a compact and rugged Michelson interferometer, operating between roughly 500 and $2000 \mathrm{~cm}^{-1}(5.0-20.0 \mu \mathrm{m})$ with $1-\mathrm{cm}^{-1}$ spectral resolution. Downwelling atmospheric emission in the entire bandwidth is viewed simultaneously with a single $\mathrm{LN}_{2}$-cooled $\mathrm{HgCdT} \theta$ detector. A Fast FCiviier Transform (FFT) of the measured interferogram then yields the emission power spectrum.
Our present design calls for viewing two blackbody calbration targets immediately following each complote atmospheric emission measurement. Collection of each spectrum takes about 1 second, and wo average 100 such spectra to reduce random nolse. With this technique, callbrated atmospheric spectra are collected about once every 6-10 mirutes. We now have operated the instrument with the MWSR at the First ISCCP(a) Regional Experiment (FIRE) experiments in Coffeyville, Kansas, during November-December in 1991 and during PROBE.

\section{Observations}

The MWSR operated continuously through PROBE and, except for periods of rain, the data appear to be of high quality. Because of the problems in obtaining $\mathrm{LN}_{2}$, the FTIR database is not as large, but about 20 days of highquality calibrated spectra were taken. On two occasions during which all of the PROBE instruments were operating. down-looking radiometric observations from ER-2 aircraft were also taken over the Kavieng site. Examples of WPL radiometric data taken during PROBE are shown in Figures 1 and 2.

Figure 1 is a 24-hr time series of PWW and ICL data; Figure 2a is a FTIR spectrum during the known (from lidar observations) cirrus conditions; and Figure $2 b$ is a FTIR spectrum during clear conditions.

(a) International Satollite Cloud Climatology Project. 


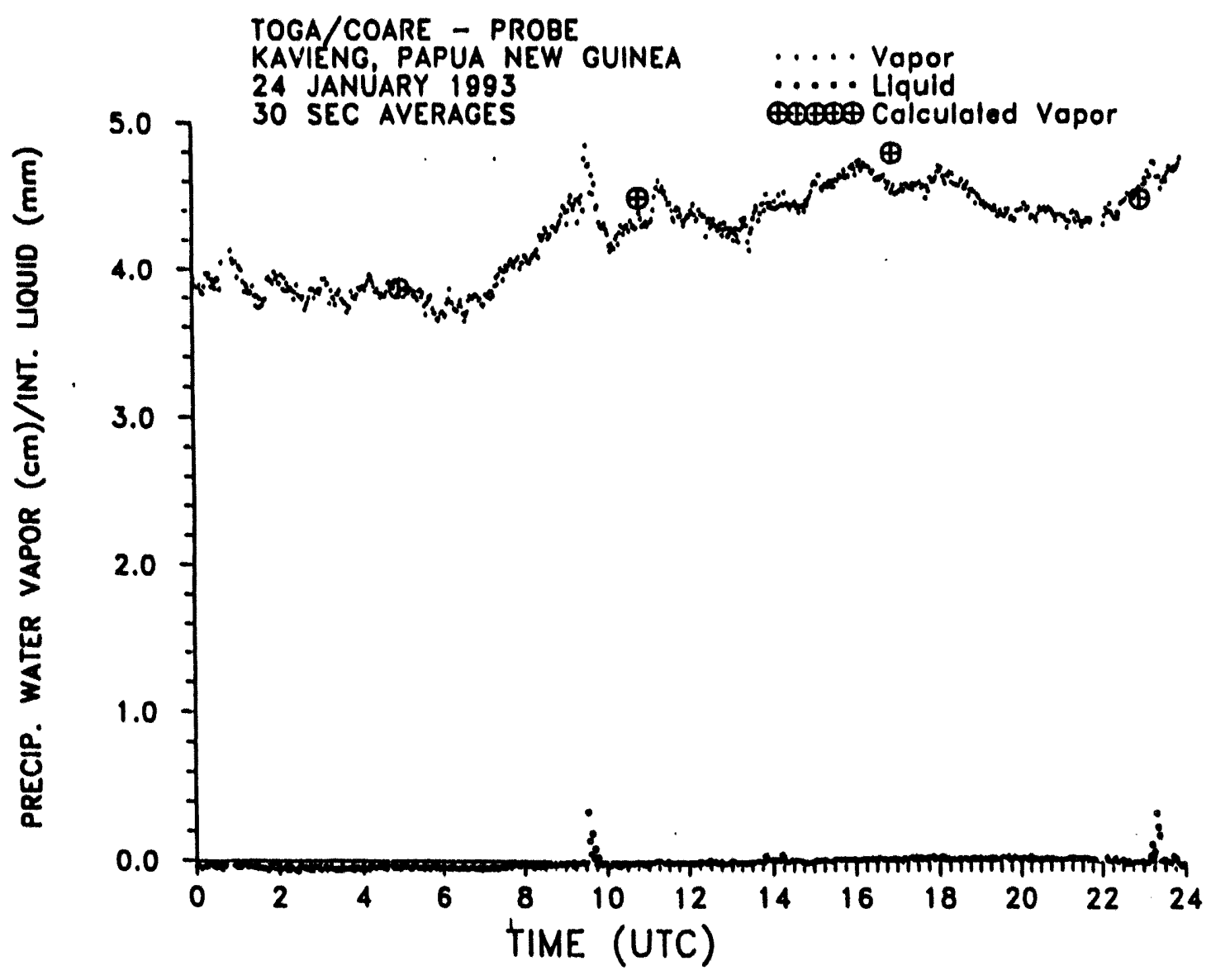

Figure 1. A 24-hr time series of precipitable water vapor and integrated cloud liquid derived from the MWSR, Kavieng. Papua Now Guinea, 1/24/93. The crossed circles are PWV values derived from CLASS radiosondes.

\section{Plans}

Data that will be of immediate interest to WPL's radiometric analysis are CLASS observations of temperature and water vapor and lidar measurements of cloud height. Wo plan to investigate the following scientific questions:

1. Can tropical cirrus clouds be obeerved from the ground in narrow-band infrared spentra?
2. To what accuracy can clear-sky radiance be calculated from temperature, water vapor, and pressure profiles? Can we calculate radiance during cloudy conditions?

3. Are FTIRs and MWSRs useful for remole sensing of temperature, water vapor, and clouds in a tropical atmosphere? as complements to CLASS and RASS? for satellite validation and calibration?

4. How do short-term fluctuations of PWV and ICL compare with continental observations? 

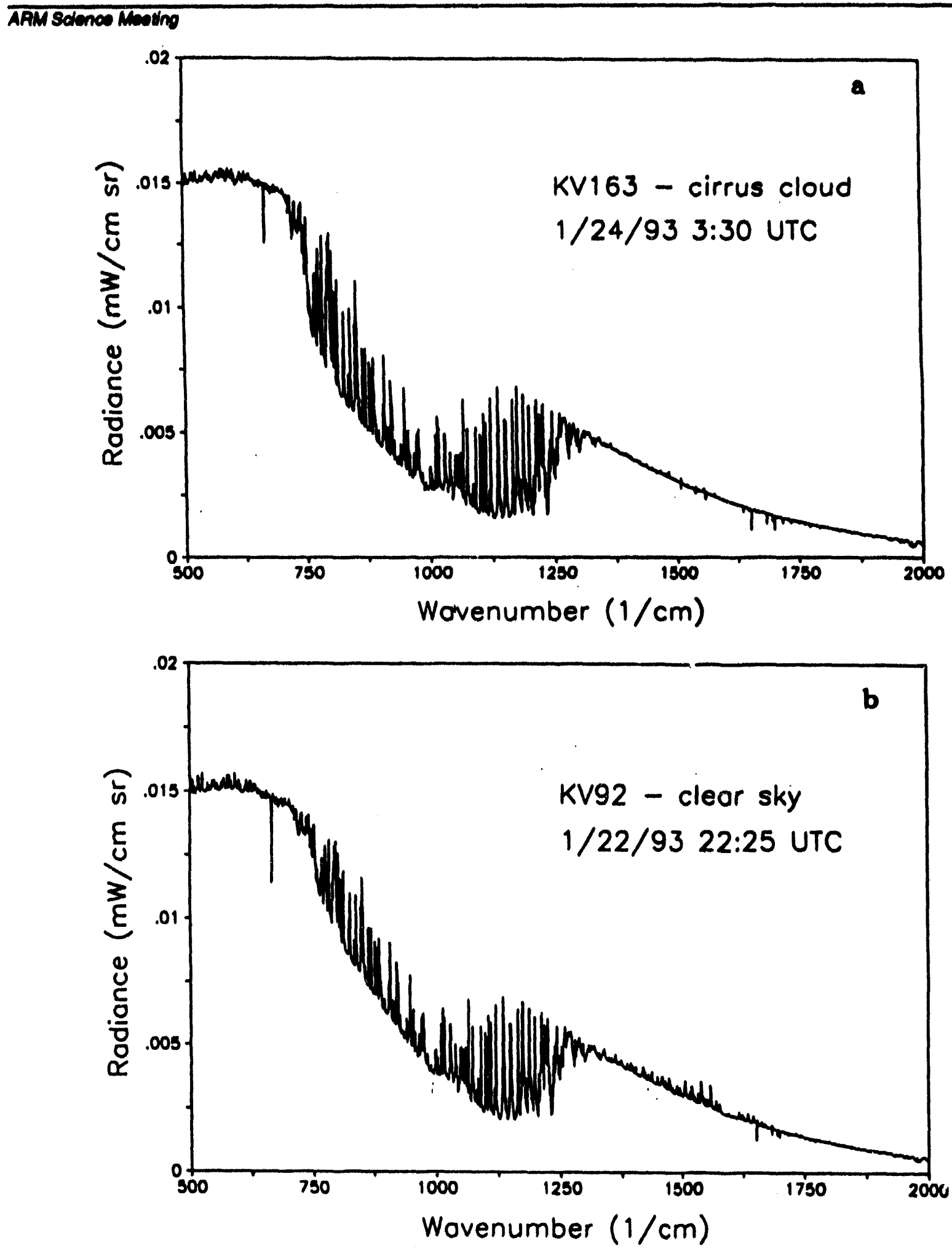

Flgure 2. (a)FTIR spectrum of infrared radiance taken during the prosence of cirrus clouds. Kavieng, Papua Now Guinea, 0300 UTC, 1/24/93, (b) FTIR spectrum of infrared radiance taken during clear conditions, 1/22/93, 2225 UTC. 

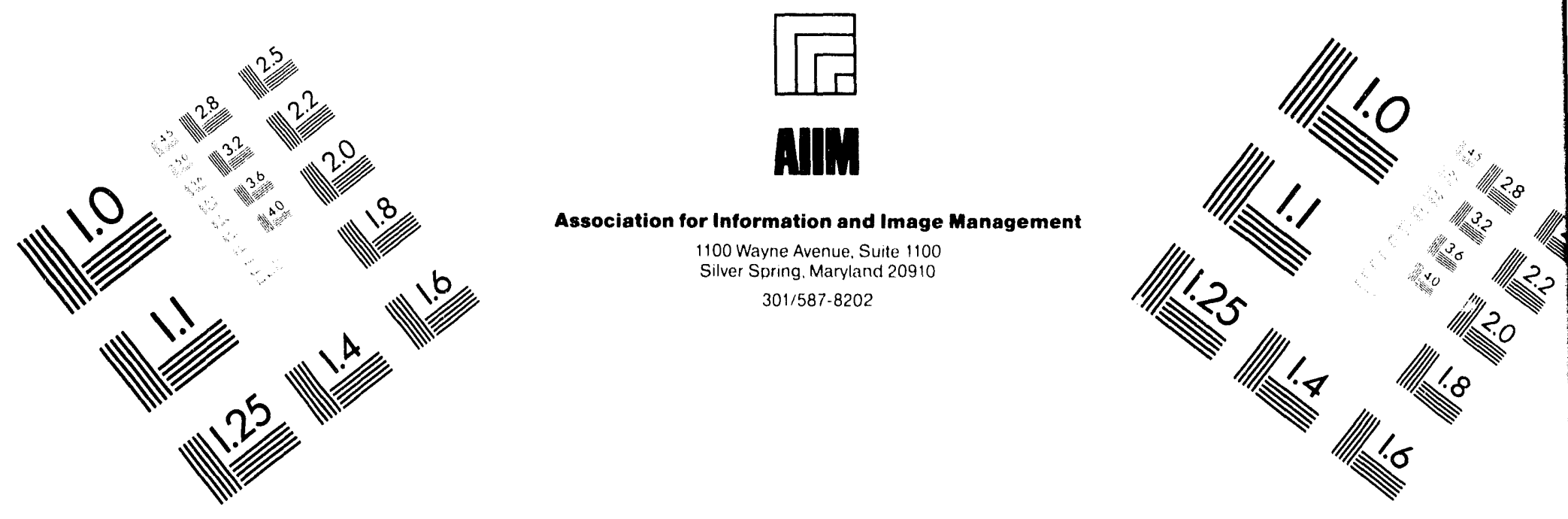

\section{Centimeter}

${ }_{1}$ Inches
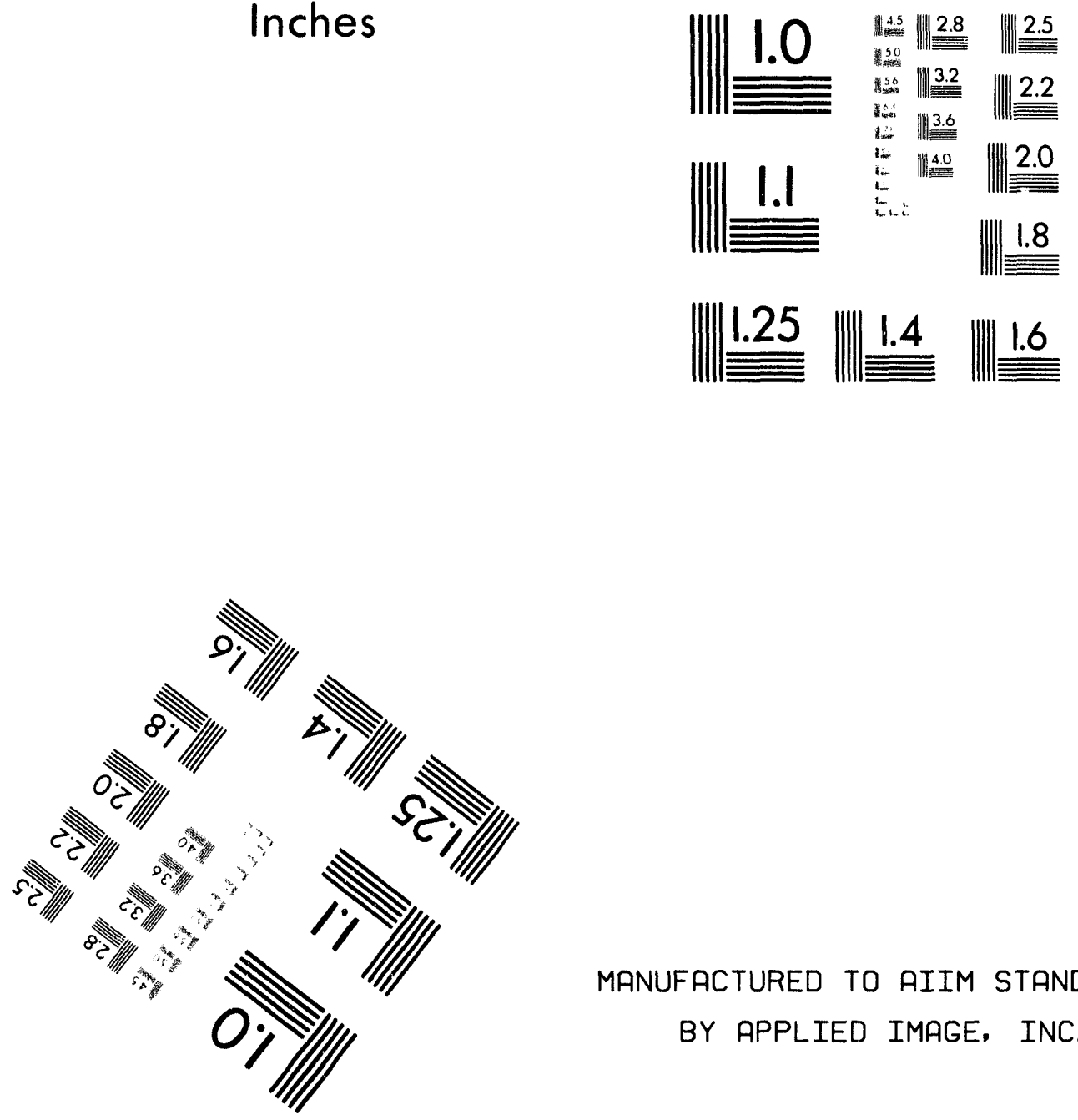

MANUFACTURED TO AIIM STANDARDS

BY APPLIED IMAGE, INC.

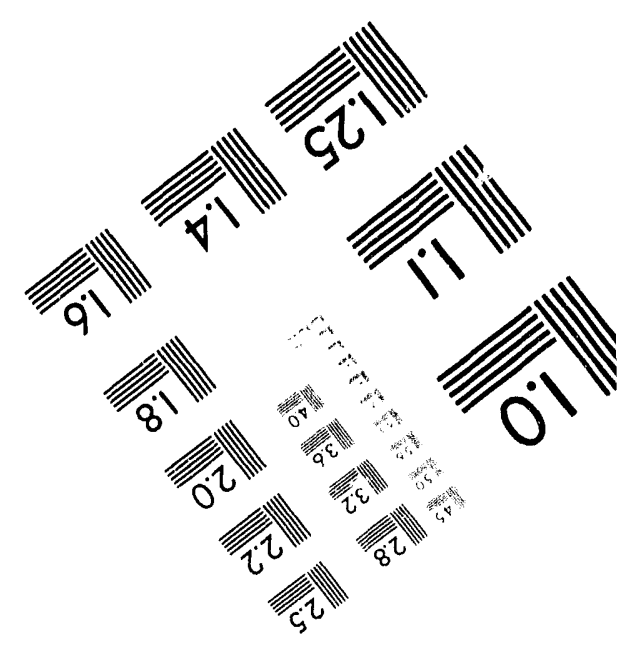



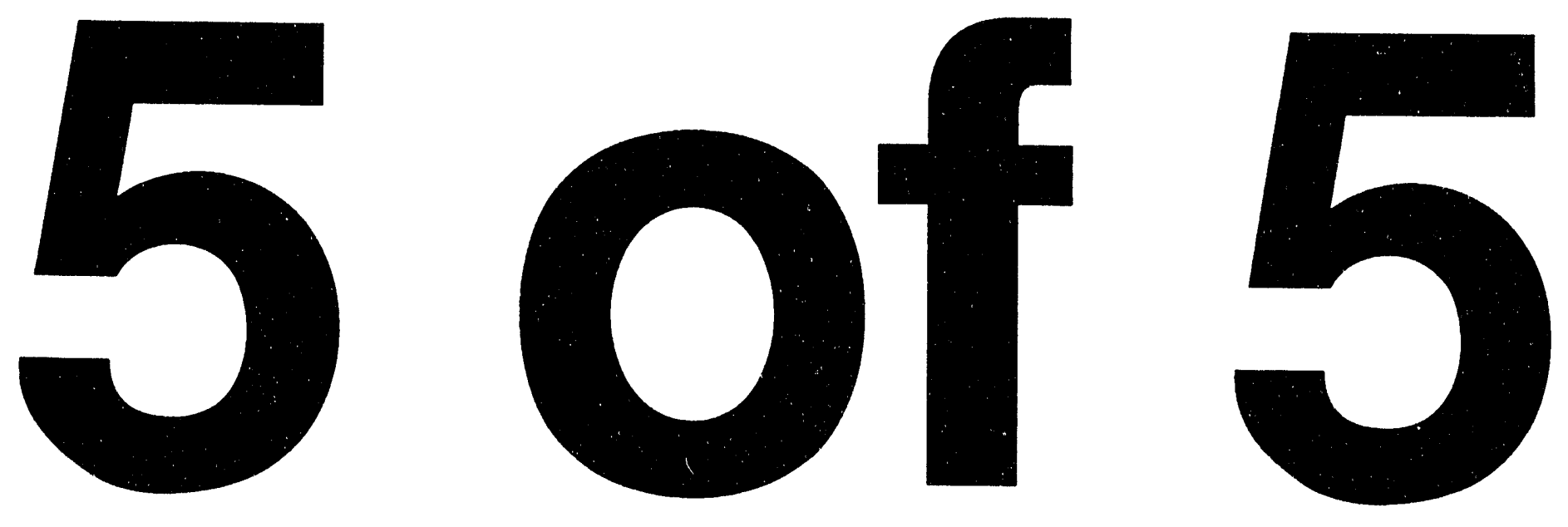


\section{References}

Clements, W., T. Ackerman, and D. Renné. 1992. Tropical Westem Pacific Project: Status. Proceedings oithe Second Atmospheric Radiation Measurement (ARM) Science Team Meoting, pp. 145-150. October 26-30, 1991, Denver, Colorado. CONF-9110336, U.S. Department of Energy, Washington, D.C.

Hogg, D. C., F. O. Guirand, J. B. Snider, M. T. Decker, and E. R. Westwater. 1983. A Steerable Dual-Channel Microwave Radiometer for Measurement of Water Vapor and Liquid in the Troposphere. J. Appl. Meteorol. 22:789806.

Revercomb, H. E., F. A. Best, R. G. Dedecker, T. P. Dirkx, R. A. Herbsleb, R. O. Knuteson, J. F. Short, and W. L. Smith. 1992. High Spectral Resolution Fourier Transform
Infrared (FTIR) Instruments for the Atmospheric Radiation Measurement Program: Focus on the Atmospheric Emitted Radiance Interierometer. Proceedings of the Second Atmospheric Radiation Measurement (ARM) Science Team Meoting, pp. 121-125. October 26-30, 1991, Denver, Colorado. CONF-9110336, U.S. Department of Energy, Washington, D.C.

Shaw, J. A., J. H. Churnside, and E. R. Westwater. 1991. An Infrared Spectrometer for Ground-Based Profiling of Atmospheric Temperature and Humidity. Proc., SPIE Int'I Symp. on Optical Appl. Sci. and Engineering, 1540, 681686. July 21-26, 1991, San Diego, California.

Westwater, E. R., and O. N. Strand. 1968. Statistical information content of radiation measurements used in indirect sensing. J. Atmos. Sci. 25:750-758. 

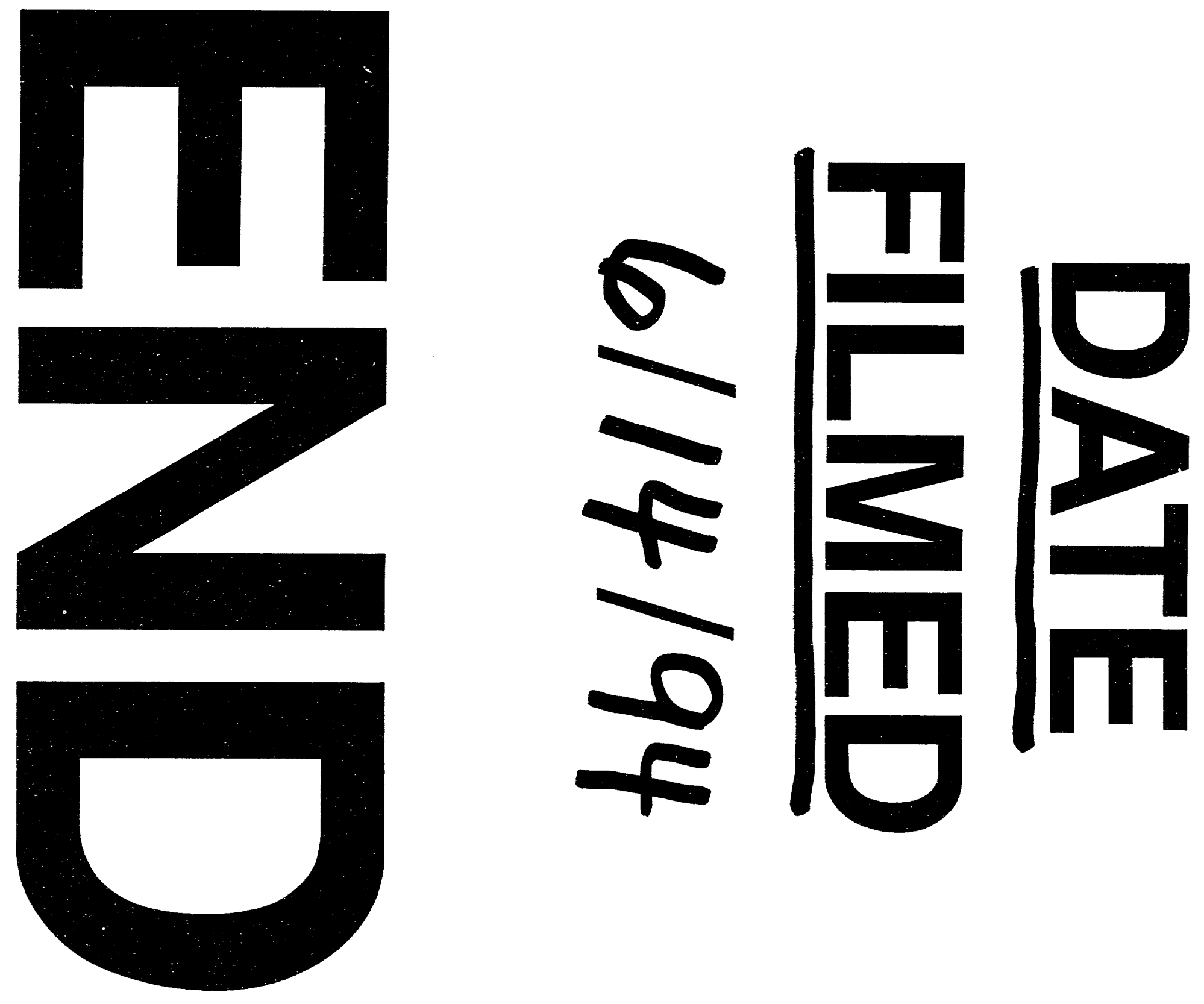
